Beiträge aus der Nordwestdeutschen Forstlichen Versuchsanstalt Band 13

Pilotstudie zu den lokalen Auswirkungen des Klimawandels auf die Forstwirtschaft in ausgewählten Regionen Sachsen-Anhalts

S. Fleck, M. Albert, P. Plašil, R. Nagel, J. Sutmöller, B. Ahrends, M. Schmidt, J. Evers, J. Hansen, M. Overbeck, W. Schmidt, H. Spellmann, H. Meesenburg
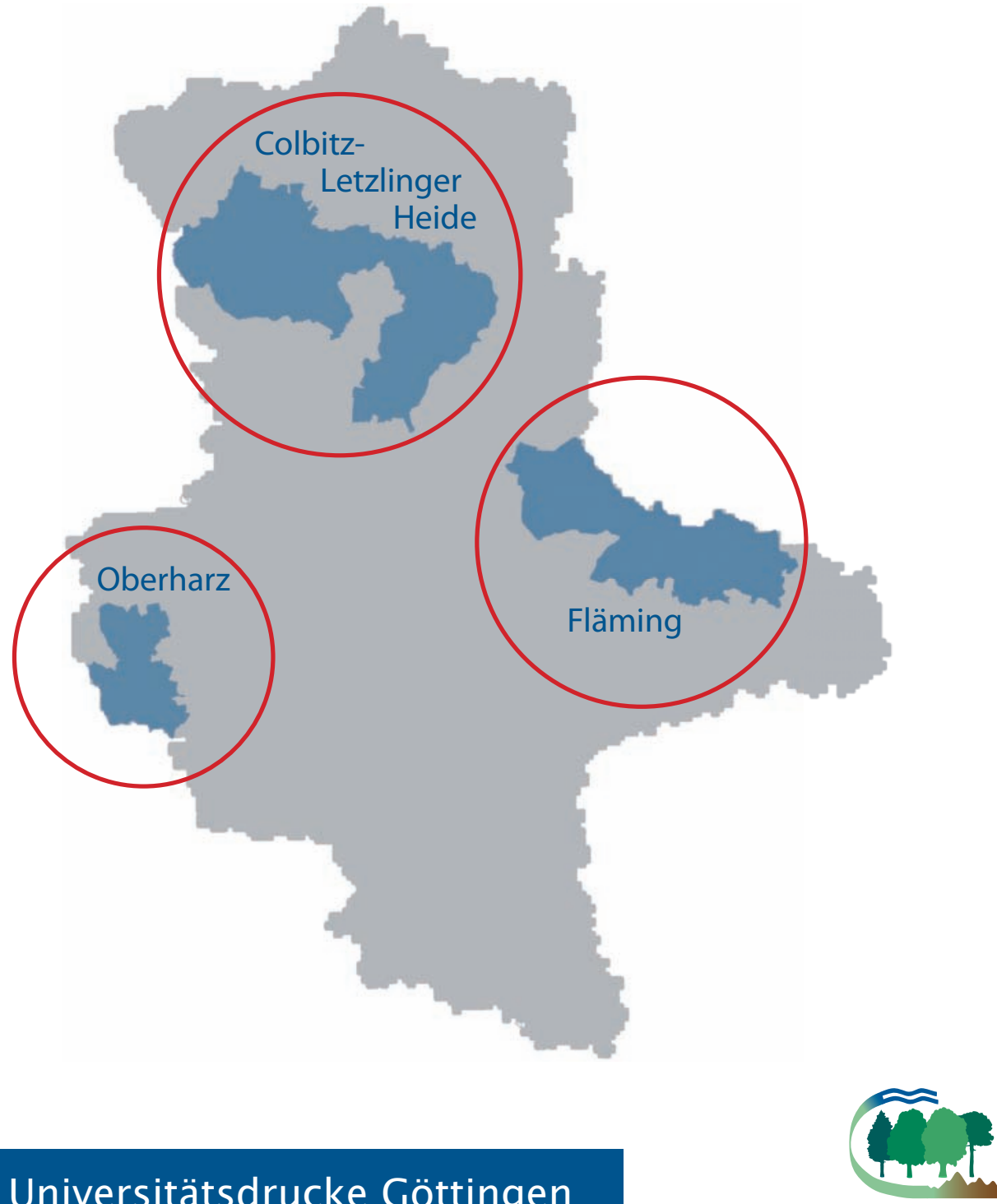

Universitätsdrucke Göttingen 

Stefan Fleck, Matthias Albert, Pavel Plašil, Ralf Nagel, Johannes Sutmöller, Bernd Ahrends, Matthias Schmidt, Jan Evers, Jan Hansen, Marc Overbeck, Wolfgang Schmidt, Hermann Spellmann, Henning Meesenburg Pilotstudie zu den lokalen Auswirkungen des Klimawandels auf die Forstwirtschaft in ausgewählten Regionen Sachsen-Anhalts

Dieses Werk ist lizenziert unter einer Creative Commons Namensnennung - Weitergabe unter gleichen Bedingungen 4.0 International Lizenz.

(c) (1) (2) 
erschienen als Band 13 der Reihe

„Beiträge aus der Nordwestdeutschen Forstlichen Versuchsanstalt“ in den Universitätsdrucken im Universitätsverlag Göttingen 2015 
Stefan Fleck, Matthias Albert, Pavel Plašil, Ralf Nagel, Johannes Sutmöller, Bernd Ahrends, Matthias Schmidt, Jan Evers, Jan Hansen, Marc Overbeck, Wolfgang Schmidt, Hermann Spellmann, Henning Meesenburg

Pilotstudie zu den lokalen Auswirkungen des Klimawandels auf die Forstwirtschaft in ausgewählten Regionen Sachsen-Anhalts

Beiträge aus der

Nordwestdeutschen

Forstlichen Versuchsanstalt

Band 13

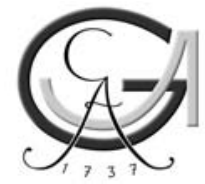

Universitätsverlag Göttingen 2015 


\section{Bibliographische Information der Deutschen Nationalbibliothek}

Die Deutsche Nationalbibliothek verzeichnet diese Publikation in der Deutschen Nationalbibliographie; detaillierte bibliographische Daten sind im Internet über $<$ http://dnb.dnb.de $>$ abrufbar.

Global Forest Decimal Classification: 421.1, 422.2, 431.5, 450, 453, 546, 547

\section{Herausgeber der Reibe:}

Nordwestdeutsche Forstliche Versuchsanstalt (NW-FVA)

Grätzelstr. 2, D-37079 Göttingen

Tel.: +49 (0)551-69401-0, Fax: +49 (0)551-69401-160

E-Mail: zentrale@nw-fva.de

www.nw-fva.de

Schriftleitung der Reihe: Prof. Dr. Hermann Spellmann

Redaktion der Reihe: Inge Kehr, Ulrike Gaertner

Die „Pilotstudie zu den lokalen Auswirkungen des Klimawandels auf die Forstwirtschaft in ausgewählten Regionen Sachsen-Anhalts“ wurde von dem Ministerium für Landwirtschaft und Umwelt des Landes Sachsen-Anhalt gefördert.

Projektlaufzeit: 1. Januar 2012 bis 31. März 2013

Titelgrafik: Etta Paar

Dieses Buch ist auch als freie Onlineversion über die Homepage der NW-FVA, des Verlags sowie über den Göttinger Universitätskatalog (GUK)

bei der Niedersächsischen Staats- und Universitätsbibliothek Göttingen

(http://www.sub.uni-goettingen.de) erreichbar.

Es gelten die Lizenzbestimmungen der Onlineversion.

(C) 2015 Universitätsverlag Göttingen

http://univerlag.uni-goettingen.de

ISBN: 978-3-86395-239-6

ISSN: 1865-6994 


\section{Inhaltsverzeichnis}

Zusammenfassung 1

Abstract 3

1 Gefährdung der Wälder in Sachsen-Anhalt $\quad 7$

2 Untersuchungsziel und Arbeitshypothesen 9

3 Modellregionen 11

4 Material und Methoden $\quad 13$

$\begin{array}{lll}4.1 & \text { Bodenwasser } & 13\end{array}$

$\begin{array}{lll}4.2 & \text { Klima } & 18\end{array}$

4.2.1 Auswahl der Klimavarianten 19

4.2.2 Methodik und Definition des Trockenstressrisikos 20

4.3 Modellierung des Standort-Leistungsbezuges 22

4.3.1 Projektion der Standortleistungsfähigkeit 31

4.3.2 Bewertung des Standort-Leistungs-Modells 35

4.4 Veränderung der Stickstoffeinträge 36

4.4.1 Datengrundlagen 36

$\begin{array}{ll}\text { 4.4.2 Depositionsszenarien } & 37\end{array}$

4.5 Grundwasserflurabstände 40

4.6 Methodik der Prognose des Sturmschadensrisikos 40

$\begin{array}{lll}\text { 4.6.1 Datengrundlage } & 40\end{array}$

4.6.1.1 Baumböhenschätzung bei Zielstärke als Eingangsgröße zur
Sturmschadensmodellierung

4.6.1.2 Durchmesser-und Baumböhenschätzung der Ist-Bestockung 51

4.6.1.3 Geländeinformationen als Eingangsgrößen zur Sturmschadens-
modellierung

4.6.1.4 Geländewasserhaushalt als Eingangsgröße zur Sturmschadens-
modellierung

4.6.2 Statistisches Sturmschadensmodell 62

4.6.3 Risikokarten $\quad 65$

4.7 Waldbrandrisiko 66

4.8 Methodik der Prognose des Buchdruckerbefallsrisikos $\quad 70$

$\begin{array}{lll}\text { 4.8.1 Datengrundlage } & 70\end{array}$ 
4.8.2 Statistisches Modell zur Prognose des Befallsrisikos durch den Buchdrucker

4.8.2.1 Modelleffekte 72

4.8.2.2 Sensitivitätsanalyse 75

81

4.9 Methodik der Beurteilung weiterer biotischer Risiken 83

5 Gefährdungspotenziale in der Region Oberharz $\quad 85$

5.1 Veränderungen des Trockenstressrisikos $\quad 85$

5.2 Veränderungen der Stickstoffeinträge 88

5.3 Projektion der Standortleistungsfähigkeit für die Region Oberharz 89

5.4 Gefährdungspotenziale durch Winterstürme 92

5.4.1 Potenzielle Vulnerabilität durch Winterstürme bei Zielstärke 93

5.4.2 Aktuelle Gefährdungspotenziale durch Winterstürme 98

$\begin{array}{lll}5.5 & \text { Veränderungen des Waldbrandrisikos } & 100\end{array}$

5.6 Gefährdungspotenziale durch Buchdruckerbefall bei Fichte im Oberharz 104

5.6.1 Gefährdungspotenziale durch Buchdruckerbefall für eine potenzielle Bestockung 104

5.6.2 Gefährdungspotenziale der aktuellen Bestockung durch Buchdruckerbefall

5.7 Zustände und Wirkungsgefüge bei biotischen Risiken 111

5.8 Konsequenzen für die künftige Waldbewirtschaftung in der Region Oberharz

5.8.1 Waldbauliche Ausgangslage 112

5.8.2 Wichtigste Wachstumstrends durch den Klimawandel und Risikoveränderungen 115

5.8.3 Stabilisierung der vorhandenen Wälder in der Region Oberharz 116 5.8.3.1 Fichtenbestände 116

5.8.3.2 Buchenbestände 120

5.8.3.3 Sonstige Bestandestypen 121

5.8.4 Standortsgemäßer Waldumbau 123

6 Gefährdungspotenziale in der Region Fläming 127

$\begin{array}{lll}6.1 & \text { Veränderungen des Trockenstressrisikos } & 127\end{array}$

6.2 Veränderungen der Stickstoffeinträge 132

6.3 Projektion der Standortleistungsfähigkeit für die Region Fläming 133

6.4 Sturmschadensrisiko 139

6.4.1 Potenzielle Vulnerabilität durch Winterstürme bei Zielstärke 139

6.4.2 Aktuelle Gefährdungspotenziale durch Winterstürme 141

6.5 Veränderungen des Waldbrandrisikos 142

6.6 Zustände und Wirkungsgefüge bei biotischen Risiken 147 
6.7 Konsequenzen für die künftige Waldbewirtschaftung in der

Region Fläming

6.7.1 Waldbauliche Ausgangslage

6.7.2 Wichtigste Wachstumstrends durch den Klimawandel und Risikoveränderungen

6.7.3 Stabilisierung der vorhandenen Wälder in der Region Fläming

\subsubsection{Kiefernbestände}

153

6.7.3.2 Eichenbestände

156

6.7.3.3 Buchenbestände

158

6.7.4 Standortsgemäßer Waldumbau

7 Gefährdungspotenziale in der Region Colbitz-Letzlinger Heide

7.1 Veränderungen des Trockenstressrisikos

7.2 Veränderungen der Stickstoffeinträge

7.3 Projektion der Standortleistungsfähigkeit für die

Region Colbitz-Letzlinger Heide

7.4 Sturmschadensrisiko

7.4.1 Potenzielle Vulnerabilität durch Winterstürme bei Zielstärke

7.4.2 Aktuelle Gefährdungspotenziale durch Winterstürme

7.5 Veränderungen des Waldbrandrisikos

7.6 Zustände und Wirkungsgefüge bei biotischen Risiken

7.7 Konsequenzen für die künftige Waldbewirtschaftung in der

Region Colbitz-Letzlinger Heide

7.7.1 Waldbauliche Ausgangslage

7.7.2 Wichtigste Wachstumstrends durch den Klimawandel und Risikoveränderungen

7.7.3 Stabilisierung der vorhandenen Wälder in der

Region Colbitz-Letzlinger Heide

7.7.3.1 Douglasienbestände

7.7.3.2 Weichlaubholzbestände

7.7.4 Standortsgemäßer Waldumbau

Literatur

Abkürzungsverzeichnis

Danksagung

Autoren

Anhang 



\title{
Pilotstudie zu den lokalen Auswirkungen des Klimawandels auf die Forstwirtschaft in ausgewählten Regionen Sachsen-Anhalts
}

\author{
Local effects of climate change on forestry in selected \\ regions of Saxony-Anhalt, Germany: a pilot study
}

\section{Zusammenfassung}

Die vorliegenden Klimaprojektionen lassen für Sachsen-Anhalt deutliche Veränderungen des Klimas erwarten, die das Wuchspotenzial und die biotischen sowie abiotischen Risiken für die Wälder verändern werden. Für die Regionen Oberharz, Fläming und Colbitz-Letzlinger Heide wurde eine Analyse der durch den Klimawandel zu erwartenden möglichen Veränderungen des Trockenstressrisikos, des Standort-Leistungsbezuges, des Sturmschadens-, des Waldbrand-, des Buchdruckerbefallsrisikos sowie weiterer biotischer Gefährdungen vorgenommen und waldbauliche Konsequenzen abgeleitet.

Für die Studie wurde das verfügbare Datenmaterial in jeweils größtmöglicher Auflösung aufbereitet. In der Regel beziehen sich die Ergebnisse daher auf die forstlichen Bewirtschaftungseinheiten. Ursache-Wirkungsbeziehungen wurden mittels physikalisch basierter Wirkmodelle (Waldbrandrisiko) oder über statistische Beziehungen (Standort-Leistungsbezug, Sturmschadensrisiko, biotische Risiken) hergeleitet. Für die Projektion der zukünftigen Entwicklung der Wuchspotenziale und Gefährdungen der Wälder in den Regionen wurde das statistische regionale Klimamodell WETTREG2010 auf der Grundlage des IPCC-SRES-Emissionsszenarios A1B und des globalen Klimamodells ECHAM5 verwendet. Neben dem 
Einfluss von Klimaveränderungen wurde auch der Effekt veränderter Stickstoffeinträge berücksichtigt. Die Ergebnisse werden jeweils für die Perioden 2011 - 2040, 2041 - 2070 und 2071 - 2100 im Vergleich zu den aktuellen Verhältnissen dargestellt.

Das Trockenstressrisiko wird in allen drei untersuchten Regionen bis 2100 deutlich ansteigen. Dabei wird ein vorwiegend hohes Risiko für die Baumarten Fichte und Buche in den Regionen Fläming und Colbitz-Letzlinger Heide erwartet, während im Oberharz ein Anstieg von einem derzeit geringen auf ein meist mittleres Trockenstressrisiko erwartet wird. Bei Kiefer, Eiche und Douglasie wird ein derzeit überwiegend geringes Risiko zu einem meist mittleren, teilweise auch hohen Risiko ansteigen.

Die Standortleistungsfähigkeit wird sich je nach Region und Baumart unterschiedlich entwickeln. Die Projektionen ergeben für das Wuchspotenzial der Kiefer in den Regionen Fläming und Colbitz-Letzlinger Heide einen Anstieg in der Periode 2011 - 2040, auf den ein Rückgang unter das derzeitige Niveau in der Periode 2071 - 2100 folgt. Fichte und Buche lassen in der Region Oberharz einen Anstieg der Standortleistungsfähigkeit erwarten, der in der Periode 2071 - 2100 stagniert bzw. auf einigen Standorten leicht zurückgeht. Für Eiche ist mit einem leichten Rückgang des Wuchspotenzials in den Regionen Fläming und Colbitz-Letzlinger Heide zu rechnen, während das der Douglasie im Oberharz eher zunimmt und in den Tieflandregionen eher abnimmt.

Das Risiko für Sturmschäden durch Winterstürme dürfte für alle Baumarten zunehmen. Es ist jedoch zu berücksichtigen, dass Aussagen über die zukünftige Entwicklung von Sturmstärken und -häufigkeiten derzeit sehr unsicher sind. Hauptrisikogebiet wird weiterhin der Oberharz sein, wo vor allem die Fichte auf einem größeren Anteil der Standorte ein hohes Risiko aufweist.

Das Waldbrandrisiko wird in allen untersuchten Regionen bis zum Ende des Jahrhunderts stark ansteigen. Insbesondere in den Regionen Fläming und ColbitzLetzlinger Heide wird die Anzahl an Tagen mit erhöhtem Waldbrandrisiko stark ansteigen und gegen Ende des Jahrhunderts mehr als doppelt so hoch liegen wie derzeit. Besonders betroffen sind gering bestockte lichte Kiefernbestände, die in diesen Regionen am stärksten vertreten sind. Im Oberharz wird das Waldbrandrisiko ebenfalls ansteigen, aber deutlich geringer als in den Tieflandregionen bleiben.

Das Buchdruckerbefallsrisiko wird im Hauptverbreitungsgebiet der Fichte im Oberharz aufgrund des erhöhten Wärmeangebotes und schlechterer Wasserversorgung stark zunehmen. Dabei steigt das Risiko mit dem Alter der Bestände und dem jeweiligen Mischungsanteil der Fichte. Für die Tieflandregionen wurde aufgrund geringer Fichtenanteile an der Bestockung keine Bewertung vorgenommen.

Für andere Schaderreger können derzeit keine quantitativen Abschätzungen über zukünftige Risiken in den Modellregionen getroffen werden. Jedoch lässt sich aus den in der Vergangenheit aufgetretenen Schäden und den gefundenen Faktoren ableiten, dass die in den Untersuchungsregionen vorkommenden Schaderreger in 
der Häufigkeit ihres Auftretens und im Schadausmaß grundsätzlich zunehmen werden. Für die Kiefernbestände des Fläming und der Colbitz-Letzlinger Heide ist aufgrund des durch den Klimawandel bedingten Anstiegs des Trockenstressrisikos mit einem vermehrten Auftreten von Nonne, Kiefernspinner und Forleule zu rechnen. In den Eichenbeständen der Regionen Fläming und Colbitz-Letzlinger Heide ist ein Anstieg der Populationen der Eichenfraßgesellschaft zu erwarten. Bei vermehrtem Schädlingsbefall ist generell auch mit einer Zunahme von Sekundärschädlingen zu rechnen, von denen insbesondere pilzliche Erreger von einer Temperaturerhöhung profitieren dürften.

Als Konsequenz für die zukünftige Bewirtschaftung der Wälder in den Modellregionen wird mit erster Priorität eine Stabilisierung der vorhandenen Wälder empfohlen. Diese beinhaltet die Erhöhung der Einzelbaumstabilität, die Sicherung der Vitalität und eine Verbesserung der Bestandesstrukturen einschließlich der Verringerung der Wasserkonkurrenz in trockenstressgefährdeten Beständen. In vielen Fällen bietet sich eine gestaffelte Durchforstung zur Erreichung der waldbaulichen Ziele an. Langfristig trägt ein standortsgemäßer Waldumbau zu einer Anpassung an die Risiken des zu erwartenden Klimawandels bei. Dabei muss sich die Auswahl der Baumarten an den heutigen und zukünftigen Standort- und Klimaverhältnissen orientieren. Die Erhöhung des Anteils von Mischbaumarten und die Einbringung von anbauwürdigen eingeführten Baumarten sind dabei eingeschlossen.

Stichworte: Oberharz, Fläming, Colbitz-Letzlinger Heide, biotische Risiken, Trockenstressrisiko, Sturmschadensrisiko, Standort-Leistungsbezug, Waldbrandrisiko

\section{Abstract}

The climate projections available for Saxony-Anhalt indicate that distinct changes in climate can be expected, which will alter the growth potential and both the biotic and abiotic forest risks. For the three regions of the Upper Harz Mountains, Fläming, and Colbitz-Letzling Heath, an analysis was undertaken of the expected potential climate change-induced shifts in the site-productivity relationship, the risk of drought stress, storm damage, forest fire and European bark beetle (Ips typographus L.) infestation as well as other biological threats. From this analysis, the silvicultural consequences were derived.

For the study, the available data were processed at the greatest resolution possible. Therefore, as a rule, the results relate to forest management units. Causeeffect relationships were derived using mechanistic physical models (forest fire risk) or statistical relationships (site-productivity relationship, risk of storm damage, biotic risks). For the projection of the future development of the growth potential, and threats to forests in the region, the statistical regional climate model WETTREG2010, based on the IPCC-SRES emissions scenario A1B and the global 
climate model ECHAM5, was used. In addition to the influence of climate changes, the effect of changes in nitrogen inputs was also considered. The results are presented for the periods 2011 - 2040, 2041 - 2070 and 2071 - 2100 and compared to the actual conditions.

In all three regions studied, the risk of drought stress will clearly increase up to 2100. Whereas primarily a high risk for the tree species Norway spruce and European beech in Fläming, and Colbitz-Letzling Heath is expected, in the Upper Harz Mountains an increase in the risk of drought stress from a currently low to a mostly moderate level is expected. The currently predominantly low risk of drought stress for Scots pine, European oak (Quercus petraea LIEBL., Quercus robur L.) and Douglas fir will increase to a mostly moderate, but sometimes even high risk.

The site productivity will develop differently depending on region and tree species. The projections show an increase in the growth potential of Scots pine in Fläming, and in Colbitz-Letzling Heath in the period 2011 - 2040, followed by a decline below the current level in the period 2071 - 2100. The site productivity of Norway spruce and European beech in the Upper Harz Mountains is expected to increase, and then, in the period $2071-2100$, remain constant or even decline slightly on some sites. The growth potential of European oak is likely to decrease slightly in the Fläming, and in the Colbitz-Letzling Health, while that of Douglas fir will tend to increase in the Upper Harz Mountains and decrease in the lowland regions.

The risk of storm damage from winter storms should increase markedly for all tree species. However, it must be recognised that predictions of the future development of the strength and frequency of storms are currently very uncertain. The main risk area will continue to be the Upper Harz Mountains, where primarily Norway spruce will be subjected to a high risk on a greater proportion of the sites.

The risk of forest fire will increase markedly in all the regions investigated through to the end of the century. In the Fläming and Colbitz-Letzling Heath regions, in particular, the number of days with an enhanced fire risk will increase markedly and, towards the end of the century, will be more than double the current number. Low density, and hence more open Scots pine stands, the most common stands in these regions, will be particularly affected. In the Upper Harz Mountains, the risk of forest fire will also increase, but much less so than in the lowland regions.

The risk of European spruce bark beetle infestation in the main distribution range of Norway spruce in the Upper Harz Mountains will increase markedly due to increased temperatures and poorer water availability. Furthermore, the risk will increase with stand age and percentage of Norway spruce in the species mix. In view of the low percentage of Norway spruce in the lowland forests, these regions were not included in the analysis.

Currently, it is not possible to make quantitative estimates of future risks from other pests in the regions studied. Nevertheless, based on damage that has occurred 
in the past and from the factors found, we deduce that the frequency of occurrence of pests and the extent of damage they cause will essentially increase in the regions studied. For the Scots pine stands in Fläming, and the Colbitz-Letzling Heath, a greater occurrence of the nun moth (Lymantria monacha L.), pine-tree lapped moth (Dendrolimus pini L.) and the pine beauty (Panolisflammea DENIS \& SCHIFFERMÜLLER) is expected as a result of the increase in drought stress risk arising from climate change. In the European oak stands in Fläming, and the Colbitz-Letzling Heath, a rise in the populations of oak-feeding defoliators is anticipated. In the event of an increase in pest infestation one can generally also expect an increase in secondary pests of which fungal pathogens, in particular, will benefit from higher temperatures.

Consequently, for the future management of the forests in the regions studied we recommend that the existing forests be stabilised as a first priority. This will involve enhancing the stability of individual trees, ensuring vitality and improving stand structure, which will include a reduction in competition for water in stands threatened by drought stress. In many cases, the silvicultural aims can be achieved by a graded thinning. In the long term, site-specific forest conversion-will assist the adaptation of forests to the risks arising from the anticipated climate change. Here, the selection of tree species must be based on the current and future site and climate conditions. The increase in the proportion of admixed tree species and the inclusion of suitable introduced tree species for planting will be integral elements.

Keywords: Upper Harz Mountains, Fläming, Colbitz-Letzling Heath, biotic risks, drought stress risk, storm damage risk, site-productivity relationship, forest fire risk 



\section{Gefährdung der Wälder in Sachsen-Anhalt}

Sämtliche Klimaprojektionen lassen für Deutschland einen deutlichen Temperaturanstieg bei gleichzeitig veränderten jährlichen Niederschlagsverteilungen erwarten. Dabei ist das projizierte Ausmaß des Klimawandels regional sehr unterschiedlich und steigt allgemein mit zunehmender Kontinentalität. Für das Land SachsenAnhalt liegt bereits eine Vulnerabilitätsstudie aus dem Jahre 2009 vor, die für ausgewählte Sektoren erste Erkenntnisse zu den Folgen des Klimawandels aufbereitet hat und Handlungsoptionen zur Anpassung aufzeigt (KROPP et al. 2009). Eine zweite Studie entstand auf der Grundlage aktueller Daten im Jahr 2012 (SUTMÖLLER et al. 2013). Diese Studien verfolgen methodisch einen großräumigen Ansatz, um regionale Gefährdungsschwerpunkte und Anpassungsnotwendigkeiten herauszuarbeiten und politische Weichenstellungen vorzubereiten. Nach den Ergebnissen der Studien ist die langfristig operierende Forstwirtschaft in einem besonderen Maße vom Klimawandel betroffen, weil sich die Produktionsgrundlagen, Risiken und Ertragsaussichten wesentlich verändern.

Forstbetriebliches Handeln vollzieht sich auf der Ebene des einzelnen Waldbestandes. Dort stellt sich im Zeichen des Klimawandels konkret die Frage, wie ein vorhandener Bestand stabilisiert werden kann, wie sich etwaige Risiken verteilen bzw. begrenzen lassen sowie ob und mit welchen Baumarten ein Waldumbau notwendig ist. Für dieses operationale Handeln fehlen den Forstbetrieben bisher wissenschaftlich abgesicherte Arbeitsgrundlagen, die hoch aufgelöst Klima-, Standorts-, Bestandes- und Risikoinformationen miteinander verknüpfen und in Entscheidungshilfen verdichten. An dieser Stelle setzt das Projekt an, um in ausgewählten Regionen Sachsen-Anhalts Datenverfügbarkeit und Prognosemöglichkeiten auszuloten. 



\section{Untersuchungsziel und Arbeitshypothesen}

Die Forstbetriebe in Sachsen-Anhalt müssen sich auf eine Zunahme der abiotischen und biotischen Gefahren sowie veränderte Standort-Leistungs-Beziehungen einstellen (PLAŠIL u. HABERMANN 2012, SUTMÖLLER et al. 2013). In diesem durch ein hohes $\mathrm{Maß}$ an Unsicherheiten geprägten Umfeld ist es das Ziel der Untersuchung, hoch aufgelöst Zustände und Wirkungsgefüge zu analysieren, szenariobasiert Entwicklungen abzuschätzen und Anpassungsstrategien abzuleiten, die lokale Entscheidungen ermöglichen. Es wird davon ausgegangen, dass regional aufbereitetes Erfahrungswissen zu Waldschutzfragen und Modellschätzungen, die auf hoch aufgelösten Eingangsdaten der unabhängigen Variablen beruhen, wertvolle Hilfestellungen für waldbauliche Entscheidungen der Forstbetriebe bieten.

Beim Erfahrungswissen zum Waldschutz wird von folgenden Grundannahmen ausgegangen:

1. Klima und Witterung beeinflussen grundlegend die Populationsdynamik von Insekten und anderen Schaderregern. Sie bestimmen die geografische Verbreitung der Arten sowie deren Populationsdichte und das Gradationsgeschehen.

2. Das Klima hat einen wesentlichen Einfluss auf die Disposition der Waldbestände. Unter Trockenstress lassen die Abwehrkräfte der Bäume nach.

3. Für die wechselwarmen Insekten ist die Temperatur von besonderer Bedeutung. Obere und untere letale Grenzwerte der Temperatur entscheiden darüber, ob ein Insekt überlebt und ein enger Temperaturbereich bestimmt das Wachstum, die Entwicklung und die Reproduktion.

4. Die Feuchtigkeit modifiziert die Wirkungen der Temperatur.

5. Die Gradationen der Insekten gehen in der Regel von bestimmten Standorten aus und sind an besonders sensitive Entwicklungsstadien der Bestände gebunden.

Im Rahmen der Modellbildung werden folgende Annahmen getroffen:

1. Die Standortskonstanz ist auch für mittelfristige Zeiträume nicht mehr gewährleistet, weil sich bereits im Laufe einer Umtriebszeit die klimatischen Bedingungen und Stoffeinträge zu stark verändern.

2. Die Auswirkungen des Klimawandels auf das Waldwachstum und auf biotische und abiotische Risiken müssen getrennt betrachtet und analysiert werden.

3. Prognosen über die zukünftige Waldentwicklung erfolgen im Analogieschluss. Dabei sollen die Zustände an einem Standort unter zukünftigen 
Bedingungen über die Zustände an Standorten beschrieben werden, die bereits heute diese oder ähnliche Bedingungen aufweisen. 


\section{Modellregionen}

Das Land Sachsen-Anhalt ist in drei natürliche Großlandschaften gegliedert, die sich sowohl geomorphologisch als auch klimatisch deutlich voneinander unterscheiden. Diese Großlandschaften, die ihrerseits sehr unterschiedliche Landschaftseinheiten erkennen lassen, sind das Norddeutsche Tiefland, das Hügelland und das Mittelgebirge. Die größte Waldfläche liegt im Tiefland, der höchste Bewaldungsanteil ist im Harz zu verzeichnen. Das Hügelland muss als waldarmer Naturraum bezeichnet werden.

Als Modellregionen wurden der Oberharz, der Fläming und die Colbitz-Letzlinger Heide ausgewählt, weil sie wichtige Standorte und Bestandestypen repräsentieren und schon unter heutigen Bedingungen aufgrund ihrer Waldschutzrisiken eine besondere Aufmerksamkeit genießen (s. Abb. 1).

Der Oberharz liegt im Südwesten des Landes und umfasst die plateauartigen Lagen zwischen 500 und $600 \mathrm{~m}$ über NN, die das zentrale Massiv des Hochharzes umschließen, und die östlich angrenzenden, allmählich abfallenden Abdachungen des Unterharzplateaus. Die Region wird überwiegend durch Jahresmitteltemperaturen von $5-6^{\circ} \mathrm{C}$ und hohe Jahresniederschläge von $800-1000 \mathrm{~mm}$ geprägt. Die Unterharzlagen zeichnen sich durch mildere, trockenere Klimabedingungen aus. Die Plateaulagen bestehen vornehmlich aus Tonschiefern, Quarziten und Grauwacken aus dem Tertiär sowie inselartig vorkommenden devonischen Kalksteinen. Im Einzugsgebiet der Bode befinden sich mehrere Trinkwassertalsperren. Der Waldanteil beläuft sich in den montanen Plateaulagen auf $93 \%$. Es dominieren Fichtenwälder auf Standorten mit mittlerer Nährstoffversorgung. Der Waldanteil auf den nährstoffkräftigeren Unterharzplateaustandorten ist aufgrund der großen Rodungen um die Ortslagen mit $69 \%$ für die Region Harz vergleichsweise niedrig.

Der Fläming liegt im Osten Sachsen-Anhalts. Das Regionalklima unterscheidet sich von seinem Umland durch höhere Jahresniederschläge von 560-620 mm. Es trägt noch eher subatlantische Züge. Die Landschaft wurde wesentlich durch die Saaleeiszeit geprägt und ist durch eine vollständige glaziale Serie gekennzeichnet. Im Osten bis nach Brandenburg hinein erhebt sich die Stauchendmoräne des Warthestadiums. Im Südwesten schließen sich die Landschaftseinheiten des Burger Vorflämings und des Roßlau-Wittenberger Vorflämings an. Als Böden überwiegen mittlere bis ärmere Sande und lehmige Sande als Braunerden und Braunpodsole. Der Waldanteil liegt bei über $50 \%$, die dominierende Baumart ist die Kiefer. Weiterhin ist der Fläming der wichtigste Grundwasserneubildungsraum für das östliche Sachsen-Anhalt.

Die Colbitz-Letzlinger Heide liegt nördlich von Magdeburg im Süden der altmärkischen Moränenlandschaft. Sie befindet sich im Übergangsbereich zwischen 
der subkontinentalen Elbniederung und dem subatlantischen Klima der niedersächsischen Heiden. Die Jahresniederschläge erreichen 550 bis $600 \mathrm{~mm}$, die Jahresmitteltemperaturen liegen bei $8,5^{\circ} \mathrm{C}$. Die Landschaft ist von warthestadialen Ablagerungen geprägt. Neben stark übersandeten Grundmoränenflächen überwiegen die trockeneren Sanderflächen. Es herrschen Sand-Podsole vor, auf denen überwiegend Kiefern-Reinbestände stocken. Unter der Colbitz-Letzlinger Heide befindet sich ein ausgedehnter Grundwasseraquifer, der stark für die Trinkwassergewinnung beansprucht wird. Das Wasserwerk Colbitz versorgt allein 700.000 Menschen mit Trinkwasser.

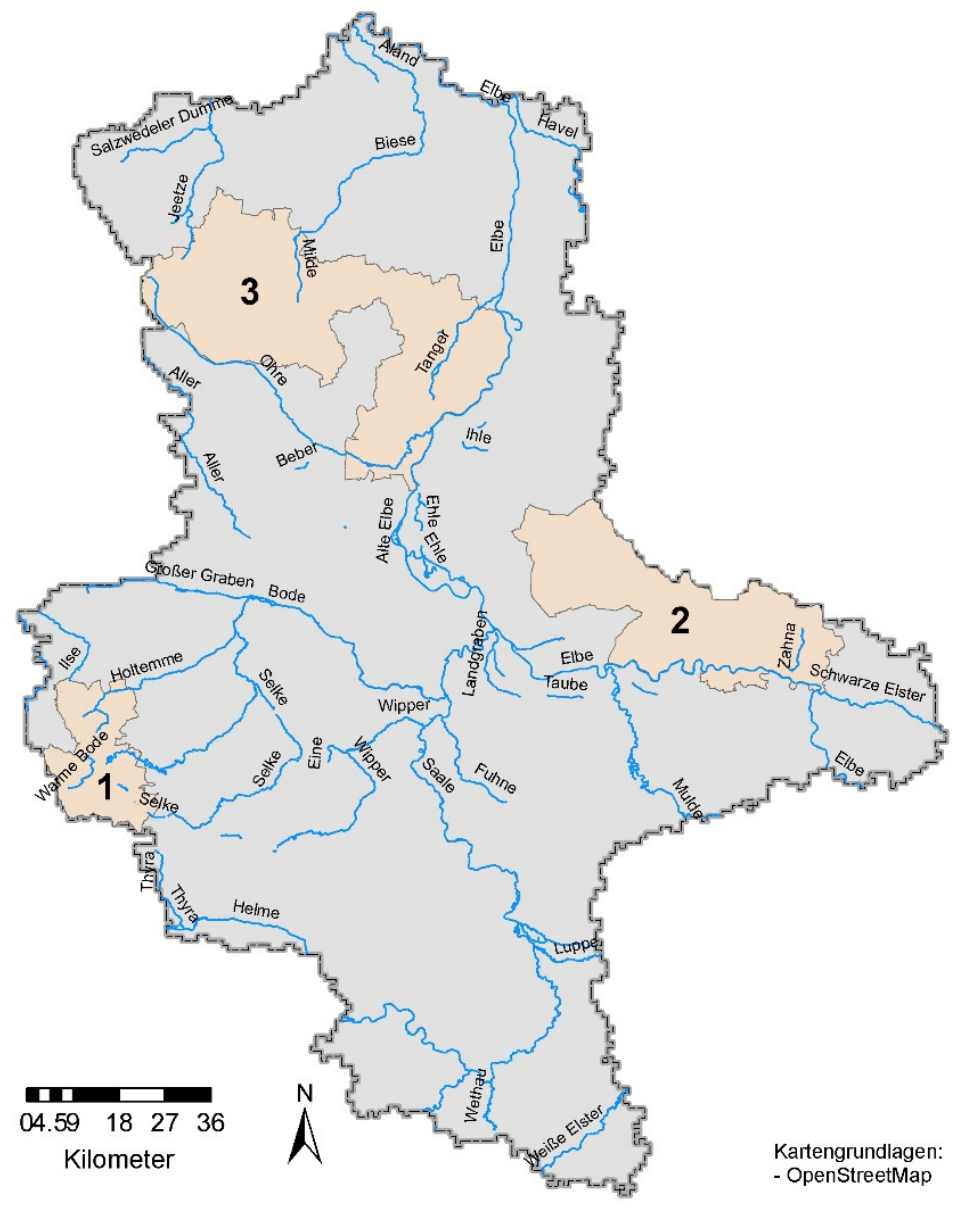

Abbildung 1: Lage der Modellregionen Oberharz (1), Fläming (2) und Colbitz-Letzlinger Heide (3); (Kartengrundlagen: (C) OpenStreetMap-Mitwirkende, ODbL (www.openstreetmap.org/ copyright)) 


\section{Material und Methoden}

\subsection{Bodenwasser}

Die nutzbare Feldkapazität (nFK) ist ein $\mathrm{Maß}$ für das pflanzennutzbare Bodenwasser und eine wichtige Größe zur Einschätzung des Wasserhaushaltes von Wäldern. Im Zusammenhang mit dem Klimawandel und der Beurteilung der Baumarteneignung am jeweiligen Standort ist die $\mathrm{nFK}$ von entscheidender Bedeutung, da diese Wassergehalte in der Regel zu Beginn der Vegetationsperiode im Boden gespeichert und den Pflanzen damit in der Vegetationsperiode zur Verfügung stehen. Die nFK ist die Menge des Bodenwassers, die in Poren mit einem Äquivalentdurchmesser zwischen $50 \mu \mathrm{m}$ und $0,2 \mu \mathrm{m}$ oder bei einer definierten Saugspannung $(\mathrm{pF})$ zwischen 1,8 und 4,2 gebunden ist. Sie berechnet sich aus der Differenz zwischen Feldkapazität und permanentem Welkepunkt (AG BODEN 2005). Die nFK kann anhand von Kennwerttabellen aus der Bodenart, der Trockenrohdichte des Feinbodens, dem Grobbodenanteil und dem Humusgehalt geschätzt werden (AG BODEN 2005).

Flächendeckende Werte für die nFK liegen mit der Vorläufigen Bodenkarte 1:50.000 von Sachsen-Anhalt vor (VBK 50, LANDESAMT FÜR GEOLOGIE UND BERGWESEN 2009). Hier wurden Informationen aus der forstlichen Standortskartierung und anderen Quellen digital aufbereitet und die nFK für übergeordnete Polygone relativ einheitlicher Bodenformen abgeschätzt. Eine unabhängige Validierung der nFK-Werte aus der VBK 50 kann über die Ergebnisse der 76 BZE IIProfile (Bodenzustandserhebung) vorgenommen werden. An den BZE II-Profilen liegen die für die nFK-Schätzung nach der AG BODEN (2005) erforderlichen Parameter mit Ausnahme der Bodenart analytisch bestimmt vor. Das Ergebnis der Gegenüberstellung der VBK 50 und der BZE II ist in Abbildung 2 dargestellt.

Die Beziehung zwischen der nFK aus der BZE II und der nFK aus der VBK 50 ist zwar signifikant, es wird jedoch über eine lineare Regression nur knapp $15 \%$ der Streuung erklärt. Die Streuung der Werte ist z. T. sehr stark und es werden an Standorten mit geringer nFK deutlich höhere Werte aus der VBK 50 als aus der BZE II geschätzt. Damit überschätzt die VBK 50 Standorte mit geringer nFK im Vergleich zur BZE II. In mehreren Fällen weichen die Werte der VBK 50 um das bis zu Vierfache von den Werten der BZE II ab. Möglicherweise führen eher allgemeine Angaben zu Skelettanteilen, Bodenarten und Trockenrohdichten sowie übergeordnete Zusammenfassungen von Einzelstandorten in der VBK 50 zu diesen hohen Abweichungen und der allgemein geringen Übereinstimmung. 


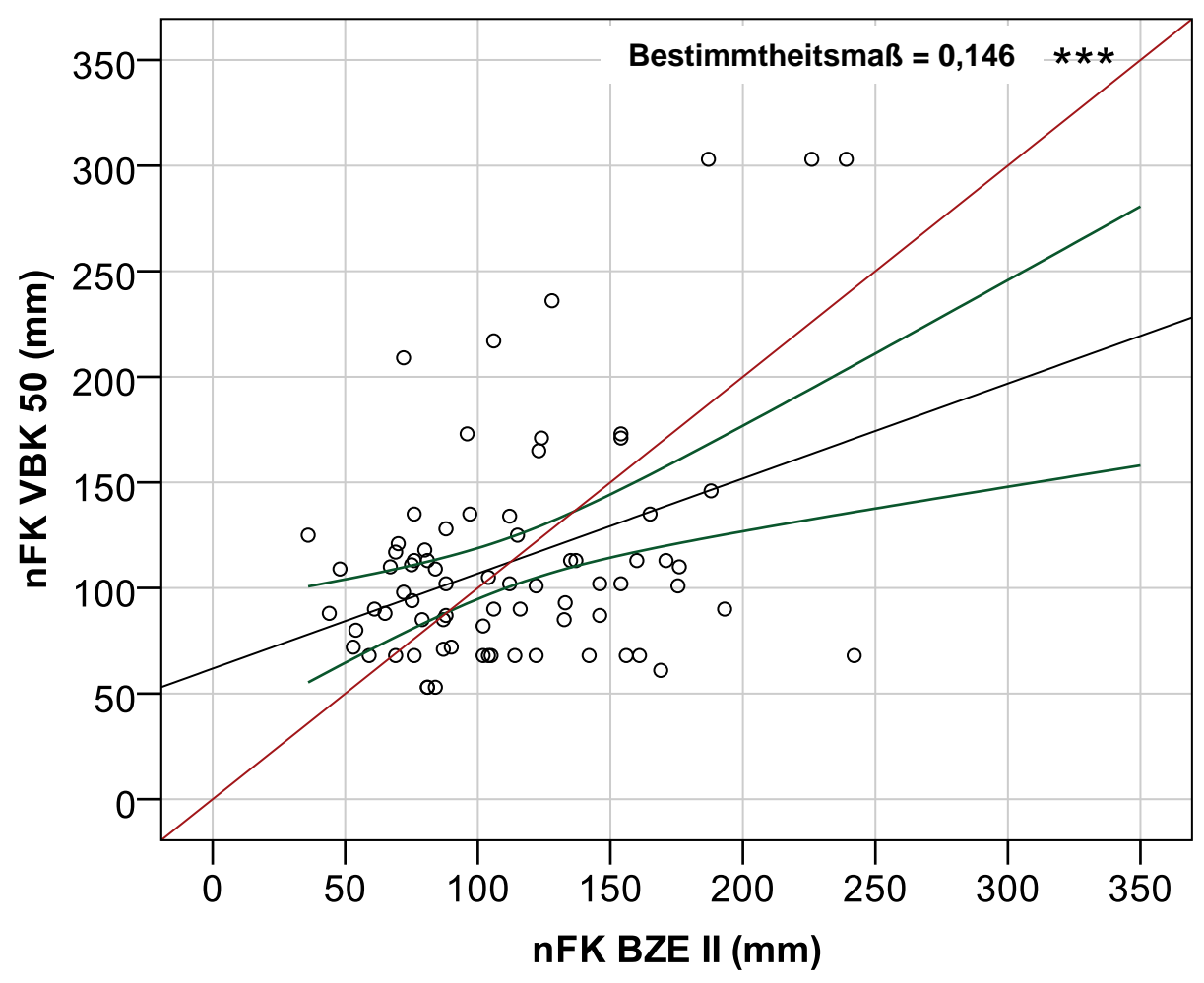

Abbildung 2: Nutzbare Feldkapazität (nFK) in $\mathrm{mm}$ bis $90 \mathrm{~cm}$ Bodentiefe aus der Bodenzustandserbebung II (BZE II) und aus der Vorläufigen Bodenkarte 50 (VBK 50) (rote Linie: 1:1, schwarze Linie: Regressionsgerade, grüne Linien: $95 \%$ Konfidenzintervall, $N=75$ )

Genauere Angaben zu den die nFK bestimmenden Parametern sind über Merkmalsspiegel vorhanden, die für die wichtigsten Lokalbodenformen der forstlichen Standortskarte erstellt wurden. Merkmalsspiegel geben für bestimmte Parameter (Merkmale) mittlere Verhältnisse der jeweiligen Lokalbodenform an. Es gibt z. B. Angaben zur Schichtmächtigkeit, Bodenart (KA 5 konform (AG BODEN 2005)), zum Skelettanteil, zur Trockenrohdichte und zum Humusgehalt. Der Katalog von Merkmalsspiegeln für Lokalbodenformen in Sachsen-Anhalt wird zurzeit digital aufbereitet (SCHMIDT et al. 2015). Beispielhaft ist der Merkmalsspiegel mit den für die nFK relevanten Parametern für die häufig vorkommende Lokalbodenform „Nedlitzer Sand-Braunerde" angegeben (s. Abb. 3). 
Merkmalsspiegel für Standortsformen in Waldnaturräumen nach Stammeigenschaften

Stamm-Bodenform (Haupt- und Lokalbodenform): NeS Nedlitzer Sand-Braunerde

KMgCaP-Serie III Ökol. Nährkraftstufe M"'

Bodensyst. Einheit nach KA5: Norm-Braunerde aus Sand Natürliche Gleichgewichts-Humusform: Mo Moder

\begin{tabular}{l}
$\begin{array}{l}\text { Normalprofil nach mittleren Horizont- und Schichtmerkmalen, einschl. Kombination mit Grund-/Stauwasserform } \\
\begin{array}{l}\text { Symbol } \\
\text { in Ableit- } \\
\text { datei } \\
\text { einschl. v. }\end{array} \\
\begin{array}{l}\text { Zusatz- } \\
\text { merkmalen }\end{array}\end{array}$ \\
\hline orig.
\end{tabular}

Abbildung 3: Merkmalsspiegel zur Lokalbodenform „Nedlitzer Sand-Braunerde“ (Auszug)

Datenbankgestützt wurde über die Angaben in den Merkmalsspiegeln eine nFK berechnet, die dann über die dazugehörige Lokalbodenform den Polygonen der digitalen forstlichen Standortskarte der Waldfläche zugeordnet wurde.

Die nFK aus den Merkmalsspiegeln der kartierten Lokalbodenform aus der digitalen forstlichen Standortskarte wurde dann den Ergebnissen der nFK aus der BZE II gegenübergestellt (s. Abb. 4).

Die signifikante Beziehung ist mit knapp $40 \%$ erklärter Streuung deutlich straffer als die Beziehung zwischen der aus der VBK 50 geschätzten nFK und der an den BZE-Profilen erhobenen nFK. Wie erwartet spiegeln sich die Unschärfen aus standörtlicher Heterogenität, dem Zwang zur Vereinheitlichung und Klassenbildung in der Standortskartierung und die hohe Lokalbodenvielfalt im System der sachsen-anhaltischen Standortskartierung in diesem Ergebnis wider. Lokalbodenformen von kartierten Flächen müssen mittlere Verhältnisse abbilden. Es ist daher nicht auszuschließen, dass sich an Teilflächen im Polygon einer kartierten Lokalbodenform auch andere Lokalbodenformen ableiten lassen. Zum anderen besteht die Schwierigkeit, im Zuge einer Kartierung aus dem umfangreichen Katalog der möglichen Lokalbodenformen (ca. 1500 ohne Sonderstandorte) möglichst einheitlich die zutreffende Einheit zuzuordnen. Trotz dieser Unschärfen wurden die bereits formulierten Merkmalsspiegel für die kartierten Lokalbodenformen für die datenbankgestützte Berechnung der nFK und eine entsprechende Regionalisierung verwendet. 


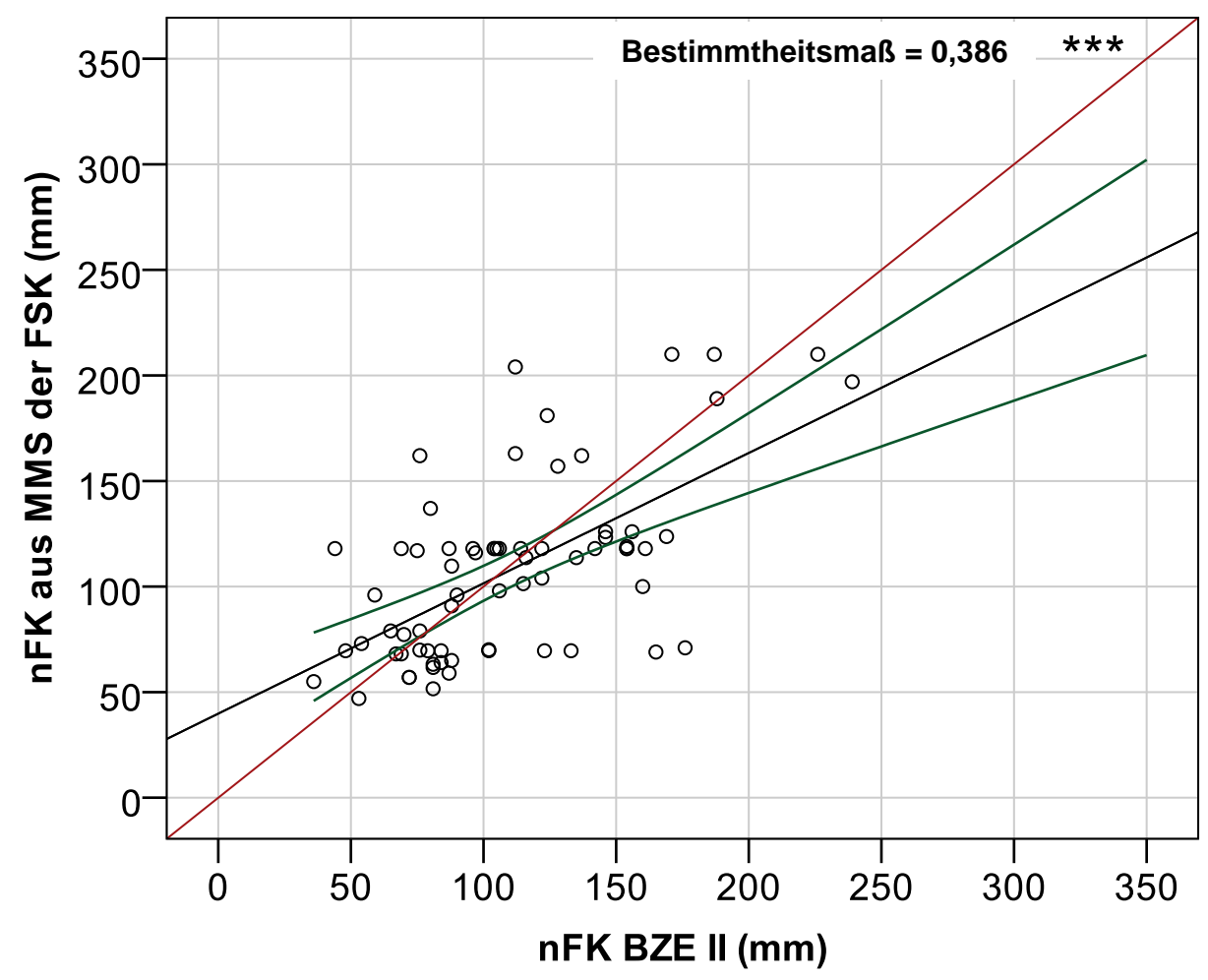

Abbildung 4: Nutzbare Feldkapazität (nFK) bis $90 \mathrm{~cm}$ Bodentiefe aus der Bodenzustandserbebung II (BZE II) und aus den Merkmalsspiegeln (MMS) der Lokalbodenformen aus der forstlichen Standortskartierung (rote Linie: 1:1, schwarze Linie: Regressionsgerade, grüne Linien: $95 \%$ Konfidenzintervall, $N=69$ )

Weiterhin wurde an den BZE II-Profilen die nFK anhand der Merkmalsspiegel der punktbezogenen Lokalbodenform berechnet. Die punktbezogene Lokalbodenform ergibt sich direkt aus dem BZE-Profil mit seinen spezifischen Eigenschaften und wurde einheitlich durch erfahrene Standortskartierer aus Sachsen-Anhalt ermittelt. Anschließend wurde die sich aus der punktbezogenen Lokalbodenform ergebende $\mathrm{nFK}$ aus dem Merkmalsspiegel der aus der BZE II berechneten nFK gegenübergestellt (s. Abb. 5). Die signifikante Regression erklärt $65 \%$ der Streuung. Damit zeigt sich, dass über die Merkmalsspiegel die nFK von Lokalbodenformen hinreichend genau abgeschätzt werden kann. Es liegen allerdings nicht für alle BZE II-Profile Merkmalsspiegel vor. Es fehlen 6 Merkmalsspiegel von insgesamt 76 BZE II-Standorten. Ein Moorstandort ist aus der Berechnung ausgenommen worden. Die relativ enge Beziehung zwischen der nFK aus den Merkmalsspiegeln und der BZE II zeigt, dass die Zuordnung von Lokalbodenformen zu konkreten Profilen bezüglich der nFK belastbar ist. 


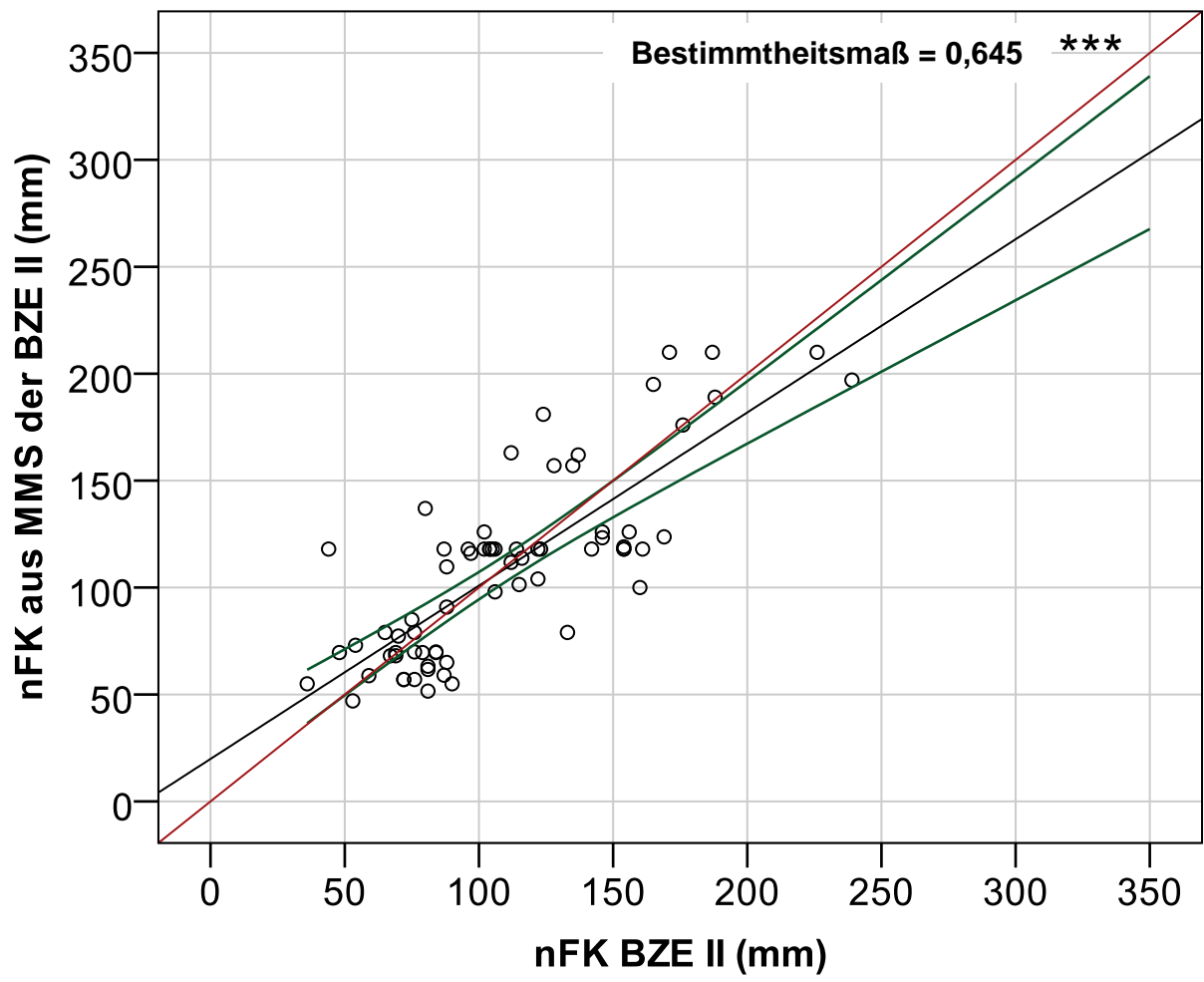

Abbildung 5: Nutz̧bare Feldkapazität (nFK) bis $90 \mathrm{~cm}$ Bodentiefe aus der Bodenzustandserhebung II (BZE II) und aus den Merkmalsspiegeln (MMS) der sich aus der BZE II Profilansprache ergebenden Lokalbodenform (rote Linie: 1:1, schwarze Linie: Regressionsgerade, grüne Linien: $95 \%$ Konfidenzintervall, $N=69$ )

Um eine möglichst hohe Abdeckung der Waldfläche in den Modellregionen mit Standortsinformationen zu erzielen, wurden die Datensätze der digitalen Standortskartierung auf dem aktuellen Stand des Landeszentrums Wald Sachsen-Anhalt in Halberstadt und die VBK 50 (LANDESAMT FÜR GEOLOGIE UND BERGWESEN 2009) verschnitten. Aus dieser Verschneidung ergaben sich insgesamt 1700 über Abkürzungen verschlüsselte Lokalbodenformen inklusive Sonderstandorten (z. B. Aufschüttungen, Freiflächen, waldfrei).

Aus der Verteilung der Lokalbodenformen hinsichtlich ihrer flächenbezogenen Relevanz zur Waldfläche wird deutlich, dass sich durch eine Beschreibung von $20 \%$ der flächenbedeutsamsten Lokalbodenformen bereits $80 \%$ der Waldfläche mit einer nFK belegen lassen (s. Abb. 6).

Daher wurden die Lokalbodenformen anhand ihrer flächenbezogenen Relevanz sortiert und über die Merkmalsspiegel, sofern vorhanden, mit einer Datenbank gestützt berechneten $\mathrm{nFK}$ belegt (Durchwurzelungstiefe $100 \mathrm{~cm}$ und $140 \mathrm{~cm}$ ). Über 
360 Lokalbodenformen konnte bereits $85 \%$ der Waldfläche Sachsen-Anhalts eine nFK zugewiesen werden. Alle Lokalbodenformen mit über 300 ha Vorkommen in Sachsen-Anhalt sind abgedeckt.

Weitere Verbesserungsmöglichkeiten ergeben sich aus dem Fortschritt der Digitalisierung der forstlichen Standortskartierung, einer weiteren Aufbereitung der Merkmalsspiegel und Zuweisung einer nFK für Lokalbodenformen mit fehlenden Merkmalsspiegeln über die Hauptbodenform.

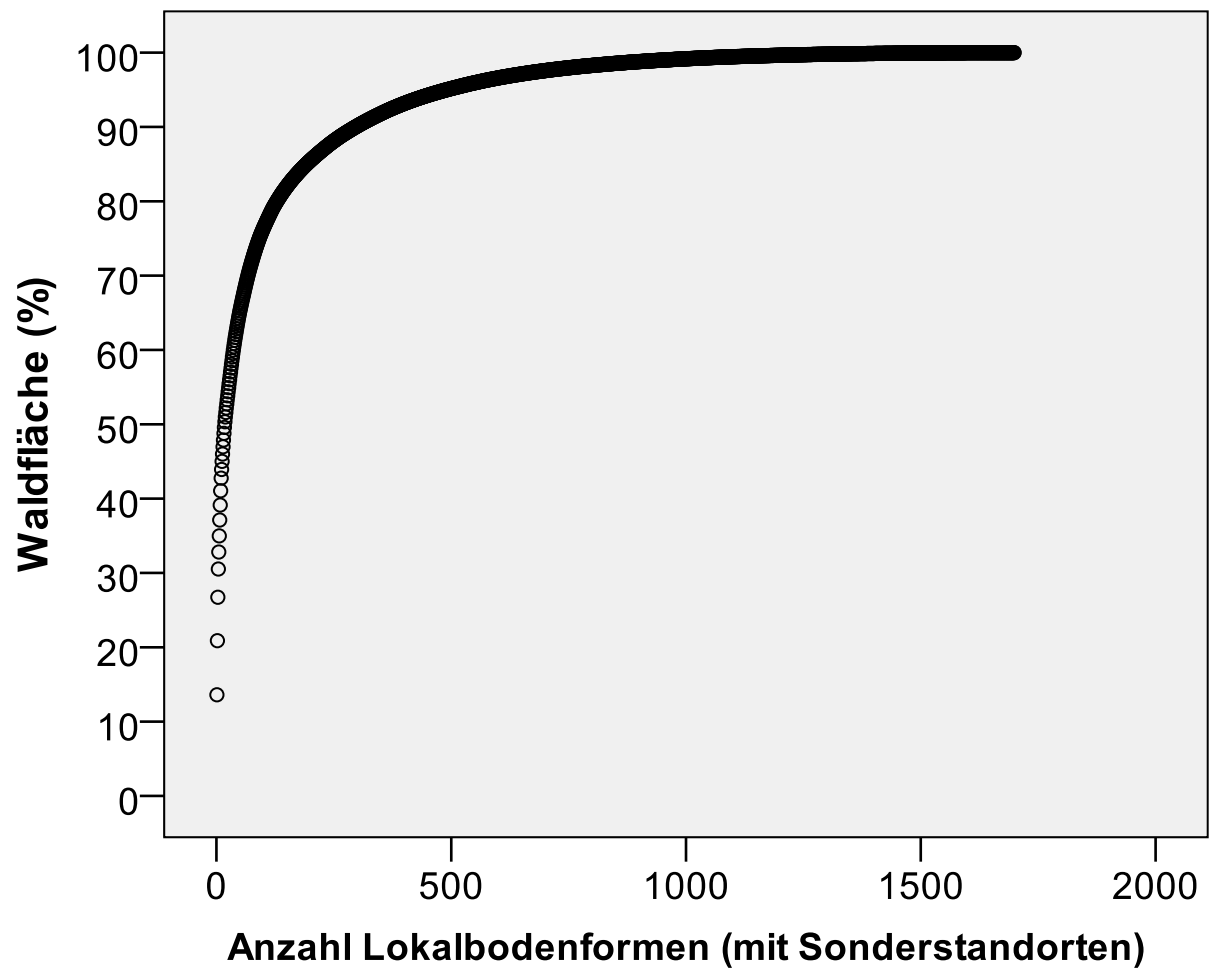

Abbildung 6: Kartierte Lokalbodenformen mit Sonderstandorten in Sachsen-Anbalt und Abdeckung der $W$ aldfläche $[\%]$

\subsection{Klima}

Für das Land Sachsen-Anhalt wurde im Rahmen der Klimastudie (SUTMÖLLER et al. 2013) das Anbaurisiko der Hauptbaumarten auf Grundlage des Klimaszenarios A1B mit dem regionalen Klimamodell WETTREG2010 (KREIENKAMP et al. 2012) abgeschätzt und in Kartenform aufbereitet. Die Risikoeinstufung erfolgte anhand der berechneten Klimatischen Wasserbilanz (KWB) in der Vegetationsperiode, die mit dem pflanzenverfügbaren Bodenwasser verrechnet wurde (SPELLMANN et al. 2007). Die nutzbare Feldkapazität (nFK) wurde anhand der vorläufigen Bodenkarte 
(VBK 50 im Maßstab 1:50.000) für eine Durchwurzelungstiefe von $100 \mathrm{~cm}$ bestimmt.

Im Rahmen dieser Studie wurde für die Baumarten Kiefer, Fichte, Eiche, Buche und Douglasie das Trockenstressrisiko nach dem gleichen methodischen Ansatz abgeschätzt. Allerdings diente die abgeleitete nFK der forstlichen Standortskartierung als Grundlage, um die Trockenstressgefährdung zu bestimmen (s. Kap. 4.1). Folglich wurden in den Modellregionen nur die Waldflächen in die Auswertung mit einbezogen, die mit einer $\mathrm{nFK}$ aus der forstlichen Standortkartierung belegt werden konnte. Die Waldflächen, für die keine forstliche Standortkartierung vorliegt, wurden nicht berücksichtigt. Die Auswertung zum Trockenstressrisiko erfolgte auf Grundlage der Bestandesgeometrien, so dass für jede Waldfläche eine flächengewichtete $\mathrm{nFK}$ berechnet werden musste.

Das baumartenspezifische Trockenstressrisiko wird in Anlehnung an SPELLMANN et al. (2011) berechnet. Um die zeitliche Dynamik des Klimawandels darzustellen, werden die 30-jährigen Perioden 1971 - 2000, 1981 - 2010, 2011 - 2040, 2041 - 2070 und 2071 - 2100 getrennt ausgewertet. Dabei basiert das Mittel der Periode 1971 - 2000 auf regionalisierten Messdaten des Deutschen Wetterdienstes, während das Trockenstressrisiko der nachfolgenden Perioden auf Grundlage der Projektionsdaten des regionalen Klimamodells WETTREG2010 abgeschätzt wird.

\subsubsection{Auswabl der Klimavarianten}

Über das Ausmaß der erwarteten Klimaänderungen und ihre regionale Ausprägung besteht Unsicherheit. Dies findet in den verschiedenen Klimaszenarien seinen Ausdruck. Für diese Studie wurde das Klimaszenario A1B ausgewählt. Alle Auswertungen werden auf Basis der regionalisierten Klimaprojektionen mit dem regionalen Klimamodell WETTREG2010 vorgenommen (KREIENKAMP et al. 2012).

Das Modell WETTREG2010 stellt für jedes Klimaszenario zehn Varianten zur Verfügung, deren Eintrittswahrscheinlichkeit gleich groß ist. Damit wird ein Bereich möglicher Klimaentwicklungen definiert, dessen Unsicherheit mit zunehmender Simulationsdauer stetig zunimmt. Insbesondere die Projektion der Niederschlagshöhen unterliegt je nach Klimavariante deutlichen Schwankungen.

Für die Abschätzung des Anbaurisikos der Hauptbaumarten wird die Summe aus pflanzenverfügbarem Bodenwasser (nFK = nutzbare Feldkapazität) und Klimatischer Wasserbilanz (KWB) in der Vegetationszeit herangezogen (SPELLMANN et al. 2007). Hierzu wurden die Klimavariablen und die berechnete potenzielle Evapotranspiration mit Hilfe des hydrologischen Modells WaSiM-ETH (SCHULLA u. JASPER 2012) für alle zehn Varianten des Szenarios A1B flächendeckend für das Land Sachsen-Anhalt auf ein $200 \times 200 \mathrm{~m}$ Raster regionalisiert und die KWB berechnet. Der Beginn der Vegetationsperiode wurde baumartenspezifisch nach MENZEL u. FABIAN (1999) und das Ende der Vegetationsperiode nach v. WILPERT (1990) bestimmt. 
Die Auswahl der Klimavariante erfolgte analog zur Vorgehensweise bei der Klimastudie Sachsen-Anhalts (SUTMÖLLER et. al. 2013). Um die Vergleichbarkeit der Ergebnisse zwischen den drei Modellregionen zu gewährleisten, erfolgte die Auswahl der Variante auf Grundlage der Ergebnisse für die gesamte Landesfläche.

In Tabelle 1 sind für die 30-jährigen Untersuchungsperioden die Mittelwerte der zehn Varianten bestehend aus KWB und nFK für das Land Sachsen-Anhalt aufgelistet. Die Auswahl der für die Abschätzung des Anbaurisikos verwendeten Variante erfolgte anhand der Periode 2071 - 2100. Da die Spannweite zwischen den Varianten relativ gering ist, wurde die Variante 6, die in der Periode 2071 - 2100 mit -263 mm das höchste mittlere Defizit aufweist, zur Abschätzung des zukünftigen Trockenstressrisikos betrachtet.

Tabelle 1: $\quad$ Summe der Klimatischen Wasserbilanz und der nutzbaren Feldkapazität in der Vegetationsperiode für die V arianten des Klimaszenarios A1B (WETTREG2010) in mm

\begin{tabular}{lrrrrrrrrrr}
\hline Variante & 00 & 01 & 02 & 03 & 04 & 05 & 06 & 07 & 08 & 09 \\
\hline $1971-2000$ & 11 & 7 & 4 & -2 & -12 & -16 & 39 & -4 & 18 & 12 \\
$1981-2010$ & -1 & -4 & -10 & 9 & -6 & -5 & 32 & 14 & 5 & 14 \\
$2011-2040$ & -45 & -46 & -67 & -77 & -51 & -53 & -51 & -24 & -48 & -60 \\
$2041-2070$ & -167 & -156 & -185 & -148 & -157 & -167 & -151 & -176 & -170 & -131 \\
$2071-2100$ & -226 & -244 & -234 & -233 & -238 & -223 & -263 & -231 & -253 & -239 \\
$\varnothing 2011-2100$ & -146 & -149 & -162 & -153 & -149 & -148 & -155 & -144 & -157 & -143 \\
\hline
\end{tabular}

\subsubsection{Methodik und Definition des Trockenstressrisikos}

In Anlehnung an SPELLMANN et al. (2011) wird das pflanzenspezifische Trockenstressrisiko für die Hauptbaumarten abgeschätzt. Als Standortsmerkmal wird hierzu die Summe aus nFK und KWB in der Vegetationszeit herangezogen. Die potenzielle Evapotranspiration wurde für eine normierte Grasvegetation (Grasreferenzverdunstung) nach Vorgabe des Deutschen Wetterdienstes (ALLEN et al. 1994) berechnet. Die Risikoklassifizierung erfolgte auf der Grundlage von Expertenwissen und bisherigen Anbauerfahrungen.

Das Trockenstressrisiko wird für Kiefer, Eiche und Douglasie am geringsten eingeschätzt. Da die Kiefer in Sachsen-Anhalt jedoch die Baumart mit der größten Verbreitung ist, erfolgt die Darstellung des Trockenstressrisikos separat. Für die Buche wird von einem gegenüber der Kiefer deutlich höheren Trockenstressrisiko ausgegangen, während die Fichte das höchste Risiko aufweist. In Abänderung zu 
SPELLMANN et al. (2011) wird die Douglasie in der Risikoeinstufung gutachtlich mit der Eiche gleichgestellt.

Auf Grundlage der WETTREG2010-Ergebnisse werden die Klimavarianten mit einem abstands- und höhenabhängigen Regionalisierungsverfahren interpoliert. Die räumliche Auflösung beträgt 200 x 200 Meter. Anhand der regionalisierten Wetterund Klimadaten können dann die klimatischen Verhältnisse detailliert und kleinräumig beschrieben werden.

In Abbildung 7 (links) ist exemplarisch für die Modellregion Oberharz die Klimatische Wasserbilanz (KWB) für die Vegetationsperiode von Ende April bis Anfang Oktober am Beispiel der 30-jährigen Periode 1970 - 2000 dargestellt. Bereits heute tritt in den unteren Lagen des Oberharzes im Mittel ein leichtes Defizit in der KWB auf (hellrot). Nur die mittleren und hohen Lagen des Harzes weisen auch während der Vegetationsperiode einen Wasserüberschuss auf (grau bzw. hellblau).

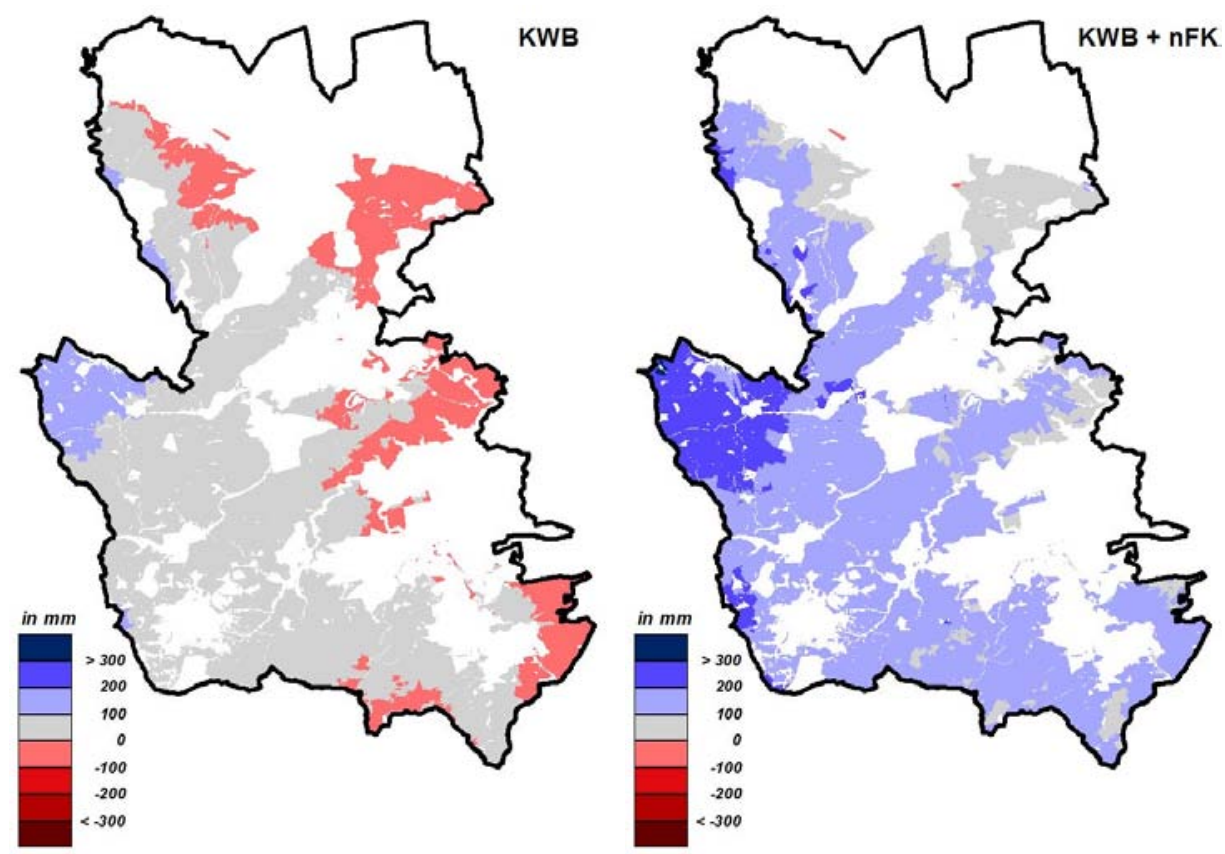

Abbildung 7: Klimatische Wasserbilan₹ (KWB, links) und Summe aus KWB und nutzbarer Feldkapazität (nFK, rechts) am Beispiel der 30-jährigen Periode 1970 - 2000 (Mittelwert der Vegetationsperiode) für die Modellregion Oberharz.

Verschneidet man die berechnete KWB mit dem pflanzenverfügbaren Bodenwasser (nFK) aus der forstlichen Standortskarte, so sind auf fast allen Bestandesflächen positive Wasserbilanzen zu verzeichnen (s. Abb. 7, rechts). Folglich ist die Menge des pflanzenverfügbaren Bodenwassers noch ausreichend, um unter heutigen 
Klimabedingungen die Defizite in der Klimatischen Wasserbilanz während der Vegetationsperiode in der Modellregion Oberharz auszugleichen.

Die Klassifizierung des Trockenstressrisikos wird nach SUTMÖLLER et al. (2013) vorgenommen (s. Tab. 2). Das geringe Trockenstressrisiko der Fichte wird bei Werten von $>-80 \mathrm{~mm}$, bei der Buche von $>-100 \mathrm{~mm}$ und bei der Kiefer, Eiche und Douglasie von $>-150 \mathrm{~mm}$ ausgewiesen. Das mittlere Trockenstressrisiko bei der Fichte reicht von -80 bis $-250 \mathrm{~mm}$, bei der Buche von -100 bis $-300 \mathrm{~mm}$ und bei der Kiefer, Eiche und Douglasie von -150 bis $-400 \mathrm{~mm}$. Höhere Defizite werden mit einem hohen Trockenstressrisiko belegt.

Tabelle 2: $\quad$ Definition des baumartensperifischen Trockenstressrisikos (Summe der KWB $+n F K$ in der Vegetationszeit in mm, in Anlehnung an SPELLMANN et al. 2011)

\begin{tabular}{crrr}
\hline $\begin{array}{c}\text { Trocken- } \\
\text { stressrisiko }\end{array}$ & \multicolumn{1}{c}{ Fichte } & \multicolumn{1}{c}{ Buche } & Kiefer / Eiche / Douglasie \\
\hline gering & $>-80 \mathrm{~mm}$ & $>-100 \mathrm{~mm}$ & $>-150 \mathrm{~mm}$ \\
mittel & -80 bis $-250 \mathrm{~mm}$ & -100 bis $-300 \mathrm{~mm}$ & -150 bis $-400 \mathrm{~mm}$ \\
hoch & $<-250 \mathrm{~mm}$ & $<-300 \mathrm{~mm}$ & $<-400 \mathrm{~mm}$ \\
\hline
\end{tabular}

Aufgrund der langen Produktionszeiten der untersuchten Hauptbaumarten ist für den Entscheidungsprozess bei der Baumartenwahl die Klimaperiode mit dem höchsten Trockenstressrisiko ausschlaggebend. Um die Veränderung des Trockenstressrisikos einschätzen zu können, wird das unter heutigen gemessenen Klimabedingungen (Periode 1971 - 2000) geschätzte Trockenstressrisiko mit den Verhältnissen in der Periode 2071 - 2100 verglichen.

\subsection{Modellierung des Standort-Leistungsbezuges}

Der Standort-Leistungsbezug sollte so umfassend durch ein statistisches Modell beschrieben werden, dass die Variabilität des Leistungsspektrums auf einem Standort quantifiziert werden kann. Für viele waldwachstumskundliche Fragestellungen sind Schätzungen des Erwartungswertes einer Bonitätsverteilung aussagekräftig. Für eine spezifische Kombination von Standortseigenschaften sind die beobachteten Höhenbonitäten approximativ normalverteilt, so dass ihr Erwartungswert mit einem Standardregressionsmodell geschätzt werden kann.

Ziel der Modellierung ist es, die Standortleistungsfähigkeit für jeden beliebigen Forstort und verschiedene Baumarten in Abhängigkeit von Boden- und Klimavariablen aber ohne Kenntnis von Bestandesmerkmalen zu schätzen. Somit wird es möglich, die Standortleistungsfähigkeit unabhängig von der aktuell vorhandenen Baumart in die Zukunft zu projizieren. Die klimatischen Daten des Parametrisie- 
rungsdatensatzes stammen von der retrospektiven Modellierung der Klimanormalperiode 1961 bis 1990 des Modells WETTREG (SPEKAT et al. 2007) in der Version 2006. Die Stickstoffdepositionsraten wurden modelliert mit einer modifizierten Version von MAKEDEP (vgl. ALVETEG et al. 1998 AHRENDS et al. 2008, AHRENDS 2010). Wie in ALBERT u. SCHMIDT (2010 a u. b, 2012) beschrieben, wird folgende Modellspezifikation auf Basis von verallgemeinerten additiven Modellen $G A M$ (WOOD 2006) für die Baumarten Buche, Eiche, Fichte und Kiefer verwendet und an einem bundesweiten Datensatz im Statistikpaket R 2.10.0 (R DEVELOPMENT CORE TEAM 2006) unter Verwendung der Bibliothek mgcv 1.6-0 (WOOD 2006) parametrisiert:

$$
\begin{aligned}
\operatorname{hg} 100_{i}= & \alpha_{1}+\mathrm{Nähr}_{i}^{\mathrm{T} \beta}+\mathrm{f}_{1}\left(\mathrm{Tempsum}_{i}\right)+\mathrm{f}_{2}\left(\mathrm{KWB}_{i}\right)+\mathrm{f}_{3}\left(\mathrm{nFK}_{i}\right)+\mathrm{f}_{4}\left(\mathrm{Ndep}_{i}\right) \\
& +\mathrm{f}_{5}\left(\mathrm{RW}_{i}, \mathrm{HW}_{i}\right)+\varepsilon_{i}
\end{aligned}
$$

mit:

$$
\begin{array}{ll}
\operatorname{hg}_{100_{i}} & \text { Mittelhöhenbonität im Alter } 100 \text { an Stichprobenpunkt } i[\mathrm{~m}] \\
\mathrm{Nähr}_{i}^{\mathrm{T}} & =\text { Indikatorenvektor für klassifizierte Bodennährstoffe } \\
\beta & =\text { zu Nähr gehörender Parametervektor } \\
\mathrm{KWB}_{i} & =\text { klimatische Wasserbilanz in der Vegetationszeit }(\mathrm{VZ})[\mathrm{mm}] \\
\mathrm{Tempsum}_{i}= & \text { Temperatursumme in der VZ }\left[{ }^{\circ} \mathrm{C}\right] \\
\mathrm{Ndep}_{i} & =\text { mittlere Stickstoffdeposition }[\mathrm{eq} / \mathrm{ha} / \mathrm{a}] \\
\mathrm{nFK}_{i} & =\text { nutzbare Feldkapazität auf } 1,4 \mathrm{~m} \text { Bodentiefe }[\mathrm{mm}] \\
\mathrm{RW}_{i}, \mathrm{HW}_{i}= & \text { Gauss-Krüger-Lagekoordinaten } \\
\alpha_{1} & =\text { Regressionskoeffizient (Interzept) } \\
\mathrm{f}_{1}, \mathrm{f}_{2}, \mathrm{f}_{3}, \mathrm{f}_{4}= & \text { eindimensionale glättende Funktionen } \\
\mathrm{f}_{5} & =\text { zweidimensionale glättende Funktion zur Beschreibung des räumlichen } \\
& \text { Trends }
\end{array}
$$

Bei der Baumart Douglasie führen die untereinander korrelierten Prädiktoren bei gleichzeitig deutlich geringerer Datengrundlage als bei den anderen Baumarten dazu, dass sich die Modelleffekte nicht voneinander trennen lassen. Insbesondere der Effekt der Klimatischen Wasserbilanz zeigt bei simultaner Schätzung einen unplausiblen Verlauf, wonach zunehmende Trockenheit zu einer gesteigerten Standortleistungsfähigkeit führen würde. Für die Gewährleistung eines biologisch plausiblen Modellverhaltens werden deshalb die Modelleffekte der Variablen Temperatursumme (Tempsum) und Klimatische Wasserbilanz (KWB) mittels einer Vorschätzung bestimmt. Anschließend gehen diese Modelleffekte als Konstanten in ein finales Modell ein, in dem lediglich noch die Effekte der übrigen Prädiktoren geschätzt werden. Darüber hinaus ist die Variable nutzbare Feldkapazität (nFK) im Douglasienmodell nicht signifikant. Für die Douglasie wird somit folgendes Modell definiert: 
$\operatorname{hg} 100_{i}=\alpha_{1}+\mathrm{Nähr}_{i}^{\mathrm{T} \beta}+\hat{\mathrm{f}}_{1}\left(\mathrm{Tempsum}_{\mathrm{i}}\right)+\hat{\mathrm{f}}_{2}\left(\mathrm{KWB}_{\mathrm{i}}\right)+\mathrm{f}_{4}\left(\mathrm{Ndep}_{i}\right)+\mathrm{f}_{5}\left(\mathrm{RW}_{i}, \mathrm{HW}_{i}\right)+\varepsilon_{i}$

mit:

$\hat{f}_{1}, \hat{f}_{2}=$ mittels Vorschätzung bestimmte und konstant gesetzte Effekte; übrige Variablen wir für (1) spezifiziert

Die Kenngrößen der Modellgüte in Tabelle 3 weisen mittlere Bestimmtheitsmaße aus, der Standardfehler liegt je nach Baumart meist deutlich unter der Differenz einer Ertragsklasse und ein Bias ist nicht vorhanden.

Tabelle 3: $\quad$ Kenngrößen der Modellgüte (se = Standardfebler)

\begin{tabular}{lccc}
\hline Baumart & $\mathrm{R}^{2}$ & se $[\mathrm{m}]$ & Bias [m] \\
\hline Fichte & 0,44 & 3,1 & $-7,3 \mathrm{e}-14$ \\
Buche & 0,31 & 3,4 & $1,6 \mathrm{e}-14$ \\
Kiefer & 0,45 & 2,6 & $-4,0 \mathrm{e}-15$ \\
Eiche & 0,33 & 3,1 & $-9,0 \mathrm{e}-17$ \\
Douglasie & 0,40 & 5,0 & $8,7 \mathrm{e}-15$ \\
\hline
\end{tabular}

Zum Verständnis des Modellverhaltens unter sich verändernden Klimabedingungen sind die partiellen Effekte der Klimatischen Wasserbilanz, der Temperatursumme, der nutzbaren Feldkapazität und der Stickstoffdeposition von Interesse (s. Abb. 8 bis Abb. 11).

Bei der Interpretation der partiellen Effekte muss die Korrelation der Prädiktorvariablen untereinander beachtet werden. Da beispielsweise Temperaturveränderungen fast immer mit Veränderungen der Klimatischen Wasserbilanz einhergehen, darf nicht vom Temperatureffekt direkt auf die Veränderung der Standortleistungsfähigkeit geschlossen werden. Des Weiteren dürfen die Effekte in den Randbereichen der Beobachtungswerte nicht überinterpretiert werden, da sie mit nur wenigen Daten belegt sind. In Abbildung 8 sind die unterschiedlichen Temperaturbereiche, in denen Datensätze der einzelnen Baumarten vorliegen, gut erkennbar. Fichte und Buche zeigen im unteren Temperaturbereich nur geringe Reaktionen der Leistungsfähigkeit, während die übrigen Baumarten auch vom Temperaturanstieg im unteren Wertebereich ihrer jetzigen Vorkommen profitieren. Ab einer Temperatursumme von ca. $1500{ }^{\circ} \mathrm{C}$ in der Vegetationszeit reagiert die Fichte stark, die Buche ab ca. $1800^{\circ} \mathrm{C}$. Für alle Baumarten zeigt der Temperatureffekt im oberen Bereich ein Abflachen, d. h. steigende Temperatursummen scheinen sich hier nicht mehr leistungssteigernd auszuwirken. 


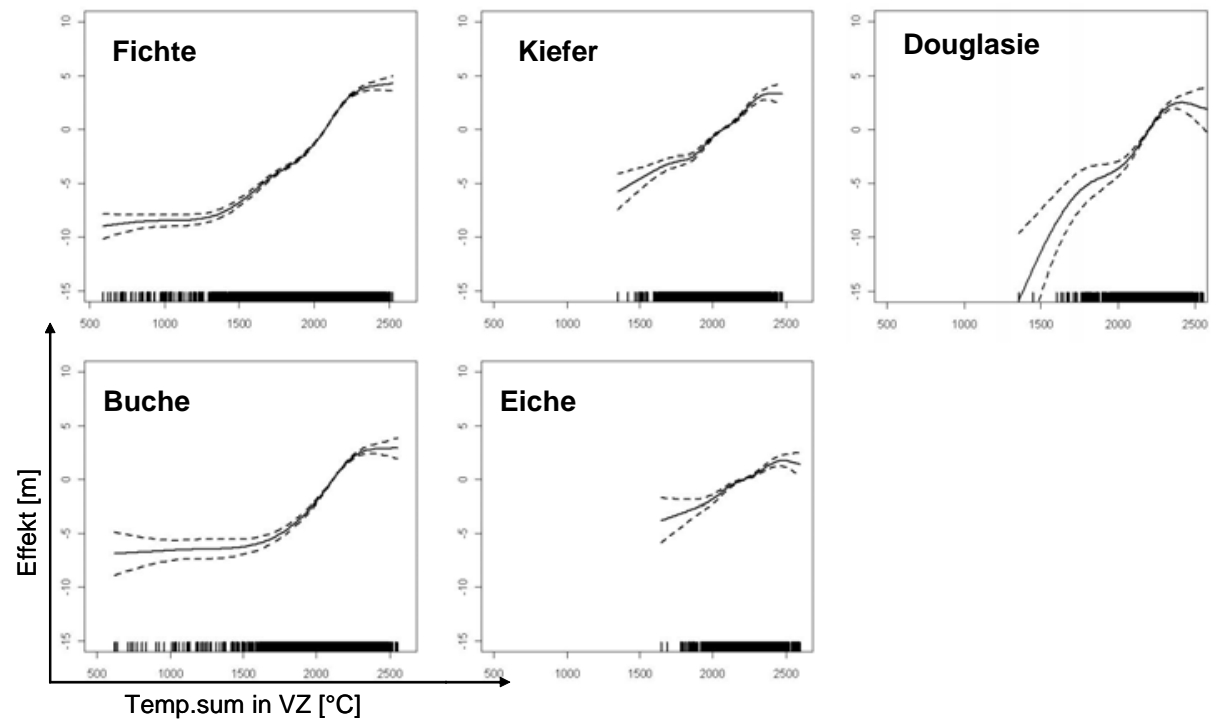

Abbildung 8: Nicht-linearer Effekt der Temperatursumme in der Vegetationszeit $\left[{ }^{\circ} \mathrm{C}\right]$ auf die Mittelböhenbonität $[\mathrm{m}]$ der fünf Baumarten. Die gestrichelten Linien beschreiben punktweise Konfidenzintervalle (5\% Irrtumswabrscheinlichkeit). Die Striche auf der Abszisse markieren Datensätze mit der entsprechenden Ausprägung.
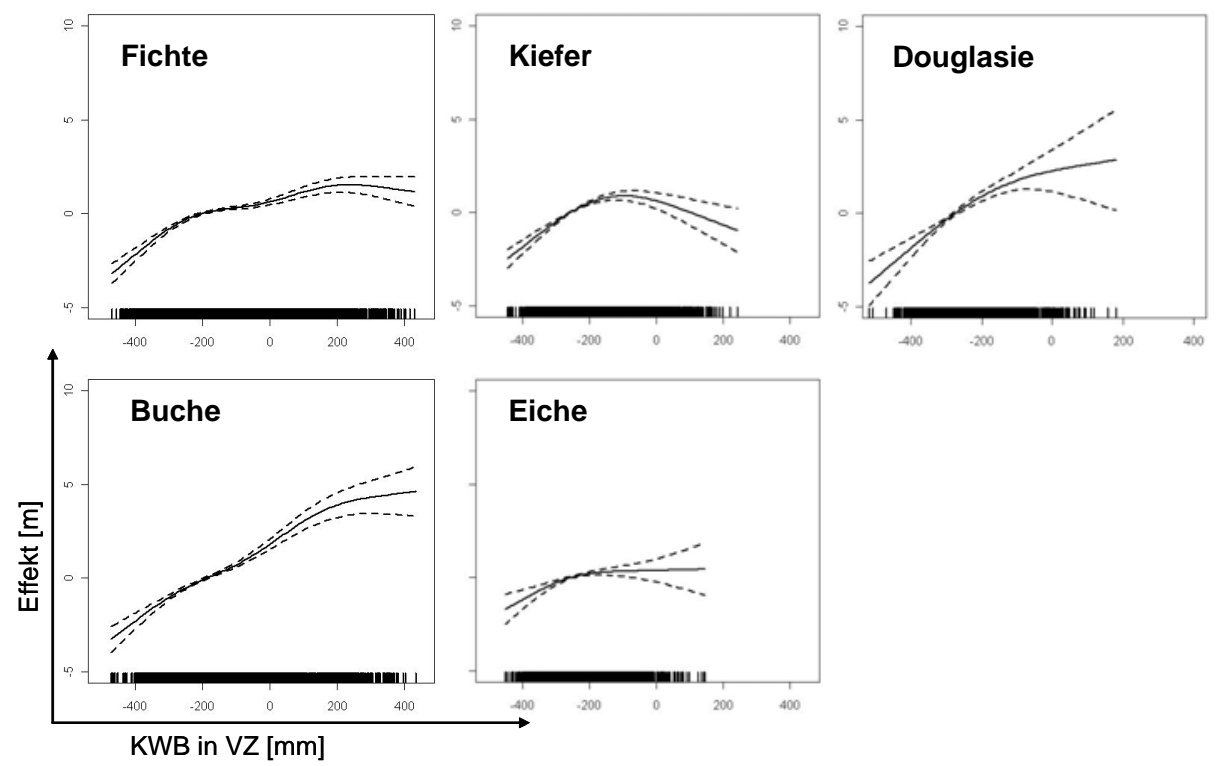

Abbildung 9: Nicht-linearer Effekt der Klimatischen Wasserbilanz KWB in der Vegetationszeit [mm] auf die Mittelhöhenbonität $[\mathrm{m}]$ der fünf Baumarten 
Der Effekt der Klimatischen Wasserbilanz ist für die Baumart Buche über den gesamten Wertebereich fast gleichmäßig ansteigend und erst im oberen Bereich abflachend (s. Abb. 9). Die Effekte bei den anderen Baumarten zeigen bereits bei ca. -200 mm ein deutliches Abflachen. Der nicht monoton steigende Effekt der Klimatischen Wasserbilanz bei der Baumart Kiefer erscheint biologisch nicht sinnvoll, ist jedoch durch die vorliegenden (unbalancierten) Kombinationen der einzelnen Einflussfaktoren im Parametrisierungsdatensatz erklärbar. Kiefernbestände mit einer Klimatischen Wasserbilanz größer $-100 \mathrm{~mm}$ befinden sich überwiegend auf Berglandstandorten, die im Mittel eine geringere Bonität aufweisen. Diese geringere Leistungsfähigkeit trotz besserer Wasserversorgung als im Tiefland wird im Modell eben auch durch das Absinken des Effektes der Klimatischen Wasserbilanz beschrieben und nicht ausschließlich über den Temperatureffekt.

Der Effekt der nutzbaren Feldkapazität auf die Standortleistungsfähigkeit ist im Vergleich zum Effekt der Temperatursumme und der Klimatischen Wasserbilanz recht gering (s. Abb. 10). Die nutzbare Feldkapazität ist dennoch eine wichtige Kenngröße, da sie hilft, zwischen Standorten mit ähnlichen Niederschlags- und Temperaturverhältnissen aber unterschiedlichen Bodenarten zu differenzieren.

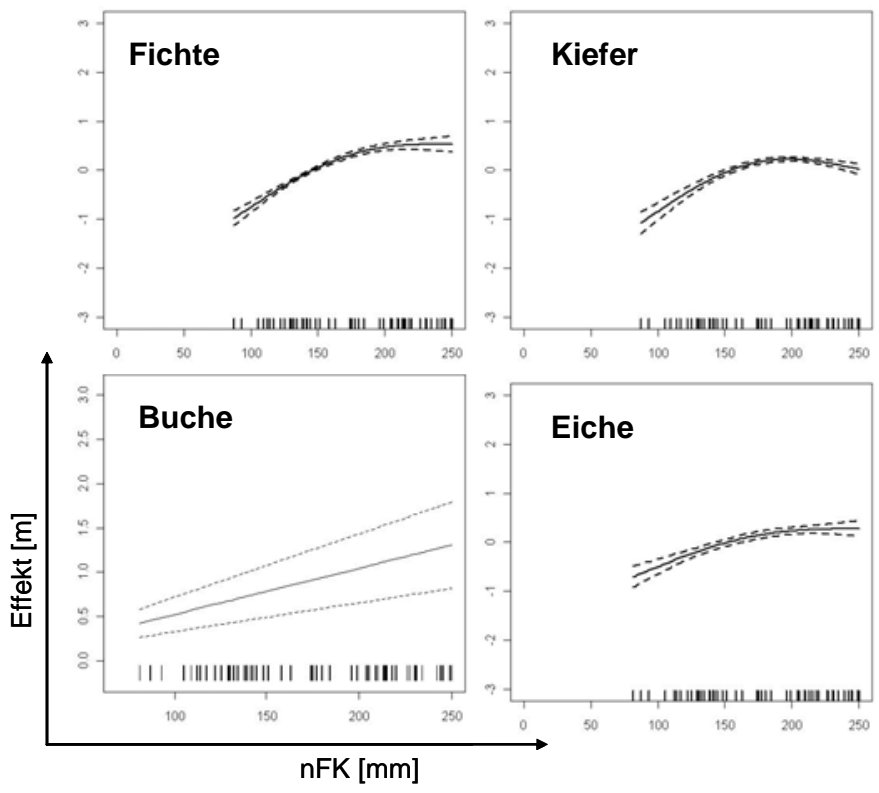

Abbildung 10: Nicht-linearer bzw. linearer (Buche) Effekt der nutzbaren Feldkapazität nFK [mm] auf die Mittelhöhenbonität $[m]$ der vier Baumarten

Die Stickstoffdepositionsrate hat einen großen Effekt auf die Standortleistungsfähigkeit (s. Abb. 11). Allerdings weist sie eine starke räumliche Abhängigkeit auf, mit Werten in Nordwestdeutschland zwischen 6000 und $8000 \mathrm{eq} / \mathrm{ha} / \mathrm{a}$ und Werten zwischen 700 und $5000 \mathrm{eq} / \mathrm{ha} / \mathrm{a}$ im übrigen Bundesgebiet (AHRENDS 2010). 

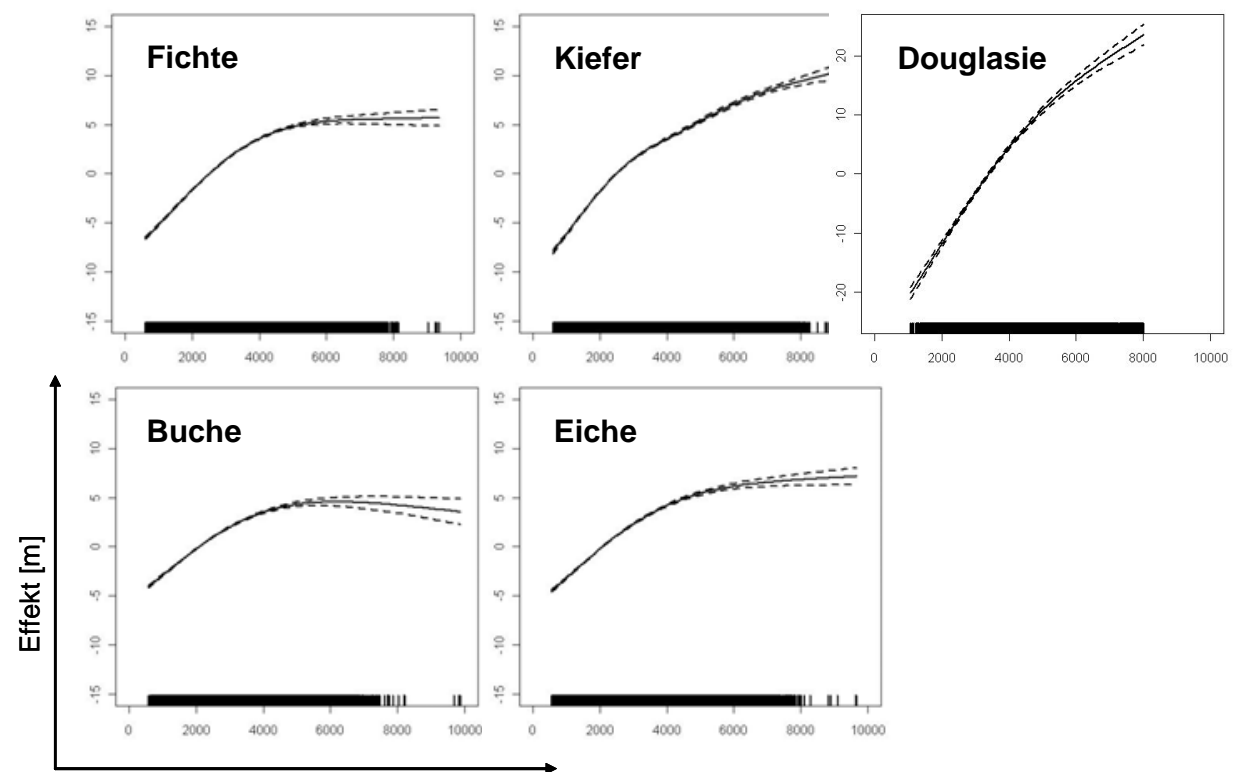

ndep $[\mathrm{eq} / \mathrm{ha} / \mathrm{a}]$

Abbildung 11: Nicht-linearer Effekt der Stickstoffdepositionsrate [eq/ha/ a] auf die Mittelhöhenbonität $[m]$ der fünf Baumarten

Besonders zu beachten ist, dass das Standort-Leistungs-Modell nicht zwischen grundwasserbeeinflussten und grundwasserfernen Standorten unterscheidet. Das Leistungspotenzial von grundwasserbeeinflussten Standorten wird somit tendenziell zu gering geschätzt. Diese Modelllimitationen müssen bei der Interpretation einzelner Ergebnisse berücksichtigt werden.

Das verwendete Standort-Leistungs-Modell ist auf der Basis von mit WETTREG2006 modellierten Klimawerten des retrospektiven Laufes der Klimanormalperiode 1961 bis 1990 unter dem Szenario A1B in der trockenen Variante parametrisiert worden (ALBERT u. SCHMIDT 2010a und b, 2012). Eine Anwendung des Modells zur Schätzung der heutigen und zukünftigen Standortleistungsfähigkeit in den drei Modellregionen Oberharz, Fläming und Colbitz-Letzlinger Heide auf der Basis der Messdaten des Deutschen Wetterdienstes (DWD), deren Verwendung in dieser Studie vorgeschrieben wurde, kann zu erheblichen Verzerrungen der Schätzergebnisse führen. Ähnliche Verzerrungen ergeben sich aus den Unterschieden der bei der Parametrisierung verwendeten Stickstoffdeposition (Ndep_Gauger) nach GAUGER et al. (2008) und der in der Simulation verwendeten Stickstoffdeposition (Ndep) nach BULTJES et al. (2011). In Abbildung 12 sind die Verzerrungen für die drei Modellparameter Temperatursumme in der Vegetationszeit (Temp.sum in VZ), Klimatische Wasserbilanz in der Vegetationszeit (KWB in VZ) und Stickstoffdeposition (Ndep) dargestellt. 

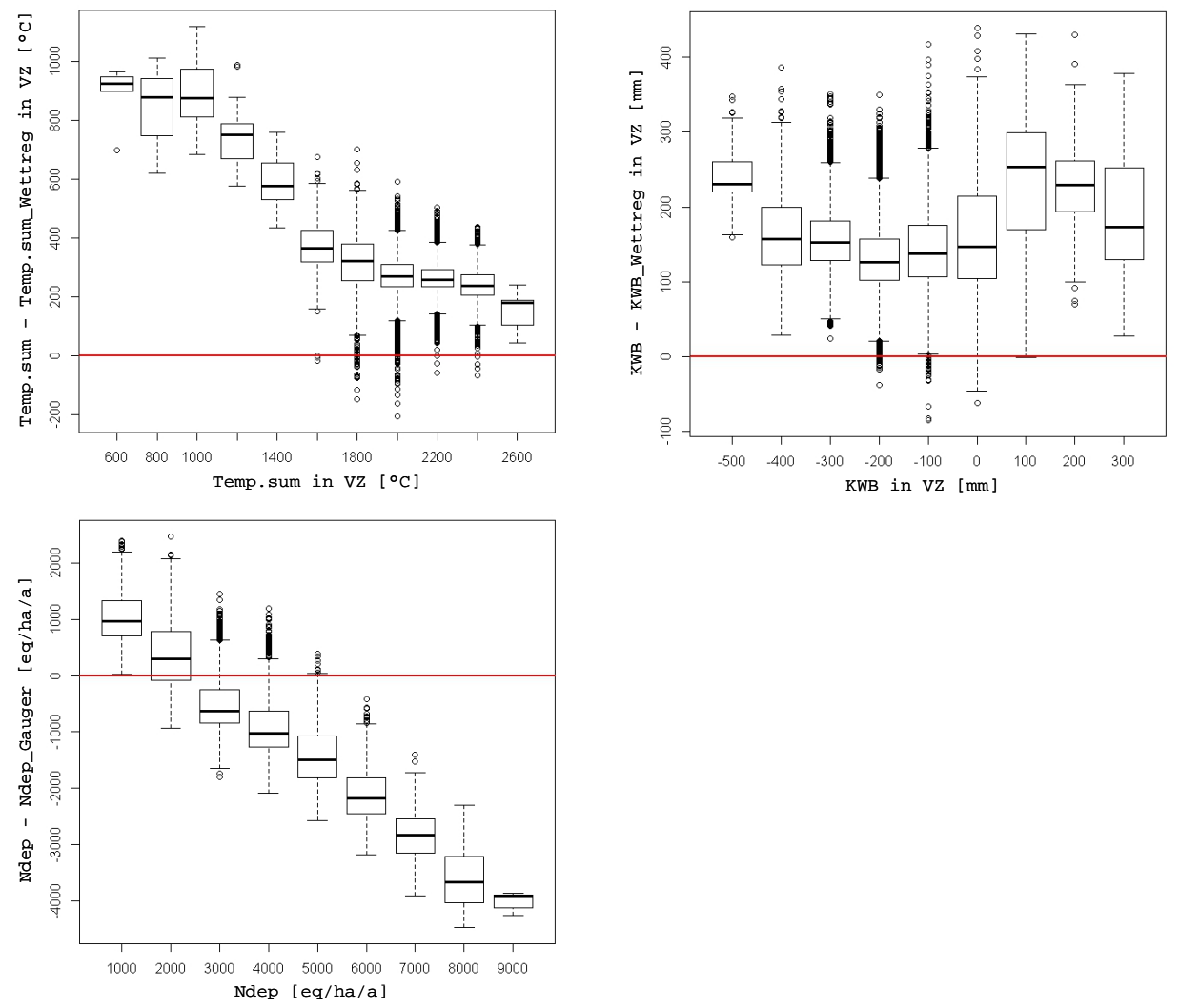

Abbildung 12: Verzerrung aufgrund der Unterschiede in den Parametrisierungs- und Anwendungsdatensätzen des Standort-Leistungs-Modells der Baumart Kiefer dargestellt anhand der Temperatursumme in der Vegetationszeit (oben links), der Klimatischen Wasserbilanz. (oben rechts) und der Stickstoffdeposition (unten). Auf der Ordinate aufgetragen ist die Differenz, zwischen den Werten der Anwendungsdaten Messwerte des DWD zu Temperatursumme in VZ (Temp.sum) und Klimatischer W asserbilanz (KWB) bzw. der Stickstoffdeposition (Ndep) nach BULTJES et al. (2011)) und den Parametrisierungsdaten des Klimamodells WETTREG2006 bžw. der Stickstoffdeposition (Ndep_Gauger) nach GAUGER et al. (2008).

Die zum Teil erhebliche Verzerrung für die drei betreffenden Modellparameter hat ihre Ursachen in den unterschiedlichen Datenquellen (WETTREG2006 vs. DWD, Ndep vs. Ndep_Gauger), in der unterschiedlichen Bestimmung der Vegetationszeit (im Parametrisierungsdatensatz wurde diese baumartspezifisch bestimmt, im Anwendungsdatensatz liegt einheitlich die Vegetationszeit der Fichte zugrunde, des Weiteren wurde die Temperatur für das Vegetationszeitabbruchkriterium von $10{ }^{\circ} \mathrm{C}$ auf $5^{\circ} \mathrm{C}$ abgesenkt) und in der unterschiedlichen Berechnung der Klimatischen 
Wasserbilanz (die potenzielle Evapotranspiration wurde im Parametrisierungsdatensatz baumartspezifisch berechnet, im Anwendungsdatensatz ist die Grasreferenzverdunstung nach ALLEN et al. (1994) die Grundlage).

Als Konsequenz dieser Verzerrungen wurde folgende allgemeine Korrekturfunktion als verallgemeinertes additives Modell für die drei zu korrigierenden Modellvariablen und für die fünf Baumarten Buche, Eiche, Fichte, Kiefer und Douglasie getrennt parametrisiert:

$$
\begin{aligned}
& \log \left(X_{P_{i}}\right)=a_{0}+f_{1}\left(X_{A_{i}}\right)+f_{2}\left(H N N_{i}\right)+f_{3}\left(l \text { lon }_{i}, \text { lat }_{i}\right)+\varepsilon_{i} \\
& \text { wobei } X_{P_{i}} \sim \text { Gamma und } \varepsilon_{i} \sim N(0, G) \\
& \text { mit: } \\
& X_{P i}=\text { Modellvariable des Parametrisierungsdatensatzes (hier Wettreg2006 A1B } \\
& \text { trocken bzw. N-Deposition nach GAUGER) an Stichprobenpunkt i } \\
& X_{A i}=\text { Modellvariable des Anwendungsdatensatzes (hier DWD-Werte bzw. N- } \\
& \text { Deposition nach BULTJES) } \\
& H N N_{i}=\text { Seehöhe des Stichprobenpunktes i } \\
& \text { lon }_{i} \text { lat }_{i}=\text { geografische Koordinaten des Stichprobenpunktes } \mathrm{i} \\
& a_{0}=\text { Interzept } \\
& f_{1}, f_{2}=\text { eindimensionale glättende Funktionen } \\
& f_{3}=\text { zweidimensionale glättende Funktion }
\end{aligned}
$$

Die logarithmische Transformation der Korrekturfunktionen ist zur Stabilisierung der Varianz notwendig. Die Residuen sind heteroskedastisch und werden durch diese Transformation homogenisiert. Alle Modellkoeffizienten sind hochsignifikant. Einen Überblick über die Modellgüte bieten die Bestimmtheitsmaße in Tabelle 4.

Tabelle 4: $\quad$ Kennwerte der Modellgüte für die Korrekturfunktionen der Temperatursumme in der Vegetationszeit (Temp.sum), der Klimatischen W asserbilanz in der V egetationszeit (KWB) und der Stickstoffdeposition (Ndep)

\begin{tabular}{lrrrrr}
\hline & Buche & Eiche & Fichte & Kiefer & Douglasie \\
\hline $\mathrm{N}_{\text {Temp.sum,KWB }}$ & 102.956 & 102.956 & 102.956 & 102.956 & 102.956 \\
$\mathrm{R}_{\text {adj }}$ (Temp.sum) & 0,903 & 0,907 & 0,906 & 0,904 & 0,906 \\
$\mathrm{R}_{\text {adj }}^{2}$ (KWB) & 0,924 & 0,929 & 0,920 & 0,926 & 0,920 \\
$\mathrm{~N}_{\text {Ndep }}$ & 35.859 & 20.457 & 57.070 & 35.101 & 4.585 \\
$\mathrm{R}^{2}$ adj $(\mathrm{Ndep})$ & 0,521 & 0,664 & 0,735 & 0,810 & 0,930 \\
\hline
\end{tabular}


Die spezifizierten Korrekturfunktionen beheben die durch die drei oben erwähnten Unterschiede zwischen Parametrisierungs- und Anwendungsdatensatz entstandenen Verzerrungen weitgehend (s. Abb. 13).

Die weiterhin bestehende Verzerrung im unteren Temperatursummenbereich und für hohe Werte der Klimatischen Wasserbilanz und der Stickstoffdeposition kommt aufgrund der geringen Anzahl von Beobachtungen in diesen Wertebereichen zustande. Insgesamt kann nach der Korrektur der Eingangsdaten das StandortLeistungs-Modell weitgehend verzerrungsfreie Schätzungen für die drei Regionen Oberharz, Fläming und Colbitz-Letzlinger Heide bereitstellen.
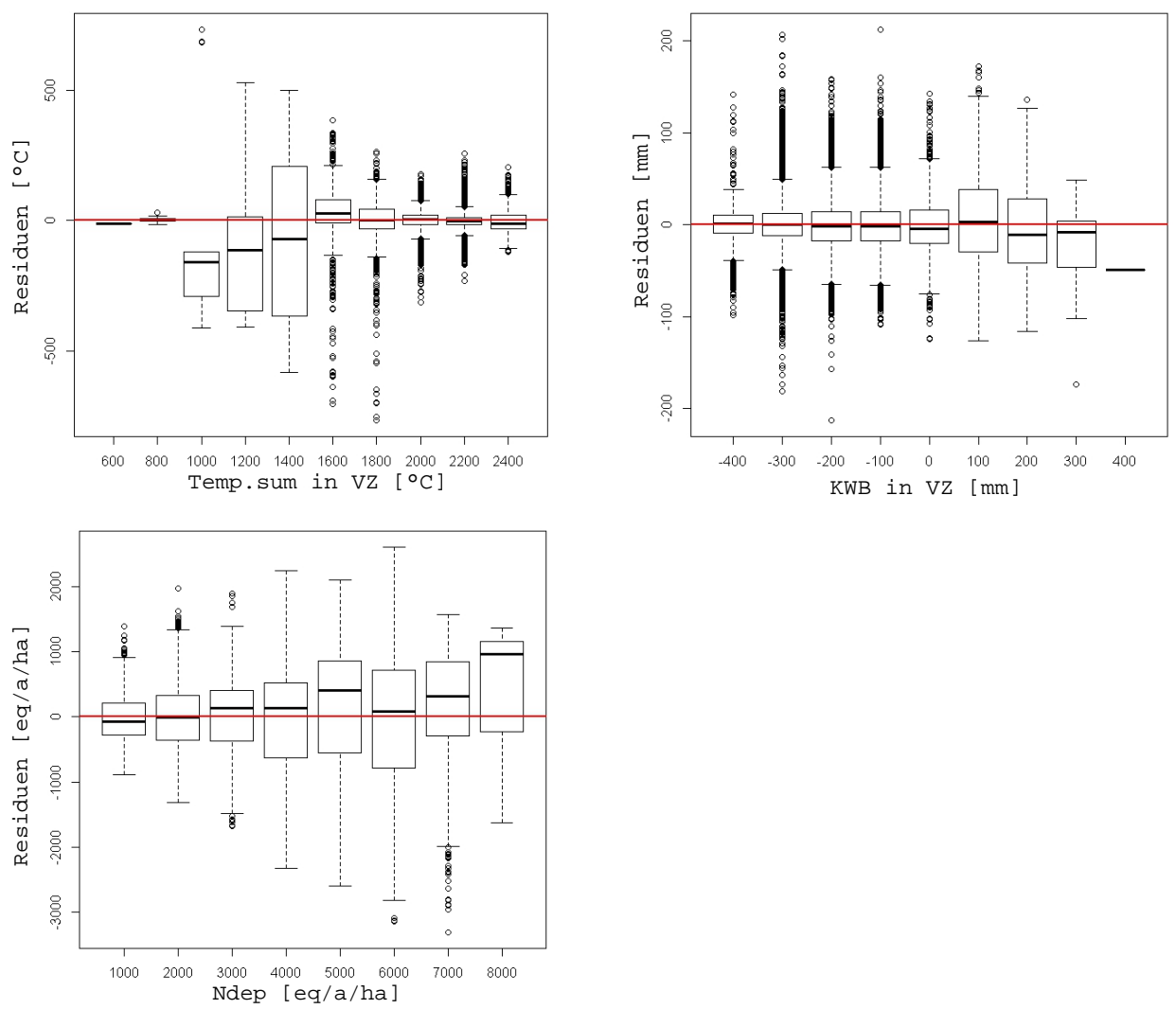

Abbildung 13: Boxplots der drei Modellparameter Temperatursumme in der Vegetationszeit (oben links; Temp.sum $\left[{ }^{\circ} \mathrm{C}\right]$ ), Klimatische $W$ asserbilanz (oben rechts; KWB $[\mathrm{mm}]$ ) und der Stickstoffdeposition (unten; Ndep [eq/a/ha]) nach der Biaskorrektur des Kiefern-Datensatzes 


\subsubsection{Projektion der Standortleistungsfähigkeit}

Das Standort-Leistungs-Modell schätzt den Erwartungswert der Standortsleistungsfähigkeit, definiert als Mittelwert der Höhenbonität, die eine Baumart hypothetisch nach 100 Jahren unter konstanten aktuellen oder zukünftigen Standortsbedingungen erreichen würde. Tatsächlich wird ein Bestand jedoch aufgrund u. a. sich verändernder Klimabedingungen ein variables Höhenwuchspotenzial über das Bestandesleben aufweisen. Ein Anwendungsbereich des Standort-Leistungs-Modells ist somit eine Abschätzung des standortspezifischen Wuchspotenzials verschiedener Baumarten unter Klimawandel, z. B. eine zeitliche Analyse der Veränderung des StandortLeistungs-Potenzials, eine Gegenüberstellung der projizierten Auswirkungen unterschiedlicher Klimaszenarien oder räumliche Analysen und Vergleiche von z. B. Standortsgruppen.

Zur Analyse der Standortleistungsfähigkeit unter Klimawandel werden Szenariosimulationen eingesetzt. Dabei gilt es, die Unsicherheiten zukünftiger Waldentwicklungen zu quantifizieren und abzuschätzen. Die Wahl des Klimaszenarios hat bei der Projektion der Standortleistungsfähigkeit einen entscheidenden Einfluss. In dieser Untersuchung wird das Klimaszenario A1B, projiziert mit dem regionalen Klimamodell WETTREG2010 (KREIENKAMP et al. 2012), verwendet. Für die Projektion der Standortleistungsfähigkeit wird die Variante 05 herangezogen, die im Mittel über alle Perioden eine mittlere Ausprägung bzgl. der Trockenheitsentwicklung aufweist (SUTMÖLLER et al. 2013). Die dem Standort-Leistungs-Modell übergebenen Werte der Klimaparameter werden wie folgt für die drei Projektionsperioden 2011 - 2040, 2041 - 2070 und 2071 - 2100 ermittelt:

$$
K_{x_{j}}=K_{D W D \_1971 \_2000_{j}}+\left(K_{\bmod _{-} X_{j}}-K_{\text {mod_1981_2010 }}\right)
$$

mit:

$K_{x j}=30$-jähriger Mittelwert des Klimaparameters in Projektionsperiode $x$ an Standort $j$

$K_{D W D \_1971 \_2000 j}=30$-jähriger Mittelwert basierend auf den korrigierten DWDMesswerten in der Referenzperiode 1971 - 2000 an Standort j

$\left(K_{\text {mod_Xj }}-K_{\text {mod_1981_2010j }}\right)=$ Signal des Klimaszenarios; Differenz des 30-jährigen Mittelwertes des Klimaparameters basierend auf der WETTREG2010-Projektion in Projektionsperiode $x$ an Standort $j$ und des 30-jährigen Mittelwertes des Klimaparameters basierend auf der WETTREG2010-Projektion in der Referenzperiode 1981 - 2010 an Standort j 
Die Klimabedingungen zum Status quo werden durch die korrigierten DWD-Messwerte der Referenzperiode 1971 - 2000 definiert. Die zukünftigen projizierten Klimabedingungen ergeben sich aus diesen DWD-Referenzwerten und dem Klimasignal der WETTREG2010-Projektion. Es besteht hierbei eine zeitliche Diskrepanz zwischen der DWD-Referenzperiode 1971 - 2000 und der WETTREG-Referenzperiode 1981 - 2010. Diese zeitliche Diskrepanz kann zu Verzerrungen bei den projizierten Standortleistungspotenzialen gegenüber dem geschätzten Status quo-Standortleistungspotenzial führen.

Für die konkreten Szenariosimulationen mit dem Standort-Leistungs-Modell verändern sich laut unterstelltem Klimaszenario die Temperatursummen und Klimatischen Wasserbilanzen der Standorte, während die übrigen erklärenden Modellvariablen als konstant angenommen werden. Der Boden wird zu Beginn der Vegetationszeit als wassergesättigt angesehen, die nutzbare Feldkapazität steht somit in vollem Umfang zur Verfügung. Abbildung 15 zeigt für die Level II-Flächen (Klötze, Nedlitz, Lange Bramke) einen deutlichen Rückgang der Gesamt-N-Depositionen. Würde dieser Rückgang direkt an das Standort-Leistungs-Modell weitergegeben, würden sich wahrscheinlich unrealistische Wachstumsreduzierungen ergeben. Hier muss die Beziehung zwischen den $\mathrm{C} / \mathrm{N}-$ Verhältnissen sowie der N-Deposition und dem Waldwachstum beachtet werden. Insbesondere die C/N-Verhältnisse im Oberboden haben sich unter dem Einfluss hoher atmosphärischer Stickstoffeinträge verändert. So konnten z. B. bei den Untersuchungen von BRINKMANN u. NIEDER (2002) in niedersächsischen Kiefernforsten in den Untersuchungsbeständen mit den höchsten N-Depositionen (Region Weser-Ems) auch die niedrigsten C/N-Verhältnisse in den L- und Of-Horizonten statistisch nachgewiesen werden. Die N-Vorräte waren in diesen Horizonten durchschnittlich um ca. $200 \mathrm{~kg} \mathrm{~N} \mathrm{ha}^{-1}$ gegenüber den anderen Untersuchungsgebieten in Niedersachsen erhöht. Von einer systematischen Verengung der C/N-Verhältnisse berichten auch die Langzeituntersuchungen von PRIETZEL et al. (1997) und ZEZSCHWITZ (1985). Letztlich hängt das Stickstoffangebot an einem Standort von der Stickstoffmineralisation, der Nitrifikation und der Stickstoffdeposition ab. Entsprechend wurde die Stickstoffdeposition als konservative Abschätzung für die zukünftigen Projektionen im Standort-Leistungs-Modell als konstant angenommen.

Die Szenariosimulation wird für alle forstlichen Teilflächen (Bestandeseinheiten) in den drei Regionen Oberharz, Fläming und Colbitz-Letzlinger Heide durchgeführt, für die vollständige Datensätze zur Verfügung stehen. Im Einzelnen sind dies 6.826 Waldflächen im Oberharz mit einer Gesamtfläche von 21.086 ha, im Fläming 7.991 Waldflächen (30.226 ha) und in der Colbitz-Letzlinger Heide 11.906 Waldflächen (40.615 ha). Tabelle 5 gibt einen Überblick über die Baumartenanteile. 
Tabelle 5: $\quad$ Flächenanteile der Baumartengruppen in den drei Regionen

\begin{tabular}{|c|c|c|c|c|c|c|}
\hline & Fläc & nanteil & er Baumar & tengruppe & {$[\%]^{1)}$} & NA2)/Blöße \\
\hline & Buche $^{3)}$ & Eiche & Fichte $^{4)}$ & Kiefer $^{5)}$ & Douglasie & \\
\hline Oberharz & 15,6 & 1,4 & 80,4 & 2,1 & 0,5 & 4,5 \\
\hline Fläming & 9,8 & 9,9 & 1,6 & 77,5 & 1,2 & 34,2 \\
\hline $\begin{array}{l}\text { Colbitz-Letzlinger } \\
\text { Heide }\end{array}$ & 5,2 & 6,8 & 1,3 & 85,5 & 1,2 & 23,0 \\
\hline $\begin{array}{l}\text { 1) Bei der Berechnung } \\
\text { artenanteile beziehe }\end{array}$ & $\begin{array}{l}\text { rd jeweils } \\
\text { ich auf de }\end{array}$ & $\begin{array}{l}\text { Hauptba } \\
\text { henant }\end{array}$ & $\begin{array}{l}\text { art die g } \\
\text { mit zur I }\end{array}$ & $\begin{array}{l}\text { te Fläch } \\
\text { gung ste }\end{array}$ & $\begin{array}{l}\text { er Teilfläche } \\
\text { den Baumar }\end{array}$ & $\begin{array}{l}\text { reordnet; die Baum } \\
\text { ngaben. }\end{array}$ \\
\hline 2) NA: keine Angabe & Ir Baumart fi & lie Teilflä & he verfügbar. & & & \\
\hline 3) Buche beinhaltet au & h ALh und $A$ & & & & & \\
\hline 4) Fichte beinhaltet au & Kodierung & onstiges I & idelholz“. & & & \\
\hline 5) Kiefer beinhaltet au & Lärche & & & & & \\
\hline
\end{tabular}

Das Standortleistungspotenzial für den Status quo und die drei Projektionsperioden wird für alle Teilflächen geschätzt. Zum einen werden dabei die real dort stockenden Baumarten als Grundlage genommen, zum anderen werden für einen Baumartenvergleich hypothetisch alle Flächen jeweils einer Baumart zugeordnet. Für eine bessere Vergleichbarkeit der Baumarten wird die Wuchsleistung anhand der Leistungsklasse (durchschnittlicher Gesamtzuwachs zum Zeitpunkt der Kulmination $\left.d G Z_{\text {max }}\right)$ charakterisiert. Die vom Standort-Leistungs-Modell geschätzten Mittelhöhenbonitäten im Alter 100 (hg100) werden mit Hilfe folgender Übergangsfunktionen, die aus Ertragstafelwerten (SCHOBER 1987) hergeleitet wurden, umgerechnet:

$$
d G Z_{\max }=a_{1} \cdot(h g 100)^{2}+a_{2} \cdot(h g 100)
$$

Die baumartspezifischen Modellkoeffizienten und das Bestimmtheitsmaß für die Übergangsfunktion (4) sind in Tabelle 6 aufgelistet.

Tabelle 6: $\quad$ Baumartsperifische Modellkoeffizienten und Modellgüte für die Übergangsfunktion (4)

\begin{tabular}{lrrrrr}
\hline & Buche & Eiche & Fichte & Kiefer & Douglasie \\
\hline$a_{1}$ & 0,0038 & 0,0107 & 0,0078 & 0,0071 & 0,0099 \\
$a_{2}$ & 0,157 & $-0,016$ & 0,1034 & 0,0902 & $-0,0297$ \\
$\mathrm{R}_{\text {adj }}$ & 0,998 & 0,999 & 0,999 & 0,999 & 0,999 \\
\hline
\end{tabular}


Die Kenntnis der Werte der Modellparameter und der Entwicklung der klimatischen Größen in den drei Projektionsperioden ermöglicht eine bessere Interpretierbarkeit der mit dem Standort-Leistungs-Modell geschätzten heutigen und zukünftigen Wuchspotenziale zwischen den Baumarten und zwischen den drei Regionen.

Der Oberharz ist die kühlste und feuchteste Region. Die Böden weisen die geringste mittlere nutzbare Feldkapazität auf, sind aber zu 95\% Standorte mindestens mittlerer Nährstoffversorgung. Die Stickstoffdepositionsraten sind in der Region Oberharz am höchsten. Ebenso weist der Fläming mit einem sehr hohen Eintrag in der Nähe von Dessau eine hohe mittlere Depositionsrate auf. Und in der Colbitz-Letzlinger Heide ist der Stickstoffeintrag insgesamt am geringsten. Die Region Fläming weist die höchsten Temperaturen und größte Trockenheit auf. Bezüglich der nutzbaren Feldkapazität und der Bodennährstoffe bestehen zwischen Fläming und Colbitz-Letzlinger Heide nur geringe Unterschiede. Tabelle 7 weist die Kennwerte für die drei Regionen aus.

Tabelle 7: $\quad$ Kennwerte der Modellparameter in den drei Regionen Oberbary, Fläming, ColbitzLetzlinger Heide

\begin{tabular}{|c|c|c|c|c|}
\hline & & Oberharz & Fläming & $\begin{array}{c}\text { Colbitz-Letzlinger } \\
\text { Heide }\end{array}$ \\
\hline \multicolumn{2}{|l|}{ Modellparameter } & $\begin{array}{c}\text { 1. Quantil/Mittelw./ } \\
\text { 3. Quantil }\end{array}$ & $\begin{array}{c}\text { 1. Quantil/Mittelw./ } \\
\text { 3. Quantil }\end{array}$ & $\begin{array}{c}\text { 1. Quantil/Mittelw./ } \\
\text { 3. Quantil }\end{array}$ \\
\hline \multirow{4}{*}{$\begin{array}{l}\text { Temp.sum }\left[{ }^{\circ} \mathrm{C}\right] \\
\text { in der Veg.zeit }\end{array}$} & $1981-2010$ & 1724 / 1819 / 1904 & 2377 / 2404 / 2427 & 2319 / $2332 / 2346$ \\
\hline & $2011-2040$ & 1994 / 2050 / 2099 & 2551 / 2577 / 2602 & $2501 / 2512$ / 2524 \\
\hline & $2041-2070$ & 2281 / 2338 / 2381 & 2811 / 2838 / 2912 & 2754 / 2766 / 2777 \\
\hline & $2071-2100$ & $2521 / 2572$ / 2618 & 2972 / 2999 / 3025 & 2918 / 2928 / 2939 \\
\hline \multirow{4}{*}{$\begin{array}{l}\text { KWB [mm] in } \\
\text { der Veg.zeit }\end{array}$} & $1981-2010$ & $12 / 37 / 59$ & $-203 /-191 /-179$ & $-182 /-172 /-162$ \\
\hline & $2011-2040$ & $-72 /-47 /-18$ & $-254 /-241 /-227$ & $-229 /-221 /-212$ \\
\hline & $2041-2070$ & $-175 /-156 /-140$ & $-385 /-366 /-348$ & $-345 /-337 /-330$ \\
\hline & $2071-2100$ & $-255 /-241 /-227$ & $-437 /-418 /-400$ & $-400 /-395 /-389$ \\
\hline \multirow[t]{2}{*}{ Ndep [eq/a/ha] } & Nadelholz & $2430 / 2612 / 2770$ & 1874 / 2026 / 2126 & 1776 / 1865 / 1962 \\
\hline & Laubholz & 2373 / 2566 / 2736 & 1747 / 1881 / 1977 & 1647 / 1734 / 1831 \\
\hline $\mathrm{nFK}[\mathrm{mm}]$ & & $110 / 118$ / 125 & $126 / 137 / 146$ & $102 / 131 / 146$ \\
\hline \multirow[t]{3}{*}{ Nähr'1) } & 1 & \multirow[b]{2}{*}{$35 \%$} & \multirow[b]{2}{*}{\} $18 \%$} & \multirow[b]{2}{*}{$322 \%$} \\
\hline & 2 & & & \\
\hline & $\begin{array}{l}3 \\
4\end{array}$ & \} $95 \%$ & $382 \%$ & $378 \%$ \\
\hline
\end{tabular}

1) Die Nährstoffversorgung wurde in vier kategorische Klassen eingeteilt von $1=$ schlechteste Nährstoffversorgung bis 4 = beste Nährstoffversorgung. 


\subsubsection{Bewertung des Standort-Leistungs-Modells}

Die Funktionalitäten und Limitationen des klimasensitiven Standort-LeistungsModells wurden in Validierungsstudien ausführlich beschrieben (ALBERT $u$. SCHMIDT 2012, 2010b). Zum besseren Verständnis der in den nachfolgenden Kapiteln beschriebenen Ergebnisse in den drei Regionen Oberharz, Fläming und Colbitz-Letzlinger Heide wird an dieser Stelle die Besonderheit des für die Baumart Douglasie parametrisierten Standort-Leistungs-Modells betont. Das Douglasienmodell weist im Vergleich zu den anderen Baumarten Buche, Eiche, Fichte und Kiefer mit 4750 Beobachtungen bei weitem die geringste Datengrundlage auf. Die Effekte der erklärenden Modellparameter haben somit einen breiten Konfidenzbereich. Die Abdeckung der schon heute in Sachsen-Anhalt auftretenden Parameterwertebereiche durch den Parametrisierungsdatensatz ist ebenfalls eingeschränkt. Folglich sind die Ergebnisse des Douglasienmodells im Vergleich zu den Schätzungen anderer Baumarten mit größeren Unsicherheiten und Fehlern behaftet. Besonders im Extrapolationsbereich des Modells können unplausible Schätzungen der Standortleistungsfähigkeit resultieren.

Beleuchtet man im Vorgriff auf die unten ausführlich dargestellten Ergebnisse die Frage näher, warum die Standortleistungsfähigkeit für die Douglasie in der Colbitz-Letzlinger Heide tendenziell höher geschätzt wird als im Fläming, so wirkt sich der räumliche Trend für die Douglasie im Fläming reduzierend auf die Standortleistungsfähigkeit aus (im Mittel ein Effekt von -1,25 m), während in der ColbitzLetzlinger Heide der räumliche Trend positiv ist (im Mittel +4,3 m). Für die Buche und auch die Baumarten Eiche und Kiefer ist die Wirkung des räumlichen Trends in den beiden Regionen genau umgekehrt mit einem mittleren Effekt für die Buche von $+0,7 \mathrm{~m}$ im Fläming und -3,3 $\mathrm{m}$ in der Colbitz-Letzlinger Heide. Die Wirkung des räumlichen Trends in den beiden klimatisch ähnlichen Regionen kann somit als eine Ursache für diese gegensätzlichen Ergebnisse der Standortleistungsfähigkeit der Douglasie im Vergleich zu den Baumarten Buche, Eiche und Kiefer zwischen den beiden Regionen gesehen werden.

Des Weiteren erscheinen die absoluten Schätzwerte der Standortleistungsfähigkeit der Douglasie recht hoch. Die Temperatursumme in der Vegetationszeit liegt in den beiden Regionen Fläming und Colbitz-Letzlinger Heide bereits heute größtenteils im Extrapolationsbereich des Douglasienmodells. Der Temperatureffekt beeinflusst die geschätzte Standortleistungsfähigkeit maßgeblich und kann somit zu einer Überschätzung der dGZ $Z_{\max }$-Werte der Douglasie führen. Die relative Entwicklung der projizierten Standortleistungsfähigkeit über der Zeit bleibt davon weitestgehend unberührt. Eine Verbesserung bzw. Verschlechterung der ertragskundlichen Bedingungen für den Douglasienanbau in zukünftigen Perioden kann somit aus den Ergebnissen abgeleitet werden. 


\subsection{Veränderung der Stickstoffeinträge}

\subsubsection{Datengrundlagen}

Die Nährstoffversorgung ist neben dem Wasserhaushalt die wichtigste lokale Standortskomponente. Waldökosysteme in Mitteleuropa sind seit mehreren Jahrzehnten hohen atmosphärischen Stoffeinträgen ausgesetzt. Durch Maßnahmen zur Luftreinhaltung sind die Säureeinträge in den letzten Jahren stark zurückgegangen (FOWLER et al. 2007). Anders verhält es sich bei den atmosphärischen Einträgen von reduzierten und oxidierten Stickstoffverbindungen, die nach wie vor einen sehr hohen Stand aufweisen. Somit sind die atmosphärischen Stickstoffdepositionen mittlerweile neben dem Klimawandel zu einer der Hauptgefährdungen für die Funktionalität und Stabilität der Waldökosysteme geworden (GLATZEL et al. 1987, NILSEN 1990, MATSON et al. 2002). Als weitere unerwünschte Effekte sind die Verschiebung des Artenspektrums in der Bodenvegetation (ANDERS et al. 2002, SVERDRUP et al. 2007) und insbesondere die Austräge von Nitrat mit dem Sickerwasser (Gewässereutrophierung und -versauerung) (DISE u. WRIGHT 1995, AHRENDS et al. 2010) zu nennen.

Neben Störungen und Schädigungen der Waldökosysteme berichten zahlreiche Untersuchungen von Wachstumssteigerungen durch die atmosphärischen Stickstoffeinträge (KENK u. FISCHER 1988, NILSSON u. WIKLUND 1992, SPIECKER et al. 1996, AlBERT u. SCHMidT 2010a). Entsprechend sind bei der Modellierung des Waldwachstums die Stickstoffeinträge durch die atmosphärische Deposition von zentraler Bedeutung. So haben z. B. die Untersuchungen von LAUBHANN et al. (2009) gezeigt, dass aus einer Erhöhung der N-Deposition um $1 \mathrm{~kg} \mathrm{ha}^{-1} \mathrm{a}^{-1}$ in Abhängigkeit von der Baumart eine Zunahme des Grundflächenzuwachses zwischen $1,2 \%$ und $1,49 \%$ resultieren kann.

Da das Standort-Leistungs-Modell anhand der bundesweiten Daten der Bundeswaldinventur (BWI2) parametrisiert wurde, sind bundesweite Datengrundlagen erforderlich. Flächendeckende und räumlich detaillierte Eingangsdaten von atmosphärischen Stickstoffeinträgen in Deutschland liefern die Daten des Umweltbundesamtes (BUILTJES et al. 2011). Diese Daten werden mit einer modifizierten Version (AHRENDS et al. 2007; 2010) des Modells MAKEDEP von ALVETEG et al. (1998) gekoppelt. Beispiele für die Anwendung dieses gekoppelten Modellsystems sind bei AHrends et al. (2007, 2008, 2009, 2010), AlBERT u. SCHMidt (2010a), HAUCK et al. (2012) und JANSEN et al. (2007) zu finden. Durch Verbesserungen der Methode zur Berechnung der trockenen Deposition (Erhaltung der Massenbilanz; veränderte Parametrisierung der atmosphärischen Widerstände) unterscheiden sich die Depositionsfrachten der Datensätze von BUILTJES et al. (2011) und GAUGER et al. (2008). Da das empirische Waldwachstumsmodells von ALBERT u. SCHMIDT (2010a) mit den Daten von GAUGER et al. (2008) parametrisiert wurde, wurde eine 
Korrekturfunktion für die aktuellen Depositionsdaten des Umweltbundesamtes entwickelt (vgl. Kap. 4.3).

Abbildung 14 zeigt die räumliche Auflösung des EMEP-Rasters und die atmosphärischen Stickstoffeinträge für das Jahr 2007 in Sachsen-Anhalt.
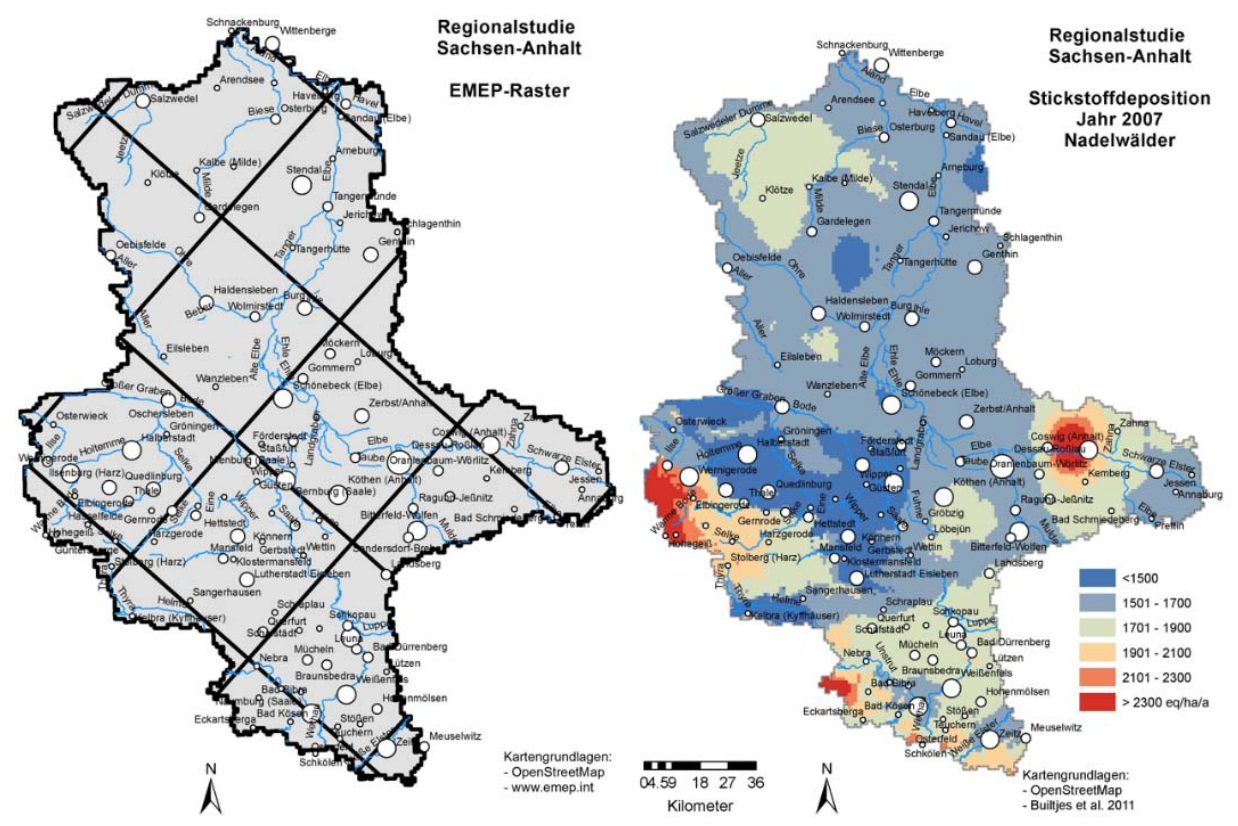

Abbildung 14: Räumliche Auflösung der EMEP-Daten (links) und regionale Verteilung der Stickstoffdepositionen im Jahr 2007 für Nadelwälder nach BUILTJES et al. (2011); (Kartengrundlagen: (C) OpenStreetMap-Mitwirkende, ODbL (www.openstreetmap.org/copy right))

\subsubsection{Depositionsszenarien}

Die Depositionsdaten von BUILTJES et al. (2011) decken nur einen relativ kurzen Zeitraum ab. Daher ist es notwendig, zeitliche Extrapolationsverfahren für die Stoffdeposition mit den Daten zu kombinieren. Dieses umfasst sowohl die Rekonstruktion der historischen Inputkurven, als auch die Entwicklung von Szenarien für die zukünftige Entwicklung der Deposition von Stickstoffkomponenten.

Für die Konstruktion von einheitlichen Depositionszeitreihen wurde für den Zeitraum von 1980 bis 2006 auf die EMEP-Daten (Co-operative Programme for Monitoring and Evaluation of the Long-range Transmission of Air Pollutants in Europe) zurückgegriffen (http://www.emep.int) und aus ihnen Kurven der relativen Deposition berechnet. Zur Berechnung der Deposition vor 1980 wurden historische Zeitreihen herangezogen (AlvETEG et al. 1998, SCHÖPP et al. 2003). Die 
Verwendung dieser einheitlichen Rekonstruktionsgrundlage ist daher von Bedeutung, da mit Hilfe dieser Methodik auch das klimasensitive Waldwachstumsmodell (ALBERT u. SCHMIDT 2010a) für ganz Deutschland parametrisiert wurde. Die Berechnung der relativen Deposition wurde wie folgt durchgeführt:

$$
D E P_{t}=D E P_{R J} \cdot\left(\frac{E M E P_{t}}{E M E P_{R J}}\right)
$$

mit:

$\operatorname{DEP}_{\mathrm{t}}=$ Deposition zum Zeitpunkt $\mathrm{t}\left[\mathrm{eq} \mathrm{m}^{-2} \mathrm{a}^{-1}\right]$

$\operatorname{DEP}_{\mathrm{RJ}}=$ Deposition im Referenzjahr $\left[\right.$ eq $\left.\mathrm{m}^{-2} \mathrm{a}^{-1}\right]$

EMEP $_{\mathrm{t}}:=$ EMEP Deposition zum Zeitpunkt $\mathrm{t}\left[\right.$ eq $\left.\mathrm{m}^{-2} \mathrm{a}^{-1}\right]$

EMEP $_{\mathrm{RJ}}:=$ EMEP Deposition im Referenzjahr $\left[\right.$ eq $\left.\mathrm{m}^{-2} \mathrm{a}^{-1}\right]$

Diese Art der Skalierung von gemessenen Daten mit dem Verhältnis (oder der Differenz) von modellierten Daten wird häufig in der Meteorologie zur Korrektur von Klimadaten angewendet, um Konsistenz mit den gemessenen Daten zu erzeugen (PIANI et al. 2010, MUDELSEE et al. 2010).

Als Referenzjahr wurde für den Datensatz von BuILTJES et al. (2011) (Zeitraum 2004 - 2007) das Jahr 2005 ausgewählt und für dieses Jahr eine mittlere Deposition berechnet. Dieser Berechnungsansatz macht die Skalierung robuster gegenüber einzelnen jährlichen Schwankungen in den modellierten Depositionsdaten. Um die Übertragbarkeit dieses Ansatzes auf Sachsen-Anhalt zu gewährleisten, wurde zunächst untersucht, ob die Depositionstrends in den EMEP-Daten mit denen auf den Level II-Flächen des forstlichen Umweltmonitorings vergleichbar sind. Abbildung 15 zeigt beispielhaft diesen Vergleich für die Level II-Flächen in SachsenAnhalt (Nedlitz, Klötze) und drei Level II-Flächen im niedersächsischen Teil des Harzes (Lange Bramke Nordhang/Südhang/Kamm).

Die angenommenen Projektionen der zukünftige Depositionsentwicklungen basieren auf den Auswertungen von AMANN et al. (2008). Tabelle 8 zeigt die Projektionen für die Emissionsentwicklung der Schadstoffe $\mathrm{SO}_{2}, \mathrm{NO}_{\mathrm{X}}$ und $\mathrm{NH}_{3}$ für das Jahr 2020 unter verschiedenen gesellschaftlichen und politischen Rahmenbedingungen. Für die Berechnungen der Deposition wurde das „wahrscheinlichste“ Szenario der gegenwärtigen Politik (CP) ausgewählt.

Tabelle 8: $\quad$ Szenarien der Emissionsentwicklung in Deutschland bis zum Jahr 2020. Das Bezugsjahr für die prozentuale Reduktion ist 2000 (AMANN et al. 2008).

\begin{tabular}{lrrr}
\hline Szenario & $\mathrm{SO}_{2}[\%]$ & $\mathrm{NO}_{\mathrm{x}}[\%]$ & $\mathrm{NH}_{3}[\%]$ \\
\hline CLE (gegenwärtige Gesetzgebung) & -31.9 & -48.1 & -5.6 \\
CP (gegenwärtige Politik) & -36.0 & -54.9 & -10.0 \\
MRR (maximale Reduktion) & -44.6 & -63.3 & -46.3 \\
\hline
\end{tabular}



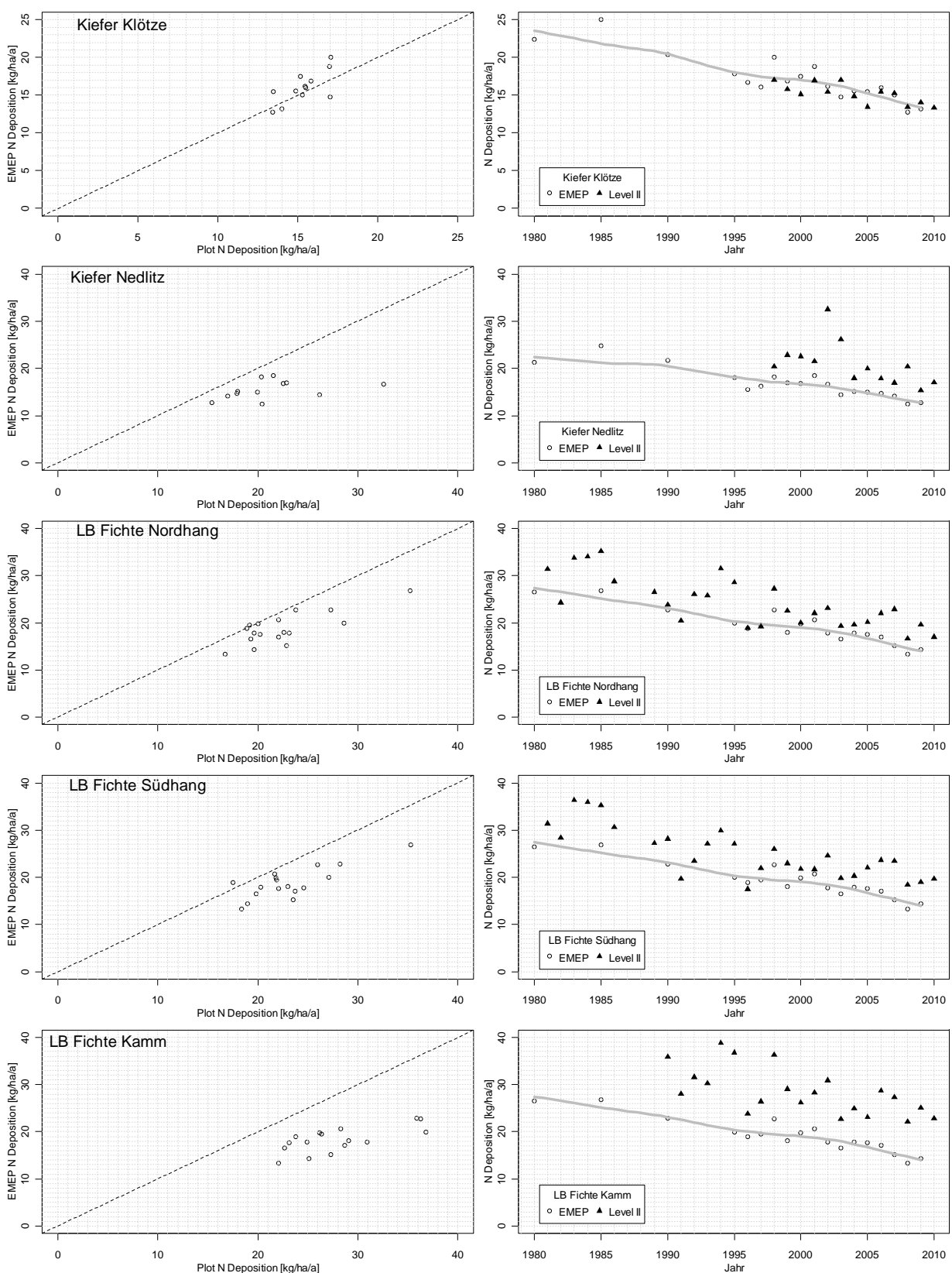

Abbildung 15: Vergleich der Gesamt-N-Deposition des EMEP-Datensatzes mit der Gesamt-N-Deposition auf den Level II-Flächen des forstlichen Umweltmonitorings (berechnet nach ULRICH 1994)

Beiträge aus der NW-FVA, Band 13, 2015 


\subsection{Grundwasserflurabstände}

Zur Initialisierung des longitudinalen Höhen-Durchmesser-Modells wird der Grundwasserflurabstand in der Vegetationsperiode benötigt. Entsprechend wurde aus der Bodenkarte für Sachsen-Anhalt (VBK 50 im Maßstab 1:50.000) der mittlere Grundwassertiefstand (MNGW) verwendet. Dieser beschreibt den Grundwassertiefstand im langjährigen Mittel (meist Juli bis Oktober) und somit ungefähr die Verhältnisse in der Vegetationsperiode. Die Grundwasserstufen (GWS) wurden wie folgt klassifiziert:

$$
G W S=\left\{\begin{array}{cc}
0 & \text { wenn } M N G W>25 d m \\
2 & \text { wenn } M N G W<9,5 d m \\
1 & \text { andernfalls }
\end{array}\right.
$$

\subsection{Methodik der Prognose des Sturmschadensrisikos}

Sturmschäden und die häufig durch sie ausgelösten Borkenkäferkalamitäten sind seit jeher die bedeutendsten Risikofaktoren für die Forstwirtschaft. Sie verursachen durch Holzentwertung, erhöhte Erntekosten und Hiebsunreifeverluste sowie die bei hohen Kalamitätsnutzungen entstehenden Holzmarktstörungen erhebliche wirtschaftliche Schäden (SCHELHAAS et al. 2003). Im Zusammenhang mit der Klimaerwärmung wird teilweise mit einem weiter steigenden Sturmrisiko und möglicherweise auch einer erhöhten Frequenz schwerer Winterstürme in West- und Mitteleuropa gerechnet. Selbst bei Annahme einer gleich bleibenden Intensität besteht seitens der Praxis eine steigende Nachfrage nach Entscheidungshilfen, um das Risikopotenzial durch Winterstürme als einen weiteren Aspekt standortsgerechten Waldbaus besser berücksichtigen zu können. Vor diesem Hintergrund erfolgt eine Prognose der aktuellen und potenziellen Vulnerabilität des Waldes gegenüber Winterstürmen.

\subsubsection{Datengrundlage}

\subsubsection{Baumhöhenschätzung bei Zielstärke als Eingangsgröße zur Sturmschadens- modellierung}

Als Datenbasis für eine Einschätzung der Vulnerabilität des Waldes in den drei Modellregionen gegenüber Winterstürmen werden Informationen über die Bestockung benötigt. Dabei geht die Struktur der Bestände vereinfachend über den Brusthöhendurchmesser (BHD) und die Baumhöhe von zielstarken Bäumen bzw. von Grundflächenmittelstämmen als Prädiktor in das Sturmschadensmodell ein. Darauf aufbauend werden zwei unterschiedliche Arten von Gefährdungsprognosen durchgeführt: 
1. Potenzielle Vulnerabilität: Einschätzung des potenziellen Sturmschadensrisikos von zielstarken Bäumen für die Baumarten Fichte, Douglasie, Kiefer, Buche und Eiche.

2. Aktuelle Vulnerabilität: Einschätzung des Sturmschadensrisikos der Baumarten im Hauptbestand der aktuellen Bestockung anhand von Grundflächenmittelstämmen.

Die Prognose des potenziellen Risikos bei Erreichen der Zielstärke liefert Informationen für die langfristige Waldbauplanung und hier vor allem für die Baumartenwahl. Für eine möglichst realitätsnahe Prognose des Risikopotenzials ist dabei eine standortsensitive Modellierung der Baumhöhe notwendig. Zu diesem Zweck war es erforderlich, in einem ersten Schritt das Alter bei Erreichen der Zielstärke in Abhängigkeit von den Standortseigenschaften zu prognostizieren. Als Zielstärken wurden die folgenden Werte im Anhalt an die „Clusterstudie Forst und Holz SachsenAnhalt" (Rüther et al. 2008) verwendet:

- Eiche: $65 \mathrm{~cm}$

- Buche: $55 \mathrm{~cm}$

- Fichte: $45 \mathrm{~cm}$

- Douglasie: $55 \mathrm{~cm}$

- Kiefer: $45 \mathrm{~cm}$

Für die standortsensitive Schätzung des Alters bei Zielstärke wurde ein verallgemeinertes additives Regressionsmodell GAM genutzt (WOOD 2006, 2011). Die Datengrundlage des Modells sind alle erstmaligen Betriebsinventuren in Niedersachsen. In Sachsen-Anhalt steht keine der niedersächsischen Betriebsinventur vergleichbare Datenbasis (ca. 50.000 konzentrische Stichprobenpunkte) zur Verfügung, so dass das in Niedersachsen parametrisierte Modell verwendet wurde:

$$
\begin{aligned}
& \log \left\{E\left(A l t \_Z s t_{i j}\right)\right\}=f_{1}\left(B H D_{i j}\right)+f_{2}\left(n F K_{j}\right) Z_{j}+f_{3}\left(H N N_{j}\right)+G W \_ \text {class }{ }^{T} \beta_{1} \\
& +N Z \text { _classj }{ }^{T} \beta_{2}+f_{4}\left(\text { Rechtswert }{ }_{j} \text {, Hochwert }\right) \\
& \text { Alt_Zstij } \sim \text { Gamma } \\
& E\left(A l t Z Z s t_{i j}\right) \quad=\text { Erwartungswert des Alters von Baum } i \text { in Bestand } j \\
& \mathrm{BHD}_{i j} \quad=\mathrm{BHD} \text { von Baum } i \text { in Bestand } j \\
& n \mathrm{FK}_{j} \quad=\text { Flächengewichtete nutzbare Feldkapazität in Bestand } j \\
& Z_{i} \quad=\text { Kategorische Variable zur Definition des Grundwassereinflusses in } \\
& \text { Bestand } k \text { mit den Kategorien: „Grundwassereinfluss“, „kein } \\
& \text { Grundwassereinfluss“ } \\
& H \mathrm{HN}_{j} \quad=\text { Seehöhe des Zentroides von Bestand } j
\end{aligned}
$$




\begin{tabular}{|c|c|c|}
\hline$G W_{-}$class $_{j}$ & $=$ & $\begin{array}{l}\text { Vektor der kategorischen Variable zur Definition des Grundwasser- } \\
\text { einflusses in Bestand } j \text { mit den Kategorien: } \\
\text { kein Grundwassereinfluss: Flurabstand }>2,5 \mathrm{~m} \text {, } \\
\text { schwach - mäßiger Grundwassereinfluss: Flurabstand 2,5-0,95 m, } \\
\text { mittlerer - starker Grundwassereinfluss: Flurabstand }<0,95 \mathrm{~m} \text {. }\end{array}$ \\
\hline$N Z \_$class $j$ & $=$ & $\begin{array}{l}\text { Vektor der kategorischen Variable zur Definition der Nährstoffaus- } \\
\text { stattung in Bestand } j \text { auf der Grundlage der Ansprache der Nähr- } \\
\text { stoffziffer inklusive Variante nach der niedersächsischen Standorts- } \\
\text { kartierung. }\end{array}$ \\
\hline $\mathrm{RW}_{j}, \mathrm{HW}_{j}$ & $=$ & $\begin{array}{l}\text { Rechts- und Hochwert des Zentroides von Bestand } j \text { im Gauss- } \\
\text { Krüger-Koordinatensystem }\end{array}$ \\
\hline$f_{1}, f_{2}, f_{3}$, & $=$ & $\begin{array}{l}\text { Eindimensionale glättende Regressionssplines zur Erfassung nicht- } \\
\text { linearer Effekte metrischer Kovariablen }\end{array}$ \\
\hline$f_{4}$ & $=$ & $\begin{array}{l}\text { Zweidimensionaler glättender Regressionsspline zur Erfassung } \\
\text { räumlicher Autokorrelation }\end{array}$ \\
\hline$\beta_{1}, \beta_{2}$ & $=$ & Koeffizientenvektoren \\
\hline
\end{tabular}

Spezifische Modelle liegen für die Baumarten(gruppen) Eiche, Buche, ALh (Anderes Laubholz mit hoher Umtriebszeit), ALn (Anderes Laubholz mit niedriger Umtriebszeit), Fichte, Douglasie, Kiefer und Lärche vor. Da das Modell der Schätzung des Alters bei Erreichen der Zielstärke dient, wurden nur Bäume mit mindestens $30 \mathrm{~cm}$ BHD bei der Modellentwicklung verwendet. Beschrieben ist das Modell mit der maximalen Anzahl an Kovariablen, wie es z. B. für die Buche parametrisiert wurde (s. Gl. 7). Teilweise sind bei anderen Baumarten nicht alle dargestellten Modelleffekte signifikant. Die Nährstoffziffer und die Kategorie des Grundwassereinflusses wurden nach sachlogischen und statistischen Gesichtspunkten baumartenspezifisch zu Klassen zusammengefasst. Für die Anwendung in Sachsen-Anhalt wurde die Nährstoffziffer entsprechend der niedersächsischen Standortskartierung verschlüsselt. Die Übersetzung erfolgte nach SCHMIDT et al. (2015). In den Fällen, in denen mehrere Standortstypen innerhalb eines Bestandes auftraten, wurde die nutzbare Feldkapazität als flächengewichtetes Mittel berechnet (s. Kap. 4.1).

Das Modell berücksichtigt durch den räumlichen Trend $f_{4}$ regionale Unterschiede in Niedersachen, die sich nicht über kausale Variablen beschreiben lassen. Bei der Modellanwendung in Sachsen-Anhalt befindet man sich somit geografisch betrachtet im Extrapolationsbereich. Zur Prognose wurden daher Varianten der räumlich expliziten Modelle verwendet, in denen der Effekt der räumlichen Lage nicht berücksichtigt wird. Die Schätzungen mit diesen Modellen entsprechen damit mittleren bedingten Erwartungswerten, die auf dem jeweiligen Standort in Niedersachsen zu erwarten wären. Die Darstellung der resultierenden Alter bei Zielstärke über der Seehöhe spiegelt die unterschiedlichen Wärmebedürfnisse der Baumarten wider (s. Abb. 16 und Abb. 17). Die Seehöhenstufen sind hier und in allen weiteren Auswertungen durch ihre obere Grenze definiert. 


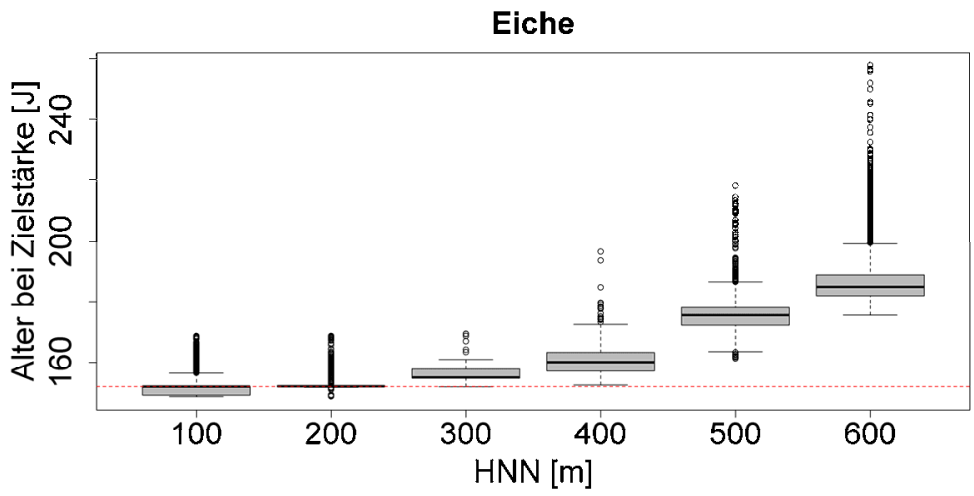

Buche

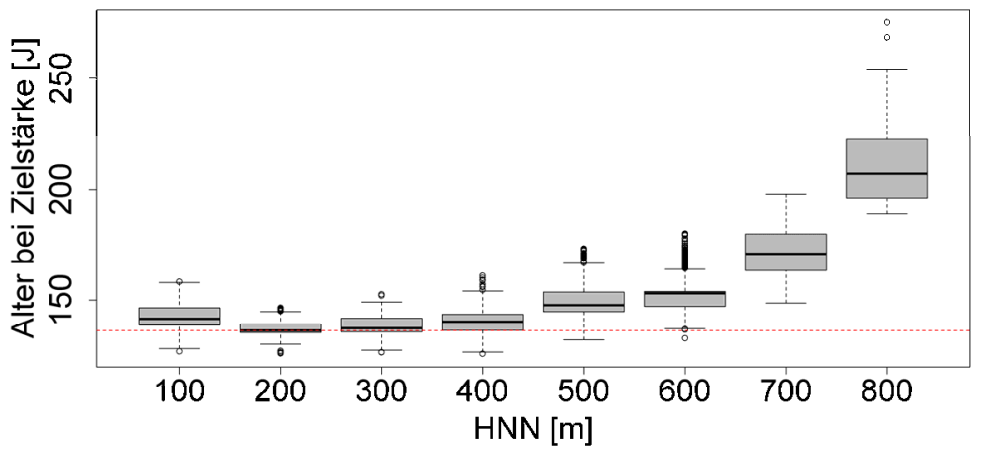

Fichte

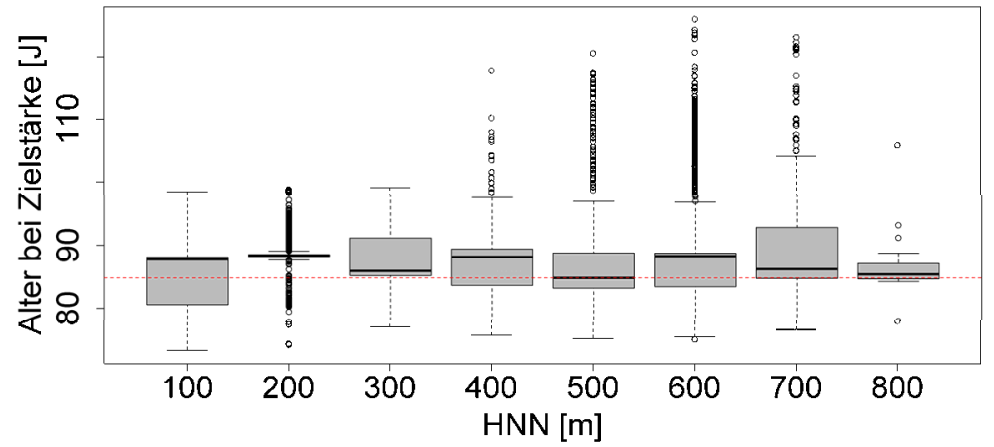

Abbildung 16: Verteilungen des prognostizierten Alters bei Zielstärke [J] über der Seeböhe HNN [m] für Eiche, Buche und Fichte

Während Eiche und Buche einen zunehmenden Trend über der Seehöhe zeigen, weisen Fichte, Douglasie und Kiefer kaum einen Effekt der Seehöhe auf. Allerdings treten bei der Douglasie in der Colbitz-Letzlinger Heide und bei der Kiefer in der Colbitz-Letzlinger Heide und im Fläming im Mittel höhere Alter bei Zielstärke auf 
als im Harz. Für Eiche, Douglasie und Kiefer ist der dargestellte Wertebereich auf unter $600 \mathrm{~m}$ beschränkt, wobei die Schätzungen für diese Baumarten bereits ab ca. $400 \mathrm{~m}$ im Extrapolationsbereich liegen. Dabei liegt der Großteil der Bestände unterhalb von $100 \mathrm{~m}$ in der Region Colbitz-Letzlinger Heide, während der Großteil der Bestände der Höhenstufe $200 \mathrm{~m}$ in der Region Fläming liegt. Die Werte ab der Höhenstufe $300 \mathrm{~m}$ stammen ausschließlich aus der Region Oberharz. Die bedingte Streuung innerhalb der Höhenstufen resultiert aus den Standortseffekten, die nicht über den Höhengradienten erfasst werden.
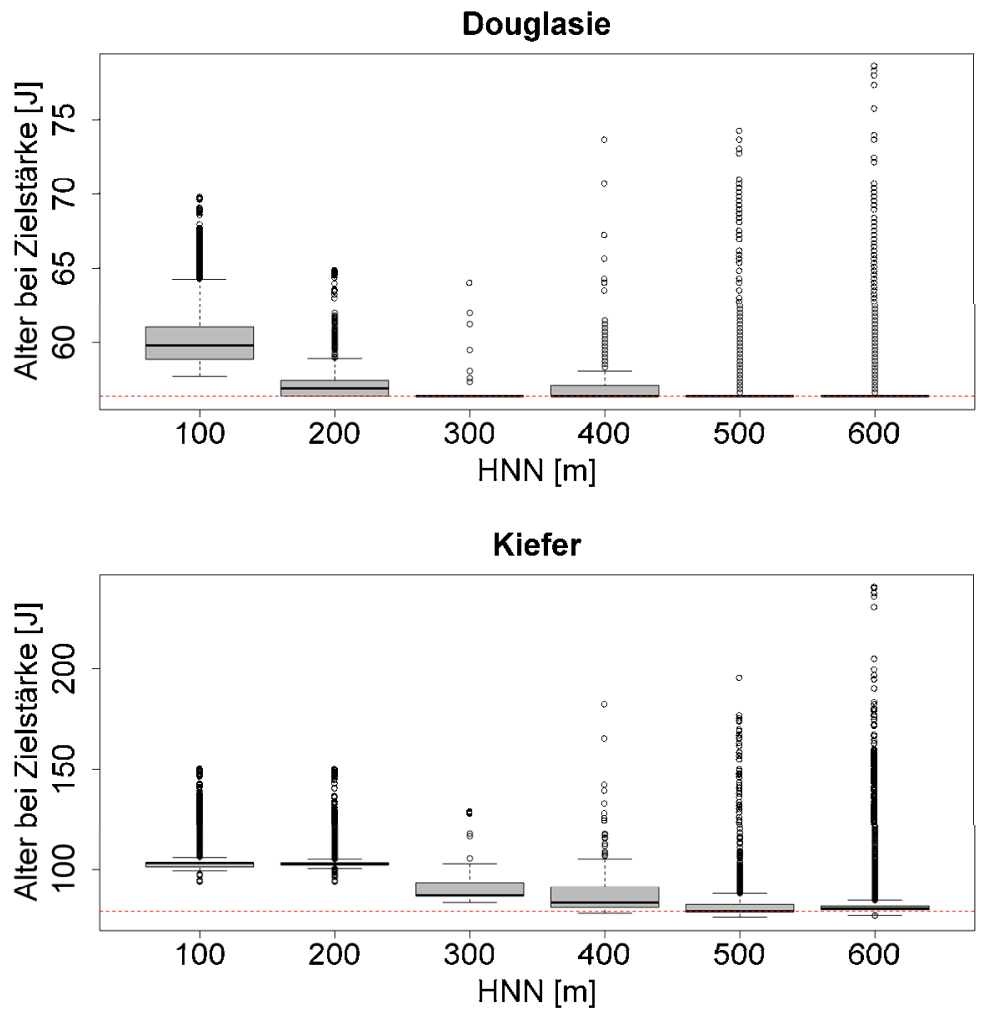

Abbildung 17: Verteilungen des prognostizierten Alters bei Zielstärke [J] über der Seeböhe HNN [m] für Douglasie und Kiefer

Die gesamte Spreitung der Alter bei Zielstärke spiegelt die Effekte der unterschiedlichen Standortsbedingungen über die drei Modellregionen hinweg wider (s. Tab. 9). Insgesamt zeigen Eiche, Buche und Kiefer eine größere Variabilität der prognostizierten Alter, wobei bei der Interpretation der unterschiedliche Ausschnitt des betrachteten Seehöhenbereiches zu beachten ist. 
Tabelle 9: $\quad$ Perzentile der Verteilungen des prognostizierten Alters bei Zielstärke für die Baumarten Eiche $(65 \mathrm{~cm})$, Buche $(55 \mathrm{~cm})$, Fichte $(45 \mathrm{~cm})$, Douglasie $(55 \mathrm{~cm})$ und Kiefer $(45 \mathrm{~cm})$. Für Eiche, Douglasie und Kiefer werden nur Bestände unterbalb von 600 und für Buche unterbalb von $800 \mathrm{~m}$ ü. NN betrachtet.

\begin{tabular}{lrrrrrrrrrrr}
\hline & \multicolumn{10}{c}{ Quantile J] } \\
& $0 \%$ & $10 \%$ & $20 \%$ & $30 \%$ & $40 \%$ & $50 \%$ & $60 \%$ & $70 \%$ & $80 \%$ & $90 \%$ & $100 \%$ \\
\hline Eiche & 148,6 & 149 & 151,7 & 151,9 & 152 & 152 & 152,1 & 160,7 & 172,9 & 182,6 & 257,8 \\
Buche & 125,8 & 135,8 & 137,0 & 138,8 & 140,3 & 141,8 & 144,1 & 146,7 & 149,8 & 153,2 & 275,0 \\
Fichte & 73,4 & 80,2 & 82,1 & 84,7 & 87,8 & 88 & 88,2 & 88,4 & 88,7 & 91,8 & 147,2 \\
Douglasie & 56,4 & 56,4 & 56,4 & 56,7 & 57,5 & 58,3 & 59,0 & 59,7 & 60,3 & 61,8 & 78,6 \\
Kiefer & 76,4 & 79,9 & 87,1 & 99,5 & 101,4 & 102,9 & 103,2 & 103,3 & 103,3 & 114 & 240,8 \\
\hline
\end{tabular}

Auf der Basis der Zielstärke (BHD), des zugehörigen Alters sowie von Standortsparametern wie der Temperatursumme in der Vegetationszeit, dem Ariditätsindex und weiteren Bodenparametern wurde anschließend die Baumhöhe bei Zielstärke geschätzt, die in Kombination mit der Zielstärke als Prädiktor in das Modell zur Sturmschadensmodellierung eingeht. Zur Schätzung der Baumhöhe wurde ein longitudinales Höhen-Durchmesser-Modell (SCHMIDT 2010) verwendet, das für Eiche, Buche, Fichte, Douglasie und Kiefer parametrisiert ist. Das Modell ist u. a. auf der Datenbasis der Bundeswaldinventur entwickelt worden und erlaubt deutschlandweit Baumhöhenschätzungen, die sensitiv gegenüber der Temperatursumme in der Vegetationszeit und dem Ariditätsindex nach DE MARTONNE (1926) sind:

$$
\begin{aligned}
\operatorname{In}\left\{E\left(H_{k t i}\right)\right\}= & \widehat{p_{1 a}}+p_{2 a}\left(1-e^{-\widehat{p_{3 a}} A l t e r_{k t i}}\right)^{\widehat{p_{4 a}}}+f_{2 a}\left(d_{-} r e I_{k t i}\right)+f_{3 a}\left(K J_{k i}\right)+f_{4 a}\left(\text { Tempsum }_{k}\right)+f_{5 a}\left(\text { Arid }_{k}\right) \\
& +f_{6 a}\left(R W_{k}, H W_{k}\right)-p_{0 b} x_{k t i}-p_{1 b} A / t e r_{k t i} x_{k t i}-p_{2 b} H N N_{k} x_{k t i}
\end{aligned}
$$

\begin{tabular}{|c|c|c|}
\hline$E\left(H_{k t j}\right)$ & $=$ & $\begin{array}{l}\text { Erwartungswert der Baumhöhe von Baum } i \text { in Bestand } k \text { zum } \\
\text { Zeitpunkt } t \text { mit } H_{k t i} \sim N\left(\exp \left(\eta_{k t i}\right), \sigma 2\right)\end{array}$ \\
\hline Alter $_{k t i}[\mathrm{~J}]$ & $=$ & Alter von Baum $i$ in Bestand $k$ zum Zeitpunkt $t$ \\
\hline d_relkti & $=$ & $\begin{array}{l}\text { Relativer BHD: Verhältnis des BHD von Baum } i \text { in Bestand } k \text { zum } \\
\text { Zeitpunkt } t \text { in Relation zum zugehörigen Grundflächenmittel- } \\
\text { stamm }\end{array}$ \\
\hline$K J_{k i}$ & $=$ & Keimjahr von Baum $i$ in Bestand $k$ \\
\hline $\begin{array}{l}\text { Tempsumk } \\
{\left[{ }^{\circ} \mathrm{C}\right]}\end{array}$ & $=$ & $\begin{array}{l}\text { Mittlere Temperatursumme in der Vegetationszeit in Bestand kim } \\
\text { Zeitraum } 1961 \text { - } 1990\end{array}$ \\
\hline Arid $_{k}$ & $=$ & $\begin{array}{l}\text { Mittlerer Ariditätsindex nach DE MARTONNE (1926) in Bestand } k \\
\text { im Zeitraum } 1961 \text { - } 1990\end{array}$ \\
\hline$H N N_{k}[\mathrm{~m}]$ & $=$ & Seehöhe des Zentroides von Bestand $k$ \\
\hline$R W_{k}, H W_{k}$ & $=$ & $\begin{array}{l}\text { Gauss-Krüger Rechts- und Hochwert des Zentroides von Bestand } \\
k\end{array}$ \\
\hline
\end{tabular}




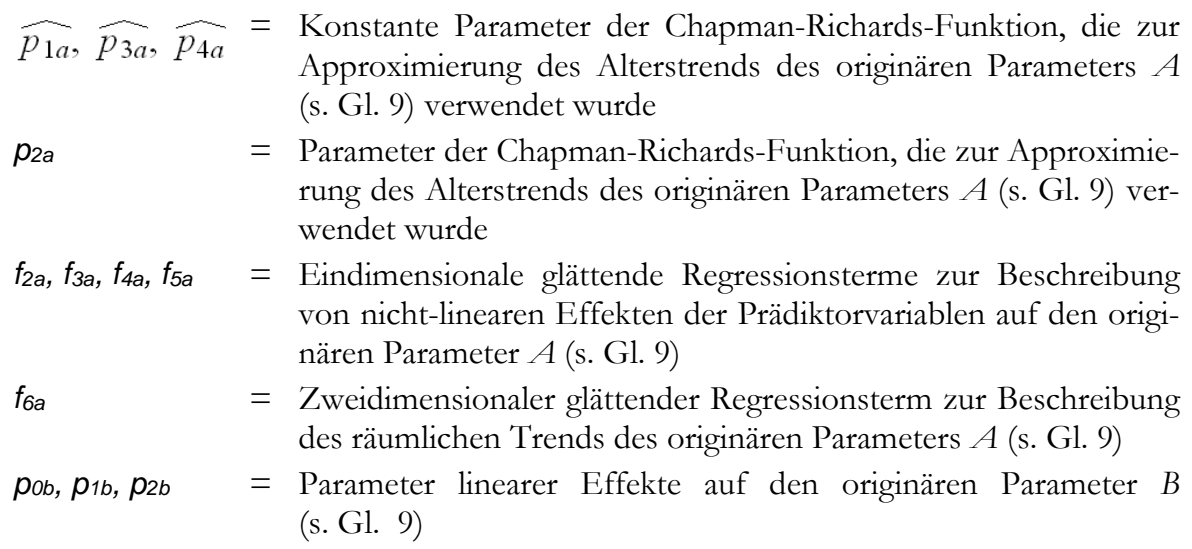

Die Variablen Temperatursumme in der Vegetationszeit und Ariditätsindex werden über deutschlandweit gültige Regionalisierungsmodelle in Abhängigkeit von der Seehöhe und der geografischen Lage geschätzt. Das originäre Höhen-DurchmesserModell ist eine von LAPPI (1997) modifizierte Form der Korf-Funktion, die sich durch die biologische Interpretierbarkeit und eine relativ geringe Kollinearität der Parameter $A$ und $B$ auszeichnet (s. Gl. 9). Das Modell wurde in einem ersten Entwicklungsschritt als gemischtes lineares Modell mit Zufallseffekten auf der Ebene des Bestandes und des Inventurzeitpunktes innerhalb von Beständen parametrisiert, wobei verschiedene Kombinationen der Parameter $C$ und $\lambda$ getestet bzw. als Konstanten im Modell verwendet wurden. Die solchermaßen heuristisch ermittelte optimale Parameterkombination von $C$ und $\lambda$ wurde in allen folgenden Parametrisierungsschritten konstant gesetzt, so dass das Modell linear ist und verallgemeinerte additive Modelle zur Quantifizierung der Standortseffekte verwendet werden konnten (s. Gl. 8):

$$
\ln \left(H_{k t i}\right)=A_{k t}-B_{k t} x_{k t i}+\varepsilon_{k t i}
$$

mit $x_{k t i}$ :

$$
x_{k t i}=\frac{\left(B H D_{k t i}+\lambda\right)^{-C}-(30+\lambda)^{-C}}{(10+\lambda)^{-C}-(30+\lambda)^{-C}}
$$

und:

$$
\begin{array}{rl}
E\left(H_{k t i}\right)= & \text { Erwartungswert der Baumhöhe von Baum } i \text { in Bestand } k \text { zum Zeit- } \\
& \text { punkt } t \text { mit } H_{k t i} \sim N\left(\exp \left(\eta_{k t i}\right), \sigma 2\right) \\
B H D_{k t i} & B H D \text { von Baum } i \text { in Bestand } k \text { zum Zeitpunkt } t \\
A_{k t,} B_{k t,}, \lambda= & \text { Parameter des Höhen-Durchmesser-Modells für Parzelle } k \text { zum Zeit- } \\
& \text { punkt } t
\end{array}
$$


Mit Hilfe einer zweiten Modellstufe, die anhand der Betriebsinventur bzw. der forstlichen Standortskartierung in Niedersachsen entwickelt worden ist, erfolgten Schätzungen der Baumhöhe bei Zielstärke, die zusätzlich gegenüber den Bodenparametern nutzbare Feldkapazität, Nährstoffziffer und der kategorischen Variablen Grundwassereinfluss sensitiv sind (s. Gl. 10). Die logarithmierten Erwartungswerte der Höhenschätzung aus dem klimasensitiven Modell (s. Gl. 9) gehen dabei als Kovariable in die zweite bodensensitive Modellstufe ein. In der zweiten Modellstufe erfolgt somit eine Modifikation der Schätzung aus Modell Gleichung 8 unter der Berücksichtigung von Bodenparametern. Auch in diesem Fall wurde die Prognose ohne den Effekt der geografischen Lage $f_{2 a}$ durchgeführt, um valide Schätzungen im geografischen Extrapolationsbereich bzw. für Sachsen-Anhalt zu ermöglichen:

$$
\begin{aligned}
& \ln \left\{E\left(H_{k f i}\right)\right\}=\operatorname{In}\left\{\widehat{\left.E\left(H_{k t i}\right)\right\}}\right\}+f_{1 a}\left(n F K_{k}\right) Z_{k}+G W_{-} c l a s s_{k}^{T} \beta_{1}+N Z_{-} c l a s s_{k}^{T} \beta_{2}+f_{2 a}\left(R W_{k}, H W_{k}\right) \\
& E\left(H_{k t}\right)=\text { Erwartungswert der Baumhöhe von Baum } i \text { in Bestand } k \text { zum Zeit- } \\
& \text { punkt } t \text { mit } H_{k t i} \sim N\left(\exp \left(\eta_{k t i t}\right), \sigma 2\right) \\
& \widehat{E\left(H_{k t i}\right)}=\begin{array}{l}
\text { Schätzung des Erwartungswert der Baumhöhe von Baum i in Bestand } k \\
\text { zum Zeitpunkt } t \text { mit Hilfe der ersten Modellstufe (s. Gl. 8). }
\end{array} \\
& n F K_{k} \quad=\text { Nutzbare Feldkapazität in Bestand } k \\
& Z_{k} \quad=\text { Kategorische Variable zur Definition des Grundwassereinflusses in } \\
& \text { Bestand k mit den Kategorien: „Grundwassereinfluss“, „kein Grund- } \\
& \text { GW_class } k=\text { Vektor der kategorischen Variable zur Definition des Grundwasserein- } \\
& \text { flusses in Bestand } k \text { mit den Kategorien: } \\
& \text { kein Grundwassereinfluss: Flurabstand }>2,5 \mathrm{~m} \text {, } \\
& \text { schwach - mäßiger Grundwassereinfluss: Flurabstand 2,5-0,95 m, } \\
& \text { mittlerer - starker Grundwassereinfluss: Flurabstand }<0,95 \mathrm{~m} \text {. } \\
& \text { NZ_class } \text { k }_{\text {a }}=\text { Vektor der kategorische Variable zur Definition der Nährstoffausstat- } \\
& \text { tung in Bestand } k \text { auf der Grundlage der Ansprache der Nährstoffziffer } \\
& \text { inklusive Variante nach der niedersächsischen Standortskartierung } \\
& R W_{k}, H W_{k}=\text { Rechts- und Hochwert des Zentroides von Bestand } k \text { im Gauss- } \\
& \text { Krüger-Koordinatensystem } \\
& f_{1 a}=\text { Eindimensionaler glättender Regressionsspline zur Erfassung des nicht- } \\
& \text { linearen Effektes der nFK } \\
& f_{2 a} \quad=\text { Zweidimensionaler glättender Regressionsspline zur Erfassung räum- } \\
& \text { licher Autokorrelation } \\
& \beta_{1,} \beta_{2} \quad=\text { Koeffizientenvektoren }
\end{aligned}
$$


Die resultierenden Baumhöhen bei Zielstärke weisen für alle Baumarten in den Höhenstufen 100 und $200 \mathrm{~m}$ die geringsten Werte auf (s. Abb. 18 und Abb. 19). Dabei liegt der Großteil der Bestände unterhalb von $100 \mathrm{~m}$ in der Region ColbitzLetzlinger Heide, während der Großteil der Bestände der Höhenstufe $200 \mathrm{~m}$ in der Region Fläming liegt. Die im Mittel besseren Wuchsbedingungen im Fläming gegenüber der Colbitz-Letzlinger Heide zeigen sich bei allen Baumarten in allerdings unterschiedlichem Ausmaß in den resultierenden größeren Baumhöhen bei Erreichen der Zielstärke. Die Unterschiede dürfen nicht mit Bonitätsunterschieden gleichgesetzt werden, da das Alter bei Erreichen der Zielstärke nicht konstant ist und mit abnehmender Standortsgüte zunimmt. Bei der Schätzung wurden baumartenspezifisch obere Grenzwerte bzgl. der Seehöhe gesetzt, um die Extrapolation auf ein vertretbares Niveau zu begrenzen. Dabei treten Extrapolationen bei der Schätzung der Hilfsgröße „Alter bei Zielstärke“ deutlich eher auf als bei der eigentlichen Baumhöhenschätzung. Schätzungen für die Höhenstufen 800 und $900 \mathrm{~m}$ wurden daher nur für die Fichte durchgeführt. In diesen Höhenstufen fallen die Baumhöhenschätzungen auf bzw. noch unter das Niveau in den Höhenstufen 100 und $200 \mathrm{~m}$. Die Bestände in den Höhenstufen von mehr als $300 \mathrm{~m}$ liegen ausschließlich in der Region Oberharz. Hier zeigt sich über alle Baumarten hinweg, dass die Baumhöhen bei Erreichen der Zielstärke mit steigender Seehöhe sinken, was in der Verschlechterung der Standortsbedingungen, d. h. vor allem der sinkenden Temperatursumme, begründet ist. In den unteren und montanen Harzlagen resultieren die im Schnitt deutlich besseren Standortsbedingungen bzgl. der Niederschlagssummen und der Nährstoffversorgung in größeren Baumhöhenschätzungen im Vergleich zur Colbitz-Letzlinger Heide und dem Fläming. Entsprechend der ökologischen Bedürfnisse der Baumarten sind die Unterschiede bei Buche und Fichte besonders ausgeprägt, während sie bei Eiche und insbesondere Kiefer deutlich geringer ausfallen. 

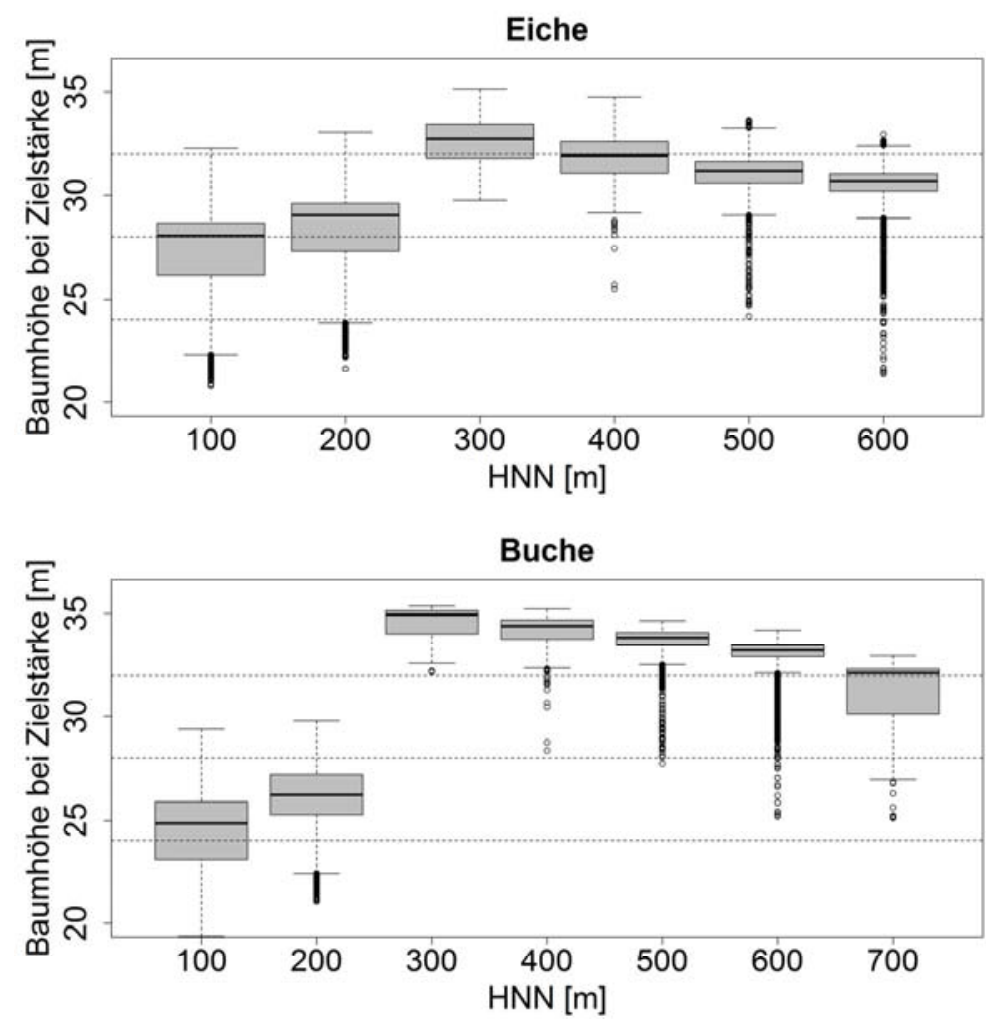

Fichte

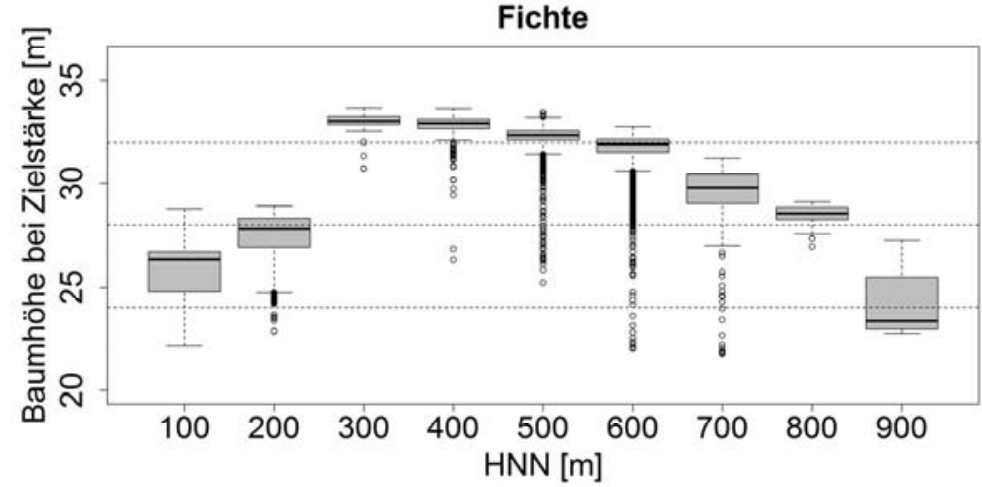

Abbildung 18: Verteilungen derprognostizierten Baumböhe bei Zielstärke über der Seehöhe (HNN in m) für Eiche, Buche und Fichte 

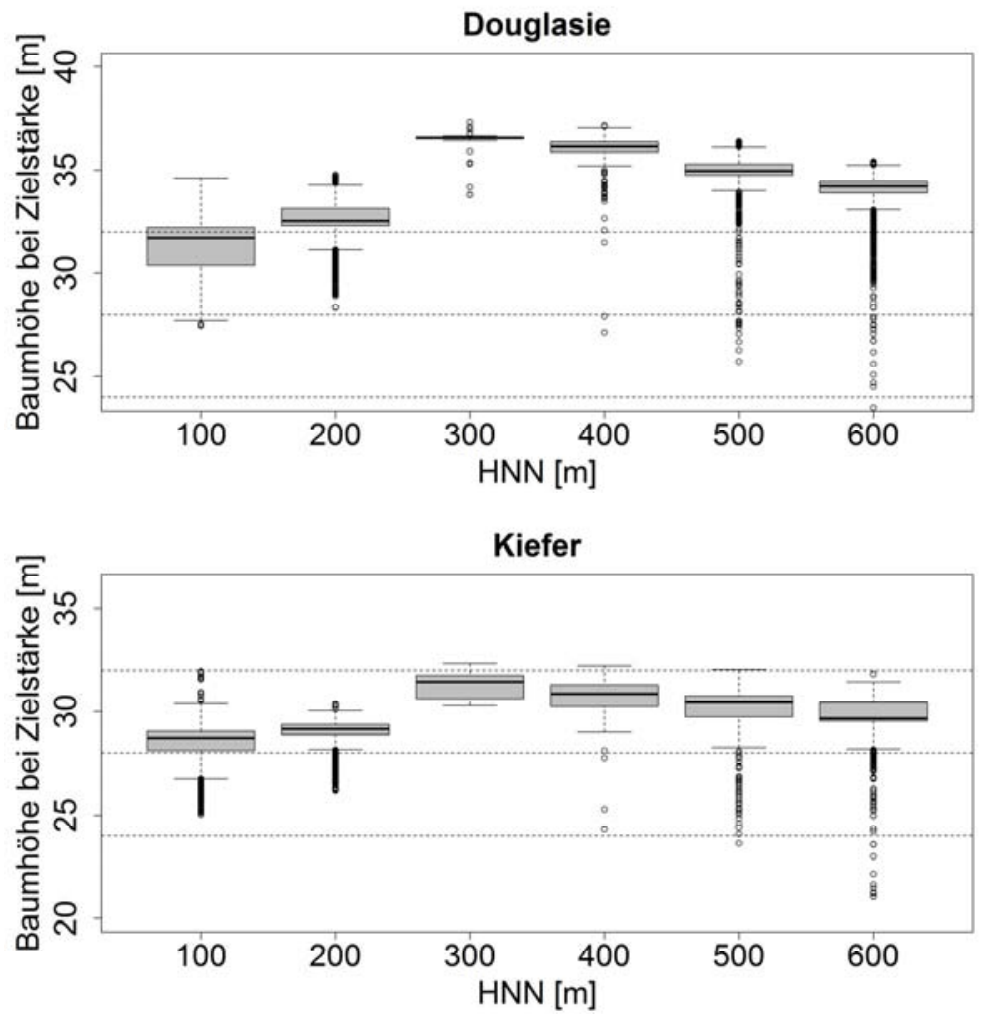

Abbildung 19: Verteilungen derprognostizierten Baumböhe bei Zielstärke über der Seeböbe (HNN in m) für Douglasie und Kiefer

Die Gesamtverteilungen der Baumhöhe bei Zielstärke spiegeln die unterschiedlichen Standortsbedingungen über alle drei Modellregionen hinweg wider (s. Tab. 10). Die Differenz zwischen den $10 \%$ - und $90 \%$-Perzentilen kennzeichnet Buche und Fichte als Baumarten, die deutlich auf die Standortsunterschiede reagieren, während Eiche, Douglasie und Kiefer in abnehmender Reihenfolge weniger starke Unterschiede aufweisen. Dabei profitieren Fichte und Buche besonders von den günstigeren Niederschlagsverhältnissen im Harz, da sie gleichzeitig geringere Ansprüche bzgl. der Temperatur stellen. Insbesondere die Eiche reagiert demgegenüber stärker auf sinkende Temperatursummen. Eiche und Douglasie profitieren zwar grundsätzlich ebenfalls von höheren Niederschlagssummen und einer besseren Nährstoffausstattung im Harz. Die Unterschiede zur Colbitz-Letzlinger Heide und zum Fläming sind jedoch durch die höheren Wärmebedürfnisse geringer. Die Kiefer weist allgemein die geringste Sensitivität gegenüber den Standortsbedingungen auf, was in der geringsten Differenz von nur 2,6 m zwischen dem $10 \%$ - und $90 \%$-Perzentil deutlich wird. Auch hier sind die Unterschiede der betrachteten Höhenlage beim Vergleich der Baumarten zu berücksichtigen. 
Tabelle 10: $\quad$ Perzentile der Verteilungen der prognostizierten Baumböhe bei Zielstärke für die Baumarten Eiche $(65 \mathrm{~cm})$, Buche $(55 \mathrm{~cm})$, Fichte $(45 \mathrm{~cm})$, Douglasie $(55 \mathrm{~cm})$ und Kiefer $(45 \mathrm{~cm})$. Für Eiche, Douglasie und Kiefer werden nur Bestände unterhalb von 600 und für Buche unterbalb von $700 m$ ü. NN betrachtet.

\begin{tabular}{lcccccccccccc}
\hline & \multicolumn{10}{c}{ Quantile [m] } \\
& $0 \%$ & $10 \%$ & $20 \%$ & $30 \%$ & $40 \%$ & $50 \%$ & $60 \%$ & $70 \%$ & $80 \%$ & $90 \%$ & $100 \%$ \\
\hline Eiche & 20,8 & 25,1 & 26,7 & 27,9 & 28,4 & 28,7 & 29,2 & 29,8 & 30,6 & 31,2 & 35,1 \\
Buche & 19,4 & 22,6 & 23,8 & 24,8 & 25,5 & 26,0 & 27,0 & 28,1 & 32,9 & 33,6 & 35,4 \\
Fichte & 21,8 & 24,4 & 25,4 & 26,3 & 26,6 & 26,9 & 27,7 & 28,4 & 31,6 & 32,3 & 33,7 \\
Douglasie & 21,8 & 29,8 & 30,9 & 31,7 & 32,1 & 32,3 & 32,6 & 33,3 & 34,1 & 34,7 & 37,3 \\
Kiefer & 21,0 & 27,8 & 28,3 & 28,7 & 28,9 & 29,1 & 29,2 & 29,4 & 29,6 & 30,4 & 32,3 \\
\hline
\end{tabular}

Insgesamt resultieren Baumhöhen, die sowohl vom absoluten Niveau als auch von der Sensitivität gegenüber den Standortsparametern eine realitätsnahe Schätzung der Normbestockung beim Erreichen der Zielstärke darstellen.

\subsubsection{Durchmesser- und Baumböhenschätrung der Ist-Bestockung}

Die standortsensitive Prognose der Baumhöhe für die Grundflächenmittelstämme (Dg) der aktuellen Bestockung wurde ebenfalls mit dem longitudinalen HöhenDurchmesser-Modell (SCHMIDT 2010) bzw. der nachgeschalteten boden-sensitiven Modellstufe durchgeführt (s. G1. 8 und Gl. 10). In diesem Fall wurden in einem ersten Schritt die Grundflächenmittelstämme aller in der Hauptschicht eines Bestandes vorkommenden Baumarten in Abhängigkeit ihres Alters geschätzt. Dabei wurden in Mischbeständen maximal die fünf wichtigsten Baumarten entsprechend ihres Anteils in der Forsteinrichtung berücksichtigt. Als Alter wurde einheitlich das auf das Stichjahr 2006 korrigierte Alter aus der aktuellen Forsteinrichtung verwendet. Zur Schätzung wurde ein auf der Grundlage der niedersächsischen Betriebsinventur parametrisiertes verallgemeinertes additives gemischtes Regressionsmodell GAMM (PINHEIRO et al. 2012, VENABLES u. RIPLEY 2002, WOOD 2006, 2011) mit Zufallseffekten auf den Ebenen Forstamt und Revier innerhalb von Forstamt verwendet (s. Gl. 11): 


$$
\begin{aligned}
\log \left\{E\left(D g_{i j k}\right)\right\}= & f_{1}\left(A / t_{i j k}\right) Z_{1 i j k}+B S_{i j k}+f_{2}\left(n F K_{i j k}\right) Z_{2 i j k}+f_{3}\left(H N N_{i j k}\right)+ \\
& G W_{-} \text {class }_{i j k}{ }^{T} \beta_{1}+N Z_{-} \text {class }_{j j k}{ }^{T} \beta_{2}+b_{0 i}+b_{0 i j}+b_{1 i} A l t_{i j k}+b_{1 i j} A l t_{i j k} \\
\text { mit: } & {\left[\begin{array}{l}
b_{0 i} \\
b_{1 i}
\end{array}\right] \sim N\left(0, \Psi_{1}\right) \text { und }\left[\begin{array}{l}
b_{0 i j} \\
b_{1 i j}
\end{array}\right] \sim N\left(0, \Psi_{2}\right) \text { und } }
\end{aligned}
$$

$E\left(D g_{i k}\right) \quad=$ Erwartungswert des Grundflächenmittelstammes der betrachteten Bestandesschicht in Bestand $k$ in Revier $j$ und Forstamt $i$ mit $D g_{i j k} \sim$ Gamma

Alt $t_{j k} \quad=$ Alter der betrachteten Bestandesschicht in Bestand $k$ in Revier $j$ und Forstamt $i$

$B S_{i j k} \quad=$ Kategorische Variable zur Definition der Bestandesschicht in Bestand $k$ in Revier $j$ und Forstamt $i$ mit den Kategorien: „Hauptbestand“, „Überhalt" und „Unterstand/Nachwuchs"

$n F K_{j j k}=$ Flächengewichtete nutzbare Feldkapazität in Bestand $k$ in Revier $j$ und Forstamt $i$

$Z_{1 i j k} \quad=$ Kategorische Variable zur Definition der Bestandesschicht in Bestand $k$ in Revier $j$ und Forstamt $i$ mit den Kategorien: „Hauptbestand/Überhalt" und „Unterstand/Nachwuchs“"

$Z_{2 i j k} \quad=$ Kategorische Variable zur Definition des Grundwassereinflusses in Bestand $k$ in Revier $j$ und Forstamt $i$ mit den Kategorien: „Grundwassereinfluss“, „kein Grundwassereinfluss“

$H N N_{j}=$ Seehöhe des Zentroides von Bestand $k$ in Revier $j$ und Forstamt $i$

GW_class $j=$ Vektor der kategorischen Variable zur Definition des Grundwassereinflusses in Bestand $k$ in Revier $j$ und Forstamt $i$ mit den Kategorien: kein Grundwassereinfluss: Flurabstand $>2,5 \mathrm{~m}$, schwach - mäßiger Grundwassereinfluss: Flurabstand 2,5-0,95 m, mittlerer - starker Grundwassereinfluss: Flurabstand $<0,95 \mathrm{~m}$

$N Z_{\text {class }}$ = Vektor der kategorischen Variable zur Definition der Nährstoffausstattung in Bestand $k$ in Revier $j$ und Forstamt $i$ auf der Grundlage der Ansprache der Nährstoffziffer inklusive Variante nach der niedersächsischen Standortskartierung

$f_{1} f_{2}=$ Eindimensionale glättende Regressionssplines zur Erfassung des nichtlinearen Effektes metrischer Kovariablen

$f_{3}=$ Zweidimensionaler glättender Regressionsspline zur Erfassung räumlicher Autokorrelation

$\beta_{1}, \beta_{2}=$ Koeffizientenvektoren

$\mathrm{b}_{i}, \mathrm{~b}_{i j} \quad=$ Vektoren von Zufallseffekten auf der Ebene des Forstamtes und des Reviers innerhalb des Forstamtes mit unbekannter positiv-definiter Kovarianzmatrix, wobei unterstellt wird, dass die Zufallseffekte unabhängig und identisch normalverteilt sind. 
Das Modell ist für die Baumarten(gruppen) Eiche, Buche, ALh, ALn, Fichte, Douglasie, Kiefer und Lärche parametrisiert und erlaubt separate Schätzungen für den Hauptbestand, Überhalt sowie Nachwuchs/Unterstand. In der vorliegenden Anwendung erfolgen die Schätzungen für die aktuelle Bestockung nur für den Hauptbestand. Regionale Unterschiede in Niedersachsen werden in dem Modell (s. Gl. 11) über die räumlichen Einheiten Forstamt und Revier bzw. die zugehörigen Zufallseffekte erfasst. Für die Anwendung in Sachsen-Anhalt wurden die Zufallseffekte auf Null gesetzt, so dass die Schätzungen bedingten mittleren Erwartungswerten in Niedersachsen unter den jeweiligen Standortsverhältnissen entsprechen. Durch die Verwendung der aktuellen Datengrundlage der Betriebsinventuren in Niedersachen werden realitätsnahe Werte für die Grundflächenmittelstämme prognostiziert. Die Modellanwendung stellt damit einen bedeutenden Fortschritt gegenüber der Ertragstafelschätzung dar. Bei der Anwendung der Ertragstafeln kommt es in jüngeren Beständen zu einer deutlichen Unterschätzung des Dg, während in älteren Beständen der Effekt der Zielstärkennutzung vernachlässigt wird. Darüber hinaus erlaubt das Modell statistisch abgesicherte Schätzungen für hohe Altersbereiche, die durch die Ertragstafeln nicht abgedeckt sind.

Die resultierenden Altersverläufe der Schätzungen spiegeln die unterschiedliche Wuchsdynamik, aber auch die unterschiedlichen waldbaulichen Behandlungskonzepte der Baumarten wider (s. Abb. 20 bis Abb. 22). Die bedingte Streuung innerhalb der Altersstufen ist Ausdruck der unterschiedlichen Standortsbedingungen innerhalb der Datengrundlage. 


\section{Eiche}

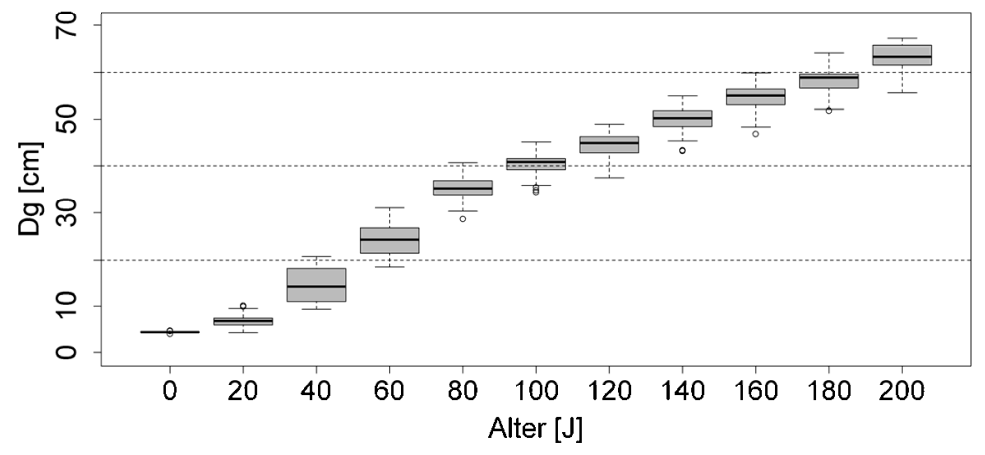

Buche
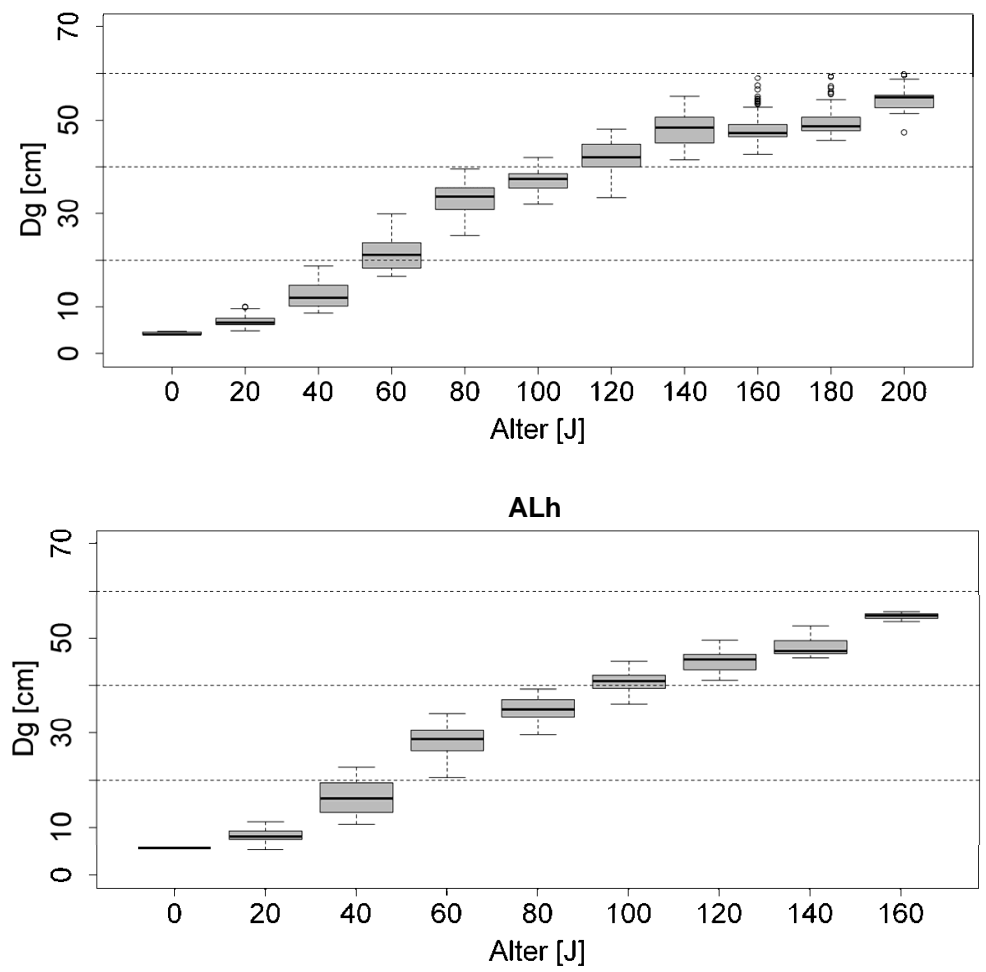

Abbildung 20: Verteilungen des prognostiz̨ierten Grundflächenmittelstammes Dg [cm] über dem Bestandesalter $[J]$ im Hauptbestand für Eiche, Buche, und ALh (Laubholz mit hoher Umtriebszeit). Berücksichtigt sind alle Bestände, in denen die jeweilige Baumart die Hauptbaumart ist. 

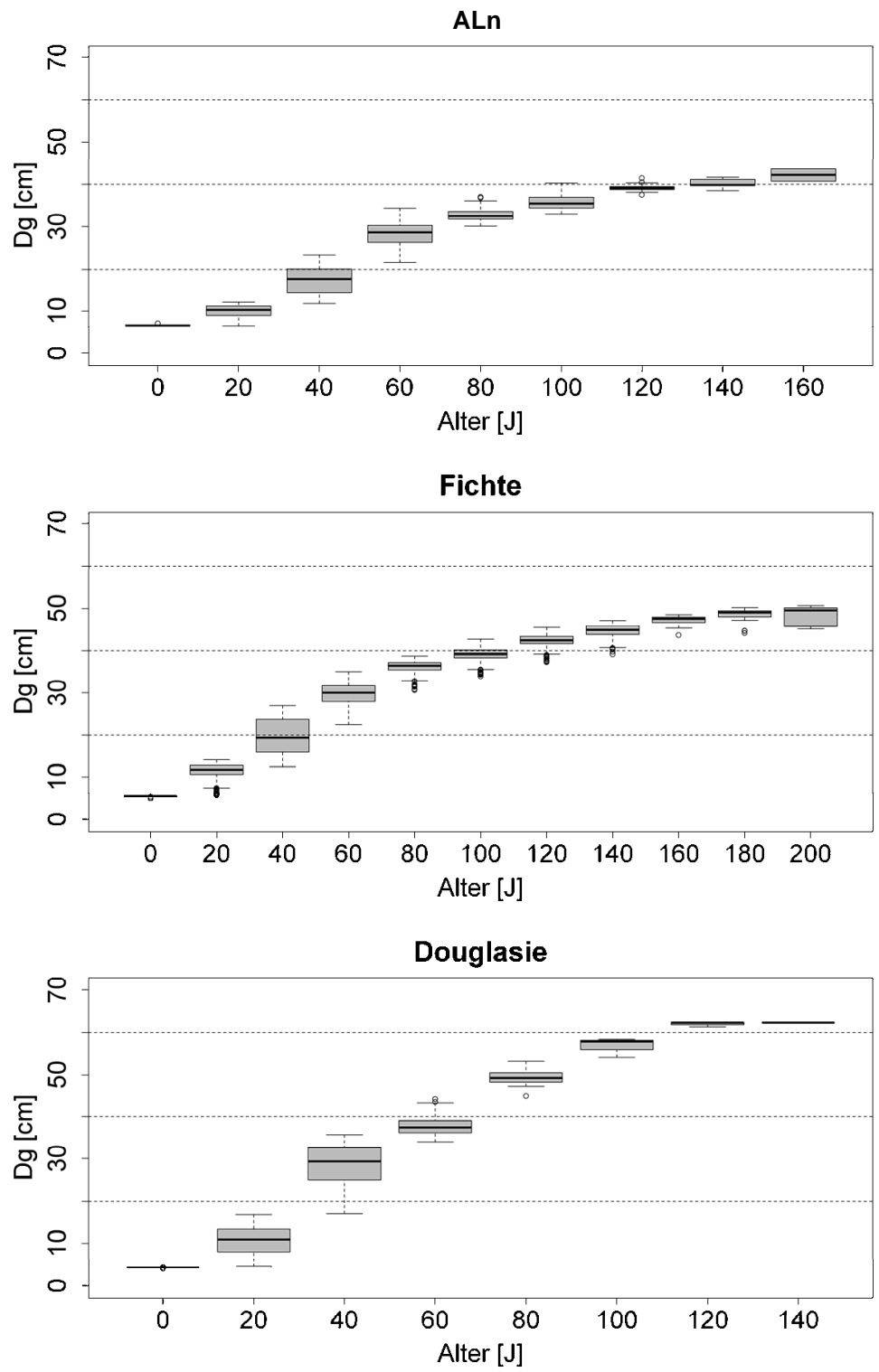

Abbildung 21: Verteilungen des prognostizierten Grundflächenmittelstammes Dg [cm] über dem Bestandesalter [J] im Hauptbestand für ALn, Fichte und Douglasie. Berücksichtigt sind alle Bestände, in denen die jeweilige Baumart die Hauptbaumart ist. 

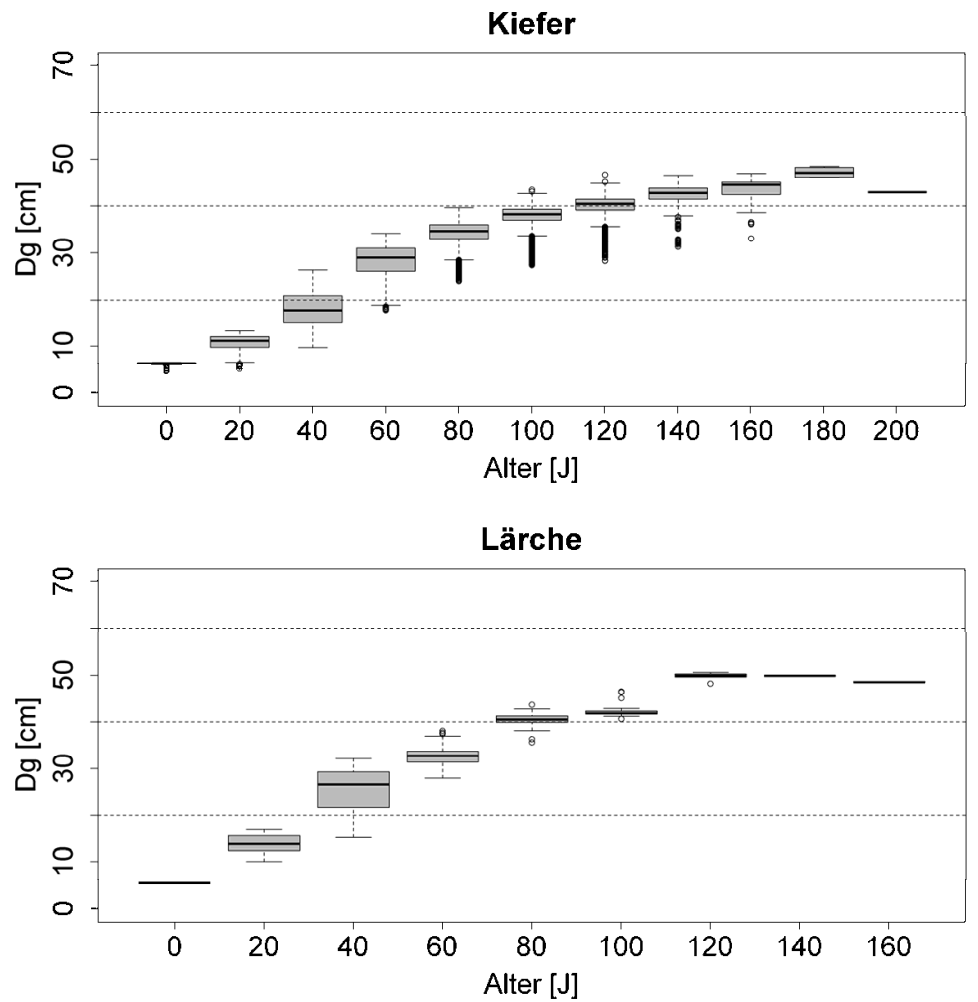

Abbildung 22: Verteilungen des prognostizierten Grundflächenmittelstammes Dg [cm] über dem Bestandesalter $[J]$ im Hauptbestand für Kiefer und Lärche. Berücksichtigt sind alle Bestände, in denen die jeweilige Baumart die Hauptbaumart ist.

Ausgehend von den prognostizierten Grundflächenmittelstämmen, dem zugehörigen Alter sowie den Standortsparametern der Bestände wurden die zugehörigen Mittelhöhen $(\mathrm{Hg})$ getrennt nach Baumarten im Hauptbestand geschätzt (s. Abb. 23 bis Abb. 25). Die Schätzungen erfolgten mit Hilfe des 2-stufigen longitudinalen Höhen-Durchmesser-Modells (SCHMIDT 2010) (s. Gl. 8 und G1. 10) unter Berücksichtigung von klimatischen und edaphischen Standortsparametern. Die Höhenschätzung für die Laubholzarten mit niedriger Umtriebszeit (ALn) erfolgte mit dem für Eiche parametrisierten, für die Laubholzarten mit hoher Umtriebszeit (ALh) mit dem für Buche parametrisierten und für Lärche mit dem für Fichte parametrisierten Modellen. 

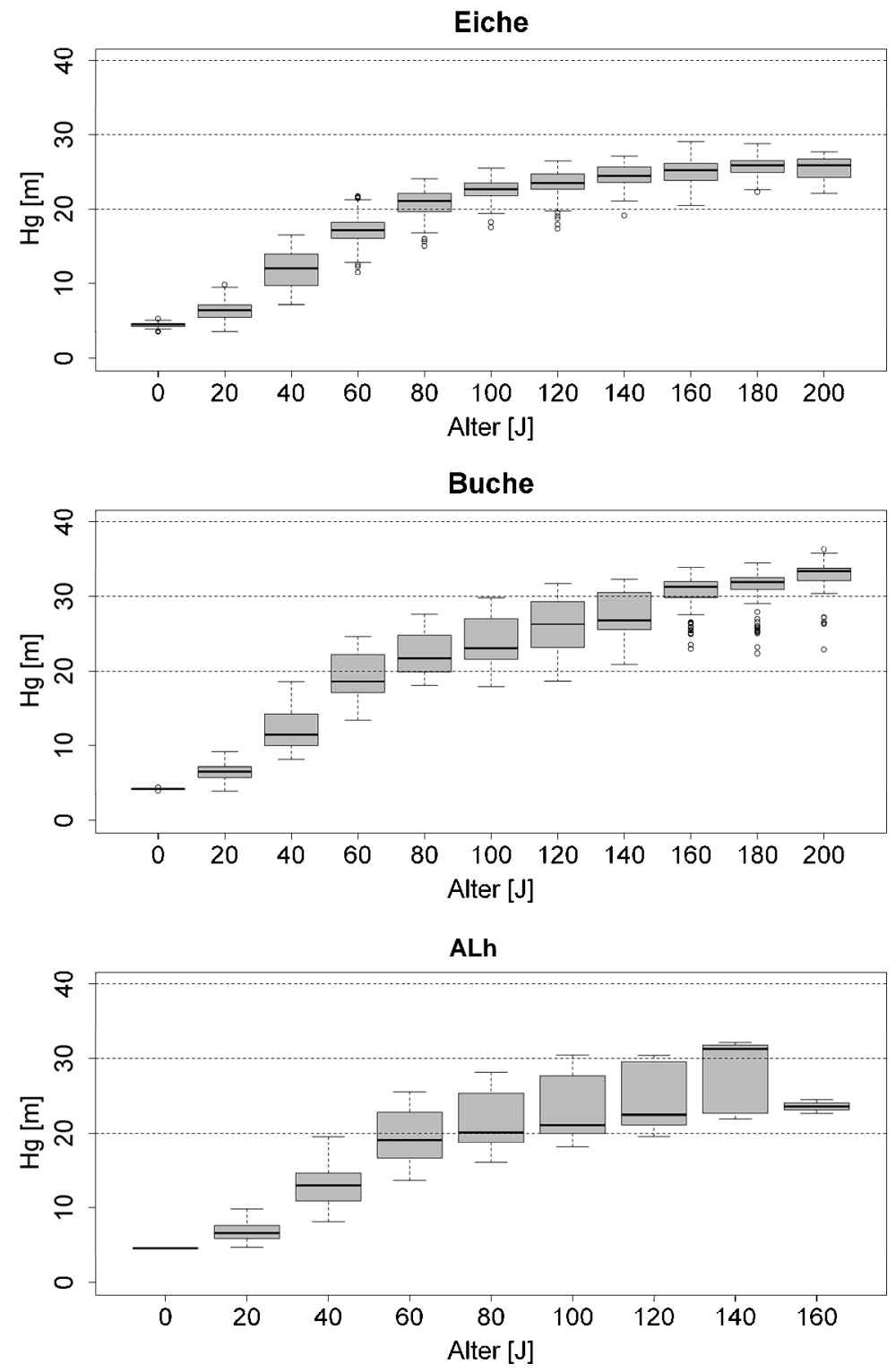

Abbildung 23: Verteilungen der prognostizierten Grundflächenmittelhöhe $\mathrm{Hg}[\mathrm{m}]$ über dem Bestandesalter [J] im Hauptbestand für Eiche, Buche und ALh (Laubholz mit hoher Umtriebszeit). Berücksichtigt sind alle Bestände, in denen die jeweilige Baumart die Hauptbaumart ist. 


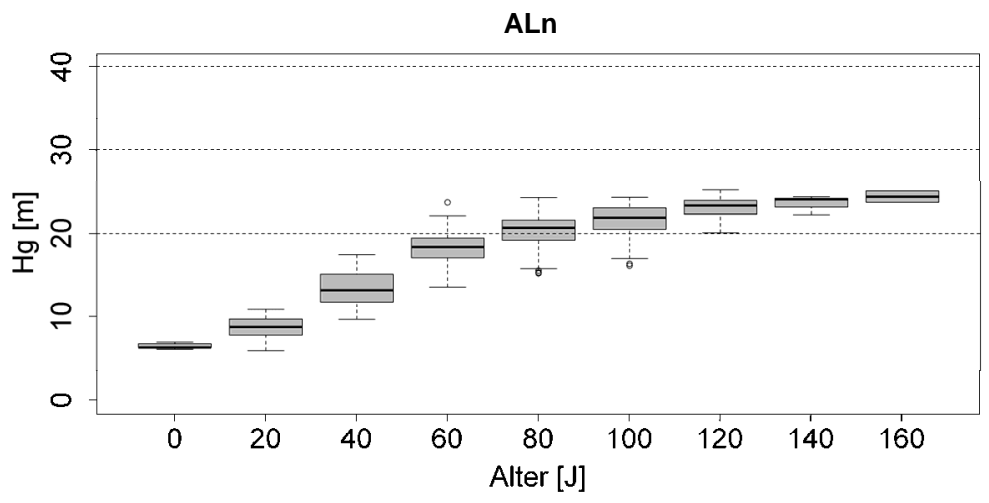

Fichte
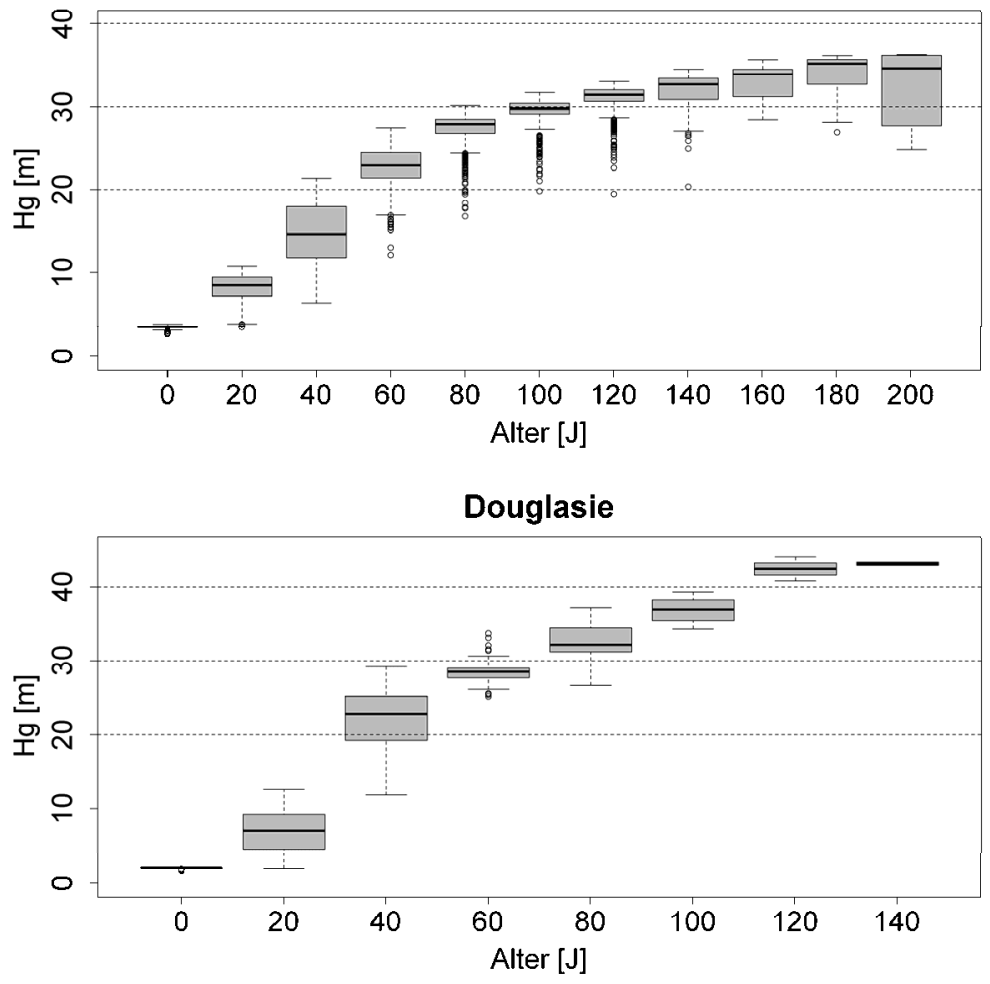

Abbildung 24: Verteilungen der prognostizierten Grundflächenmittelhöhe $\mathrm{Hg}[\mathrm{m}]$ über dem Bestandesalter JJ] im Hauptbestand für ALn, Fichte und Douglasie. Berücksichtigt sind alle Bestände, in denen die jeweilige Baumart die Hauptbaumart ist. 

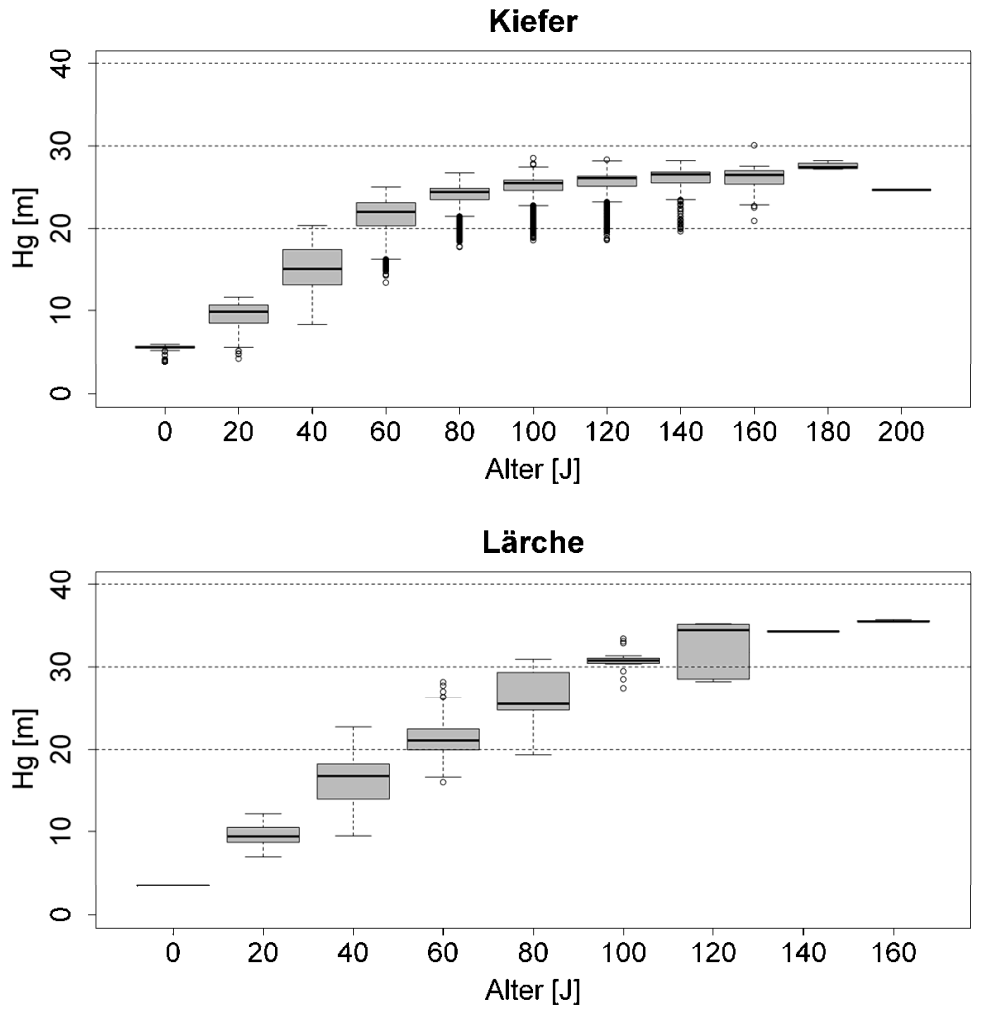

Abbildung 25: Verteilungen der prognostizierten Grundflächenmittelhöhe $\mathrm{Hg}[\mathrm{m}]$ über dem Bestandesalter $[J]$ im Hauptbestand für Kiefer und Lärche. Berücksichtigt sind alle Bestände, in denen die jeweilige Baumart die Hauptbaumart ist.

Die Leistungsrelationen der Baumarten in den drei Modellregionen weisen deutliche Unterschiede auf (s. Abb. 26). So haben Lärche, Fichte, ALh und Buche im Durchschnitt im Alter 100 im Harz deutlich größere Höhen als Eiche, ALn und Kiefer. Bei der Interpretation der Schätzungen für die Ist-Bestockung ist allerdings zu beachten, dass sich die Standortsparameter für die Baumarten innerhalb einer Modellregion deutlich unterscheiden können. So stockt die Buche in der Region Oberharz im Durchschnitt auf deutlich nährstoffreicheren Standorten als die Fichte. Die Douglasie weist - wie zu erwarten - in allen drei Modellregionen die höchste Höhenwuchsleistung auf, wobei die absolute Leistung von den Beständen im Harz, über die im Fläming zu den Beständen in der Colbitz-Letzlinger Heide abnimmt.

Im Fläming sind die Unterschiede in den Wuchsrelationen im Vergleich zum Oberharz deutlich schwächer ausgeprägt. Eiche und Buche liegen im Alter 100 ungefähr auf einem Niveau. Die Unterschiede zwischen Kiefer, Lärche und Fichte sind relativ gering, wobei die Bonitäten von Fichte und vor allem Lärche eher im Bereich 
der besten Kiefernbonitäten liegen. Insgesamt weisen Kiefer, Lärche und Fichte absolute Höhenbonitäten auf, die etwas höher sind als die von Eiche und Buche.
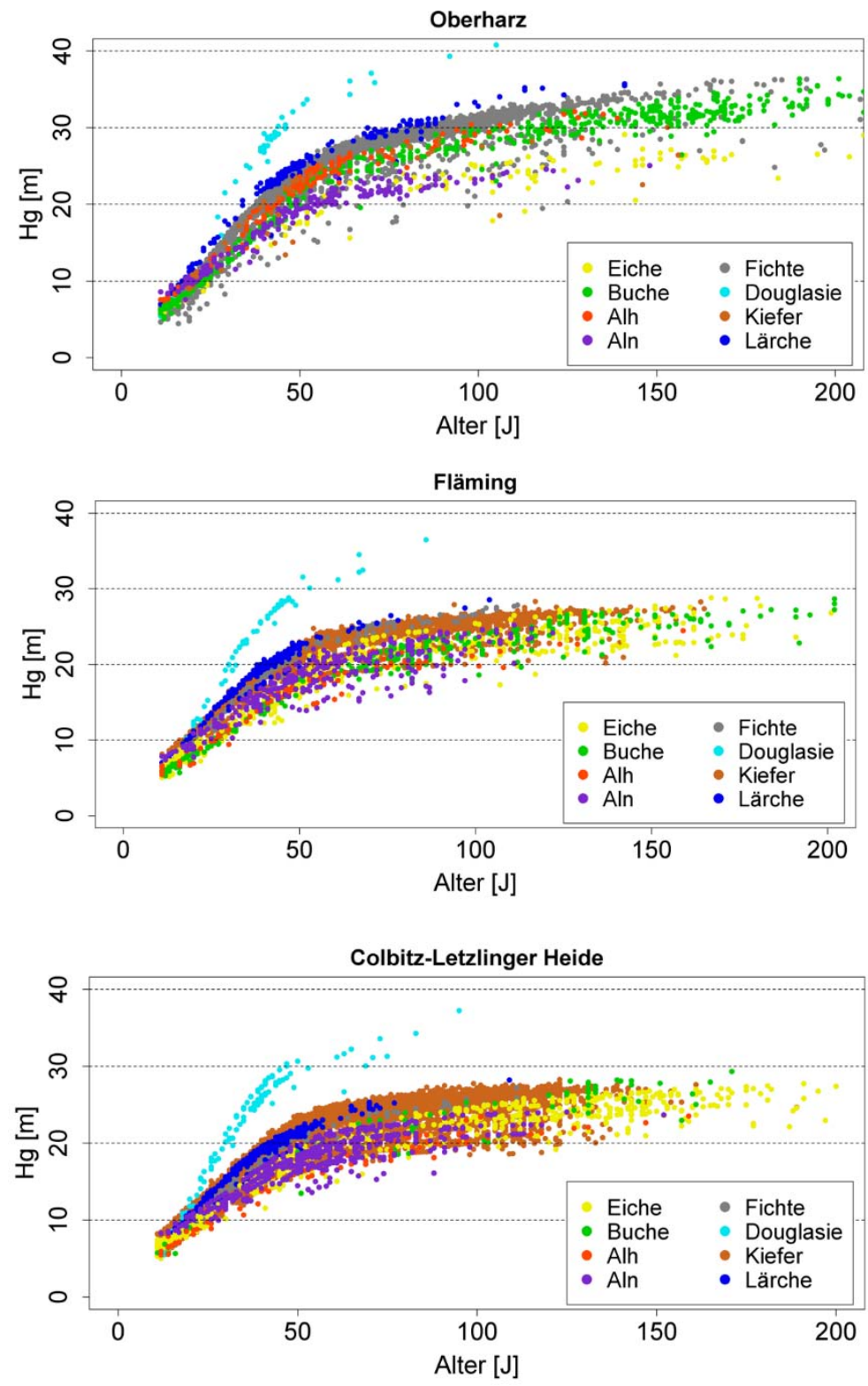

Abbildung 26: Prognostizierte Grundflächenmittelhöhe $\mathrm{Hg}[\mathrm{m}]$ über dem Bestandesalter $[\mathrm{J}]$ im Hauptbestand für Eiche, Buche, ALh, ALn, Fichte, Douglasie, Kiefer und Lärche in den Modellregionen Oberhar₹, Fläming und Colbitz-Letzlinger Heide. Berücksichtigt sind alle Bestände, in denen die jeweilige Baumart die Hauptbaumart ist. 
In der Colbitz-Letzlinger Heide treten mit Ausnahme der Douglasie ebenfalls relativ geringe Bonitätsunterschiede zwischen den Baumarten auf. Die Bonitäten der Nadelholzarten liegen auch hier im Schnitt etwas über denen von Eiche und Buche. Die Kiefer holt in den Bonitätsrelationen gegenüber dem Fläming weiter auf, so dass die besten Kiefernbonitäten jetzt über denen der Fichte und Lärche liegen. Wie im Harz müsste für eine detaillierte Interpretation der Höhenschätzungen auch in den übrigen Modellregionen berücksichtigt werden, wie sich die Baumarten auf die unterschiedlichen Standorte verteilen.

Als Ergebnis stehen damit standortsensitive Schätzungen der Baumhöhe bei Erreichen der Zielstärke für jeden Modellbestand zur Abschätzung der

1. potenziellen Vulnerabilität gegenüber Sturmschäden für die Baumarten Fichte, Douglasie, Kiefer, Buche und Eiche

sowie des Grundflächenmittelstammes und der zugehörigen Mittelhöhe für jeden Bestand der aktuellen Forsteinrichtung zur Abschätzung der

2. aktuellen Vulnerabilität gegenüber Sturmschäden für die Baumarten Fichte, Douglasie, Kiefer, Lärche, Buche, Eiche, ALh und ALn

zur Verfügung. Damit ist eine bestmögliche Initialisierung des Sturmschadensmodells für eine möglichst realistische und sensitive Abschätzung der Vulnerabilität gegenüber Winterstürmen in Abhängigkeit der Bestockung gegeben.

\subsubsection{Geländeinformationen als Eingangsgrößen zur Sturmschadensmodellierung}

Zur Erfassung der Exposition und Exponiertheit von Waldbeständen aufgrund der Geländemorphologie werden Varianten des Topex-to-Distance-Index (Topex, SCOTT u. MITCHELL 2005) verwendet. Im hier verwendeten Modellansatz werden anstelle eines Topex-Wertes vier verschiedene Werte verwendet, die die Exponiertheit in bestimmten Himmelsrichtungen beschreiben. Die Berechnung der TopexWerte erfolgte für eine Grenzdistanz von $1000 \mathrm{~m}$ flächendeckend für SachsenAnhalt für Rasterzellen von 10 x 10 m Auflösung auf der Grundlage eines digitalen Geländemodells (SUTMÖLLER et al. 2013). In der vorliegenden Prognose wurde jeweils ein Wintersturm aus exakt südwestlicher bzw. nordwestlicher Richtung unterstellt und die resultierenden Schadwahrscheinlichkeiten anschließend gemittelt. Dabei wurden jedem Bestand der Forsteinrichtung vereinfachend die Topex-Werte der Rasterzelle zugeordnet, in der sich sein Zentroid befindet.

Exemplarisch werden die Verteilungen des modifizierten Topex „Top_to_Dis_1“ für die unterstellte südwestliche Hauptsturmrichtung getrennt nach Modellregionen und nach führenden Baumartengruppen entsprechend der Forsteinrichtung dargestellt (s. Tab. 11). Die sehr viel größere Spreitung (Differenz zwischen dem $10 \%$ - und $90 \%$-Perzentil) der Topex-Werte im Oberharz spiegelt die deutlich differenziertere Topographie der Modellregion wider. Auch wird deutlich, dass Fichte und Buche (ALh), die schwerpunktmäßig im Oberharz vorkommen, eine größere Spreitung (Differenz zwischen dem $10 \%$ - und $90 \%$-Perzentil) 
der Topex-Werte aufweisen. Die unterschiedliche Verteilung der Baumarten auf exponierte und geschützte Standorte ist bei der späteren Interpretation der Prognose des Sturmschadensrisikos zu beachten.

Tabelle 11: Verteilungen des Top_to_Dis_1 nach Modellregionen und führenden Baumartengruppen

\begin{tabular}{lrrrrrrrrrrrr}
\hline & \multicolumn{10}{c}{ Quantile [Grad*10] } \\
& $0 \%$ & $10 \%$ & $20 \%$ & $30 \%$ & $40 \%$ & $50 \%$ & $60 \%$ & $70 \%$ & $80 \%$ & $90 \%$ & $100 \%$ \\
\hline Oberharz & -114 & 5 & 20 & 35 & 50 & 66 & 87 & 117 & 166 & 247 & 1335 \\
Fläming & -35 & -4 & -1 & 1 & 3 & 6 & 10 & 15 & 23 & 39 & 299 \\
Colbitz-Letzlinger & -33 & -2 & 1 & 2 & 5 & 9 & 14 & 21 & 31 & 50 & 415 \\
Heide & & & & & & & & & & & \\
\hline Eiche & -114 & -3 & 0 & 1 & 3 & 5 & 9 & 15 & 23 & 44 & 508 \\
Buche & -47 & -4 & 2 & 7 & 14 & 27 & 50 & 86 & 167 & 289 & 682 \\
ALh & -21 & -1 & 1 & 2 & 4 & 10 & 23 & 55 & 139 & 268 & 887 \\
ALn & -27 & 0 & 1 & 3 & 7 & 14 & 21 & 32 & 61 & 112 & 1111 \\
Fichte & -85 & 3 & 16 & 29 & 42 & 58 & 77 & 103 & 147 & 226 & 799 \\
Douglasie & -16 & -2 & 1 & 5 & 8 & 13 & 22 & 32 & 47 & 71 & 576 \\
Kiefer & -110 & -3 & 0 & 2 & 4 & 8 & 13 & 20 & 29 & 48 & 512 \\
Lärche & -38 & -2 & 2 & 5 & 10 & 16 & 24 & 34 & 49 & 83 & 481 \\
\hline & & & & & & & & & &
\end{tabular}

\subsubsection{Geländewasserhaushalt als Eingangsgröße zur Sturmschadensmodellierung}

Als Information zum Geländewasserhaushalt kann die Vernässungsstufe des Standortes in einer zweiten Stufe des Sturmschadensmodells für die Fichte sensitiv abgebildet werden (SCHMIDT et al. 2010). Die Vernässungsstufe weist dabei die drei Kategorien „vernässend“, „wenig vernässend“ und „nicht vernässend“ auf, die gutachtlich anhand der hydromorphen und semihydromorphen Lokalbodenformen abgeleitet wurden.

\subsubsection{Statistisches Sturmschadensmodell}

Das verwendete empirische Sturmschadensmodell (s. Gl. 12) ist auf der Grundlage von Schadansprachen in der BWI 2 nach dem Wintersturm „Lothar“ in BadenWürttemberg entwickelt worden (SCHMIDT et al. 2010, SUTMÖLLER et al. 2013). Das Modell erlaubt Schätzungen, die sensitiv gegenüber der Baumart(gruppe), dem BHD, der Baumhöhe sowie der Exposition und Exponiertheit des Geländes sind (SCHMIDT et al. 2010). Soweit Informationen zur Vernässungsstufe vorliegen, kann ihr Effekt auf die Sturmschadenswahrscheinlichkeit für die Fichte ebenfalls abgebildet werden (s. Gl. 13). Die regionalen Unterschiede in der Windgeschwindigkeit werden im verwendeten Sturmmodell durch eine räumliche Trendfunktion bzw. durch die Formulierung eines speziellen verallgemeinerten additiven Regressionsmodells (s. Gl. 12) beschrieben. Dabei wird angenommen, dass regionale Unterschiede in der Sturmschadenswahrscheinlichkeit, die nicht über die Kovariablen 
Baumart(gruppe), BHD, Baumhöhe sowie die Topex-Indizes beschrieben werden können, vor allem durch regional variierende Windgeschwindigkeiten bedingt sind. Die geografische Lage dient somit als Proxy-Variable für die Windgeschwindigkeit.

Unter der Annahme, dass die geografische Lage als Ersatz für die regionale Windgeschwindigkeit fungiert, lassen sich in Simulationen die Auswirkungen verschiedener Windgeschwindigkeiten prognostizieren. Anstelle von konkreten mittleren oder maximalen Windgeschwindigkeiten können allerdings lediglich qualitative Vorgaben gemacht werden. In der vorliegenden Untersuchung wurden bei der Prognose der Vulnerabilität die Bedingungen in Luv des Nordschwarzwaldes unterstellt. Derartig extreme Bedingungen sind auf größere Gebiete angewendet ein sehr unwahrscheinliches Szenario. In der folgenden Untersuchung geht es aber darum, eine möglichst hohe Trennschärfe zwischen Standorten bzw. Beständen zu erreichen, was eine Verwendung von extremen Randbedingungen voraussetzt. Eine realitätsnahe Prognose des Risikopotenzials würde dagegen realistische meteorologische Prognosen über die Intensität und Frequenz von Winterstürmen voraussetzen.

Die Überprägung der Strömungsparameter durch die Topografie wird durch die Topex-Indizes abgebildet, die ebenfalls als Proxy-Variablen fungieren. Die Selektion der spezifischen Himmelsrichtungen erfolgte im Rahmen der Modellselektion unter statistischen Gesichtspunkten (SCHMIDT et al. 2010).

Unter dieser Annahme lassen sich in Szenariosimulationen auch Winterstürme mit einer abweichenden Hauptwindrichtung berücksichtigen, in dem die Himmelsrichtungen der Topex-Indizes so um einen einheitlichen Winkel gedreht werden, dass die unterstellte Hauptwindrichtung durch die Himmelsrichtungen des Top_to_Dis_1 genau eingefasst wird. In der vorliegenden Untersuchung wurden die Windrichtungen Südwest und Nordwest zur Berechnung der Topex-Werte verwendet. Das anhand der Sturmschadensdaten der BWI 2 in Baden-Württemberg parametrisierte Modell lässt sich wie folgt beschreiben:

$$
\begin{aligned}
& \mathrm{g}\left(\pi_{\mathrm{ijk}}\right)=\mathrm{BA}_{\mathrm{ijk}}^{\mathrm{T}} \alpha+\log \left(\frac{\mathrm{BHD}_{\mathrm{ijk}} \mathrm{BA}_{\mathrm{ijk}}^{\mathrm{T}} \delta}{\mathrm{h}_{\mathrm{ijk}}-\mathrm{BA}_{\mathrm{ijk}}^{\mathrm{T}} \gamma}\right)+\beta_{1} \text { Top_to_Dist_1 } 1_{\mathrm{ij}}+\beta_{2} \text { Top_to_Dist_2 } 2_{\mathrm{ij}} \\
& +\beta_{3} \text { Top_to_Dist_3 } 3_{\mathrm{ij}}+\beta_{4} \text { Top_to_Dist_ } 4_{\mathrm{ij}}+f\left(\mathrm{RW}_{\mathrm{ij}} ; \mathrm{HW}_{\mathrm{ij}}\right)
\end{aligned}
$$

mit:

$$
\begin{aligned}
\pi_{\mathrm{ijk}}= & \text { Erwartungswert der Sturmschadenswahrscheinlichkeit } \\
& \pi_{\mathrm{ijk}}=\mathrm{E}\left(\mathrm{y}_{\mathrm{ijk}}\right) \text { von Baum } \mathrm{k} \text { in Traktecke ij mit i=1...4, } \\
& \mathrm{j}=1 \ldots 4516 \text { und } \mathrm{y}_{\mathrm{ijk}} \sim \operatorname{Bernoulli}\left(\pi_{\mathrm{ijk}}\right) \\
g(.)= & \text { Logistische Verknüpfungsfunktion } \\
\mathrm{BA}_{\mathrm{ijk}}= & \text { Ein Vektor zur Kodierung der Baumartengruppe von Baum }{ }_{\mathrm{ijk}} \\
& {[\text { Fichte, Tanne / Douglasie, Kiefer / Lärchen, Buche / Eichen, }} \\
& \text { übrige Laubholzarten] }
\end{aligned}
$$

Beiträge aus der NW-FVA, Band 13, 2015 


$$
\begin{aligned}
& \mathrm{BHD}_{\mathrm{ijk}}=\text { Brusthöhendurchmesser von Baum } \mathrm{ijk} 1999[\mathrm{~cm}] \\
& \mathrm{h}_{\mathrm{ijk}} \quad=\text { Baumhöhe von Baum } \mathrm{ijk}_{\mathrm{ijk}} 1999[\mathrm{~m}] \\
& \text { Top_to_Dist_1ij = Modifizierter Topex-to-Distance-Index auf den Expositionen } \\
& 270 \text { und } 240 \text { für Traktecke } \mathrm{ij}_{\mathrm{ij}} \text { [Grad * 10] } \\
& \text { Top_to_Dist_2ij = Modifizierter Topex-to-Distance-Index auf den Expositionen } \\
& 90 \text { und } 60 \text { für Traktecke }{ }_{\mathrm{ij}}[\mathrm{Grad} * 10 \text { ] } \\
& \text { Top_to_Dist_3ij = Modifizierter Topex-to-Distance-Index auf den Expositionen } \\
& 320 \text { und } 190 \text { für Traktecke } \mathrm{ij}_{\mathrm{ij}} \text { [Grad * 10] } \\
& \text { Top_to_Dist_4ij = Modifizierter Topex-to-Distance-Index auf den Expositionen } \\
& 140 \text { und } 10 \text { für Traktecke } \mathrm{i}_{\mathrm{ij}} \text { [Grad * 10] } \\
& \mathrm{RW}_{\mathrm{ij}} \quad=\text { Rechtswert des Mittelpunktes von Traktecke } \mathrm{ij}_{\mathrm{ij}} \text { (Gauß-Krüger- } \\
& \text { Koordinate) } \\
& \mathrm{HW}_{\mathrm{ij}} \quad=\text { Hochwert des Mittelpunktes von Traktecke }{ }_{\mathrm{ij}} \text { (Gauß-Krüger- } \\
& \text { Koordinate) } \\
& \alpha, \delta, \gamma, \quad=\text { Vektoren von Regressionskoeffizienten } \\
& \beta_{1}, \beta_{2}, \beta_{3}, \beta_{4}=\text { Regressionskoeffizienten } \\
& \underline{\mathrm{f}} \quad=\text { Zweidimensionaler glättender Regressionsspline zur Erfassung } \\
& \text { räumlicher Autokorrelation }
\end{aligned}
$$

Das nachgeschaltete Modell zur Beschreibung des Effektes der Vernässungsstufe auf die Sturmschadenswahrscheinlichkeit bei der Fichte lässt sich wie folgt beschreiben:

$$
g\left(\pi_{i j k}\right)=g\left(\hat{\pi}_{i j k}\right)+W B_{i j}^{T} \beta_{5 W B}
$$

mit:

$$
\begin{aligned}
& \pi_{i j k} \quad=\text { Erwartungswert } \operatorname{der} \quad \text { Sturmschadenswahrscheinlichkeit } \\
& \pi_{i j k}=E\left(y_{i j k}\right) \text { von Baum } k \text { in Traktecke } i j \text { mit } i=1 \ldots 4 \text {, } \\
& j=1 . .2026 \text { und } y_{i j k} \sim \operatorname{Bernoulli}\left(\pi_{i j k}\right) \\
& \hat{\pi}_{i j k} \quad=\text { Vorschätzung des Erwartungswertes der Sturmschadenswahr- } \\
& j=1 \ldots 2026 \text { und } y_{i j k} \sim \operatorname{Bernoulli}\left(\pi_{i j k}\right) \text { auf Basis des Modells (Gl. 8) } \\
& g(.) \quad=\text { Logistische Verknüpfungsfunktion } \\
& \beta_{5 W B} \quad=\text { Ein Vektor von Regressionskoeffizienten } \\
& \mathrm{WB}_{\mathrm{ij}} \quad=\text { Ein Vektor zur Kodierung der Vernässungsstufe an Traktecke } i j \\
& \text { [nicht vernässend, wenig vernässend, vernässend] }
\end{aligned}
$$




\subsubsection{Risikokarten}

Aus der direkten Verschneidung von Standortsparametern und abiotischen und biotischen Risiken resultiert eine für praktische Planungszwecke nicht mehr operationale Anzahl von Kombinationen. Für eine Anwendung der Risikoprognosen in der strategischen Forstplanung ist daher eine Zusammenfassung der kontinuierlichen Sturmschadenswahrscheinlichkeiten in wenige Klassen notwendig. Dabei sollte der Detaillierungsgrad dem Bedarf der praktischen Forstwirtschaft angepasst werden, die nur für eine begrenzte Anzahl von Standort-Risiko-Kombinationen spezifische Maßnahmen umsetzen kann. Allerdings resultiert selbst bei Zuweisung weniger Risikoklassen zu Standorten bzw. Beständen eine gegenüber der aktuellen Waldbauplanung sehr viel feinere Planungsgrundlage für einen an den Klimawandel adaptierten Waldbau auf standörtlicher Grundlage.

Für die Klassifizierung der Risikoprognosen werden Grenzwerte benötigt. Dabei wurde auf eine Paralleluntersuchung im niedersächsischen Harz zurückgegriffen. Die dort betrachtete Fläche von 72.435 ha weist aufgrund ihrer stärkeren Reliefbildung eine größere Bandbreite bzgl. der Exponiertheit bzw. Sturmschadenswahrscheinlichkeit von Beständen auf, sodass es sinnvoll erscheint Klassengrenzwerte aus dieser Datenbasis abzuleiten. Die Prognose von Sturmschadenswahrscheinlichkeiten für die Fichte bei Zielstärke $(45 \mathrm{~cm})$ erfolgte dabei in hoher Auflösung für Rasterflächen von 0,25 ha Größe, so dass von einer sehr genauen Erfassung der Sturmschadenswahrscheinlichkeit ausgegangen werden kann. Gutachtlich wurden die folgenden Risikoklassen zugeordnet (s. Abb. 27):

- Geringes Sturmschadensrisiko: Risikowerte unterhalb des 15\%-Quantils der Häufigkeitsverteilung der Sturmschadenswahrscheinlichkeiten der Fichte bei Zielstärke $(45 \mathrm{~cm})$.

- Mittleres Sturmschadensrisiko: Risikowerte oberhalb des $15 \%$-Quantils und unterhalb des $85 \%$-Quantils der Häufigkeitsverteilung der Sturmschadenswahrscheinlichkeiten der Fichte bei Zielstärke $(45 \mathrm{~cm})$.

- Hohes Sturmschadensrisiko: Risikowerte oberhalb des $85 \%$-Quantils und unterhalb des $95 \%$-Quantils der Häufigkeitsverteilung der Sturmschadenswahrscheinlichkeiten der Fichte bei Zielstärke $(45 \mathrm{~cm})$.

- Sehr hohes Sturmschadensrisiko: Risikowerte oberhalb des 95\%-Quantils der Häufigkeitsverteilung der Sturmschadenswahrscheinlichkeiten der Fichte bei Zielstärke $(45 \mathrm{~cm})$.

Die anhand der Fichte abgeleiteten Grenzwerte werden für die Klassifizierung aller baumartenspezifischen Risiken verwendet, um einen einheitlichen Bewertungsmaßstab zu gewährleisten. Die Grenzwerte wurden für die Fichte abgeleitet, da diese Baumart die größte Sensitivität gegenüber den Risiko bestimmenden Einflussfaktoren aufweist. 


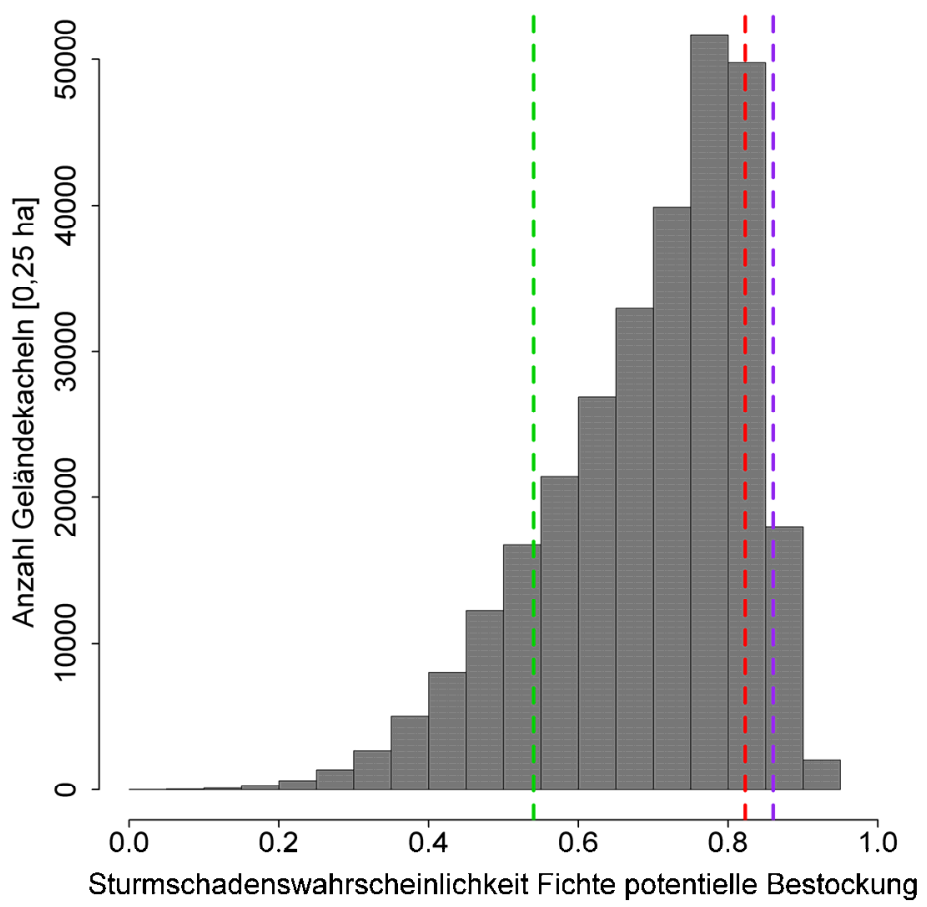

Abbildung 27: Histogramm von Sturmschadenswahrscheinlichkeiten der Fichte bei Zielstärke (45 cm) für Rasterflächen von 0,25 ha Größe im niedersächsischen Harz.

\subsection{Waldbrandrisiko}

Neben dem traditionell in Sachsen-Anhalt verwendeten Waldbrandindex M68 (KÄSE 1969) wurde das Waldbrandrisiko in dieser Studie aus Gründen der Vergleichbarkeit und um die Voraussagen auf eine breitere Basis zu stellen auch mit dem national und international gebräuchlicheren Forest Fire Weather Index (FWI, VAN WAGNER 1987) abgeschätzt (SUTMÖLLER et al. 2013). Angesichts der Tatsache, dass über $90 \%$ der Waldbrände in Deutschland durch menschliche Aktivitäten im Wald ausgelöst werden und nur weniger als $10 \%$ auf natürlichen Ursachen beruhen (BLE 2012), muss generell hinterfragt werden, ob das Waldbrandrisiko allein aufgrund klimatischer Waldbrandindizes abgeschätzt werden kann, da diese ja nichts über die Häufigkeit und Art der menschlichen Aktivitäten aussagen. Unbestreitbar sagen sie aber etwas über die Prädisposition der Vegetation aus, indem sie die Materialfeuchte von Boden, Streu, Unterwuchs und Bestand abschätzen, die eine wesentliche Voraussetzung für die Zündbereitschaft ist. Wie ausschlaggebend die klimatisch bedingte Prädisposition für das tatsächliche Auftreten von Waldbränden ist, lässt sich in Abbildung 28 nachvollziehen. 
Die Anzahl von Risikotagen mit extrem trockener Streu in den Waldbeständen wird hier durch den für alle Waldstandorte der Waldzustandserhebung berechneten Streufeuchtecode (FFMC, Fine Fuel Moisture Code, VAN WAGNER 1987) wiedergegeben. Die Überschreitung des Schwellenwerts von 92 entspricht dabei einem Absinken des Wassergehalts in der am Boden liegenden Feinstreu unter $9 \%$. Beim Vergleich mit der Zahl der jährlichen Waldbrände in Sachsen-Anhalt erkennt man durchaus Parallelen, die bei einer überwiegenden Abhängigkeit von menschlichen Aktivitäten nicht zu erwarten wären, es sei denn, dass diese die eigentliche Zündung auslösenden Aktivitäten zu jedem Zeitpunkt etwa gleich häufig vorkommen, so dass sie letztlich in der Betrachtung der Waldbrandhäufigkeit unberücksichtigt bleiben können. Dass es dennoch auch Jahre mit hoher Anzahl streutrockener Tage gegeben hat, in denen die Zahl der Waldbrände relativ gering war, lässt sich einerseits auf das mögliche Ausbleiben der stochastisch verteilten Zündursache zurückführen, andererseits aber auch auf den generell abnehmenden Trend der Waldbrandzahlen, der auf die bessere Vorbeugung und Erkennung von entstehenden Waldbränden zurückgeführt wird (KÖNIG 2007).

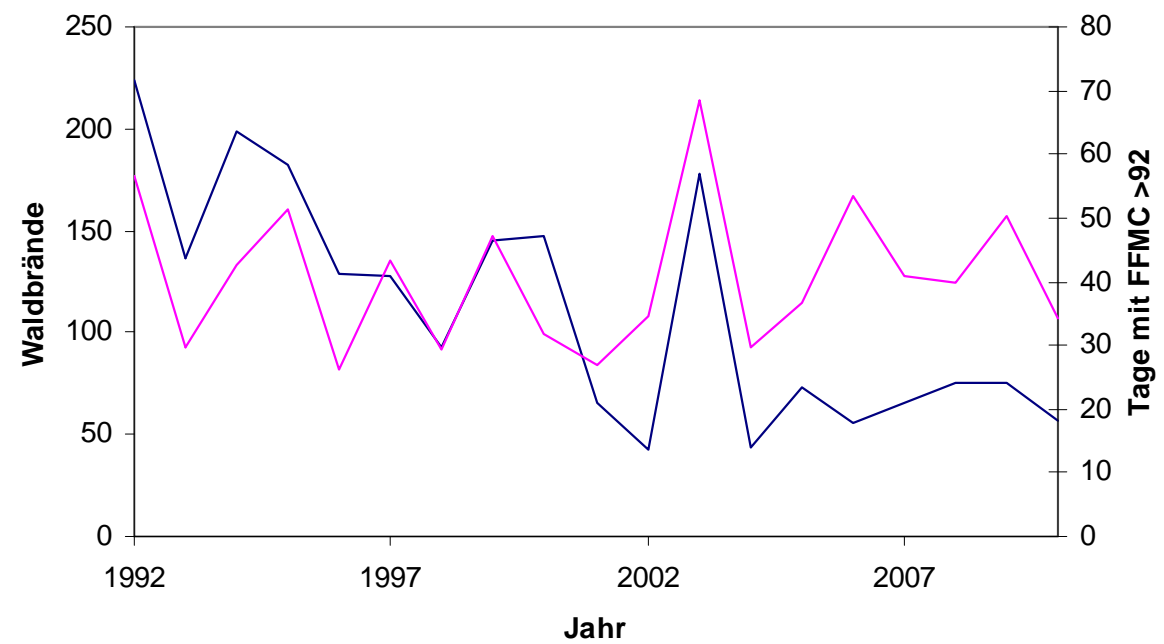

Anzahl Brände —_ Tage mit FFMC >92

Abbildung 28: Jährliche Anzabl von Waldbränden (blau) und von Tagen erböbten Waldbrandrisikos (rot, FFMC = Streufeuchtezabl) in Sachsen-Anhalt

Insgesamt ging die Zahl der Waldbrände in Sachsen-Anhalt von 1992 bis 2010 von 224 auf 57 zurück. Gleichzeitig sank auch der Anteil anthropogen verursachter Waldbrände an der Gesamtzahl der Waldbrände von ca. $60 \%$ auf etwa $50 \%$, während der Anteil natürlicher Ursachen zunahm (s. Abb. 29). Trotz dieses erfreulichen, generell rückläufigen Trends sticht aber das Jahr 2003 in der zweiten Hälfte des Betrachtungszeitraums mit 178 Bränden aus der Reihe der Jahre 2001 - 2010 mit 
durchweg ca. 60 Bränden heraus. In diesem Jahr mit der bei weitem höchsten Waldbrandgefährdung (69 Tage mit FFMC > 92) war die verbesserte Vorbeugung demnach nicht so wirksam wie in den anderen Jahren. Dies legt die Vermutung nahe, dass die verbesserten Vorbeugungsmaßnahmen in Normaljahren eine Reduktion der Waldbrandzahlen bewirken können, jedoch in extrem trockenen Sommern aber nicht hinreichend für die Vermeidung von Waldbränden sind, so dass dann die klimatische Prädisposition für das Auftreten von Waldbränden ausschlaggebend wird. Dementsprechend liegt die Rechtfertigung für die Verwendung klimatischer Waldbrandindizes vor allem darin, dass sie das Auftreten klimatischer Extrembedingungen charakterisieren können, die unabhängig von Vorbeugungsmaßnahmen zum verstärkten Auftreten von Waldbränden führen.

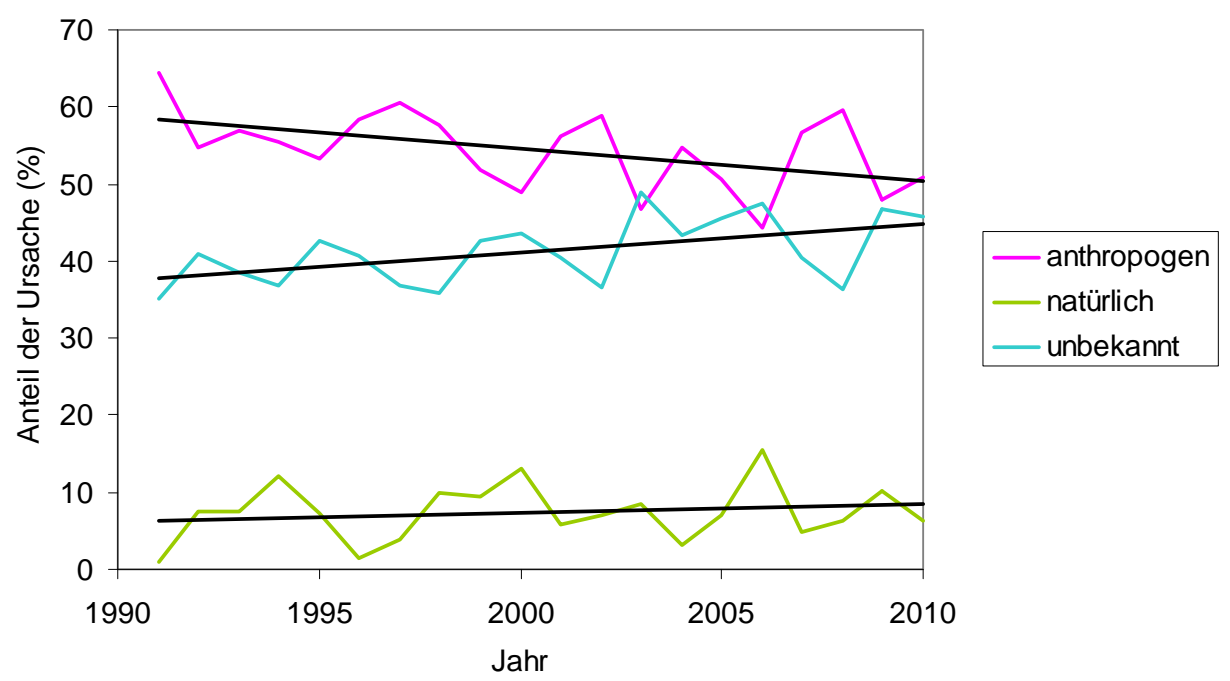

Abbildung 29: Anteil von anthropogenen und natürlichen Ursachen an den Waldbränden in SachsenAnbalt [\%]

Um das Auftreten klimatischer Extrembedingungen mit dem M68-Index und dem FWI zu quantifizieren, werden in der regionalen Auswertung nur die Risikotage betrachtet, an denen die jeweilige Ausgabegröße des M68 bzw. des FWI einen kritischen Schwellenwert überschreitet. Beim M68 gilt als kritischer Schwellenwert für die Waldbrandkennziffer (WBKZ) ein Wert von 4000 (KÄSE 1969). Beim FWI wurde aus den verschiedenen Maßzahlen für Oberbodenfeuchte (DC), Humusfeuchte (DMC) und Streufeuchte (FFMC) der FFMC ausgewählt, weil die Streufeuchte für die Zündbereitschaft entscheidender ist als die Bodenfeuchte. Eine der Risikoschwelle der WBKZ entsprechende Risikoschwelle des FFMC liegt etwa bei 92 (entsprechend einer Unterschreitung der Streufeuchte von $9 \%$ ). Im Unterschied zur WBKZ ist der FFMC aber nicht nach oben offen, sondern erreicht einen Maximalwert bei 99, der einer Streufeuchte von $2 \%$ entspricht. 
Als Grundlage der Waldbrandindex-Berechnung für den aktuellen Zustand wurden die Messwerte der in und um Sachsen-Anhalt herum liegenden DWD-Klimastationen für den Zeitraum 2004 - 2010 verwendet, für den auch die Einzeldaten zu Waldbränden vom Landesamt für Umwelt (LAU) zur Verfügung gestellt wurden. Die Klimadaten wurden mittels inverser Distanzwichtung auf den WaldflächenMittelpunkt jeder Gemeinde interpoliert, außerdem wurde bezüglich der mittleren Geländehöhe der Waldgebiete jeder Gemeinde eine Höhenkorrektur durchgeführt. Die Bezugnahme auf die Gemeindegrenzen ist eine Konsequenz der auf die Gemeindegrenzen bezogenen Waldbrandstatistik. Bei den Index-Vorhersagen auf Basis des WETTREG2010 Klimamodells wurde dagegen auf eine räumliche Interpolation der Szenarien verzichtet. Hier wurde stattdessen eine Interpolation der mit WETTREG2010 berechneten Waldbrandindexwerte auf Basis des nearest neighbour-Verfahrens vorgenommen. Durch die Anwendung dieses Verfahrens werden zufällige räumliche Unterschiede innerhalb der Szenarien nicht überbewertet, es sind jedoch aufgrund mangelnder Regressionsbeziehung keine Höhenkorrekturen möglich, so dass bei großen Höhenunterschieden nur dann lagegenaue Ergebnisse erzielt werden, wenn die Dichte der Klimastationen die Variabilität der Geländehöhen widerspiegelt. Insbesondere im klimastationsfreien Oberharz sind die Ergebnisse daher als regionsspezifische Durchschnittswerte zu interpretieren.

Waldbrandindizes sind nicht in der Lage, die lokale Bedeutung zusätzlicher Risikofaktoren abzubilden. Für drei Risikofaktoren wurde deshalb die räumliche Verteilung innerhalb der Regionen kartiert:

1. Direkte Sonneneinstrahlung

Da über Waldbrandindizes der regional bedeutsame Effekt der unterschiedlichen Sonneneinstrahlung etwa auf Nord- und Südhängen nicht berücksichtigt werden kann, wurde untersucht, ob sich in den jeweiligen Regionen Gebiete mit relativ erhöhtem Risiko aufgrund der topographisch bedingten längeren Sonnenscheindauer befinden.

2. Globalstrahlungsenergie

Ebenfalls topographisch bedingt unterscheidet sich auch die täglich auf die Fläche einfallende Globalstrahlungsenergie während der Hauptwaldbrandperiode (1.4. - 31.8. jedes Jahres). Die Globalstrahlungsenergie enthält außer der direkten Sonneneinstrahlung auch die diffuse Himmelsstrahlung. Bei diesen Berechnungen wurde von einem durchschnittlichen Anteil von $40 \%$ diffuser Strahlung ausgegangen und die eintreffende Globalstrahlung anhand von Flächenneigung, Hangrichtung und Sonnenlauf berechnet.

3. Locker bestockte Kiefernjungbestände

Da mehr als die Hälfte der in Sachsen-Anhalt auftretenden Waldbrände Kiefernwälder betroffen haben und diese besonders bis zum Alter von 40 Jahren und bei geringem Bestockungsgrad aufgrund der leichter entzünd- 
lichen Streu waldbrandanfälliger sind (MISSBACH 1972), wurden Kiefernbestände unter 40 Jahren bei einem Bestockungsgrad kleiner oder gleich 0,7 als lokale Risikogebiete ausgewiesen.

\subsection{Methodik der Prognose des Buchdruckerbefallsrisikos}

Der Klimawandel wird auf vielen Standorten zu periodischem Wassermangel und damit zu einer physiologischen Schwächung der Waldbäume führen. Dies wird bei der Fichte die Gefährdung durch Borkenkäfer wesentlich erhöhen. Zudem werden die steigenden Wärmesummen in der Vegetationszeit einen Anstieg der mittleren Generationenzahl der Borkenkäfer pro Jahr bzw. einen exponentiellen Anstieg der Populationen bewirken (WERMELINGER u. SEIFERT 1998, BAIER et al. 2007). Zur Beurteilung des Befallsrisikos der Fichte durch Borkenkäfer wurde ein statistisches Modell weiterentwickelt, das bisher auf der Grundlage von Hiebsauswertungen im niedersächsischen Harz parametrisiert worden war (OVERBECK u. SCHMIDT 2011). Dazu wurde die Datenbasis um Hiebsauswertungen aus den übrigen niedersächsischen Berglandregionen und der Modellregion Oberharz ergänzt.

\subsubsection{Datengrundlage}

Das für die Region Oberharz verwendete Modell erstellt eine Prognose des 10-jährigen Buchdrucker-Befallsrisikos für Flächen mit Fichtenanteil. Da der zur Berechnung verwendete Modellansatz ein Bestandesmodell ist, wurden unten genannte Bestandes- und Standortsparameter mit den Daten der Befallsflächen des Buchdruckers verschnitten. Als befallen galten dabei Flächen, bei denen mehr als $1 \%$ des Einschlags auf der Teilfläche Käferholz aufwies. Aus den insgesamt 6.826 Teilflächen im Untersuchungsgebiet Oberharz wiesen 3.173 Teilflächen zum Simulationszeitpunkt Fichtenbestockung im Hauptbestand auf und gingen somit in die Auswertung ein. Durch den Sturm Kyrill (2007) vernichtete Bestände und nicht zuzuordnende Daten sowie Bestände, die jünger als 30 Jahre oder älter als 180 Jahre alt waren, wurden aus dem Datenpool entfernt. Zum Zeitpunkt der Modellberechnung wurden für den Bereich des Oberharzes 42,3\% der Teilflächen als befallen bezeichnet.

Die Prädiktoren, die im Modell das Niveau des Buchdruckerbefallsrisikos bestimmen (s. Tab. 12), lassen sich untergliedern in die Bestandesparameter:

- Bestandesalter der Fichte

- Mischungsprozent der Fichte

und die Standortsparameter:

- Mittelwert der Temperatursumme in der Vegetationszeit der Fichte in den vier Projektionsperioden

- Nutzbare Feldkapazität (nFK) 
- Topex zur Beschreibung der Südexponiertheit

- Geografische Lage (Gauß-Krüger-Koordinaten)

Tabelle 12: Verteilungen unabhängiger Variablen des Modells zur Prognose des Buchdruckerbefallsrisikos in der Modellregion Oberharz für die aktuell mit Fichte (Stichjahr 2006) bestockten Flächen (Werte der Ist-Bestockung) und die gesamte Waldfläche (potenzielle Bestockung)

Ist-Bestockung

$$
\text { Quantile }
$$

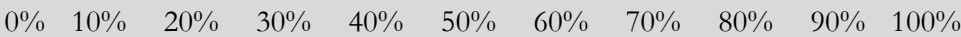

$\begin{array}{llllllllllll}\text { Mischungsprozent } & 1 & 27 & 67 & 87 & 94 & 100 & 100 & 100 & 100 & 100 & 100\end{array}$

Fichte [\%]

Bestandesalter der

Fichte [J]

$\begin{array}{lllllllllll}0 & 18 & 24 & 34 & 41 & 49 & 57 & 72 & 86 & 106 & 212\end{array}$

Temperatursumme in der Vegetationszeit $\left[{ }^{\circ} \mathrm{C}\right]$ :

\begin{tabular}{lrrrrrrrrrrr}
$1981-2010$ & 1197 & 1672 & 1700 & 1731 & 1752 & 1807 & 1853 & 1882 & 1911 & 1959 & 2167 \\
$2011-2040$ & 1521 & 1921 & 1971 & 2002 & 2023 & 2056 & 2072 & 2084 & 2103 & 2154 & 2362 \\
$2041-2070$ & 1933 & 2229 & 2263 & 2286 & 2321 & 2343 & 2353 & 2367 & 2385 & 2422 & 2612 \\
$2071-2100$ & 2238 & 2470 & 2501 & 2524 & 2550 & 2572 & 2590 & 2605 & 2622 & 2660 & 2807 \\
\hline Topex & -9 & -1 & -1 & 0 & 1 & 2 & 3 & 5 & 8 & 13 & 42 \\
$\mathrm{nFK}[\mathrm{mm}]$ & 24 & 91 & 102 & 114 & 115 & 121 & 123 & 123 & 126 & 143 & 206 \\
\hline
\end{tabular}

Potenzielle Bestockung

Quantile

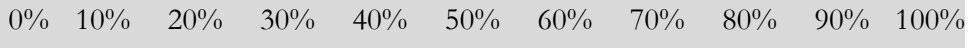

Temperatursumme in der Vegetationszeit $\left[{ }^{\circ} \mathrm{C}\right]$ :

\begin{tabular}{lrrrrrrrrrrr}
$1981-2010$ & 1197 & 1679 & 1707 & 1738 & 1763 & 1835 & 1862 & 1893 & 1920 & 1984 & 2207 \\
$2011-2040$ & 1521 & 1935 & 1978 & 2016 & 2027 & 2067 & 2075 & 2090 & 2111 & 2180 & 2406 \\
$2041-2070$ & 1933 & 2237 & 2270 & 2295 & 2327 & 2348 & 2357 & 2373 & 2392 & 2443 & 2647 \\
$2071-2100$ & 2238 & 2478 & 2507 & 2532 & 2556 & 2580 & 2595 & 2611 & 2629 & 2676 & 2829 \\
\hline Topex & -15 & -1 & 0 & 0 & 1 & 2 & 4 & 5 & 8 & 13 & 67 \\
nFK $[\mathrm{mm}]$ & 24 & 93 & 103 & 114 & 116 & 122 & 123 & 123 & 126 & 143 & 304 \\
\hline
\end{tabular}

Über die sich ändernden Temperatursummen wird der Effekt des projizierten Klimawandels auf das Befallsrisiko prognostiziert. Die Temperatursummen wurden unter Verwendung der spezifischen jährlichen Vegetationsperioden der Fichte bilanziert und anschließend für jede der vier Projektionsperioden gemittelt. Der TopexIndex dient als Proxyvariable, um kleinräumige reliefbedingte Unterschiede im Strahlungshaushalt der Bestände zu erfassen. Die Topex-Werte wurden für die Expositionen S, SW, SO bei einer Grenzdistanz von $150 \mathrm{~m}$ berechnet und 
anschließend gemittelt. Die Berechnung der Topex-Werte erfolgte auf der Grundlage eines digitalen Geländemodells mit $10 \mathrm{~m}$ Auflösung. Jedem Bestand wurde vereinfachend der Topexwert der $10 \times 10 \mathrm{~m}$ Rasterzelle zugeordnet, in der sein Bestandeszentroid liegt. Die Standortsdaten Temperatursumme in der Vegetationszeit sowie die geografische Lage eines Bestandes beziehen sich ebenfalls auf die Koordinaten des Bestandeszentroids. Die nFK resultiert aus der Verschneidung der Bestandesgeometrien mit den Standortspolygonen. Treten mehrere Standortseinheiten innerhalb eines Bestandes auf, wurde der flächengewichtete Mittelwert der nFK verwendet (s. Kap. 4.1).

Entsprechend der Vorgehensweise zur Sturmschadensmodellierung werden unterschiedliche Ansätze für die Unterstützung der kurz- bis mittelfristigen bzw. langfristigen Planung gewählt:

- Die Prognose des Befallsrisikos für verschiedene potenzielle Bestockungen für die gesamte Waldfläche der Region Oberharz.

- Die Prognose des Befallsrisikos für die aktuelle Bestockung für alle Bestände, in denen die Fichte entsprechend der Forsteinrichtung als Haupt- oder Mischbaumart vorkommt.

Das Befallsrisiko für die Ist-Bestockung wird unter Verwendung der aktuellen Bestandesalter und Mischungsprozente im Stichjahr 2006 prognostiziert. Das Befallsrisiko für potenzielle Bestockungen wird einheitlich für ein Bestandesalter von 92 Jahren und für ein Fichtenmischungsprozent von $100 \%$ geschätzt. Das Alter 92 ist der Mittelwert der Schätzungen des Alters bei Zielstärke (s. Gl. 7) aller Bestände der Region Oberharz.

\subsubsection{Statistisches Modell zur Prognose des Befallsrisikos durch den Buchdrucker}

Als Modelltyp zum Buchdruckerbefallsrisiko wird ein verallgemeinertes additives gemischtes logistisches Regressionsmodell GAMM verwendet. Das Modell quantifiziert das 10-jährige Befallsrisiko der Fichte auf Bestandesebene in Abhängigkeit des Bestandesalters der Fichte, des Fichtenmischungsanteils, der Temperatursumme in der Vegetationszeit der Fichte, der nutzbaren Feldkapazität (nFK), der Südexponiertheit sowie der geografischen Lage (s. Gl. 14).

$$
\begin{aligned}
& g\left(\pi_{i j}\right)=\beta_{0}+f_{1}\left(\text { Alter }_{i j}\right)+f_{2}\left(\text { Misch }_{i j}\right)+f_{3}\left(\text { Tempsum }_{i j}\right) \\
& +f_{4}\left(n F K_{i j}\right)+f_{5}\left(\text { Topex }_{i j}\right)+f_{6}\left(\text { RW }_{i j}, H W_{i j}\right)+b_{j}
\end{aligned}
$$

wobei $\pi_{i j}=\mathrm{E}\left(\mathrm{y}_{i j}\right)$ mit $\mathrm{y}_{i j} \sim \operatorname{binomial}\left(1, \pi_{i j}\right)$ und $b_{j} \sim \mathrm{N}\left(0, \sigma^{2}\right)$ und $j=1, \ldots, 107$. 
mit:

\begin{tabular}{|c|c|c|}
\hline$g()$. & $=$ & Logistische Verknüpfungsfunktion \\
\hline$\pi_{i j}$ & $=$ & $\begin{array}{l}\text { Erwartungswert } \mathrm{E}\left(\mathrm{y}_{\mathrm{ij}}\right) \text { der Wahrscheinlichkeit eines Borken- } \\
\text { käferbefalls in Bestand } i \text { in Revier } j \text { innerhalb von } 10 \text { Jahren }\end{array}$ \\
\hline$f_{1}, f_{2}, f_{3}, f_{4}, f_{5}$ & $=$ & $\begin{array}{l}\text { Eindimensionale glättende Regressionssplines zur Erfassung } \\
\text { des nicht-linearen Effektes metrischer Kovariablen }\end{array}$ \\
\hline$f_{6}$ & $=$ & $\begin{array}{l}\text { Zweidimensionaler glättender Regressionsspline zur Erfas- } \\
\text { sung räumlicher Autokorrelation }\end{array}$ \\
\hline Alter $_{i j}$ & $=$ & Alter [J] von Bestand $i$ in Revier $j$ \\
\hline Misch $_{i j}$ & $=$ & Mischungsprozent der Fichte $[\%]$ in Bestand $i$ in Revier $j$ \\
\hline Tempsum $_{i j}$ & $=$ & $\begin{array}{l}\text { Mittlere Temperatursumme }\left[{ }^{\circ} \mathrm{C}\right] \text { innerhalb der Vegetations- } \\
\text { periode der Fichte im Zeitraum } 1981-2010 \text { von Bestand } i \text { in } \\
\text { Revier } j\end{array}$ \\
\hline$n F K_{i j}$ & $=$ & $\begin{array}{l}\text { Flächengewichtete nutzbare Feldkapazität [mm] von Bestand } i \\
\text { Revier } j\end{array}$ \\
\hline Topex $i j$ & $=$ & $\begin{array}{l}\text { Mittelwert der Topexwerte auf den Expositionen S, SW, } \\
\text { SO }\left[^{\circ}\right] \text { von Bestand } i \text { Revier } j\end{array}$ \\
\hline$b_{j}$ & $=$ & $\begin{array}{l}\text { Ein Vektor von Zufallseffekten zur Erfassung des Revier- } \\
\text { effektes mit unbekannter positiv-definiter Kovarianzmatrix } \psi \\
\text { mit } b_{j} \sim \mathrm{N}(0, \psi) \text {, wobei unterstellt wird, dass die Zufallseffekte } \\
\text { unabhängig und identisch normalverteilt sind }\end{array}$ \\
\hline
\end{tabular}

Bei den meisten biotischen Schadrisiken ist mit einer deutlichen Überprägung durch die forstliche Bewirtschaftung zu rechnen. Allerdings liegen im Allgemeinen keine Informationen über potenzielle Einflussgrößen wie die jeweilige Holzmarktsituation, unterschiedliche Aufarbeitungsverfahren, die Erschließungsdichte und die Intensität von Forstschutzmaßnahmen vor. In dem verwendeten Regressionsmodell wird versucht, diese Effekte über Zufallseffekte auf der Ebene des Reviers zu quantifizieren. Dieser Vorgehensweise liegt die Annahme zugrunde, dass der direkte menschliche Einfluss im Bereich der „,sauberen Forstwirtschaft“ eng mit der Revierzugehörigkeit korreliert ist. Über die Zufallseffekte lassen sich diese Abweichung von einem „Populationsmittel“" quantifizieren und die Effekte unbekannter „Störvariablen“ können weitestgehend ausgeschaltet werden, ohne dass eine Kausalität wie bei den festen Effekten abgebildet wird. Für möglichst realistische Einschätzungen des aktuellen Risikos sollten die Zufallseffekte bei der Prognose berücksichtigt werden. Für Prognosen im Bereich der strategischen Planung sollte der direkte menschliche Einfluss unterdrückt bzw. nur die festen Modelleffekte der Bestandes- und Standortsparameter abgebildet werden. In der vorliegenden Untersuchung erfolgt daher eine Prognose unter ausschließlicher Verwendung der festen Effekte. Im Rahmen der Weiterentwicklung des Modells wurde deutlich, dass neben 
den zufälligen (unstrukturierten) Zufallseffekten, zusätzlich räumlich korrelierte (strukturierte) Abweichungen auftreten (FAHRMEIR et al. 2007), die nicht den kausalen Effekten innerhalb des Modells zugeordnet werden können. Dieser räumlich strukturierte Fehler wird über eine zweidimensionale glättende Trendfunktion $f_{6}$ als Funktion der geografischen Lage erfasst. Der Effekt kann dahingehend interpretiert werden, dass er die Effekte aller räumlich korrelierten Variablen beinhaltet, die nicht oder nur unzureichend über die bereits im Modell integrierten kausalen Variablen erfasst werden. Ergänzend werden statistische Kenngrößen des Modells aufgeführt (s. Tab. 13).

Abschließend werden die festen Effekte der kausalen Variablen auf der Ebene des linearen Prädiktors dargestellt, um die Unterschiede zur bisher veröffentlichten Version und hier insbesondere die erhöhte Sensitivität gegenüber verschiedenen Prädiktorvariablen darzustellen (OvERBECK u. SCHMIDT 2011). Ergänzend dienen $95 \%$-Konfidenzintervalle der Darstellung der Signifikanz der Modelleffekte. Bei der Interpretation der Effekte ist zu beachten, dass nur eine qualitative Aussage bezüglich des resultierenden Risikos zulässig ist, da der lineare Prädiktor erst mit Hilfe der inversen Verknüpfungsfunktion in Befallswahrscheinlichkeiten transformiert werden muss.

Tabelle 13: $\quad$ Statistische Kenngrößen des Modells zur Prognose des Befallsrisikos durch den Buchdrucker (s. Gl. 14)

\begin{tabular}{lrrrrr}
\hline Feste Modelleffekte & & & & & \\
& Koeffizient & Std.-Fehler & t-Wert & p-Wert & \\
\hline Interzept & -1.7617 & 0.1049 & -16.79 & $<2 \mathrm{e}-16$ & $* * *$
\end{tabular}

\begin{tabular}{lrrrr} 
& Freiheitsgrade & F-Wert & p-Wert & \\
\hline$f_{1}$ (Alter) & 4.839 & 236.856 & $<2 \mathrm{e}-16$ & $* * *$ \\
$f_{2}$ (Misch) & 4.233 & 118.02 & $<2 \mathrm{e}-16$ & $* * *$ \\
$f_{3}($ Tempsum $)$ & 1.911 & 9.979 & 0.000077 & $* * *$ \\
$f_{4}($ nFK $)$ & 2.946 & 4.885 & 0.00237 & $* *$ \\
$f_{5}($ Topex $)$ & 3.43 & 7.622 & 0.000021 & $* * *$ \\
$f_{6}($ RW, HW $)$ & 13.964 & 5.056 & $1.60 \mathrm{e}-09$ & $* * *$
\end{tabular}

Zufällige Modelleffekte auf Revierebene

$2.5 \%$-Quantil Erwartungswert $\quad 97.5 \%$-Quantil

\begin{tabular}{lccc}
$\begin{array}{l}\text { Standardabw. } \\
\text { Interzept }\end{array}$ & 0.772 & 0.947 & 1.162 \\
\hline
\end{tabular}

\begin{tabular}{lll}
\hline R-sq. $($ adj $)=$ & $0.2 \quad$ Anzahl Reviere: $107 \quad$ Anzahl Beobachtungen: 12938 \\
\hline
\end{tabular}




\subsubsection{Modelleffekte}

Das Bestandesalter ist neben dem Mischungsprozent die Variable mit dem stärksten Effekt auf das Befallsrisiko. Ab einem Bestandesalter von ca. 35 Jahren bis zu ca. 100 Jahren ist ein annährend linear ansteigender Effekt zu beobachten (s. Abb. 30). Ab ca. 100 Jahren ist ein erst konstanter und dann leicht absinkender Effekt zu beobachten, der allerdings kaum signifikant ist (graues Band des Konfidenzintervalls). Aus Expertensicht wurde angenommen, dass das Risiko mit steigendem Alter nicht sinkt, was bei der Modellanwendung durch eine Approximation des originalen Effektes erreicht wird, die ab einem Alter von 130 Jahren einen konstanten Wert unterstellt (rot gestrichelte Linie). Unter Anwendung des approximierten Effektes resultiert somit bei sonst konstanten Einflussfaktoren bis zu einem Alter von 130 Jahren ein monoton steigendes und anschließend konstantes Befallsrisiko.

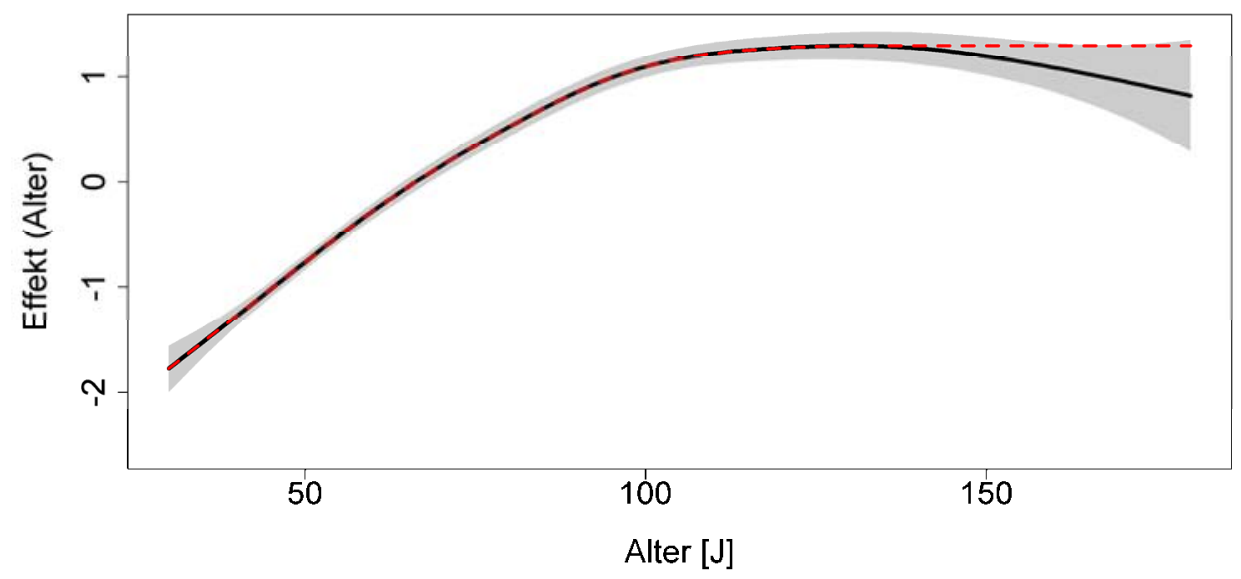

Abbildung 30: Modelleffekt des Bestandesalters auf das 10-jährige Befallsrisiko der Fichte durch den Buchdrucker mit Darstellung des $95 \%$-Konfidenzintervalls (graues Band). Der originale Effekt ist durch die schwarze Linie und eine zugehörige Approximation, die ein Absinken des Risikos mit steigendem Alter verbindert, durch die rot gestrichelte Linie dargestellt.

Der Effekt des Mischungsanteiles der Fichte verläuft monoton steigend, wobei der Gradient der Zunahme mit steigendem Mischungsanteil abnimmt (s. Abb. 31). Somit resultiert bei sonst konstanten Einflussfaktoren über den gesamten Wertebereich ein monoton steigendes Befallsrisiko mit steigendem Mischungsanteil der Fichte. Die Amplitude des Effektes über den gesamten Wertebereich des Mischungsprozentes ist relativ hoch und beträgt ungefähr 2/3 der Amplitude des Effektes des Bestandesalters. 


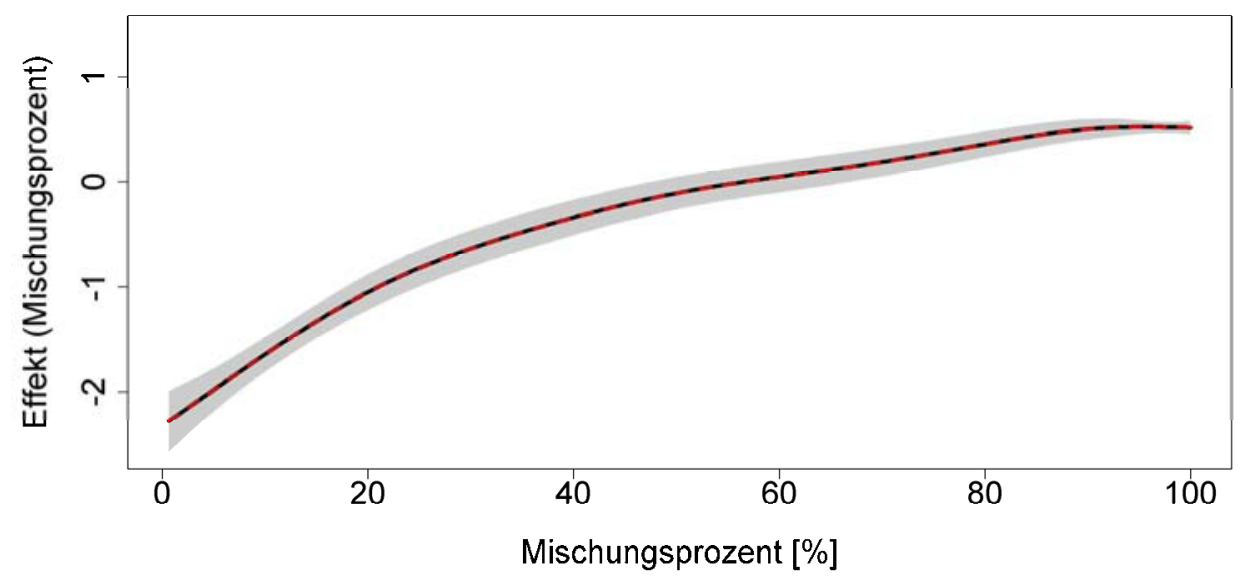

Abbildung 31: Modelleffekt des Mischungsprozentes der Fichte auf das 10-jährige Befallsrisiko der Fichte durch den Buchdrucker mit Darstellung des $95 \%$-Konfidenzintervalls (graues Band). Der originale Effekt ist durch die schwarze Linie und eine zugehörige Approximation, die ein Absinken des Risikos mit steigendem Mischungsprozent verbindert, durch die rot gestrichelte Linie dargestellt.

Der Effekt der Temperatursumme ist über den gesamten Wertebereich monoton steigend, wobei zwei annähernd lineare Bereiche unterhalb und oberhalb von ca. $1950{ }^{\circ} \mathrm{C}$ erkennbar sind (s. Abb. 32). Dabei ist der Gradient der Zunahme oberhalb von ca. $1950 \mathrm{C}^{\circ}$ deutlich stärker als unterhalb. Die Amplitude des Modelleffektes über den gesamten Wertebereich der Temperatursumme ist etwas geringer als die Amplitude des Effektes des Mischungsprozentes.

Die senkrechten gestrichelten Linien markieren die Maximalwerte der Temperatursumme in der Region Oberharz für die vier Projektionsperioden (s. Abb. 32). Es wird deutlich, dass es bis zur Periode 2011 - 2040 nicht zu Extrapolationen kommt. Die Prognosen für die Periode 2041 - 2070 liegen für nur knapp $10 \%$ und für die Periode 2071 - 2100 für etwa $95 \%$ der Bestände im Extrapolationsbereich. Bis einschließlich der Periode 2041 - 2070 sind somit für die Mehrzahl der Bestände der Region Oberharz Prognosen innerhalb des Parametrisierungsbereiches des Modells möglich. Diese Tatsache liegt darin begründet, dass Bestände in den tieferen Lagen des niedersächsischen Harzes und Weserberglandes bereits aktuell Temperatursummen aufweisen, wie sie von vielen Beständen der Region Oberharz erst in den Perioden 2011 - 2040 bzw. 2041 - 2070 erreicht werden. Für Prognosen im Extrapolationsbereich erfolgt eine lineare Extrapolation des Modelleffektes. Bei sonst konstanten Einflussfaktoren resultiert über den gesamten Wertebereich ein monoton steigendes Befallsrisiko mit steigender Temperatursumme. 


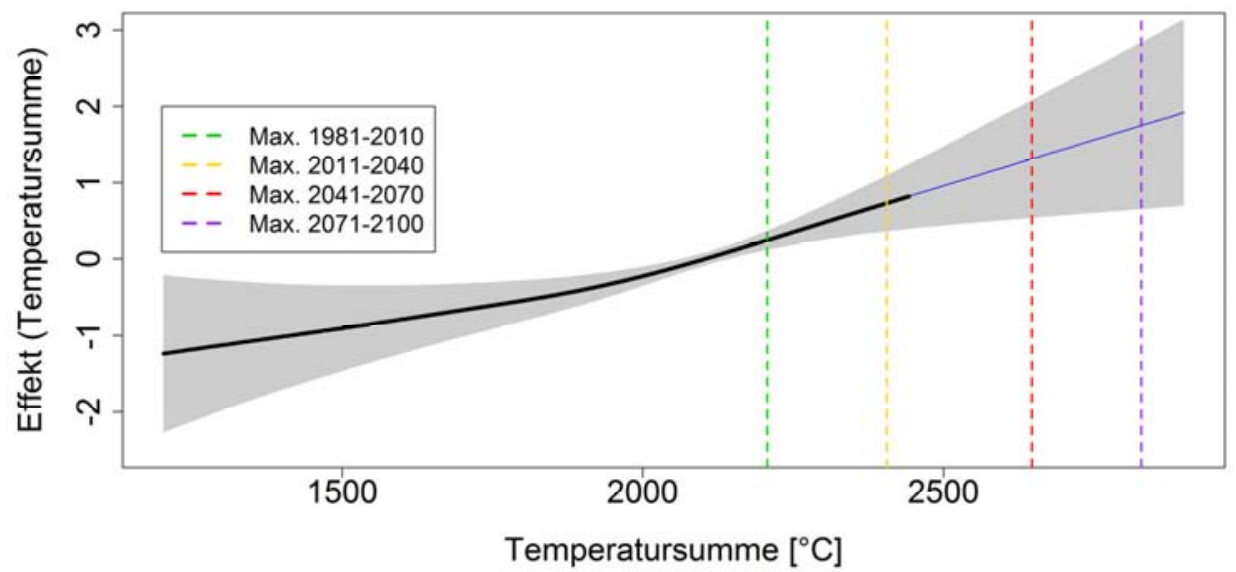

Abbildung 32: Modelleffekt der Temperatursumme in der Vegetationszeit $\left[{ }^{\circ} \mathrm{C}\right]$ auf das 10-jährige Befallsrisiko der Fichte durch den Buchdrucker mit Darstellung des $95 \%$-Konfidenzintervalls (graues Band). Zusätzlich sind die Maximalwerte der Temperatursumme für die 4 Projektionszeiträume dargestellt. Der Modelleffekt der Temperatursumme ist für den Parametrisierungsbereich durch die fette schwarze Linie und für den Extrapolationsbereich durch die düne blaue Linie dargestellt.

Der Modelleffekt der nFK weist ein schwach ausgeprägtes Maximum bei ca. $118 \mathrm{~mm}$ auf. Der mit sinkender nFK schwach abfallende Modelleffekt ist nicht signifikant, was anhand des breiten Konfidenzbereiches deutlich wird. Für diesen Bereich wurde der originale Modelleffekt daher durch eine Konstante approximiert, die dem Effekt für $118 \mathrm{~mm} n \mathrm{nK}$ entspricht. Somit resultieren bei sonst konstanten Einflussfaktoren unterhalb von $118 \mathrm{~mm}$ nFK konstante Befallsrisiken auf dem Niveau des Maximalwertes. Oberhalb von 118 mm nFK weist der originale Modelleffekt ebenfalls einen schwach sinkenden Trend auf. Das relativ enge Konfidenzband kennzeichnet den sinkenden Effekt im Bereich zwischen 118 und ca. $180 \mathrm{~mm}$ als signifikant. Gleichzeitig kann ein mit steigender nFK sinkender Modelleffekt aus Expertensicht als plausibel bezeichnet werden. Im Vergleich mit den Prädiktoren Bestandesalter, Mischungsprozent und Temperatursumme ist die nFK als Variable mit nachrangiger Bedeutung zu beurteilen (s. Abb. 33).

Der Modelleffekt des Topex zur Erfassung der Südexponiertheit weist ein schwach ausgeprägtes Maximum bei ca. $3^{\circ}$ auf. Der sinkende Trend mit zunehmender Südexponiertheit (sinkendem Topex) ist kaum signifikant und wird durch eine Konstante approximiert, die dem Effekt für einen Topex von $3^{\circ}$ entspricht. Somit resultieren bei sonst konstanten Einflussfaktoren mit steigender Südexponiertheit konstante Befallsrisiken auf dem Niveau des Maximalwertes (s. Abb. 34). Mit zunehmender Beschattung durch vorgelagerte Hänge oder zunehmender Neigung auf Nordhängen (steigende Topex-Werte) resultiert ebenfalls ein sinkender Modelleffekt. Das Konfidenzband zwischen 3 und ca. $20^{\circ}$ ist relativ eng und der Trend stimmt hier mit Expertenwissen überein. Im Vergleich mit den Prädiktoren 
Bestandesalter, Mischungsprozent und Temperatursumme ist der Topex als Variable mit nachrangiger Bedeutung zu beurteilen (s. Abb. 34).

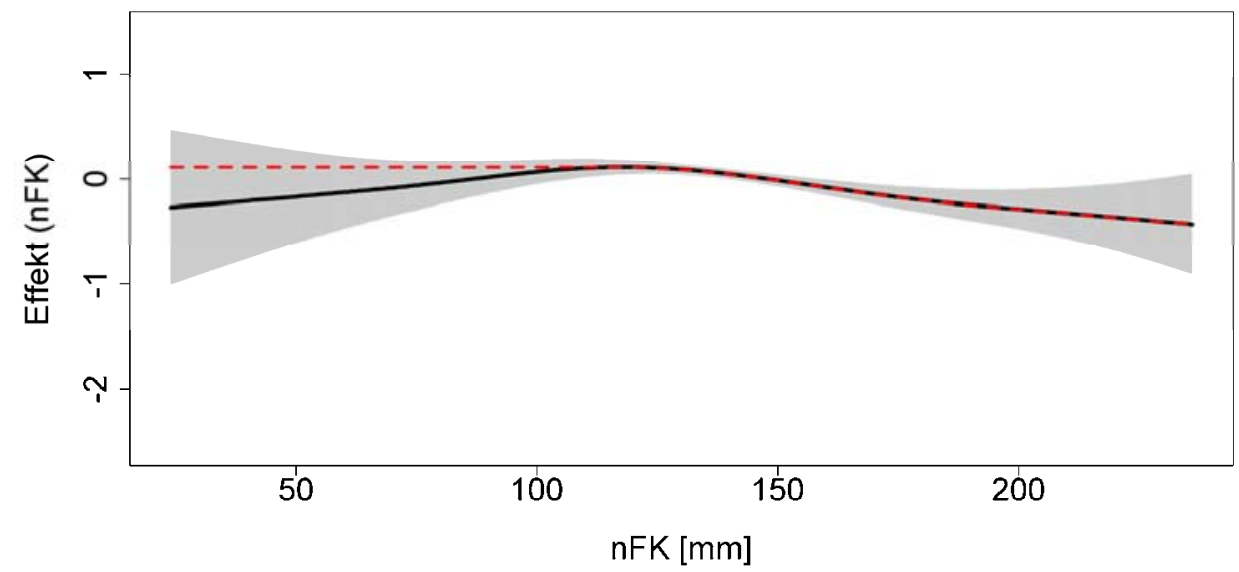

Abbildung 33: Modelleffekt der nutzbaren Feldkapazität ( $\mathrm{FFK}$ ) auf das 10-jährige Befallsrisiko der Fichte durch den Buchdrucker mit Darstellung des $95 \%$-Konfidenzintervalls (graues Band). Der originale Effekt ist durch die schwarze Linie und eine zugehörige Approximation, die ein Absinken des Risikos mit sinkender $n F K$ verbindert, durch die rot gestrichelte Linie dargestellt.

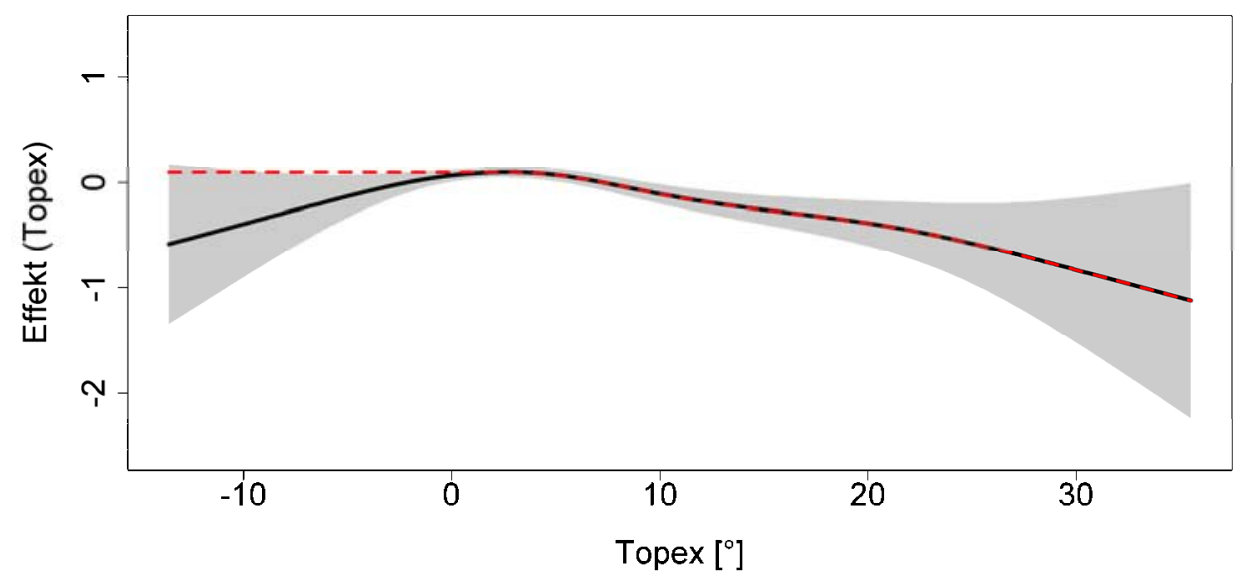

Abbildung 34: Modelleffekt des Topex zur Erfassung der Südexponiertheit auf das 10-jährige Befallsrisiko der Fichte durch den Buchdrucker mit Darstellung des $95 \%$-Konfidenzintervalls (graues Band). Der originale Effekt ist durch die schwarze Linie und eine zugehörige Approximation, die ein Absinken des Risikos mit sinkendem Topex verbindert, durch die rot gestrichelte Linie dargestellt. 
Der Modelleffekt der geografischen Lage verdeutlicht, dass unter konstanten Bedingungen bzw. konstanten kausalen Einflussgrößen beträchtliche regionale Unterschiede auftreten (s. Abb. 35). So ist der Bereich um Osnabrück, der Solling, aber auch der südlichste Teil des niedersächsischen Harzes durch ein relativ geringes Niveau des Befallsrisikos gekennzeichnet. Mittlere Werte werden für den Hils, den südlichen Bramwald und zentrale Bereiche des niedersächsischen Harzes geschätzt. Noch höhere Werte treten im Kaufunger Wald, in den nordöstlichen Teilen des niedersächsischen Harzes sowie in den westlichen und südlichen Teilen der Modellregion Sachsen-Anhaltischer Oberharz auf. Das höchste Niveau weisen die nordöstlichen Bereiche der Modellregion Oberharz auf.

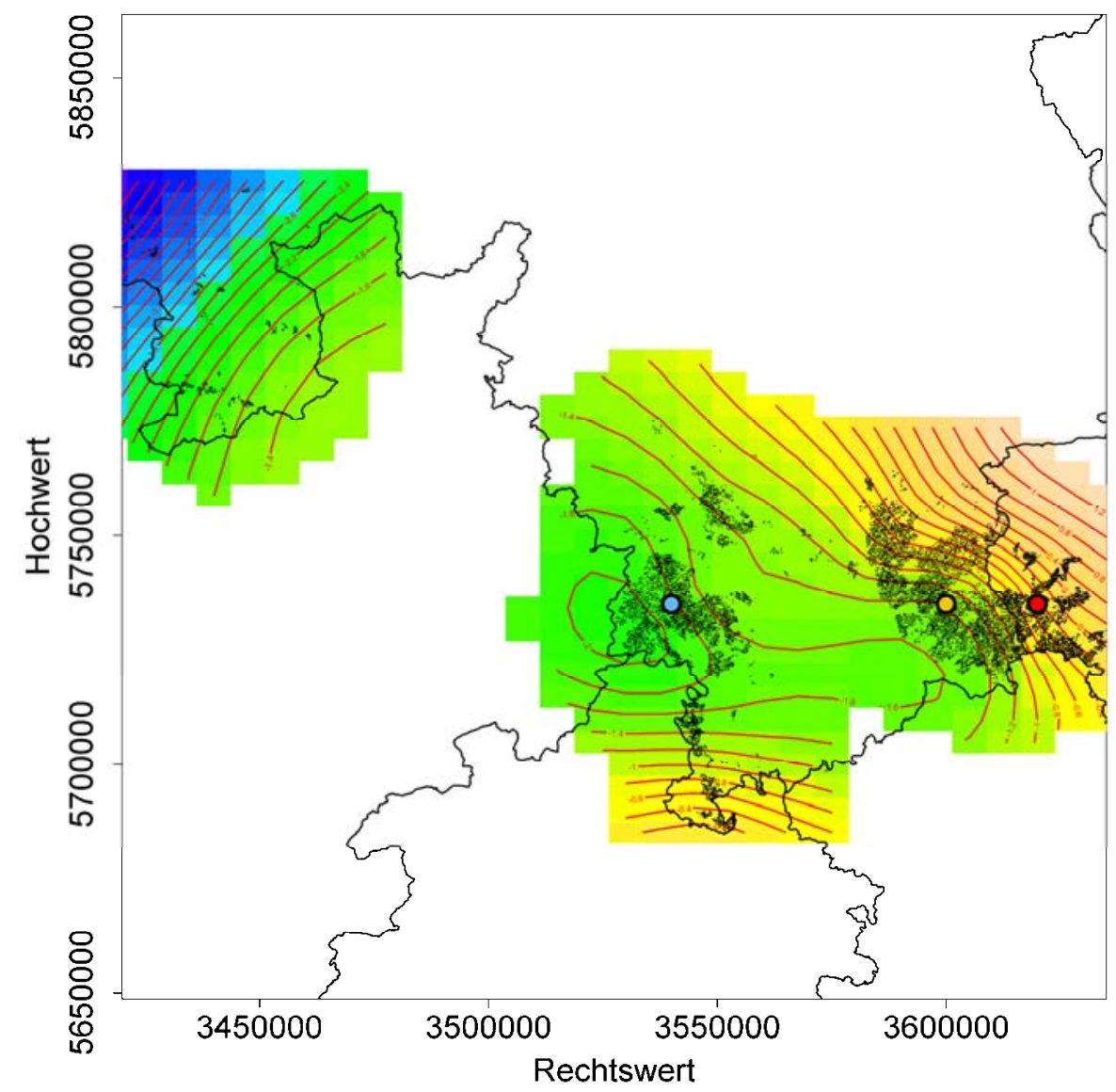

Abbildung 35: Modellprognosen auf der Ebene des linearen Prädiktors unter konstanten Bedingungen, wobei alle Prädiktoren mit Ausnahme der geografischen Lage ibren Mittelwerten in der Datenbasis entsprechen. Die Hintergrundfarbe kennzeichnet das Niveau der Prognose, wobei die Werte von blau über grün zu gelb zunehmen. Die drei schwarz geränderten Punkte markieren Positionen, für die im Rabmen einer Sensitivitätsanalyse exemplarische Prognosen durchgeführt werden. 
Wie bereits erläutert, können diese regionalen Niveauunterschiede weder über kausale Kovariablen noch über die Zufallseffekte auf der Revierebene hinreichend erfasst werden. Potenzielle Ursachen wie die Effekte übergeordneter Organisationseinheiten oder nicht ausreichend genaue Informationen bzgl. der Standortsbedingungen können letztlich nicht eindeutig identifiziert werden. Für eine theoretisch fundierte Auswertung und für eine höhere Prognosegenauigkeit ist die Modellierung dieser räumlich korrelierten Fehlerkomponente jedoch unabdingbar. So würde ihre Nichtbeachtung zu einer Verletzung der Unabhängigkeitsannahme führen. Für die Modellanwendung wird unterstellt, dass der räumliche Effekt eher auf großräumige standörtliche Unterschied zurückführen ist. Der Effekt wird somit im Gegensatz zu den Zufallseffekten der Revierebene in den Prognosen berücksichtigt.

Die mit der Revierebene korrelierten zufälligen Abweichungen im Befallsniveau streuen relativ symmetrisch um den Mittelwert Null wie es der Annahme in der Theorie der gemischten Modelle entspricht (s. Abb. 36). Die Werte der neun Reviere des Forstbetriebes Oberharz weichen alle positiv vom Median der 107 Reviere ab und weisen damit ein höheres Befallsniveau auf, als man es aufgrund der Prognose unter ausschließlicher Verwendung der festen Modelleffekte erwarten würde. Die Werte von fünf Revieren liegen innerhalb des Interquartilbereiches und weisen somit geringfügige bis moderate Abweichungen auf. Die Werte der vier übrigen Reviere liegen außerhalb des Interquartilbereiches und weisen somit deutlichere Abweichungen auf.

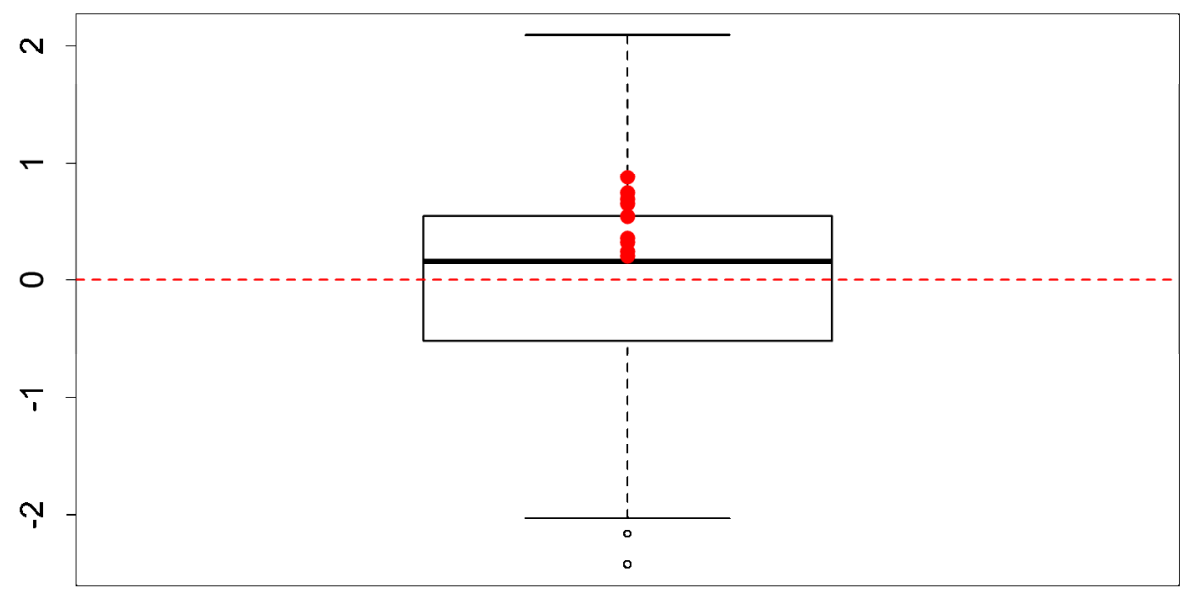

Abbildung 36: Boxplot der Zufallseffekte auf Revierebene. Die Box kennzeichnet den Interquartilbereich und die sogenannten Antennen markieren die äußersten Beobachtungen, die gerade noch in der 1,5-fachen Interquartilsdifferenz, außerbalb der Box liegen. Rote Punkte markieren die Zufallseffekete der neun Reviere des Forstbetriebes Oberhar\%. 


\subsubsection{Sensitivitätsanalyse}

Die statistische Analyse wird ergänzt durch exemplarische Modellprognosen unter „ceteris paribus“-Bedingungen. Dazu wird neben dem Alter und der geografischen Lage nur jeweils die Kovariable variiert, deren Effekt auf das Befallsrisiko abgebildet werden soll. Alle übrigen Kovariablen gehen mit den nachfolgend aufgeführten Konstanten in die Schätzung ein:

- Mischungsprozent: $100 \%$

- Temperatursumme in der Vegetationszeit: $2080{ }^{\circ} \mathrm{C}$

- Nutzbare Feldkapazität (nFK): 140 mm

- Topex: 0 (ebene Lage)

Die Werte der Temperatursumme und der nFK entsprechen den Mittelwerten in der Parametrisierungsdatenbasis. Der regionale Effekt wird beispielhaft anhand von drei Orten im Solling, im niedersächsischen Harz und in der Modellregion Oberharz dargestellt (s. Abb. 35).

Mit steigender Temperatursumme ist ein deutlicher Anstieg des Befallsrisikos zu beobachten (s. Abb. 37). Die Temperatursummen entsprechen im Harz unter den aktuellen Klimabedingungen Seehöhen von ungefähr $780 \mathrm{~m}$ (Temperatursumme = $\left.1600{ }^{\circ} \mathrm{C}\right), 510 \mathrm{~m}\left(2080^{\circ} \mathrm{C}\right)$ bzw. $250 \mathrm{~m}\left(2400^{\circ} \mathrm{C}\right)$ ü. NN. Zusätzlich wird jeweils innerhalb einer Einzelgrafik deutlich, dass auch die geografische Lage das Befallsrisiko sehr stark bestimmt. Die exemplarisch ausgewählten geografischen Orte repräsentieren das Befallsniveau unter konstanten Nebenbedingungen, das entlang der Konturlinie zu erwarten wäre, auf der der jeweilige Ort liegt (s. Abb. 35).
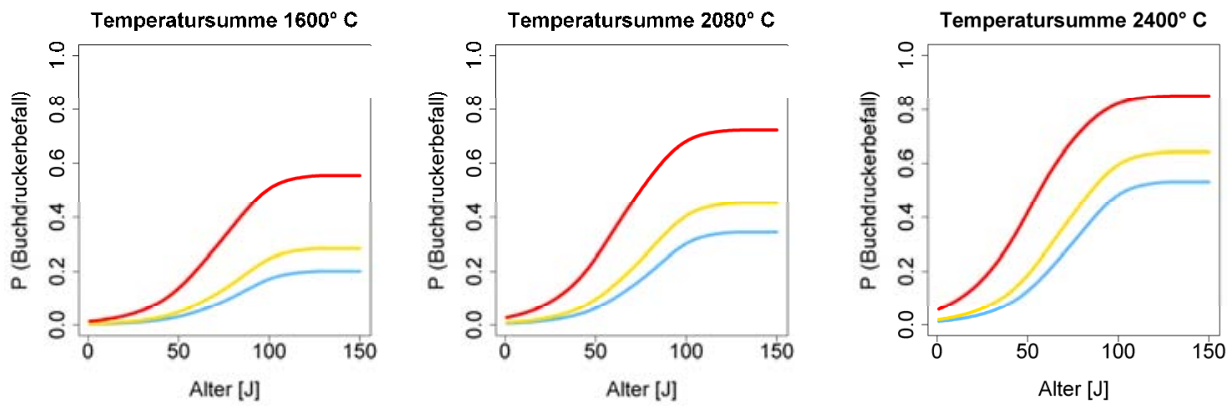

Abbildung 37: Prognostizierte Befallsrisiken für drei unterschiedliche geografische Orte und Temperatursummen in Abhängigkeit vom Alter $[J]$ bei sonst konstanten Nebenbedingungen (Mischungsprozent: 100 \%, Topex: 0, $n F K: 140$ mm). Die Farben der verschiedenen Risikoverläufe entsprechen den Farben der zugehörigen Punkte in Abbildung 35 (rot: exemplarische Lage im Sachsen-Anhaltischen Hary gelb: exemplarische Lage im niedersächsischen Hary, blau: exemplarische Lage im Solling). 
Mit sinkendem Mischungsprozent der Fichte sinkt das Befallsrisiko. Dabei resultiert aus der Reduktion von 100 auf $67 \%$ ein geringeres Absinken des Risikos als aus der Reduktion von 67 auf $33 \%$ (s. Abb. 38).
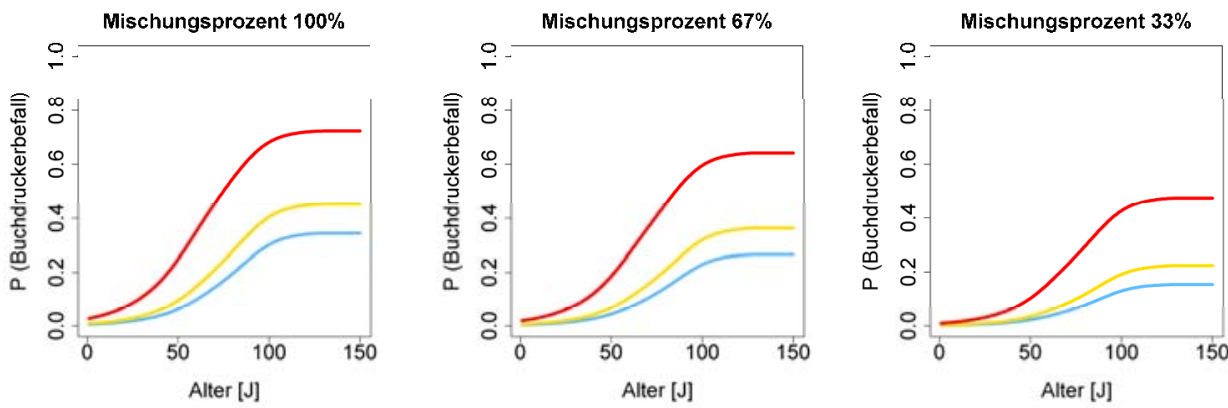

Abbildung 38: Prognostizierte Befallsrisiken für drei unterschiedliche geografische Orte und Mischungsprozente in Abhängigkeit vom Alter [J] bei sonst konstanten Nebenbedingungen (Temperatursumme: $2080^{\circ} \mathrm{C}$, Topex: 0, $n$ FK: $140 \mathrm{~mm}$ ). Die Farben der verschiedenen Risikoverläufe entsprechen den Farben der zugehörigen Punkte in Abbildung 35 (rot: exemplarische Lage im Sachsen-Anhaltischen Harz, gelb: exemplarische Lage im niedersächsischen Harv, blau: exemplarische Lage im Solling).

Mit steigender nutzbarer Feldkapazität und steigendem Topex (sinkender Südexponiertheit) sinkt das Befallsrisiko (s. Abb. 39). Im Vergleich mit den übrigen Kovariablen sind die Effekte der nFK und des Topex auf das Befallsrisiko relativ schwach.
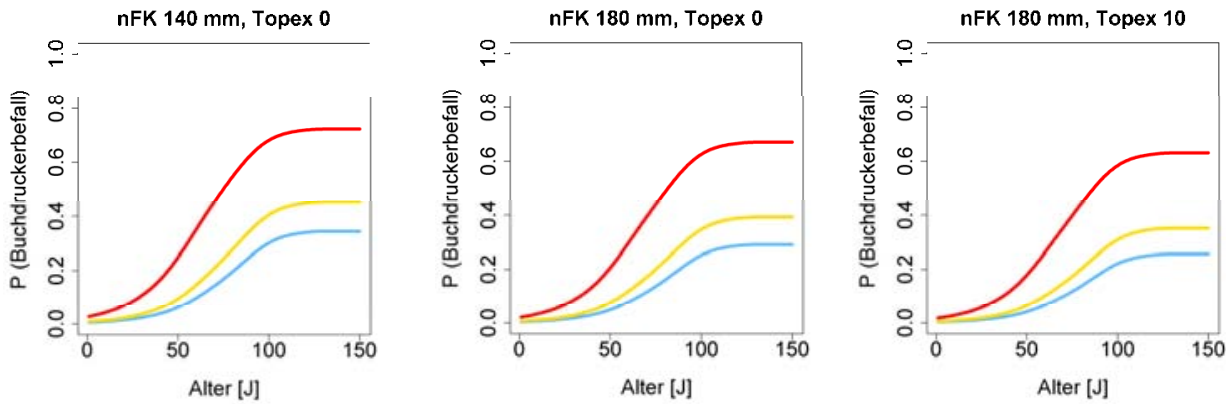

Abbildung 39: Prognostizierte Befallsrisiken für drei unterschiedliche Orte und unterschiedliche $n F K$ - und Topex-Werte in Abhängigkeit vom Alter [J] bei sonst konstanten Nebenbedingungen (Temperatursumme: $2080^{\circ} \mathrm{C}$, Mischungsprozent $100 \%$ ). Die Farben der verschiedenen Risikoverläufe entsprechen den Farben der zugehörigen Punkte in Abbildung 35 (rot: exemplarische Lage im Sachsen-Anhaltischen Hary, gelb: exemplarische Lage im niedersächsischen Hary, blau: exemplarische Lage im Solling). 


\subsection{Methodik der Beurteilung weiterer biotischer Risiken}

Auf Grundlage des generalisierten linearen Modells (GLM) und des gesicherten Erkenntnisstands über die ökologischen Ansprüche und die Populationsdynamik der wichtigsten Schadorganismen wurde der Einfluss ausgewählter Bestandes- und Standortsparameter auf das Schadvorkommen in den untersuchten Gebieten Fläming und Colbitz-Letzlinger Heide analysiert. Die betreffenden Bestandes- und Standortsparameter wurden dabei mit den Daten des Schadgeschehens der wichtigsten Schadorganismen in den untersuchten Gebieten verschnitten.

Die Variablen, die im oben genannten Modell das Niveau des Schädlingsbefalls beeinflussen, lassen sich wie folgt gliedern:

- Bestandesparameter:

- mittleres Alter der Baumart

- Mischungsanteil der jeweiligen Baumart

- Standortsparameter:

- $\quad$ nutzbare Feldkapazität (nFK)

- Nährstoffziffer

Die betreffenden Bestandesinformationen wurden aus den Forsteinrichtungsunterlagen gewonnen und auf die Teilfläche bezogen, wobei jeweils der flächengewichtete Mittelwert für die Teilfläche verwendet wurde. Informationen zum Standort selbst, wie die nutzbare Feldkapazität und Nährstoffziffer, wurden aus den Daten der Standortskartierung entnommen. Die nFK resultiert aus der Verschneidung der Bestandesgeometrien mit den Standortspolygonen. Treten mehrere Standortseinheiten innerhalb eines Bestandes auf, wurde der flächengewichtete Mittelwert der nFK verwendet (s. Kap. 4.1).

Für die Analyse des Schadvorkommens der Eichenfraßgesellschaft sowie der Kieferngroßschädlinge und der Nonne in den Beständen der untersuchten Gebiete Fläming und Colbitz-Letzlinger Heide wurden die teilflächenscharfen Informationen aus dem Waldschutzmeldewesen als Datengrundlage genutzt. Dabei wurden die Flächenangaben (ha) zum Befall von Beständen, zum Fraßgeschehen, zu eventuellen Massenvermehrungen und zu Bekämpfungen von Schadorganismen für die letzten 20 Jahre teilflächenscharf aufgenommen. Diese als Befallsgeschehen definierten Parameter wurden anschließend mit den Bestandes- und Standortsinformationen verschnitten.

Um einen Befall zu bestimmen, wurden gestaffelte Befallsschwellen definiert. Als befallen galten Teilflächen, die auf mehr als $1 \%, 10 \%$ oder $30 \%$ Flächenanteil Befall durch einzelne Arten aufwiesen. Die genannten Abstufungen wurden vorgenommen, um den jeweiligen Einfluss der einzelnen Variablen (Standorts- und Bestandesparameter) bei verschiedenen Befallsstärken zu untersuchen. Der Einfluss der einzelnen Parameter bei unterschiedlichen Befallsstufen wurde mit Hilfe eines 
GLM auf lineare Zusammenhänge getestet. Das genannte Modell basiert auf dem Ansatz zur Lösung regressionsanalytischer Problemstellungen und lässt normalverteilte und auch verschiedene Formen nicht-normalverteilter Zielvariablen zu (MAINDONALD u. BRAUn 2007). Dabei wurde die Richtung des Zusammenhangs zur Zielgröße, d. h. die Wahrscheinlichkeit eines Befalls auf mehr als $1 \%, 10 \%$ und 30 \% Flächenanteil der Teilfläche, analysiert und auf Signifikanz getestet. 


\section{Gefährdungspotenziale in der Region Oberharz}

\subsection{Veränderungen des Trockenstressrisikos}

Unter heutigen Klimabedingungen besteht für die Hauptbaumarten Fichte, Buche, Eiche, Kiefer und Douglasie in der Region Oberharz kein Trockenstressrisiko. Für die betrachteten 21.239 ha Waldfläche wird für alle untersuchten Baumarten ein geringes Trockenstressrisiko ausgewiesen (s. Tab. 14 und Abb. 40 bis Abb. 43).

Tabelle 14: $\quad$ Fläche [ha] der Hauptbaumarten nach den definierten Trockenstressrisikoklassen in der Region Oberbarz.

\begin{tabular}{l|rr|rr|rr}
\hline \multicolumn{1}{r|}{ Periode } & \multicolumn{2}{|c|}{ Fichte } & \multicolumn{2}{|c}{ Buche } & \multicolumn{2}{|c}{ Kiefer / Eiche / Douglasie } \\
Risiko & $1971-2000$ & $2071-2100$ & $1971-2000$ & $2071-2100$ & heute & $2071-2100$ \\
\hline gering & 21.239 & 511 & 21.239 & 1.221 & 21.239 & 9.541 \\
mittel & 0 & 20.658 & 0 & 20.013 & 0 & 11.698 \\
hoch & 0 & 70 & 0 & 5 & 0 & 0 \\
\hline
\end{tabular}

Unter der Annahme des Klimaszenarios A1B wird sich das Trockenstressrisiko bis zum Ende des Jahrhunderts für die Wälder in der Region Oberharz erhöhen. Dabei nimmt das Gefährdungspotenzial besonders für die Fichte und die Buche zu. Für die Periode 2071 - 2100 werden für die Fichte zwar nur 70 ha mit einem hohen Trockenstressrisiko belegt, aber für über $97 \%$ (20.658 ha) der Fläche wird ein mittleres Trockenstressrisiko erwartet (s. Tab. 14 und Abb. 41). Nur in den höchsten Lagen des Modellgebietes werden auch in Zukunft die Bestände über eine ausreichende Wasserversorgung verfügen.

Für die Buche sieht es nur wenig besser aus, da ca. 94 \% (20.013 ha) der Waldstandorte ein mittleres Trockenstressrisiko zum Ende des Jahrhunderts aufweisen (s. Abb. 42). Die Kiefer, Eiche und Douglasie sind hingegen in Zukunft auf rund $55 \%$ mit einem mittleren Trockenstressrisiko belegt (s. Tab. 14). Dies betrifft vornehmlich Bestände in den unteren Lagen und Standorte mit geringer nFK (s. Abb. 40 und Abb. 43). Auf 9.541 ha ist auch langfristig mit keinem nennenswerten Trockenstressrisiko für die Kiefer, die Eiche und die Douglasie zu rechnen. 
Kiefer
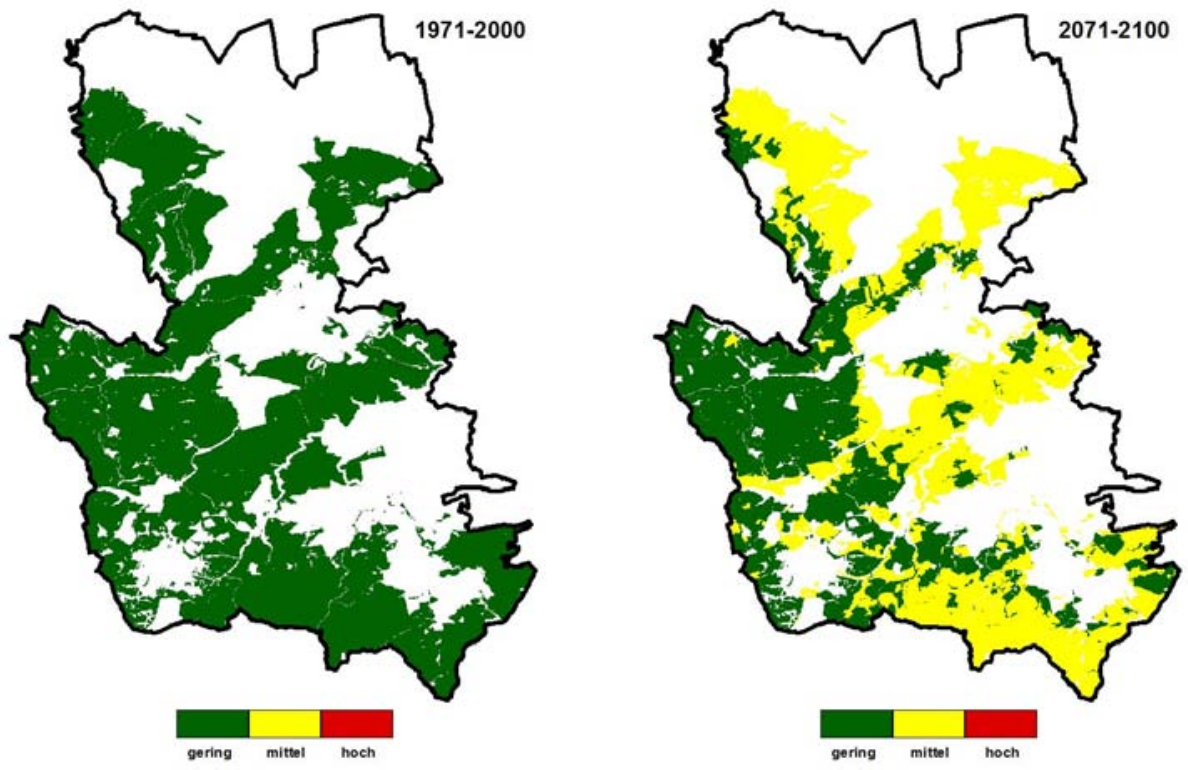

Abbildung 40: Trockenstressrisiko für die Kiefer in der Region Oberbarz.

Fichte
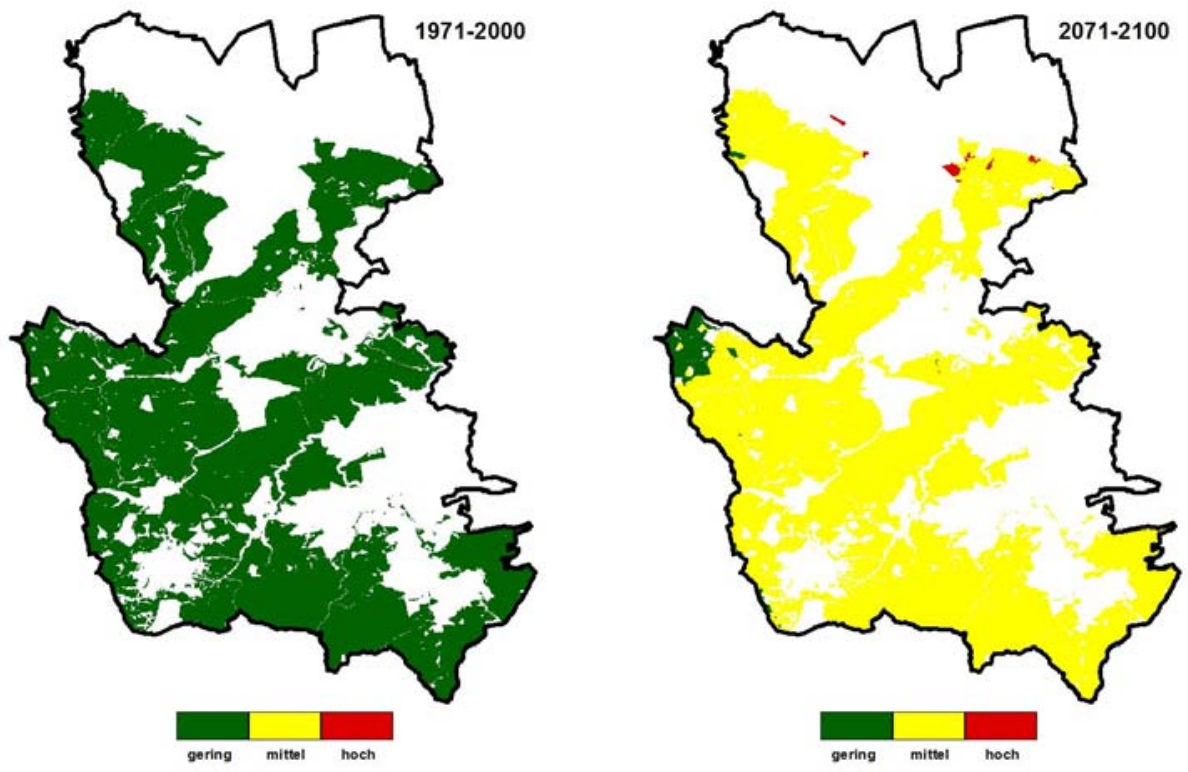

Abbildung 41: Trockenstressrisiko für die Fichte in der Region Oberharz. 
Buche
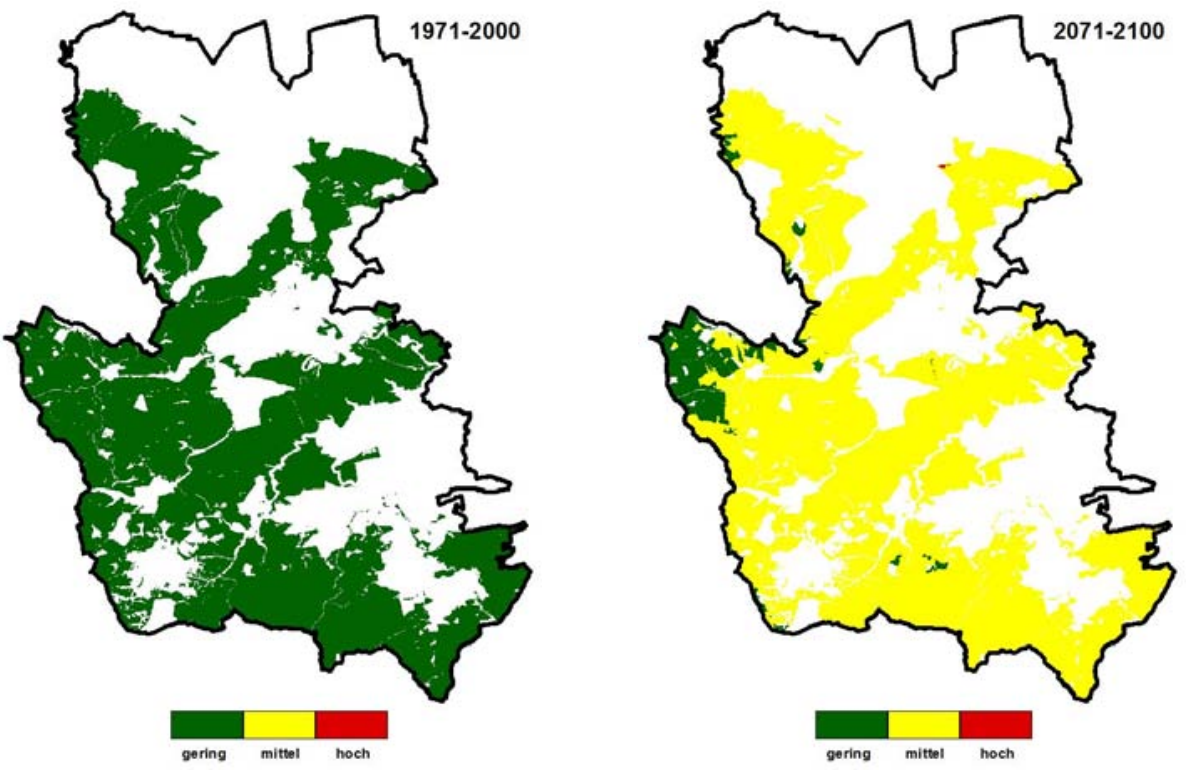

Abbildung 42: Trockenstressrisiko für die Buche in der Region Oberharz.

Eiche und Douglasie
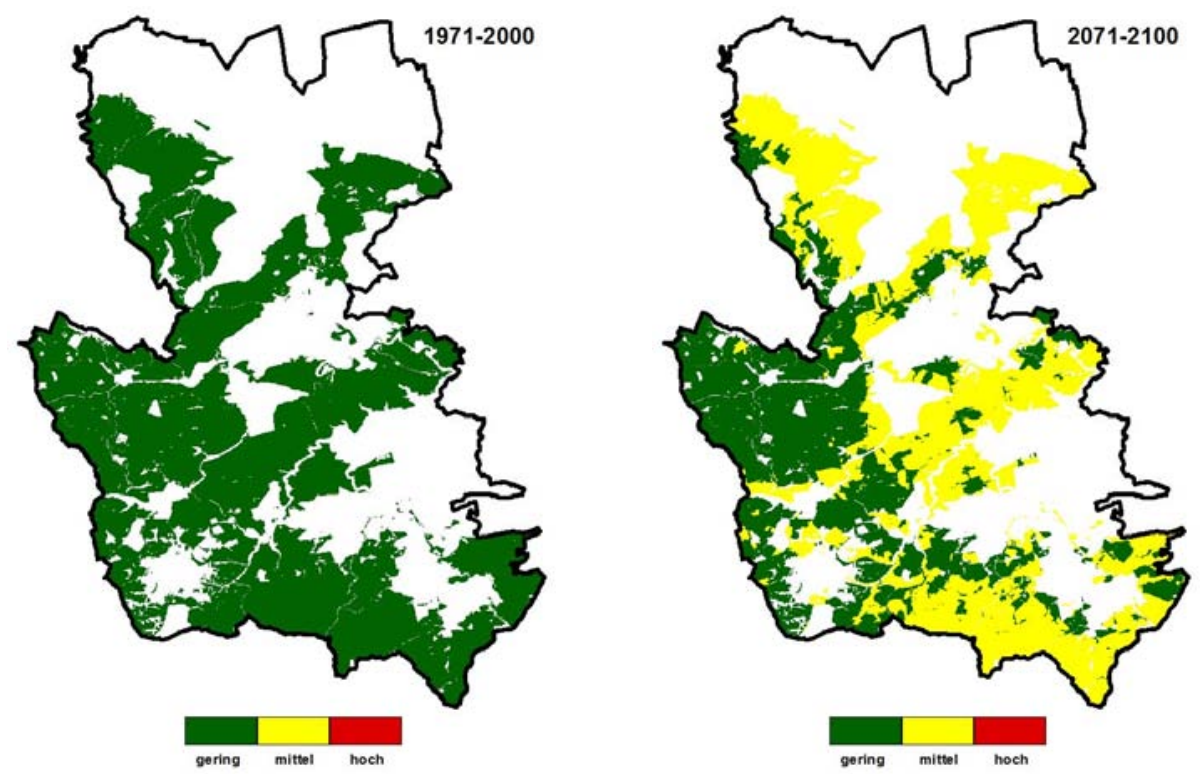

Abbildung 43: Trockenstressrisiko für die Eiche und Douglasie in der Region Oberharz. 
In Anhang 1 bis Anhang 4 ist die zeitliche Entwicklung des Trockenstressrisikos für die untersuchten Perioden dargestellt. Die Auswertung zeigt, dass bis zum Jahr 2070 nur mit einer verhältnismäßig geringen Zunahme des Gefährdungspotenzials durch Trockenstress zu rechnen ist. Erst in der letzten Periode 2071 - 2100 nimmt dieses Risiko deutlich zu.

Obwohl auch die Wälder in der Region Oberharz in Zukunft vermehrt unter Trockenstressbedingungen wachsen werden, wird sich das Wasserbilanzdefizit im Vergleich zu den Hügel- und Tieflandbereichen relativ moderat entwickeln. Insgesamt wird das Trockenstressrisiko in Abhängigkeit von der Baumart als gering bis mittel eingestuft.

\subsection{Veränderungen der Stickstoffeinträge}

Abbildung 44 zeigt die atmosphärischen Stickstoffeinträge für die Zeiträume 1981 - 2010 und 2071 - 2100. Es ergibt sich eine höhere großräumige Belastungssituation mit beträchtlichen Einträgen im Westen des Untersuchungsgebietes. Die geringeren Einträge im östlichen Gebietsabschnitt sind das Resultat der Orographie von Sachsen-Anhalt (Regenschatten des Harzes) und damit der deutlich geringeren Stoffeinträge mit der Feuchtdeposition. Insgesamt ist der Oberharz im Vergleich der Modellregionen jedoch durch die höchsten Stickstoffeinträge gekennzeichnet. Zum Vergleich der beiden Zeiträume ist anzumerken, dass die mittlere Deposition zwischen 1981 bis 2010 nicht die aktuelle Eintragssituation widerspiegelt, da die NDepositionen seit Anfang der 80er-Jahre z. T. erheblich zurückgegangen sind (s. Abb. 15). Für die Schätzung der N-Depositionen in der Periode 2071 - 2100 wurde als „wahrscheinlichstes“ Szenario die Umsetzung der gegenwärtigen Politik (CP) bis zum Jahre 2020 angenommen (s. Tab. 8).
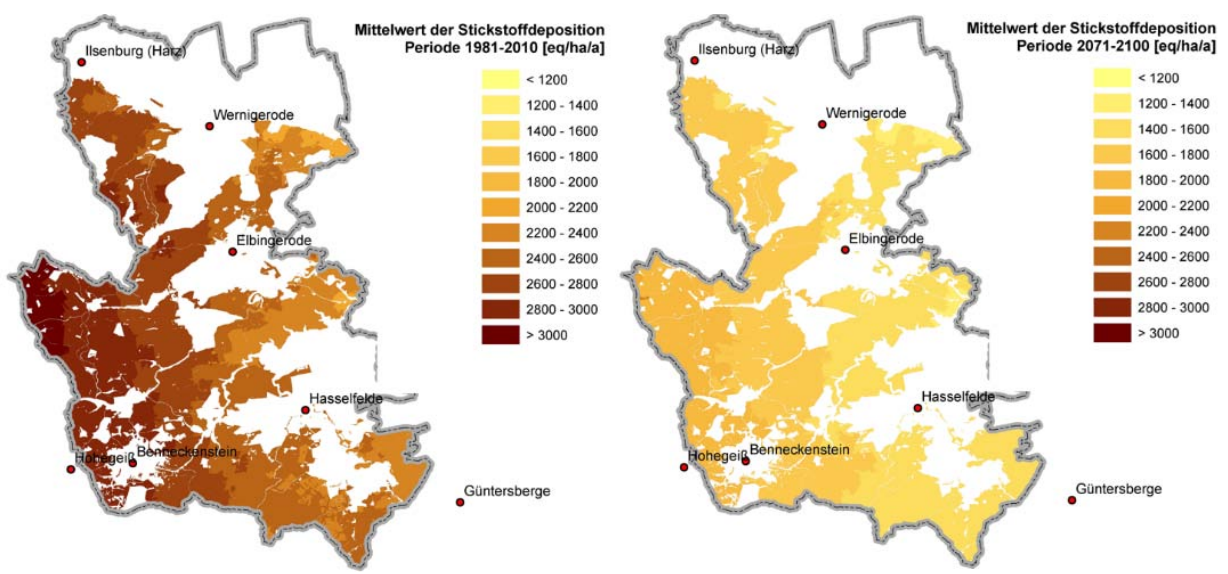

Abbildung 44: Regionale Verteilung der Stickstoffdepositionen Mittelwerte für die Zeiträume von 1981 - 2010 und 2071 - 2100) in der Region Oberhary. 


\subsection{Projektion der Standortleistungsfähigkeit für die Region Oberharz}

Die Fichte dominiert in der Region Oberharz als Hauptbaumart mit einem Flächenanteil von nahezu $80 \%$. Die Projektion der Standortleistungsfähigkeit auf den gegenwärtig mit Fichte bestockten Beständen erscheint daher aussagekräftig, um die zukünftige Wuchsleistung gerade der jüngeren Bestände abschätzen zu können.

Abbildung 45 zeigt die Standortleistungsfähigkeit für die Baumart Fichte zum Status quo und für die drei Projektionsperioden. Das heutige räumliche Mosaik der Standortleistungsfähigkeit für Fichte wird überwiegend bestimmt durch die höhenzonale Gliederung und damit durch die mit zunehmender Seehöhe zurückgehende Temperatur bzw. ansteigenden Niederschläge. Dieses räumliche Muster des Wuchspotenzials bleibt auch unter den Klimaprojektionen erhalten. Die Standortleistungsfähigkeit verbessert sich bis 2070 auf fast allen Standorten. In der letzten Projektionsperiode 2071-2100 werden für die höheren Lagen im Nordwesten und Westen weiterhin Verbesserungen geschätzt, während in den mittleren Lagen eine Stagnation bzw. vereinzelt sogar wieder ein Rückgang der Standortleistungsfähigkeit für Fichte projiziert wird. In den mittleren Lagen scheint somit der positive Effekt des verbesserten Temperaturangebotes durch den negativen Effekt bei zurückgehenden Niederschlägen überkompensiert zu werden und folglich bereits in einer Verminderung der Standortleistungsfähigkeit zu resultieren.

Neben der Analyse der Standortleistungsfähigkeit für die reale Fichtenbestockung ist ein Baumartenvergleich bezüglich des Wuchspotenzials in der Region Oberharz aufschlussreich. Dafür werden allen Teilflächen jeweils die zum Anbau als Hauptbaumart in Frage kommende Fichte, Buche und Douglasie zugewiesen und die baumartspezifische Standortleistungsfähigkeit geschätzt. Abbildung 46 und Abbildung 47 fassen die Ergebnisse in Form von Häufigkeitsverteilungen zusammen. Die Histogramme und die Mittelwerte des durchschnittlichen Gesamtzuwachses $\left(\mathrm{dGZ}_{\max }\right)$ zeigen für die Standortleistungsfähigkeit aller drei Baumarten eine Zunahme vom Status quo bis zum Jahr 2100. Der Anstieg der dGZ $Z_{\max }$-Mittelwerte um $41 \%$ vom Status quo bis zur Projektionsperiode 2071 - 2100 offenbart, dass die Fichte relativ am meisten von den veränderten Klimabedingungen profitiert, gefolgt von der Douglasie mit $36 \%$ und der Buche mit 29,5\%. In absoluten Werten zeigt die Douglasie deutlich den höchsten durchschnittlichen Gesamtzuwachs, allerdings muss hierbei die modellbedingte Unsicherheit einer möglichen Überschätzung beachtet werden. 

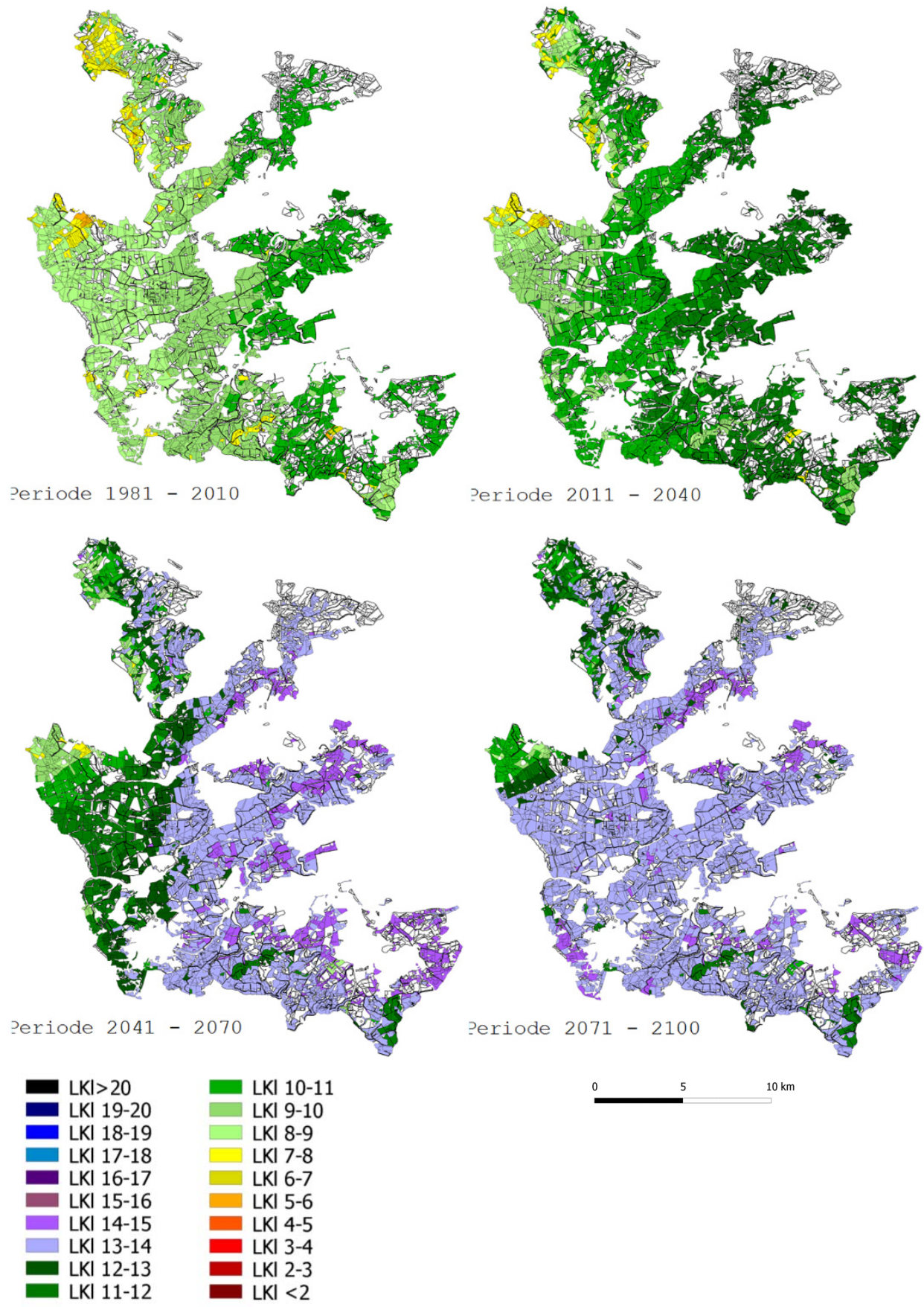

Abbildung 45: Projektion der Standortleistungsfähigkeit für gegenwärtig mit Fichte bestockte Teilflächen in der Region Oberhary. 

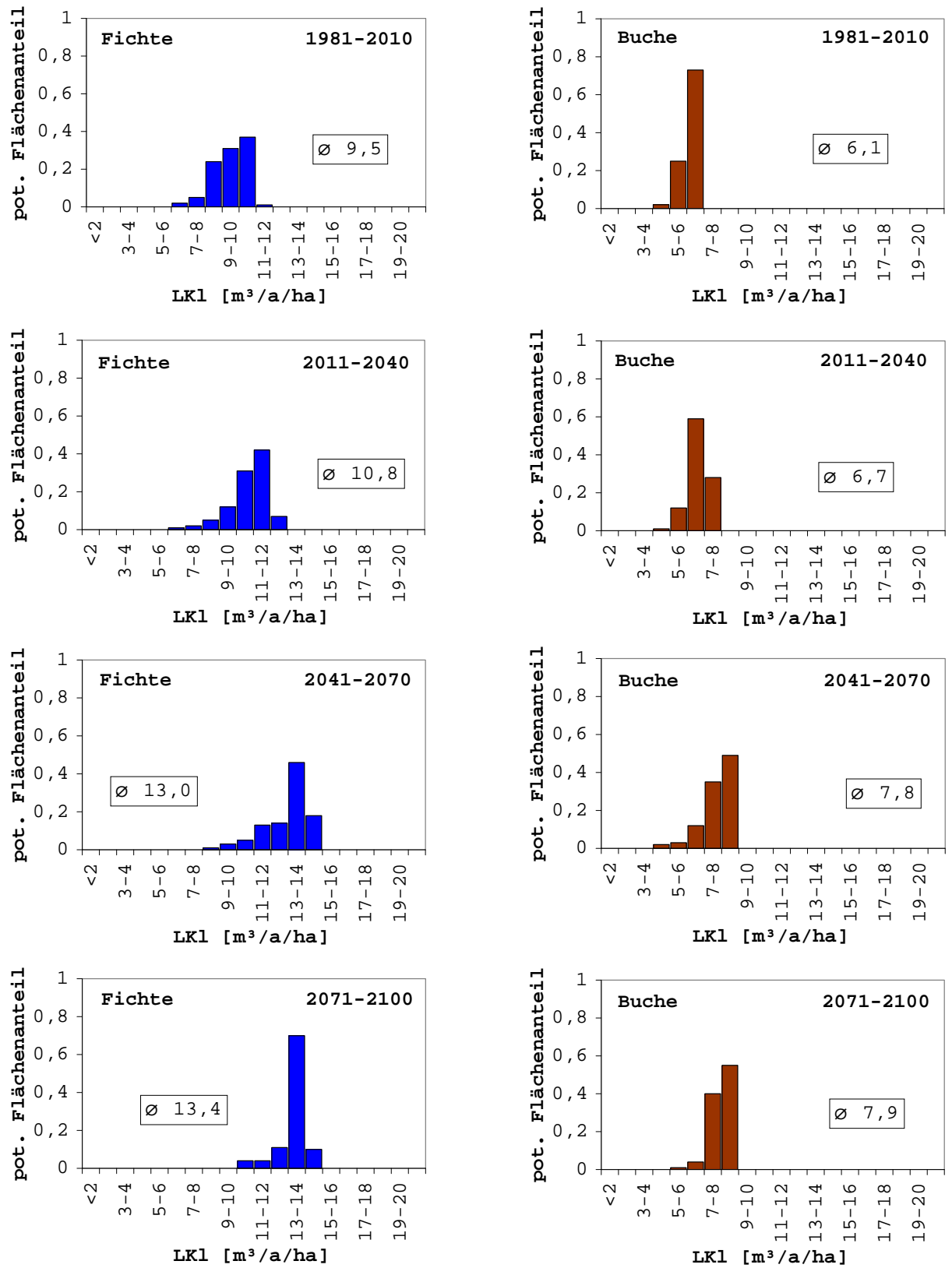

Abbildung 46: Poten₹ielle Flächenanteile der Standortleistungsfähigkeit $\left[\mathrm{m}^{3} / \mathrm{a} / \mathrm{ha}\right]$ für die Baumarten Fichte (links) und Buche (rechts) für den Status quo (1981 - 2010) und die drei Projektionsperioden in der Region Oberhar: Jeder Teilfläche wurde bypothetisch die jewreilige Baumart zugeordnet. In den Grafiken ebenfalls angegeben ist der arithmetische Mittelwert o des durchschnittlichen Gesamtruwachses (dGZ $\left.Z_{\text {max }}\right)$. 


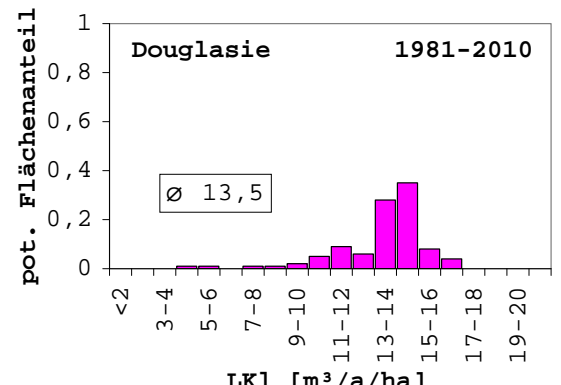

LKI $\left[\mathrm{m}^{3} / \mathrm{a} / \mathrm{ha}\right]$

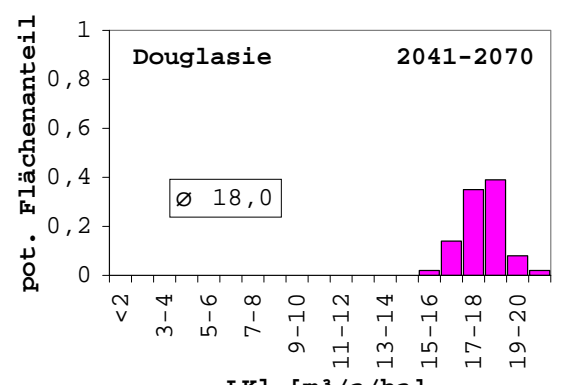

LKI $\left[\mathrm{m}^{3} / \mathrm{a} / \mathrm{ha}\right]$

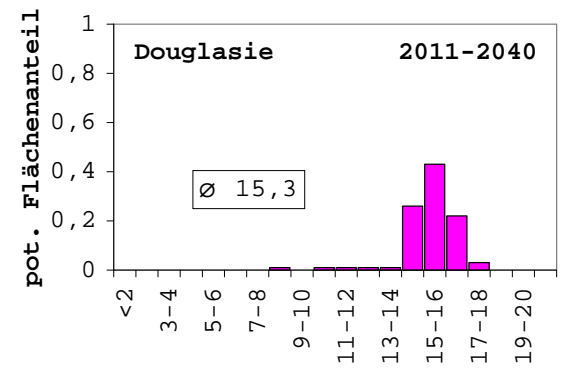

LKL $\left[\mathrm{m}^{3} / \mathrm{a} / \mathrm{ha}\right]$

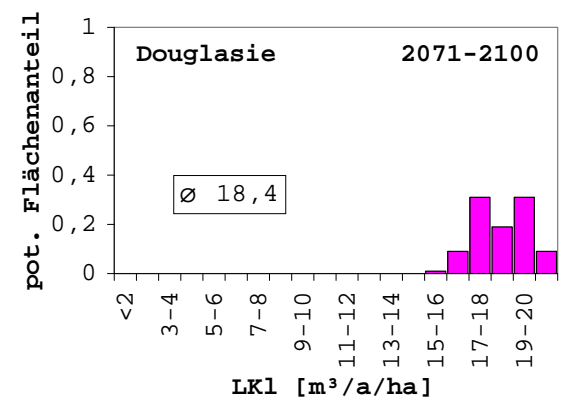

Abbildung 47: Potenqielle Flächenanteile der Standortleistungsfähigkeit $\left[\mathrm{m}^{3} / \mathrm{a} / \mathrm{ha}\right]$ für die Baumart Douglasie für den Status quo (1981 - 2010) und die drei Projektionsperioden in der Region Oberhar:. Jeder Teilfläche wurde hypothetisch die Baumart Douglasie zugeordnet. In den Grafiken ebenfalls angegeben ist der arithmetische Mittelwert o des durchschnittlichen Gesamtruwachses (dGZ max $)$.

\subsection{Gefährdungspotenziale durch Winterstürme}

Im Rahmen der Studie wurde die Sturmschadenswahrscheinlichkeit für die Modellbäume bei Zielstärke und die Grundflächenmittelstämme der aktuellen Bestockung für jeden Bestand geschätzt. Die Topex-Werte wurden für die Zentroide der zugehörigen Bestandespolygone geschätzt. Auf dieser Datengrundlage lassen sich Karten zur Entscheidungsunterstützung der forstlichen Praxis erstellen. Entsprechend der Fragestellung können die potenzielle und die aktuelle Bestockung in Risikoklassen eingeteilt werden, um das spezifische Sturmschadensrisiko bei waldbaulichen Maßnahmen zu berücksichtigen. Insbesondere für Flächen mit besonders hohem oder niedrigem Risiko sollten besonders angepasste Maßnahmenfolgen erarbeitet werden, um das standörtliche Potenzial optimal auszunutzen. Als ein Beispiel kann die Absenkung der Zielstärke auf besonders gefährdeten und ihre Anhebung auf besonders geschützten Standorten genannt werden. Auch erlaubt die bestandesscharfe Zuordnung von Risiken die einzelbestandsweise Anpassung der Baumartenwahl. Üblicherweise erfolgt die Zuweisung von Bestandeszieltypen - in denen die Baum- 
artenanteile definiert sind - anhand der Nährstoffversorgung und des Wasserhaushaltes eines Standortes und getrennt nach Klimaregionen, um regionale Unterschiede zu berücksichtigen. Die bestandesscharfe Prognose von Risiken erweitert die Standortsdefinition um weitere Dimensionen, die bei einem Waldbau auf standörtlicher Grundlage berücksichtigt werden sollten. Waldentwicklungstypen sollten Standorten somit zusätzlich unter Berücksichtigung wichtiger Risiken zugeordnet werden. Daher werden Karten des Sturmschadensrisikos für die drei Modellregionen sowie summarische Flächenverteilungen nach Risikostufen dargestellt.

\subsubsection{Potenzielle Vulnerabilität durch Winterstürme bei Zielstärke}

Für eine Interpretation der Flächenverteilung nach Risikostufen ist ein Vergleich zwischen den Baumarten und den drei Modellregionen sinnvoll. Allerdings hat die Fichte für die Forstwirtschaft in den Modellregionen Fläming und Colbitz-Letzlinger Heide keine Bedeutung. Zu Vergleichszwecken wurden daher Ergebnisse einer Vergleichsuntersuchung im Landeswald des niedersächsischen Harzes verwendet (s. Abb. 48). Die untersuchte Fläche der Region Oberharz umfasst 21.239 ha im Vergleich zu 72.435 ha im niedersächsischen Harz. Die insgesamt größere Reliefbildung im niedersächsischen Harz wird am höheren Anteil von besonders geschützten Beständen aber auch am höheren Anteil von Beständen in der höchsten Risikostufe 0,95 deutlich. Im Mittel weist die Region Oberharz ein deutlich höheres Risiko von 0,77 gegenüber 0,69 auf, was in erster Linie auf den größeren Anteil von Plateaulagen zurückgeführt werden kann.

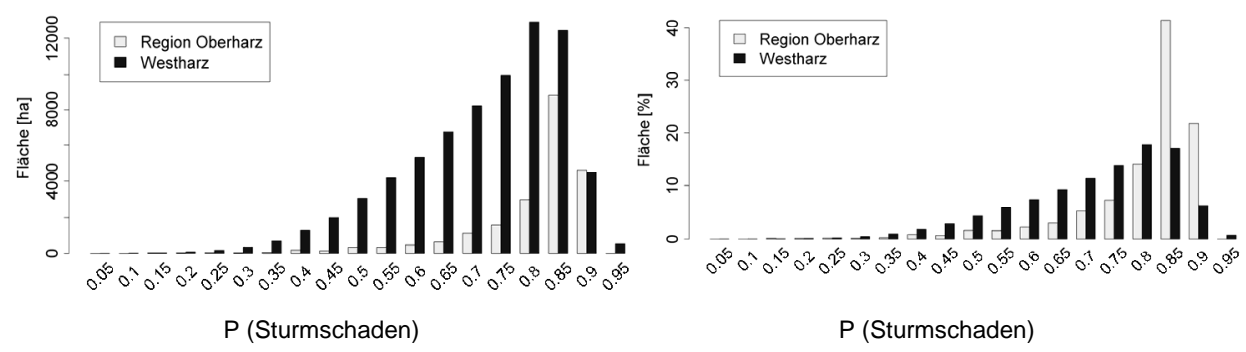

Abbildung 48: Absolute [ha] und prozentuale [\%] Flächenverteilung einer potenziellen Fichtenbestockung (Zielstärke $45 \mathrm{~cm}$ ) nach Sturmschaden-Risikostufen in der Modellregion Oberharz und im niedersächsischen Harz, (Landeswald)

Die Eiche weist in allen drei Modellregionen relativ ähnliche Verteilungen auf, wobei die prozentualen Anteile in den sehr niedrigen und sehr hohen Risikostufen im Oberharz am höchsten sind (s. Abb. 49). In der Region Oberharz werden nur Bestände unterhalb von $600 \mathrm{~m}$ Seehöhe betrachtet. Als Erklärung sind die deutlich größeren Reliefunterschiede im Harz zu nennen. Der größere Anteil in den höheren Risikostufen ist aber auch auf die größeren Baumhöhen zurückzuführen, die die Eiche auf vielen Standorten im Oberharz bei Erreichen der Zielstärke aufweisen 
würde (s. Abb. 18). Insgesamt sind die Unterschiede zwischen den Regionen für die Eiche relativ gering, was auch an den Mittelwerten von 0,30 für den Oberharz, 0,31 für den Fläming und 0,27 für die Colbitz-Letzlinger Heide deutlich wird. Die etwas höheren Risiken im Fläming gegenüber der Colbitz-Letzlinger Heide sind auf die besseren Standorte bzw. die daraus resultierenden größeren Baumhöhen zurückzuführen.

Die Buche weist im Oberharz deutlich höhere Risiken auf als im Fläming und der Colbitz-Letzlinger Heide (s. Abb. 49). Die Mittelwerte betragen 0,41 im Oberharz, 0,25 im Fläming und 0,24 in der Colbitz-Letzlinger Heide. Neben den reliefbedingten Unterschieden sind hier vor allem die gegenüber der Eiche sehr viel größeren Unterschiede in der Wuchsleistung zwischen der Region Oberharz sowie den Regionen Fläming und Colbitz-Letzlinger Heide zu nennen (s. Abb. 18). In den Regionen Fläming und Colbitz-Letzlinger Heide liegen die Baumhöhen bei $55 \mathrm{~cm}$ Zielstärke auch deutlich unter denen der Eiche bei $65 \mathrm{~cm}$ Zielstärke, woraus die hier geringeren Risiken der Buche resultieren.

Eiche
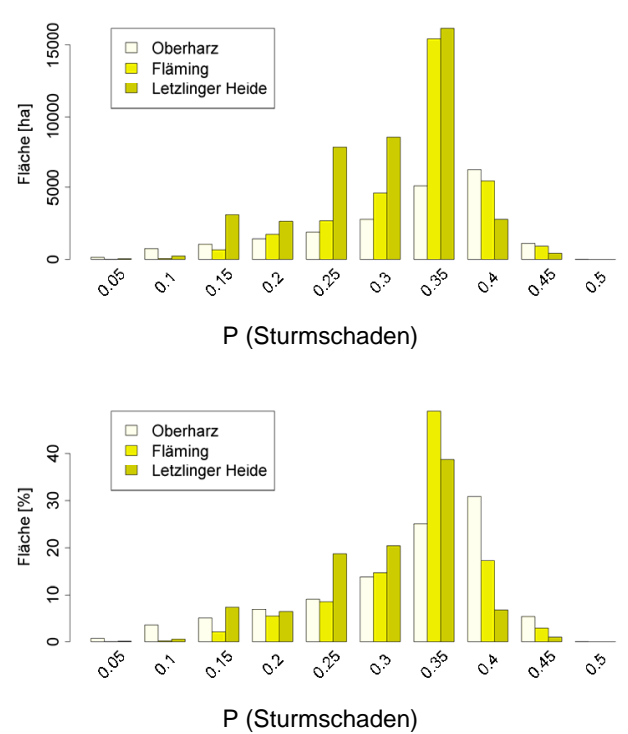

Buche
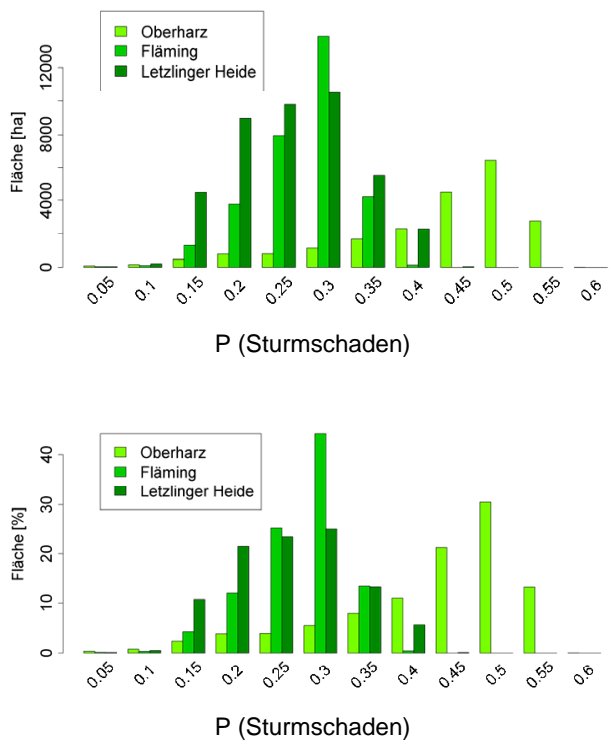

Abbildung 49: Absolute [ha] und relative [\%] Flächenverteilungen potenzieller Bestockungen von Eiche (Zielstärke $65 \mathrm{~cm}$ ) und Buche $(55 \mathrm{~cm})$ nach Sturmschaden-Risikestufen in den Modellregionen Oberhary, Fläming und Colbitz-Letzlinger Heide. Für Eiche sind nur Bestände unterhalb von 600 m und für Buche nur Bestände unterhalb von 700 m berücksichtigt.

Douglasie und vor allem Kiefer weisen im Vergleich zu Fichte und vor allem Buche relativ geringe regionale Unterschiede bzgl. der Baumhöhe bei Zielstärke auf (s. Abb. 18 und Abb. 19). Die Verteilungen nach Sturmschaden-Risikostufen werden daher deutlich von der unterschiedlichen Topographie der Regionen geprägt 
(s. Abb. 50). Die Region Oberharz weist für beide Baumarten die höchsten Flächenanteile mit geringeren Risiken auf. Dagegen sind die Verteilungen im Fläming und der Colbitz-Letzlinger Heide deutlich enger und werden von wenigen Klassen dominiert. Die Form der Verteilungen im Fläming und der Colbitz-Letzlinger Heide ist für die Kiefer sehr ähnlich. Dagegen liegt der Schwerpunkt bei der Douglasie im Fläming bei etwas höheren Werten als in der Colbitz-Letzlinger Heide. Die Mittelwerte des Sturmschadensrisikos betragen für die Kiefer 0,47 im Harz, 0,53 im Fläming und 0,52 in der Colbitz-Letzlinger Heide und für die Douglasie 0,68 im Harz, 0,75 im Fläming und 0,73 in der Colbitz-Letzlinger Heide.

Kiefer
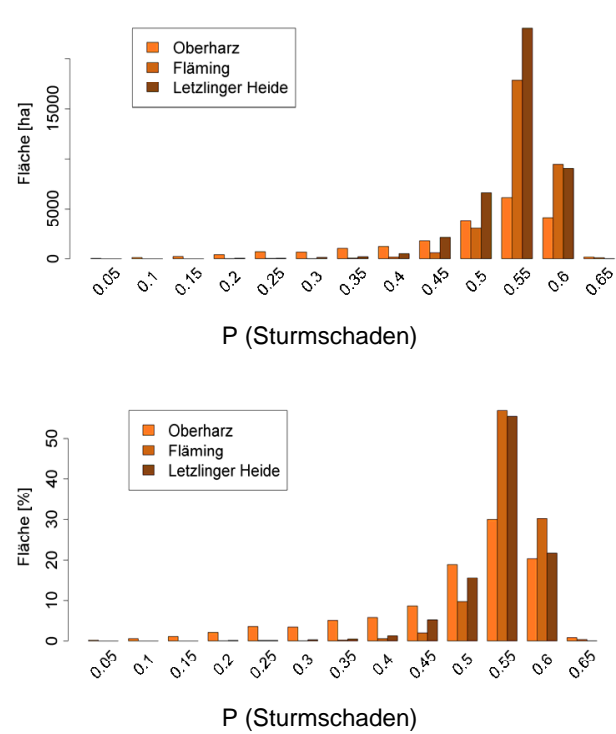

Douglasie
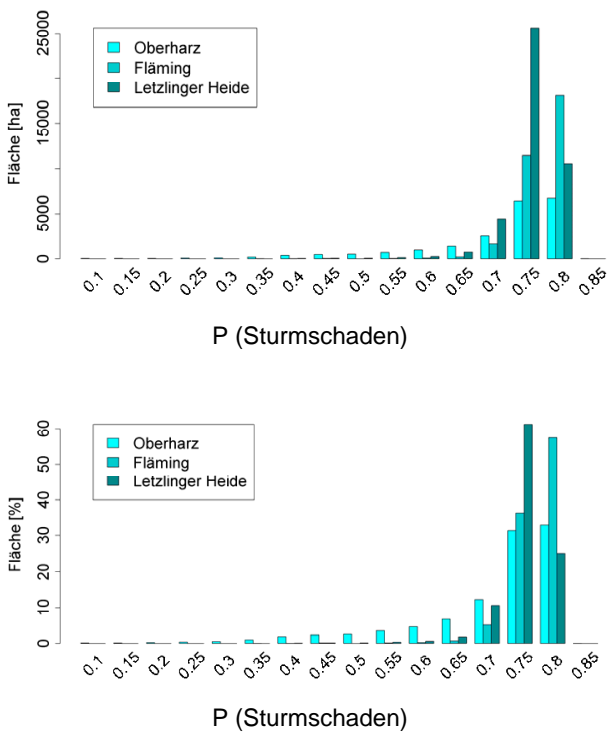

Abbildung 50: Absolute [ha] und relative [\%] Flächenverteilungen potenzieller Bestockungen von Kiefer (Zielstärke $45 \mathrm{~cm}$ ) und Douglasie $(55 \mathrm{~cm})$ nach Sturmschaden-Risikostufen in den Modellregionen Oberbarz, Fläming und Colbitz-Letzlinger Heide. Für beide Baumarten sind nur Bestände unterhalb von 600 m berücksichtigt.

Der Vergleich der potenziellen Bestockung bei Zielstärke zwischen den Baumarten zeigt im Oberharz ein im Mittel steigendes Risiko von Eiche über Buche, Kiefer und Douglasie zu Fichte (s. Abb. 51). Dabei weist die Douglasie trotz ihrer höheren Zielstärke von $55 \mathrm{~cm}$ und den zugehörigen größeren Höhen (s. Abb. 19) ein im Mittel geringeres Risiko auf als die Fichte. Der Unterschied zwischen Eiche und Buche ist vor allem auf die geringeren Höhen der Eiche im Oberharz zurückzuführen (s. Abb. 18), da der Modelleffekt beider Baumarten identisch ist (s. Gl. 12). Die Kiefer weist bei einer Zielstärke von $45 \mathrm{~cm}$ ein Risikoniveau auf, das im Mittel etwas höher ist als das der Buche bei $55 \mathrm{~cm}$ Zielstärke. Insgesamt ist die Kiefer - bezüglich 
des Sturmschadensrisikos - der Buche somit ähnlicher als der Douglasie und der Fichte. Die Mittelwerte des Sturmschadensrisikos betragen für die Eiche 0,30, für die Buche 0,41, für die Kiefer 0,47, für die Douglasie 0,68 und für die Fichte 0,77.

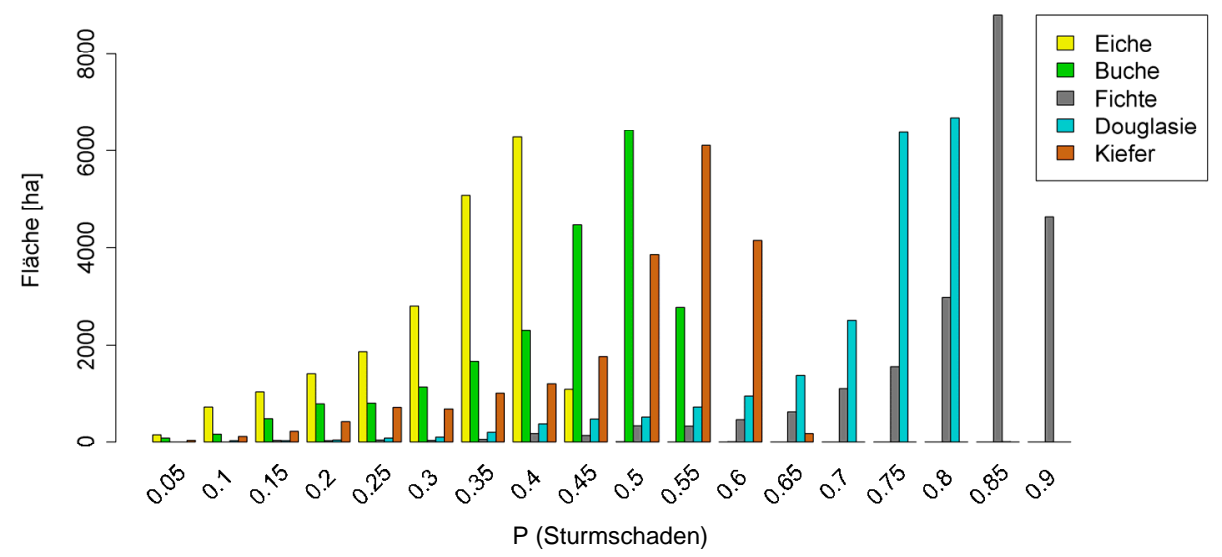

Abbildung 51: Flächenverteilungen [ha] potenqieller Bestockungen bei baumartensperifischen Zielstärken nach Sturmschaden-Risikostufen und Baumarten in der Modellregion Oberhar:. Für Eiche, Douglasie und Kiefer sind nur Bestände unterhalb von 600 m und für Buche nur Bestände unterhalb von 700 m berücksichtigt.

Die Klassifizierung anhand der vier Risikostufen führt zu einer Vereinfachung des Musters des prognostizierten Sturmschadensrisikos (s. Abb. 52, rechts versus links). Dabei wird davon ausgegangen, dass trotz der notwendigen Vereinfachung eine für die Planung ausreichende Informationsgrundlage bereitgestellt wird.

Eine potenzielle Bestockung der Fichte bei Zielstärke würde zu $6 \%$ in die Klasse „geringes Risiko“, zu $49 \%$ in die Klasse „mittleres Risiko“, zu $38 \%$ in die Klasse „hohes Risiko“ und zu $7 \%$ in die Klasse „sehr hohes Risiko“ eingeteilt werden. Die Flächenanteile mit niedrigem und mittlerem Risiko liegen damit deutlich unter und die Flächenanteile mit sehr hohem und vor allem hohem Risiko deutlich über den Werten, die im niedersächsischen Harz auftreten. Als Ursache kann der höhere Anteil von Plateaulagen in der Region Oberharz vermutet werden, auf denen die Fichte überwiegend in die Klasse „hohes Risiko" eingestuft wird (s. Abb. 52). 
10 Sturmschadensklassen mit identischer Klassenbelegung:

$0,09-0,60$

$0,61-0,70$

$0,71-0,76$

$0,77-0,79$

$0,80-0,81$

0,82

$0,83-0,84$

0,85

0,86

$0,87-0,92$
Fichte

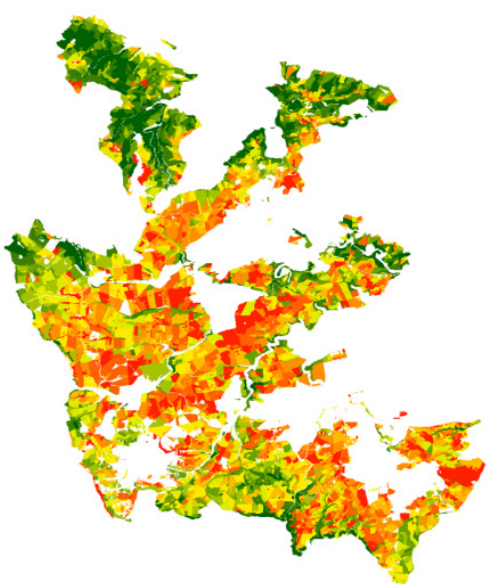

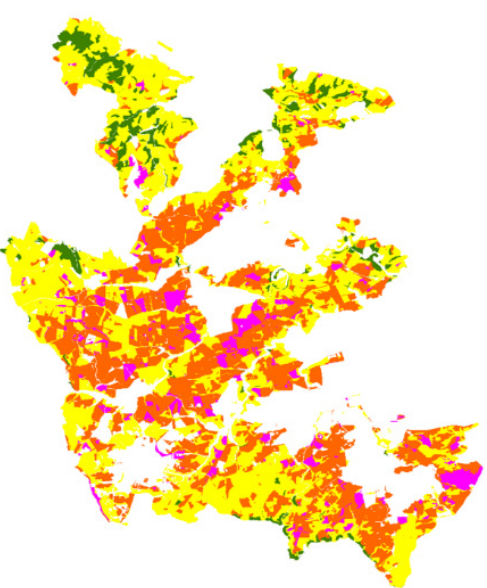

Abbildung 52: Karten des Sturmschadensrisikos derpotenziellen Fichtenbestockung bei Zielstärke (45 cm) in der Region Oberharz mit Einteilungen in 10 (links) und 4 (rechts) Risikoklassen (grün: niedriges, gelb: mittleres, rot: hohes und pink: sehr hohes Sturmschadensrisiko)

Eine potenzielle Douglasienbestockung bei Zielstärke $(55 \mathrm{~cm}$ ) würde zu $89 \%$ in die Klasse „mittleres Risiko“ und zu $11 \%$ in die Klasse „niedriges Risiko“ eingestuft (s. Abb. 53). Ein Baumartenwechsel von der Fichte zur Douglasie würde somit trotz der höheren Zielstärke zu einer Absenkung der Risikoklasse in den für die Fichte mit hohem und sehr hohem Risiko bewerteten Beständen führen.

Eine potenzielle Buchenbestockung bei Zielstärke $(55 \mathrm{~cm})$ würde zu $100 \%$ in die Klasse „geringes Risiko“ eingestuft (s. Abb. 53), so dass ein Baumartenwechsel von der Fichte zur Buche eine Absenkung der Risikoklasse in allen für die Fichte mit mittlerem bis sehr hohem Risiko bewerteten Beständen bedeuten würde. Die Absenkung ist dabei umso deutlicher je höher die Risikoklasse der Fichte ist. 

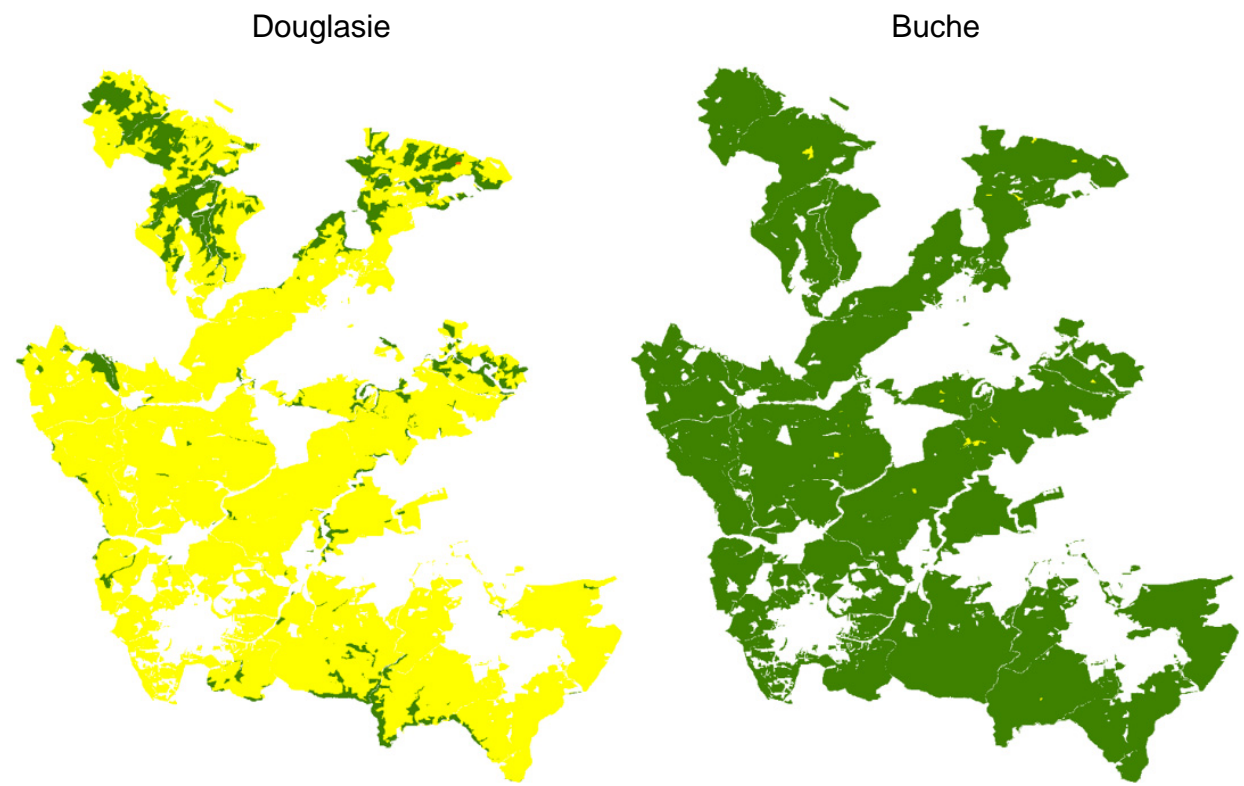

Abbildung 53: Karten des Sturmschadensrisikos der potenziellen Douglasien- und Buchenbestockung bei Zielstärke $(55 \mathrm{~cm})$ in der Region Oberharz unter Anwendung von 4 anhand der Fichte abgeleiteten Risikoklassen (grün: niedriges, gelb: mittleres, rot: hohes und pink: sehr hohes Sturmschadensrisiko)

\subsubsection{Aktuelle Gefährdungspotenziale durch Winterstürme}

Die Abschätzung des aktuellen Gefährdungspotenzials anhand der Informationen aus der Forsteinrichtung dient in erster Linie der Identifizierung von aktuell besonders gefährdeten Beständen. Die Identifizierung der Bestände erlaubt die Anpassung von Maßnahmen im Rahmen der kurz- und mittelfristigen Planung, wie eine Absenkung der Zielstärke oder eine beschleunigte Räumung von Restbestockungen. Die Darstellung der Prognoseergebnisse erfolgt erneut über Flächenverteilungen nach Risikostufen und Risikokarten.

Die Flächenverteilungen nach Risikostufen werden in der Region Oberharz von der Fichte dominiert, wobei abgesehen von der untersten Risikostufe mittlere bis hohe Risikostufen vorherrschen (s. Abb. 54). Dargestellt sind die vier flächenmäßig wichtigsten Baumarten Fichte, Buche, ALn und Lärche.

Die aktuelle Fichtenbestockung weist einen Mittelwert der Sturmschadenswahrscheinlichkeit von 0,53, die aktuelle Lärchenbestockung von 0,36, die aktuelle Buchenbestockung von 0,26 und die aktuelle Bestockung des ALn von 0,10 auf. Bestände aus Lärche, Buche und ALn erfordern damit bzgl. des Sturmschadensrisikos keine speziellen waldbaulichen Maßnahmen. Dagegen erscheint es sinnvoll, 
in Abhängigkeit von der Risikoaffinität des Bewirtschafters und weiterer Restriktionen bestimmte Anteile besonders gefährdeter Fichtenbestände waldbaulich gesondert zu behandeln. Ein besonderer Fokus sollte dabei selbstverständlich auf den erntereifen Beständen liegen.

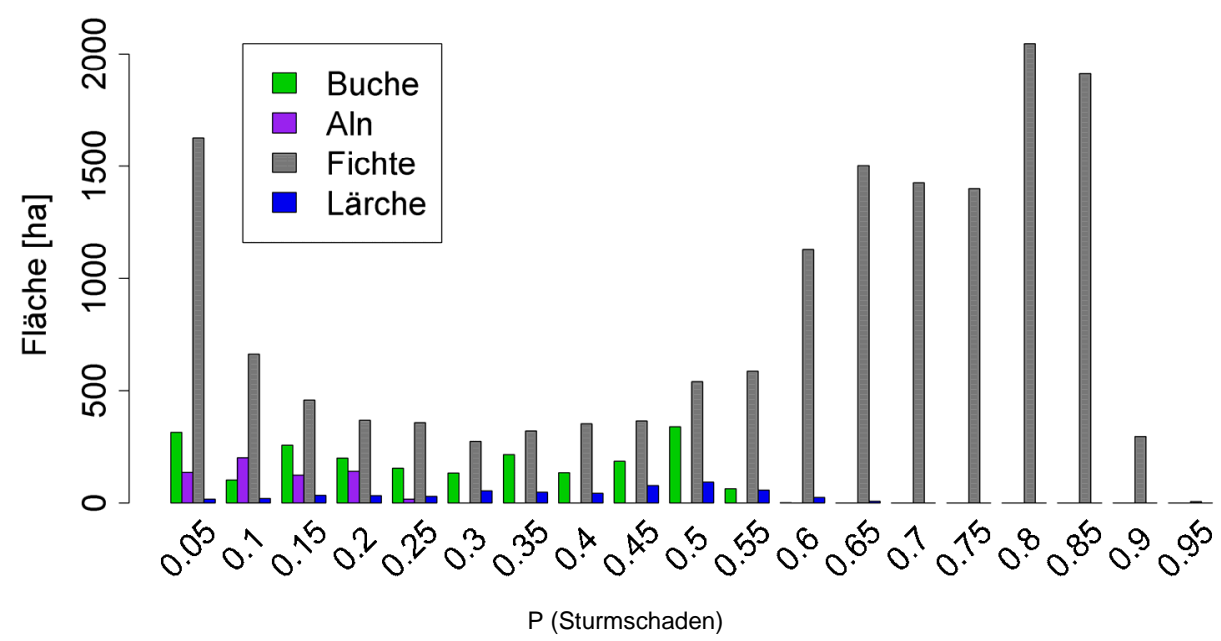

Abbildung 54: Flächenverteilungen der aktuellen Bestockungen [ba] nach Sturmschaden-Risikostufen und Baumarten in der Modellregion Oberbarz.

Die Prognose der aktuellen Sturmschadensrisiken dient vor allem der Identifizierung besonders gefährdeter Bestände, um im Rahmen der kurz- und mittelfristigen Planung konkrete Maßnahmen wie z. B. eine Absenkung der Zielstärke oder die Wahl der Hiebsform für die Abnutzung zu planen. Daher ist es nicht notwendig, eine Klassifizierung vorzunehmen, wie sie beispielsweise für die operationale Zuweisung strategischer Maßnahmen anhand des potenziellen Risikos notwendig ist. Für die kartographische Darstellung wurde daher lediglich eine relativ feine Klassifizierung in zehn Risikoklassen gewählt (s. Abb. 55).

Anhand der Risikokarte für die aktuelle Fichtenbestockung in der Region Oberharz (s. Abb. 55 links) lassen sich besonders gefährdete Bestände identifizieren. Der Vergleich mit der Risikokarte für die aktuelle Buchenbestockung (s. Abb. 55 rechts) unterstreicht, dass die Berücksichtigung des Sturmschadensrisikos in der kurz- bis mittelfristigen Planung ausschließlich Fichtenbestände betreffen sollte. 
10 Sturmschadensklassen mit einheitlicher Klassenbreite:

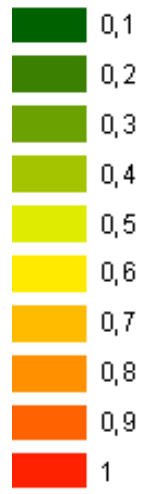

Fichte

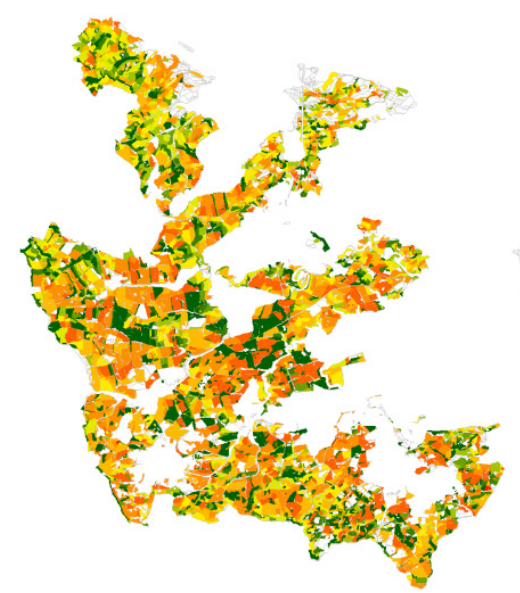

Buche

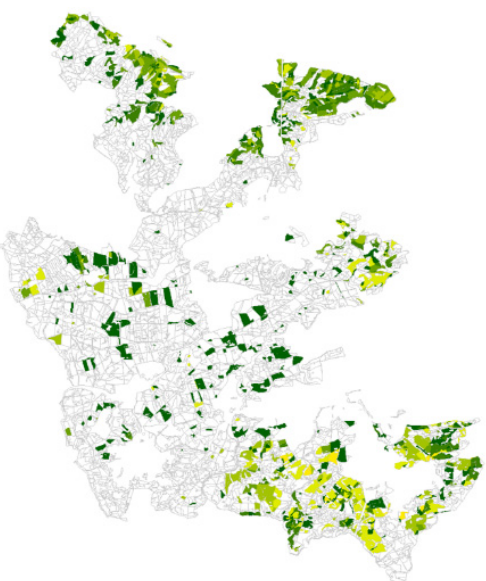

Abbildung 55: Karten des Sturmschadensrisikos der aktuellen Fichten- und Buchenbestockung in der Region Oberharz mit einer Einteilung in zehn Risikoklassen

\subsection{Veränderungen des Waldbrandrisikos}

Das klimatische Waldbrandrisiko im Oberharz war in den Jahren 2004 - 2010 gering: Auf Basis des M68 und des FWI wird die Anzahl der Tage mit erhöhtem Waldbrandrisiko für alle Gemeinden übereinstimmend mit weniger als 15 (FWI) bzw. weniger als zehn Tagen im Jahr (M68) angegeben (s. Abb. 56 und Abb. 57). Die Variation zwischen den Gemeindegebieten ist vernachlässigbar gering.

\section{Jährliche Tage mit WBKZ > 4000}
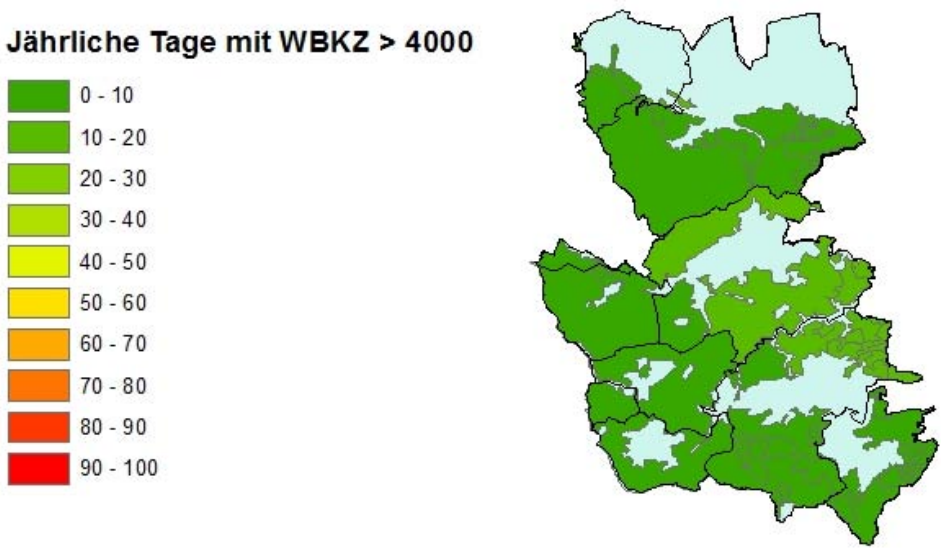

Abbildung 56: Jährliche Tage mit erböbtem Waldbrandrisiko im Oberbarz nach Berechnung mit dem M68-Waldbrandindex (WBKZ = Waldbrandkenniiffer) 
Jährliche Tage mit FFMC > 92

\begin{tabular}{|c|}
\hline $0-10$ \\
\hline $10-20$ \\
\hline $20-30$ \\
\hline $30-40$ \\
\hline $40-50$ \\
\hline $50-60$ \\
\hline $60-70$ \\
\hline $70-80$ \\
\hline $80-90$ \\
\hline $90-100$ \\
\hline
\end{tabular}

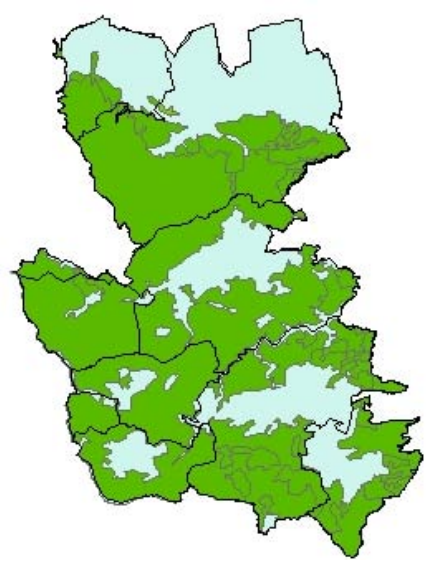

Abbildung 57: Jährliche Tage mit erböbtem Waldbrandrisiko im Oberharz nach Berechnung mit dem Fire Weather Index (FWI) (FFMC = Strenfeuchtezahl)

Zu dieser sehr geringen Gefährdungseinschätzung haben die im Bergland regelmäßigeren Niederschläge und höhenlagenabhängig niedrigeren Temperaturen beigetragen.

Tatsächlich gab es in diesem Referenzzeitraum auch keinen einzigen Waldbrand im Oberharz, so dass der Eindruck entstehen könnte, Waldbrände kämen in der Region Oberharz gar nicht vor. Dies ist jedoch nur gültig für den kurzen Zeitraum der vorliegenden Waldbrandstatistik, die ja keine Extremjahre wie etwa das Jahr 2003 enthält (s. Abb. 58).
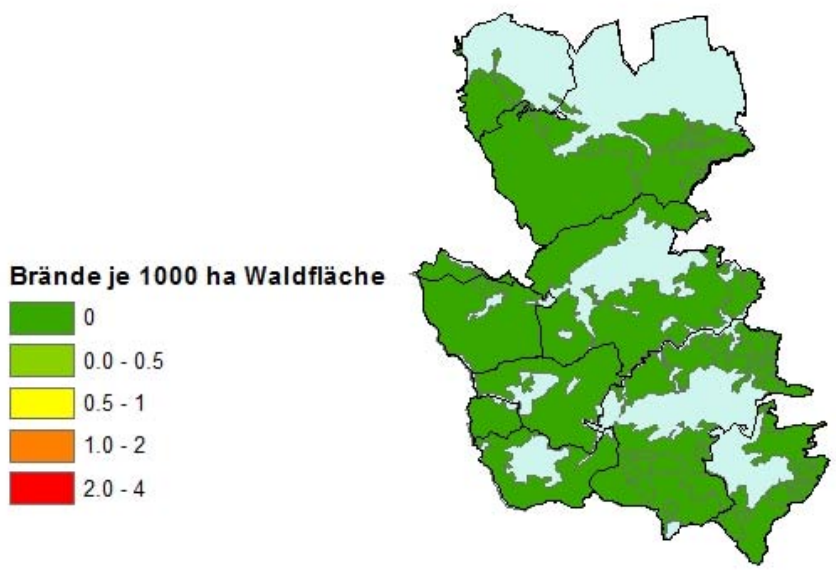

Abbildung 58: Waldbrände je 1000 ba Waldfläche in der Region Oberharz im Zeitraum 2004 - 2010 
Für die Zukunft ist nach Berechnungen mit M68-Index und FWI mit einer allmählichen und stetigen Zunahme des Waldbrandrisikos im Oberharz zu rechnen. Während für den Hauptteil der Region im Zeitraum 2011 - 2040 noch weniger als 20 Tage im Jahr mit erhöhtem Waldbrandrisiko erwartet werden, nimmt die Zahl solcher Risikotage bis zum Zeitraum 2071 - 2100 voraussichtlich auf jährlich bis zu 50 Tage (M68) bzw. 55 Tage (FWI) zu (s. Abb. 59 und Abb. 60).

Waldbrandkennziffer: 30-Jahres-Projektionen bis 2040, 2070 und 2100

\section{Jährliche Tage mit WBKZ $>4000$}
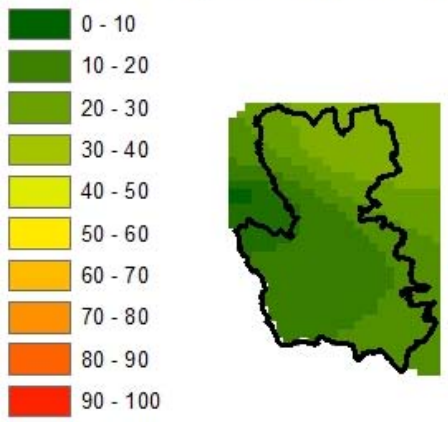

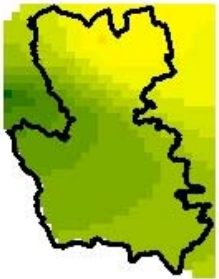

2041-2070

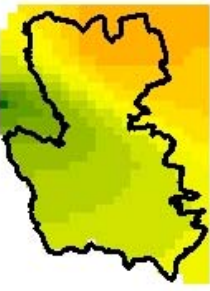

$2071-2100$

Abbildung 59: Erwartete jährliche Zabl von Tagen mit erböhtem Waldbrandrisiko auf Basis des M68Indexes (Tage, an denen die Waldbrandkennziffer (WBKZ) über 4000 liegt)

Fine Fuel Moisture Code: 30-Jahres-Projektionen bis 2040, 2070 und 2100

\section{Jährliche Tage mit FFMC > 92}

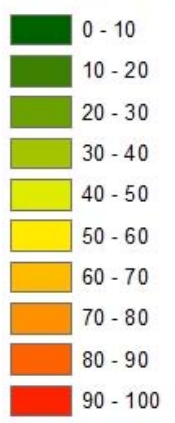

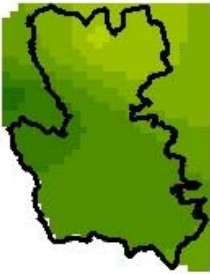

2011-2040

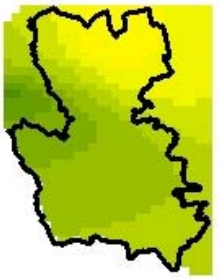

2041-2070

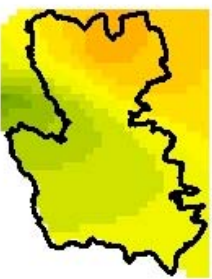

2071-2100

Abbildung 60: Erwartete jährliche Zabl von Tagen mit erböhtem Waldbrandrisiko auf Basis des FWI (Tage, an denen der Fine Fuel Moisture Code (FFMC = Streufeuchtezabl) über 92 liegt) 
Tendenziell nimmt das Waldbrandrisiko im Oberharz nach Osten aufgrund der abnehmenden Niederschläge zu. Der Nord-Süd-Gradient in den Vorhersagen für die Region dagegen wird aufgrund der zu geringen Klimastationsdichte in den Hochlagen des Harzes vermutlich überschätzt (SUTMÖLLER et al. 2013). Der nördlichste Teil der Region dürfte demnach einem nur etwas höheren Waldbrandrisiko ausgesetzt sein als der Mittelteil.

Schon die Steigerung auf bis zu 20 Risikotage bedeutet, dass die waldbrandfreie Periode der letzten Jahre in den kommenden 30 Jahren beendet wird und dass es gelegentlich zu Waldbränden kommen kann, wie sie sonst bisher nur aus sehr trockenen Jahren bzw. aus den anderen beiden Untersuchungsregionen bekannt sind. Mit der erwarteten Steigerung auf im räumlichen Durchschnitt bis zu 30 Tage (2041 - 2070) und bis zu 40 Tage (2071 - 2100) entwickelt sich der Oberharz dann zu einer Region mit ähnlicher Gefährdungseinstufung wie die am stärksten waldbrandgefährdeten Regionen in Sachsen-Anhalt heute.

Zusätzliche Risikofaktoren:

In einem bewegten Gelände wie dem Oberharz kann die Frage der Exposition (Südhang/Nordhang) und der damit verbundenen Sonneneinstrahlung eine größere Rolle für die Entstehung von Waldbränden spielen als im Flachland. Während nur wenige Hänge im Südosten und Nordosten des Gebiets so frei liegen, dass bis zu 16 Stunden direkter Sonneneinstrahlung möglich sind (s. Abb. 61), kann den größten Teil des Oberharzes in Sachsen-Anhalt bis zu 15 Stunden lang Sonnenschein erreichen. Besonders im Nordwesten ist die potenzielle direkte Sonneneinstrahlung dagegen aufgrund der Topographie meist auf weniger als 14 oder 13 Stunden begrenzt, so dass hier von einem regional verminderten strahlungsbedingten Risiko ausgegangen werden kann.

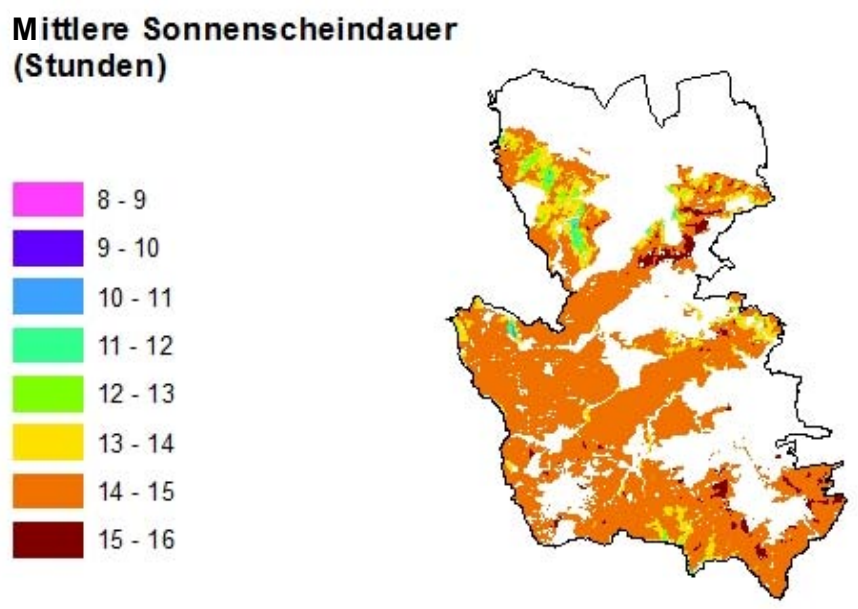

Abbildung 61: Mittlere potenzielle Sonnenscheindauer während der Monate April bis August auf den Waldflächen im Oberharz. 
Ein ähnliches Bild zeigt sich auch bei der Globalstrahlung (s. Abb. 62), die besonders auf den nordexponierten Lagen im Nordteil des Gebiets gering ist. Auf den südexponierten Lagen und im Süd- und Mittelteil der Region werden dagegen häufig besonders hohe mittlere Globalstrahlungswerte über $220 \mathrm{~W} / \mathrm{m}^{2}$ erreicht. Die erhöhten Globalstrahlungswerte sind ein zusätzlicher Risikofaktor in der Region Oberharz, der diese Region von den anderen unterscheidet.

Im nördlichen Oberharz liegt das einzige, mit 0,8 ha aber recht überschaubare Risikogebiet mit Kiefern im Alter von weniger als 40 Jahren und einem aktuellen Bestockungsgrad unter 0,7 .

\section{Mittlere Globalstrahlung 1. April - 31. August $\left(\mathrm{W} / \mathrm{m}^{2}\right)$}

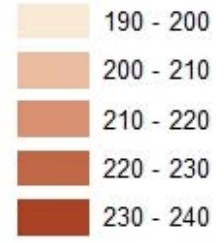

Risikogebiete (ha)

0,8

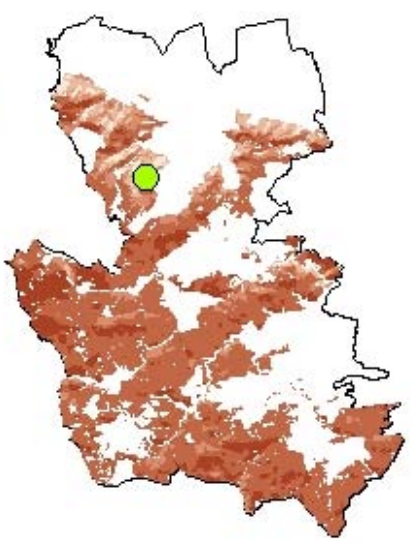

Abbildung 62: Regionale Verteilung der mittleren Globalstrablung $\left(W / m^{2}\right)$ auf $W$ aldflächen im Oberharz während der Monate April bis August und Lage von Kiefernjungbeständen geringer Bestockungsdichte (lokale Risikogebiete)

\subsection{Gefährdungspotenziale durch Buchdruckerbefall bei Fichte im Oberharz}

\subsubsection{Gefährdungspotenziale durch Buchdruckerbefall für eine potenzielle Bestockung}

Für die Abschätzung der potenziellen Vulnerabilität wurden die Bestockungsparameter für jeden Bestand konstant auf ein Alter von 92 Jahren und einen Fichtenmischungsanteil von $100 \%$ gesetzt.

Die Abschätzung des Gefährdungspotenzials für eine potenzielle Bestockung dient dazu, standörtliche Unterschiede bzgl. des Buchdruckerbefallsrisikos zu identifizieren und bei der langfristigen strategischen Forstplanung zu berücksichtigen. Der projizierte Klimawandel resultiert in einem deutlichen Anstieg der Temperatursumme in der Vegetationszeit (s. Abb. 63). Die Veränderung des Gefährdungspotenzials als Effekt des Temperaturanstiegs wird in Form von Zeitreihen für die vier Projektionsperioden dargestellt. 


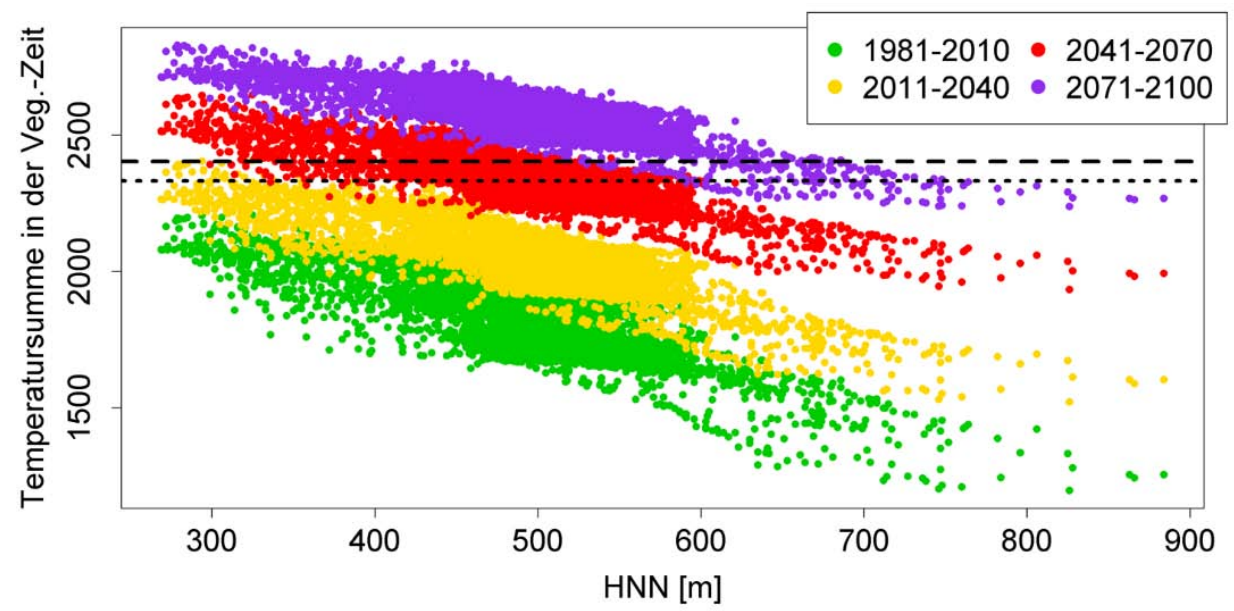

Abbildung 63: Temperatursumme $\left[{ }^{\circ} \mathrm{C}\right]$ in der Vegetationszeit der Fichte über der Seeböhe (HNN in m) getrennt nach Klimaperioden. Die kurz-gestrichelte Linie markiert die mittlere Temperatur im Fläming und die lang-gestrichelte Linie die mittlere Temperatur in der ColbitzLetzlinger Heide in der Periode 1981 - 2010.

Zu Vergleichszwecken ist zusätzlich die Temperatursumme in der Vegetationszeit der Fichte in der Periode 1981 - 2010 für den Fläming und die Colbitz-Letzlinger Heide dargestellt. Danach sind beispielsweise für die Periode 2041 - 2070 auf $500 \mathrm{~m}$ im Oberharz Temperatursummen zu erwarten, wie sie in der Periode 1981 - 2010 im Mittel im Fläming herrschen. Die mittlere Temperatursumme in der ColbitzLetzlinger Heide in der Periode 1981 - 2010 wird im Oberharz auf ca. $620 \mathrm{~m}$ in der Periode 2071 - 2100 erreicht.

Als Effekt der projizierten Temperaturerhöhung findet eine Verlagerung der Flächenverteilung hin zu den höheren Stufen des potenziellen Befallsrisikos statt (s. Abb. 64). Gleichzeitig nimmt die Spreitung der Verteilungen über der Zeit ab. Die Mittelwerte des Befallsrisikos betragen 0,55 für die Periode 1981 - 2010, 0,64 für die Periode 2011 - 2040, 0,77 für die Periode 2041 - 2070 und 0,85 für die Periode $2071-2100$. 


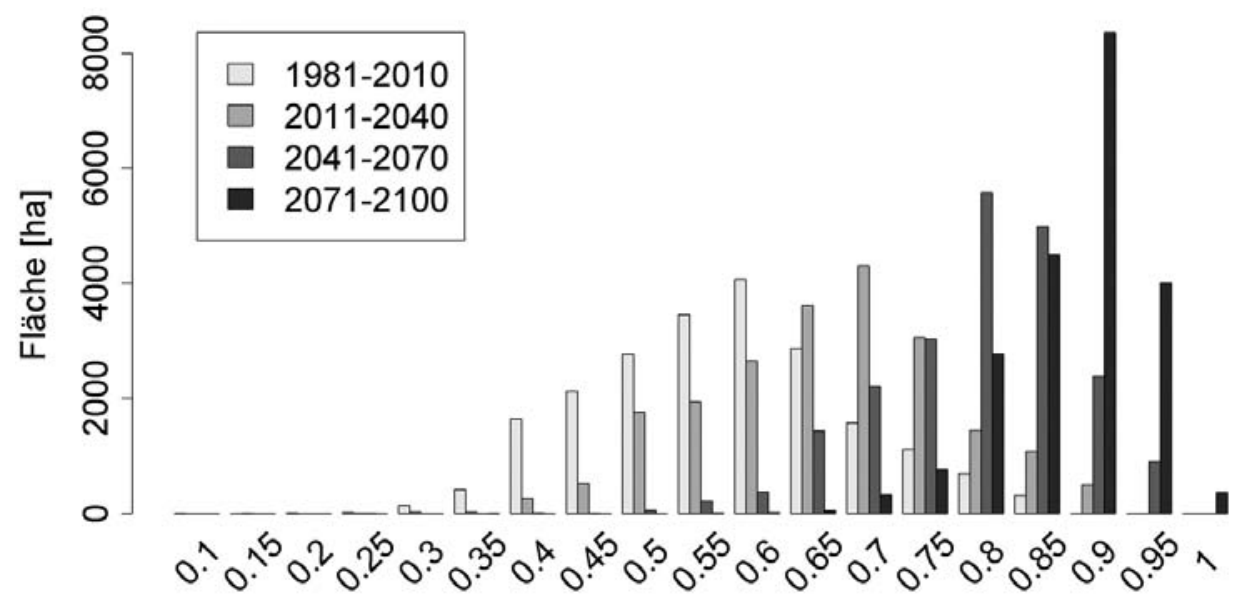

Abbildung 64: Flächenverteilungen der potenziellen Fichten-Bestockung (Zielstärke $45 \mathrm{~cm}$ ) [ha] nach Buchdruckerbefall-Risikostufen in der Modellregion Oberhar getrennt nach Klimaperioden

Die Ableitung von Grenzwerten zur Klassifizierung der Risikoprognosen erfolgt anhand der Prognosen für die Periode 2041 - 2070 (s. Abb. 65). Dabei werden alle Bestände mit Werten unterhalb des 15 \%-Quantils der Risiko-Häufigkeitsverteilung als gering gefährdet (Risikoklasse 1) eingestuft. Bestände mit Werten oberhalb des $85 \%$ - und unterhalb des $95 \%$-Quantils weisen ein hohes Risiko (Risikoklasse 3) und Bestände oberhalb des $95 \%$-Quantils ein sehr hohes Risiko (Risikoklasse 4) auf. Bestände mit Werten zwischen dem $15 \%$ - und dem $85 \%$-Quantil wird ein mittleres Risiko (Risikoklasse 2) zugeordnet (s. Abb. 65). Eine Ableitung von Grenzwerten, die sich an einer umfassenden ökonomischen Bewertung von Risiken orientiert, erscheint derzeit kaum möglich. Für die Forstwirtschaft sind letztlich die mit den verschiedenen Risikoklassen verknüpften waldbaulichen Maßnahmen wie eine risikoadaptierte Zuordnung von Bestandeszieltypen entscheidend. 


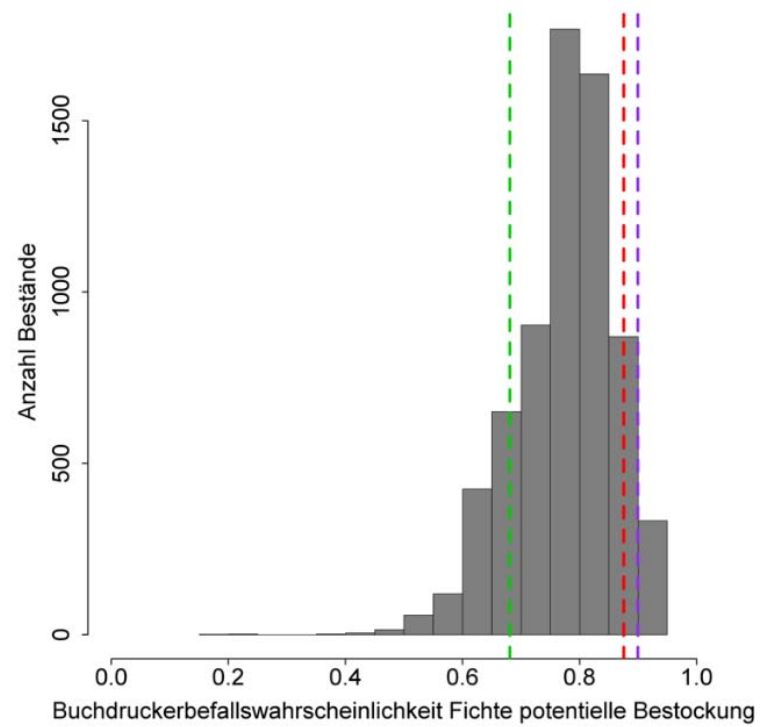

Abbildung 65: Histogramm von Wabrscheinlichkeiten des Buchdruckerbefallsrisikos der Fichte bei Zielstärke $(45 \mathrm{~cm})$ für alle Bestände in der Modellregion Oberbarz in der Projektionsperiode 2041 - 2070. Die grün gestrichelte Linie markiert die Obergrenze der Klasse „niedriges Befallsrisiko (Risikoklasse 1)". Werte zwischen der grünen und roten Linie werden als „mittleres Befallsrisiko (Risikoklasse 2)", Werte zwischen der roten und violetten Linie als „hohes Befallsrisiko (Risikoklasse 3)" und oberbalb der violetten Linie als „sehr hohes Befallsrisiko (Risikoklasse 4)" eingestuft.

In der ersten Projektionsperiode treten im Oberharz nur die Risikoklassen 1 und 2 mit 87 bzw. 13 \% der Fläche auf (s. Abb. 66). Auch noch in der Periode 2011 - 2040 entfällt der mit $65 \%$ größte Flächenanteil auf die Risikoklasse 1, während auf die Risikoklassen 2 und 3 lediglich 33 bzw. $2 \%$ entfallen. In der Periode 2041 - 2070 dominiert definitionsgemäß erstmals die Risikoklasse 2 mit $70 \%$ der Fläche. In der Periode 2071 - 2100 entfällt mit 34 und $21 \%$ erstmals mehr als die Hälfte der Fläche auf die Risikoklassen hoch und sehr hoch. In die mittlere Risikoklasse entfallen immerhin noch $44 \%$ während ein geringes Risiko nur auf $1 \%$ bzw. für die höchsten Lagen des Oberharzes prognostiziert wird. Insgesamt werden die Muster des Befallsrisikos in erster Linie durch den Höhengradienten der Temperatursumme und die regionalen Unterschiede bestimmt. Der regionale Trend führt dabei zu einem Anstieg des Risikos von Südwest nach Nordost (s. Abb. 66). In diesem Zusammenhang kann vermutet werden, dass die flächengewichtete Mittelwertbildung der nFK in den Bestandespolygonen zu einer gewissen Nivellierung der Befallsprognosen führt. Für eine bessere Erfassung kleinräumiger Standortsunterschiede sollten die Prognosen daher zukünftig nicht mehr auf der Ebene von Bestandesgeometrien, sondern auf der von Standortsgeometrien erfolgen. 

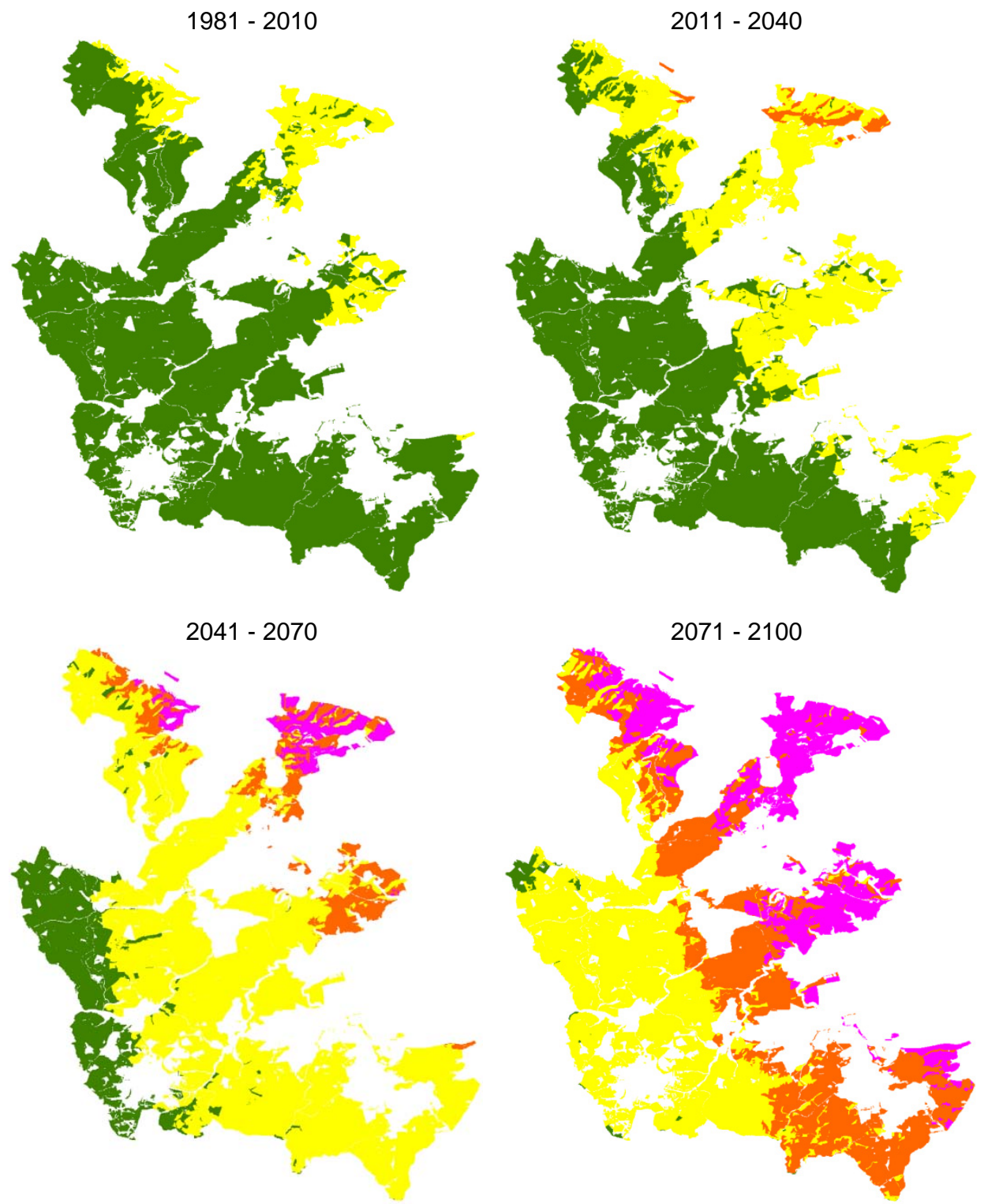

Abbildung 66: Karten des Buchdruckerbefallsrisikos einer potenqiellen Fichtenbestockung in der Region Oberbarz für die verschiedenen Projektionszeiträume mit einer Einteilung in 4 Risikoklassen (grün: niedriges, gelb: mittleres, rot: hohes und pink: sehr hohes Sturmschadensrisiko) 


\subsubsection{Gefährdungspotenziale der aktuellen Bestockung durch Buchdruckerbefall}

Für die Prognose des Gefährdungspotenzials der aktuellen Bestockung wurden das Bestandesalter und das Mischungsprozent der Fichte entsprechend der Forsteinrichtung im Stichjahr 2006 zur Initialisierung des Modells (s. Gl. 14) verwendet. Diese Werte wurden vereinfachend auch für die Prognosen in zukünftigen Klimaperioden verwendet, so dass der Altersklassenaufbau und die Mischungsprozente der Bestockung über der Zeit konstant sind. Eine Fortschreibung der Bestandesalter und Mischungsprozente hätte eine vollständige Wachstumssimulation erfordert, um ein realistisches Endnutzungs- und Verjüngungsszenario abzubilden.

Die nFK, der Topex zur Erfassung der Südexposition sowie die Temperatursummen der einzelnen Bestände entsprechen den Werten, wie sie zur Prognose des Befallsrisikos für eine potenzielle Bestockung verwendet wurden. Für die Herleitung der Flächenverteilung nach Risikostufen wurden in Mischbeständen nur die Anteilflächen der Fichte berücksichtigt.

Aufgrund der Altersspreitung der aktuellen Fichtenbestockung resultiert zu allen Projektionszeiträumen eine breite Flächenverteilung nach Risikostufen (s. Abb. 67). Der relativ hohe Anteil junger Bestände (s. Tab. 12) führt in den ersten beiden Klimaperioden zu vergleichsweise hohen Anteilen der niedrigen Risikostufen. Aufgrund der projizierten Klimaerwärmung nehmen diese Anteile insbesondere in der Periode 2071 - 2100 stark ab und es wird eine starke Zunahme für die hohen Risikostufen prognostiziert. Die Verlagerung der Verteilungen hin zu höheren Risikostufen wird auch an den Mittelwerten von 0,26 für die Periode 1981 - 2010, von 0,32 für die Periode 2011 - 2040, von 0,44 für die Periode 2041 - 2070 und von 0,54 für die Periode 2071 - 2100 deutlich.

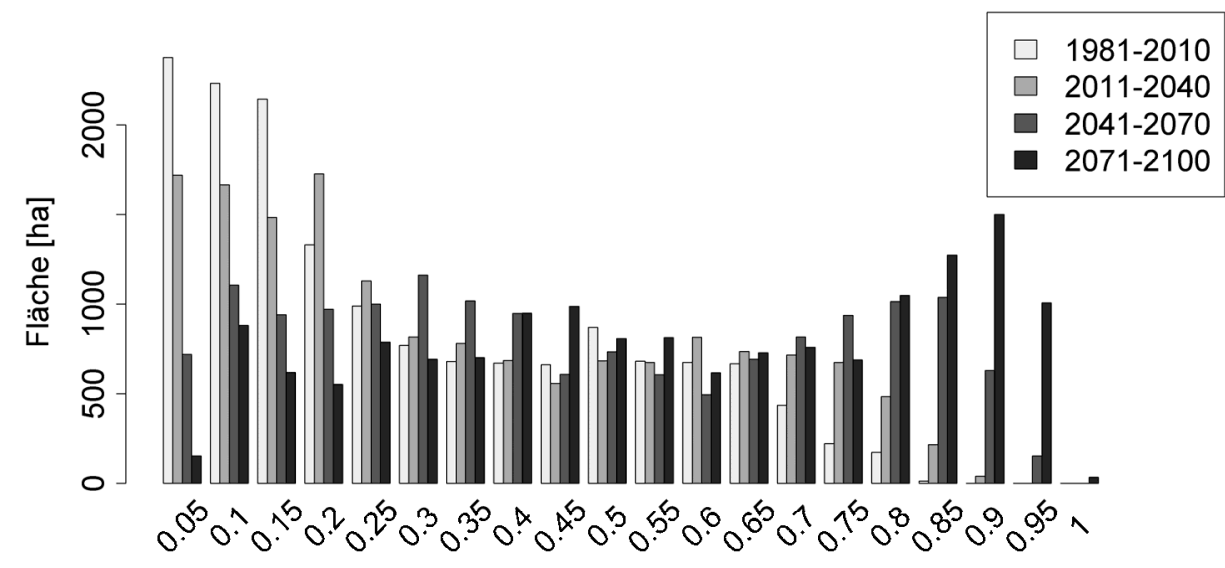

Abbildung 67: Flächenverteilungen der aktuellen Fichten-Bestockung [ha] nach Buchdruckerbefall-Risikostufen in der Modellregion Oberbarz getrennt nach Projektionszeiträumen

Beiträge aus der NW-FVA, Band 13, 2015 
Die Prognose des aktuellen Befallsrisikos dient vor allem der Identifizierung besonders gefährdeter Bestände, um im Rahmen der kurz- und mittelfristigen Planung konkrete Maßnahmen wie z. B. eine Absenkung der Zielstärke zu planen. Daher ist es nicht notwendig, eine Klassifizierung vorzunehmen, wie sie beispielsweise für die operationale Zuweisung strategischer Maßnahmen anhand des potenziellen Risikos notwendig ist. Für die kartographische Darstellung wurde daher lediglich eine relativ feine Einteilung in zehn Risikoklassen gewählt (s. Abb. 68).

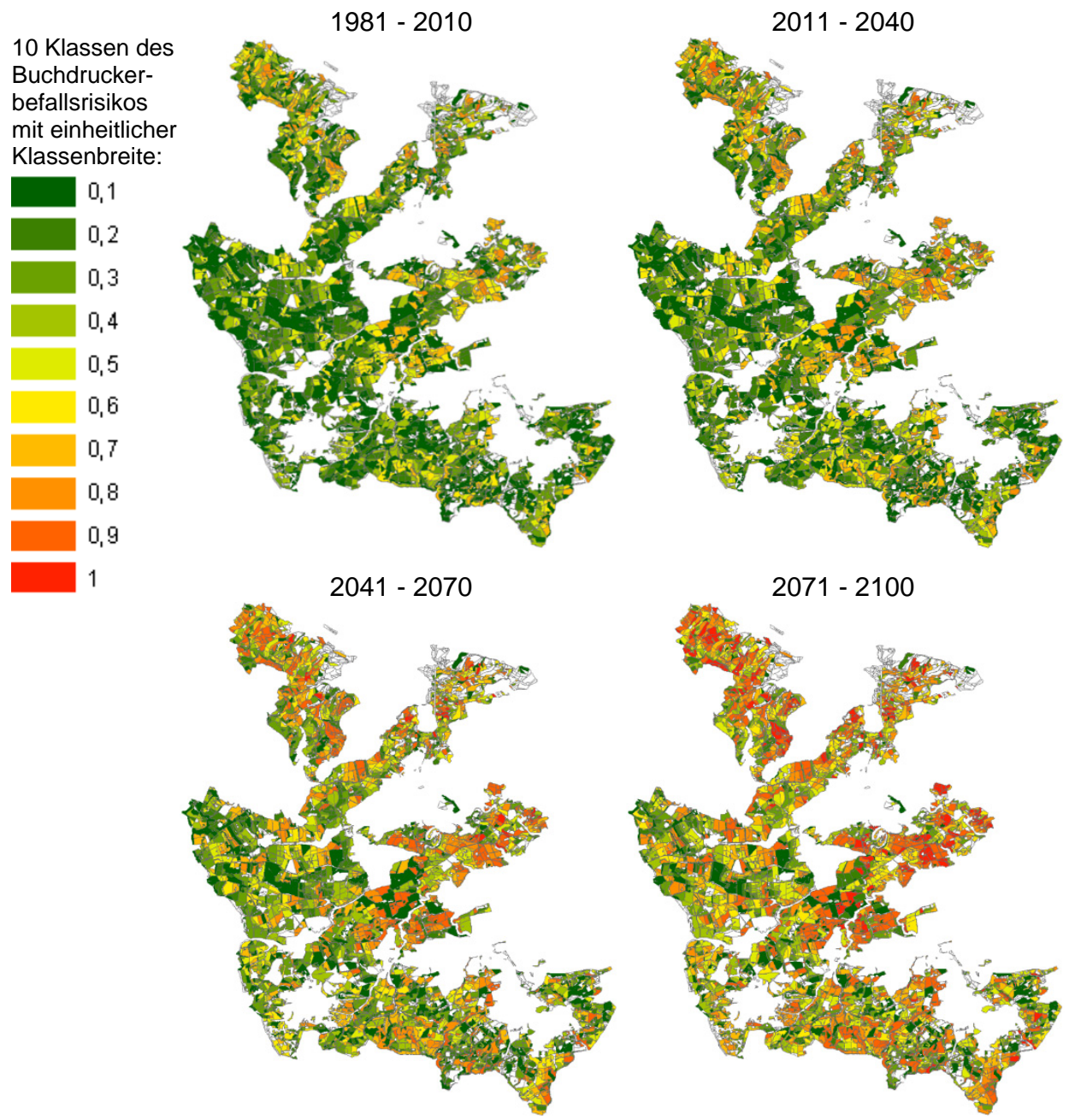

Abbildung 68: Karten des Buchdruckerbefallsrisikos der aktuellen Fichtenbestockung in der Region Oberharz für die verschiedenen Projektionszeiträume mit einer Einteilung in zehn Risikoklassen 
Der Effekt der Temperatursumme, ihrer projizierten Änderung sowie der geografische Effekt sind zwar erkennbar, werden jedoch durch den Effekt des bestandesspezifischen Alters und Mischungsprozentes der aktuellen Bestockung überlagert. Überdurchschnittlich viele Bestände mit hohen aktuellen Befallsrisiken sind dabei im Bereich der Reviere Wernigerode, Elbingerode und Rübeland zu finden.

\subsection{Zustände und Wirkungsgefüge bei biotischen Risiken}

Die Populationsdynamik von Borkenkäfern sowie die Disposition von Waldbeständen wird von Klima und Wetter sowohl direkt als auch indirekt beeinflusst. Die Populationsdichte und das Gradationsgeschehen des Buchdruckers beruhen daher nicht nur auf klimatischen Abhängigkeiten an sich, sondern auch auf der Baum- bzw. Bestandesdisposition, auf Koinzidenzen, natürlichen Feinden und Parasiten sowie der inter- und intraspezifischen Konkurrenz (SKUHRAVÝ 2002).

Die am häufigsten vertretenen Baumarten auf der analysierten Fläche von 21.239 ha in der untersuchten Region Oberharz sind die Fichte mit 76,8 \% und die Buche mit 14,9\%. Auf insgesamt 6.826 Teilflächen wurde das Befallsvorkommen der Schädlinge im Laufe der Jahre 2002 - 2012 untersucht. Von allen Insektenarten, die bei der Fichte Schäden verursachten, spielten die rindenbrütenden Borkenkäfer die größte Rolle. Im Rahmen der durchgeführten Analysen (s. Kap. 4.8.1) wurde eine Fläche als befallen definiert, wenn mehr als $1 \%$ des Fichteneinschlags auf der Teilfläche als Käferholz verbucht war. Ein so definierter Befall wurde bei 42,3 \% aller Bestände mit Fichtenvorkommen festgestellt.

Zur Beurteilung des Befallsrisikos der Fichte durch Borkenkäfer wurde ein statistisches Modell angewandt, das auf der Grundlage von Hiebsauswertungen parametrisiert wurde (s. Kap. 4.8.2). Mit Hilfe der durchgeführten Modellanalyse wurde festgestellt, dass das 10-jährige Befallsrisiko durch den Buchdrucker vor allem mit steigendem Alter und Mischungsanteil der Fichte sowie weiterhin bei steigenden Temperatursummen in der Vegetationszeit zunimmt; bei zunehmendem Topex zur Erfassung der Südexponiertheit, d. h. bei zunehmender Beschattung, reduziert sich hingegen das Befallsrisiko. Dies wurde schon von SKUHRAVÝ (2002) beschrieben, der auf eine besondere Gefahr durch Buchdruckerbefall für reine Fichtenbestände in niedrigen Lagen hinwies. Der Einfluss der nFK hat sich im Verhältnis dazu als nur schwach signifikant herausgestellt, bei abnehmender nFK, besonders in den Bereichen mit flachgründigen Standorten, steigt das Risiko eines Befalls durch den Buchdrucker.

Die Populationsentwicklung des Buchdruckers und damit auch die Bestandesschäden werden neben der warmen Witterung während der Vegetationsperiode auch durch ausreichendes Brutmaterial, also Windwurf- und Bruchholz, begünstigt (RICHTER 1965, VELDMANN 2000). Nach der Analyse der Gefahr durch das Sturmschadensrisiko (s. Kap. 5.4) im Bereich des Oberharzes ist zu erwarten, dass vor 
allem in den mittleren Plateaulagen, wo ein mittleres und vor allem hohes Sturmschadensrisiko vorliegt, eine solche Gefährdung entstehen und zu großflächigen Borkenkäfer-Kalamitäten führen kann. Vergleichbare Folgen sind ebenfalls zu erwarten, wenn die Regeln der sauberen Waldwirtschaft im täglichen Forstbetrieb nicht ausreichend beachtet werden. Für den gesamten Bereich des Oberharzes ist zu erwarten, dass der Buchdrucker auch zukünftig Schäden verursachen wird. Besonders nach flächigen Störungen muss man in Fichtenkulturen ebenfalls mit verstärkten Schäden durch den Großen Braunen Rüsselkäfer (Hylobius abietis L.) rechnen.

Insbesondere in den mittleren und unteren Höhenlagen spielt neben dem Buchdrucker auch das Vorkommen der Nonne (Lymantria monacha L.) im untersuchten Bereich eine Rolle. Besonders gefährdet sind Fichtenbestände, weil dort schon ein einmaliger Kahlfraß durch die Nonne zum Absterben der Bestände ausreicht (ŠVESTKA et al. 1998). Nach der Analyse der Informationen aus dem Waldschutzmeldewesen wurde zwar festgestellt, dass in der untersuchten Region Oberharz keine Befallsgeschehen gemeldet wurden. Trotzdem hat sich aus dem Monitoring mittels Pheromonfallen ein Vorkommen der Nonne in bis zu $700 \mathrm{~m}$ ü. NN ergeben. Das stärkste Vorkommen wurde in einer Höhenlage von ca. $600 \mathrm{~m}$ im Bereich des damaligen Forstamtes Hasselfelde verzeichnet, wo im Jahr 1995 ein maximaler Fang von 413 männlichen Faltern der Nonne erreicht wurde. Damit wurde die übliche Schadschwelle zwar nicht erreicht, dennoch ist aber davon auszugehen, dass aufgrund der Ergebnisse aus Kapitel 5.1 (Veränderungen des Trockenstressrisikos) ein zunehmendes Risiko vor allem für die unteren und mittleren Lagen der im Oberharz stockenden Fichtenbestände vorliegt. Insgesamt lagen für das Schadvorkommen der Nonne keine ausreichenden Datensätze für die Analyse eines Wirkungsgefüges vor.

Ein erhöhtes Trockenstressrisiko trägt zur Zunahme der Schäden durch den dunklen Hallimasch (Armillaria ostoyae ROMAGN.) bei (ŠvESTKA et al. 1998). Sie sind in mittleren und niedrigeren Lagen des Harzes für alle Altersklassen der Fichte zu erwarten. Primärer Pilzbefall begünstigt generell auch weitere Schäden an Fichten, da geschwächte Bäume bevorzugt von Borkenkäfern befallen werden (ŠVESTKA et al. 1998).

\subsection{Konsequenzen für die künftige Waldbewirtschaftung in der Region Oberharz}

\subsubsection{Waldbauliche Ausgangslage}

Die Waldflächen der Region Oberharz reichen von den unteren Harzlagen bei Ilsenburg und Wernigerode im Nordosten bis in ober- und hochmontane Bereiche an den Grenzen zum Nationalpark Harz. Die betrachteten Waldflächen liegen hauptsächlich in montanen mittleren feuchten und sehr feuchten Lagen (Mf und Mff) zwischen 500 und $650 \mathrm{~m}$ ü. NN. Die standörtlichen Voraussetzungen sind hinsichtlich der Nährstoffversorgung relativ günstig. Über ein Drittel der Standorte ist der 
Nährkraftstufe „kräftig“ (K) zuzuordnen. Es handelt sich meist um terrestrische Standorte, auf denen sich die günstige Nährkraftstufe mindestens mit der Wasserhaushaltsstufe „mittelfrisch“ (2) kombiniert. Den größten Teil der Standorte machen Böden mittlerer Nährkraft aus, die hauptsächlich ebenfalls kombiniert mit einer mittleren Wasserversorgung vorkommen. Mineralische Nassstandorte haben eine gewisse Bedeutung in Plateaulagen. Standorte geringer Nährkraft sind in dem betrachteten Flächenzuschnitt relativ selten.

Die aktuelle Hauptbaumart ist mit großem Abstand und einem Flächenanteil von ca. $80 \%$ im Hauptbestand die Fichte. Sie stockt überwiegend in Reinbeständen, die in mittleren Altern auch aufgrund der früheren Behandlung wenig Begleitbaumarten und keinen nennenswerten Unter- und Zwischenstand aus Laubbaumarten aufweisen (ausgewiesener Unterstand aus Buche, Birke, Eberesche etc. nur 135 ha, $<1 \%$ ). Die Buche nimmt als bedeutendste Laubbaumart ca. $12 \%$ der Fläche ein. Ihr folgen die Weichlaubbaumarten, Edellaubbaumarten, Lärchen und Eichen, die aber jede für sich kaum einen Anteil von $2 \%$ erreichen (s. Abb. 69).

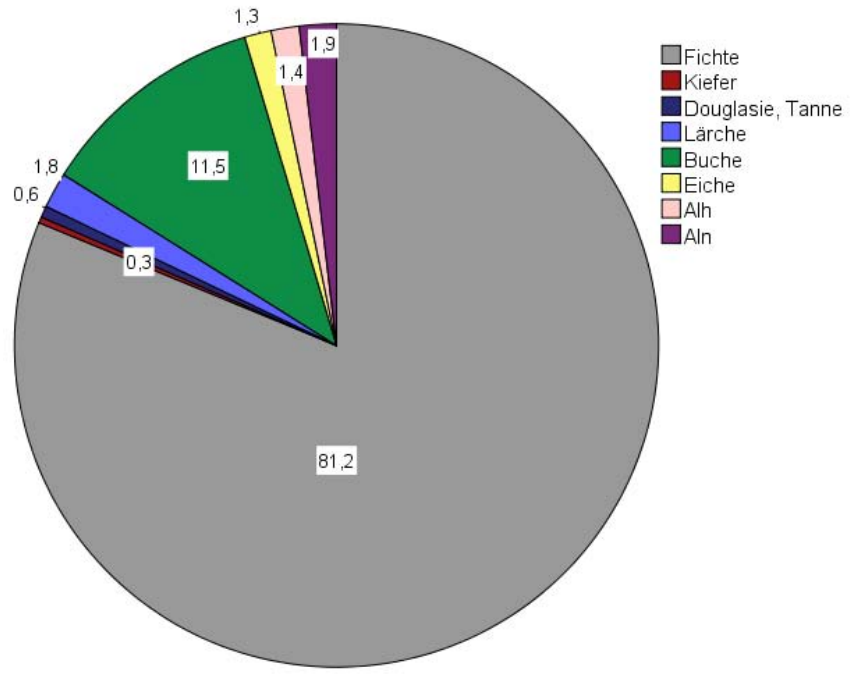

Abbildung 69: Flächenanteile [\%] der Baumarten im Hauptbestand in der Region Oberharz $(A L h=$ Laubholz, mit hoher Umtriebszeit, ALn = Laubholz, mit niedriger Umtriebszeit; im Datenspeicher W ald erfasste Flächen, Privatwald z. T. Stand 1994)

Der Altersaufbau der Fichte zeigt eine deutliche Überbesetzung der III. Altersklasse (s. Abb. 70). Ansonsten ist er aber sehr ausgeglichen, wenn man die über 100-jährigen Bestände zusammen mit der V. Altersklasse betrachtet. Ab der ausgehenden IV. Altersklasse bestehen erfahrungsgemäß Nutzungsmöglichkeiten für zielstarkes Holz, so dass sich nahezu ein Drittel der Fichtenfläche in dem dafür relevanten Altersbereich befindet. Die erste Altersklasse zeigt, dass die Fichte im Oberharz die Baumart mit der größten Verjüngungsfläche auf Freiflächen geblieben ist. Der 
Altersklassenaufbau der Buchenbestände ist bis zur VII. Altersklasse relativ ausgeglichen. Der sehr hohe Anteil der über 140-jährigen Bestände ist häufig deren schlechter Qualität und geringen Durchmessern geschuldet, weshalb Nutzungen und Verjüngung über längere Zeiträume unterblieben waren.

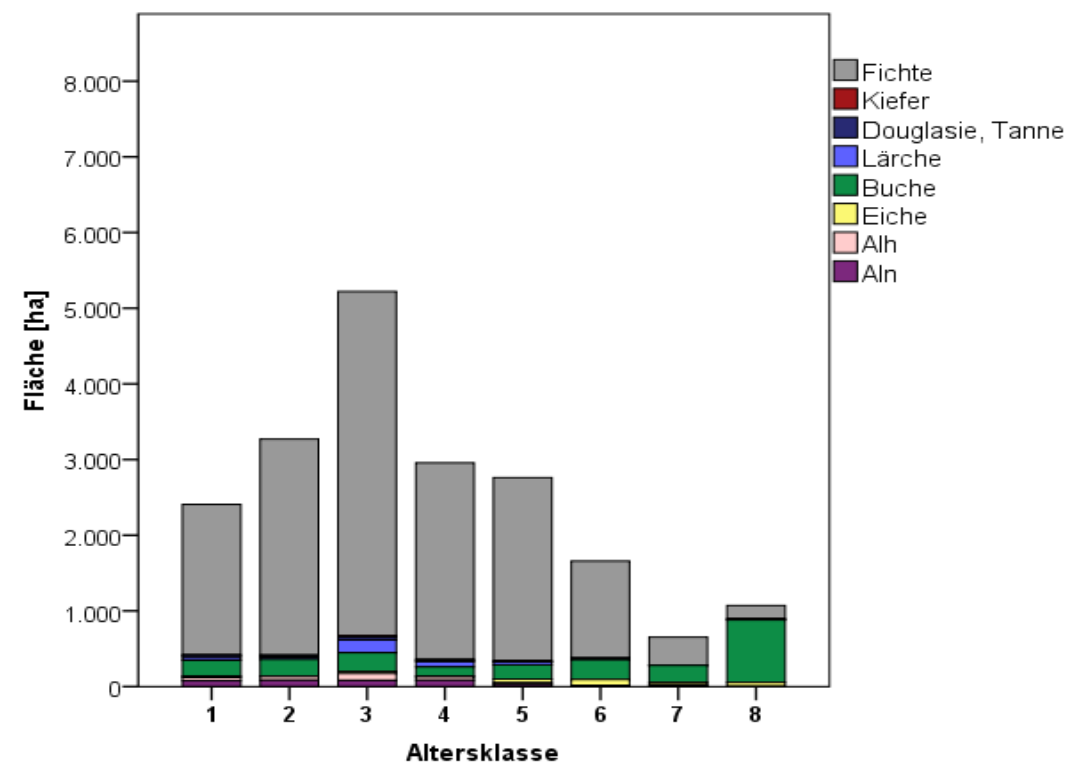

Abbildung 70: Altersklassenverteilung in der Region Oberharz, (eine Altersklasse umfasst 20 Jahre; ALh $=$ Laubholz mit hoher Umtriebszeit, ALn = Laubholz mit niedriger Umtriebszeit; im Datenspeicher W ald erfasste Flächen, Privatwald ₹. T. Stand 1994)

Betrachtet man den Nachwuchs unter Schirm, so zeigt sich, dass sich die Laubholzvermehrung in der Vergangenheit auf Voranbauten konzentrierte. Ca. 1.500 ha der Waldfläche der Region weisen mittlerweile eine gesicherte Verjüngung unter Altbestandsschirm auf, was einem Anteil von ca. $7 \%$ der Waldfläche entspricht (s. Abb. 71). Damit ist bereits ein nicht unerheblicher Teil der älteren Bestände verjüngt. Die Rotbuche ist mit einem Anteil von über $70 \%$ und Laubholz insgesamt mit nahezu $80 \%$ am planmäßigen Waldumbau unter Fichtenaltholzschirm beteiligt. Der Anteil der Fichtennaturverjüngung unter Schirm beträgt ca. 16\%. Andere Nadelbaumarten wie die Douglasie sind aufgrund des notwendigen, kostspieligen Zaunschutzes bislang nur sehr gering vertreten. 


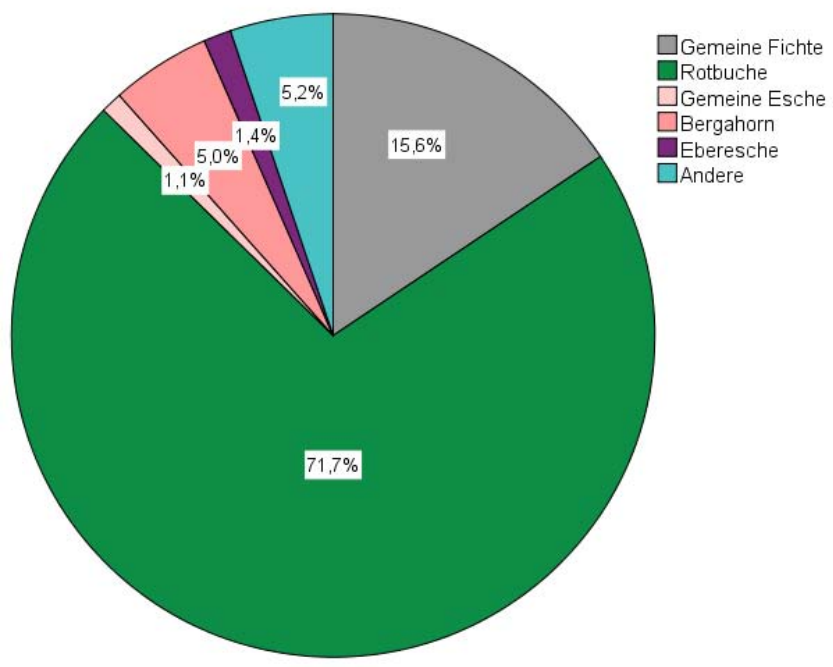

Abbildung 71: Flächenanteile [\%] der Baumarten im überschirmten Nachwuchs in der Region Oberharz. (im Datenspeicher Wald erfasste Flächen, Privatwald ₹. T. Stand 1994)

\subsubsection{Wichtigste Wachstumstrends durch den Klimawandel und Risikoveränderungen}

Die erwartete Trockenstressgefährdung der Fichte nimmt bis zum Ende des Jahrhunderts zu und erreicht auf nahezu der gesamten Waldfläche der Region ein „,mittleres Risiko“. Ähnliches zeigt die Prognose des Trockenstressrisikos für die Buche. Nur ca. $6 \%$ der Fläche weisen zum Ende des Jahrhunderts noch ein geringes Risiko auf. Für die weniger empfindlichen Baumarten Douglasie, Eiche und Kiefer verbleiben immerhin ca. $40 \%$ der Fläche mit einem geringen Trockenstressrisiko, während für $60 \%$ der Waldfläche ein mittleres Trockenstressrisiko ausgewiesen wird.

Bei nach wie vor ausreichender Wasserversorgung profitiert die Fichte im Wachstum am stärksten von den ansteigenden Temperaturen. Das absolut höchste Leistungspotenzial wird auf den meisten Standorten der Region die Douglasie aufweisen und dies bei nach derzeitigem Wissensstand überschaubaren Risiken durch Trockenstress und Windwurf.

Allein für die Fichte besteht bei Erreichen einer Zielstärke von $45 \mathrm{~cm}$ und entsprechender Baumhöhen auf einem größeren Teil der Flächen ein potenziell hohes Windwurfrisiko. Nach den Ergebnissen der Modellierung würden ca. $38 \%$ der Fläche eine hohe und ca. $7 \%$ der Fläche eine sehr hohe potenzielle Sturmwurfgefährdung aufweisen. Betroffen wären bei den unterstellten Sturmrichtungen Nordwest und Südwest vor allem Plateaulagen, weite ungeschützte Täler sowie breite Höhenrücken, die sich vor allem im Zentrum der Region befinden und von Südwest nach Nordost verlaufen (Oberharzer Hochtal von Elend - Benneckenstein 
bis Elbingerode bzw. bis zur Rappbode-Talsperre sowie Bereiche südwestlich und v. a. östlich um Stiege). Sehr geschützte Lagen mit ausgesprochen geringer Windwurfgefährdung würde es nur auf einem Flächenanteil von $6 \%$ geben. Aktuell besteht eine erhöhte Sturmwurfgefährdung für ca. 2.000 ha hiebsreife Fichtenaltbestände.

Zukünftig wird für ältere Fichtenbestände die Gefahr des Befalls durch Buchdrucker wegen der steigenden Temperaturen auch in den mittleren und höheren Lagen stark zunehmen. Dies gilt bis zum Zeitraum 2041 - 2070 vor allem für die schon früher verstärkt vom Borkenkäfer befallenen Bereiche im Nordosten der Region. Die vornehmlich gefährdeten Reviere Elbingerode und Rübeland zeichnen sich ansonsten durch ein gutes Leistungspotenzial der Fichte aus und sind in größeren Bereichen auch eher weniger stark durch Windwurf gefährdet. Bis zum Ende des Jahrhunderts zeichnet sich vor allem aufgrund der steigenden Temperaturen für alle Waldflächen des Oberharzes östlich einer Linie von Ilsenburg Königshütte - Trautenstein - Stiege bei einer Bestockung mit zielstarken, reinen Fichtenbeständen eine hohe oder sogar sehr hohe potenzielle Borkenkäfergefährdung ab. Insgesamt wäre davon mehr als die Hälfte der Waldfläche der Region Oberharz betroffen.

Die Waldbrandgefahr würde nach derzeitigen Prognosen ebenfalls deutlich zunehmen und gegen Ende des Jahrhunderts ein Niveau erreichen, das in etwa dem der heute am stärksten gefährdeten Tieflandregionen Sachsen-Anhalts entspricht.

Die Antworten auf die komplexen Herausforderungen liegen nach derzeitigem Stand der Forschung in Maßnahmen zur Förderung der Stabilität, der Senkung der Risiken, der Verkürzung der Gefährdungszeiträume und der Risikostreuung. Aufgrund der Langlebigkeit der Wälder steht planmäßig jährlich nur auf ca. 1,0 bis 1,5 \% der Waldfläche eine Entscheidung über die Baumartenwahl an. Der Waldumbau hat daher zunächst noch gegenüber der Stabilisierung und Risikominderung der vorhandenen Wälder eine geringere Priorität für die Forstbetriebe. In der strategischen Planung gewinnt er hingegen zunehmend an Bedeutung, weil er mit großen Investitionen und Auswirkungen auf die künftigen Handlungsspielräume der Forstbetriebe verbunden ist. Für seine operationelle Umsetzung bedarf es räumlich konkreter Entscheidungshilfen.

\subsubsection{Stabilisierung der vorbandenen Wälder in der Region Oberharz.}

\subsubsection{Fichtenbestände}

Fichtenbestände bilden derzeit den weit überwiegenden Teil der Waldfläche der Region. Nach den Klimaprojektionen wird die Fichte im Oberharz im Unterschied zu den Tieflandregionen auch in Zukunft noch leistungsfähig und standortsgemäß sein. Allein schon aufgrund der derzeitigen Altersstruktur wird sie hier mittel- bis langfristig die Hauptbaumart bleiben, da über die Hälfte der Fichtenbestände noch 
jünger als 60 Jahre sind, knapp $30 \%$ sogar jünger als 40 Jahre. Eine nachhaltige Fichtenwirtschaft muss allerdings auch angemessen die für den Harz quantifizierten potenziellen Risiken berücksichtigen. Diese sind nicht mit realen Risiken gleichzusetzen, da sie von bestimmten Annahmen ausgehen: Sturmstärken wie beim Orkan „Lothar" in Baden-Württemberg, Bestandeshöhen aller Fichtenbestände bei Zielstärke, Buchdrucker-Kalamitätsnutzungen ab einem Anteil von $1 \%$ an der Nutzungsmasse. Sehr wohl erlauben sie aber eine standörtliche Differenzierung bei der Wahl geeigneter waldbaulicher Maßnahmen, um den Gefährdungen zu begegnen.

Für die vorhandenen Fichtenbestände besteht das vordringliche waldbauliche Ziel darin, sie möglichst stabil erwachsen zu lassen und ihre Disposition gegenüber den ausgewiesenen Risiken möglichst gering zu halten. Zu den vorrangigen Anliegen der Bestandespflege zählen deshalb die Erhöhung der Einzelbaumstabilität, die Sicherung der Vitalität und der Erhalt bzw. die Förderung der Mischbaumarten. Besonders wirksam sind diejenigen Maßnahmen, die in Anlehnung an den Wachstumsgang der Fichte in den frühen Bestandesentwicklungsphasen zielgerichtet durchgeführt werden.

Im Rahmen der Jungwuchspflege stellen flächige, stammzahlreiche, wenig differenzierte Fichten-Naturverjüngungen unter lichtem Schirm und besonders auf kalamitätsbedingten Freiflächen eine besondere Herausforderung dar. Für sie sollte eine frühzeitige Stammzahlreduktion auf ca. 2.000 vitale Fichten/ha bei Oberhöhen von 1,5 bis $2,5 \mathrm{~m}$ das Regelverfahren sein, um die Einzelbaumstabilität durch bessere $\mathrm{h} / \mathrm{d}$-Werte und längere grüne Kronen zu erhöhen und gleichzeitig die Wasserkonkurrenz zu entspannen. Neben der Stabilitätserhöhung ermöglicht diese Maßnahme eine äußerst effektive Förderung vorhandener Mischbaumarten, die in das Kollektiv der verbleibenden Bäume integriert werden (RUMPF u. DiTGES 2008). Außerdem zeichnete sich diese Maßnahme in länger beobachteten Versuchen durch eine effektive Förderung des Durchmesserwachstums aus, was zu einer Verkürzung der Produktionszeit und geringeren Baumhöhen bei Zielstärke führt. Die Sturmwurfgefährdung der Altbestände wird durch die geringeren Endhöhen vermindert, ebenso wie der gesamte Gefährdungszeitraum mit der Verkürzung der Produktionszeiten abnimmt. Auf Standorten mit sehr hohem Sturmwurfrisiko ist die Stammzahl der vorhandenen Fichtennaturverjüngungen ggf. sogar auf ca. 1000 je ha abzusenken. Vor allem auf Standorten mit potenziell hohem Sturmwurfrisiko bzw. mittelfristig hohem Borkenkäferrisiko ist im Zuge der Stammzahlreduktionen der Erhaltung und Förderung der vorhandenen Mischbaumarten Vorrang einzuräumen.

In älteren Fichtennaturverjüngungen, bei denen der günstigste Zeitpunkt für einen Pflegeeingriff bereits verpasst wurde, sollte im Oberhöhenbereich zwischen 2,5 und $6 \mathrm{~m}$ wegen der besonders ungünstigen Arbeitsbedingungen (Kosten, Ergonomie) zunächst auf eine Läuterung verzichtet werden. Bei einer Oberhöhe von 6 bis $8 \mathrm{~m}$ ist dann zu prüfen, ob die Differenzierung ausreicht, um bis zur Erstdurchforstung vollständig auf einen Eingriff zu verzichten. Von unzureichender Differen- 
zierung ist am ehesten bei nicht überschirmten Fichtennaturverjüngungen auszugehen und wenn sich in unmittelbarer Nachbarschaft $(\mathrm{r}=\mathrm{ca} .2 \mathrm{~m})$ von 150 bis 250 auszuwählenden potenziellen Z-Baumanwärtern jeweils mindestens ein Konkurrent befindet, der ebenso hoch oder fast so hoch wie der Z-Baumanwärter ist. In diesen Fällen sollte zur Stabilisierung der Bestände eine Ausleseläuterung zur Förderung der Z-Baumanwärter erfolgen, die ggf. mit einem Schälschutz kombiniert wird.

In gepflanzten Fichtenjungbeständen beschränken sich die Läuterungseingriffe i. d. R. auf Mischwuchsregulierungen zur Förderung der Mischbaumarten. Sie ist besonders für beigemischte Edellaubbaumarten von großer Bedeutung, deren Zuwachsgrößen sehr früh kulminieren. In jedem Fall sind die Pionierbaumarten als Begleitbaumarten in angemessenem Umfang zu erhalten, weil sie aufgrund ihrer häufigen Fruktifikation, ihrer Fähigkeit, Freiflächen zu besiedeln, sowie ihren geringen Wasser- und Nährstoffansprüchen eine Art Rückversicherung im Falle von Kalamitäten darstellen (v. LÜPKE 2004).

Entscheidend für die Stabilität, Struktur und Wertleistung der Bestände ist die anschließende Durchforstungsphase. Die Feinerschließung durch Rückegassen im Abstand von $20 \mathrm{~m}$ (Gassenrand zu Gassenrand) sollte bereits 2 bis 3 Jahre vor der Erstdurchforstung erfolgen. In der Regel finden in Fichtenbeständen aus ökonomischen Gründen Hochdurchforstungen Anwendung, die möglichst früh einsetzen (ab Oberhöhen von 12-14 m). Bei dieser Durchforstungsart konzentriert sich die Pflege auf die Förderung von 150 bis 250 vitalen, qualitativ guten Zukunftsbäumen je ha durch Entnahme qualitativ schlechterer Bäume im Herrschenden. Die Anzahl der Z-Bäume darf bei dieser frühen Auswahl nicht zu niedrig bemessen sein, weil es im Laufe der Bestandesentwicklung nicht nur das soziale und qualitative Umsetzen gibt (SCHOBER 1988), sondern weil auch die Ausfallrisiken durch den Klimawandel steigen. Mischbaumarten sind zu erhalten und zu fördern. Auf Standorten mit hohem Trockenstressrisiko (trockene, mäßig sommertrockene oder wechselfeuchte Standorte) können aber auch starke Niederdurchforstungen mit geringeren Deckungsbeiträgen angebracht sein, die neben herrschenden Bäumen anfangs auch schwächere Bestandesglieder entnehmen, um die Wasserkonkurrenz kurzfristig zu senken (KOHLER et al. 2010, GEBHARDT et al. 2012). Langfristig haben sie keinen entscheidenden Effekt auf die Verdunstungsraten, weil die verbleibenden Bäume mit dem Ausbau ihrer Kronen reagieren. Bezüglich der Durchforstungsstärke sind grundsätzlich gestaffelte Durchforstungen zu empfehlen, bei denen die ersten drei Eingriffe die Bestandesdichte deutlich absenken $\left(\mathrm{B}^{\circ}>0,7\right.$ bis 0,8$)$. Anschließend folgt bis zum Abschluss des Haupthöhenwachstums eine Übergangsphase in der Durchforstungsstärke $\left(\mathrm{B}^{\circ}>0,8\right.$ bis 0,9$)$ und danach schließen sich nur noch mäßig starke Eingriffe $\left(\mathrm{B}^{\circ}=1,0\right)$ bis zum Einsetzen der Zielstärkennutzung an. Für die gestaffelte Durchforstung spricht, dass sie dem Wachstumsgang und der Reaktionsfähigkeit der Baumarten folgt, die Kronen der bestveranlagten Bäume frühzeitig ausbaut und ihren Zuwachs fördert und hierdurch die Produktions- und Gefährdungszeiträume deutlich gesenkt werden können (SPELLMANN 2001, 2004; SPELLMANN 
u. SCHMidT 2003; NAGEL u. SPELLMANN 2008). Außerdem wird so in Baumhölzern über 20 m Oberhöhe die Ausbreitung einer üppigen Bodenvegetation unterbunden, die sich im Falle einer starken Vergrasung sehr negativ auf den Bodenwasserhaushalt auswirken kann. Vorbeugend gegen Borkenkäferbefall ist bei allen Holzerntemaßnahmen das Derbholz bis zu einer Stärke von $10 \mathrm{~cm}$ aufzuarbeiten, um das Angebot an bruttauglichem Material in den Beständen gering zu halten.

In Altbeständen soll mit einsetzender Zielstärkennutzung und Verjüngung der Bestände eine nachträgliche schematische Verdichtung des Erschließungsnetzes auf Rückegassenabstände von 20 m vermieden werden. Besonders in Kombination mit einer gleichzeitigen Zielstärkennutzung führt dies zu einer Destabilisierung. Außerdem ist darauf zu achten, dass in den Beständen über längere Zeit ein Stützgerüst aus gut bekronten Fichten verbleibt. Jeder einzelne Eingriff sollte eine Entnahmemenge von $60 \mathrm{Efm} /$ ha bei einer Nutzungswiederkehr von drei bis fünf Jahren nicht überschreiten. Für instabile Bestände, sehr vorratsreiche Bestände mit hohem Zielstärkenanteil und fortschreitender Entwertung sowie die Restvorräte bei weit fortgeschrittener Zielstärkennutzung ist auch in Abhängigkeit des geplanten Folgebestandes eine saumartige oder flächige Nutzung ggf. risikoärmer.

In der Region Oberharz sind vor allem die Fichtenbestände der III. (41-60 Jahre) bzw. der beginnenden IV. Altersklasse mit einem überproportionalen Flächenanteil vertreten. Der Grundsatz der Nachhaltigkeit gebietet es, vorsichtig mit den hier konzentrierten Holzvorräten umzugehen und das Ertragsvermögen der Bestände optimal auszunutzen. Dies ist i. d. R. nur mit langen Nutzungs- und Verjüngungszeiträumen möglich, was für die einzelnen Bestände frühzeitige Weichenstellungen bezüglich ihrer Abnutzung und des angestrebten Verjüngungsziels bedeutet. Konkret heißt dies, in gut vorgepflegten stabilen Beständen ist auf risikoärmeren Standorten möglichst frühzeitig, ca. ab einem Bestandesalter von 70 Jahren, mit der Zielstärkennutzung zu beginnen. Der Einstieg in die Zielstärkennutzung sollte in strukturreicheren Bestandesteilen gewählt werden. Dabei ist ein Stützgefüge aus einer ausreichenden Anzahl stabiler, starker Bäume zu erhalten. Die Eingriffsstärke sollte $60 \mathrm{Efm} / \mathrm{ha}$ bei Nutzungsintervallen von ca. vier Jahren nicht überschreiten. Hierdurch soll möglichst lange eine geordnete Nutzung sichergestellt werden, bevor die Bestockungsgrade soweit abgesenkt sind $\left(\mathrm{B}^{\circ}<0,6\right)$, dass eine saumweise oder kleinflächige Abnutzung aus Stabilitätsgründen angezeigt ist. Eine Alternative stellen die räumlich strenger geordneten zonenweisen Zielstärkennutzungen gegen die Hauptwindrichtung in einer Tiefe von zwei Baumlängen dar, die eine saumfemelartige Verjüngung erlauben (RICHTER 1995). In labilen Fichtenaltbeständen oder bei großflächigen, starkholzreichen Fichtenaltholzkomplexen wird es sich schließlich nicht vermeiden lassen, die Vorräte im Zuge von Saumschlägen und begrenzten Kahlschlägen schneller zu nutzen und zu verjüngen.

Grundsätzlich ist eine stärkere Differenzierung der angestrebten Zieldurchmesser nach den Kriterien Wuchsleistung (Ertragsklasse), Bestandesrisiko (Trockenstress, Sturm, Borkenkäfer, Waldgefüge) und Qualität (Entwertungsgefahr durch 
Rotfäule) zu empfehlen (SPELLMANN 2013). Gerade bei einem unausgeglichenen Altersklassenaufbau wie im Oberharz ist ein solches Vorgehen angebracht, um die Endnutzungen bei möglichst geringem Gesamtrisiko zu strecken und so die Liquidität der Forstbetriebe zu erhalten (s. Abb. 72).

I. Ekl. und besser

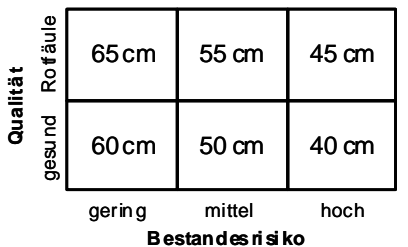

I.5 Ekl. - II.5 Ekl.

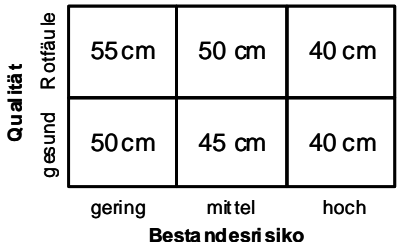

III. Ekl. und schlechter

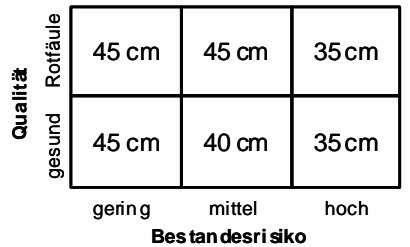

Abbildung 72: Variable Zielstärken für Fichte in der Region Oberharz in Abhängigkeit von Wuchsleistung (Ekl. = Ertragsklasse), Qualität und Bestandesrisiken (SPELLMANN 2013)

\subsubsection{Buchenbestände}

In Reinbeständen aber auch als Mischbaumart ist die Buche die mit Abstand wichtigste Laubbaumart der Region. Nach Einschätzung der Standortskartierung wäre sie unter den gegenwärtigen Bedingungen auf den meisten Standorten die Hauptbaumart der Stamm-Vegetationsform. Die Buche wird auf vielen Standorten des Harzes sogar mit einem besseren Wachstum auf die erwartete Temperaturerhöhung reagieren. Da dies aber auch für das Wachstum der anderen Baumarten zutrifft bzw. im Falle der Douglasie zumindest keine Verschlechterung zu erwarten ist, wird ihr absolutes Leistungsniveau weiterhin deutlich hinter dem standortsgemäßer Nadelbaumarten zurückbleiben. Bezüglich des Sturmwurfrisikos wird die Buche auf den allermeisten Standorten als stabil eingeschätzt, bezüglich des Trockenstressrisikos wird ihre heute ganz überwiegend geringe Gefährdung bis zum Ende des Jahrhunderts auf ein mittleres Niveau steigen. Hervorzuheben ist die positive Wirkung von Buchen-Mischungsanteilen auf eine Senkung der Borkenkäferbefallswahrscheinlichkeit von Fichtenbeständen, ebenso wie ihr günstiger Einfluss auf den Wasserhaushalt von Nadelholzbeständen, wenn sie im Hauptbestand mit wirksamen Mischungsanteilen vertreten ist.

Aktuell ist in der Region Oberharz die Qualität der Buchenbestände in allen Bestandesentwicklungsphasen häufig unbefriedigend. Wesentliche Ursachen hierfür sind die seltene Fruktifikation der Buche bis in die achtziger Jahre des letzten Jahrhunderts, Wildschäden sowie Schneedruck und Eisbruch. Durch relativ stammzahlarme Voranbauten, Ausfälle und Wildschäden weisen auch die jüngeren Bestände vielerorts schlechte Schaftformen und Schäden auf.

Bezüglich der Bestandespflege empfiehlt sich in der Dickungsphase ein Protzenaushieb, um die Bestandesqualität zu verbessern und eine Labilisierung der Bestände bei einer späteren Entnahme in der Durchforstungsphase zu vermeiden. Bei großen 
Qualitätsmängeln ist ein besonderes Augenmerk auf die Erhaltung der wenigen gut veranlagten Bäume zu richten, und es sind ggf. mehrere Eingriffe notwendig, um den Bestandesschluss nicht dauerhaft zu unterbrechen. Nach dem Erreichen einer astfreien Schaftlänge von 7-9 m beginnt die Durchforstungsphase mit der Auswahl von 100 bis 140 Z-Bäumen je ha, bei geringer Anzahl gut veranlagter Exemplare auch in Gruppen von zwei bis drei Bäumen. Anfangs sollen die Z-Bäume im Zuge von starken Hochdurchforstungen durch die Entnahme von zunächst einem bis drei, später einem bis zwei Bedrängern gefördert werden. Eingriffe in die Zwischenfelder dienen vorrangig der Entnahme starker, qualitativ schlechter Bäume. Der Unterstand ist zu erhalten. Die Z-Bäume sind vor jeder Durchforstung kritisch hinsichtlich ihrer weiteren Förderungswürdigkeit zu überprüfen. Ab einer Bestandesoberhöhe von ca. $26 \mathrm{~m}$ nimmt die Eingriffsstärke ab und geht zu einer mäßigen Hochdurchforstung über. In bisher nicht zielgerecht bzw. zu schwach gepflegten Beständen kann aufgrund des lange anhaltenden, guten Reaktionsvermögens der Buche auch noch in dieser Pflegephase ein Kollektiv von 80 bis 120 qualitativ gut veranlagten, vitalen Bäumen ausgewählt und lichtwuchsartig - das bedeutet kein Kronenkontakt mehr zu Nachbarbäumen - freigestellt werden.

Die Zielstärkennutzung beginnt, sobald ca. zehn Bäume je ha die individuelle Hiebsreife erreicht haben. Die Durchmesserdifferenzierung der Wertträger erlaubt i. d. R. eine zeitlich gestreckte und räumlich konzentrierte Zielstärkennutzung. Die jeweils anzustrebende Zielstärke ist stark von Qualität, Wüchsigkeit und Entwertungsgefahr abhängig. Sie beträgt bei guter Qualität und geringer Entwertungsgefahr $\geq 65 \mathrm{~cm}$, bei schlechter Qualität liegt sie darunter.

Grundsätzlich sollte der Nutzungs- und Verjüngungsfortschritt räumlich geordnet von innen nach außen bzw. von der Bestandesmitte zu den Rückegassen erfolgen. Als Hiebsform ist der Femelschlag dem Großschirmschlag vorzuziehen, um strukturreichere Bestände zu erziehen und lichtbedürftigere Mischbaumarten (Edellaubbaumarten bzw. Nadelbaumarten) vor dem Auflaufen der Buchen-Naturverjüngung zu verjüngen bzw. einzubringen. Die Femel sollten eine Anfangsgröße ab 0,3 ha aufweisen, weitestgehend schirmfrei sein und für die Etablierung der lichtbedürftigeren Baumarten kontinuierlich gerändelt werden. Für ein differenziertes Vorgehen sprechen auch die derzeit nach mehreren trockenen Sommern und häufigem Fruktifizieren zu beobachtenden schlechten Kronenzustände der Altbuchen in den gleichmäßig großschirmschlagartig aufgelichteten Beständen (MLU LSA 2012).

\subsubsection{Sonstige Bestandestypen}

Edellaubbaumarten sind im Oberharz auf den nährstoffreicheren Standorten mit nur geringen Flächenanteilen vertreten. Häufig kommen sie als Mischbaumart zur Buche vor. Die Esche ist derzeit fast ausnahmslos sehr stark durch das von dem pilzlichen Erreger Chalara fraxinea hervorgerufene Eschentriebsterben betroffen. Für die Förderung dieser Baumart im Zuge von Durchforstungsmaßnahmen ist deshalb vor 
allem die Vitalität ausschlaggebend. Nähere Empfehlungen zum waldbaulichen Vorgehen in befallenen Eschen-Rein- und Mischbeständen werden zurzeit erarbeitet. Der Bergahorn ist im Oberharz auf einem breiten Standortsspektrum standortheimisch. Er zeichnet sich durch eine reichliche Samenproduktion und eine gute Samenverbreitung aus. Mischungsanteile und auch Einzelexemplare sind eine wertvolle Beimischung in Fichten- und Buchenbeständen. Sie sind durch eine bevorzugte Pflege zu erhalten. Dabei sind auch deutliche Zugeständnisse in Bezug auf die Qualität der Bergahorne zu vertreten. Wirtschaftlich interessant ist der Bergahorn ab der Nährkraftstufe K-/M+. Ähnliches trifft für den selteneren Spitzahorn zu, für den aufgrund seiner ökologischen Eigenschaften eine besonders gute Anpassungsfähigkeit an Klimawandel prognostiziert wird (v. LÜPKE 2004).

Bestände mit Europäischer Lärche haben einen deutlichen Schwerpunkt im Altersbereich zwischen 40 und 60 Jahren. Bei dieser sehr lichtbedürftigen Baumart muss die Bestandespflege mit Ausleseläuterungen zugunsten der bestveranlagten 150 bis 200 Z-Baumanwärter bereits in der Stangenholzphase bei Oberhöhen von 6 bis $8 \mathrm{~m}$ einsetzen und mit starken Hochdurchforstungen ab Oberhöhen von $12 \mathrm{~m}$ fortgesetzt werden. Die Durchforstungsstärke geht dann ab Oberhöhen von $20 \mathrm{~m}$ zu mäßigen Hochdurchforstungen über. In der gesamten Pflegephase darf es nicht versäumt werden, bedrängende bzw. aus dem Unterstand nachdrängende Schattbaumarten zu entnehmen, da andernfalls die Lärche im Zuwachs stagniert. Gerade auf Sonnhängen und etwas trockeneren, nicht zu nährstoffarmen Standorten ist sie eine standortsgemäße Baumart, die durch ihre gute Samenproduktion und -verbreitung sowie ihre hervorragende Anpassung an Freiflächenverhältnisse ein waldbauliches Potenzial für die Besiedlung kalamitätsbedingter Blößen darstellt.

Weichlaubbäume, wie die heimischen Birkenarten, Eberesche und Aspe, kommen hauptsächlich als Pionier- und Begleitbaumarten in den verschiedenen Bestandestypen, hauptsächlich in Fichtenbeständen vor. Bestandesbildend sind sie als meist stabile Bestockung auf Sonderstandorten verbreitet. Auf die Notwendigkeit zur Erhaltung und Förderung angemessener Anteile dieser Baumarten zur Risikovorsorge mit Blick auf die steigenden Gefahren durch den Klimawandel wurde bereits hingewiesen. Die Erle als die Hauptbaumart bachbegleitender Waldgesellschaften ist ebenfalls in all ihren vorhandenen Vorkommen zu erhalten. Gerade an Gewässerrändern ist sie durch großzügige Freistellung zu fördern bzw. es muss ihrer Wiederausbreitung entsprechend Raum geben werden. Weitere Entscheidungshilfen zur Pflege und Entwicklung von Sandbirke und Roterle sind in Kapitel 7.7.3.2 für die Region Colbitz-Letzlinger Heide zusammengefasst. 


\subsubsection{Standortsgemäßer Waldumbau}

Die Baumartenwahl ist die wichtigste langfristige Entscheidung im Forstbetrieb. Mit ihr werden für die Dauer des Produktionszeitraumes Aufwand und Ertrag, die Lieferung von Infrastrukturleistungen, die Gestaltung des Landschaftsbildes und die Übernahme bestimmter Produktionsrisiken festgelegt (SPEIDEL 1991). Gerade unter dem Gesichtspunkt der Risikobegrenzung hat sich die Erkenntnis durchgesetzt, dass die Baumarten standortsgemäß sein müssen. Folgt man der Definition von v. LÜPKE (1995), dann ist dann eine Baumart ,standortsgemäß, wenn ihre Bedürfnisse an Strahlung, Wärme, Wasser und Nährstoffen durch Boden und Klima des Anbauortes gut erfüllt sind. Dies äußert sich in Gesundheit, Vitalität und gutem Wachstum."

Die Einschätzung, ob eine Baumart standortsgemäß ist, beruhte bisher i. d. R. auf der gutachtlichen Einschätzung der Wasser- und Nährstoffversorgung der Standorte sowie der jeweiligen klimatischen Gegebenheiten. In diesem Sinne existiert mit den „Waldbauempfehlungen des MLU LSA“ (MLU LSA 2003) eine für die heutigen Bedingungen geeignete, differenzierte Grundlage für die Bewirtschaftung des Landeswaldes und zur Beratung und Förderung des Privatwaldes, die geeignet ist, sowohl ökologischen Belangen als auch den Anforderungen an die Produktivität und Wirtschaftlichkeit der Wälder Rechnung zu tragen. In diesen Empfehlungen wird bei den möglichen Bestandeszieltypen zwischen ,naturnah“, „naturnah bis standortsgerecht“ und „standortsgerecht“ unterschieden, was einer Wertung und damit Prioritätensetzung gleichkommt. Besonders unter dem Einfluss der klimatischen Änderungen und der damit verbundenen Risiken stößt ein solches Vorgehen an seine Grenzen. Für die Zukunft kann jedenfalls nicht von einer generellen Bevorzugung der naturnahesten Bestockung ausgegangen werden, weil die Umweltveränderungen ohnehin Auswirkungen auf die Einschätzung der Naturnähe haben und die Risikoprofile bestimmter Standorte sich verschieben werden. Außerdem sind in jedem Fall die waldbaulichen Ausgangslagen zu beachten. Für die wichtigsten Klimastufen und Stamm-Standortsformengruppen der Region Oberharz wurden die bestehenden Bestandeszieltypenempfehlungen in Tabelle 15 zusammengestellt und ergänzt, wobei auf eine Rangfolge der Bestandeszieltypen i. S. der gegenwärtigen Naturnäheeinschätzung verzichtet wurde.

Die im Rahmen dieser Studie eingesetzten Modelle zur klimasensitiven Beschreibung der Leistungsänderungen der Hauptbaumarten, der Abschätzung ihrer zu erwartenden Trockenstressgefährdung sowie der potenziellen Sturmschadensgefährdung und des Borkenkäferbefallsrisikos für Fichtenbestände liefern wichtige Entscheidungshilfen zur standortsgemäßen Baumartenwahl unter sich ändernden ökologischen Rahmenbedingungen. Abgesehen von Standorten mit einer ausgewiesen hohen Risikogefährdung, Zwangsstandorten und bereits derzeitigen oder sich durch den Klimawandel abzeichnenden Grenzstandorten gibt es nicht nur eine richtige Lösung, sondern i. d. R. mehrere Anbaualternativen. 
Diese schließen auch die wenigen anbauwürdigen, ökologisch zuträglichen eingeführten Baumarten ein, in erster Linie die im Harz seit mehreren Generationen bewährte Douglasie und die in den Bestandeszieltypen ebenfalls bereits berücksichtigte Europäische Lärche. Daneben kämen zur Erweiterung der Palette weitere Baumarten für einen bemessenen Anbau in Mischbeständen auf einem geeigneten Standortsspektrum in Betracht. Von den Nadelbaumarten wären hier die Küstentanne, die Weißtanne und die Japanlärche zu nennen (ОтTO 1993, SPELLMANN 1994), die innerhalb des Bestandeszieltyps „Buche-Nadelbäume (BU-NDB)“ in größeren Mischungsanteilen, ggf. sogar als führende Baumart eine Rolle spielen könnten:

- die Küstentanne bei mittlerer und ziemlich armer Nährkraftstufe auf trockeneren Standorten, aber auch noch bei Staufeuchte

- die Japanlärche bei gleicher Nährstoffversorgung auf feuchten bzw. bei mäßig staunassen Standorten (keine Gefährdung durch Lärchenkrebs)

- die Weißtanne auf mittel bis besser wasserversorgten bzw. staufeuchten Standorten und mindestens mittlerer Nährkraft.

Eine Bereicherung wäre die Roteiche in Mischung mit Buche, die unter dem Gesichtspunkt des Klimawandels in den unteren und mittleren feuchten Lagen des Oberharzes die Baumartenpalette erweitern könnte, ebenso wie zukünftig in Höhenlagen bis $500 \mathrm{~m}$ auch die heimischen Eichen als führende Baumart in Betracht kämen (s. hervorgehobene Ergänzungen in s. Tab. 15).

Von den Weichlaubbaumarten sind vor allem Birke, Eberesche und Aspe als Vorwaldbaumarten und als Begleitbaumarten mit einem in jedem Fall umzusetzenden Mischungsanteil von ca. $10 \%$ in fast allen Bestandeszieltypen zu berücksichtigen.

Für die konkrete Baumartenplanung liefert die flächenbezogene, standortspezifische Risikoeinschätzung insbesondere bezüglich des Sturmwurfrisikos und der Gefährdung durch Fïchtenborkenkäfer eine Entscheidungshilfe für das Ranking der Baumarten. Die Risikokarten der vorliegenden Arbeit bieten hierfür eine erste Grundlage. Danach wäre für die aktuell von hoher bzw. sehr hoher Windwurfgefährdung betroffenen ca. 2.000 ha Fichtenaltbestände ein Umbau der Reinbestände in strukturreichere Mischbestände unter Einbeziehung schon vorhandener bzw. noch ankommender Fichtennaturverjüngung angezeigt. Unter Beachtung der Standortskartierung kämen als Mischbaumarten vor allem Buche, Douglasie, Lärche oder Bergahorn in Betracht. Unterhalb von $500 \mathrm{~m}$ käme auch ein Umbau in EichenBuchen- oder Roteichen-Buchen-Mischbestände in Frage. Bei potenziell hoher Gefährdung durch Borkenkäfer (Bezugszeitraum 2041 - 2070) müssten zumindest wirksame Anteile risikomindernder Mischbaumarten, vorzugsweise Buche, im Folgebestand etabliert werden. Die höchste Priorität für einen Baumartenwechsel 
besitzen Fichtenbestände, auf denen sich hohe Risiken für Sturmwurf und Borkenkäferbefall (Bezugszeitraum 2041 - 2070) überlagern (einige Teilbereiche v. a nordöstlich von Elbingerode sowie Bereiche um die Rappbode-Talsperre).

Hohe Artendiversität bedeutet oft höhere Stabilität, fast immer aber höhere Elastizität zum Ausgleich von Störungen (OTTO 1994, KNOKE et al. 2005). Dementsprechend sind Mischbestände gegenüber biotischen und abiotischen Störungen weniger anfällig als Reinbestände. Die für alle verbreiteten Kombinationen aus Klimastufe und Stamm-Standortsformengruppe berücksichtigten MischbestandsZieltypen würden neben höherer Stabilität und Diversifizierung vielfach auch zu mehr Naturnähe führen, da die Buche die wichtigste dabei verwendete Mischbaumart ist. Mit der Buche, aber auch unter den anderen Baumarten gibt es fast keine spannungsfreien Mischungen. Daher sind bei der Begründung von Mischbeständen die Standortsansprüche und das Konkurrenzverhalten der Baumarten streng zu beachten, um den Erfolg der Mischungen zu sichern, den Pflegeaufwand zu begrenzen und natürliche Entwicklungen nutzen zu können. Wichtige Entscheidungshilfen bieten die standortsabhängige Leistungsfähigkeit der Baumarten, der Kulminationszeitpunkt ihrer Zuwachsgrößen, ihr Schattenerträgnis, die Fähigkeit ihrer Kronen, Freiräume zu nutzen bzw. Seitendruck zu ertragen und ihr Standraumbedarf beim Erreichen bestimmter Zieldurchmesser. Unter Berücksichtigung dieser Gesichtspunkte ist es in gleichaltrigen Mischungen meist empfehlenswert, die Baumarten gruppen- bis horstweise oder kleinflächig zu mischen. Die betriebswirtschaftliche Bedeutung unterschiedlicher Mischungsanteile der jeweils leistungsfähigeren Baumart darf dabei nicht unterschätzt werden. Dieser Aspekt hat erhebliche Auswirkungen auf die Begründungskosten, die Pflegeintensität und die Werterträge (SPELLMANN 2010).

Trotz der Vorteile natürlicher Waldverjüngungen darf die biologische Rationalisierung jedoch nicht dazu führen, dass bereits heute bzw. zukünftig nicht standortsgemäße Fichten-Naturverjüngung einfach übernommen wird. Solche Verjüngungen sollten mit Douglasie oder Küstentanne überpflanzt werden, um die Risiken zu senken. Vorzugsweise sollte der Waldumbau durch Voranbauten erfolgen, bei denen die Lichtansprüche der Baumarten beachtet werden müssen. Störungslöcher bieten die Möglichkeit, standortsgemäße Baumarten frühzeitig einzubringen. Sie sollten größer als 0,3 ha sein, um den Voranbauten gute Entwicklungsmöglichkeiten zu bieten. Bei der künstlichen Bestandesbegründung ist auf die Verwendung geeigneter Herkünfte zu achten, da die meisten Baumarten große Verbreitungsgebiete haben, innerhalb derer sich die Teilpopulationen an die unterschiedlichen Standortsbedingungen genetisch angepasst haben (BOLTE u. DEGEN 2010). 
Tabelle 15: Bestandesqieltypen für die wichtigsten Klimastufen und Standortsformengruppen der Region Oberharz; Ergänzungen blau bervorgehoben (nach MLU LSA 2003, verändert)

\begin{tabular}{|c|c|c|c|c|}
\hline $\begin{array}{l}\text { Klima- } \\
\text { stufe }\end{array}$ & $\begin{array}{l}\text { Stamm- } \\
\text { StaO- } \\
\text { formen- } \\
\text { Gruppe }\end{array}$ & $\begin{array}{c}\begin{array}{c}\text { Flächen- } \\
\text { anteil }\end{array} \\
{[\%]}\end{array}$ & Stamm-Vegetationsform & Bestandeszieltypen \\
\hline Uf & K2 & 7 & $\begin{array}{l}\text { Perlgras-Goldnessel-TEi- } \\
\text { Bu-Wald }\end{array}$ & $\begin{array}{l}\text { BU-EI, EI-BU, REI-BU, BU- } \\
\text { BAH, BU-NDB, FI-BU, ELÄ- } \\
\text { SCH, DGL-BU }\end{array}$ \\
\hline \multirow[t]{5}{*}{ Mf } & $\mathrm{K} 1$ & 1 & Farn-Goldnessel-Bu-Wald & BU, BU-ELB, BU-BAH, BU-NDB \\
\hline & K2 & 16 & $\begin{array}{l}\text { Zahnwurz-Goldnessel- } \\
\text { (Fi)-Bu-Wald }\end{array}$ & $\begin{array}{l}\text { BU, BU-NDB, BU-BAH, EI-BU, } \\
\text { REI-BU }\end{array}$ \\
\hline & K3 & 2 & $\begin{array}{l}\text { Hainrispen-Goldnessel- } \\
\text { (Fi)-Bu-Wald }\end{array}$ & $\begin{array}{l}\text { BU, BU-EI, EI-BU, REI-BU, } \\
\text { BU-NDB }\end{array}$ \\
\hline & NM2 & 3 & $\begin{array}{l}\text { Rasenschmielen-Waldhain- } \\
\text { simsen-Fi-(RER)-Bu-Wald }\end{array}$ & $\begin{array}{l}\text { BU-NDB, BU-BAH, EI-ELB, FI- } \\
\text { BU, FI-WLB }\end{array}$ \\
\hline & M2 & 6 & $\begin{array}{l}\text { Waldreitgras-Hainsimsen- } \\
\text { (Fi)-Bu-Wald mit } \\
\text { Drahtschmiele }\end{array}$ & BU, BU-NDB, FI-BU, BU-BAH \\
\hline \multirow[t]{4}{*}{ Mff } & $\mathrm{K} 2$ & 10 & $\begin{array}{l}\text { Waldmeister-Wald- } \\
\text { schwingel-Fi-Bu-Wald }\end{array}$ & $\begin{array}{l}\text { BU-NDB, BU-BAH, BU, FI-BU, } \\
\text { FI-DGL }\end{array}$ \\
\hline & M2 & 28 & $\begin{array}{l}\text { Harzlabkraut-Hain- } \\
\text { simsen.Fi-Bu-Wald }\end{array}$ & $\begin{array}{l}\text { BU-NDB, BU, FI-BU, FI-DGL, } \\
\text { DGL-BU }\end{array}$ \\
\hline & M3 & 3 & $\begin{array}{l}\text { Drahtschmielen-Hain- } \\
\text { simsen-Fi-Bu-Wald mit } \\
\text { Heidelbeere }\end{array}$ & BU-NDB, BU, FI-BU, ELÄ-SCH \\
\hline & A3 & 2 & $\begin{array}{l}\text { Weißmoos-Heidelbeer- } \\
\text { (Bu)-Fi-Wald }\end{array}$ & FI-BU, FI, FI-WLB, KI, BI \\
\hline Hff & M2 & 3 & $\begin{array}{l}\text { Harzlabkraut-Wollreitgras- } \\
\text { (Bu)-Fi-Wald }\end{array}$ & FI-BU, FI \\
\hline Sonstige* & & 19 & & \\
\hline
\end{tabular}

* Standorte mit einem Flächenanteil $<1 \%$ 


\section{Gefährdungspotenziale in der Region Fläming}

\subsection{Veränderungen des Trockenstressrisikos}

Die Modellregion Fläming umfasst insgesamt 31.395 ha Waldfläche. Aktuell stocken auf mehr als der Hälfte der Flächen Kiefern gefolgt von Eiche und Buche. Die Fichte wurde im Fläming nur vereinzelt angebaut.

Bereits unter heutigen klimatischen Bedingungen weisen viele Standorte in der Modellregion ein Defizit in der Wasserversorgung auf. Dieses ist jedoch für die Kiefer als wichtigster Baumart der Region noch unbedenklich. Auf nur knapp $2 \%$ der Waldfläche ist unter den heutigen Klimabedingungen mit einem mittleren Trockenstressrisiko für die Kiefer zu rechnen (s. Tab. 16). Dies betrifft einige Bestände im Südosten der Modellregion, die auf Standorten mit geringer Bodenwasserspeicherkapazität stocken (s. Abb. 73). Der Großteil der Waldfläche im Fläming ist für die Kiefer (und dementsprechend auch für Eiche und Douglasie) mit einem geringem Risiko behaftet (s. Abb. 76).

Tabelle 16: Fläche [ba] der Hauptbaumarten nach den definierten Trockenstressrisikoklassen in der Region Fläming

\begin{tabular}{l|rr|rr|rr}
\multicolumn{1}{r|}{ Periode } & \multicolumn{2}{|c|}{ Fichte } & \multicolumn{2}{|c|}{ Buche } & \multicolumn{2}{c}{ Kiefer / Eiche / Douglasie } \\
Risiko & $1971-2000$ & $2071-2100$ & $1971-2000$ & $2071-2100$ & heute & $2071-2100$ \\
\hline gering & 18.689 & 106 & 26.412 & 106 & 30.788 & 121 \\
mittel & 12.706 & 146 & 4.983 & 461 & 607 & 28.561 \\
hoch & 0 & 31.143 & 0 & 30.828 & 0 & 2.713 \\
\hline
\end{tabular}

Für die Buche ist die Wasserversorgung bereits heute auf rund $15 \%$ der Waldfläche unzureichend. Dementsprechend sind 4.983 ha mit einem mittleren Trockenstressrisiko belegt (s. Tab. 16). Hiervon sind überwiegend Bestände im östlichen Bereich der Modellregion mit den geringsten Niederschlagssummen in der Vegetationsperiode betroffen, sowie generell Flächen mit geringer Bodenwasserspeicherkapazität (s. Abb. 75). Ein hohes Trockenstressrisiko wird unter heutigen Klimabedingungen weder für die Buche noch für die Fichte ausgewiesen. Allerdings ist die Fichte auf rund $40 \%$ der Waldfläche (12.706 ha) mit einem mittleren Risiko behaftet (s. Abb. 74). Hiervon sind insbesondere die östlichen Bereiche des Modellgebietes Fläming fast flächendeckend betroffen (s. Abb. 74). Im Vergleich zur Buche wird die mittlere Risikoeinstufung für die Fichte auch im übrigen Modellgebiet auf vielen Flächen ausgewiesen. 
Kiefer
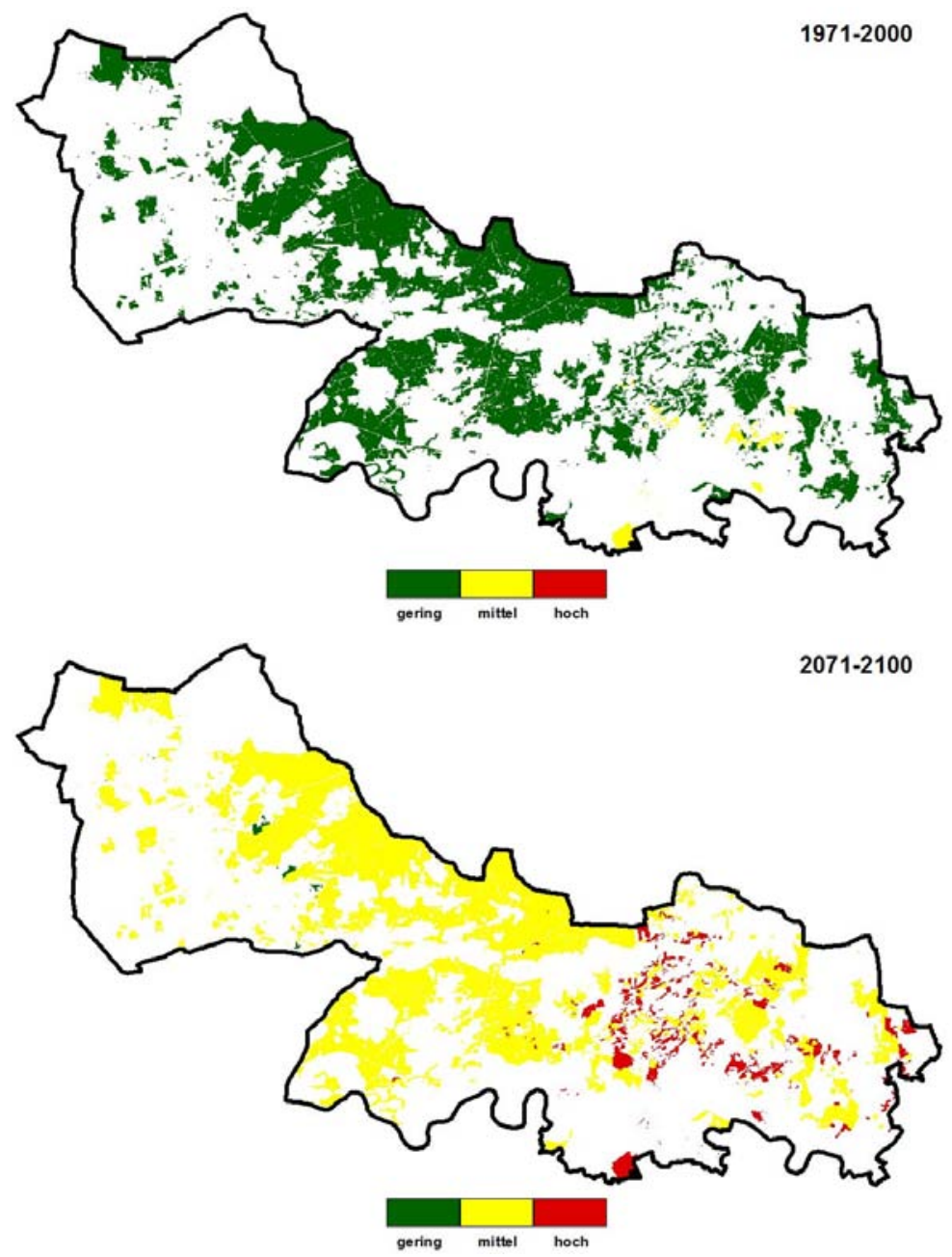

Abbildung 73: Trockenstressrisiko für die Kiefer in der Region Fläming

Bis zum Ende des Jahrhunderts wird das Gefährdungspotenzial durch Trockenheit in der Modellregion Fläming für die Buche und Fichte auf fast allen Waldflächen (ca. $99 \%$ ) als hoch eingeschätzt (s. Tab. 16). Nur wenige Hektar Waldfläche, die über eine sehr hohe Wasserspeicherkapazität verfügen, vermindern das Wasserdefizit in der KWB soweit, dass für Buche und Fichte ein geringes bis mittleres Trockenstressrisiko besteht (s. Abb. 74 und Abb. 75). Damit dürfte die Buche, die bereits heute auf weniger als $10 \%$ der Waldfläche stockt, in Zukunft in der Modellregion Fläming weiter zurückgedrängt werden. Dementsprechend wird der Anteil trockenresistenterer Baumarten wie Kiefer, Eiche und Douglasie in dieser Region 
weiter an Bedeutung gewinnen. Allerdings wird auch für diese Baumarten das Trockenstressrisiko zunehmen. So wird auf über $90 \%$ der Waldfläche (28.561 ha) ein mittleres und auf rund $8 \%$ (2.713 ha) sogar ein hohes Trockenstressrisiko erwartet (s. Tab. 16). Die Standorte mit einem hohen Risiko für Kiefer, Eiche und Douglasie befinden sich dabei ausschließlich im östlichen Modellgebiet (s. Abb. 73 und Abb. 76).

Fichte
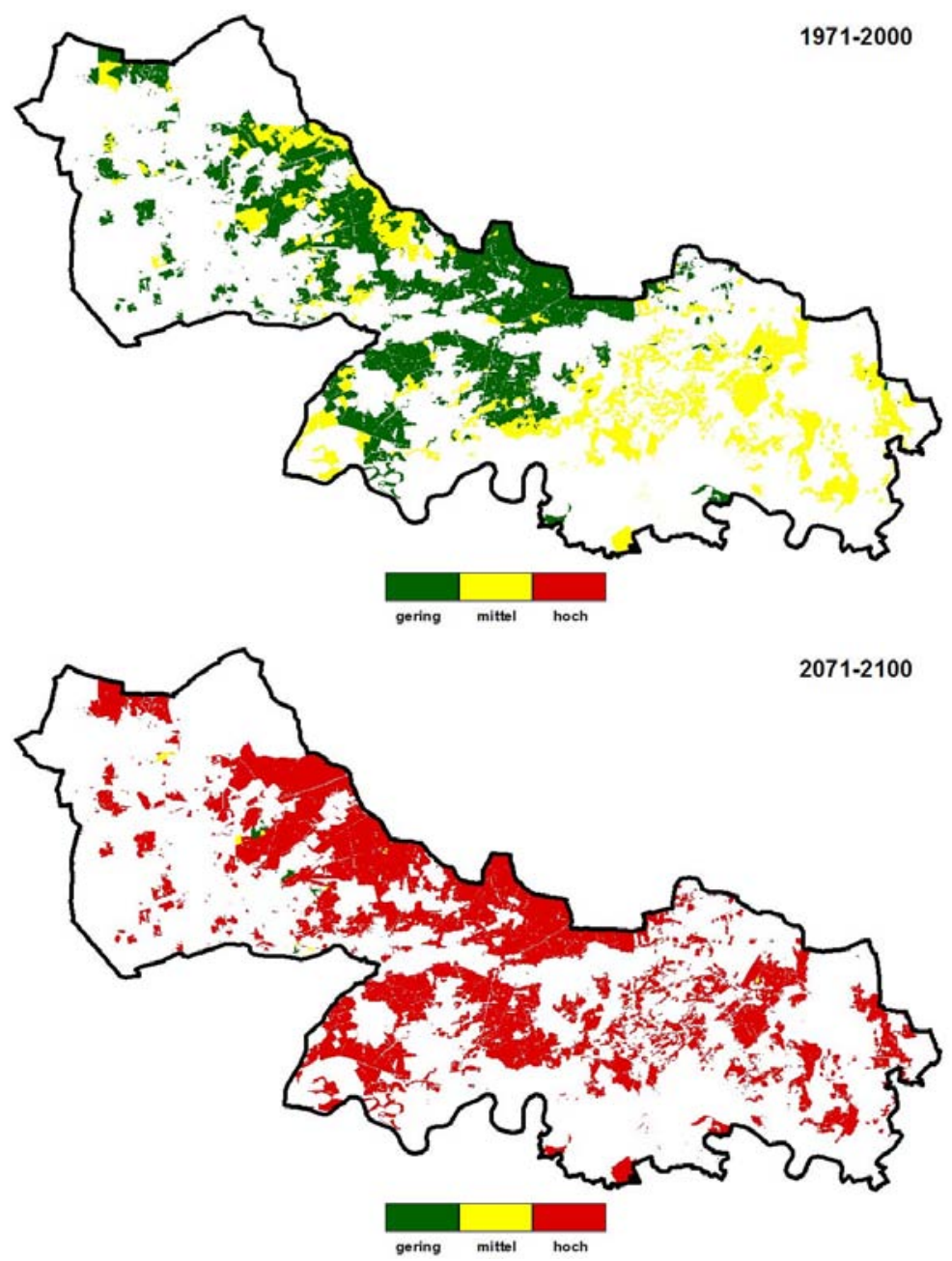

Abbildung 74: Trockenstressrisiko für die Fichte in der Region Fläming 
Buche
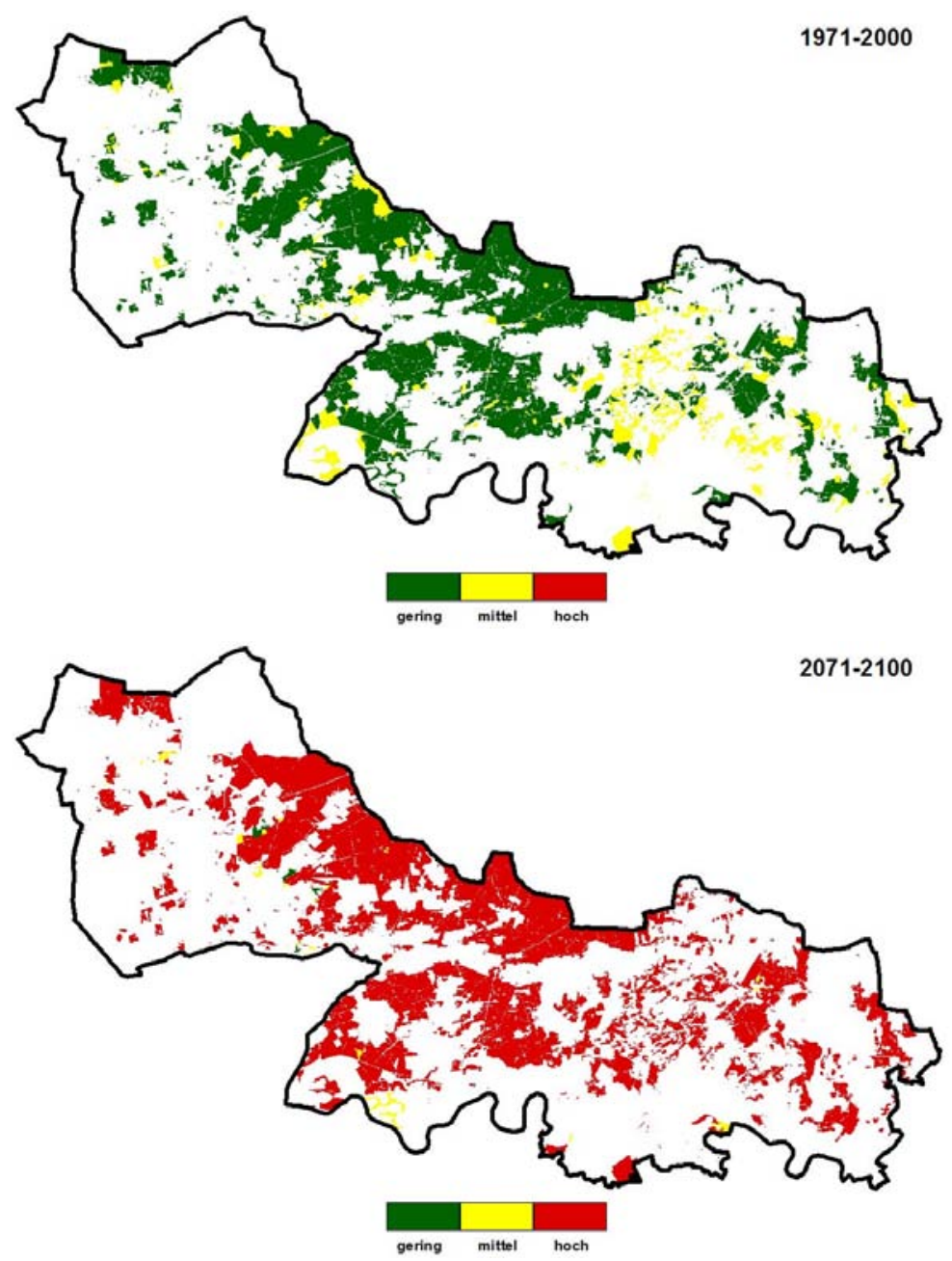

Abbildung 75: Trockenstressrisiko für die Buche in der Region Fläming 
Eiche und Douglasie
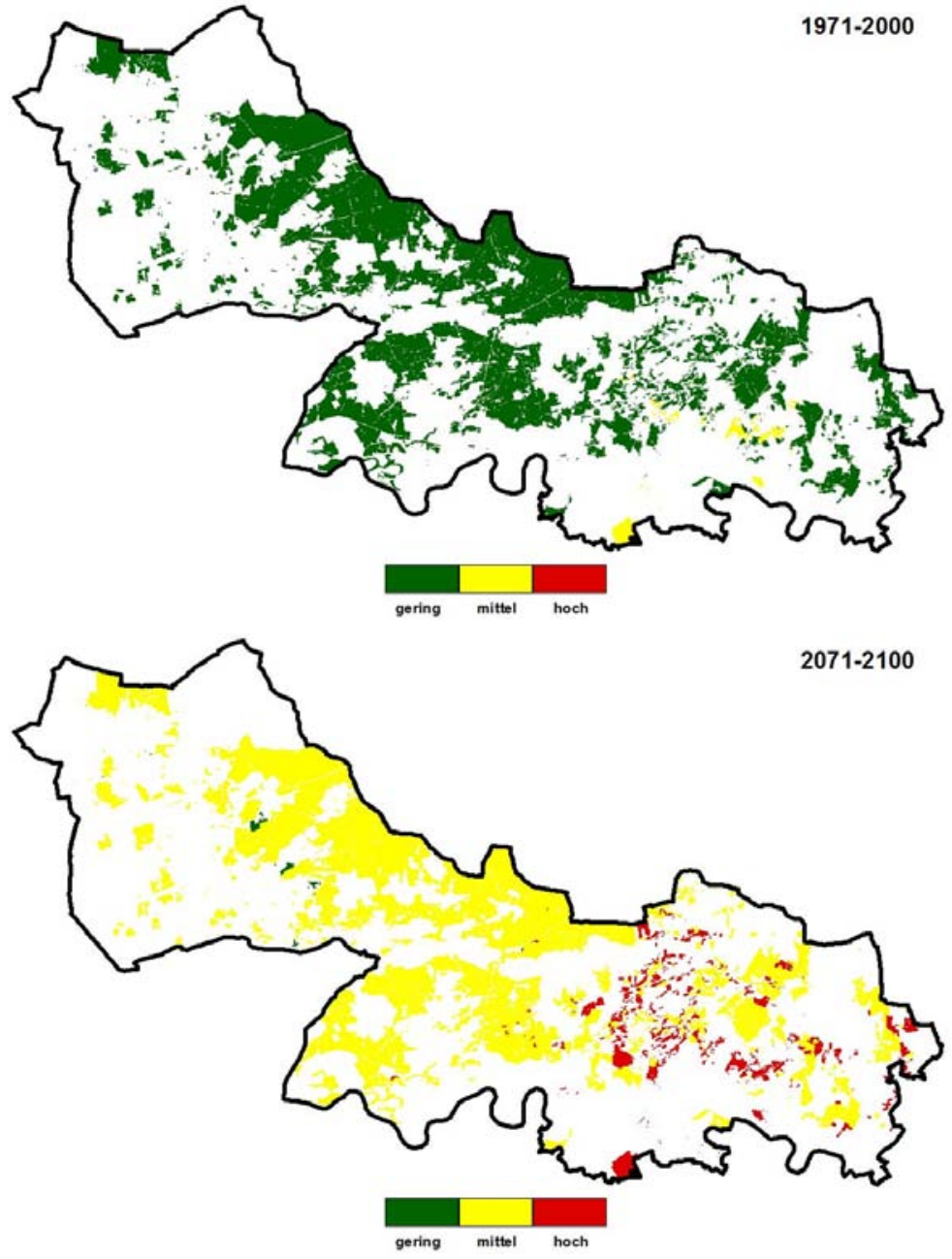

Abbildung 76: Trockenstressrisiko für die Eiche und Douglasie in der Region Fläming

In Anhang 5 bis Anhang 8 ist die zeitliche Entwicklung des Trockenstressrisikos für die untersuchten Perioden dargestellt. Die Auswertung zeigt, dass im Gegensatz zur Modellregion Oberharz das Gefährdungspotenzial durch Trockenstress bei der Fichte und Buche bereits in der Periode 2011 - 2040 signifikant zunimmt, bei der Kiefer, Eiche und Douglasie in der Periode 2041 - 2070. 


\subsection{Veränderungen der Stickstoffeinträge}

Hinsichtlich der Stickstoffeinträge (s. Abb. 77) nimmt die Region Fläming eine Mittelstellung zwischen dem stärker belasteten Harz und der Colbitz-Letzlinger Heide mit geringen Einträgen ein. Innerhalb der Region Fläming sticht insbesondere der Bereich nordwestlich von Wittenberg hervor. Hier zeigt sich die regionale Belastung durch Emissionen der SKW Stickstoffwerke Piesteritz GmbH.
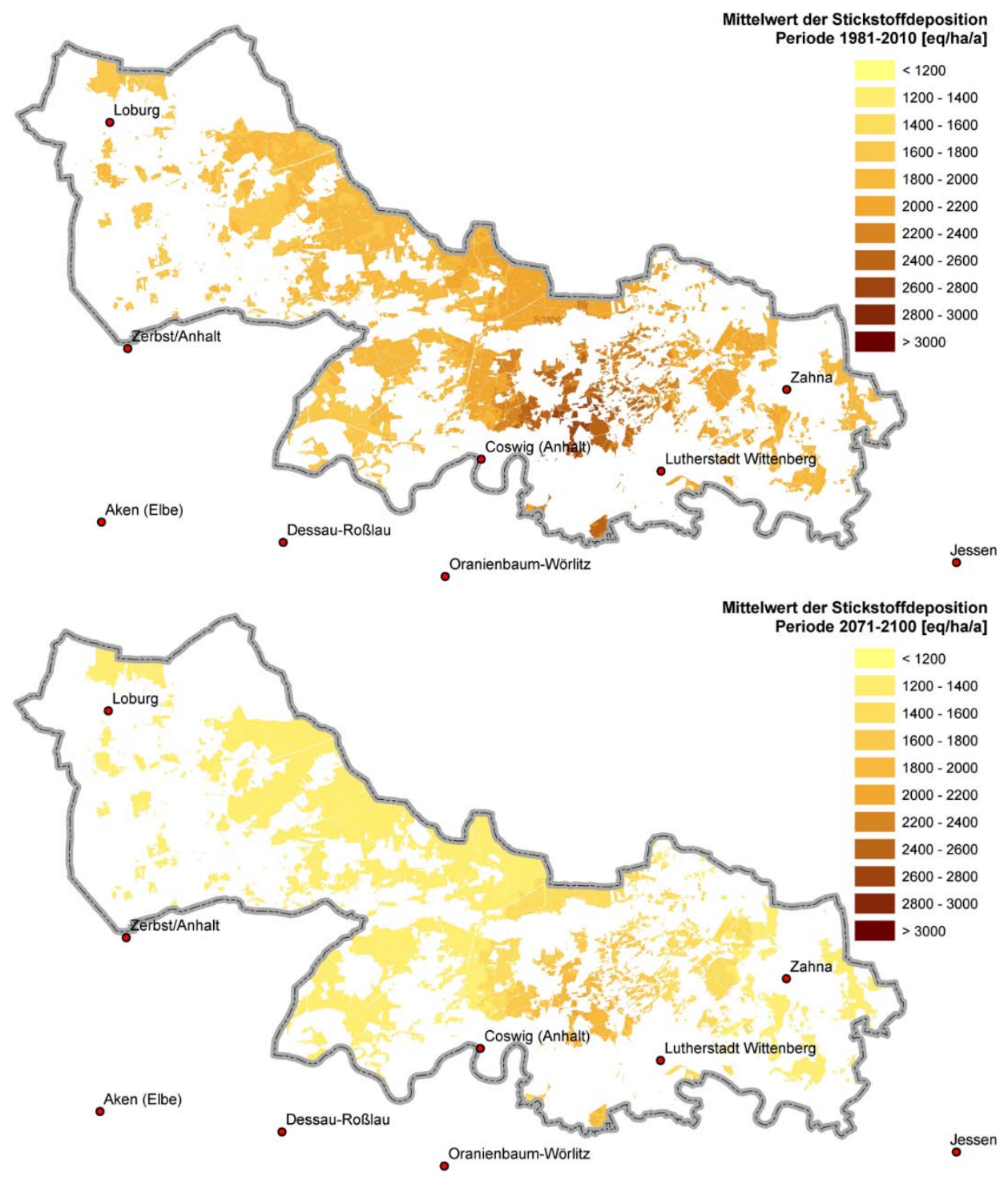

Abbildung 77: Regionale Verteilung der Stickstoffdepositionen (1981 - 2010 oben) und (2071 - 2100 unten) [eq/ba/a] in der Region Fläming 


\subsection{Projektion der Standortleistungsfähigkeit für die Region Fläming}

Die aktuelle Bestockung in der Region Fläming ist geprägt durch die Kiefer mit einem Flächenanteil von $78 \%$, gefolgt von Buche und Eiche mit $10 \%$. Die Douglasie könnte sich aufgrund der klimatischen Bedingungen als eine alternative Baumart mit hohem Ertragspotenzial erweisen. Einen direkten räumlich expliziten Vergleich der Baumarten Kiefer und Douglasie ermöglichen die Abbildung 78 und Abbildung 79. Hierbei wurden für alle Teilflächen jeweils eine hypothetische Bestockung mit Kiefer bzw. Douglasie angenommen und die Standortleistungsfähigkeit unter heutigen und zukünftigen Klimabedingungen geschätzt.

Die Standortleistungsfähigkeit für alle potenziell mit Kiefer bestockten Teilflächen in der Region Fläming zeigt für den Status quo ein räumlich ziemlich homogenes Bild, der maximale durchschnittliche Gesamtzuwachs schwankt je nach Standort zwischen 6 und $9 \mathrm{~m}^{3}$ pro Jahr und Hektar mit einem Anteil von $90 \%$ in der Klasse dGZ $\mathrm{dmax}_{\max } 7$ bis $8 \mathrm{~m}^{3} / \mathrm{a} / \mathrm{ha}$ (s. Abb. 78). Die Projektion für die Periode 2011 - 2040 zeigt eine Steigerung der Standortleistungsfähigkeit auf fast allen Flächen. Der positive Effekt auf die Wuchsleistung der Baumart Kiefer durch die für diese Region projizierte Verlängerung der Vegetationszeit und der Temperaturerhöhung wird scheinbar nicht durch den negativen Effekt durch zunehmende Trockenheit überkompensiert. Erst in der nächsten Projektionsperiode 2041 - 2070 führt das weiter zunehmende Wasserdefizit zu einem Absinken der Standortleistungsfähigkeit für die Kiefer auf ganzer Fläche. Ein weiterer Rückgang des Wuchspotenzials wird auch für die letzte Periode bis 2100 für viele Standorte projiziert. Das räumliche Muster ist zum Ende des Projektionszeitraumes wie bereits beim Status quo insgesamt sehr homogen.

Die Standortleistungsfähigkeit für die Baumart Douglasie in der Region Fläming stellt sich räumlich im Vergleich zur Kiefer etwas differenzierter dar (s. Abb. 79). Der $\mathrm{dGZ}_{\max }$ liegt bei der Douglasie zwischen 13 bis $17 \mathrm{~m}^{3} / \mathrm{a} / \mathrm{ha}$ und auch die Flächenanteile in den Klassen streuen stärker als bei der Kiefer. Für die erste Projektionsperiode 2011 - 2040 werden kaum Veränderungen der Standortleistungsfähigkeit geschätzt. Für die Periode 2041 - 2070 wird jedoch ein drastischer Rückgang der Leistungsfähigkeit für die Baumart Douglasie auf fast allen Flächen projiziert. Wie auch für die Kiefer setzt sich dieser negative Trend auf fast allen Standorten bis 2100 fort. Das räumliche Muster der Standortleistungsfähigkeit der Douglasie ist auch zum Ende der Projektionsperiode recht differenziert mit einem maximalen Anteil von knapp $60 \%$ in der Klasse dGZ $Z_{\max } 11$ bis $12 \mathrm{~m}^{3} / \mathrm{a} / \mathrm{ha}$. 

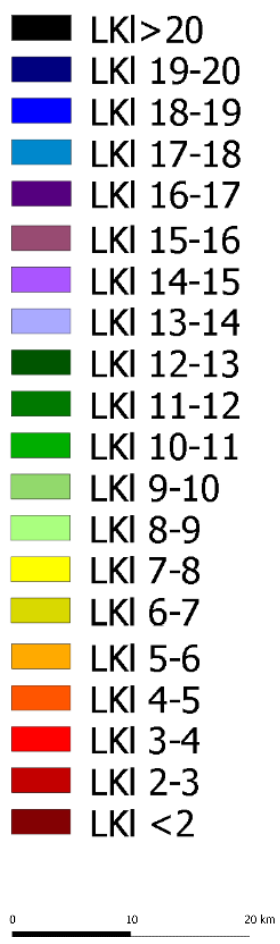

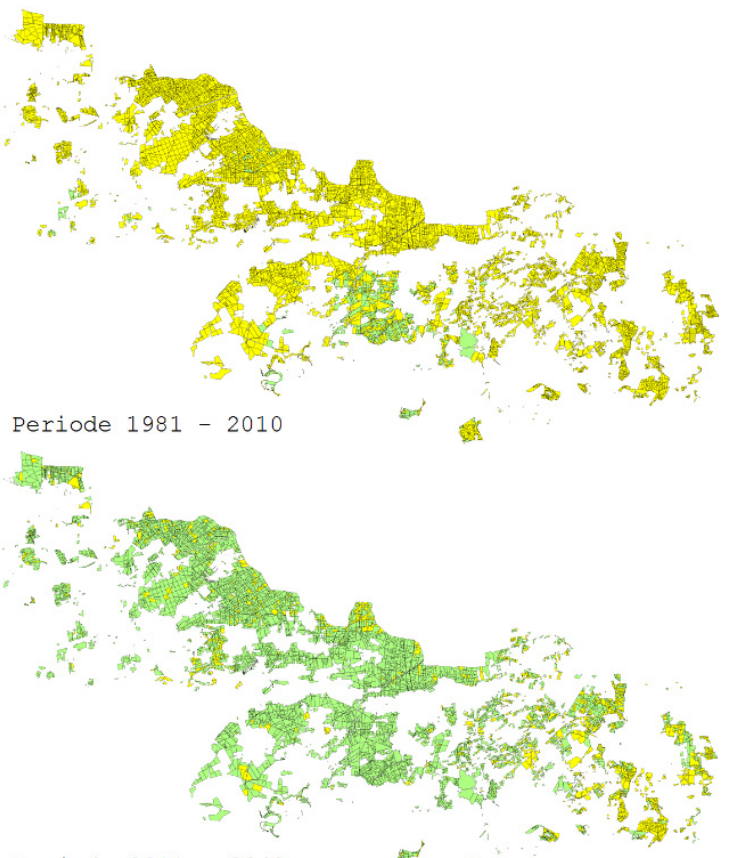

Periode 2011 - 2040 재

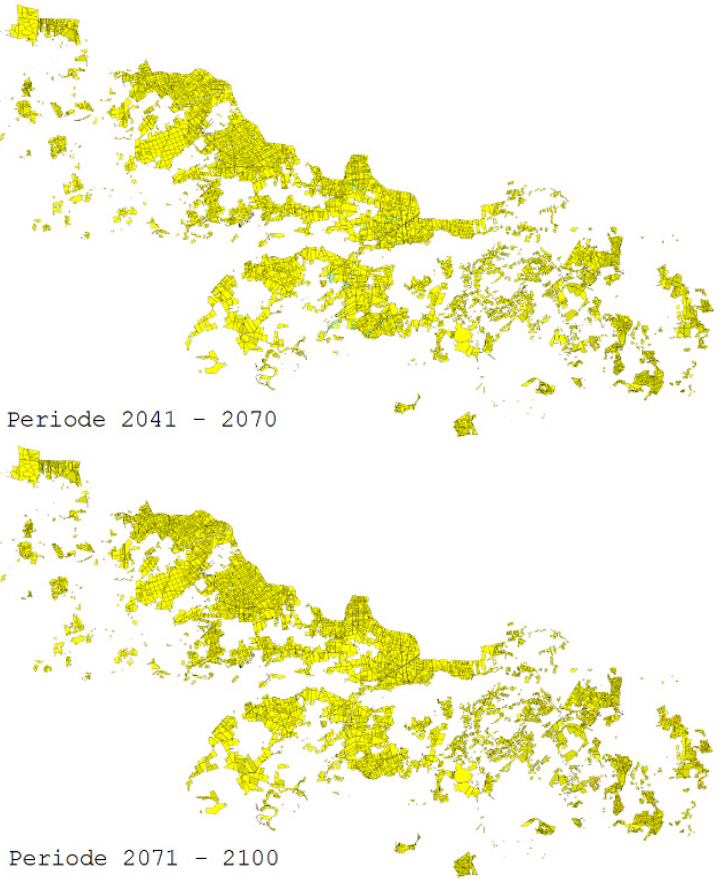

Abbildung 78: Projektion der Standortleistungsfähigkeit für potenziell mit Kiefer bestockte Teilflächen in der Region Fläming 

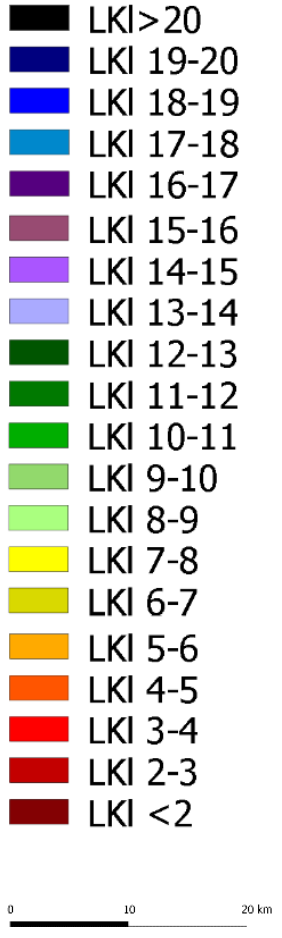
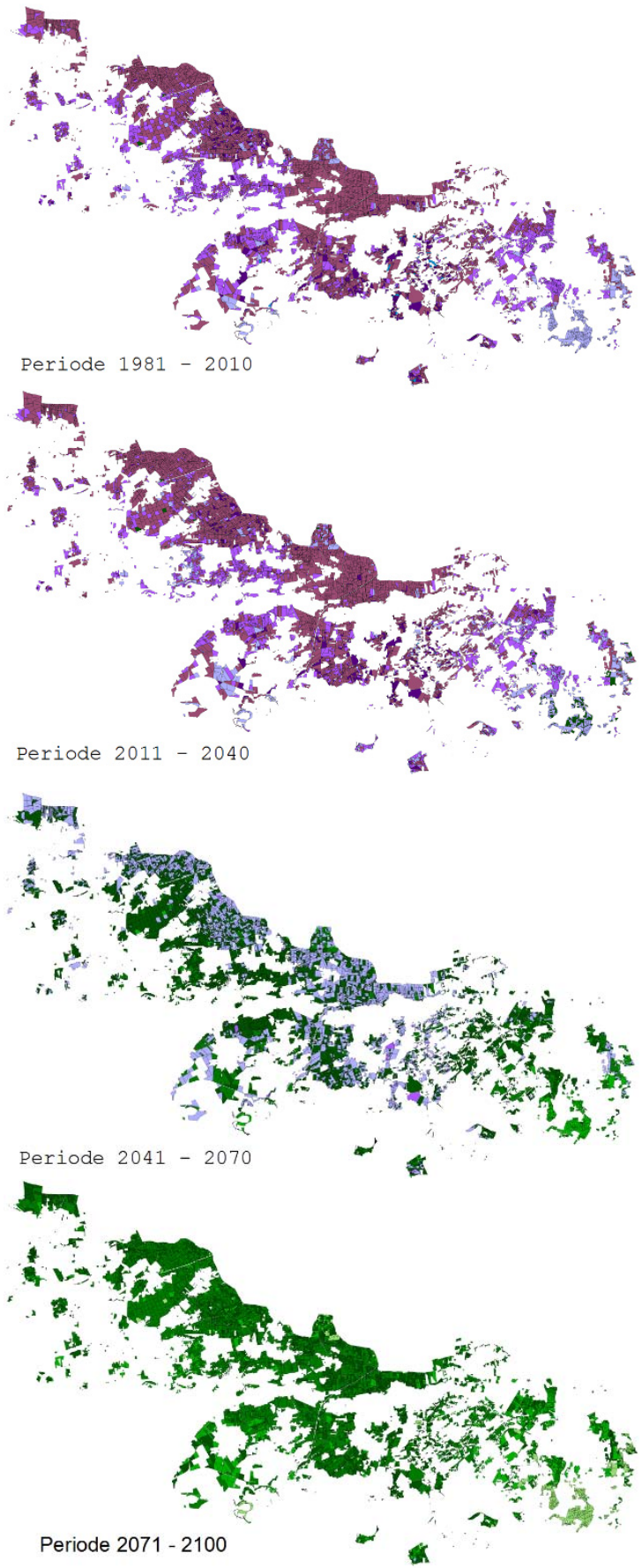

Abbildung 79: Projektion der Standortleistungsfähigkeit für poten₹iell mit Douglasie bestockte Teilflächen in der Region Fläming

Beiträge aus der NW-FVA, Band 13, 2015 
Eine quantitative Analyse der Veränderung der Standortleistungsfähigkeit für die Baumarten Kiefer, Eiche, Buche und Douglasie in der Region Fläming ermöglichen die Histogramme in Abbildung 80 und Abbildung 81. Die Standortleistungsfähigkeit der Kiefer beträgt unter gegenwärtigen Klimabedingungen in der Region Fläming im Mittel 7,6 m³/a/ha. In der Projektion bis 2100 wird ein Rückgang des dGZ $\max$ auf im Mittel 7,1 m³/a/ha geschätzt. Dies entspricht einer Abnahme um knapp $7 \%$. Die Standortleistungsfähigkeit für die Buche ist in der Referenzperiode 1981 - 2010 im Mittel mit 7,5 $\mathrm{m}^{3} / \mathrm{a} / \mathrm{ha}$ auf dem gleichen Niveau wie die Kiefer. Allerdings reagiert die Buche auf zunehmende Trockenheit mit höheren Ertragseinbußen, so dass die Standortleistungsfähigkeit bis zur dritten Projektionsperiode um $12 \%$ auf im Mittel 6,6 $\mathrm{m}^{3} / \mathrm{a} / \mathrm{ha}$ abnimmt. Das Wuchspotenzial der Eiche beträgt zum Status quo durchschnittlich $5,4 \mathrm{~m}^{3} / \mathrm{a} /$ ha und liegt damit deutlich unter den Werten von Kiefer und Buche. Die Reduktion der Standortleistungsfähigkeit für Eiche wird bis 2100 auf knapp $17 \%$ geschätzt. Die Standortleistungsfähigkeit für die Baumart Douglasie weist auf den Standorten der Region Fläming die größte Spreitung auf. Die geschätzte durchschnittliche Gesamtwuchsleistung sinkt von durchschnittlich $15,1 \mathrm{~m}^{3} / \mathrm{a} / \mathrm{ha}$ auf $11,5 \mathrm{~m}^{3} / \mathrm{a} / \mathrm{ha}$, was im Vergleich mit den anderen Baumarten relativ die größte Einbuße bedeutet (knapp $24 \%$ ), aber in Bezug auf das Wuchspotenzial gegenwärtig und zukünftig absolut den höchsten Ertrag verspricht. 

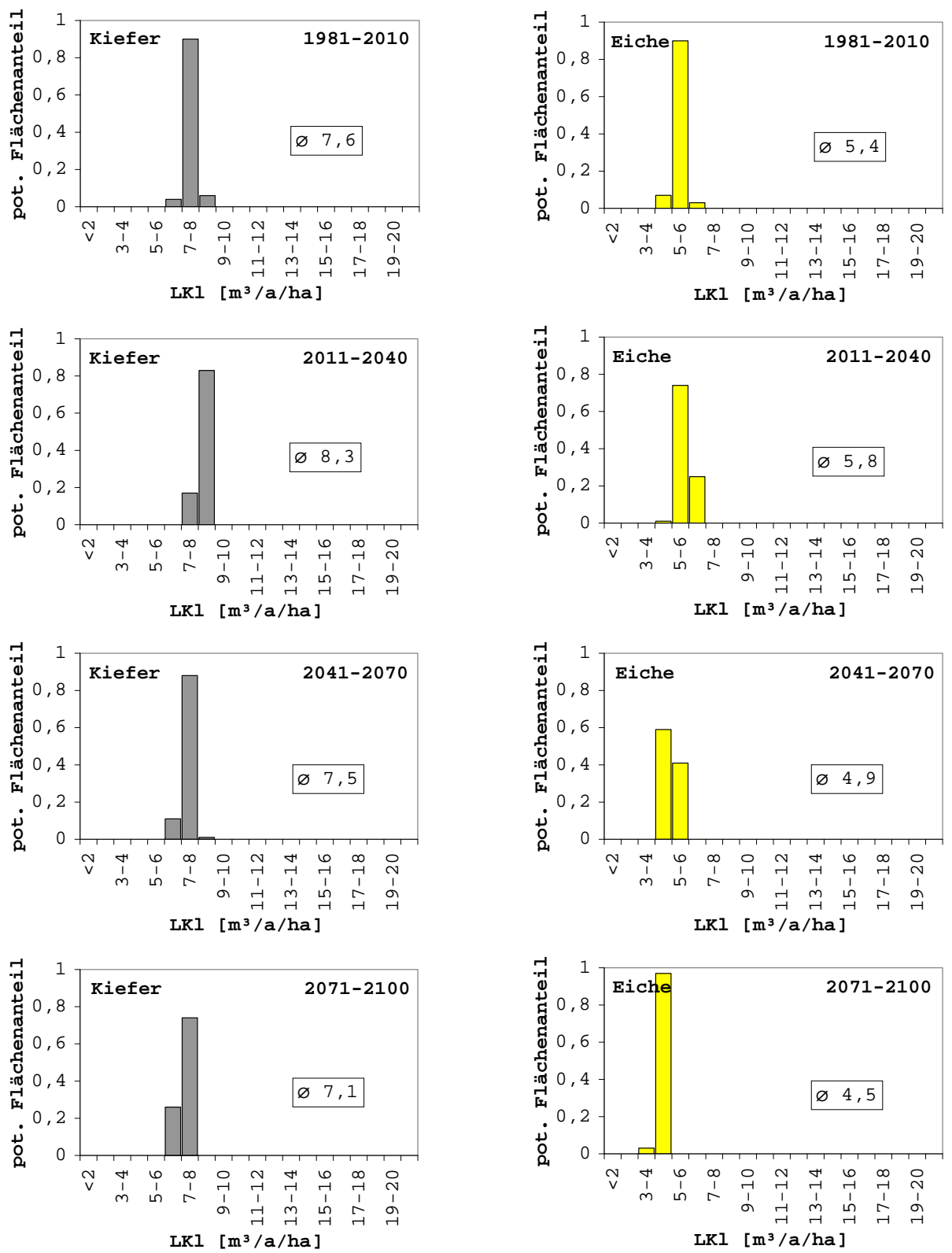

Abbildung 80: Potenzielle Flächenanteile der Standortleistungsfäbigkeit $\left[\mathrm{m}^{3} / \mathrm{a} / \mathrm{ha}\right]$ für die Baumarten Kiefer (links) und Eiche (rechts) für den Status quo (1981 - 2010) und die drei Projektionsperioden in der Region Fläming. Jeder Teilfläche wurde bypothetisch die jeweilige Baumart zugeordnet. In den Grafiken ebenfalls angegeben ist der arithmetische Mittelwert o des durchschnittlichen Gesamtruwachses (dGZ max $)$. 

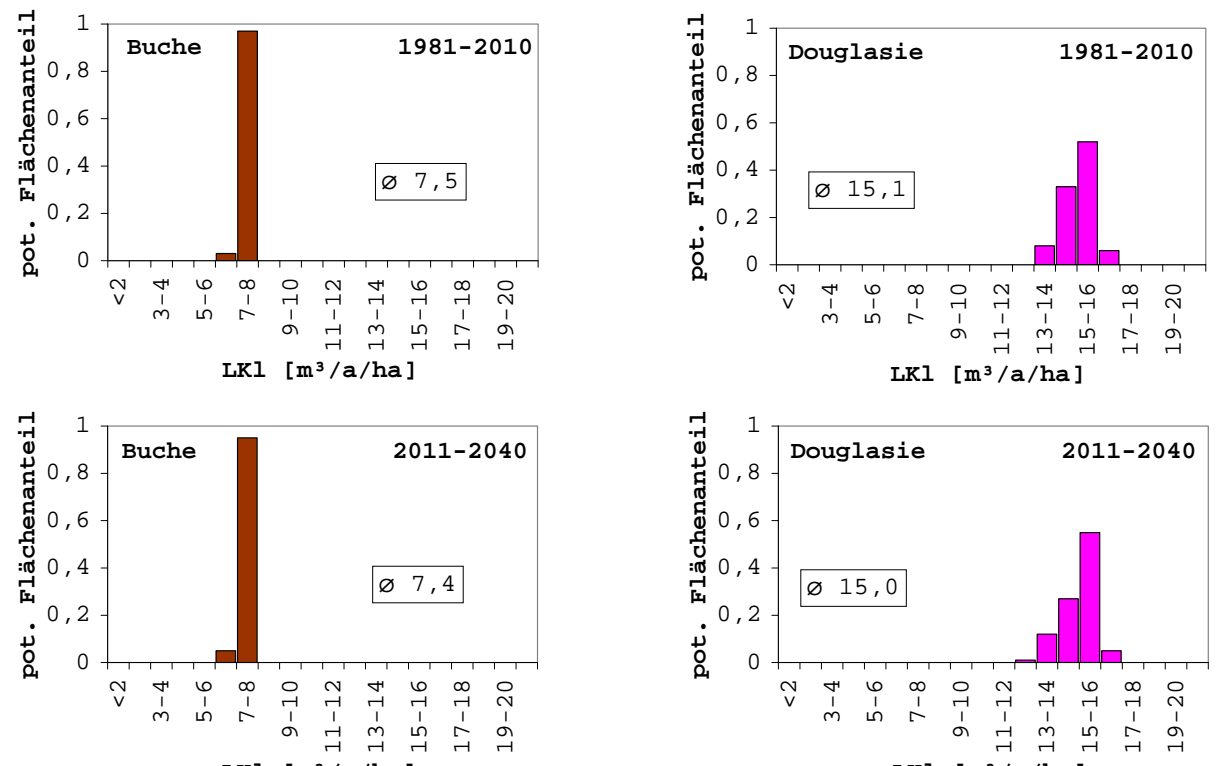

LKI $\left[\mathrm{m}^{3} / \mathrm{a} / \mathrm{ha}\right]$

LKI $\left[\mathrm{m}^{3} / \mathrm{a} / \mathrm{ha}\right]$
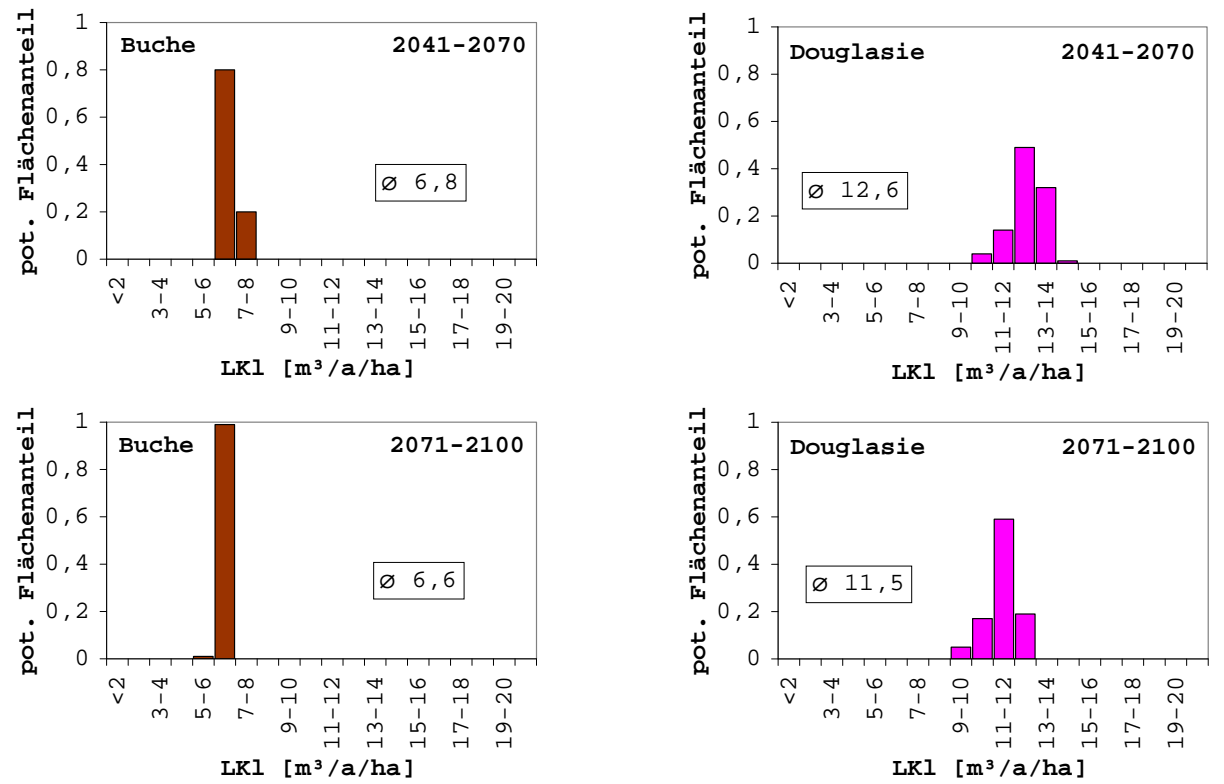

Abbildung 81: Potenqielle Flächenanteile der Standortleistungsfähigkeit $\left[\mathrm{m}^{3} / \mathrm{a} / \mathrm{ha}\right]$ für die Baumarten Buche (links) und Douglasie (rechts) für den Status quo (1981 - 2010) und die drei Projektionsperioden in der Region Fläming. Jeder Teilfläche wurde bypothetisch die jeweilige Baumart zugeordnet. In den Grafiken ebenfalls angegeben ist der arithmetische Mittelwert o des durchschnittlichen Gesamtruwachses ( $d G Z_{\text {max }}$ ). 


\subsection{Sturmschadensrisiko}

\subsubsection{Poten₹ielle Vulnerabilität durch Winterstürme bei Zielstärke}

Der Vergleich der potenziellen Bestockung bei Zielstärke zwischen den Baumarten zeigt im Fläming ein im Mittel steigendes Sturmschadensrisiko von Buche über Eiche und Kiefer zu Douglasie (s. Abb. 82). Die Mittelwerte für das Sturmschadensrisiko betragen 0,25 für die Buche, 0,31 für die Eiche, 0,53 für die Kiefer und 0,75 für die Douglasie. Kiefer und Douglasie weisen dabei sehr viel engere Verteilungen als Eiche und Buche auf. Die Kiefer nimmt somit eine Mittelstellung zwischen Buche / Eiche und Douglasie ein.

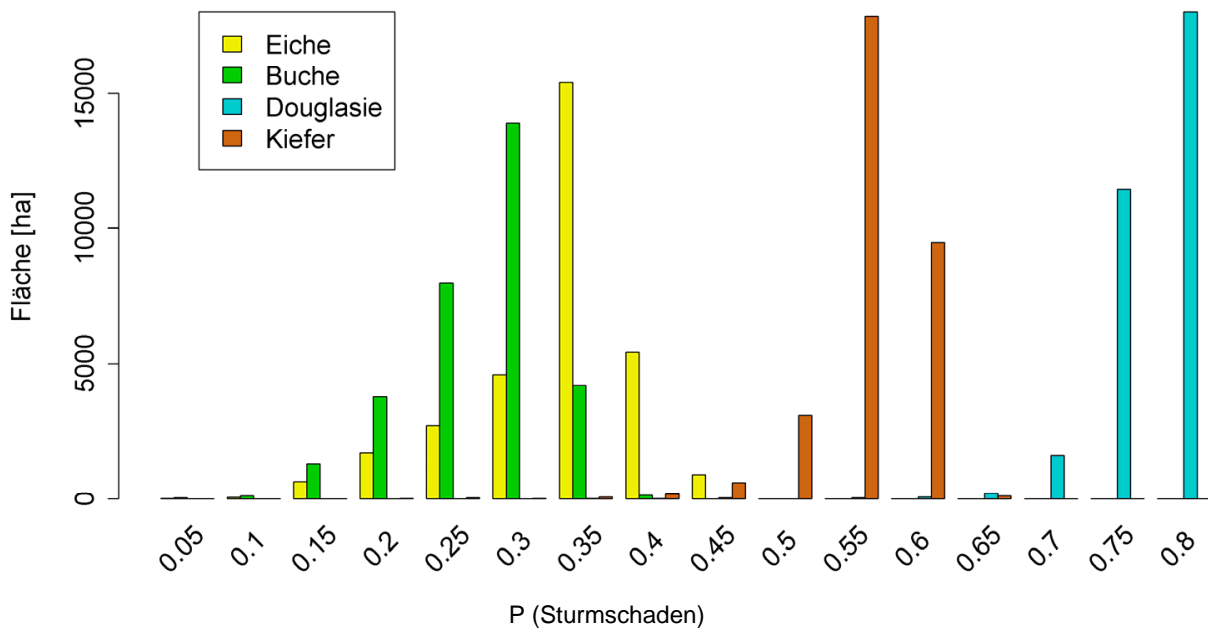

Abbildung 82: Flächenverteilungen einer potenziellen Bestockung [ha] bei baumartenspezifischen Zielstärken nach Sturmschaden-Risikostufen und Baumarten in der Modellregion Fläming

Für die Region Fläming werden Risikokarten für die Baumarten Eiche, Kiefer und Douglasie dargestellt (s. Abb. 83). Eine potenzielle Eichenbestockung bei Zielstärke $(65 \mathrm{~cm})$ würde zu $100 \%$ in die Risikoklasse „geringes Risiko“ eingestuft. Eine potenzielle Kiefernbestockung bei Zielstärke $(45 \mathrm{~cm})$ würde zu $47 \%$ in die Risikoklasse „geringes Risiko“ und zu $53 \%$ in die Risikoklasse „mittleres Risiko“ eingestuft. Eine potenzielle Douglasienbestockung bei Zielstärke $(65 \mathrm{~cm})$ würde zu $100 \%$ in die Risikoklasse „mittleres Risiko“ eingestuft. Da die Fichte als Baumart im Fläming keine Rolle spielt, ist das Risikopotenzial insgesamt geringer als im Harz. Gleichzeitig resultieren aufgrund der relativ geringen Relief- und Standortsunterschiede sehr gleichmäßige Muster der Risikoklassen. Für eine Identifizierung der geringfügigen Unterschiede des Sturmschadensrisikos wäre theoretisch eine feinere Klasseneinteilung notwendig, wobei zu prüfen wäre, ob diese Unterschiede planungsrelevant sind. Unter den betrachteten Baumarten weist die Kiefer die größte 
Sensitivität bzgl. der Risikoklassen auf, an der strategische Entscheidungen wie ein Baumartenwechsel ausgerichtet werden sollten. Dabei geht es vor allem um die Diversifizierung der Baumartenverteilung und die Erhöhung des Anteils von Mischbeständen durch die Reduktion des Kiefernanteils. Unter Risikoaspekten wäre es sinnvoll, die Douglasie in Bereichen zu etablieren, die bzgl. der Kiefernbestockung ein geringes Risiko aufweisen, selbst wenn damit eine begrenzte Verlagerung in Richtung höherer Risikoklassen verbunden ist. Die Einbringung der Eiche in Beständen, die bzgl. der Kiefernbestockung ein mittleres Risiko aufweisen, würde zu einer Absenkung der Risikoklasse führen.

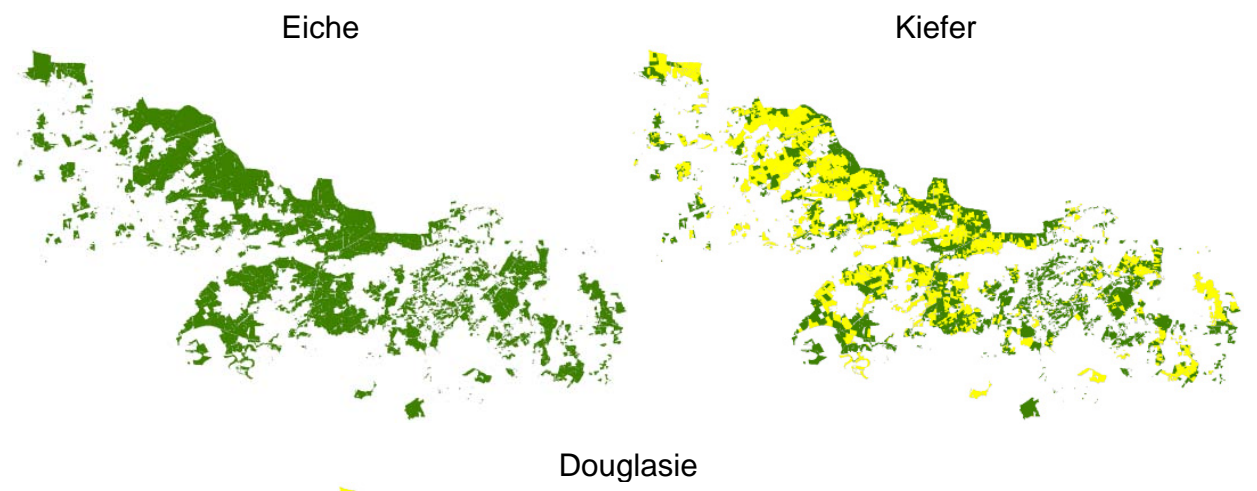

Abbildung 83: Karten des Sturmschadensrisikos der potenziellen Eichen-, Kiefern- und Douglasienbestockung bei Zielstärke (65, 45 bzw. $55 \mathrm{~cm})$ in der Region Fläming unter Anwendung von 4 anhand der Fichte abgeleiteten Risikoklassen (grün: niedriges, gelb: mittleres, rot: bohes und pink: sehr hohes Sturmschadensrisiko) 


\subsubsection{Aktuelle Gefährdungspoten₹iale durch Winterstürme}

Die Flächenverteilungen nach Risikostufen werden in der Region Fläming von der Kiefer dominiert, wobei mittlere Risikostufen vorherrschen (s. Abb. 84). Dargestellt sind die vier flächenmäßig wichtigsten Baumarten Kiefer, Eiche, Buche und ALn. Eine Adaption waldbaulicher Maßnahmen unter dem Aspekt des Sturmschadensrisikos ist weniger zwingend als in der Region Oberharz. Allerdings ließen sich anhand der Risikoprognosen und unter Berücksichtigung der Hiebsreife Kiefernbestände identifizieren, die im Rahmen eines Waldumbaus prioritär berücksichtigt werden sollten. Die Mittelwerte der Sturmschadensprognosen betragen für die aktuelle Kiefernbestockung 0,43, für die aktuelle Eichenbestockung 0,15, für die aktuelle Buchenbestockung 0,17 und für die aktuelle Bestockung des ALn 0,17.

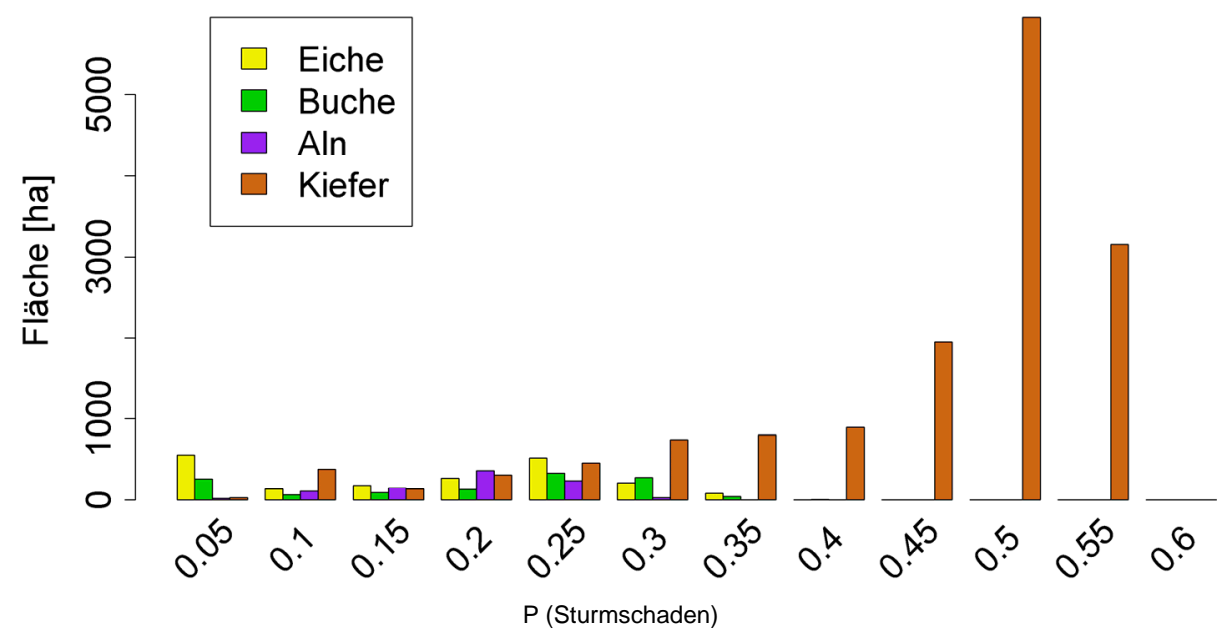

Abbildung 84: Flächenverteilungen der aktuellen Bestockung [ha] nach Sturmschaden-Risikostufen und Baumarten in der Modellregion Fläming

Die Risikokarten für die aktuelle Kiefern- und Eichenbestockung in der Region Fläming weisen aufgrund der geringeren Relief- und Standortsunterschiede sowie des insgesamt geringeren Risikoniveaus der Kiefer und Eiche eine geringere Variabilität der prognostizierten Sturmschadenswahrscheinlichkeiten auf (s. Abb. 85). Die bereits diskutierte vorrangige Behandlung von hiebsreifen Kiefernbeständen, die ein relativ hohes Sturmschadensrisiko aufweisen, im Rahmen eines Waldumbauprogrammes würde die Erstellung von großmaßstäblichen Karten erfordern. 


\section{Kiefer}

\section{mos \\ 10 Sturm-}

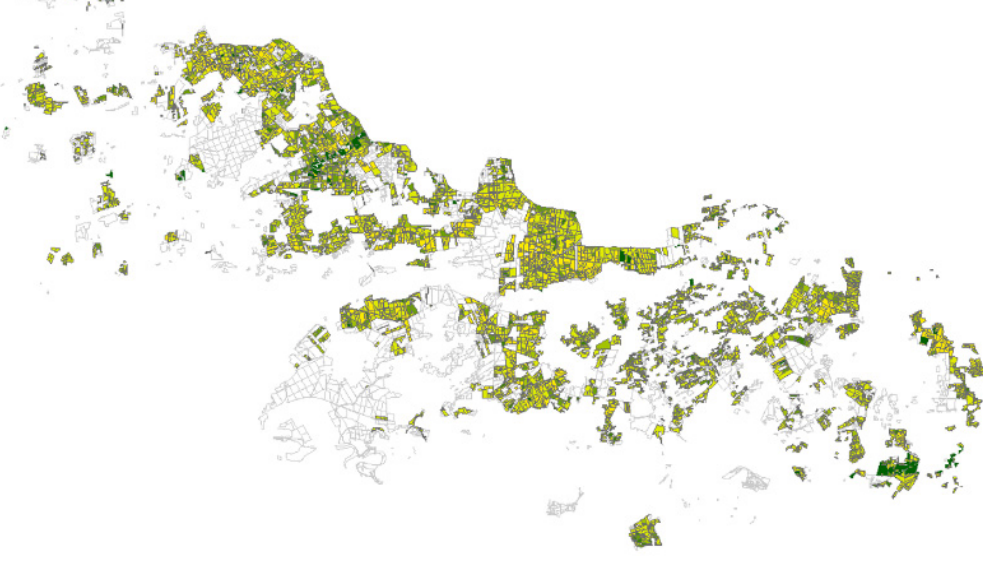

Eiche

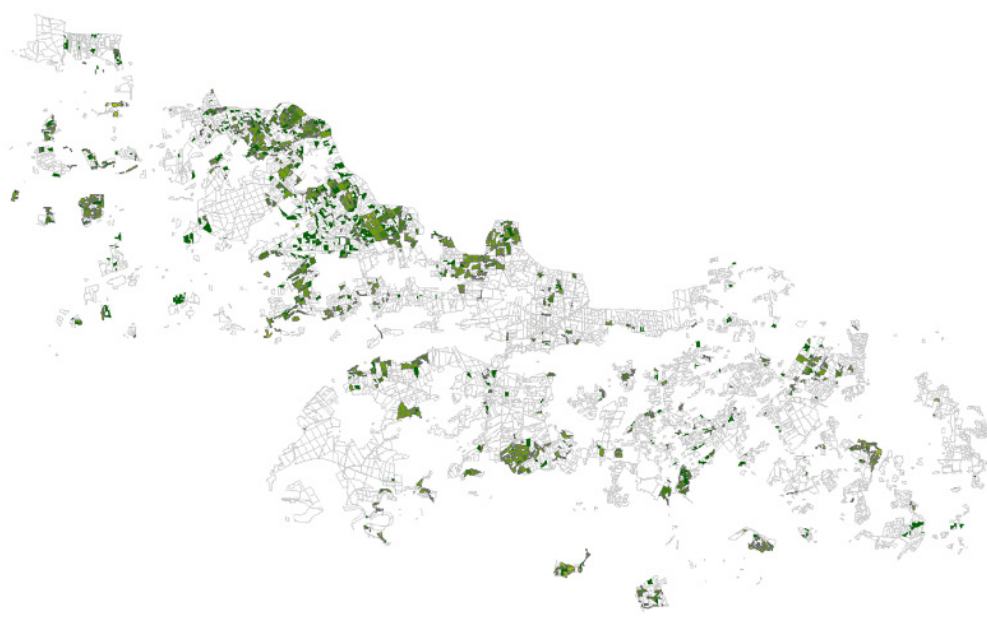

schadensklassen

mit einheitlicher

Klassenbreite:

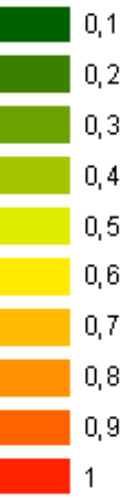

10 Sturm-

schadensklassen mit einheitlicher Klassenbreite:

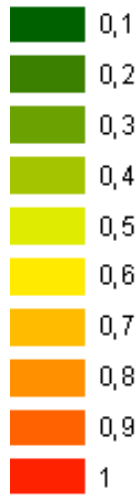

Abbildung 85: Karten des Sturmschadensrisikos der aktuellen Kiefern- und Eichenbestockung in der Region Fläming mit einer Einteilung in zehn Risikoklassen

\subsection{Veränderungen des Waldbrandrisikos}

In der Region Fläming liegt das aktuelle Waldbrandrisiko mit durchschnittlich 20-30 Tagen (M68) oder gar 30-40 Tagen erhöhter Waldbrandgefahr im Jahr (FWI) erheblich höher als etwa im Harz. Einige Waldgebiete im Ostfläming waren im Referenzzeitraum 2004 - 2010 mit bis zu 35 (M68; s. Abb. 86) bzw. 45 Risikotagen (FWI; s. Abb. 87) noch stärker gefährdet als im Westfläming. 


\section{Jährliche Tage mit WBKZ > 4000}
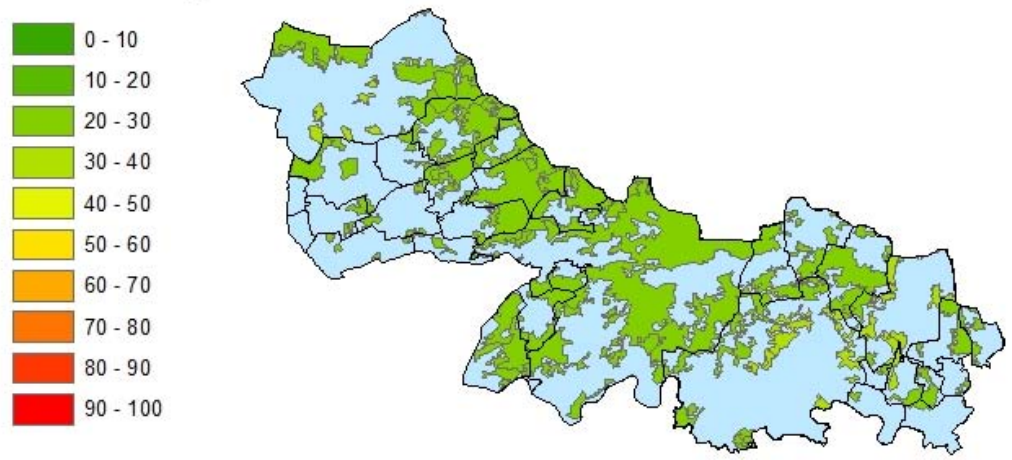

Abbildung 86: Jährliche Tage mit erböbtem Waldbrandrisiko im Fläming nach Berechnung mit dem M68Waldbrandindex (WBKZ $=$ Waldbrandkenniiffer)

\section{Jährliche Tage mit FFMC > 92}

\begin{tabular}{|l|l|}
\hline & $0-10$ \\
& $10-20$ \\
\hline & $20-30$ \\
\hline & $30-40$ \\
\hline$\square$ & $40-50$ \\
\hline$\square$ & $50-60$ \\
\hline & $60-70$ \\
\hline & $70-80$ \\
\hline & $80-90$ \\
\hline & $90-100$
\end{tabular}

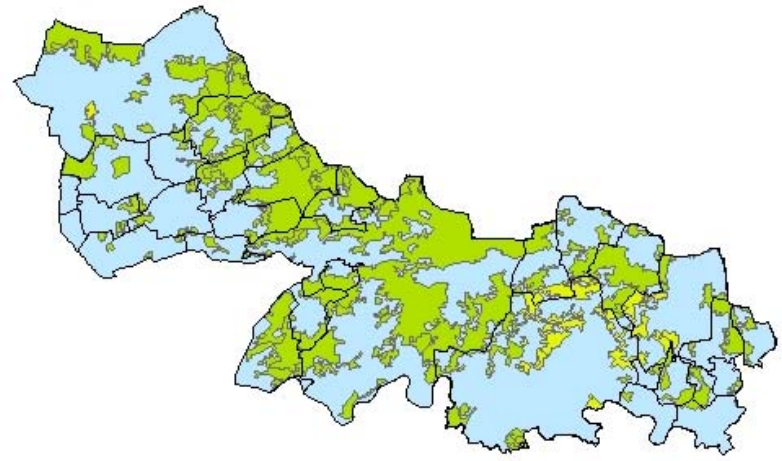

Abbildung 87: Jährliche Tage mit erböhtem Waldbrandrisiko im Fläming nach Berechnung mit dem Fire Weather Index (FWI) (FFMC = Streufeuchtezabl)

Das relativ hohe Gefährdungsniveau spiegelt sich auch im tatsächlichen Vorkommen von Waldbränden im Referenzzeitraum wider. In zwei Gemeinden kamen bis zu vier Brände je 1000 ha Waldfläche vor (s. Abb. 88). Der gesamte Fläming mit Ausnahme weniger Gemeinden war von Waldbränden betroffen. 


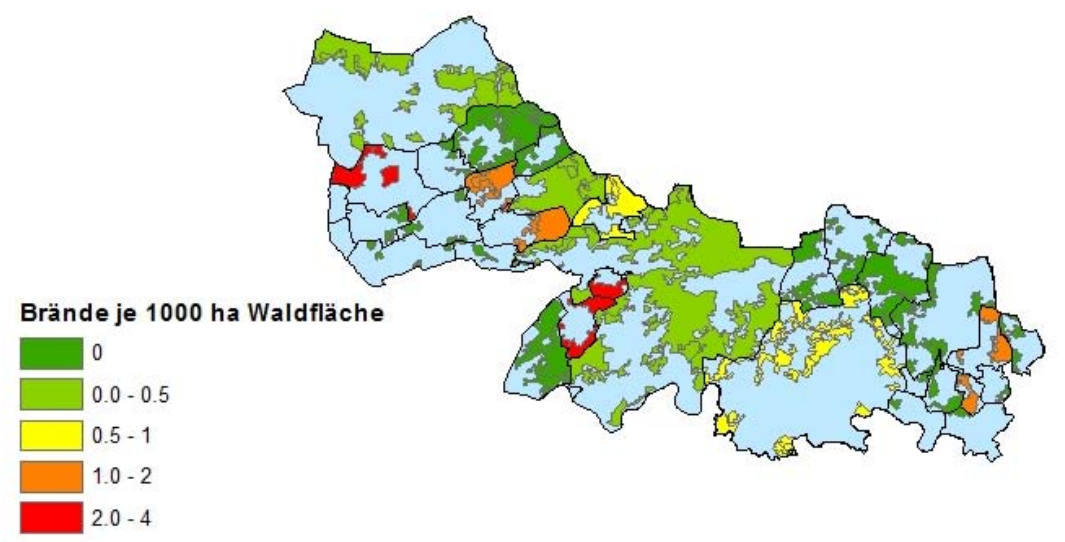

Abbildung 88: Waldbrände je 1000 ha Waldfläche in der Region Fläming im Referenzzeitraum 2004 2010

Die Vorhersage der Waldbrandgefährdung für den Fläming ergibt eine gravierende Steigerung: So ergibt sich schon im ersten Terzennium (2011 - 2040) des Simulationszeitraums mit durchschnittlich bis zu 45 (M68; s. Abb. 89) bzw. 50 (FWI; s. Abb. 90) jährlichen Tagen erhöhten Waldbrandrisikos eine über das bisher in Sachsen-Anhalt bekannte Maß hinaus gehende Gefährdung, die das Vorkommen extrem trockener Jahre nach Art des Jahres 2003 einschließt. Im zweiten 30-JahresAbschnitt (2041 - 2070) wird mit durchschnittlich etwa 60 (M68) oder 65 (FWI) Risikotagen im Jahr bereits ein extrem gesteigertes Ausmaß der Waldbrandgefährdung erreicht, das noch extremere Sommer als den Trockensommer 2003 enthält. Die Vorhersage für das dritte Terzennium (2071 - 2100: jährlich ca. 85 Risikotage) kann mit der heutigen Situation am besten verglichen werden, indem man sich vorstellt, dass es in den meisten Jahren eine noch extremere Situation als den Trockensommer 2003 gibt. Allen Vorhersagen gemeinsam ist der bereits heute existierende zunehmende Gefährdungsgradient von Westen nach Osten. 
Waldbrandkennziffer: 30-Jahres-Projektionen bis 2040, 2070 und 2100

\section{Jährliche Tage mit WBKZ > 4000}
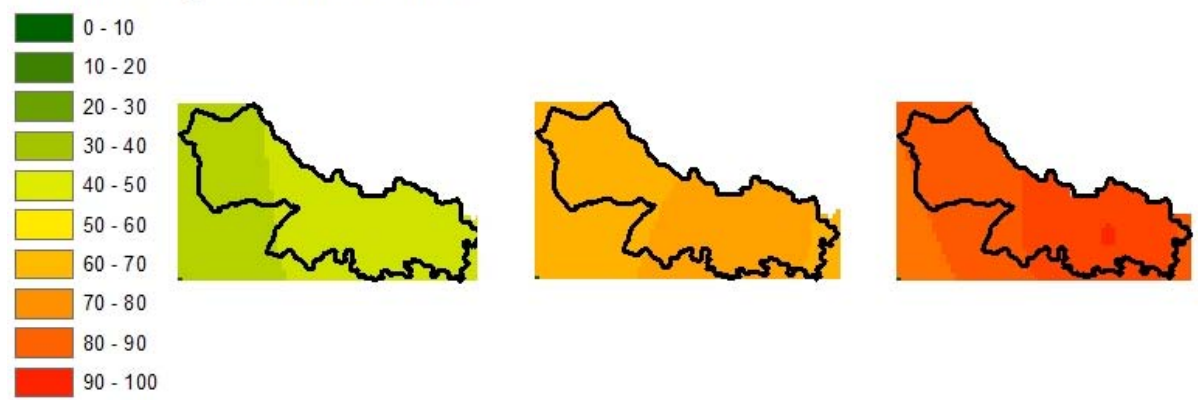

2011-2040

2041-2070

2071-2100

Abbildung 89: Erwartete jährliche Zabl von Tagen erböbten Waldbrandrisikos auf Basis des M68 (Tage, an denen die Waldbrandkenn₹iffer (WBKZ) über 4000 liegt)

Fine Fuel Moisture Code: 30-Jahres-Projektionen bis 2040, 2070 und 2100

\section{Jährliche Tage mit FFMC $>92$}
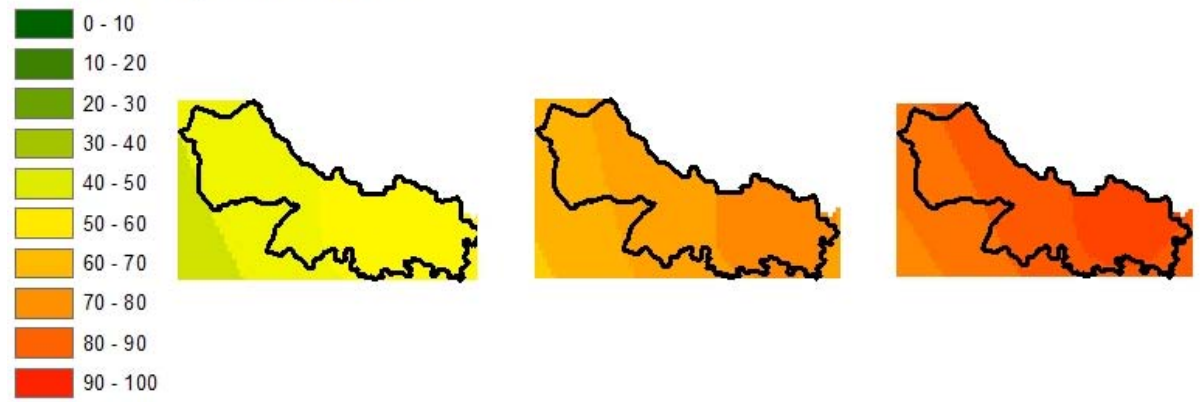

2011-2040

2041-2070

$2071-2100$

Abbildung 90: Erwartete jährliche Zabl von Tagen erböhten Waldbrandrisikos auf Basis des FWI (Tage, an denen der Fine Fuel Moisture Code (FFMC= Streufeuchtezabl) über 92 liegt)

Zusätzliche Risikofaktoren:

Bezüglich des Risikofaktors direkte Sonneneinstrahlung gibt es im Fläming nur wenig Variabilität (s. Abb. 91). Die meisten Waldflächen werden maximal 15-16 Stunden am Tag bestrahlt, ca. $25 \%$ der Waldflächen liegen wegen der Horizontabschattung etwas geschützter (14-15 Stunden). Sehr hohe Globalstrahlungswerte über $220 \mathrm{~W} \mathrm{~m}^{-2}$ werden in der Hauptwaldbrandperiode (April bis August) aber nur auf wenigen Südhängen erreicht, in den allermeisten Fällen liegt die mittlere eintreffende Globalstrahlung zwischen 210 und $220 \mathrm{~W} \mathrm{~m}^{-2}$ (s. Abb. 92). 
Mittlere Sonnenscheindauer

(Stunden)

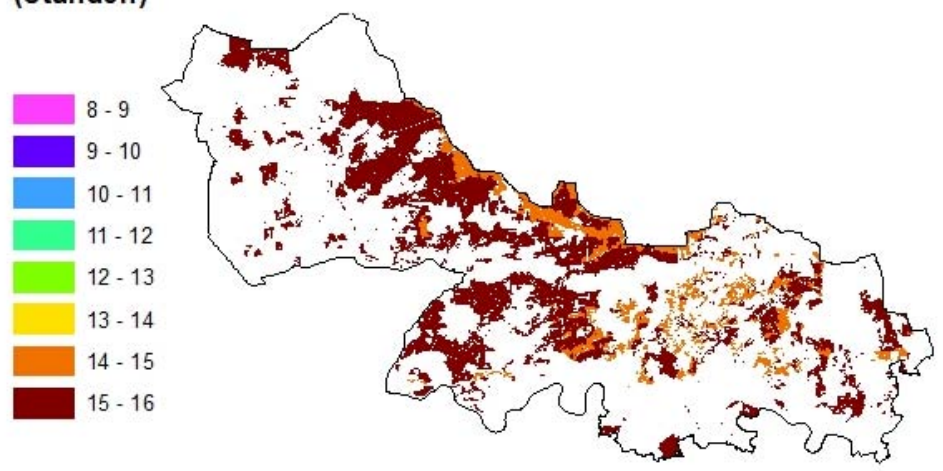

Abbildung 91: Regionale Verteilung der mittleren potenziellen Sonnenscheindauer während der Monate April bis August auf den W aldflächen im Fläming

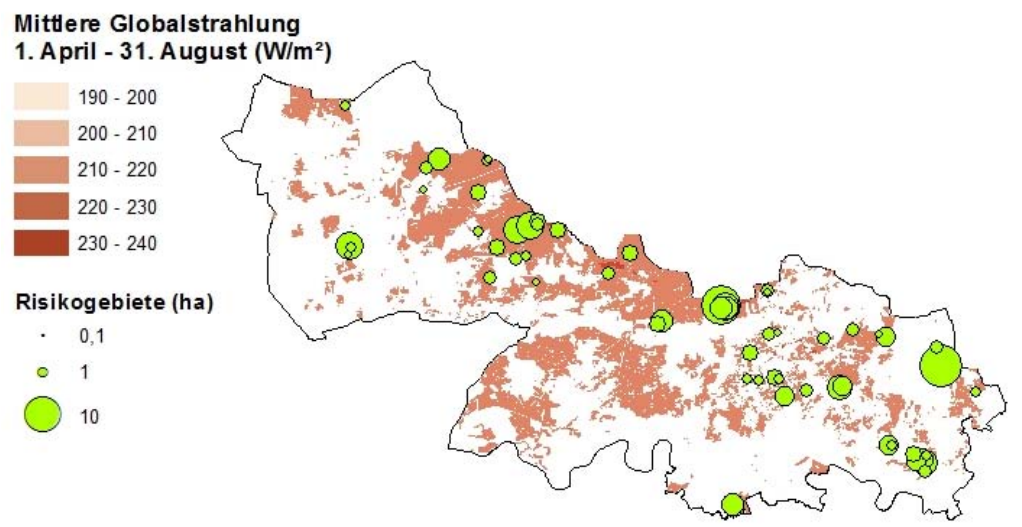

Abbildung 92: Regionale Verteilung der mittleren Globalstrablung $\left(W / m^{2}\right)$ auf $W$ aldflächen im Fläming während der Monate April bis August und Lage von Kiefernjungbeständen geringer Bestockungsdichte (lokale Risikogebiete)

Die aufgrund der leicht entzündlichen Streu besonders waldbrandanfälligen jungen Kiefernbestände sind in der Region Fläming besonders zahlreich und weit über die ganze Region verstreut. Gegenwärtig befinden sich größere lokale Risikogebiete am nördlichen und östlichen Rand der Region. 


\subsection{Zustände und Wirkungsgefüge bei biotischen Risiken}

Die am stärksten vertretenen Baumarten auf der untersuchten Fläche von 31.395 ha im Bereich des Flämings sind Kiefer mit $76 \%$ und Eiche mit ca. $10 \%$. Die standörtliche Differenzierung ist relativ stärker als in den anderen untersuchten Gebieten, was auch durch die flächenmäßig stärkere Beteiligung der Eiche deutlich wird. Dies wiederum beeinflusst dann auch die Waldschutzproblematik.

Bei den analysierten 7.991 Teilflächen wurde das Befallsvorkommen der Schädlinge im Laufe der letzten 20 Jahre untersucht. Zu den Schädlingen mit dem meisten Befallsvorkommen gehört in diesem Untersuchungsgebiet die Eichenfraßgesellschaft, wobei der Eichenwickler (Tortrix viridana L.) und der Kleine Frostspanner (Operophtera brumata L.) vorwiegend vergesellschaftet aufgetreten sind (s. Tab. 17). Darüber hinaus spielte der Eichenprozessionsspinner (Thaumetopoea processionea L.), dessen Vorkommen seit den 1990er Jahren mit steigenden Tendenz gemeldet wurde (VELDMANn u. KONTZOg 1994), eine immer bedeutendere Rolle.

Tabelle 17: Anzabl der Befallsvorkommen nach Schädlingsarten im Bereich des Flämings (1993 2012) bei verschiedenen Befallschwellen bezogen auf die Fläche

\begin{tabular}{l|ccc}
\hline $\begin{array}{r}\text { definierte Schwellen } \\
\text { für Befall }\end{array}$ & $1 \%$ & $10 \%$ & $30 \%$ \\
\hline Schadverursacher & 574 & 543 & 440 \\
\hline Eichenfraßgesellschaft: & 587 & 556 & 452 \\
Frostspanner & 605 & 574 & 471 \\
Eichenprozessionsspinner & 9 & & 9 \\
\hline Kiefergroßschädlinge und Nonne: & 10 & 9 & 10 \\
\hline Kiefernspanner & 1 & 10 & 1 \\
Kiefernspinner & 2 & 2 & 31 \\
Forleule & 38 & 37 & 2 \\
Kiefernbuschhornblattwespen & & 1 & \\
Nonne & & 2 & \\
\hline
\end{tabular}

Die Hauptschadgebiete der Eichenfraßgesellschaft lagen im Bereich des Hohen Flämings (Landesforstbetrieb (LFB) Anhalt, Revier (Rev.) Nedlitz), vereinzelt traten Schäden ebenfalls im südlichen und südwestlichen Flämingvorland auf. Dabei korrelierten die Befallsgebiete des Eichenprozessionsspinners mit denen des Eichenwicklers und Frostspanners, gingen jedoch auch noch darüber hinaus. 
Nach der Analyse auf lineare Zusammenhänge mittels eines generalisierten linearen Modells (GLM) wurde festgestellt, dass das Alter und der Mischungsanteil der Eiche sowie die nFK den größten Einfluss auf den Befall durch die Eichenfraßgesellschaft haben (s. Tab. 18). Der Einfluss der Nährstoffziffer hat sich hingegen als nicht signifikant erwiesen, jedoch war eine Tendenz erkennbar, da mit abnehmender Nährstoffziffer das Befallsrisiko steigt. Die Wahrscheinlichkeit eines Befalls durch die Eichenfraßgesellschaft nimmt mit steigendem Alter und Anteil der Eiche in den Beständen zu. Gleichzeitig kann damit gerechnet werden, dass mit abnehmendem nFK-Wert das Befallsrisiko ansteigt.

Nach SCHWANECKE u. KOPP (1994) herrschen im Bereich des Flämings mittlere bis ärmere und lehmige Sande vor. Die Ergebnisse der GLM-Analyse zeigten ein zunehmendes Befallsrisiko für ältere Bestände mit hohem Eichenanteil auf Böden mit steigendem Sand- sowie mit abnehmendem Schluffanteil.

Tabelle 18: $\quad$ Durch ein generalisiertes lineares Modell (GLM) analysierter Einfluss der Bestandes- und Standortsvariablen auf Befall durch die Eichenfraßgesellschaft im Bereich des Flämings (1993 - 2012); (+/- Richtung des Zusammenhangs zur Zielgröße (Wahrscheinlichkeit des Befalls), Signifikanz: ( ) keine, (*) gering, (**) mittel, (***) boch)

\begin{tabular}{|c|c|c|c|c|c|c|c|c|c|}
\hline \multirow{2}{*}{ Variablen } & \multicolumn{3}{|c|}{ Eichenwickler } & \multicolumn{3}{|c|}{ Frostspanner } & \multicolumn{3}{|c|}{$\begin{array}{l}\text { Eichenprozes- } \\
\text { sionsspinner }\end{array}$} \\
\hline & $1 \%$ & $10 \%$ & $30 \%$ & $1 \%$ & $10 \%$ & $30 \%$ & $1 \%$ & $10 \%$ & $30 \%$ \\
\hline \multirow{2}{*}{ Alter } & $* * *$ & $* * *$ & $* * *$ & $* * *$ & $* * *$ & $* * *$ & $* * *$ & $* * *$ & $* * *$ \\
\hline & + & + & + & + & + & + & + & + & + \\
\hline \multirow{2}{*}{ Mischungsanteil } & $* * *$ & $* * *$ & $* * *$ & $* * *$ & $* * *$ & $* * *$ & $* * *$ & $* * *$ & $* * *$ \\
\hline & + & + & + & + & + & + & + & + & + \\
\hline Nährstoffziffer & - & - & - & - & - & - & - & - & - \\
\hline \multirow{2}{*}{$\mathrm{nFK}$} & $* * *$ & $* * *$ & $* * *$ & $* * *$ & $* * *$ & $* * *$ & $* * *$ & $* * *$ & $* * *$ \\
\hline & - & - & - & - & - & - & - & - & - \\
\hline
\end{tabular}

In Zukunft ist aufgrund des zunehmenden Trockenstressrisikos (s. Kap. 6.1) im gesamten Untersuchungsgebiet des Hohen Flämings und Flämingvorlands, welches auf manchen Standorten für die Eiche prädisponierend bzw. schadensauslösend wirken kann, ein verstärktes Vorkommen des Fraßgeschehens durch die o.g. Schmetterlinge zu erwarten. Durch wiederholtes Fraßgeschehen in Kombination mit Vorkommen von pilzlichen Schaderregern wie Hallimasch und PhytophthoraArten kann man für die prädisponierten Standorte mit einer Zunahme der Eichenkomplexerkrankung rechnen.

Neben der Eichenfraßgesellschaft spielte das Vorkommen von Nonne und Kieferngroßschädlingen eine geringe Rolle. Zu ihnen zählen Forleule (Panolis flammea Denis \& Schiffermüller), Kiefernspinner (Dendrolimus pini L.), Kiefernspanner 
(Bupalus piniaria L.), Kiefernbuschhornblattwespen (Diprion spec.) und Nonne (Lymantria monacha L.). Im untersuchten Zeitraum war die Anzahl der durch Nonne befallenen Teilflächen vor allem im Bereich des südöstlichen Flämingvorlands (LFB Anhalt, Rev. Göritz; Landeszentrum Wald (LZW) Betreuungs-Forstamt (Betr.-FA) Annaburg, Rev. Wittenberg) am größten, geringere Schäden verursachten Kiefernspinner und -spanner, vereinzelt wurden dort auch Kiefernbestände durch Forleule und Kiefernbuschhornblattwespen befallen. Trotz des hohen Vorkommens von Kiefernbeständen im gesamten Untersuchungsgebiet Fläming war der Anteil der befallenen Bestände so niedrig (s. Tab. 17), dass diese Anzahl für die GLM-Analyse von befallenen Beständen durch die Kieferngroßschädlinge nicht ausreichend war, um signifikante Zusammenhänge zu Bestandes- und Standortsfaktoren nachweisen zu können.

In Anbetracht der Prognose zum Trockenstressrisiko für die Kiefernbestände im Bereich des Untersuchungsgebiets Fläming (s. Kap. 6.1) ist zu erwarten, dass ein zunehmendes Risiko durch Kieferngroßschädlinge und Nonne im gesamten Bereich des Flämings vorliegt, insbesondere auf den ärmeren sandigen Standorten des südöstlichen Flämingvorlandes. Weiterhin ist bei zunehmenden abiotischen oder biotischen Störungen mit Schäden durch den Großen Braunen Rüsselkäfer (Hylobius abietis L.) zu rechnen.

\subsection{Konsequenzen für die künftige Waldbewirtschaftung in der Region Fläming}

\subsubsection{Waldbauliche Ausgangslage}

Nach den Ergebnissen der forstlichen Standortskartierung stocken die Wälder der Region Fläming zu nahezu $60 \%$ auf grundwasserfernen Waldstandorten mittlerer Nährkraft in Kombination mit einer mittleren Wasserhaushaltsstufe (Stammstandortsformengruppe TM2). Die nächst bedeutendere Gruppe sind die ziemlich armen (Z) Sandstandorte mittlerer Wasserversorgung, die insgesamt deutlich über ein Viertel der Region ausmachen. Terrestrische nährstoffkräftige Standorte nehmen unter $5 \%$ der Fläche ein und kommen relativ konzentriert in wenigen Bereichen des Hohen Fläming vor.

Kiefernbestände bilden mit $76 \%$ der Fläche derzeit die Hauptbestockung der Region, häufig als Reinbestände bzw. mit geringen Beimischungen von Laub- und anderen Nadelbaumarten wie Fichte (s. Abb. 93). Weitere Nadelbaumarten wie Lärche, Fichte und Douglasie sind nur mit zusammen 4\% im Hauptbestand vertreten. Die wichtigsten Laubbaumarten sind Eiche mit $10 \%$ und Buche mit $6 \%$ sowie Weichlaubbäume wie Birke und Roterle mit zusammen knapp $3 \%$.

Mit ideellen Flächenanteilen von insgesamt $9 \%$ ist allerdings ein günstig zu beurteilender Unter- und Zwischenstand vorzugsweise aus Laubbäumen wie Eichen 
aus Hähersaat, Buchen, Birken und Ebereschen relativ verbreitet und vor allem in den mittelalten bis älteren Kiefernbeständen vertreten. Die flächenmäßig bedeutendste Unterstandsbaumart ist jedoch die Spätblühende Traubenkirsche (Prunus serotina), die große waldbauliche Probleme, vor allem bei der Verjüngung stärker lichtbedürftiger Baumarten, verursacht (s. Abb. 94).

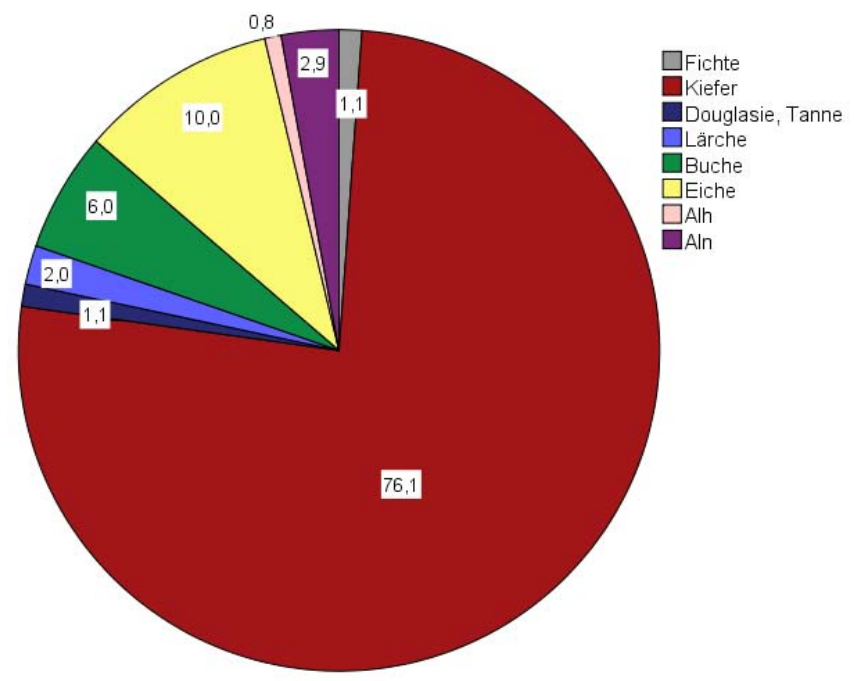

Abbildung 93: Flächenanteile [\%] der Baumarten im Hauptbestand in der Region Fläming (ALh= Laubbolz, mit hoher Umtriebszeit, ALn = Laubholz mit niedriger Umtriebszeit; im Datenspeicher W ald erfasste Flächen, Privatwald ₹. T. Stand 1994)

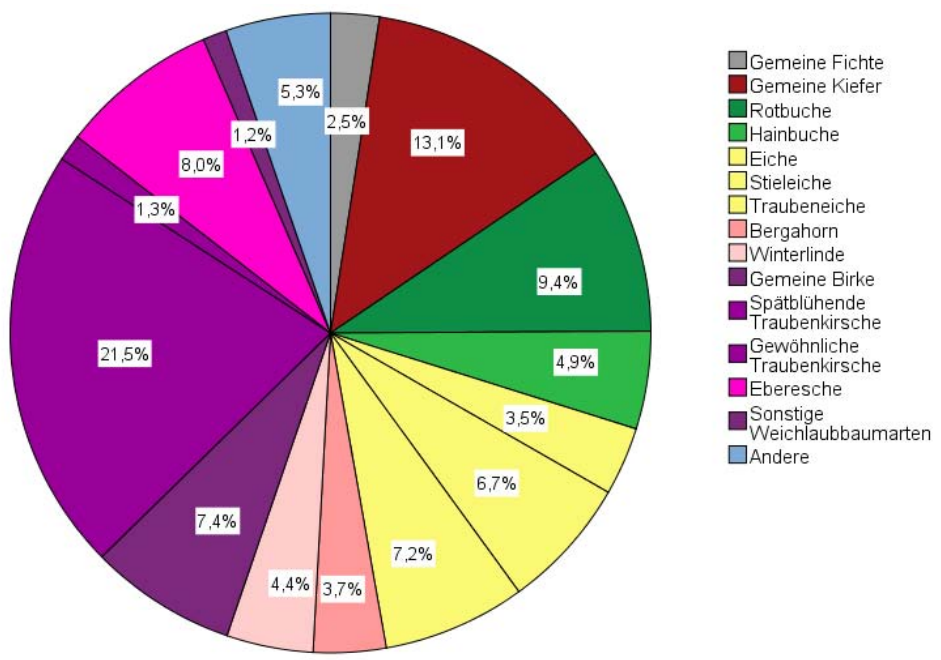

Abbildung 94: Baumartenanteile [\%] im Unterstand in der Region Fläming; Unterstand auf ca. $9 \%$ der Gesamtwaldfläche 
Die Altersklassenverteilung der Kiefer zeigt einen gewissen Überhang in der III. Altersklasse und deutlich geringere Anteile in der ersten Altersklasse (s. Abb. 95). Insgesamt ist der Altersaufbau ansonsten jedoch recht ausgeglichen, wenn man die über 100-jährigen Bestände gemeinsam betrachtet. Trotz lokal z. T. gravierender Schäden durch das Sturmereignis Kyrill ist ein bedeutender Block älterer Kiefernbestände mit hohen Anteilen von Bäumen über $40 \mathrm{~cm}$ Brusthöhendurchmesser vertreten, der zur Nutzung und Verjüngung ansteht. Die Eiche als wichtigste Laubbaumart ist ebenso wie die Buche in allen Altersklassen vertreten, mit jeweils überdurchschnittlichen Anteilen alter Bestände über 120 Jahre und unter 20-jährigen Beständen.

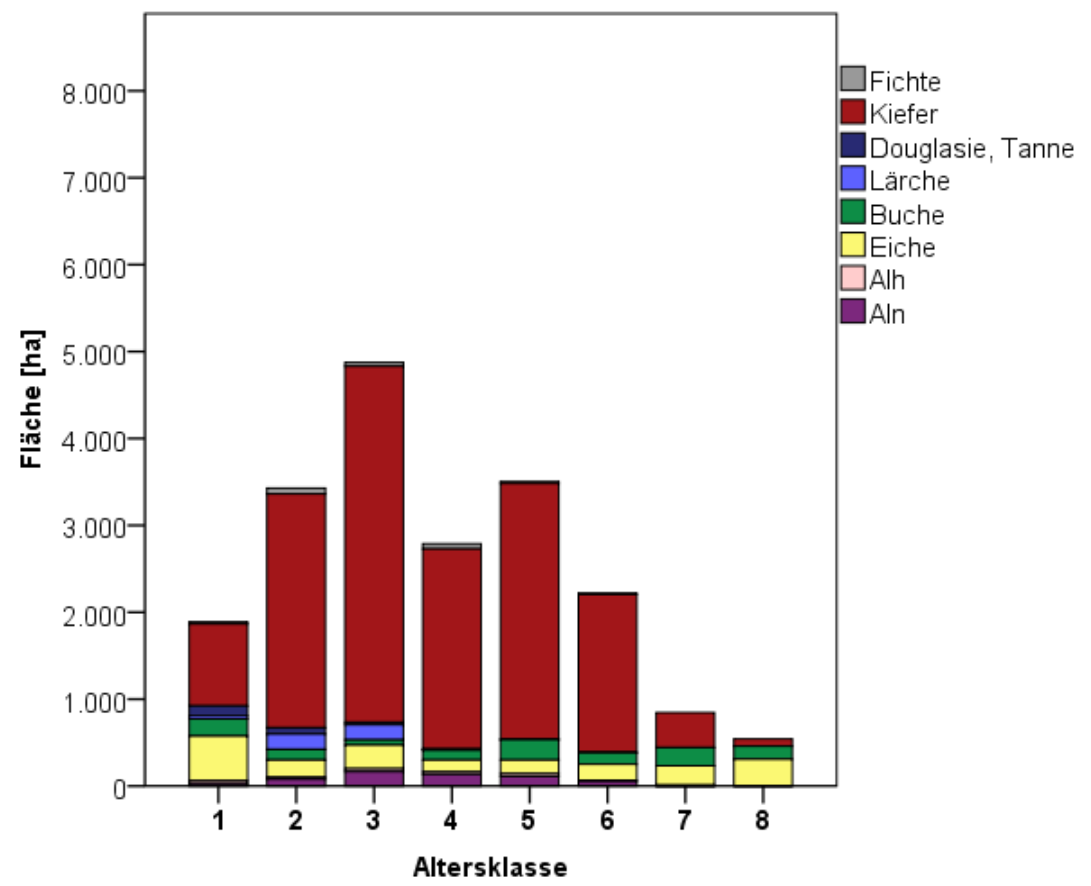

Abbildung 95: Altersklassenverteilung in der Region Fläming (eine Altersklasse umfasst 20 Jabre; ALh $=$ Laubholz mit hoher Umtriebszeit, ALn = Laubholz mit niedriger Umtriebszeit; im Datenspeicher Wald erfasste Flächen, Privatwald z. T. Stand 1994)

Zusammen mit bereits den Hauptbestand bildenden jungen Laubholzflächen ist der noch überschirmte Nachwuchs, hauptsächlich entstanden aus Voranbauten ehemaliger Kiefernreinbestände, zu betrachten (s. Abb. 96). Mehr als 6\% der gesamten Waldfläche weist bereits Verjüngung unter dem Altbestandschirm auf. Hiervon nehmen die Laubbäume, vor allem Rotbuche mit $46 \%$ und Eiche, einschließlich kleinerer Anteile an Roteiche, mit über $25 \%$, die weitaus größten Flächenanteile ein. Nadelbaumarten machen zusammen nur ca. $10 \%$ aus. Hierbei handelt es sich vor allem um Kiefer und bislang vergleichsweise sehr geringe Anteile an Douglasie. 
Zusammen ist damit in der Region in den vergangenen 20 Jahren mehr als doppelt so viel Fläche mit Laubholz verjüngt worden wie mit Nadelholz.

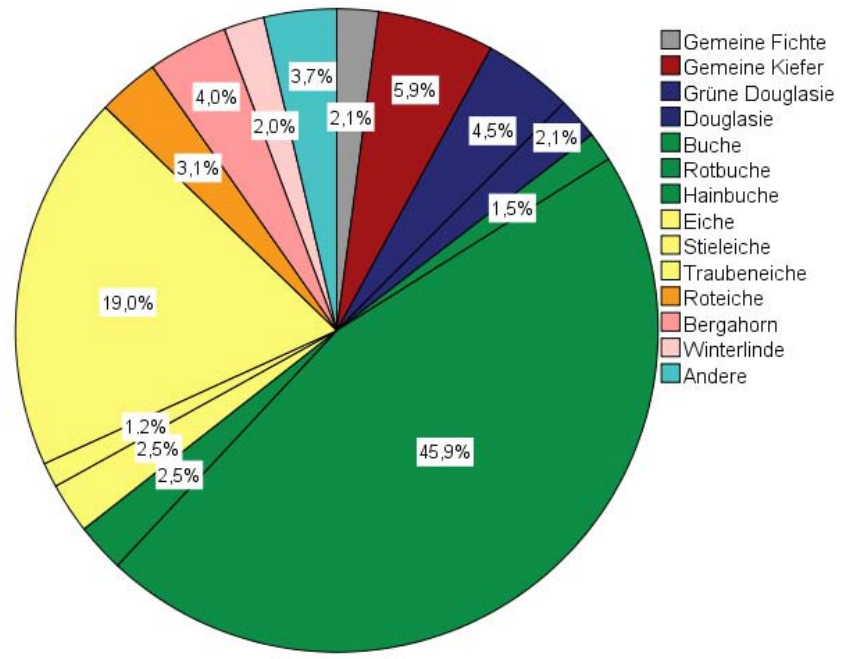

Abbildung 96: Flächenanteile [\%] der Baumarten im überschirmten Nachwuchs in der Region Fläming; Nachwuchs auf ca. $6 \%$ der Gesamtwaldfläche (im Datenspeicher Wald erfasste Flächen, Privatwald ₹. T. Stand 1994)

\subsubsection{Wichtigste Wachstumstrends durch den Klimawandel und Risikoveränderungen}

Die Trockenstressgefährdung nimmt bis zum Ende des Jahrhunderts generell stark zu. Am stärksten davon betroffen wird die Buche sein, die nach heutiger Standortseinschätzung noch auf über $70 \%$ der Fläche die Hauptbaumart der Stamm-Vegetationsform wäre. Im Zeitraum 2071 - 2100 würde fast die gesamte Waldfläche der Region für die Buche ein hohes Trockenstressrisiko aufweisen. Für die gegenüber Wasserdefiziten als toleranter eingeschätzten Baumarten Kiefer, Eiche und Douglasie stiege das Risiko bis dahin auf ein überwiegend mittleres Niveau. Nur 8 \% der Fläche, vorrangig im südöstlichen Teil der Region, würde auch für diese Baumarten eine hohe Trockenstressgefährdung aufweisen.

Bezüglich der standortsbezogenen Leistungsfähigkeit der Baumarten würde die Kiefer bis zum Ende des Jahrhunderts den relativ geringsten Leistungsabfall zeigen (Ø dGZ 7,1 statt heute 7,6), stärker ließen die Laubbaumarten Buche und Eiche nach, die in ihrem absoluten Leistungsniveau schon heute hinter der Kiefer zurückbleiben. Die absolut leistungsstärkste Baumart bliebe auch unter den veränderten Bedingungen am Ende des Jahrhunderts die Douglasie, die dann noch eine durchschnittliche $\mathrm{dGZ}_{\max }$-Leistungsklasse von 11,5 erreichen würde, wobei für diese Baumart der relativ stärkste Leistungsabfall gegenüber den heutigen Verhältnissen 
modelliert wurde. Dieser Befund bedarf aber auch noch im Vergleich zu den Ergebnissen für die Region Colbitz-Letzlinger Heide einer näheren Überprüfung.

Hinsichtlich der Sturmschadensgefährdung weisen die Laubbaumarten in der Region nur ein geringes Risiko auf. Ein auf der überwiegenden Fläche mittleres Sturmschadensrisiko ergibt sich aufgrund der großen Baumhöhen bei Erreichen einer Zielstärke von $55 \mathrm{~cm}$ für die Douglasie.

Nach den Ergebnissen der Klimaprojektionen wird die Waldbrandgefährdung in der Region sehr stark zunehmen. Bis zum Ende des Jahrhunderts soll sich danach die durchschnittliche Anzahl von Tagen mit erhöhter Waldbrandgefahr ungefähr verdoppeln. Für die älteren Eichenbestände der Region mit geringen Mischbaumartenanteilen besteht bereits heute ein erhöhtes Befallsrisiko durch die Schadinsekten der Eichenfraßgesellschaften. Aufgrund der zunehmenden Temperaturen ist von einem weiter steigenden Befallsrisiko auszugehen. Ebenso werden die Kieferngroßschädlinge, von denen im Gegensatz zur Colbitz-Letzlinger Heide im Fläming bisher keine übermäßige Gefährdung ausging, zu einer Gefahr für die Kiefernwälder werden, besonders auf den schwächeren Standorten und im Südosten der Region.

\subsubsection{Stabilisierung der vorhandenen Wälder in der Region Fläming}

\subsubsection{Kiefernbestände}

Die Kiefer ist und bleibt auf absehbare Zeit die Charakterbaumart der Region Fläming. Hierfür sprechen nicht nur ihr Altersklassenaufbau, sondern vor allem auch ihre bescheidenen Standortsansprüche und ihre gute ökologische Anpassung an die zu erwartenden Klimaänderungen. Die vielfältigen Gefährdungen, denen die Kiefer im Laufe ihres Bestandeslebens ausgesetzt ist, sind weniger eine Folge ihrer ökologischen Ansprüche, als vielmehr auf die vorherrschenden Klima- und Bodenverhältnisse in ihren Hauptanbaugebieten und auf ihre oft forstgeschichtlich bedingte Bewirtschaftung in Form ausgedehnter gleichaltriger Reinbestände zurückzuführen (ALTENKIRCH et al. 2002). Die verschiedenen Störungen wirken ausgesprochen altersspezifisch (s. Abb. 97) und lassen sich durch eine Erhöhung der Einzelbaumvitalität und eine Verbesserungen der Bestandesstrukturen begrenzen. Besonders in der auslaufenden Stangenholzphase und in der geringen Baumholzphase sind Kiefernbestände durch die Massenvermehrung von Kieferngroßschädlingen in ihrer Existenz gefährdet. Auch wenn sich die Schäden im Fläming in der Vergangenheit in Grenzen hielten, so ist doch zu befürchten, dass sich diese Situation in Folge der Klimaänderungen verschlechtert. 


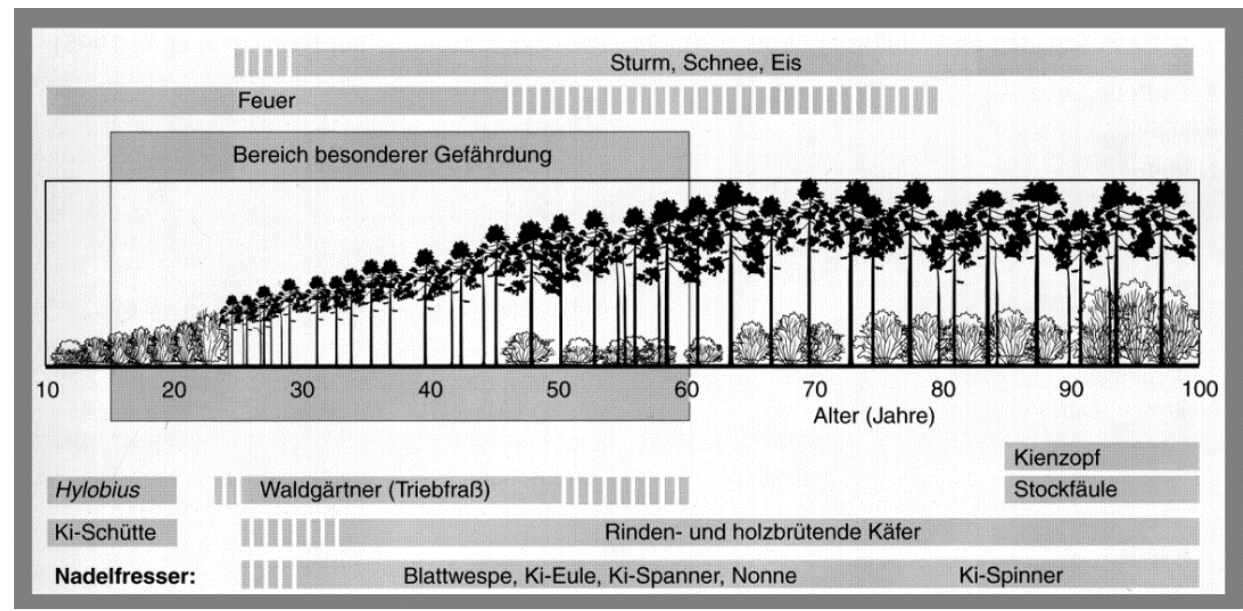

Abbildung 97: Gefährdungsprofil der Kiefer im schlagweisen Hochwald (ALTENKIRCH et al. 2002, verändert nach ОТТО 1994)

Die Risikovorsorge bei der Baumart Kiefer beginnt mit der Bestandesbegründung. Mit Blick auf die Qualitätsentwicklung sollten die Kiefernbestände so verjüngt werden, dass die Jungwüchse geschlossen erwachsen und Entwicklungsmöglichkeiten für Begleitbaumarten auf 10 bis $20 \%$ der Fläche bestehen. Insbesondere an Waldinnen- und Waldaußenrändern sind Begleitbaumarten zu erhalten und zu fördern, um der Waldbrandgefährdung entgegenzuwirken. Angemessene Wildstände sind hierfür unerlässliche Voraussetzung. Gepflanzte Roteichenriegel entlang von Hauptwegen haben sich als wirksame Maßnahme der Waldbrandvorsorge bewährt.

Im Zuge der Bestandespflege lässt sich die Vitalität und Stabilität der Kiefernbestände durch gezielte Maßnahmen sichern bzw. verbessern. Während in der Dickungs- und frühen Stangenholzphase die Qualitätsentwicklung noch Vorrang hat, ggf. Protzen entnommen werden und ansonsten die Kiefern im Dichtstand ausdifferenzieren und sich von Ästen im unteren Schaftabschnitt reinigen sollen, rücken zum Ende der Stangenholzphase die Entwicklung des Bestandesgefüges und der bestveranlagten Bäume in den Blickwinkel des Wirtschafters. Die Feinerschließung sollte möglichst 2-3 Jahre vor der Erstdurchforstung erfolgen, um das Stützgefüge der Bestände nicht auf einmal zu stark zu labilisieren. Bei der früh im Zuwachs kulminierenden Kiefer muss dann aber die Erstdurchforstung bei Oberhöhen von ca. 14 m einsetzen, um den Zuwachs auf ca. 200 bis 250 Z-Bäume/ha zu konzentrieren, lange, große Kronen zu erhalten und h/d-Werte unter $100 \mathrm{zu}$ sichern. Die Zahl der Z-Bäume darf sich nicht an der zu erwartenden Endbaumzahl bei Hiebsreife orientieren, sondern muss auch die Selbstdifferenzierung der Bestände und die Ausfallgefahren während des langen Produktionszeitraumes berücksichtigen. Bis zu einer Oberhöhe von ca. $18 \mathrm{~m}$ sollten die Bestände durch starke Eingriffe ausgeformt werden, wobei neben Bedrängern von Z-Bäumen auch Protzen und geschädigte 
Bäume zu entfernen sind. Außerdem sind die Begleit- und Mischbaumarten so zu begünstigen, dass sie im Unter- und Zwischenstand lebensfähig bleiben bzw. im Oberstand Entwicklungsmöglichkeiten haben. Zur Risikobegrenzung können insbesondere die beigemischten Laubbaumarten beitragen, die für ein feuchteres Bestandesinnenklima sorgen und deren Streu die physikalischen und chemischen Eigenschaften des Oberbodens zu Lasten der nadelfressenden Insekten verändern, die dort überwiegend überwintern. Da die Lichtbaumart Kiefer nicht in der Lage ist, einen arteigenen Unterstand zu erhalten, hängt die Durchforstungsart (Hoch- oder Niederdurchforstung) im Wesentlichen von Vermarktungs- bzw. ökonomischen Gesichtspunkten ab. Aus Gradationsgebieten der Forleule bzw. Nonne ist allerdings auch bekannt, dass diese Kiefernschädlinge bevorzugt Nadeln von unter- und zwischenständigen Kiefern fressen. Dies lässt dort starke Niederdurchforstung empfehlenswert erscheinen (HABERMANN 1995, HABERMANN u. BESTER 1997). Im Oberhöhenbereich zwischen 18 und $24 \mathrm{~m}$ empfiehlt sich dann ein Übergang zu mäßig starken Eingriffen, die dem Wachstumsgang der Kiefer folgen, die Stammzahl schonen und einen weitgehenden Kronenschluss sichern. Letzterer beugt einer zu starken Vergrasung mit hohen Verdunstungsverlusten vor und verringert die Waldbrandgefahr. Die Phase der Altdurchforstung endet, wenn zu erwarten ist, dass in den nächsten 10 Jahren angemessene Anteile des Bestandes den Zieldurchmesser erreichen und zur Zielstärkennutzung übergegangen werden kann. Der Oberstand sollte sich in dieser Phase überwiegend aus gut bekronten, wertvollen Kiefern zusammensetzen, die eine relativ große Durchmesserspreitung aufweisen, unregelmäßig über die Fläche verteilt sind und so eine zeitlich gestreckte Zielstärkennutzung erlauben. Den Unter- und Zwischenstand sollten Begleit- und Mischbaumarten mit wechselnden Anteilen und Dichten bilden. Der sich vielerorts stark ausbreitende Traubenkirschen-Unterstand lässt sich nicht mit einem vertretbaren Aufwand mechanisch entfernen. Als biologische Gegenmaßnahme bietet sich auf den besseren Standorten der Buchen- und auf den schwächeren Standorten der DouglasienVoranbau an, um die stark lichtbedürftige Traubenkirsche mit der Zeit auszudunkeln. Alternativ kämen nur noch das Abschneiden und die chemische Behandlung der Stöcke in Frage.

Mit der Zielstärkennutzung beginnt gleichzeitig die Verjüngungsphase über Naturverjüngungen bzw. Voranbauten. In der heutigen Klimastufe mäßig trockenes Tiefland (Tm) auf den ziemlich armen Standorten und in der Klimastufe trockenes Tiefland (Tt) auf den mäßig nährstoffhaltigen und armen Standorten wird auch in Zukunft die Kiefer vielfach die führende standortsgerechte Baumart sein. In ihrer Wuchsleistung und Qualität befriedigende Kiefernbestände sollten dort möglichst natürlich verjüngt werden. Dieses Vorgehen bietet erhebliche ökologische und ökonomische Vorteile gegenüber normalen Kulturen mit 8.000 - 10.000 Pflanzen/ha. Mit einer spontanen Kiefern-Naturverjüngung kann aber allgemein nicht gerechnet werden. Die dafür notwendigen Oberbodenzustände mit einer geringen Rohhumusauflage und einer lockeren Flechten-Astmoos-Bedeckung sind heute aufgrund der Stickstoffeinträge weitgehend verschwunden. Auf Standorten mit Vaccinium-, 
Deschampsia- oder Molinia-Decken ist i. d. R. eine vorherige streifen- bzw. plätzeweise Bodenbearbeitung unverzichtbar, während auf Standorten mit Reitgras-, Adlerfarnoder Brombeerdecken eine Naturverjüngung ohne Vollumbruch bzw. Herbizideinsatz ausgeschlossen ist. Die Wahl der Hiebsform, ob Zielstärkennutzung, Femelschlag, Saumschlag oder Kahlschlag hängt von der Wasserversorgung der Verjüngung (Vermeidung der Tellerwirkung), der Stabilität und Wertentwicklung der Altkiefern sowie von dem angestrebten Waldaufbau ab. Zur Vermeidung von Fällungsschäden an der Verjüngung sind eine strenge räumliche Ordnung und eine Feinerschließung unverzichtbar. Neben den Pionierbaumarten bieten sich als Mischbaumarten auf Teilflächen oder Fehlstellen ggf. Traubeneiche, Douglasie und Roteiche an.

Zur Risikovorsorge gehört allgemein auch eine Weiterentwicklung der Prognose- und Überwachungsmethoden für biotische und abiotischen Risiken. Bei den biotischen Risiken sollte das Augenmerk vor allem auf die nährstoffarmen Standorte mit schwacher Wasserversorgung gerichtet werden, die als typische Gradationsgebiete der Kieferngroßschädlinge gelten. Für die Waldbrandüberwachung bieten sich Kamerasysteme an. Gerade im Fläming mit seinen wenigen Oberflächengewässern sollte auch an den Ausbau einer ausreichenden Zahl Hydranten bzw. unterirdischen Wasserspeichern für etwaige Löscheinsätze gedacht werden.

\subsubsection{Eichenbestände}

Die Eiche ist die wichtigste Laubbaumart im Fläming. Aufgrund ihrer breiten Nährstoffamplitude und ihrer Unempfindlichkeit gegenüber Wassermangel bzw. Wasserüberschuss ist sie heute als führende oder beigemischte Baumart auf allen Standortsformen vertreten. Zum Ende des Jahrhunderts werden erhöhtes Trockenstressrisiko und ein Leistungsabfall ihre Anbauwürdigkeit im östlichen Teil der Region einschränken. Die Sturmgefährdung ist bei dieser mit ihrem Pfahlwurzelsystem gut im Boden verankerten Baumart eher zu vernachlässigen. Die zukünftig erwarteten milderen Winter und verlängerten Vegetationszeiten erhöhen die Gefahr der Koinzidenz zwischen dem Schlüpfen der Raupen der Schmetterlinge der Eichenfraßgesellschaften und dem Austreiben der Eichen. Nach den Auswertungen des Waldschutzmeldewesens steigt die Gefährdung signifikant mit dem Alter der Bestände und der Verschlechterung des Bodenwasserangebotes. Dies spricht für eine gestaffelte Durchforstung mit starken, die Bestände ausformenden Eingriffen in der Jugend und abnehmenden, die Stammzahl schonenden Durchforstungsstärken ab Oberhöhen von etwa 20 bis $24 \mathrm{~m}$.

Ein solches Vorgehen entspricht auch dem Wachstumsgang der Eiche. Die frühe Kulmination der Zuwachsgrößen und die dadurch mit dem Alter stark nachlassende Plastizität der Kronen erfordern eine früh einsetzende Pflege zur rechtzeitigen Herausarbeitung vitaler, gut entwickelter und möglichst symmetrischer Lichtkronen der Z-Bäume. Fehler in frühen Bestandesphasen sind nicht oder nur noch 
sehr schwer korrigierbar. Sie ziehen Zuwachsverluste und eine Verlängerung der Produktions- und Gefährdungszeiträume nach sich und führen somit langfristig zu betriebswirtschaftlichen Einbußen. Neben dem rechtzeitigen Beginn der Pflege erfordert die Eiche wie kaum eine andere Baumart auch während des gesamten Bestandeslebens Kontinuität und Stetigkeit in der Behandlung. In höherem Alter abrupt bzw. zu stark geführte Eingriffe bergen die Gefahr von Entwertungen durch Wasserreiser, Klebäste und Jahrringsprünge sowie von Stammzahlverlusten, ohne dass die damit verbundenen Standraumerweiterungen adäquat genutzt werden könnten. Angesichts des sehr langen Produktionszeitraumes sollte die anfängliche Z-Baumzahl deutlich über der angestrebten Endbaumzahl hiebsreifer Bestände liegen, um etwaigen Ausfällen oder einem sozialen bzw. qualitativen Umsetzen vorzubeugen. Empfohlen werden können Z-Baumzahlen von ca. 160 bis 200 Stück/ha.

Trotz hoher Bestandesbegründungskosten ist die Pflanzung mit ca. 10.000 Stück/ha der Regelfall für die Begründung von Eichenbeständen auf der Freifläche bzw. unter einem lichten Schirm mit kurzfristiger Räumung. Für teilflächige Bestandesbegründung auf Störungslöchern kommen auch Trupppflanzungen in Frage. Die Voraussetzungen hierfür sind ein ausreichendes Strahlungsangebot und eine ausreichende Luftbewegung um Frostschäden bzw. Mehltaubefall vorzubeugen. Der Mitanbau einer dienenden Baumart hat sich aufgrund der Wuchsdynamik und Konkurrenzkraft der eingebrachten Buchen, Hainbuchen oder Winterlinden häufig als problematisch erwiesen. Ihm ist ein Unterbau bei Oberhöhen von 20 bis $24 \mathrm{~m}$ vielfach vorzuziehen. Schattbaumarten in Eichenbeständen dienen nicht nur der Schaftpflege, sondern verhindern auch eine Vergrasung der Bestände mit hohen Transpirationsraten.

Das Vorgehen in der Endnutzung sollte sich nach dem Verjüngungsziel in der Folgegeneration richten. In der heutigen Klimastufe mäßig trockenes Tiefland (Tm) auf den kräftigen und mäßig nährstoffhaltigen Standorten sowie in der Klimastufe trockenes Tiefland ( $\mathrm{Tt}$ ) auf den kräftigen Standorten kann nach den Ergebnissen dieser Studie auch künftig mit Bestandeszieltypen mit führender Eiche geplant werden. Auf den schwächeren und meist auch schlechter wasserversorgten Standorten ist die Eiche hingegen nur noch als Mischbaumart vorzusehen, zumal sich kritische Stimmen zu ihrer Vitalität und Stabilität mehren (KÄTZEL et al. 2012). Für die Endnutzung in den Eichen-Altbeständen heißt dies, dass Bestände mit dem Verjüngungsziel Eiche noch keinen Nachwuchs Schatten ertragender Baumarten aufweisen und eine ausreichende Zahl zielstarker Bäume enthalten sollten, um ohne Hiebsopfer über Kleinkahlschläge oder kurzfristige Schirmschläge die nächste Eichengeneration zu etablieren. In Beständen, die nicht wieder in Eiche verjüngt werden sollen, bietet sich eine gestreckte Zielstärkennutzung an. 


\subsubsection{Buchenbestände}

Mit einem Flächenanteil von ca. $6 \%$ ist die Buche die zweitwichtigste Laubbaumart im Fläming. Die Bestände ab der zweiten Altersklasse stocken fast ausschließlich auf den besser versorgten Standorten, während in der ersten Altersklasse auch Buchenbestände aus Voranbauten unter Kiefer vertreten sind, die bis in den ziemlich armen Standortsbereich reichen. Häufig handelt es sich um alte Waldstandorte mit Habitatkontinuität, die naturschutzfachlich einen besonderen Wert haben. Der Klimawandel wird zu einer gravierenden Verschlechterung der Anbaumöglichkeiten der Buche führen. Fast in der ganzen Region wird die Wasserversorgung für die Buche unzureichend sein und es dementsprechend zu einem deutlichen Leistungsabfall kommen. Gleichzeitig ist mit einer Zunahme der biotischen Schäden durch BuchenPrachtkäfer und Buchen-Rindennekrosen (Buchen-Schleimfluss) sowie der abiotischen Schäden durch Trocknis und Sonnenbrand zu rechnen. Wegen der früher einsetzenden Vegetationszeit nimmt das Spätfrostrisiko für nicht überschirmte Buchenverjüngungen zu. Vor diesem Hintergrund muss es das Ziel der Buchenwirtschaft sein, die Buche als führende Baumart auf denjenigen Standorten wieder zu verjüngen bzw. ggf. einzubringen, wo noch eine möglichst gute, risikoarme Entwicklung der Volumen- und Werterzeugung zu erwarten ist bzw. wo naturschutzfachliche Gründe diesbezüglich deutliche Zugeständnisse rechtfertigen. Ein weiterer künftiger Anbaubereich ergibt sich in Mischung mit führender Douglasie oder Roteiche, um diese eingeführten Baumarten in die heimische Flora und Fauna zu integrieren, als dienende Baumart in Eichenbeständen sowie als Bodenschutz in Kiefernbeständen auf den wenigstens mäßig versorgten, mäßig trockenen Standorten.

Die Behandlungskonzepte für die vorhandenen Buchenbestände müssen so angelegt sein, dass sie vertikal wie horizontal gut strukturierte Bestände mit einem möglichst lange lebensfähigen Unterstand erhalten, um ein feuchteres Bestandesinnenklima zu wahren, eine Untersonnung zu verhindern und einer Verhagerung entgegenzuwirken. Hierzu ist die weitgehende Selbstdifferenzierung junger Buchenbestände im Dichtschluss ebenso zu nutzen, wie die ökologischen Eigenschaften Schattenerträgnis und Kronenplastizität in späteren Entwicklungsphasen. In der Stangenholzphase sollte man daher Buchen-Reinbestände sich selbst ausdifferenzieren lassen und lediglich von Protzen befreien, während in Buchen-Mischbeständen die Mischwuchsregulierung hinzu kommt, die dann aber bereits auf die Pflege der besten Bäume ausgerichtet sein sollte. Als Durchforstungsart bietet sich die starke Hochdurchforstung an, die zwei- oder mehrschichtige Bestände anstrebt. Bei ihr konzentriert sich die Pflege auf die Förderung der Zukunftsbäume durch Entnahme schlechter veranlagter Bäume im Herrschenden. Sie setzt beim Erreichen einer astfreien Schaftlänge von ca. 7 bis $8 \mathrm{~m}$ ein. Zu diesem Zeitpunkt sollten die relativ besten 100 bis 160 Bäume je Hektar als Z-Bäume ausgewählt, markiert und durch die Entnahme von 1 bis 3 Bedrängern in ihrer Kronenentwicklung gefördert 
werden. In qualitativ unbefriedigenden Beständen sind auch Z-Baumgruppen anzunehmen und am Rand freizustellen. Erwünschte Mischbaumarten sind anteilig bei den Z-Bäumen in die bevorzugte Pflege einzubeziehen. Ab Oberhöhen von ca. $25 \mathrm{~m}$ nimmt die Durchforstungsintensität ab. Habitatbaumgruppen werden ebenfalls in dieser Bestandesentwicklungsphase ausgewählt und dauerhaft gekennzeichnet. Die eigentliche Zielstärkennutzung beginnt, sobald ca. 10 Bäume je ha die individuelle Hiebsreife erreicht haben. Dabei ist auf eine räumliche Ordnung mit einem Nutzungs- und Verjüngungsfortschritt von innen nach außen bzw. von der Bestandesmitte zu den Rückegassen zu achten. Die ungleiche Verteilung gut und schlecht veranlagter Bäume sollte dazu genutzt werden, um zunächst in qualitativ schlechten Partien gezielt die Voraussetzungen für das Ankommen von Verjüngung zu schaffen oder um Voranbauten zu etablieren.

Für die jeweils mehr als $2 \%$ der Waldfläche in der Region Fläming einnehmenden Lärchen- und Weichlaubholz̧bestände sind die Empfehlungen aus den anderen beiden Regionen weitgehend übertragbar.

\subsubsection{Standortsgemäßer Waldumbau}

In der biologischen Produktion müssen die Forstbetriebe sowohl die Dynamik der Standorte, die ökologischen Ansprüche der Baumarten als auch die Wechselbeziehungen zwischen den Gliedern der Waldlebensgemeinschaften beachten und aufeinander abstimmen. Die Ergebnisse der Regionalstudie zeigen für den Fläming gravierende klimabedingte Standorts-, Leistungs- und Risikoverschiebungen auf, die eine Weiterentwicklung der bisherigen Bestandeszieltypenplanung notwendig machen (s. Tab. 19). Die mit Einführung des naturnahen Waldbaus in den 90erJahren des letzten Jahrhunderts in Sachsen-Anhalt vollzogene starke Anlehnung der Baumartenwahl an die Stammvegetationsformen bedarf einer Klimaanpassung. Dies betrifft insbesondere den vielerorts geplanten Baumartenwechsel von der genügsamen Kiefer zu anspruchsvolleren Baumarten und die starke Ausweitung des Buchenanbaus zur Erhöhung der Naturnähe. Angesichts der begrenzten Auswahl an auch künftig noch standortsgemäßen Baumarten, erhält die Frage nach einer stärkeren Beteiligung anbauwürdiger und ökologisch zuträglicher eingeführten Baumarten wie Douglasie, Küstentanne und Roteiche einen neuen Stellenwert. Während ökonomische Aspekte dafür sprechen, bestehen anderseits erhebliche naturschutzfachliche Vorbehalte. Es ist daher erforderlich, mit großer Sorgfalt, Augenmaß und unter Beachtung räumlicher Aspekte die Weichen neu zu stellen und den gravierenden Auswirkungen des Klimawandels auf die Wälder zu begegnen. Der ungleiche Altersklassenaufbau und die jährlich anstehenden Verjüngungsflächen erlauben ein schrittweises Vorgehen und eine ständige Rückkoppelung der Planung mit den zwischenzeitlichen Fortschritten der Klimafolgenforschung. 
Tabelle 19: Bestandeszieltypen für die wichtigsten Standorte der Region Fläming (nach MLU LSA 2003, verändert)

\begin{tabular}{|c|c|c|c|c|}
\hline $\begin{array}{l}\text { Klima- } \\
\text { stufe }\end{array}$ & $\begin{array}{l}\text { Stamm- } \\
\text { StaO- } \\
\text { formen- } \\
\text { Gruppe }\end{array}$ & $\begin{array}{c}\text { Flächen } \\
\text { anteil } \\
{[\%]}\end{array}$ & Stamm-Vegetationsform & Bestandeszieltypen \\
\hline \multirow[t]{3}{*}{$\mathrm{Tm}$} & $\mathrm{K} 2$ & 3 & $\begin{array}{l}\text { Riesenschwingel-TEi-Bu- } \\
\text { Wald }\end{array}$ & $\begin{array}{l}\text { BU-EI, EI-BU, BU-NDB, ELÄ- } \\
\text { SCH, KI-SCH }\end{array}$ \\
\hline & M2 & 45 & Hainrispen-TEi-Bu-Wald & $\begin{array}{l}\text { BU-EI, EI-BU, BU-NDB, KI-LIB, } \\
\text { KI-SCH }\end{array}$ \\
\hline & Z2 & 23 & $\begin{array}{l}\text { Sauerklee-Blaubeer-TEi- } \\
\text { Bu-Wald }\end{array}$ & $\begin{array}{l}\text { BU-EI, BU-NDB, KI-LIB, KI, } \\
\text { DGL }\end{array}$ \\
\hline \multirow[t]{3}{*}{$\mathrm{Tt}$} & $\mathrm{K} 2$ & 2 & \multicolumn{2}{|c|}{$\begin{array}{l}\text { Riesenschwingel-HBu-WLi- TEI-WLI-HBU, EI-BU, ELÄ- } \\
\begin{array}{ll}\text { TEi-Wald } & \text { SCH, KI-SCH }\end{array}\end{array}$} \\
\hline & M2 & 12 & $\begin{array}{l}\text { Hainrispen-Hainbuchen- } \\
\text { Winterlinden-TEi-Wald }\end{array}$ & $\begin{array}{l}\text { TEI-WLI-HBU, Ei-BU, KI-LIB, } \\
\text { KI-SCH, ELÄ-SCH, DGL-BU, KI }\end{array}$ \\
\hline & $\mathrm{Z} 2$ & 6 & $\begin{array}{l}\text { Sauerklee-Blaubeer-TEi- } \\
\text { Wald }\end{array}$ & EI-BI, KI-LIB, KI \\
\hline \multicolumn{2}{|c|}{ Sonstige* } & 9 & & \\
\hline
\end{tabular}

* Standorte mit einem Flächenanteil $<1 \%$

Die Schwerpunkte des standortsgemäßen Waldumbaus sind:

- im mäßig trockenen Tiefland auf mäßig nährstoffhaltigen Standorten:

- Überführung von Kiefernreinbeständen in Kiefern-Laubholz-Mischbestände unter Beteiligung von Eiche, Roteiche oder Buche und Birke

- Überführung von Kiefernreinbeständen in Kiefern-Douglasien/Küstentannen-Mischbestände unter Beteiligung von Buche und Birke

- Überführung von Kiefernreinbeständen in Douglasien/KüstentannenLaubholzmischbestände unter Beteiligung von Buche oder Roteiche und Birke

- Überführung von Buchenreinbeständen in Buchen-Nadelholzmischbestände unter Beteiligung von Douglasie, Küstentanne oder Europäischer Lärche

- im mäßig trockenen Tiefland auf ziemlich armen Standorten:

- Überführung von Kiefernreinbeständen in Kiefern-Laubholz-Mischbestände unter Beteiligung von Eiche oder Roteiche und Birke

- Überführung von Kiefernreinbeständen in Kiefern-Douglasien/Küstentannen-Mischbestände unter Beteiligung von Birke 
- Überführung von Kiefernreinbeständen in Douglasien-KüstentannenMischbestände unter Beteiligung von Birke

- im trockenen Tiefland auf mäßig nährstoffhaltigen Standorten:

- Überführung von Kiefernreinbeständen in Kiefern-Laubholz-Mischbestände unter Beteiligung von Eiche oder Roteiche und Birke

- Überführung von Kiefernreinbeständen in Kiefern-Douglasien/Küstentannen-Mischbestände unter Beteiligung von Birke

Die besser nährstoff- und meist auch besser wasserversorgten Standorte sollten der Traubeneiche bzw. der Buche vorbehalten sein. Etwaig vertretene Kiefernbestände sollten in Bestände mit führender Buche, Traubeneiche oder auch Roteiche überführt werden.

Bei den großflächig geplanten Voranbauten unter Kiefer sollte darauf geachtet werden, dass die Kiefer einen ausreichenden Altersvorsprung hat, denn sobald Buchen/Roteichen oder Douglasien/Küstentannen in ihre Kronen vordringen, stockt sie im Zuwachs und erreicht nicht mehr ihr Produktionsziel. Bei II. Ertragsklasse sollte mit Voranbauten der genannten Laubbaumarten bis zum Alter 60 Jahre, der genannten Nadelbaumarten bis zum Alter 90 Jahre gewartet werden. Dies schließt nicht aus, dass diese Baumarten auf Löchern und in qualitativ unbefriedigenden Partien bereits früher eingebracht werden. In jedem Fall ist bei Voranbauten auf eine strenge räumliche Ordnung mit Erschließungslinien, Voranbau- und Fällungszonen zu achten, damit die Voranbau-Investitionen nicht durch Fällungsund Rückeschäden wieder zunichte gemacht werden. Ebenso unverzichtbar ist eine Pflanzung der Folgebaumarten in ausreichend engen Verbänden. Dies belegen zahlreiche in ihrer Qualitätsentwicklung unbefriedigende Voranbauten unter Kiefernaltholzschirm, wo der geringe Schirmdruck nur in einem begrenzten Maße den Seitendruck durch engere Pflanzverbände auszugleichen vermochte (SPELLMANN 2008). 



\section{Gefährdungspotenziale in der Region Colbitz-Letzlinger Heide}

\subsection{Veränderungen des Trockenstressrisikos}

Die Colbitz-Letzlinger Heide weist mit 41.754 ha die größte Waldfläche der drei Modellregionen auf. Ähnlich wie im Fläming stellen die Kiefer gefolgt von der Eiche die wichtigsten Baumarten dar.

Auch die klimatischen Verhältnisse unterscheiden sich nicht wesentlich von den Bedingungen im Fläming. Folglich wird auf rund $30 \%$ der Waldfläche (12.736 ha) für die Fichte und auf etwa $21 \%$ (8.955 ha) der Fläche für die Buche ein mittleres Risiko berechnet (s. Tab. 20, Abb. 99 und Abb. 100). Diese Standorte weisen häufig eine geringe nutzbare Feldkapazität $(<100 \mathrm{~mm})$ auf, stellen unter den heutigen klimatischen Verhältnissen für die Kiefer, Eiche und Douglasie jedoch noch ein geringes Gefährdungspotenzial dar (s. Abb. 98 und Abb. 101).

Tabelle 20: $\quad$ Fläche [ha] der Hauptbaumarten nach den definierten Trockenstressrisikoklassen in der Region Colbitz-Letzlinger Heide

\begin{tabular}{l|rr|rr|rr}
\multicolumn{1}{r|}{ Periode } & \multicolumn{2}{|c|}{ Fichte } & \multicolumn{2}{|c|}{ Buche } & \multicolumn{2}{|c}{ Kiefer / Eiche / Douglasie } \\
Risiko & $1971-2000$ & $2071-2100$ & $1971-2000$ & $2071-2100$ & heute & $2071-2100$ \\
\hline gering & 29.135 & 15 & 32.916 & 15 & 41.871 & 117 \\
mittel & 12.736 & 510 & 8.955 & 3.867 & 0 & 41.754 \\
hoch & 0 & 41.346 & 0 & 37.989 & 0 & 0 \\
\hline
\end{tabular}

Zum Ende des Betrachtungszeitraumes wird das Wasserdefizit $(\mathrm{KWB}+\mathrm{nFK})$ in der Modellregion Colbitz-Letzlinger Heide deutlich zunehmen. Insbesondere der erhöhte Verdunstungsanspruch der Bestände führt dazu, dass das Defizit im Mittel um rund $250 \mathrm{~mm}$ auf verbreitet -300 bis $-350 \mathrm{~mm}$ ansteigt. Die klimatischen Bedingungen werden folglich für die Fichte auf ca. $99 \%$ und für die die Buche auf rund $90 \%$ der Waldfläche so ungünstig, dass das Risiko durch Trockenstress als hoch bewertet werden muss. Für die Kiefer, Eiche und Douglasie werden die Waldflächen in der Modellregion annähernd vollständig mit einem mittleren Trockenstressrisiko belegt.

In Anhang 9 bis Anhang 12 ist die zeitliche Entwicklung des Trockenstressrisikos für die untersuchten Perioden dargestellt. Die Auswertung zeigt, dass, 
vergleichbar zur Modellregion Fläming, das Gefährdungspotenzials durch Trockenstress bei der Fichte und Buche bereits in der Periode 2011 - 2040 signifikant zunimmt, bei der Kiefer, Eiche und Douglasie in der Periode 2041 - 2070.

Kiefer
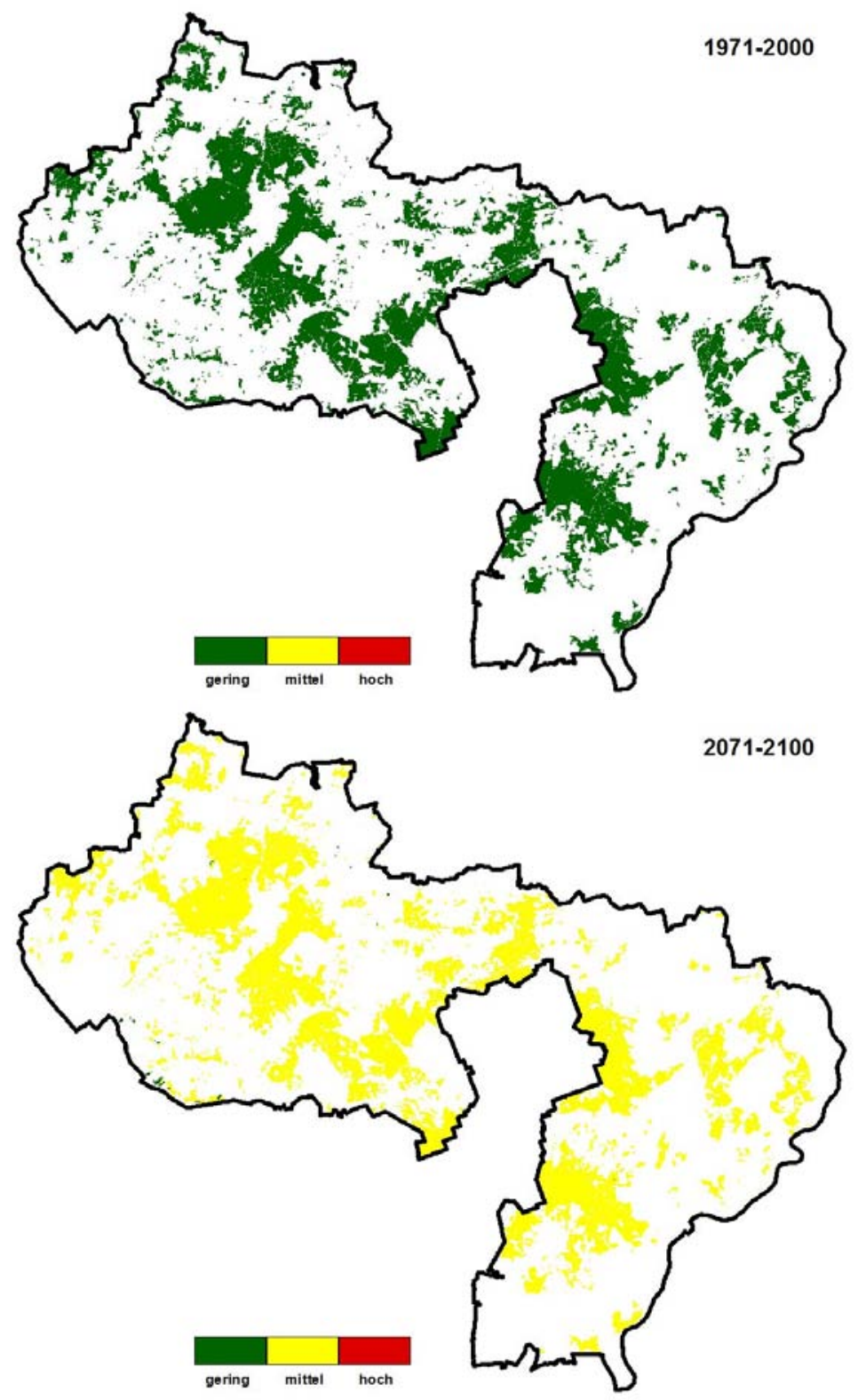

Abbildung 98: Trockenstressrisiko für die Kiefer in der Region Colbitz-Letzlinger Heide 
Fichte
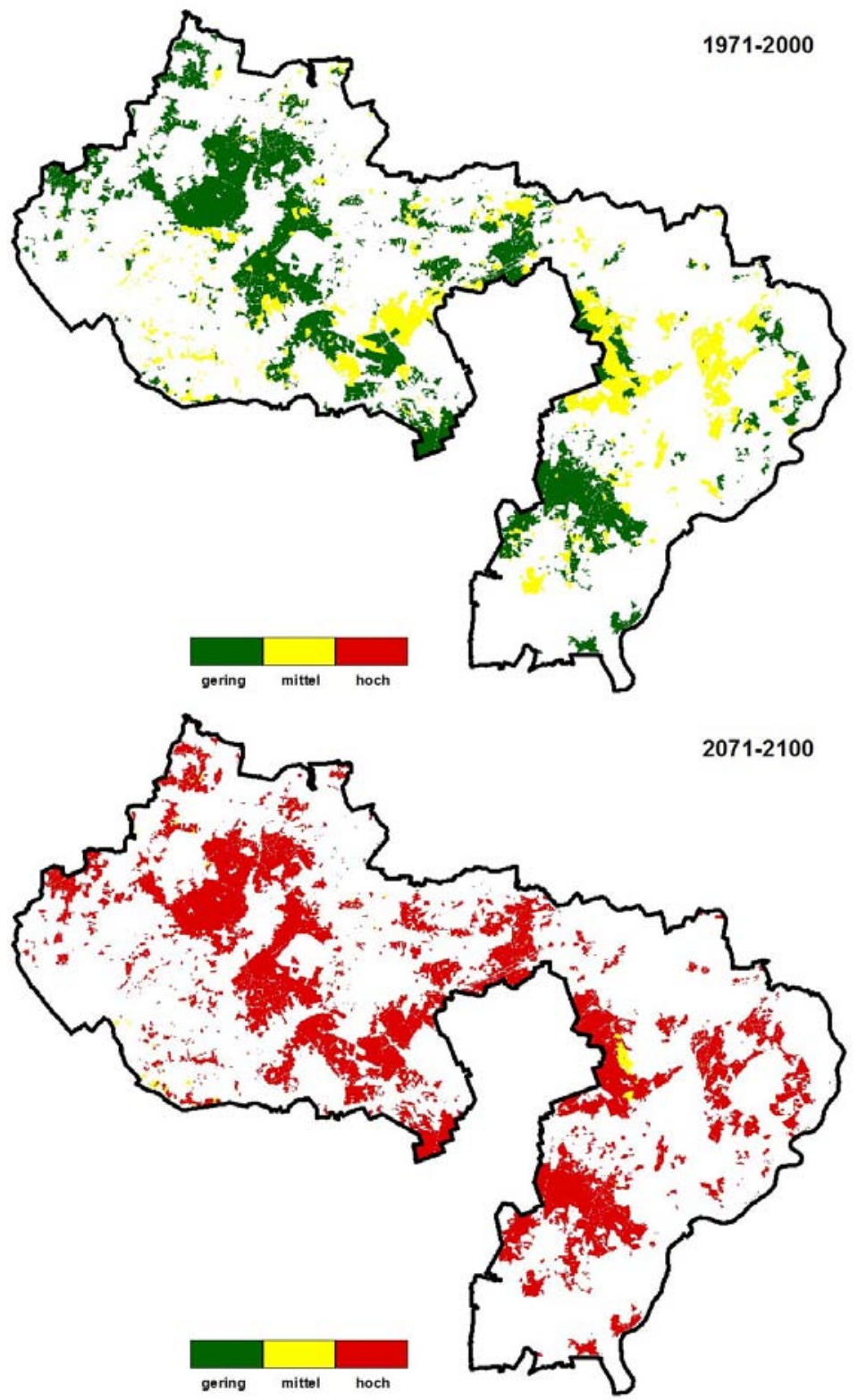

Abbildung 99: Trockenstressrisiko für die Fichte in der Region Colbitz-Letzlinger Heide 
Buche

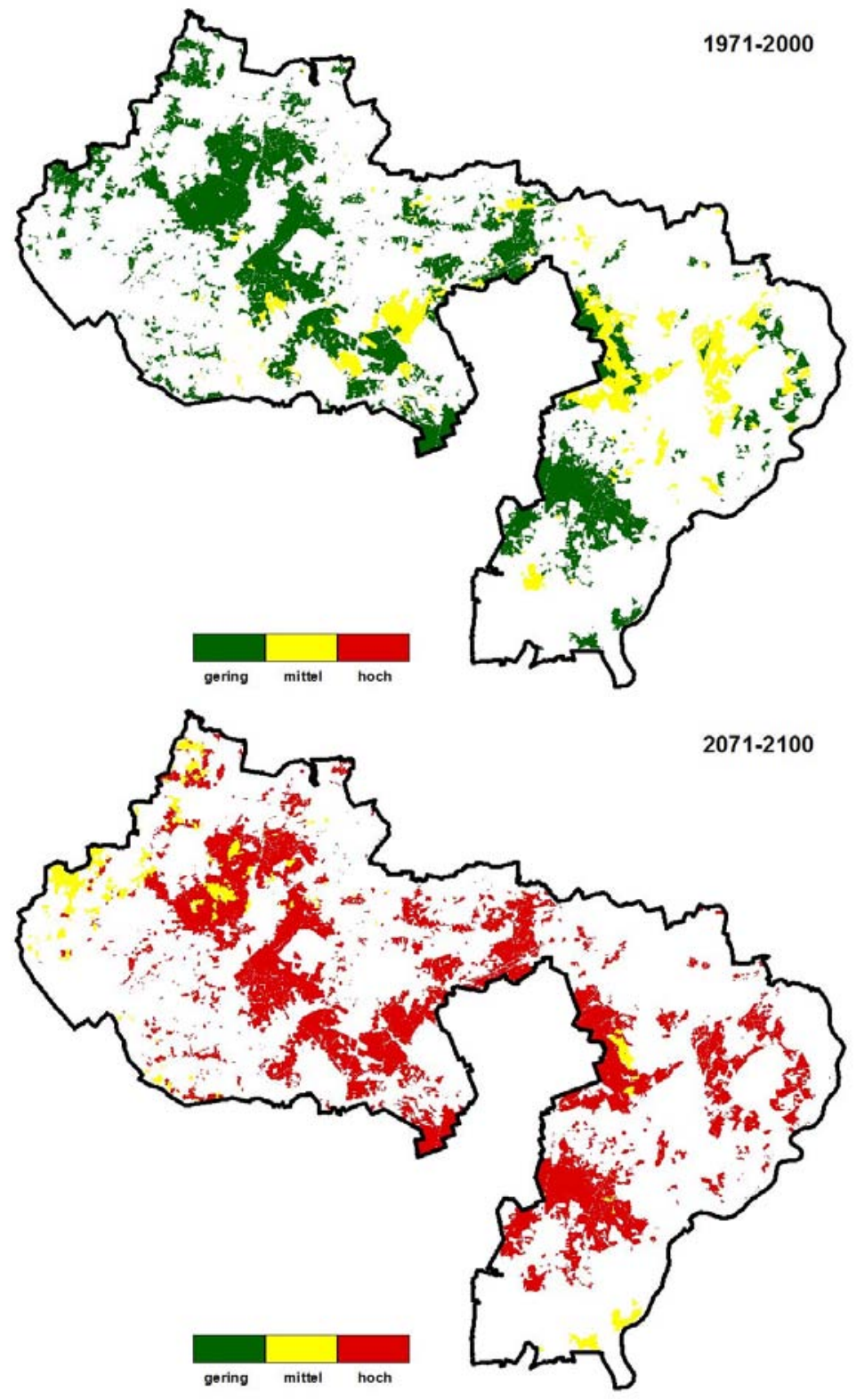

Abbildung 100: Trockenstressrisiko für die Buche in der Region Colbitz-Letzlinger Heide 
Eiche und Douglasie
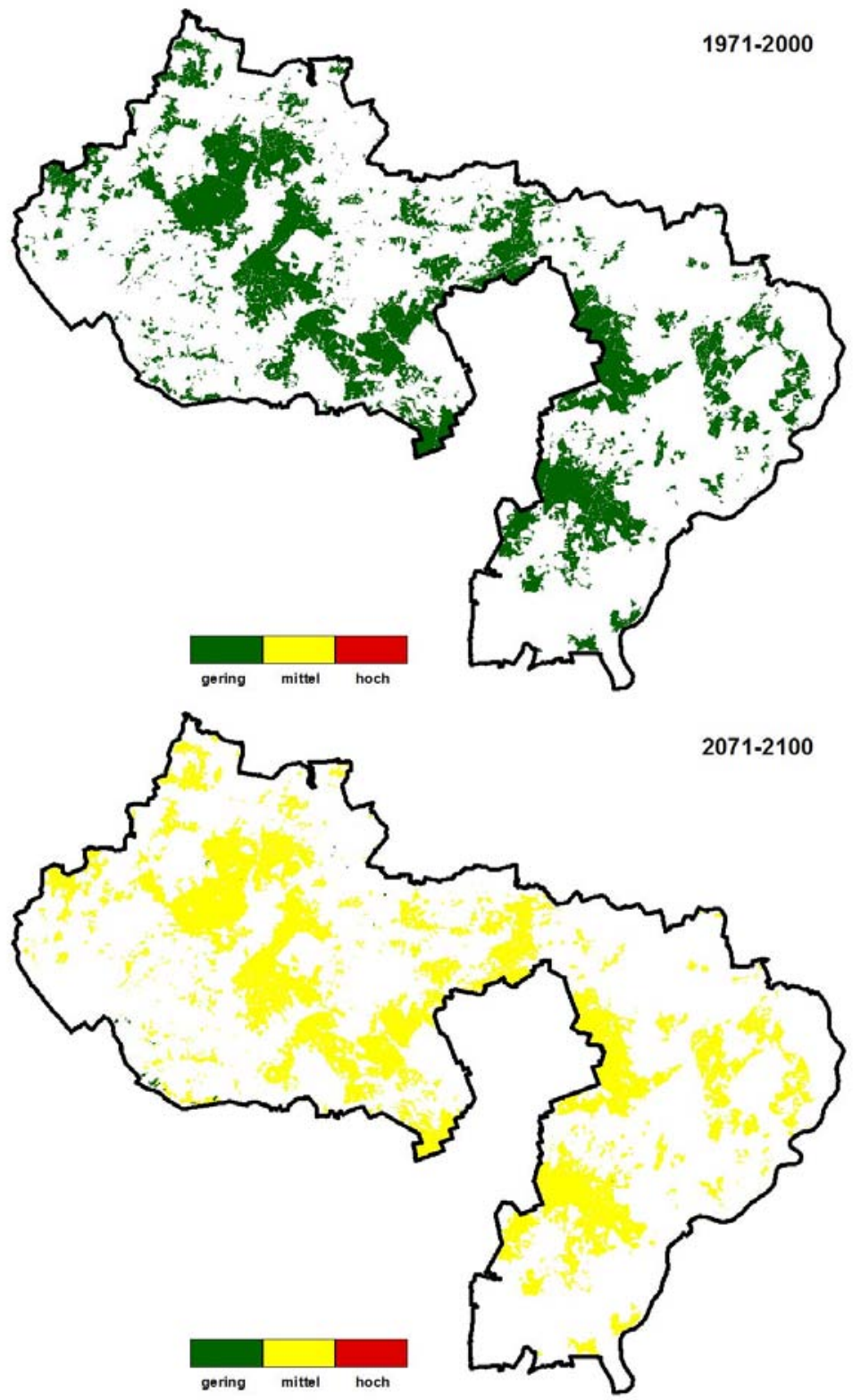

Abbildung 101: Trockenstressrisiko für die Eiche und Douglasie in der Region Colbitz-Letzlinger Heide 


\subsection{Veränderungen der Stickstoffeinträge}

Die Colbitz-Letzlinger Heide ist im Vergleich der Regionen, die Region mit den geringsten Stickstoffeinträgen (s. Abb. 14). Innerhalb dieser Region sind die höchsten Einträge im Bereich um die Stadt Klötze zu verzeichnen (s. Abb. 102).
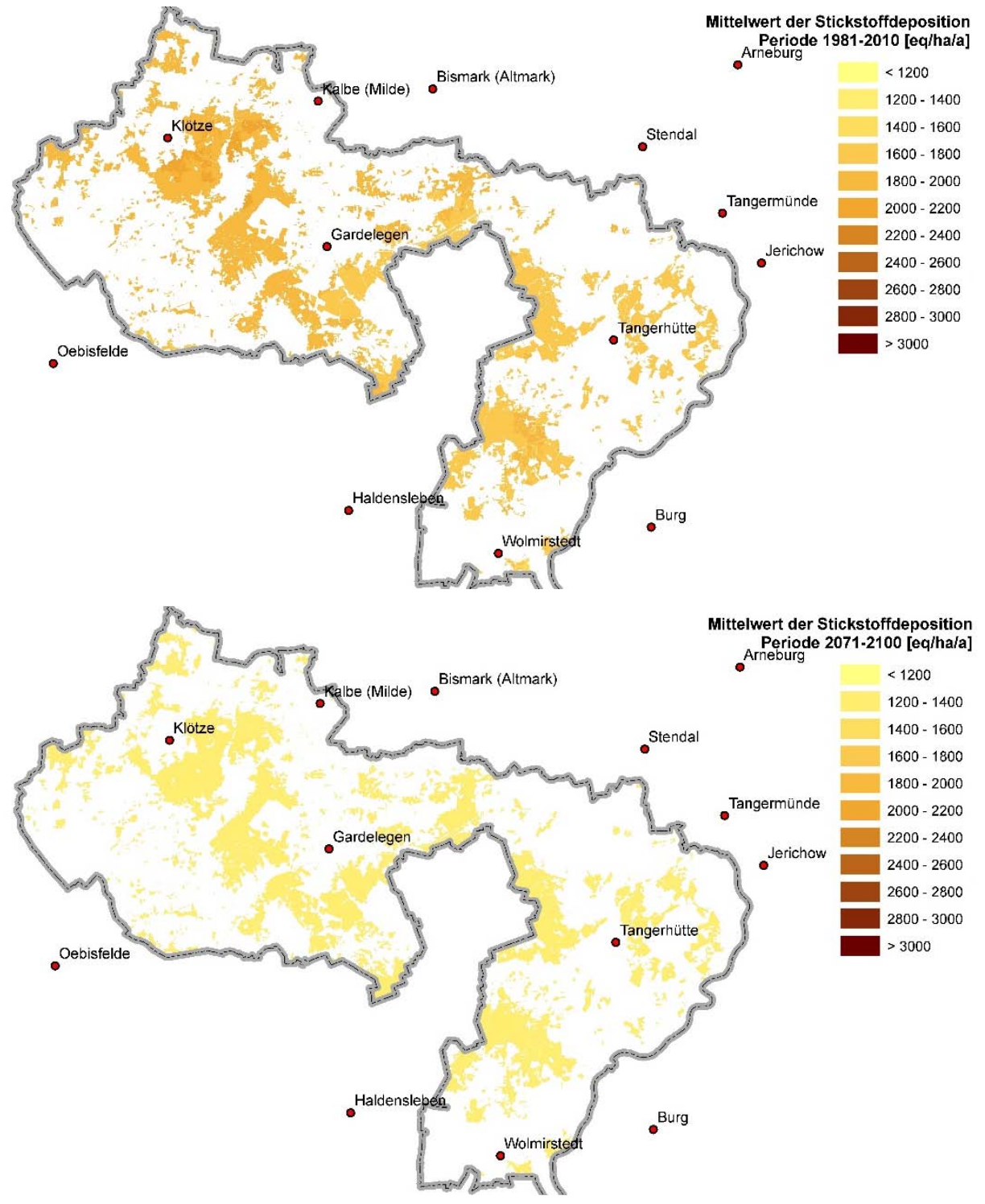

Abbildung 102: Regionale Verteilung der Stickstoffdepositionen (1981 - 2010 oben) und (2071 - 2100 unten) in der Region Colbitz-Letzlinger Heide 
Nach dem vorgegebenen Szenario (s. Tab. 8) werden sich auch in dieser Region die Stickstoffeinträge im Vergleich zur Periode 1981 - 2010 während der nächsten Jahrzehnte weiter verringern. Trotz des weiteren Eintragsrückganges reicht die Stickstoffmenge problemlos aus, um die Stickstoffversorgung der in dieser Region vorwiegend stockenden Kiefern sicherzustellen, da die Kiefer durch sehr geringe Nettonährstoffaufnahmeraten gekennzeichnet ist (KLINCK et al. 2012, HAGEMANN et al. 2008).

\subsection{Projektion der Standortleistungsfähigkeit für die Region Colbitz-Letzlinger Heide}

Mit einem Flächenanteil von $86 \%$ dominiert die Kiefer die aktuelle Bestockung in der Region Colbitz-Letzlinger Heide. Die Eiche weist einen Flächenanteil von 7 \% auf und $5 \%$ entfallen auf die Buche. Eine räumlich explizite Darstellung der Standortleistungsfähigkeit unter gegenwärtigen und zukünftigen Klimabedingungen beinhalten Abbildung 103 und Abbildung 104 für die beiden am häufigsten in der Region vorkommenden Baumarten Kiefer und Eiche. Hierbei wurden für alle Teilflächen jeweils eine hypothetische Bestockung mit Kiefer bzw. Eiche angenommen.

Die Standortleistungsfähigkeit für die Baumart Kiefer stellt sich in der Region Colbitz-Letzlinger Heide unter Status quo sehr homogen dar (s. Abb. 103). Nur für einzelne Standorte ergibt sich ein von der dGZ $\max _{\text {Kasse }}$ 6-7 nach oben bzw. unten abweichendes Wuchsleistungspotenzial. In der Projektionsperiode 2011 - 2040 zeigt sich aufgrund des verbesserten Temperaturangebotes fast überall eine leichte Steigerung der Standortleistungsfähigkeit. Durch zunehmende Trockenheit verringert sich das Wuchspotenzial in der Projektionsperiode 2041 - 2070 überall wieder annähernd auf das Ausgangsniveau und geht bis 2100 weiter leicht zurück.

Die Standortleistungsfähigkeit für die Baumart Eiche wird unter den gegenwärtigen Standortsbedingungen in der Region im Vergleich zur Kiefer etwas geringer eingeschätzt (s. Abb. 104). Räumlich ist für die Eiche eine Zweiteilung der Region mit den schwächeren Standorten im Osten zu erkennen. Dieses räumliche Muster bleibt auch in den Projektionen erhalten. In der ersten Periode profitiert die Eiche wie auch die Kiefer leicht von der Verlängerung der Vegetationszeit und den Temperaturerhöhungen. Bis zum Ende des Projektionszeitraumes 2100 wird für die Standortleistungsfähigkeit der Eiche jedoch eine deutliche Reduktion geschätzt. Die Histogramme der potenziellen Flächenanteile der Leistungsklassen von Kiefer und Eiche in Abbildung 105 bestätigen die Ergebnisse der räumlichen Analyse. Während für die Kiefer unter den bis 2100 projizierten klimatischen Bedingungen nur eine geringe Einbuße von 1,5 \% der Standortleistungsfähigkeit erwartet wird, gehen die Schätzungen für die Eiche von einem deutlichen Rückgang des Wuchspotenzials von knapp $13 \%$ aus.

Ein ähnliches Ergebnis wie für die Eiche zeigt sich für die Entwicklung der Standortleistungsfähigkeit für die Baumart Buche (s. Abb. 106). Im Mittel liegt der 
dGZ $Z_{\max }$ mit $5,3 \mathrm{~m}^{3} / \mathrm{a} / \mathrm{ha}$ am Ende des Projektionszeitraumes leicht über dem Wert der Eiche $\left(4,8 \mathrm{~m}^{3} / \mathrm{a} / \mathrm{ha}\right)$, der Rückgang gegenüber den Status quo-Bedingungen wird aber auch für die Buche auf über $10 \%$ geschätzt.

Die projizierte Standortleistungsfähigkeit der Baumart Douglasie stellt sich ähnlich wie in der Region Fläming auch in der Colbitz-Letzlinger Heide mit einer größeren Spreitung dar (s. Abb. 106). Auch hier wird verglichen mit den anderen Baumarten bis 2100 die größte relative Einbuße von über $15 \%$ geschätzt, absolut weist die Douglasie mit einem dGZ $\mathrm{max}_{\text {ax }}$-Mittelwert von $17 \mathrm{~m}^{3} / \mathrm{a} / \mathrm{ha}$ aber die deutlich höchste Standortleistungsfähigkeit auf.

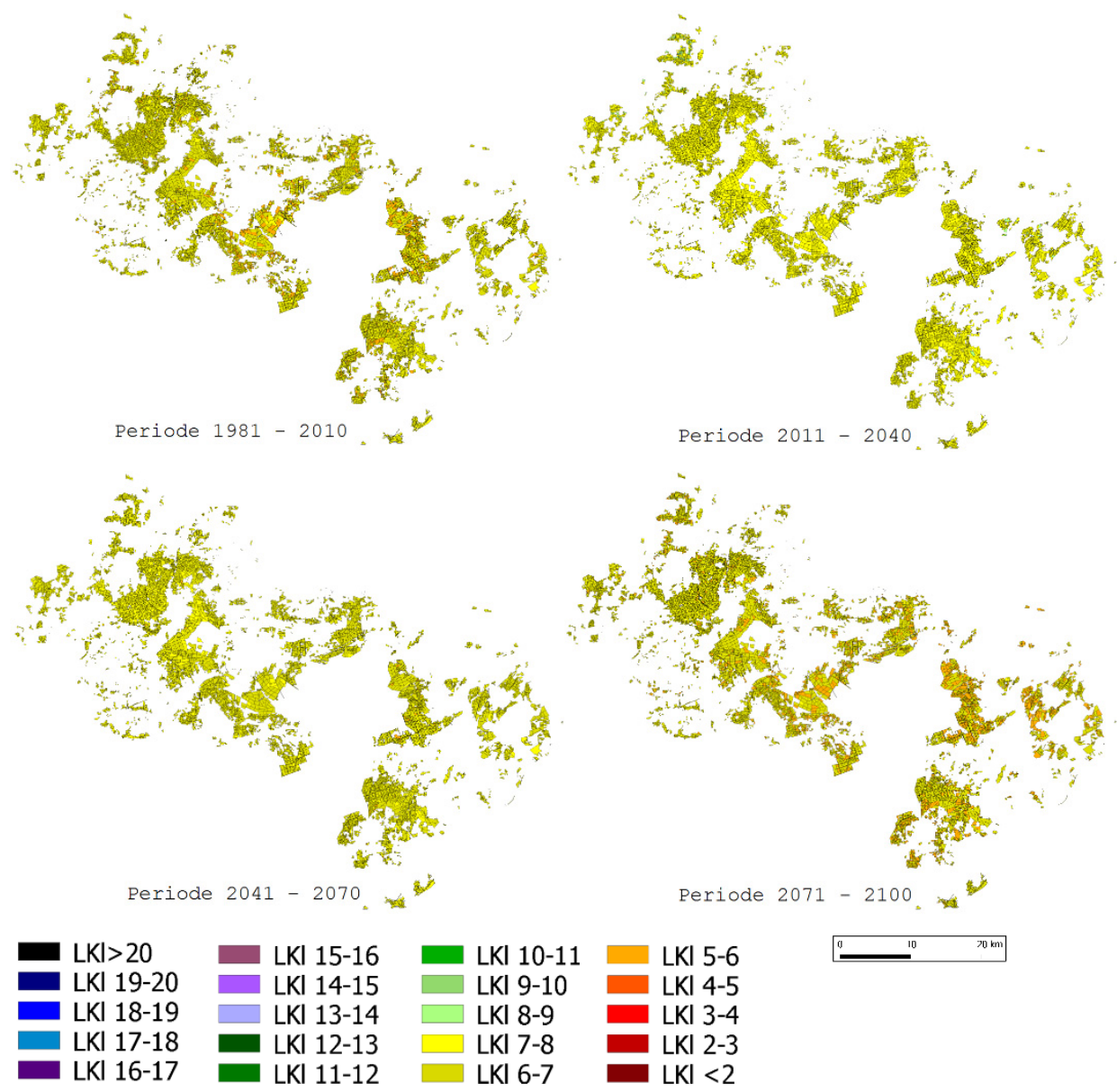

Abbildung 103: Projektion der Standortleistungsfäbigkeit für potenziell mit Kiefer bestockte Teilflächen in der Region Colbitz-Letzlinger Heide ( $L K l=$ Leistungsklasse) 

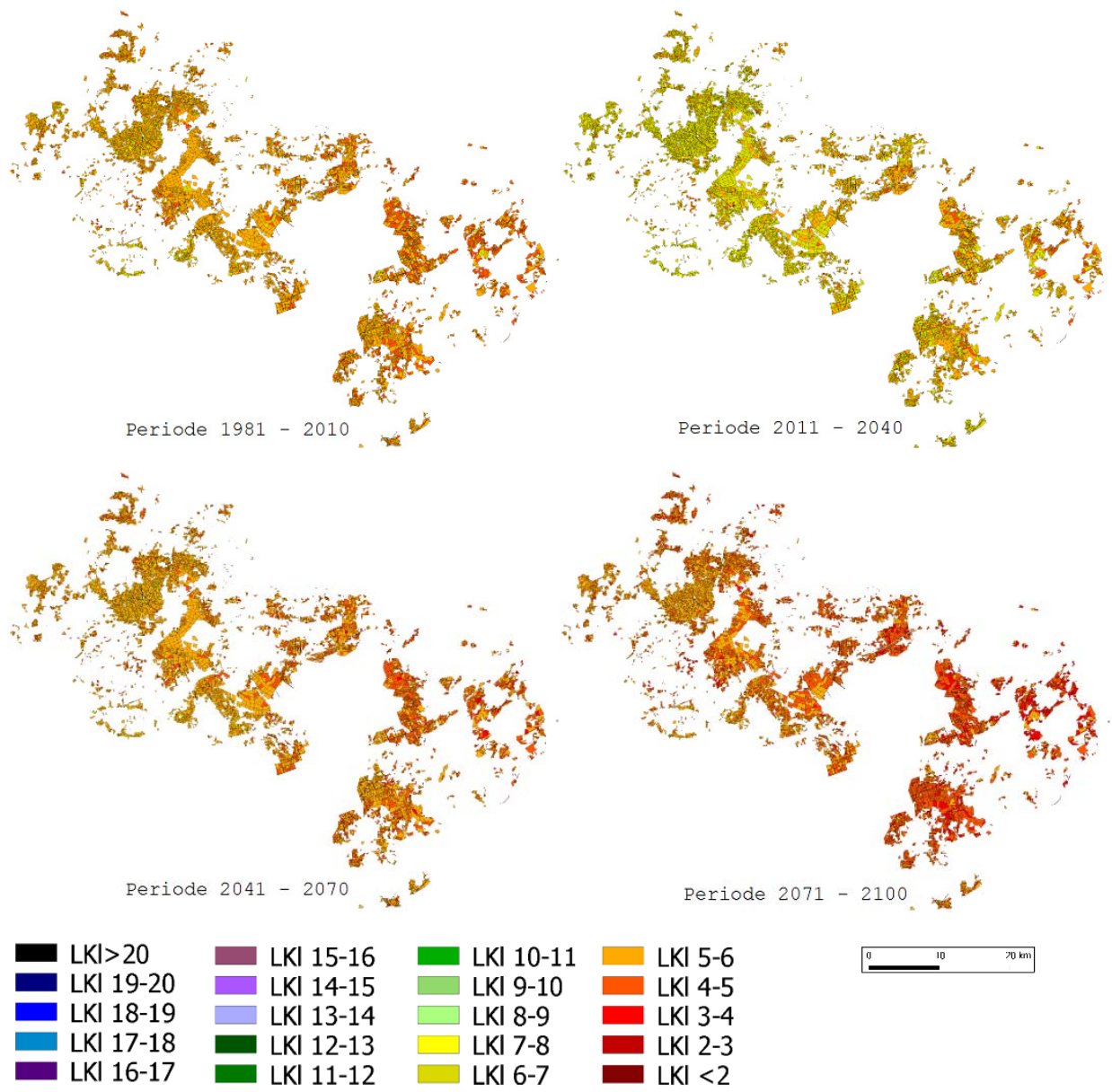

LKI 5-6

LKI 4-5

LKI 3-4

LKI 2-3

LKI 16-17

LKI 11-12

LKI 6-7 LKI $<2$

Abbildung 104: Projektion der Standortleistungsfähigkeit für potenziell mit Eiche bestockte Teilflächen in der Region Colbitz-Letzlinger Heide ( $L K l=$ Leistungsklasse) 

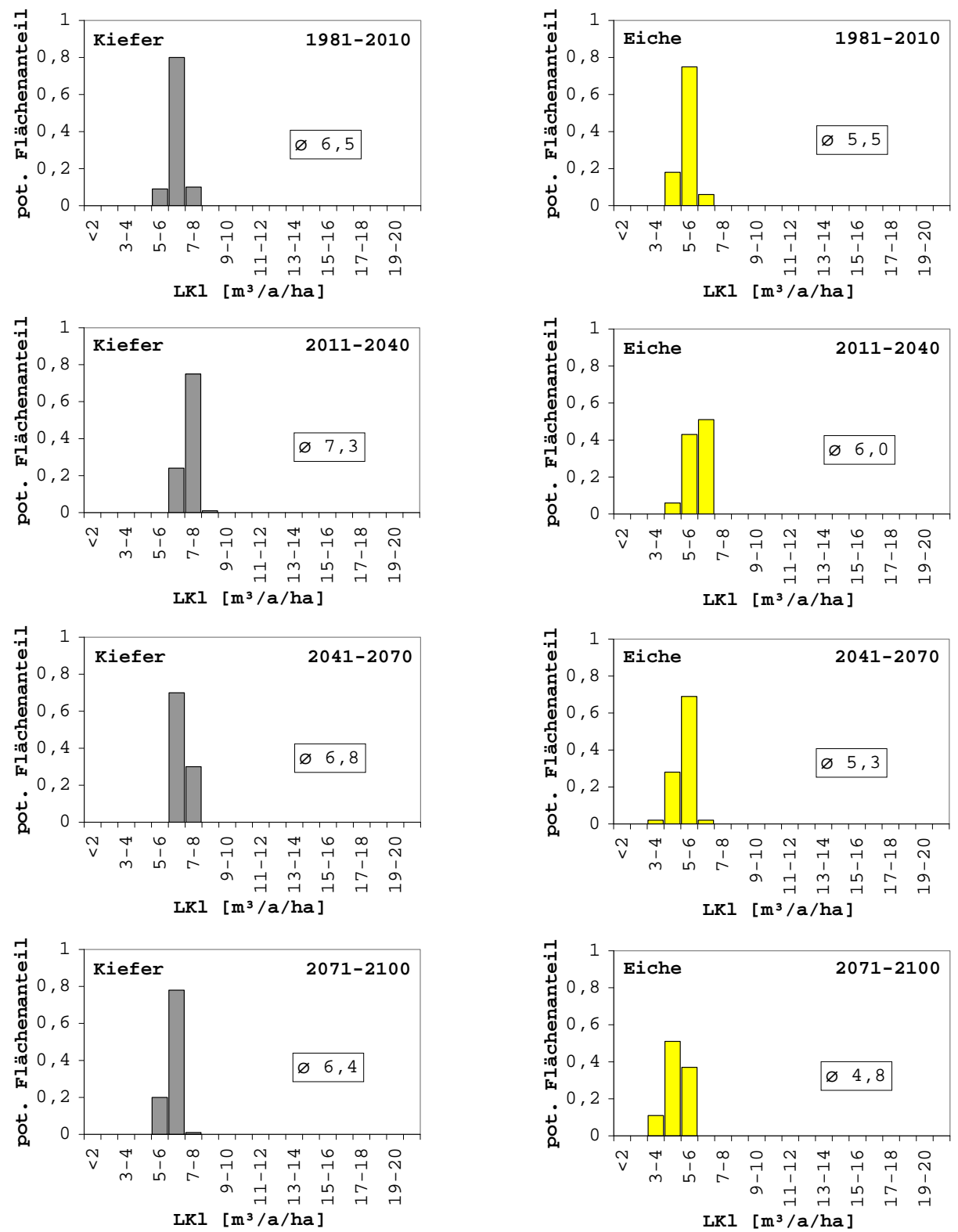

Abbildung 105: Potenzielle Flächenanteile der Standortleistungsfähigkeit für die Baumarten Kiefer (links) und Eiche (rechts) für den Status quo (1981 - 2010) und die drei Projektionsperioden. Jeder Teilfläche wurde hypothetisch die jeweilige Baumart zugeordnet. In den Grafiken ebenfalls angegeben ist der arithmetische Mittelwert o des durchschnittlichen Gesamtzuwachses (dG $Z_{\max }$ ). 

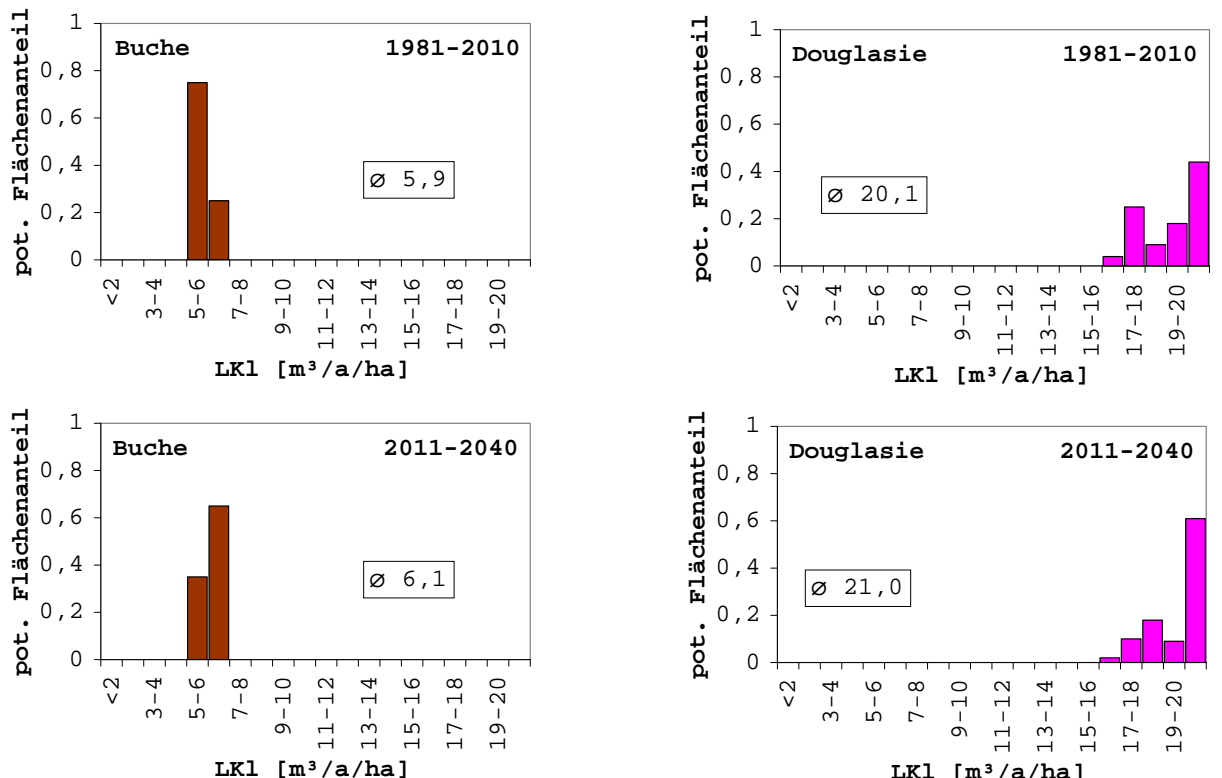

LKI $\left[\mathrm{m}^{3} / \mathrm{a} / \mathrm{ha}\right]$
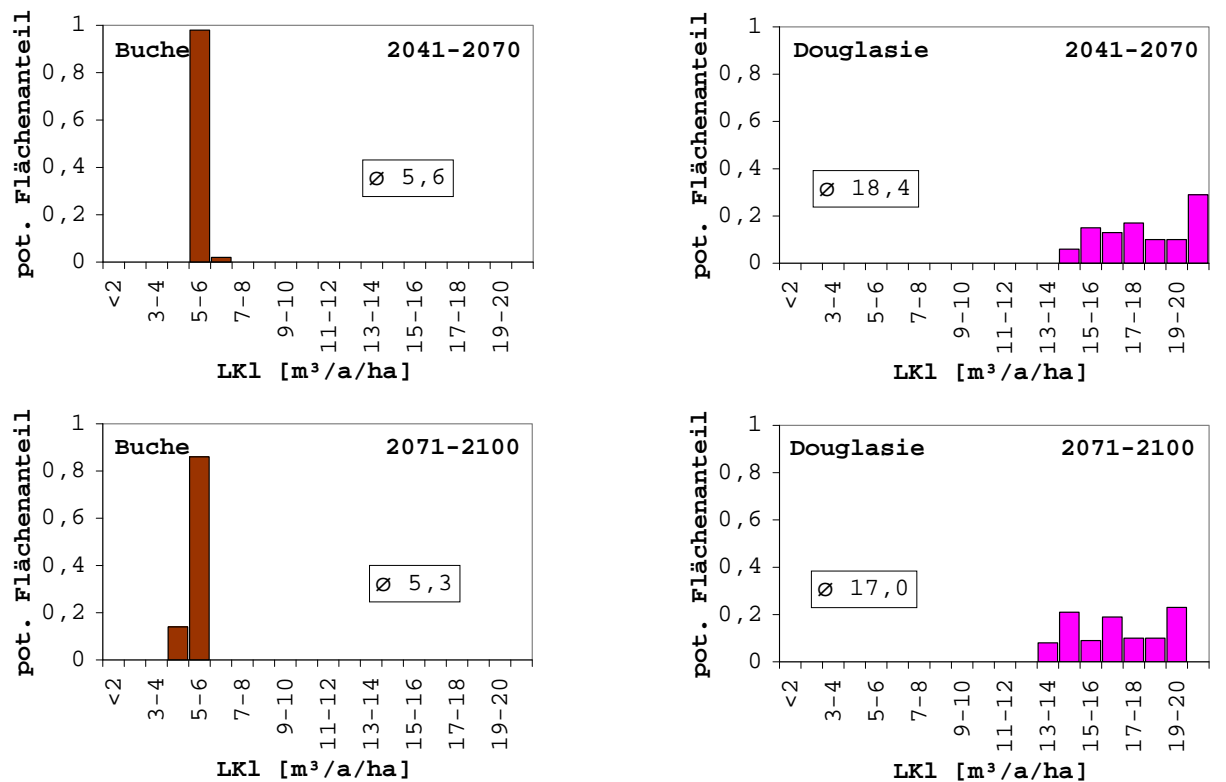

Abbildung 106: Potenzielle Flächenanteile der Standortleistungsfähigkeit für die Baumarten Buche (links) und Douglasie (rechts) für den Status quo (1981 - 2010) und die drei Projektionsperioden. Jeder Teilfläche wurde hypothetisch die jeweilige Baumart zugeordnet. In den Grafiken ebenfalls angegeben ist der arithmetische Mittelwert o des durchschnittlichen Gesamtzuwachses (dGZ max $)$. 


\subsection{Sturmschadensrisiko}

\subsubsection{Poten₹ielle Vulnerabilität durch Winterstürme bei Zielstärke}

Der Vergleich der potenziellen Bestockung bei Zielstärke zwischen den Baumarten zeigt in der Colbitz-Letzlinger Heide ein im Mittel steigendes Sturmschadensrisiko von Buche über Eiche und Kiefer zu Douglasie (s. Abb. 107). Die Mittelwerte für das Sturmschadensrisiko betragen 0,24 für die Buche, 0,27 für die Eiche, 0,52 für die Kiefer und 0,73 für die Douglasie. Kiefer und Douglasie weisen dabei sehr viel engere Verteilungen als Eiche und Buche auf. Die Kiefer nimmt somit eine Mittelstellung zwischen Buche / Eiche bzw. Douglasie ein.

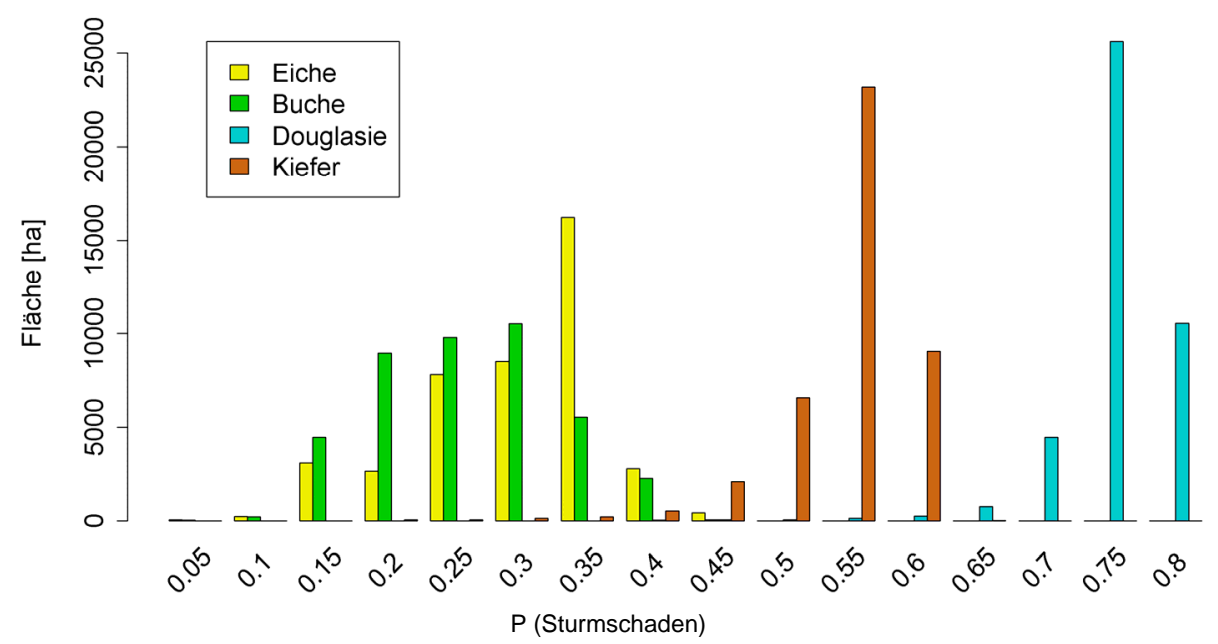

Abbildung 107: Flächenverteilungen potenzieller Bestockungen bei baumartenspezifischen Zielstärken nach Sturmschaden-Risikostufen und Baumarten in der Modellregion Colbitz-Letzlinger Heide

Für die Region Colbitz-Letzlinger Heide werden ebenfalls Risikokarten für die Baumarten Eiche, Kiefer und Douglasie dargestellt (s. Abb. 108). Eine potenzielle Eichenbestockung bei Zielstärke $(65 \mathrm{~cm})$ würde $\mathrm{zu} 100 \%$ in die Risikoklasse „geringes Risiko“ eingestuft. Eine potenzielle Kiefernbestockung bei Zielstärke $(45 \mathrm{~cm})$ würde zu $65 \%$ in die Risikoklasse „geringes Risiko“ und zu $35 \%$ in die Risikoklasse ,mittleres Risiko“ eingestuft. Eine potenzielle Douglasiebestockung bei Zielstärke $(65 \mathrm{~cm})$ würde zu $100 \%$ in die Risikoklasse „mittleres Risiko“ eingestuft. Die für den Fläming dargestellten möglichen Konsequenzen für die Einbringung von Mischbaumarten bzw. die Reduktion des Kieferanteils gelten auch für die Colbitz-Letzlinger Heide. 

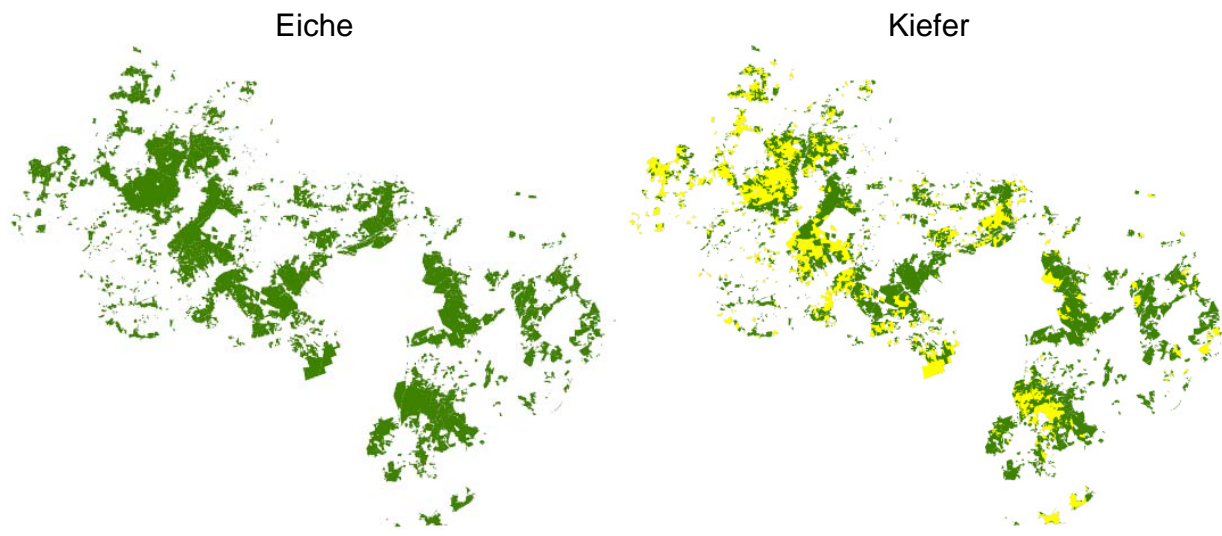

Douglasie

Abbildung 108: Karten des Sturmschadensrisikos der potenziellen Eichen-, Kiefern- und Douglasienbestockung bei Zielstärke (65, 45 bzw. $55 \mathrm{~cm})$ in der Region Colbitz-Letəlinger Heide unter Anwendung von 4 anhand der Fichte abgeleiteten Risikoklassen (grün: niedriges, gelb: mittleres, rot: hohes und pink: sehr hohes Sturmschadensrisiko)

\subsubsection{Aktuelle Gefährdungspotenziale durch Winterstürme}

Die Flächenverteilungen nach Risikostufen werden in der Region Colbitz-Letzlinger Heide von der Kiefer dominiert, wobei mittlere Risikostufen vorherrschen. Dargestellt sind die vier flächenmäßig wichtigsten Baumarten Kiefer, Lärche, Eiche und ALn (s. Abb. 109). Eine Adaption waldbaulicher Maßnahmen unter dem Aspekt des Sturmschadensrisikos ist weniger zwingend als in der Region Oberharz. Allerdings ließen sich anhand der Risikoprognosen und unter Berücksichtigung der Hiebsreife Kiefernbestände identifizieren, die im Rahmen eines Waldumbaus prioritär berücksichtigt werden sollten. Die Mittelwerte der Sturmschadensszenarien betragen für die aktuelle Kiefernbestockung 0,43, für die aktuelle Lärchenbestockung 0,27, für die aktuelle Eichenbestockung 0,17 und für die aktuelle Bestockung des ALn 0,17. 


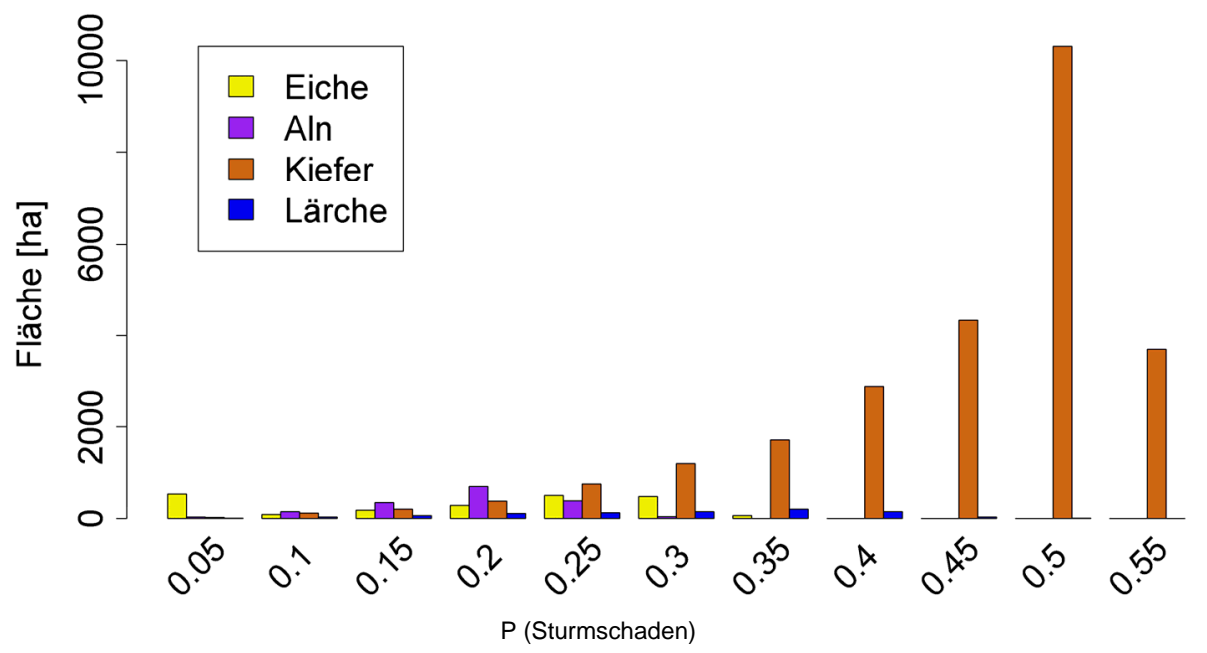

Abbildung 109: Flächenverteilungen der aktuellen Bestockung [ha] nach Sturmschaden-Risikostufen und Baumarten in der Modellregion Colbitz-Letzlinger Heide

Die Risikokarten für die aktuelle Kiefern- und Eichenbestockung in der Region Colbitz-Letzlinger Heide weisen aufgrund der geringeren Relief- und Standortsunterschiede sowie des insgesamt geringeren Risikoniveaus der Kiefer und Eiche eine geringere Variabilität der prognostizierten Sturmschadenswahrscheinlichkeiten auf (s. Abb. 110). Die bereits diskutierte vorrangige Behandlung von hiebsreifen Kiefernbeständen, die ein relativ hohes Sturmschadensrisiko aufweisen, im Rahmen eines Waldumbauprogramms würde die Erstellung von großmaßstäblichen Karten erfordern. 


\section{Kiefer}

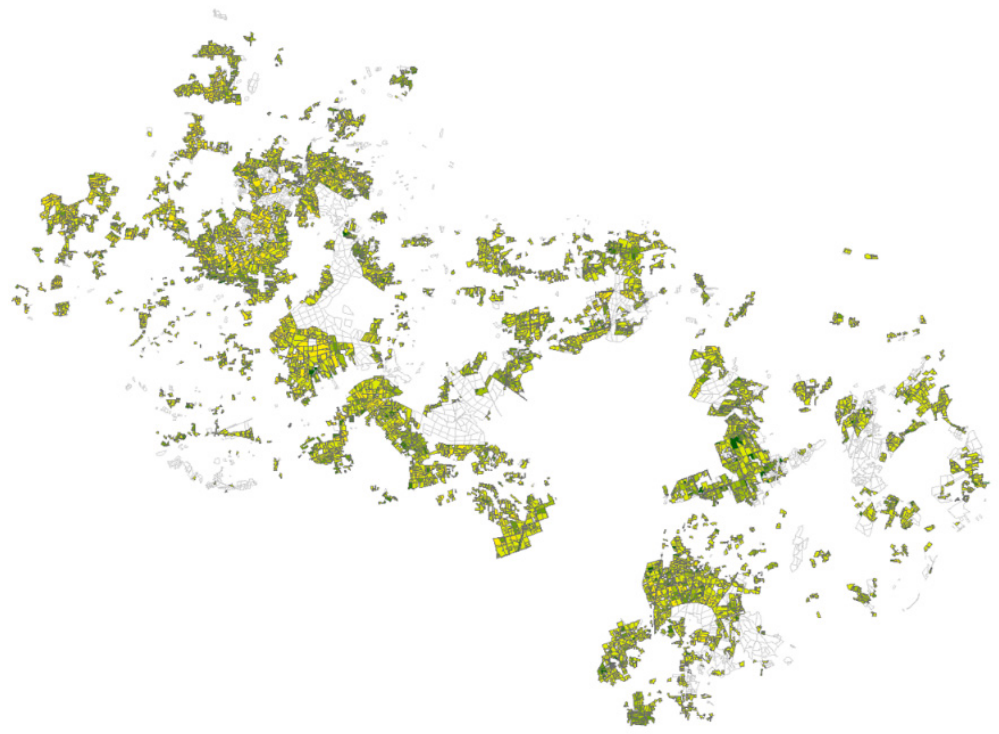

10 Sturmschadensklassen mit einheitlicher Klassenbreite:

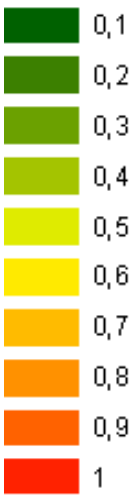

\section{Eiche}

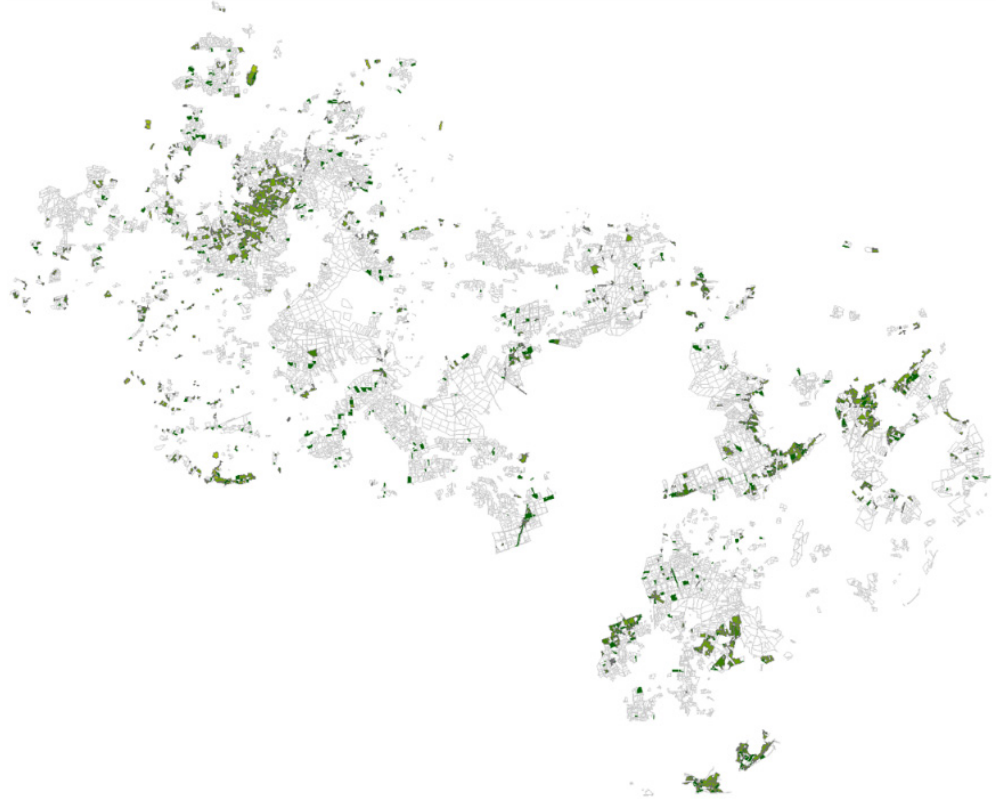

10 Sturmschadensklassen mit einheitlicher Klassenbreite:

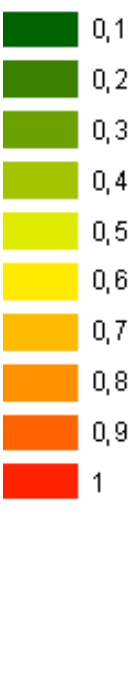

Abbildung 110: Karten des Sturmschadensrisikos der aktuellen Eichen- und Kiefernbestockung in der Region Colbitz-Letzlinger Heide mit einer Einteilung in zehn Risikoklassen 


\subsection{Veränderungen des Waldbrandrisikos}

Das Waldbrandrisiko in der Colbitz-Letzlinger Heide lag in den Jahren 2004 - 2010 mit durchschnittlich 20-30 Tagen (M68) bzw. 30-40 Tagen (FWI) in etwa auf demselben hohen Niveau wie im Fläming. Auch in der Colbitz-Letzlinger Heide gab es (in der Gemeinde Gardelegen) einzelne Waldgebiete mit bis zu 35 Risikotagen (M68; s. Abb. 111) bzw. bis zu 45 Risikotagen (FWI; s. Abb. 112). Die angrenzenden Baumbestände auf dem Truppenübungsplatz Altmark dürften ein ähnlich hohes klimatisches Waldbrandrisiko aufweisen, sie sind betriebsbedingt aber zusätzlichen Risikofaktoren ausgesetzt, wodurch sich unter Umständen Rückwirkungen auf das Gebiet der Untersuchungsregion ergeben.

\section{Jährliche Tage mit WBKZ > 4000}
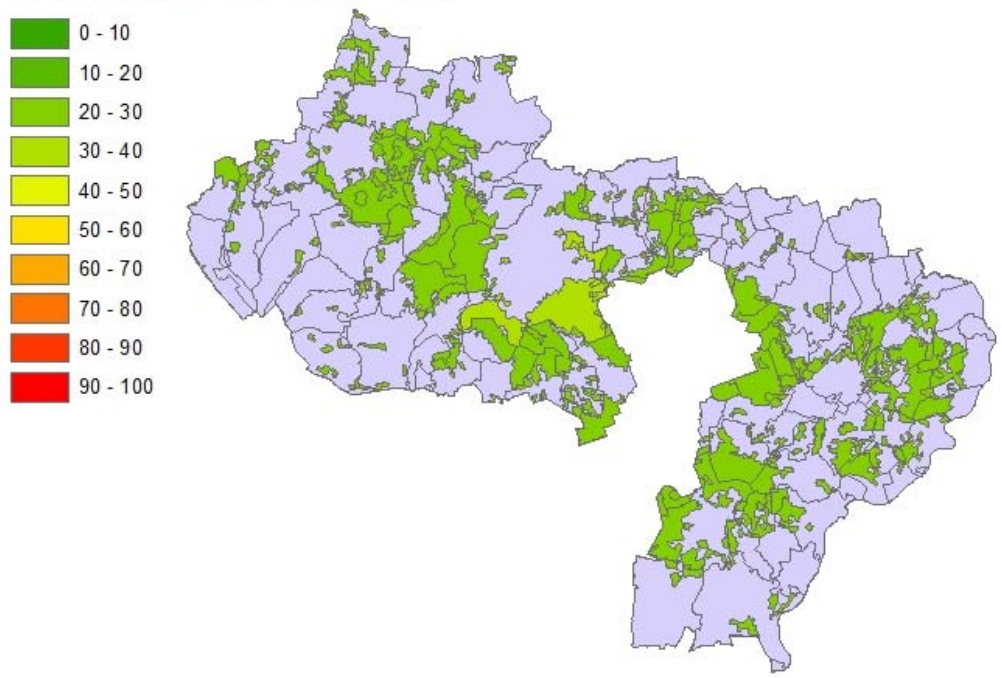

Abbildung 111:Jährliche Tage mit erböhtem Waldbrandrisiko in der Colbitz-Letəlinger Heide nach Berechnung mit dem M68-Waldbrandindex (WBKZ = Waldbrandkennziffer)

Die Zahl der im Referenzzeitraum 2004 - 2010 vorgekommenen Waldbrände war vor allem in den Randbereichen der Region mit den dort kleineren Waldgebieten verhältnismäßig hoch (bis zu vier Brände je 1000 ha Wald, .s. Abb. 113). Wenn man von diesen kleineren Waldgebieten absieht, entsprich die im Vergleich zum Umland etwas erhöhte tatsächliche Waldbrandhäufigkeit in der Gemeinde Gardelegen der für dieses Gebiet berechneten etwas höheren Gefährdungslage. 


\section{Jährliche Tage mit FFMC $>92$}

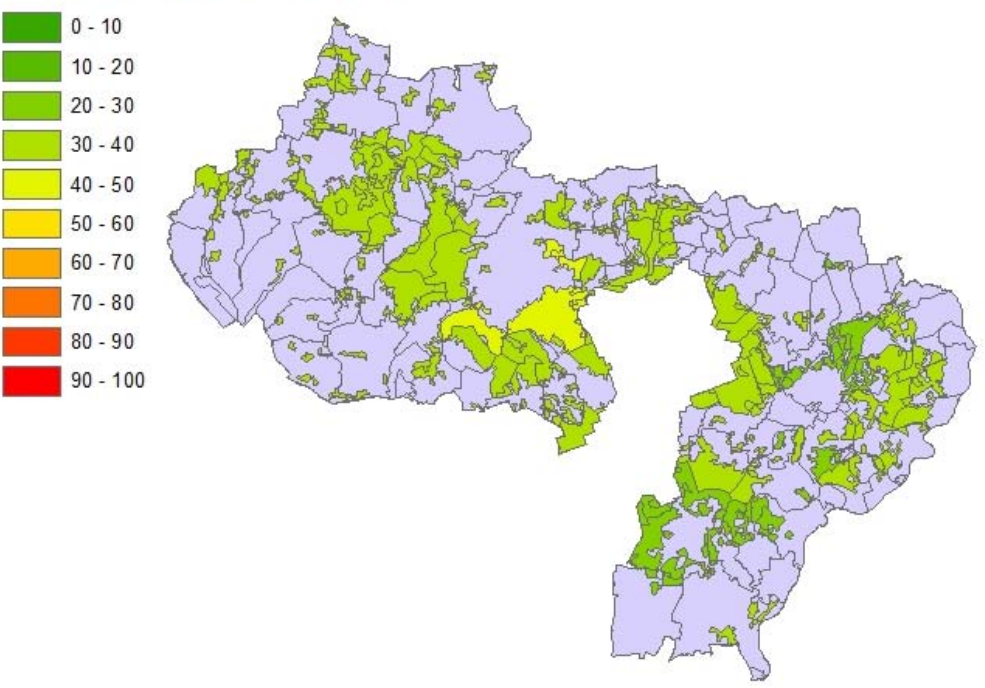

Abbildung 112: Jährliche Tage mit erböhtem Waldbrandrisiko in der Colbitz-Letzlinger Heide nach Berechnung mit dem Fire Weather Index (FWI) (FFMC = Streufeuchtezahl)

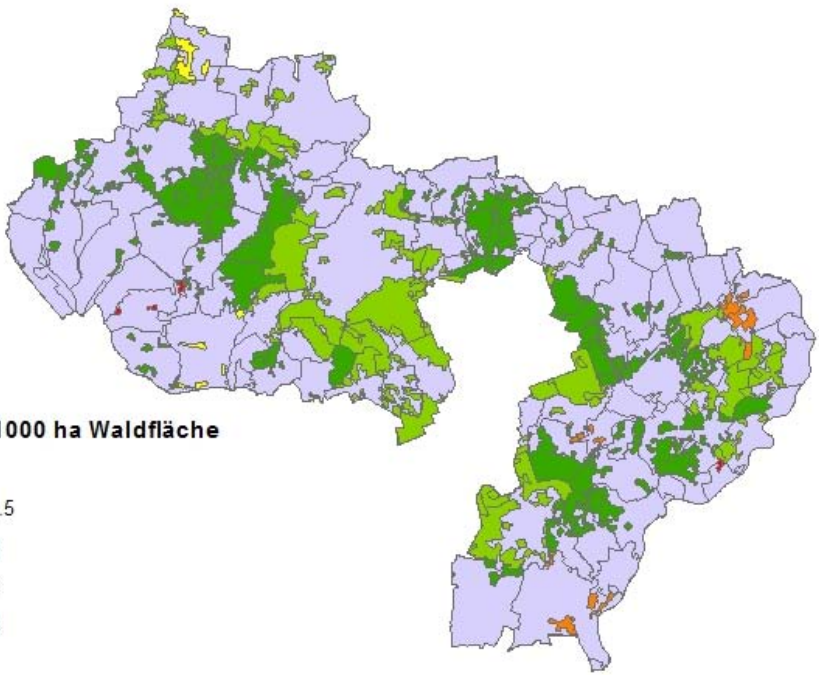

Abbildung 113: Waldbrände je 1000 ba Waldfläche in der Region Colbitz-Letzlinger Heide im Referenzzeitraum 2004 - 2010

Die Projektionen des Waldbrandrisikos für die Colbitz-Letzlinger Heide lassen eine sich beschleunigende Steigerung des Waldbandrisikos erwarten: So steigt in den ersten 30 Jahren des Projektionszeitraums die durchschnittliche Anzahl von Tagen erhöhten Waldbrandrisikos um ca. 10 Tage auf bis zu 40 (M68) bzw. bis zu 50 
Risikotage im Jahr (FWI) an (s. Abb. 114 und Abb. 115). Bereits im zweiten Terzennium (2041 - 2070) liegt die durchschnittliche erwartete Zahl von jährlichen Waldbrandrisikotagen noch weitere ca. 20 Tage höher (M68: durchschnittlich 60-65 Tage, FWI: 65-70 Tage). Bei diesem Niveau der Waldbrandgefährdung kommen Perioden wie der extrem trockene Sommer des Jahres 2003 häufig vor. Im dritten Terzennium (2071 - 2100) werden im regionalen Durchschnitt jährlich bis zu 80 Tage erhöhten Waldbrandrisikos erwartet, was einer weiteren Steigerung um 10-15 Tage entspricht. Damit würde ein Trockensommer nach Art des Jahres 2003 zu diesem Zeitpunkt als überdurchschnittlich feucht eingestuft. Die in der Grundaussage übereinstimmenden Projektionen der beiden Waldbrandindizes stimmen auch hinsichtlich der Zunahme des Waldbrandrisikos von Westen nach Osten in dieser Region überein.

Waldbrandkennziffer: 30-Jahres-Projektionen bis 2040, 2070 und 2100

\section{Jährliche Tage mit WBKZ > 4000}

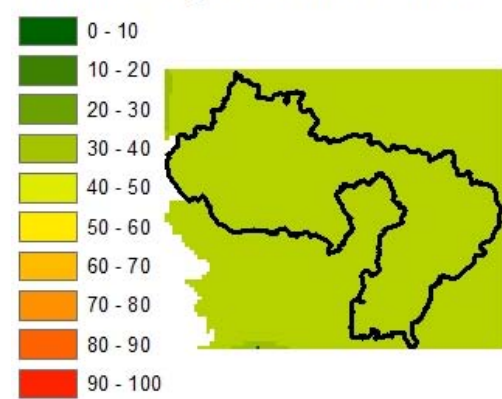

2011-2040

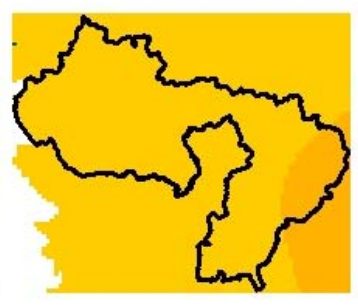

2041-2070

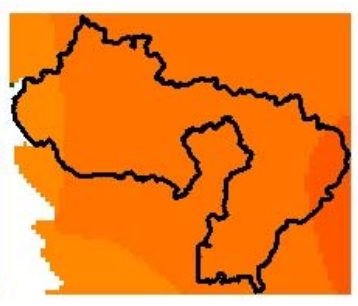

2071-2100

Abbildung 114: Erwartete jährliche Zabl von Tagen mit erböbtem Waldbrandrisiko auf Basis des M68 (Tage, an denen die Waldbrandkennziffer (WBKZ) über 4000 liegt)

Fine Fuel Moisture Code: 30-Jahres-Projektionen bis 2040, 2070 und 2100

Jährliche Tage mit FFMC $>92$

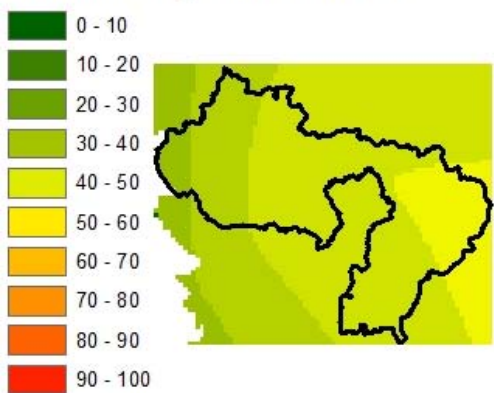

2011-2040

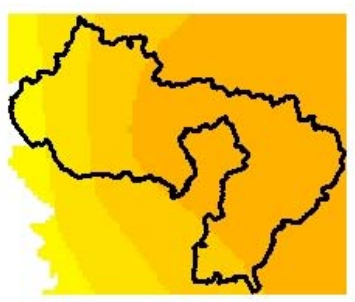

2041-2070

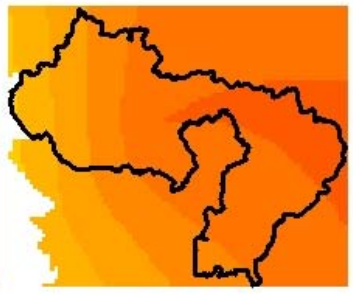

2071-2100

Abbildung 115: Erwartete jäbrliche Zabl von Tagen mit erböbtem Waldbrandrisiko auf Basis des FWI (Tage, an denen der Fine Fuel Moisture Code (FFMC = Streufeuchtezabl) über 92 liegt) 


\section{Zusätzliche Risikofaktoren:}

Die mittlere potenzielle Sonnenscheindauer auf den Waldflächen unterscheidet sich in der Colbitz-Letzlinger Heide nur wenig: Sie beträgt in den meisten Fällen 15-16 Stunden und auf etwa $30 \%$ der Fläche 14-15 Stunden (s. Abb. 116). Die zwischen dem 1.4. und 31.8. durchschnittlich eintreffende Globalstrahlung ist wegen der überwiegend ebenen, steigungslosen Waldflächen an keiner Stelle erhöht und liegt zwischen 210 und $220 \mathrm{~W} / \mathrm{m}^{2}$.

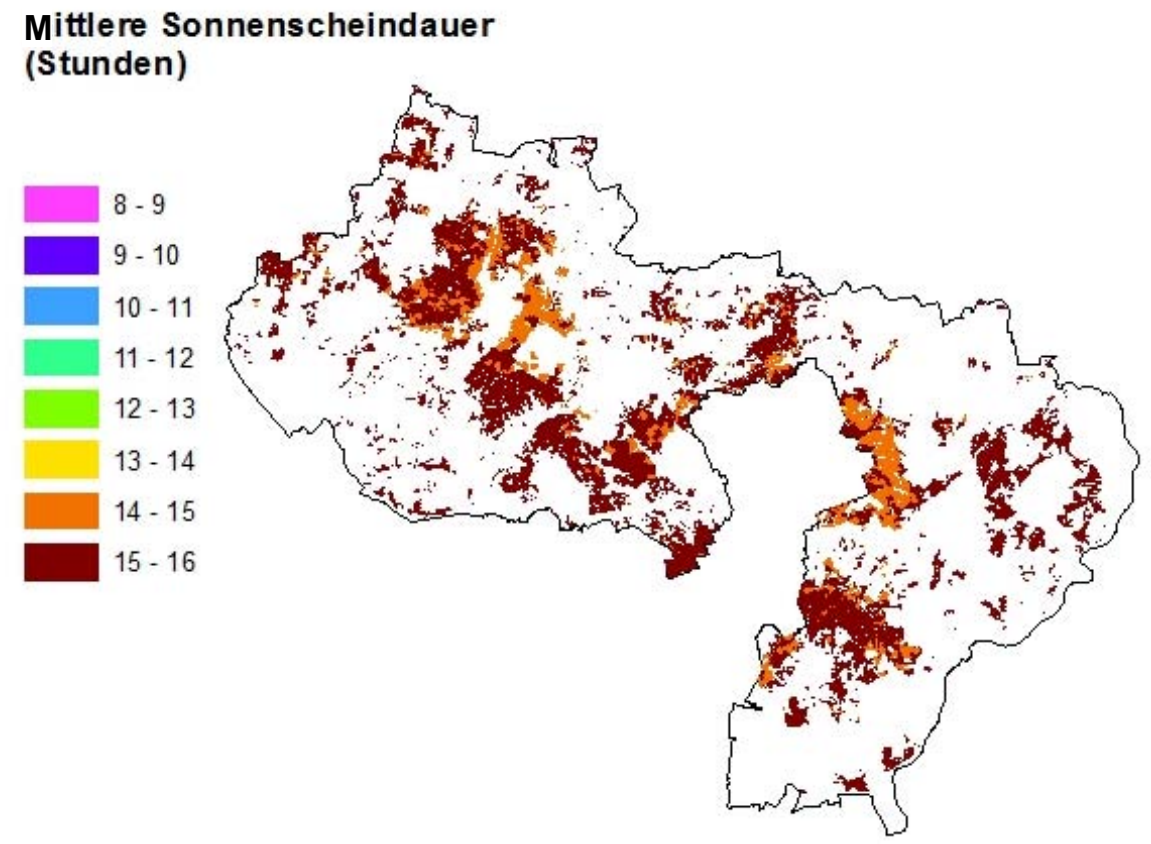

Abbildung 116: Regionale Verteilung der mittleren potenziellen Sonnenscheindauer wäbrend der Monate April bis August auf den Waldflächen in der Colbitz-Let:linger Heide

Die besonders waldbrandanfälligen Kiefernjungbestände mit geringem Bestockungsgrad kommen in der gesamten Region relativ häufig vor (s. Abb. 117). Das gegenwärtig größte dieser lokalen Risikogebiete befindet sich am Ostrand des Truppenübungsplatzes Altmark und das zweitgrößte Risikogebiet liegt in der Nähe von Zichtau im westlichen Teil der Colbitz-Letzlinger Heide. 


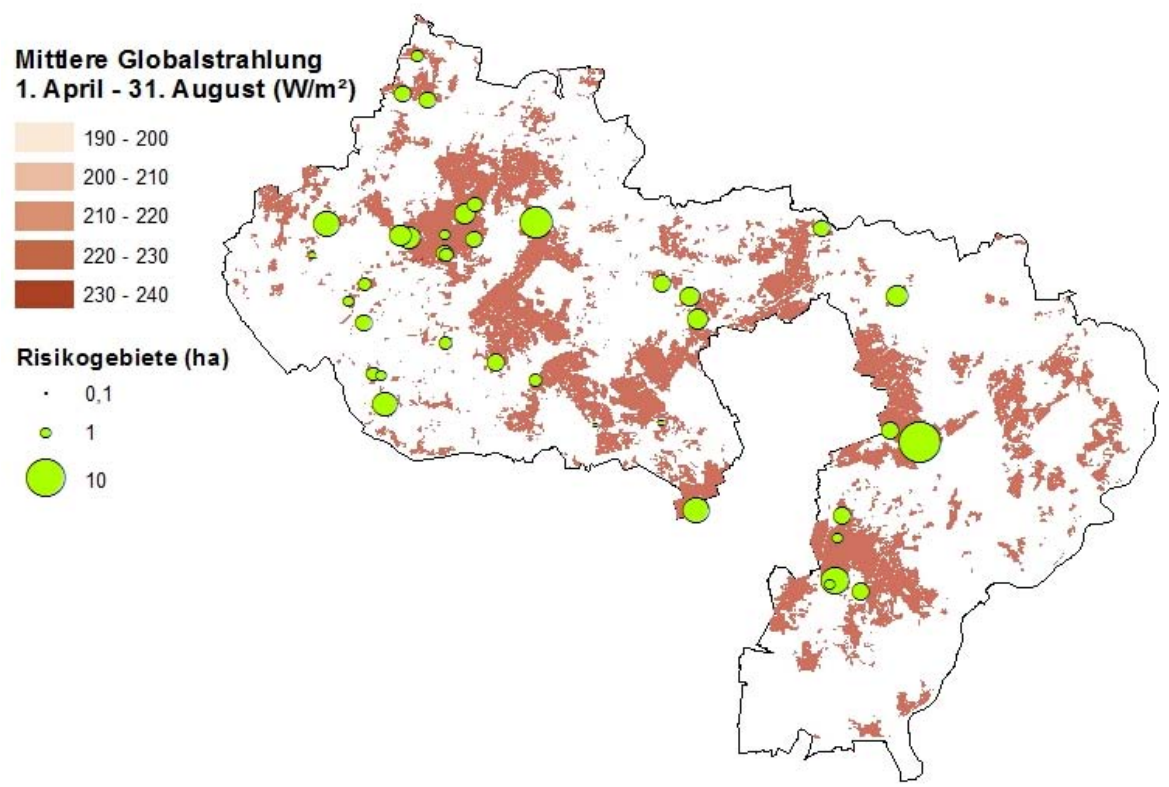

Abbildung 117: Regionale Verteilung der mittleren Globalstrablung $\left(W / m^{2}\right)$ auf Waldflächen in der Colbitz-Letzlinger Heide während der Monate April bis August und Lage von Kiefernjungbeständen geringer Bestockungsdichte (lokale Risikogebiete)

\subsection{Zustände und Wirkungsgefüge bei biotischen Risiken}

Die am stärksten vertretenen Baumarten auf der untersuchten Fläche von 40.615 ha im Bereich der Colbitz-Letzlinger Heide sind Kiefer mit $80 \%$ und Eiche mit $7 \%$. In dieser Grund- und Endmoränenlandschaft wurden schon früher größere Schäden durch die Eichenfraßgesellschaft bzw. durch Kieferngroßschädlinge und Nonne (BÜTTNER et al. 1994, EBERT 1967, WiECZOREK 1992, MAJUNKE et al. 2004, PLAŠIL u. HABERMANN 2012) sowie durch Kiefernbuschhornblattwespen (APEL et al. 2004) beobachtet. Die durchgeführten Untersuchungen wiesen ebenfalls ein starkes Vorkommen der Nonne (Lymantria monacha L.), des Kiefernspinners (Dendrolimus pini L.), der Forleule (Panolis flammea Denis \& Schiffermüller) und der Kiefernbuschhornblattwespen (Diprion spec.) im Verlauf der letzten 20 Jahre nach (s. Tab. 21). Das Befallsgeschehen durch den Kiefernspanner war zu vernachlässigen, nach EBERT $(1967,1968)$ waren seine Hauptschadgebiete ohnehin mehr im Bereich der nördlichen Altmark zu erwarten.

Die Hauptschadgebiete des am häufigsten vorkommenden Schädlings Nonne und des Kiefernspinners lagen im westlichen Bereich des Untersuchungsgebiets Colbitz-Letzlinger Heide (LZW Betr.-FA Letzlingen, Rev. Solpke, Jerchel und Wannefeld). Das Hauptschadgebiet der Forleule befand sich ebenfalls im Westen des Untersuchungsgebiets, hier traten Schäden im LZW Betr.-FA Letzlingen, Rev. 
Jerchel und Wannefeld auf. Die Schäden durch Kiefernbuschhornblattwespen waren über das gesamte untersuchte Gebiet Colbitz-Letzlinger Heide verteilt mit Schwerpunkten im westlichen Bereich (LZW Betr.-FA Letzlingen, Rev. Berge, Solpke, Wannefeld, Lüderitz und Jerchel).

Tabelle 21: Anzabl der Befallsvorkommen nach Schädlingsarten im Bereich der Colbitz-Letzlinger Heide (1993 - 2012) bei verschiedenen Befallschwellen bezogen auf die Fläche

\begin{tabular}{|c|c|c|c|}
\hline $\begin{array}{r}\text { definierte Schwellen für } \\
\text { Befall }\end{array}$ & $1 \%$ & $10 \%$ & $30 \%$ \\
\hline \multicolumn{4}{|l|}{ Eichenfraßgesellschaft: } \\
\hline Eichenwickler & 438 & 437 & 420 \\
\hline Frostspanner & 437 & 434 & 417 \\
\hline Eichenprozessionsspinner & 507 & 496 & 464 \\
\hline \multicolumn{4}{|l|}{ Kiefergroßschädlinge und Nonne: } \\
\hline Kiefernspanner & 14 & 13 & 13 \\
\hline Kiefernspinner & 791 & 791 & 781 \\
\hline Forleule & 416 & 416 & 416 \\
\hline Kiefernbuschhornblattwespen & 252 & 251 & 245 \\
\hline Nonne & 827 & 827 & 813 \\
\hline
\end{tabular}

In den im Jahr 2009 durch die Kiefernbuschhornblattwespen stark geschädigten Gebieten (LZW Betr.-FA Letzlingen, Rev. Wannefeld) kam es im Jahr 2010 zum Absterben ganzer Bestände durch das Diplodia-Triebsterben der Kiefer, welches durch den endophytisch vorkommenden Pilz Sphaeropsis sapinea (Fr.) Dyko \& B. Sutton (Synonym: Diplodia pinea) hervorgerufen wurde (LANGER et al. 2011). Der Wärme liebende Pilz wurde in den letzten Jahren verstärkt an Kiefern und auch an Douglasien (LANGER et al. 2011) in weiteren Bereichen der Colbitz-Letzlinger Heide (LZW Betr.-FA Letzlingen, Rev. Colbitz, Gardelegen und Solpke) festgestellt.

Bei der Analyse auf lineare Zusammenhänge mittels eines generalisierten linearen Modells (GLM) wurde festgestellt, dass neben dem Mischungsanteil der Kiefernbestände und deren Alter die Nährstoffziffer den größten Einfluss auf den Befall durch Kiefernspinner, Forleule und Nonne hat (s. Tab. 22). Es ist stark davon auszugehen, dass dieselben Faktoren auch das Befallsgeschehen von Kiefernspanner und Kiefernbuschhornblattwespen beeinflussen, aber die Mindestanzahl von Befallsvorkommen für eine aussagekräftige GLM-Analyse wurde hier nicht erreicht. 
Tabelle 22: $\quad$ Durch ein generalisiertes lineares Modell (GLM) analysierter Einfluss der Bestandes- und Standortsvariablen auf Befall durch Kieferngroßschädlinge und Nonne im Bereich der Colbitz-Letzlinger Heide (1993 - 2012); (+/- Richtung des Zusammenhangs zur Zielgröße (Wabrscheinlichkeit des Befalls), Signifikanz: () keine, (*) gering, (**) mittel, (***) hoch)

\begin{tabular}{|c|c|c|c|c|c|c|c|c|c|}
\hline \multirow{2}{*}{ Variablen } & \multicolumn{3}{|c|}{ Nonne } & \multicolumn{3}{|c|}{ Kiefernspinner } & \multicolumn{3}{|c|}{ Forleule } \\
\hline & $1 \%$ & $10 \%$ & $30 \%$ & $1 \%$ & $10 \%$ & $30 \%$ & $1 \%$ & $10 \%$ & $30 \%$ \\
\hline \multirow{2}{*}{ Alter } & $* * *$ & $* * *$ & $* * *$ & $* * *$ & $* * *$ & $* * *$ & $* * *$ & $* * *$ & $* * *$ \\
\hline & - & - & - & - & - & - & - & - & - \\
\hline \multirow{2}{*}{ Mischungsanteil } & $* * *$ & $* * *$ & $* * *$ & $* * *$ & $* * *$ & $* * *$ & *** & $* * *$ & $* * *$ \\
\hline & + & + & + & + & + & + & + & + & + \\
\hline \multirow{2}{*}{ Nährstoffziffer } & $* * *$ & $* * *$ & $* * *$ & *** & $* * *$ & $* * *$ & *** & *** & $* * *$ \\
\hline & - & - & - & - & - & - & - & - & - \\
\hline $\mathrm{nFK}$ & + & + & + & + & + & + & + & + & + \\
\hline
\end{tabular}

Aufgrund der negativen Richtung der Zielgröße beim Alter und der Nährstoffziffer bzw. der positiven Richtung der Zielgröße beim Mischungsanteil kann erwartet werden, dass das Befallsrisiko durch die betreffenden Schädlinge (Nonne, Kiefernspinner und Forleule) besonders hoch in Kiefernbeständen mit hohem Kiefernanteil und niedrigem Alter ist, die auf nährstoffarmen Standorten stocken.

Wenn man das durch den Klimawandel verursachte gestiegene Trockenstressrisiko (s. Kap. 7.1) berücksichtigt, so ist grundsätzlich davon auszugehen, dass die heutigen Nebenschadgebiete mit vereinzeltem Vorkommen der Kieferngroßschädlinge und vor allem der Nonne bei steigendem Trockenstressrisiko ebenfalls zu Hauptschadgebieten der betreffenden Schadinsekten werden. Zusätzlich ist zu erwarten, dass immer mehr Bestände nach einem Befall z. B. durch Kiefernbuschhornblattwespen durch das Diplodia-Triebsterben befallen werden, was für das Untersuchungsgebiet der Colbitz-Letzlinger Heide bereits nachgewiesen wurde (LANGER et al. 2011). In Anbetracht der Prognose zum Trockenstressrisiko ist davon auszugehen, dass in der Colbitz-Letzlinger Heide stockende Kiefernbestände zukünftig durch diverse Insekten- und Pilzarten destabilisiert und daher dort größere Schäden auftreten werden. Zunehmende abiotische oder biotische Störungen können in Kulturen auch Schäden durch den Großen Braunen Rüsselkäfer (Hylobius abietis L.) begünstigen.

Im Bereich des Untersuchungsgebiets Colbitz-Letzlinger Heide wurde für die Eichenfraßgesellschaft ein etwas geringeres Befallsvorkommen als im Fläming festgestellt. Das Auftreten des Eichenwicklers (Tortrix viridana L.) und des Kleinen Frostspanners (Operophtera brumata L.) war vorwiegend vergesellschaftet (s. Tab. 21). Darüber hinaus spielte der Eichenprozessionsspinner (Thaumetopoea processionea L.) 
eine immer bedeutendere Rolle. Die Hauptschadgebiete der Eichenfraßgesellschaft lagen in Bereichen mit stärkerer Eichenbestockung, nämlich sowohl in westlichen (LFB Altmark, Rev. Klötze) wie auch in östlichen Randlagen (LFB Altmark, Rev. Mahlpfuhl, Elbaue) des Untersuchungsgebietes.

Nach der Analyse auf lineare Zusammenhänge mittels GLM wurde festgestellt, dass das Alter und der Mischungsanteil der Eiche den größten Einfluss auf den Befall durch die Eichenfraßgesellschaft haben (s. Tab. 23). Der Einfluss der nFK war in diesem Untersuchungsbereich im Gegensatz zum Fläming nicht so stark signifikant. Erst in den Bereichen mit verstärktem Befall (Schwelle ab 30 \% Befall) erwies sich dieser Faktor als mittel bis hoch signifikant für ein Befallsgeschehen.

Nach SCHWANECKE u. KOPP (1994) herrschen im Bereich der Colbitz-Letzlinger Heide auf den Grund- und Endmoränen Sand-Braunerden bis -Podsole vor. Die Ergebnisse der GLM-Analyse zeigten ein zunehmendes Befallsrisiko vor allem für ältere Bestände mit hohem Eichenanteil auf tonreichen Standorten.

Im Untersuchungsbereich Colbitz-Letzlinger Heide muss man unter Berücksichtigung der Prognose zum Trockenstressrisiko (s. Kap. 7.1) in Eichenbeständen mit verstärkten Schäden durch die Eichenfraßgesellschaft rechnen. Darüber hinaus ist auf prädisponierten Standorten bei mehrmaligem Fraßgeschehen kombiniert mit pilzlichen Schaderregern wie z. B. Hallimasch und Phytophthora-Arten eine Ausweitung der Eichenkomplexerkrankung zu erwarten.

Tabelle 23: $\quad$ Durch ein generalisiertes lineares Modell (GLM) analysierter Einfluss der Bestandes- und Standortsvariablen auf Befall durch Eichenfraßgesellschaft im Bereich der Colbitz-Letzlinger Heide (1993 - 2012); (+/- Richtung des Zusammenhangs zur Zielgrößß (Wahrscheinlichkeit des Befalls), Signifikanz: () keine, (*) gering, (**) mittel, (***) hoch)

\begin{tabular}{|c|c|c|c|c|c|c|c|c|c|}
\hline \multirow{2}{*}{ Variablen } & \multicolumn{3}{|c|}{ Eichenwickler } & \multicolumn{3}{|c|}{ Frostspanner } & \multicolumn{3}{|c|}{$\begin{array}{l}\text { Eichenprozes- } \\
\text { sionsspinner }\end{array}$} \\
\hline & $1 \%$ & $10 \%$ & $30 \%$ & $1 \%$ & $10 \%$ & $30 \%$ & $1 \%$ & $10 \%$ & $30 \%$ \\
\hline \multirow{2}{*}{ Alter } & $* * *$ & $* * *$ & $* * *$ & $* * *$ & *** & $* * *$ & $* * *$ & *** & *** \\
\hline & + & + & + & + & + & + & + & + & + \\
\hline \multirow{2}{*}{ Mischungsanteil } & $* * *$ & $* * *$ & $* * *$ & $* * *$ & $* * *$ & $* * *$ & $* * *$ & *** & $* * *$ \\
\hline & + & + & + & + & + & + & + & + & + \\
\hline Nährstoffziffer & - & - & - & - & - & - & - & - & - \\
\hline \multirow{2}{*}{ nFK } & $* *$ & $* *$ & $* * *$ & $* *$ & $* *$ & $* *$ & . & . & $* *$ \\
\hline & + & + & + & + & + & + & + & + & + \\
\hline
\end{tabular}


7.7 Konsequenzen für die künftige Waldbewirtschaftung in der Region Colbitz-Letzlinger Heide

\subsubsection{Waldbauliche Ausgangslage}

Die Region Colbitz-Letzlinger Heide umfasst in der bearbeiteten Abgrenzung nicht nur den stark übersandeten Grundmoränenbereich und die Sanderflächen im Dreieck Magdeburg-Gardelegen-Tangerhütte (ohne die bedeutenden Flächenanteile des Bundes), sondern auch nennenswerte Anteile des Altmärker Endmoränenzuges im Nordwesten (Waldflächen um Klötze bis nach Beetzendorf). Diese Bereiche sind klimatisch und standörtlich insgesamt günstiger zu beurteilen als der Durchschnitt des Untersuchungsraumes. Im Vergleich zur Region Fläming weist die Region Colbitz-Letzlinger Heide eine stärkere Differenzierung in den standörtlichen Verhältnissen auf. Neben einem etwas höheren Anteil kräftiger Standorte mit mindestens mittlerer Wasserversorgung, der sich vor allem im Nordwesten konzentriert, dominieren jedoch auch hier Standorte mittlerer Nährkraftstufe und Wasserhaushaltsstufe mit Anteilen von ca. $50 \%$. Ziemlich arme terrestrische Standorte mit mittlerer, z. T. sogar frischerer Wasserhaushaltsstufe sind auf ca. $20 \%$ vertreten. Im Unterschied zum Fläming weist die Colbitz-Letzlinger Heide vor allem im Osten auch größere Anteile armer Sandstandorte (ca. $10 \%$ ) auf.

Die Kiefer ist wie im Fläming die Hauptbaumart (s. Abb. 118). Ihr Anteil im Hauptbestand erreicht sogar mehr als $80 \%$. Von den anderen Nadelbaumarten hat nur noch die Europäische Lärche eine nennenswerte Bedeutung. Sie wurde vor allem in den 60er bis 80er-Jahren des vorigen Jahrhunderts in einem etwas stärkeren Umfang angebaut. Dagegen war der bisherige Anbau von Fichte und auch Douglasie eher gering. Wichtigste Laubbaumart ist die Eiche (überwiegend Traubeneiche) mit einem Flächenanteil von ca. $7 \%$. Daneben sind Weichlaubbäume wie Roterle auf Nassstandorten und Birke vorwiegend auf trockeneren Standorten am Bestandesaufbau beteiligt. Der Anteil der Rotbuche erreicht weniger als $1 \%$.

Ein dienender Unterstand kommt nur auf ca. $6 \%$ der Gesamtfläche vor (s. Abb. 119). Dieses erwünschte Strukturelement setzt sich vor allem aus Edellaubbaumarten auf den besseren Standorten sowie Birke, Eberesche und Hähereichen auf den eher schwächeren und trockeneren Standorten zusammen. Den höchsten Anteil am Unterstand hat aber auch in dieser Tieflandregion die problematische Spätblühende Traubenkirsche. Ihr Flächenanteil ist aber bisher nicht ganz so groß wie im Fläming. 


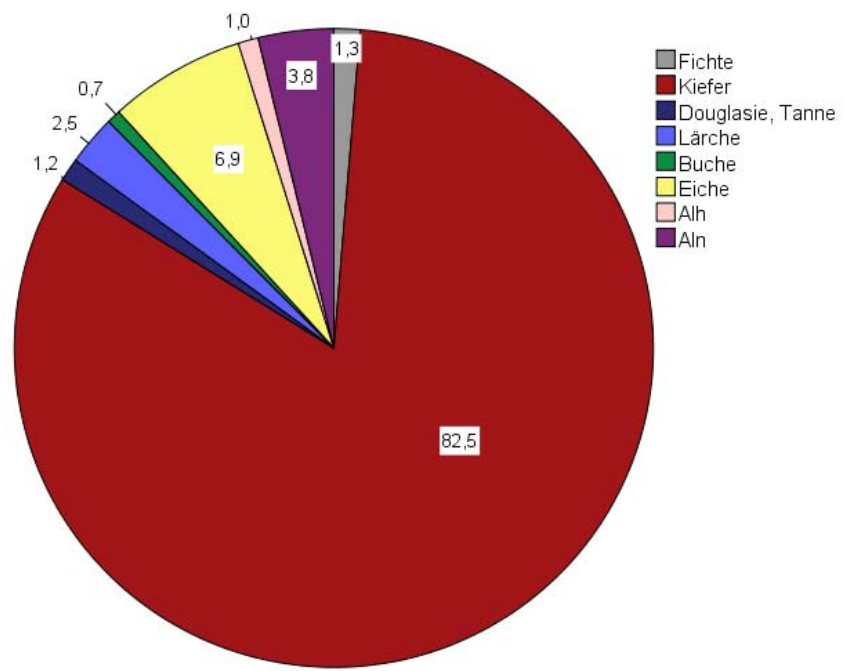

Abbildung 118: Flächenanteile [\%] der Baumarten im Hauptbestand in der Region Colbitz-Letzlinger Heide $(A L h=$ Laubholz mit hoher Umtriebszeit, $A L n=$ Laubholz. mit niedriger Umtriebszeit; im Datenspeicher W ald erfasste Flächen, Privatwald ₹. T. Stand 1994)

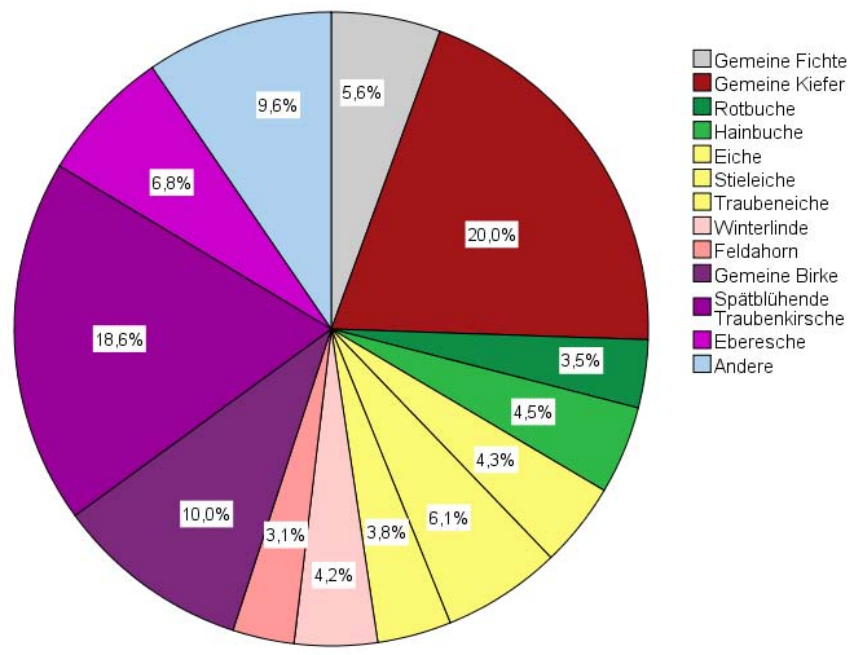

Abbildung 119: Baumartenanteile [\%] im Unterstand in der Region Colbitz-Letzlinger Heide; Unterstand auf ca. $6 \%$ der Gesamtfläche

Die Kiefernbestände der Colbitz-Letzlinger Heide wurden bis vor 20 Jahren sehr planmäßig bei Erreichen des Umtriebsalters abgenutzt und hauptsächlich wieder in Kiefer verjüngt. Dies spiegelt sich auch im weitgehend ausgeglichenen Alterklassenaufbau wider, der als Ausnahmen nur die typische Überausstattung in der dritten 
Altersklasse und einen sehr geringen Flächenanteil in der ersten Altersklasse aufweist (s. Abb. 120). Die Lärchenbestände bilden aufgrund ihres begrenzten Anbauzeitraumes einen nicht unbedeutenden Pflegeblock in der zweiten und dritten Altersklasse. Die Eiche ist stärker in den über hundertjährigen Beständen sowie mit einem großen Anteil in der ersten Altersklasse vertreten. Beim Weichlaubholz finden sich in der dritten Altersklasse noch zahlreiche Pappelflächen, während in den jüngeren Altersklassen Roterle und Birke und ab der vierten Altersklasse vor allem die Roterle vertreten ist.

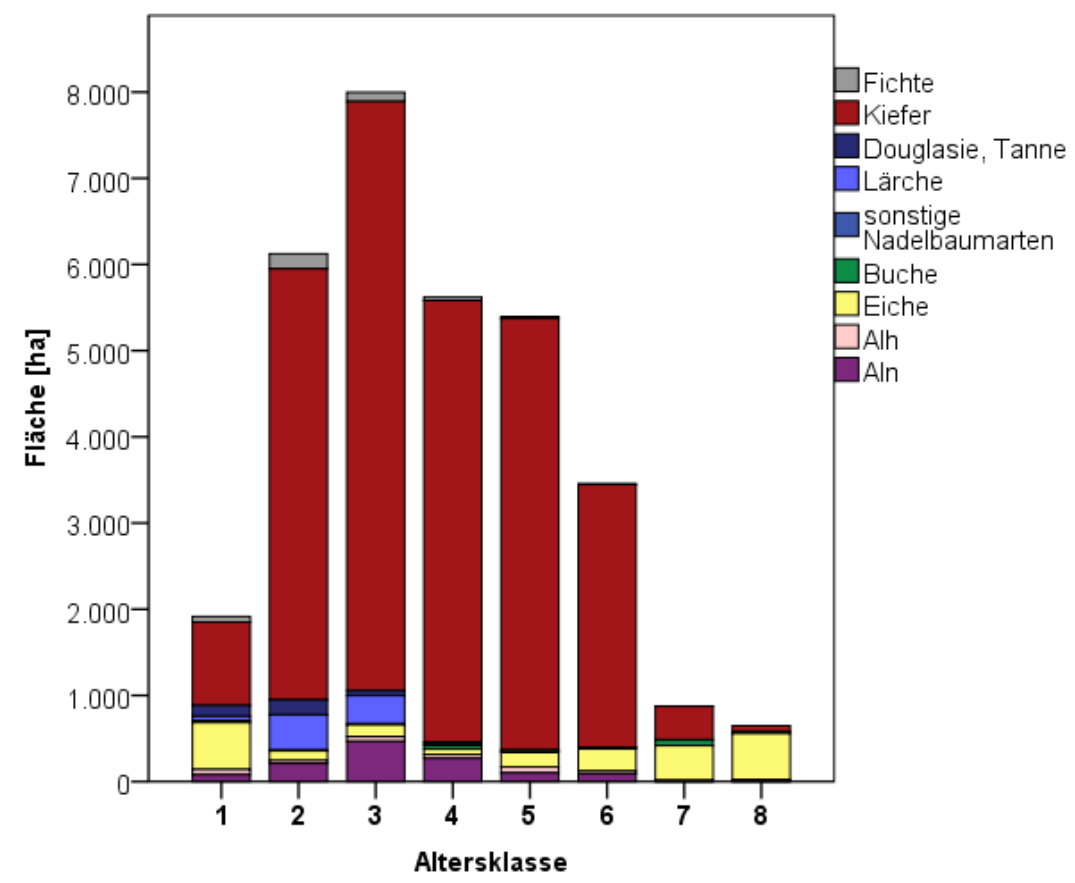

Abbildung 120: Altersklassenverteilung in der Region Colbitz-Letzlinger Heide (eine Altersklasse umfasst 20 Jahre; $A L h=$ Laubholz mit hoher Umtriebszeit, $A L n=$ Laubholz mit niedriger Umtriebszeit; im Datenspeicher W ald erfasste Flächen, Privatwald ₹. T. Stand 1994)

Auf ca. $4 \%$ der Gesamtfläche findet sich in der Region Nachwuchs unter Schirm. Seine Zusammensetzung zeigt, dass im Zuge des Waldumbaus vor allem die Eichenarten in den vergangenen 20 Jahren stark nachgezogen wurden. Zusammen mit der Roteiche $(7,5 \%)$ nehmen Trauben- und Stieleiche etwa ein Drittel der Verjüngung unter Schirm ein (s. Abb. 121). Es folgen mit jeweils etwa $20 \%$ die Kiefer und die Douglasie. Teile der Douglasien-Voranbauten, die vor allem im Nordwesten der Region stocken, sind bereits älter als 20 Jahre und wachsen noch unter dem Schirm von Kiefernaltbeständen. Die Rotbuche, die bisher über keine Anbautradition in der Region verfügt, ist mit ca. $16 \%$ an den Voranbauten beteiligt. 
Betrachtet man die I. Altersklasse und den Nachwuchs zusammen, so waren in den letzten 20 Jahren Laub- und Nadelbaumarten etwa zu gleichen Anteilen an der Waldverjüngung beteiligt. Damit blieb in der Colbitz-Letzlinger Heide der Nadelholzanteil höher als im Fläming. Auch bezogen auf die einzelnen Baumarten zeigt sich in dieser Region mit einem noch höheren Eichenanteil, einschließlich einer nennenswerten Roteichenbeteiligung, und substanziellen Anteilen der Douglasie bei gleichzeitig geringeren Buchenanteilen eine andere waldbauliche Schwerpunktsetzung.

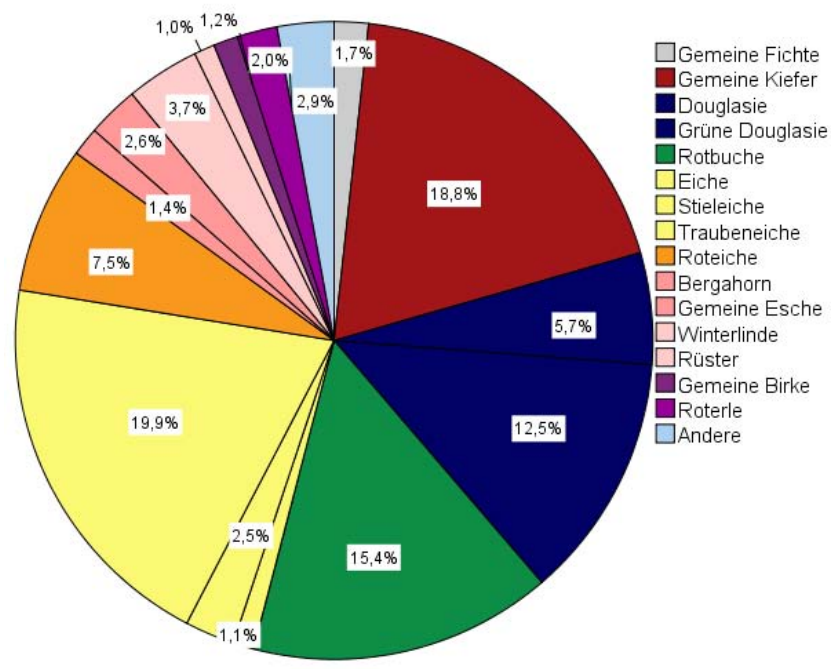

Abbildung 121: Baumartenanteile [\%] im Nachwuchs (unter Schirm) in der Region Colbitz-Letzlinger Heide; Nachwuchs auf ca. $4 \%$ der Gesamtwaldfläche

\subsubsection{Wichtigste Wachstumstrends durch den Klimawandel und Risikoveränderungen}

Auch in der Region Colbitz-Letzlinger Heide nimmt die Trockenstressgefährdung bis zum Ende des Jahrhunderts generell stark zu. Dies hat Auswirkungen vor allem auf die bisherige Buchenplanung, die sich nach heutiger Standortseinschätzung noch auf ca. $60 \%$ der Fläche an die Buche als Hauptbaumart der Stamm-Vegetationsform anlehnt. Ihr Trockenstressrisiko wird bis zum Ende des Jahrhunderts auf ca. $90 \%$ der Waldfläche als hoch einzustufen sein. Demgegenüber steigt die Gefährdung der gegenüber Wasserdefiziten weniger empfindlichen Baumarten Kiefer, Eiche und Douglasie durchgängig nur auf ein mittleres Niveau.

Deutlich negativ wird sich der Klimawandel auf die Produktivität der Wälder auswirken. Dabei wird die Kiefer bis zum Ende des Jahrhunderts den geringsten standortsbezogenen Leistungsabfall zeigen, während er bei den bereits heute hinter der Kiefer rangierenden Laubbaumarten Buche und Eiche deutlicher ausfallen wird. Die mit weitem Abstand absolut leistungsstärkste Baumart wird die Douglasie 
bleiben. Tendenziell werden die Leistungsrückgänge der Hauptbaumarten im Osten der Region mit ihren schwächeren Standorten größer sein.

Während das Sturmschadensrisiko für die Laubbaumarten gering ist und bei der Douglasie nur aufgrund der großen Baumhöhen bei Erreichen einer Zielstärke von $65 \mathrm{~cm}$ ein mittleres Niveau erreicht, wird die Waldbrandgefährdung in der Region bis zum Ende des Jahrhunderts stark zu nehmen. Die durchschnittliche Anzahl an Tagen mit erhöhter Waldbrandgefahr wird sich bis dahin in etwa verdoppeln. Bei der Eiche ist außerdem mit einem weiteren Anstieg des Befallsrisikos durch die Eichenfraßgesellschaften in den jeweils älteren Beständen zu rechnen. Ebenso wird die Gefährdung der Kiefer durch Kieferngroßschädlinge deutlich steigen, die in ihrer Populationsdynamik ebenfalls durch trockene, warme Witterung im Sommer begünstigt werden. Vor allem auf den schwächsten Standorten im Osten der Region wird sich das Risiko für die reinen Kiefernbestände erhöhen. Inwieweit Pilzinfektionen durch Kieferntriebsterben und Wurzelschwamm diese Tendenz noch verstärken, ist noch nicht abzusehen.

\subsubsection{Stabilisierung der vorhandenen Wälder in der Region Colbitz-Letzlinger Heide}

Ebenso wie im Fläming wird auch in der Region Colbitz-Letzlinger Heide die genügsame Kiefer in Zukunft die wichtigste Wirtschaftsbaumart bleiben. Sie ist an die zu erwartenden Klimaänderungen ökologisch gut angepasst, allerdings auch in erhöhtem Maße waldbrandanfällig. Zur Risikoverteilung sollte sie in Zukunft vermehrt in Mischung mit anderen Baumarten angebaut werden. Dabei erlaubt ihre Konkurrenzschwäche aber keine intensiven Mischungsformen.

Die für den Fläming gegebenen Entscheidungshilfen zur Begründung, Pflege und Entwicklung stabiler Kiefernbestände gelten uneingeschränkt auch für die ColbitzLetzlinger Heide und müssen daher hier nicht wiederholt werden. Gleiches gilt auch für die Empfehlungen für Eichenbestände. Bezüglich der Pflege der mittelalten Lärchenbestände wird auf die Ausführungen zur Region Oberharz verwiesen.

\subsubsection{Douglasienbestände}

Die Douglasie könnte in der Region in Zukunft wesentlich an Bedeutung gewinnen. Für die Douglasie spricht, dass sie standortsgerecht, bodenpfleglich, nicht über ein Normalmaß hinaus gefährdet, natürlich zu verjüngen, gut waldbaulich zu führen und leicht als Mischbaumart in heimische Ökosysteme zu integrieren ist (ОTTO 1993). Darüber hinaus überzeugt sie durch hohe Wuchsleistungen bei nur bescheidenen Standortsansprüchen und ist gut an die zu erwartenden Klimaänderungen angepasst (SPELLMANN et al. 2011).

Die heute meist im Raum Gardelegen/Klötze anzutreffenden KiefernDouglasien-Mischbestände sind entweder aus geordneten Voranbauten, aus Nachanbauten nach größeren Schadereignissen oder aus lückigen Douglasienkulturen mit 
Kiefernanflug entstanden. Ihr Entwicklungsziel sind stabile, gemischte, artenreiche, ungleichaltrige und vertikal gestufte Wälder, die eine einzelstammweise Ernte nach Zielstärke (Ki BHD 40-45 cm, Dgl BHD $65 \mathrm{~cm}$ ) und eine gezielte natürliche Verjüngung ermöglichen. Die Ausgangssituationen unterscheiden sich in den Überschirmungsverhältnissen, dem Altersvorsprung der Kiefern, ihrer Wuchsleistung und Qualität sowie in der teil- bzw. vollflächigen Einbringung der Douglasie.

In teilflächigen Nachanbauten mit einem Altersvorsprung der Kiefer von mehr als 60 Jahren ist davon auszugehen, dass beide Baumarten gemeinsam in die Zielstärke wachsen. Im Kiefernschirm sind die besten Kiefern konsequent zu pflegen. Unterschiede im Kronenschluss sind zur vertikalen Strukturierung des DouglasienNachanbaus zu nutzen. Die zu ästenden und herauszupflegenden Douglasien-ZBäume sollten einen Mindestabstand von 10-12 m zu Kiefern-Z-Bäumen haben und möglichst schirmfrei sein, damit sie ungehindert in den Oberstand einwachsen und ausreifen können. Die Pflege ist zunächst eine Ausleseläuterung im Herrschenden, später eine Hochdurchforstung mit abnehmender Durchforstungsstärke.

Bei vollflächigen Douglasiennachanbauten rechtfertigen die deutlich bessere Volumen- und Wertleistung der Douglasie nur in wüchsigen, qualitativ guten Kiefernbeständen die Erhaltung höherer Kiefernanteile von 20-50\%. Für ihren Erhalt sprechen vor allem Stabilitätsgründe, die Erhöhung der ökologischen Vielfalt und die größeren Gestaltungsspielräume bei der späteren Verjüngung dieser Bestände. Bei schlechter Qualität oder geringem Wuchs der Kiefern sollten aus denselben Gründen Kiefernanteile von 10-20 \% erhalten werden. Eine Entwicklung in Richtung Douglasienreinbestände ist zu vermeiden. Für die Kiefernbereiche sind Hochdurchforstungen zu empfehlen. Die unter ihnen stehenden Douglasien sind nicht zu pflegen und nicht zu ästen. Sie werden im Zuge der Vornutzungen entnommen, sobald sie in den Kronenraum der Kiefern vordringen. In den Douglasienbereichen müssen zunächst die qualitativ schlechten Kiefern herausgezogen werden. Die besseren Kiefern können solange verbleiben, bis sie die Entwicklung der Douglasien wesentlich beeinträchtigen (Leittriebverletzungen, Kronendeformationen). Um den Kiefernschirm möglichst lange für die Differenzierung der Douglasien ausnutzen zu können, sollten Douglasien-Z-Bäume wenig oder gar nicht, andere Douglasien dagegen länger überschirmt sein. Die mit einer Ausleseläuterung im Herrschenden zu kombinierende Ästung der Z-Bäume muss erst bei Oberhöhen von 8-10 m erfolgen, weil die Douglasien unter Schirm feinastiger und vollholziger sind. Die Z-Bäume sollten in der ersten Ästungsstufe auf 3 bis 3,5 m, ggf. auf $6 \mathrm{~m}$ geästet werden. Die Bestandespflege sollte sowohl in der Kiefer, als auch in der Douglasie als gestaffelte Hochdurchforstung erfolgen, die im mittleren bis starken Baumholz in die Zielstärkennutzung übergehen.

Die heute gleichaltrigen Douglasien-Kiefern-Mischbestände sind meist nach Kalamitäten aus lückigen Douglasien-Freiflächen-Kulturen mit Kiefern- und Birkenanflug entstanden. Die Pflege dieser Bestände muss darauf ausgerichtet sein, die besten Douglasien herauszuarbeiten und die Mischbaumarten zur Erhöhung der 
Vielfalt und der Bestandesstabilität angemessen zu fördern. Die Jungwuchspflege (bis $2 \mathrm{~m}$ Oberhöhe) sollte sich auf die Entnahme stark verbuschter bzw. verzwieselter Douglasien beschränken. Auf den Teilflächen, wo der Jungbestand 6-7 m Oberhöhe erreicht hat, muss in der Regel eine zweite negative Auslese durchgeführt werden. Dabei sind die jeweils besten Bäume hochdurchforstungsartig zu fördern und die herrschenden Baumarten gruppen- bzw. horstweise zu trennen. Die ausgewählten Douglasien-Z-Bäume sind meist relativ frei erwachsen und daher lang bekront und grobastig. Zur Sicherung ihrer Wertleistung ist eine mehrstufige Ästung mit früh einsetzender, stark in die grüne Krone eingreifende Reichhöhenästung vorzusehen. In der Kiefer beschränkt sich die Läuterung auf einen Protzenaushieb, damit die Astreinigung im Dichtstand gefördert wird. Gute Birken sind herauszupflegen. Diese Art der Pflege ist bei den ersten Durchforstungen beizubehalten. Anschließend wird die Z-Baumpflege mit abnehmender Durchforstungsstärke und sich vergrößernden Durchforstungsintervallen fortgesetzt. Ziel ist es, dass das Bestandesbild von herrschenden, gut bekronten Douglasien und Kiefern in ungleichmäßiger Verteilung geprägt wird. Eine breite Durchmesserspreitung ermöglicht später eine zeitlich gestreckte und räumlich konzentrierte Zielstärkennutzung. Der Kronenschluss sollte locker bis geschlossen sein, um ausreichend Licht zum Erhalt des Unter- und Zwischenstandes durchzulassen.

\subsubsection{Weichlaubholz̧bestände}

Sandbirke und Roterle sind die wichtigsten Vertreter dieser Baumartengruppe. Obwohl die Sandbirke eine sehr weite Standortsamplitude hat, ist sie in der Region wegen der meist höheren Konkurrenzkraft der anspruchsvolleren Baumarten vorwiegend auf feuchte oder trockene bis sehr trockene, nährstoffarme und saure Böden zurückgedrängt. Dort bildet sie natürlicherweise Reinbestände oder kommt in Mischung mit anderen Lichtbaumarten wie Kiefer und Eiche vor. Ihre flächenbezogene Volumenleistung ist relative gering. Als Vorteile gelten ihr enormes Verjüngungspotenzial, ihr schnelles Jungendwachstum und ihre geringen Ansprüche an die Standortsgüte. Entsprechend ihrem natürlichen Wachstumsgang, mit einer sehr frühen Kulmination und einem anschließend starken Abfall der Zuwachsgrößen, lässt sich höherwertiges Stammholz nur erzeugen, wenn die Pflege bereits im Jungbestand mit starken Eingriffen zur Freistellung der Kronen der vitalsten und qualitativ besten Bäume einsetzt und mit zunehmendem Alter dann in schwache Eingriffe übergeht. Dies ist erforderlich, da die Sandbirke ab einem Alter von ca. 25 Jahren auf eine Freistellung kaum noch mit einem Kronenausbau reagiert und mit der eingeschränkten Kronenentwicklung der Durchmesserzuwachs stark zurückgeht (HEIN et al. 2009). Die Förderung der Z-Bäume (ca. 100 Stück/ha) muss auf den schwachen Standorten konsequent betrieben werden, um in ca. 60 Jahren Produktionszeit Zielstärken von 40 bis $45 \mathrm{~cm}$ zu erreichen. 
Die Roterle ist die wichtigste Wirtschaftsbaumart unter den Weichlaubhölzern. Sie besiedelt ausgesprochen nasse, ganzjährig grundwasserbeeinflusste oder zeitweise überschwemmte Standorte wie Auen, Niedermoore oder Brüche. Bei guter Nährstoffversorgung zeigt sie ansprechende Wuchsleistungen. Auf Trockenheit reagiert die Roterle sehr empfindlich, weshalb in Zukunft der Entwässerung bzw. Gestaltung der Vorflut im Bereich der Roterlenstandorte eine besondere Aufmerksamkeit geschenkt werden muss. Ernte- und bringungstechnisch sind die meist nicht oder nur eingeschränkt befahrbaren Roterlen-Standorten sehr schwierig, weshalb die Bestände oftmals Pflege- und Nutzungsrückstände aufweisen. Während früher vielerorts die Roterle zur Brennholzgewinnung im Niederwaldbetrieb bewirtschaftet wurde, erfolgt die Bewirtschaftung heute fast ausschließlich als Hochwald mit dem Ziel, möglichst viel wertvolles, d. h. qualitativ gutes, stärker dimensioniertes Stammholz zu produzieren. Aufgrund des vergleichbaren Wachstumsgangs entsprechen die Pflegegrundsätze der Roterle weitgehend denjenigen der Sandbirke. Auch bei ihr muss die Bestandespflege in der Stangenholzphase einsetzen, um die Reaktionsfähigkeit der bestveranlagten 120 bis 160 Z-Bäumen/ha auf Freistellung ausnutzen zu können. Das Pflegeziel ist die Ausbildung gleichmäßiger, vitaler Z-Baumkronen, die die Voraussetzung für einen guten Stärkezuwachs sind. Nach den ersten drei starken Eingriffen nimmt mit dem Bestandesalter die Durchforstungsstärke ab, weil dann der verbleibende Bestand nicht mehr nennenswert auf weitere Freistellung reagiert und stattdessen die Gefahr der Wasserreiserbildung steigt. In bisher nicht zielgerecht gepflegten Beständen müssen daher auch Durchforstungsrückstände in mehreren mäßig starken Eingriffen nachgeholt werden. Wegen der Gefahr einer Holzentwertung durch Stammfäule sollten Produktionszeiten von 60-80 Jahren nicht überschritten werden. Auf guten Standorten lassen sich in diesem Zeitraum Stammdurchmesser von über $45 \mathrm{~cm}$ erreichen.

\subsubsection{Standortsgemäßer Waldumbau}

Die Veränderungsanalysen für die Region Colbitz-Letzlinger Heide zeigen weniger einen Anpassungsbedarf für die heutige Bestockung, als vielmehr für die bisherige Waldbauplanung auf (s. Tab. 24). 
Tabelle 24: Bestandeszieltypen für die wichtigsten Standorte der Region Colbitz-Letzlinger Heide obne organische Nassstandorte (nach MLU LSA 2003)

\begin{tabular}{|c|c|c|c|c|}
\hline $\begin{array}{l}\text { Klima- } \\
\text { stufe }\end{array}$ & $\begin{array}{l}\text { Stamm- } \\
\text { StaO- } \\
\text { formen- } \\
\text { Gruppe }\end{array}$ & $\begin{array}{c}\text { Flächen- } \\
\text { anteil } \\
{[\%]}\end{array}$ & Stamm-Vegetationsform & Bestandeszieltypen \\
\hline \multirow[t]{4}{*}{$\mathrm{Tm}$} & $\mathrm{K} 2$ & 2 & Riesenschwingel-TEi-Bu-Wald & $\begin{array}{l}\text { BU-EI, EI-BU, BU-NDB, ELÄ-SCH, } \\
\text { KI-SCH }\end{array}$ \\
\hline & M2 & 30 & Hainrispen-TEi-Bu-Wald & $\begin{array}{l}\text { BU-EI, EI-BU, BU-NDB, KI.-LIB, KI- } \\
\text { SCH }\end{array}$ \\
\hline & $\mathrm{Z} 2$ & 11 & $\begin{array}{l}\text { Sauerklee-Blaubeer-TEi-Bu- } \\
\text { Wald }\end{array}$ & BU-EI, BU-NDB, KI-LIB, KI, DGL \\
\hline & $\mathrm{A} 2$ & 9 & Blaubeer-Bu-Kiefern-Wald & KI-SCH, KI, KI-LIB \\
\hline \multirow[t]{5}{*}{$\mathrm{Tt}$} & K2 & 3 & $\begin{array}{l}\text { Riesenschwingel-HBu-WLi-TEi- } \\
\text { Wald }\end{array}$ & $\begin{array}{l}\text { TEI-WLI-HBU, EI-BU, ELÄ-SCH, KI- } \\
\text { SCH }\end{array}$ \\
\hline & M1 & 1 & Hainrispen-HBu-WLi-TEi-Wald & $\begin{array}{l}\text { SEI-HBU, EI-BU, KI-SCH, DGL-BU, } \\
\text { ELÄ-SCH }\end{array}$ \\
\hline & M2 & 18 & $\begin{array}{l}\text { Hainrispen-Hainbuchen- } \\
\text { Winterlinden-TEi-Wald }\end{array}$ & $\begin{array}{l}\text { TEI-WLI-HBU, Ei-BU, KI-LIB, KI- } \\
\text { SCH, ELÄ-SCH, DGL-BU, KI }\end{array}$ \\
\hline & $\mathrm{Z1}$ & 2 & $\begin{array}{l}\text { Pfeifengras-Sauerklee-Blaubeer- } \\
\text { Ei-Wald }\end{array}$ & EI-BI, KI-LIB, KI-SCH, KI-DGL, KI \\
\hline & $\mathrm{Z} 2$ & 6 & Sauerklee-Blaubeer-TEi-Wald & EI-BI, KI-LIB, KI \\
\hline \multicolumn{2}{|c|}{ Sonstige* } & 18 & & \\
\hline
\end{tabular}

Die Erwartung, dass die Erholung ehemals devastierter Standorte durch eine langjährige Bestockung mit Kiefer in mehreren Waldgenerationen und die Stickstoffeinträge aus der Luft vielerorts einen Baumartenwechsel von der genügsamen Kiefer zu anspruchsvolleren Baumarten ermöglicht, findet ihre Grenzen in den Klimaprojektionen. Dies betrifft vor allem die Laubholzvermehrung zugunsten der Buche. Als führende Baumart sollte sie auf den kräftigen Standorten mit guter Wasserversorgung gehalten werden und als Mischbaumart die Integration anbauwürdiger, eingeführter Baumarten wie Douglasie, Küstentanne und Roteiche in die heimische Fauna und Flora unterstützen. Außerdem können Mischungsanteile dieser Schattbaumart dazu beitragen, das Bestandesklima in Kiefernbeständen auf Standorten mit heute mindestens mittlerer Feuchtestufe zu verbessern und ggf. die Vitalität bzw. Ausbreitung der Spätblühenden Traubenkirsche einzuschränken. Demgegenüber wird der Anbau von Douglasie, Küstentanne und Roteiche an Bedeutung gewinnen. Die Douglasie bietet sich für ein breites Standortsspektrum von ziemlich arm bis kräftig sowohl im mäßig trockenen, als auch im trockenen Tiefland an. Gleiches gilt auch für die Küstentanne, die an die erwarteten Klimaänderungen ebenfalls gut 
angepasst ist und sich für mittlere und ziemlich arme Standorte in beiden Klimastufen anbietet. Die Roteiche kann auf den mittleren und ziemlich armen Standorten der Untersuchungsregion teilweise an die Stelle der Kiefer bzw. der Traubeneiche treten und dazu beitragen, die Ertragserwartungen zu sichern und das Waldbrandrisiko zu begrenzen. Die Ausweitung des Anbaus dieser eingeführten Baumarten muss aber mit Augenmaß, räumlicher Ordnung und unter Beachtung naturschutzfachlicher Aspekte erfolgen. Leitbaumart der Colbitz-Letzlinger Heide wird in jedem Fall die Kiefer bleiben. Auch die Traubeneiche wird voraussichtlich ihre Stellung als wichtigste Laubbaumart bewahren und zwar als führende Baumart auf den mittleren und kräftigen Standorten mit besserer Bodenfeuchte und als Mischbaumart auf den schwächeren, schlechter mit Wasser versorgten Standorten. Zur Begrenzung der Risiken sollte allgemein der Beteiligung der Pionierbaumarten in den Waldentwicklungszielen mehr Beachtung geschenkt und diese auch aktiver in die Bewirtschaftung einbezogen werden. Für die Zukunft der Erlenwirtschaft wird es entscheidend sein, ob es gelingt, bei zu erwartend feuchteren Wintern und trockeneren Sommern durch geeignete Wasserführung ein Austrocknen der organischen Nassstandorte zu verhindern.

Die Schwerpunkte des standortsgemäßen Waldumbaus entsprechen denjenigen im Fläming. Es sind dies:

- im mäßig trockenen Tiefland auf mäßig nährstoffhaltigen Standorten:

- Überführung von Kiefernreinbeständen in Kiefern-Laubholz-Mischbestände unter Beteiligung von Eiche, Roteiche oder Buche und Birke

- Überführung von Kiefernreinbeständen in Kiefern-Douglasien/Küstentannen-Mischbestände unter Beteiligung von Buche und Birke

- Überführung von Kiefernreinbeständen in Douglasien/KüstentannenLaubholzmischbestände unter Beteiligung von Buche oder Roteiche und Birke

- Überführung von Buchenreinbeständen in Buchen-Nadelholzmischbestände unter Beteiligung von Douglasie, Küstentanne oder Europäischer Lärche

- im mäßig trockenen Tiefland auf ziemlich armen Standorten:

- Überführung von Kiefernreinbeständen in Kiefern-Laubholz-Mischbestände unter Beteiligung von Eiche oder Roteiche und Birke

- Überführung von Kiefernreinbeständen in Kiefern-Douglasien/Küstentannen-Mischbestände unter Beteiligung von Birke

- Überführung von Kiefernreinbeständen in Douglasien-KüstentannenMischbestände unter Beteiligung von Birke

- im trockenen Tiefland auf mäßig nährstoffhaltigen Standorten: 
- Überführung von Kiefernreinbeständen in Kiefern-Laubholz-Mischbestände unter Beteiligung von Eiche oder Roteiche und Birke

- Überführung von Kiefernreinbeständen in Kiefern-Douglasien/Küstentannen-Mischbestände unter Beteiligung von Birke

Die für die Region Fläming gegebenen Empfehlungen zum Voranbau unter Kiefer gelten auch für die Region Colbitz-Letzlinger Heide. 


\section{Literatur}

AG BODEN (2005): Bodenkundliche Kartieranleitung. Bundesanstalt für Geowissenschaften und Rohstoffe (Hrsg.), 5. Aufl., Hannover. 438 S.

AHrendS, B. (2010): Entwicklung eines dynamischen Standortmodells auf der Basis flächenhafter Standortsdaten. 3. Zwischenbericht des BMBF-Verbundprojektes „Anpassungsstrategien für eine nachhaltige Waldbewirtschaftung unter sich wandelnden Klimabedingungen - Decision Support System Wald und Klimawandel", 10-19

Ahrends, B.; Döring, C.; Jansen, M. u. Meesenburg, H. (2008): Unterschiedliche Nutzungsszenarien und ihre Auswirkungen auf die Basensättigung im Wurzelraum - Ergebnisse von Szenarienvergleichen in Teileinzugsgebieten der Großen Bramke. Forst und Holz 63 (12), 32-36

Ahrends, B.; Döring, C.; Jansen, M.; Meesenburg, H. u. Beese, F. (2007): Kopplung dynamischer Modelle für die flächenhafte Abschätzung der Stoffdeposition im Wald. Mitt. DBG 110 (2), $421-422$

Ahrends, B.; Meesenburg, H.; Döring, C. u. JAnsen, M. (2009): Assessment of forest management effects on N-cycling at three basins in the Upper Harz Mountains, Germany. Landschaftsökologie und Umweltforschung 50, 3-7

Ahrends, B.; Meesenburg, H.; Döring, C. u. Jansen, M. (2010): A spatio-temporal modelling approach for assessment of management effects in forest catchments. Status and Perspectives of Hydrology in Small Basins, IAHS Publ. 336, 32-37

Albert, M. u. SCHMidT, M. (2010a): Climate-sensitive modelling of site-productivity relationships for Norway spruce (Picea abies (L.) Karst.) and common beech (Fagus sylvatica L.). For. Ecol. Manage. 259, 739-749

Albert, M. u. SChMidT, M. (2010b): Dynamik der Standort-Leistungs-Beziehungen von Buche, Eiche, Fichte, Kiefer und Douglasie unter Klimaveränderungen in Deutschland. Tagungsband der Jahrestagung der Sektion Ertragskunde im DVFFA vom 17.-19.05.2010 in Körbecke, 66-81

Albert, M. u. SChMidt, M. (2012): Standort-Leistungs-Modelle für die Entwicklung von waldbaulichen Anpassungsstrategien unter Klimawandel. Archiv f. Forstwesen u. Landsch.ökol. 46 (2), $57-71$

Allen, R. G.; Smith, M.; Perrier, A. u. Pereira, L. S. (1994): An update for definition of reference evapotranspiration. ICID Bulletin 43, 2

Altenkirch, W.; Majunke, C. u. Ohnesorg, B. (2002): Waldschutz auf ökologischer Grundlage. Ulmer Verlag Stuttgart, $434 \mathrm{~S}$.

Alveteg, M.; Walse, C. u. Warfvinge, P. (1998): Reconstructing historic atmospheric deposition and nutrient uptake from present day values using MAKEDEP. Water, Air, and Soil Pollution, $104,269-283$

Amann, M.; Bertok, I.; Cofala, J.; Heyes, C.; Klimont, Z.; Rafaj, P.; Schöpp, W. u. Wagner, F. (2008): National Emission Ceilings for 2020 based on the 2008 Climate \& Energy Package. NEC Scenario Analysis Report Nr. 6, International Institute for Applied Systems Analysis (IIASA, Laxenburg, $72 \mathrm{~S}$.

Anders, S.; Beck, W.; Bolte, A.; Hofmann, G.; Jenssen, M.; Krakau, U.-K. u. Müller, J. (2002): Ökologie und Vegetation der Wälder Nordostdeutschlands. Dr. Kessel. Oberwinter, 283 S.

APEL, K.-H.; HAUSWIRTH, M.; RÖS, M. u. WENK, M. (2004): Schadgebiete der wichtigsten nadelfressenden Forstschadinsekten der Kiefer und Risikobewertung von Waldgebieten für das Nordostdeutsche Tiefland. Beitr. Forstwirtschaft Landschaftsökol. 38, 14-18

Baier, P.; Pennerstorfer, J. u. Schopf, A. (2007): PHENIPS-A comprehensive phenology model of Ips typographus (L.) (Col., Scolytinae) as a tool for hazard rating of bark beetle infestation. For. Ecol. Manage. 249, 171-186

Beiträge aus der NW-FVA, Band 13, 2015 
BLE (2012):Waldbrandstatistik der Bundesrepublik Deutschland für das Jahr 2011. Bundesanstalt für Landwirtschaft und Ernährung, Bonn

Bolte, A. u. Degen, B. (2010): Anpassung der Wälder an den Klimawandel: Optionen und Grenzen. Landbauforschung - vTI Agriculture and Forestry Research 3, 60, 111-118

BRINKMANN, S. u. NiedER, R. (2002): Critical Loads für eutrophierenden Stickstoff - Weiterentwicklung des Ansatzes unter besonderer Berücksichtigung der Umsetzung im Boden (Humusschicht). Inst. f. Geoökologie, Techn. Univ Braunschweig. Braunschweig, 116 S.

Builtjes, P.; Hendriks, E.; Koenen, M.; SchaAp, M.; Banzhaf, S.; Kerschbaumer, A.; Gauger, T.; Nagel, H.-D.; Scheuschner, T. u. Schlutow, A. (2011): Erfassung, Prognose und Bewertung von Stoffeinträgen und ihren Wirkungen in Deutschland (Modelling of Air Pollutants and Ecosystem Impact - MAPESI). Texte Umweltbundesamt, Dessau-Roßlau. $97 \mathrm{~S}$.

Büttner, K.; Hennig, R.; Kneitz, G.; Majunke, C. u. Schrötter, H. (1994): Waldhygiene: Die Hauptschadgebiete des Kiefernspanners im nordostdeutschen Tiefland. Eberswalde, 20: 65-95

De Martonne, E. (1926): Une nouvelle fonction climatologique: l' indice d' aridité. La Météorologie $2,449-458$

Dise, N. u. Wright, R. F. (1995): Nitrogen leaching from European forests in relation to nitrogen deposition. For. Ecol. Manage. 71, 153-161

EBERT, W. (1967): Ergebnisse arealkundlicher Untersuchungen über die wichtigsten Kiefernbestandesschädlinge im nördlichen Tiefland der DDR. Die Sozialistische Forstwirtschaft, 361-364

EBERT, W. (1968): Die Schadgebiete unserer wichtigsten Kiefernbestandesschädlinge im Tiefland der DDR. Archiv für Forstwesen, Berlin. 17, 125-144

FAhrmeIR, L.; KNeIB, T. u. LANG, S. (2007): Regression: Modelle, Methoden und Anwendungen. Berlin Heidelberg, Springer: 490

Fowler, D.; Smith, R.; Muller, J.; Cape, J. N.; Sutton, M.; Erisman, J. W. u. Fagerli, H. (2007): Long term trends in sulphur and nitrogen deposition in europe and the cause of non-linearities. Water Air Soil Pollut.: Focus 7, 41-47

Gauger, T.; Haenel, H.-D.; Rösemann, C.; NAgel, H.-D.; Becker, R.; Kraft, P.; Schlutow, A.; SChÜtZe, G.; Weigelt-KirChNer, R. u. ANSHELm, F. (2008): Nationale Umsetzung UNECELuftreinhaltekonvention (Wirkung). Abschlussbericht zum UFOPLAN-Vorhaben FKZ 20463 252. Im Auftrag des Umweltbundesamtes, gefördert vom Bundesministerium f. Umwelt, Naturschutz und Reaktorsicherheit. Dessau-Roßlau.

Gebhardt, T.; Grams, T.; HäBerle, K.-H.; Matyssek, R.; Schulz, C.; Grimmeisen, W. u. Ammer, C. (2012): Helfen Durchforstungen bei Trockenheit? LWF aktuell 87, 8-10

Glatzel, G.; KazDA, M.; Grill, D.; Halbwachs, G. u. KatZensteiner, K. (1987): Ernährungsstörungen bei Fichte als Komplexwirkung von Nadelschäden und erhöhter Stickstoffdeposition ein Wirkungsmechanismus des Waldsterbens? Allg. Forst- u. J.-Ztg. 158 (5/6), 91-97

Habermann, M. (1995): Zur Massenvermehrung der Nonne (Lymantria monacha L.) in Kiefernbeständen (Pinus sylvestris L.) des Niedersächsischen Flachlandes. Forst Holz 50, 558-564

Habermann, M. u. BeEsTer, R. (1997): Einfluss von Bestandesstrukturen und Nadelphysiologie auf die Entstehung von Massenvermehrungen der Nonne (Lymantria monacha L.) in Kiefernbeständen (Pinus sylvestris L.) eines Dauerschadgebietes in Niedersachsen. Allg. Forst- u. J.-Ztg. 168, 157-162

Hagemann, H.; Bilke, G.; MuraCh, D. u. SChulte, A. (2008): Bilanzierung und Bewertung von Nährelemententzügen durch Vollbaumnutzungsstrategien bei der Kiefer (Pinus sylvestris) in Brandenburg. Archiv f. Forstwesen u. Landsch.ökol. 42, 16-25

Hauck, M.; Zimmermann, J.; Mascha, J.; Dulamsuren, C.; Bade, C.; Ahrends, B. u. Leuschner, C. (2012): Rapid recovery of stem growth at reduced $\mathrm{SO}_{2}$ levels suggests a major contribution of foliar damage in the pollutant-caused dieback of Norway spruce during the late 20th century. Environ. Pollut. 164, 132-141

Hein, S.; Winterhalter, D.; Wilhelm, G. J. u. Kohnle, U. (2009): Wertholzproduktion mit der Sandbirke (Betula pendula Roth): waldbauliche Grenzen und Möglichkeiten. Allg. Forst- u. J.-Ztg. 180, 206-218 
Jansen, M.; Döring, C.; Ahrends, B.; Meesendurg, H.; Meiwes, K.-J. u. Beese, F. (2007): Kopplung dynamischer Modelle für die Bodenschutzkalkung im Wald. Mitt. DBG 110 (2), 483-484

KÄSE, H. (1969): Ein Vorschlag für eine Methode zur Bestimmung und Vorhersage der Waldbrandgefährdung mit Hilfe komplexer Kennziffern. Abh. des meteorol. Dienstes der Deutschen Demokratischen Republik 94 (13)

KÄTZEL, R.; BECKER, F. u. LÖFFLER, S. (2012): Zehn Jahre genetisches Monitoring in Eichenbeständen Brandenburgs. Forstarchiv 83, 26-33

KENK, G. u. FisCHER, H. (1988): Evidence from nitrogen fertilisation in the forests of Germany. Environ. Pollut. 54, 199-218

KLinck, U.; Rademacher, P.; SCheler, B.; Wagner, M.; Fleck, S.; Ahrends, B. u. Meesendurg, H. (2012): Ökosystembilanzen auf forstwirtschaftlich genutzten Flächen. GeoBerichte 23, $163-174$

KNoKe, T.; STIMm, B.; Ammer, C. u. Moog, M. (2005): Mixed forests reconsidered: A forest economics contribution on an ecological concept. For. Ecol. Manage. 213, 102-116

Kohler, M.; SoHN, J.; NÄGELE, G. u. BAuhus, J. (2010): Can drought tolerance of Norway spruce (Picea abies (L.) Karts.) be increased through thinning? Eur. J. Forest Res. 129, 1109-1118

KÖNIG, H.-C. (2007): Waldbrandschutz: Kompendium für Forst und Feuerwehr. Grimm, Berlin

KREIENKAMP, F.; SPEKAT, A. u. ENKE, W. (2012): Durchführung einer Untersuchung zu den Folgen des Klimawandels in Sachsen-Anhalt. Bericht, Los 1.1 und 2.1. Climate and Environment Consulting Potsdam GmbH im Auftrag des Landesamtes für Umweltschutz Sachsen-Anhalt, Halle (Saale)

Kropp, J.; Roithmeier, O.; Hattermann, F.; Rachimow, C.; LÜttger, A.; Wechung, F.; Lasch, P.; Christiansen, E.; ReYer, C.; SuCKOW, F.; Gutsch, M.; Holsten, A.; KARTSCHAll, T.; WodinSKi, M.; Hauf, Y.; Conradt, T.; Österle, H.; Walther, C.; Lissner, T.; LuX, N.; TekKen, V.; Ritschie, S.; Kossak, J.; Klaus, M.; VetTer, L. C. L. T. u. Klose, M. (2009): Klimawandel in Sachsen-Anhalt-Verletzlichkeiten gegenüber den Folgen des Klimawandels. Technical report, Abschlussbericht des Potsdam-Instituts für Klimafolgenforschung (PIK)

Langer, G.; Bressem, U. u. Habermann, M. (2011): Diplodia-Triebsterben der Kiefer und endophytischer Nachweis des Erregers Sphaeropsis sapinea. AFZ-DerWald (11), 28-31

LAPPI, J. (1997): A longitudinal analysis of height/diameter curves. Forest Sci. 43, 555-570

LANDESAMT FÜr GEOlOGIE UND BERGWESEN (2009): Vorläufige Bodenkarte 1:50.000 (VBK 50), Landesamt für Geologie und Bergwesen Sachsen-Anhalt, Halle

Laubhann, D.; Sterba, H.; Reinds, G. J. u. DeVries, W. (2009): The impact of atmospheric deposition and climate on forest growth in European monitoring plots: An individual tree growth model. For. Ecol. Manage. 258, 1751-1761

LÜPKE, B. v. (1995): Waldbau unter ökonomischen Zwängen? Vortrag anläßlich des Forstökonomischen Kolloquiums "Forstwirtschaft im Umbruch" am 12.5.1995 in Göttingen

LÜPKE, B. v. (2004): Risikominderung durch Mischwälder und naturnaher Waldbau: ein Spannungsfeld. Forstarchiv 75, 43-50

Maindonald, J. u. Braun, J. (2007): Data analysis and graphics Using R. An example-based approach. $2^{\text {nd }}$ edition, Cambridge University Press, 502 S.

Majunke, C.; MÖLLER, K. u. FunKe, M. (2004): Waldschutz-Merkblatt Nr. 52 - Die Nonne. Landesforstanstalt Eberswalde, Abteilung Waldschutz, $24 \mathrm{~S}$.

Matson, P.; LOHSE, K. A. u. Hall, S. J. (2002): The globalisation of nitrogen exposition: Consequences for terrestrial ecosystems. Ambio 31, 113-119

Menzel, A. u. Fabian, P. (1999): Growing season extended in Europe. Nature 397

MissBACH, K. (1972): Waldbrand Verhütung und Bekämpfung. Deutscher Landwirtschaftsverlag, Berlin

MLU LSA (2003): Waldbauempfehlungen des MLU LSA. Rd.-Erl. des Ministeriums für Landwirtschaft und Umwelt Sachsen-Anhalt. Magdeburg

Beiträge aus der NW-FVA, Band 13, 2015 
MLU LSA (2012): Waldzustandsbericht Sachsen-Anhalt 2012. Ministerium für Landwirtschaft und Umwelt Sachsen-Anhalt u. Nordwestdeutsche Forstliche Versuchsanstalt (Hrsg.), 31 S.

Mudelsee, M.; Chirila, D.; Deutschländer, T.; Döring, C.; Haerter, J.; Hagemann, S.; Hoffmann, H.; Jacob, D.; Krahé, P.; Lohmann, G.; Moseley, C.; Nilson, E.; Panferov, O.; Rath, T. u. Tinz, B. (2010): Climate Model Bias Correction und die Deutsche Anpassungsstrategie. Mitt. DMG 03, 2-7

Nagel, R.-V. u. Spellmann, H. (2008): Wachstum, Behandlung und Ertrag von Reinbeständen der Rotbuche (Fagus sylvatica L.) in Nordwestdeutschland. In: Nordwestdt. Forstl. Versuchsanstalt (Hrsg.) 2008: Ergebnisse angewandter Forschung zur Buche. Beitr. Nordwestdt. Forstl. Versuchsanstalt 3, 221-265

NiLSEN, P. (1990): Effect of nitrogen on drought of Norway spruce and Scots pine. Sci. Total Environ. 96, 189-198

NiLSSON, L.-O. u. WikLund, K. (1992): Influence of nutrient and water stress on Norway spruce production in south Sweden - the role of air pollutants. Plant and Soil 147, 251-265

Отто, H. J. (1993): Fremdländische Baumarten in der Waldbauplanung. Forst Holz 48, 454-456

Отто, H. J. (1994): Ökologischer Waldbau - Walddynamische Prozesse bei Fichte und Kiefer im Rahmen waldbaulicher Rationalisierung. Der Wald 44, 364-367, 408-411

Overbeck, M. u. SCHMidT, M (2011): Modelling infestation risk of Norway spruce by Ips typographus (L.) in the Lower Saxon Harz Mountains (Germany). For. Ecol. Manage. 266, 115-125

Piani, C.; HAerter, J. O. u. Coppola, E. (2010): Statistical bias correction for daily precipitation in regional climate models over Europe. Theor. Appl. Climatol. 99, 187-192

Pinheiro, J.; Bates, D.; Debroy, S.; Sarkar, D. and the R Development Core Team (2012). nlme: Linear and Nonlinear Mixed Effects Models. R package version 3.1-105

Plašil, P. u. HABERMAnN, M. (2012): Beurteilung verschiedener Waldschutzrisiken in Sachsen-Anhalt. AFZ/Der Wald 67 (22), 26-29

Prietzel, J.; Kolb, E. u. Rehfuess, K. E. (1997): Langzeituntersuchung ehemals streugenutzter Kiefernökosysteme in der Oberpfalz: Veränderungen von bodenchemischen Eigenschaften und der Nährelementversorgung der Bestände. Forstw. Cbl. 116, 269-290

R Development Core Team (2006): R. A language and environment for statistical computing. R Foundation for Statistical Computing, Vienna

RichTER, D. (1965): Zum Auftreten des Großen Fichtenborkenkäfers (Ips typographus L.). Die Sozialistische Forstwirtschaft, (Sonderdruck, Heft 2) (15), 49-54

RiCHTER, J. (1995): Der Übergang zur Zielstärkennutzung in gleichaltrigen Fichtenbeständen. Forst Holz 50 (13), 414-415

RÜther, B.; Hansen, J.; Spellmann, H.; Nagel, J.; Möhring, B.; Schmidt-Walter, P. u. Dieter, M. (2008): Clusterstudie Forst und Holz Sachsen-Anhalt. Nordwestdeutsche Forstliche Versuchsanstalt (Hrsg.), $60 \mathrm{~S}$.

RumpF, H. u. DitGes, J. (2008): Jugendwachstum von Fichtennaturverjüngung in Abhängigkeit von Überschirmungsdichte und Pflegestrategie. Forst Holz 63 (12), 20-25

SChelhaAs, M.-J.; NABUURS, G.-J. u. SCHUCK, A. (2003): Natural disturbances in the European forests in the 19th and 20th centuries. Global Change Biol. 9, 1620-1633

SCHMidT, M. (2010): Ein standortsensitives, longitudinales Höhen-Durchmesser-Modell als eine Lösung für das Standort-Leistungs-Problem in Deutschland. Jahrestagung der Sektion Ertragskunde im DVFFA in Körbecke am Möhnesee, 131-152

Schmidt, M.; Hanewinkel, M.; Kändler, G.; Kublin, E. u. Kohnle, U. (2010): An inventorybased approach for modeling single-tree storm damage - experiences with the winter storm of 1999 in southwestern Germany. Can. J. For Res. 40 (8), 1636-1652 (doi:10.1139/X10-099)

Schmidt, W.; Stüber, V.; Ullrich, T.; Paar, U.; Evers, J.; Dammann, K.; Hövelmann, T. u. SCHMIDT, M. (2015.): Synopse der Hauptmerkmale der forstlichen Standortskartierungsverfahren der Nordwestdeutschen Bundesländer. Beiträge aus der NW-FVA, Band 12, $136 \mathrm{~S}$. 
SCHOBER, R. (1988): Von Zukunfts- und Elitebäumen. Allg. Forst- u. J.-Ztg. 159 (11/12), 239-248

SCHOBER, R. (1987): Ertragstafeln wichtiger Baumarten. J.D. Sauerländer's Verlag, Frankfurt/Main. $154 \mathrm{~S}$.

Schöpp, W.; Posch, M.; Mylona, S. u. Johansson, M. (2003): Long-term development of acid deposition (1880-2030) in sensitive freshwater regions in Europe. Hydrol. Earth System Sci. 7 (4), 436-446

Schulda, J. u. JASPER, K. (2007): Model Description WaSiM-ETH, Technical Report, unveröff. http://www.wasim.ch/downloads/doku/wasim/wasim_2007_en.pdf.

SCHWANECKE, W. u. Kopp, D. (1994): Forstliche Wuchsgebiete und Wuchsbezirke des Landes SachsenAnhalt (Naturraumareale auf der Grundlage der forstlichen Standortserkundung). Im Auftrag der Forstlichen Landesanstalt Sachsen-Anhalt. Haferfeld, 203 S.

SCOTT, R. E. u. MitCHELL, S. J. (2005): Empirical modelling of windthrow risk in partially harvested stands using tree neighbourhood and stand attributes. For. Ecol. Manage. 218, 193-209. doi:10.1016/j.foreco.2005.07.012

SkUHRAvÝ, V. (2002): Lýkožrout smrkový (Ips typographus L.) a jeho kalamity. Der Buchdrucker und seine Kalamitäten. Agrostroj, Praha. 196 pp., 125 obr.

SpeIDEL, G. (1991): Planung im Forstbetrieb. Hamburg u. Berlin, 267 S.

SpeKat, A.; ENKE, W. u. KREIENKAMP, F. (2007): Neuentwicklung von regional hoch aufge-lösten Wetterlagen für Deutschland und Bereitstellung regionaler Klimaszenarien auf der Basis mit dem Regionalisierungsmodell WETTREG auf der Basis von globalen Klimasimula-tionen mit ECHAM/MPI-OM T63L31 2010 bis 2100 für die SRES-Szenarios B1, A1B und A2; FuE-Vorhaben Förderkennzeichen 20441 138, Publikationen des Umweltbundesamtes

SpellmanN, H. (1994): Ertragskundliche Aspekte des Fremdländeranbaus. Allg. Forst- u. J.-Ztg. 165, 27-34

SpellmanN, H. (2001): Bewirtschaftung der Eiche auf der Grundlage waldwachstumskundlicher Untersuchungen in Nordwestdeutschland. Beitr. Forstwirtschaft und Landschaftsökol. 35, $145-152$

Spellmann, H. (2004): Ursachen-Wirkungs-Beziehungen am Beispiel der Douglasie, waldwachstumskundliche Entscheidungshilfen für Waldbewirtschaftung und Forstplanung. Allg. Forst- u. J.-Ztg. $175,142-150$

Spellmann, H. (2008): Die Kiefer - ein Auslaufmodell? - Beiträge für eine zielgerichtete Entwicklung. In: Nordwestdt. Forstliche Versuchsanstalt (Hrsg.) 2008: Die Waldkiefer - Fachtagung zum Baum des Jahres 2007. Beitr. Nordwestdt. Forstl. Versuchsanstalt 2, 63-78

SpellmanN, H. (2010): Nachhaltige Waldbewirtschaftung auf ökologischen Grundlagen. In: Depenhauer, O. u. Möhring, B. (Hrsg.): Waldeigentum. Bibliothek des Eigentums 8. SpringerVerlag, Berlin, Heidelberg, 99-116

Spellmann, H. (2013): Masse statt Klasse? Waldbauliche Konsequenzen aus einer veränderten Rohholznachfrage. AFZ 68, 10-15

Spellmann, H. u. SChmidT, M. (2003): Massen-, Sorten- und Wertertrag der Fichte in Abhängigkeit von der Bestandesbehandlung. Forst Holz 58, 412-419

Spellmann, H.; Albert M.; Schmidt M.; Sutmöller, J. u. Overbeck, M. (2011): Waldbauliche Anpassungsstrategien für veränderte Klimaverhältnisse. AFZ/Der Wald 11, 19-23

Spellmann, H.; SutMÖller, J. u. MeESEnburg, H. (2007): Risikovorsorge im Zeichen des Klimawandels. AFZ/Der Wald 23, 1246-1249

Spiecker, H.; MieliKäIneN, K.; KÖHL, M. u. SkovsgaArd, J. P. (1996): Growth trends in European forests.Springer Verlag Berlin, Heidelberg, New York, $372 \mathrm{~S}$.

SutMÖller J.; Ahrends, B.; Schmidt, M.; Albert, M.; Fleck, S.; Plašil, P.; Hansen, J.; Overbeck, M.; NAgel, R.-V.; SPellmann, H. u. MeESEnburg, H. (2013): Durchführung einer Untersuchung $\mathrm{zu}$ den Folgen des Klimawandels in Sachsen-Anhalt. Los 3: Forstwirtschaft. In: Klimafolgenstudie 2012 Forstwirtschaft, Berichte des Landesamtes für Umweltschutz Sachsen-Anhalt, Heft 8/2013

Beiträge aus der NW-FVA, Band 13, 2015 
Sverdrup, H.; Belyazid, S.; Nihlgard, B. u. Ericson, L. (2007): Modelling change in ground vegetation response to acid and nitrogen pollution, climate change and forest management at in Sweden 1500-2100 A.D. Water, Air and Soil Pollution: Focus 7, 163-179

ŠvestKa, M.; Hochmut, R. u. JANČAŘíK, V. (1998): Praktické metody ochrany lesa. Lesnická práce, Kostelec nad Černými lesy. $311 \mathrm{~S}$.

UlRICH, B. (1994): Nutrient and Acid-Base Budget of Central European Forest Ecosytems. In: Godbold, D. u. Hüttermann, A.: Effects of Acid Rain on Forest Processes. Wiley-Liss. New York, $1-50$

VAN WAGNER, C. E. (1987): Development and structure of the Canadian forest fire weather index. Forestry Technical Report 35, Canadian Forestry Service

VeldmanN, G. (2000): Kritische Einschätzung der Buchdrucker-Befallsentwicklung im Nationalpark Hochharz. In: Beiträge zur Waldschutzforschung in Thüringen. Mitt. Landesanstalt für Wald und Forstwirtschaft, Gotha. 17, 163-172

Veldmann, G. u. Kontzog, H.-G. (1994): Waldschutzsituation in Sachsen-Anhalt 1993/94. Der Wald, Berlin, 44 (4), 128-129

Venables, W. N. u. Ripley, B. D. (2002): Modern Applied Statistics with S. $4^{\text {th }}$ edition. Springer, New York. ISBN 0-387-95457-0

Wermelinger, B. u. SEIFERT, M. (1998): Analysis of the temperature dependent development of the spruce bark beetle Ips typographus (L.) (Col. Scolytidae). J. Appl. Entomol. 122, 185-191

WieczoreK, H.-H. (1992): Umweltschäden in der DDR: dargestellt an einer Analyse von Bekämpfungsaktionen gegen den Forstschädling Nonne (Lymantria monacha L.) in den Jahren 1980 bis 1984. Berlin: Duncker \& Humboldt, 236 S.

WiLPERT, K. V. (1990): Die Jahrringstruktur von Fichten in Abhängigkeit vom Bodenwasserhaushalt auf Pseudogley und Parabraunerde. Freiburger Bodenkundliche Abh. 24

Wood, S. N. (2006): Generalized additive models: an introduction with R. Chapman \& Hall/CRC, Boca Raton, $391 \mathrm{~S}$.

WooD, S. N. (2011): Fast stable restricted maximum likelihood and marginal likelihood estimation of semiparametric generalized linear models. J. Royal Stat. Soc. (B) 73(1), 3-36

ZEZsCHWITZ, E. V. (1985): Qualitätsänderungen des Waldhumus. Forstw. Cbl. 104, 205-220 


\section{Abkürzungsverzeichnis}

ALh Anderes Laubholz mit hoher Umtriebszeit

ALn Anderes Laubholz mit niedriger Umtriebszeit

$\mathrm{B}^{\circ} \quad$ Bestockungsgrad

Betr.-FA Betreuungs-Forstamt

BHD Brusthöhendurchmesser

BWI Bundeswaldinventur

BZE Bodenzustandserhebung

BZE II zweite Bodenzustandserhebung

DC Oberbodenfeuchte

Dg Durchmesser des Grundflächenmittelstammes

dGZmax durchschnittlicher Gesamtzuwachs zum Zeitpunkt der Kulmination

DMC Humusfeuchte

DWD Deutscher Wetterdienst

Efm Erntefestmeter

Ekl Ertragsklasse

FFMC Streufeuchtecode (Fine Fuel Moisture Code)

FWI Forest Fire Weather Index

GAM verallgemeinertes additives Modell (generalized additive model)

GAMM verallgemeinertes additives gemischtes Modell (generalized additive mixed model)

GLM generalisiertes lineares Modell

GWS Grundwasserstufen

ha Hektar

$\mathrm{Hg} \quad$ Mittelhöhe

hg100 Mittelhöhenbonitäten im Alter 100

HNN Höhe ü. NN

KA Kartieranleitung

KWB Klimatische Wasserbilanz

LFB Landesforstbetrieb

LKL Leistungsklasse

LZW Landeszentrum Wald

Beiträge aus der NW-FVA, Band 13, 2015 
M68 Waldbrandindex nach KäsE (1969)

MLU LSA Ministerium für Landwirtschaft und Umwelt des Landes Sachsen-Anhalt

MNGW mittlerer Grundwassertiefstand

Ndep Stickstoffdeposition

nFK nutzbare Feldkapazität

$\mathrm{pF} \quad$ Saugspannung

Rev. $\quad$ Revier

Tempsum Temperatursumme

VBK 50 Vorläufige Bodenkarte von Sachsen-Anhalt im Maßstab 1:50.000

VZ Vegetationszeit

WBKZ Waldbrandkennziffer 


\section{Danksagung}

Die Studie wurde durch die umfangreiche Bereitstellung von Datenmaterial aus verschiedenen Quellen erst möglich gemacht. Insbesondere bedanken wir uns bei Frau Paul, Landesforstbetrieb Sachsen Anhalt, Halberstadt für die Bereitstellung verschiedener Datenquellen. Des Weiteren möchten wir uns bei den Forstamtsleitern und Revierförstern aus den in dieser Studie untersuchten Gebieten, die uns die Waldschutzunterlagen zur Verfügung gestellt haben, bedanken. 



\section{Autoren}

\section{Dr. Stefan Fleck}

Nordwestdeutsche Forstliche

Versuchsanstalt (NW-FVA)

Abt. Umweltkontrolle

Grätzelstraße 2

D-37079 Göttingen

stefan.fleck@nw-fva.de

Dr. Pavel Plašil

NW-FVA, Abt. Waldschutz

Grätzelstraße 2

D-37079 Göttingen

pavel.plasil@nw-fva.de

Johannes Sutmöller

NW-FVA, Abt. Umweltkontrolle

Grätzelstraße 2

D-37079 Göttingen

johannes.sutmöller@nw-fva.de

\section{Dr. Matthias Schmidt}

NW-FVA, Abt. Waldwachstum

Grätzelstraße 2

D-37079 Göttingen

matthias.schmidt@nw-fva.de

\section{Dr. Jan Hansen}

NW-FVA, Abt. Waldwachstum

Grätzelstraße 2

D-37079 Göttingen

jan.hansen@nw-fva.de

\section{Dr. Matthias Albert}

Nordwestdeutsche Forstliche

Versuchsanstalt (NW-FVA)

Abt. Waldwachstum

Grätzelstraße 2

D-37079 Göttingen

matthias.albert@nw-fva.de

\section{Ralf Nagel}

NW-FVA, Abt. Waldwachstum

Grätzelstraße 2

D-37079 Göttingen

ralf.nagel@nw-fva.de

\section{Dr. Bernd Ahrends}

NW-FVA, Abt. Umweltkontrolle

Grätzelstraße 2

D-37079 Göttingen

bernd.ahrends@nw-fva.de

\section{Dr. Jan Evers}

NW-FVA, Abt. Umweltkontrolle

Grätzelstraße 2

D-37079 Göttingen

jan.evers@nw-fva.de

\section{Dr. Marc Overbeck}

Ehem. NW-FVA,

Abt. Waldwachstum

marc.overbeck@nlf.niedersachsen.de 


\section{Wolfgang Schmidt}

Ehem. NW-FVA,

Abt. Umweltkontrolle

Krausenstrasse 17

D-08523 Plauen

\section{Prof. Dr. Hermann Spellmann}

NW-FVA, Abt. Waldwachstum

Grätzelstraße 2

D-37079 Göttingen

hermann.spellmann@nw-fva.de

\section{Dr. Henning Meesenburg}

NW-FVA, Abt. Umweltkontrolle

Grätzelstraße 2

D-37079 Göttingen

henning.meesenburg@nw-fva.de 


\section{Anhang}

Anhang 1: $\quad$ Trockenstressrisiko für die Kiefer in der Region Oberharz 210

Anhang 2: $\quad$ Trockenstressrisiko für die Fichte in der Region Oberharz 211

Anhang 3: $\quad$ Trockenstressrisiko für die Buche in der Region Oberharz 212

Anhang 4: $\quad$ Trockenstressrisiko für die Eiche und Douglasie in der Region Oberharz 213

Anhang 5: $\quad$ Trockenstressrisiko für die Kiefer in der Region Fläming 214

Anhang 6: $\quad$ Trockenstressrisiko für die Fichte in der Region Fläming 215

Anhang 7: $\quad$ Trockenstressrisiko für die Buche in der Region Fläming 216

Anhang 8: $\quad$ Trockenstressrisiko für die Eiche und Douglasie in der Region Fläming

Anhang 9: $\quad$ Trockenstressrisiko für die Kiefer in der Region Colbitz-Letzlinger Heide

Anhang 10: $\quad$ Trockenstressrisiko für die Fichte in der Region Colbitz-Letzlinger Heide

Anhang 11: $\quad$ Trockenstressrisiko für die Buche in der Region Colbitz-Letzlinger Heide

Anhang 12: $\quad$ Trockenstressrisiko für die Eiche und Douglasie in der Region Colbitz-Letzlinger Heide 


\section{Kiefer}
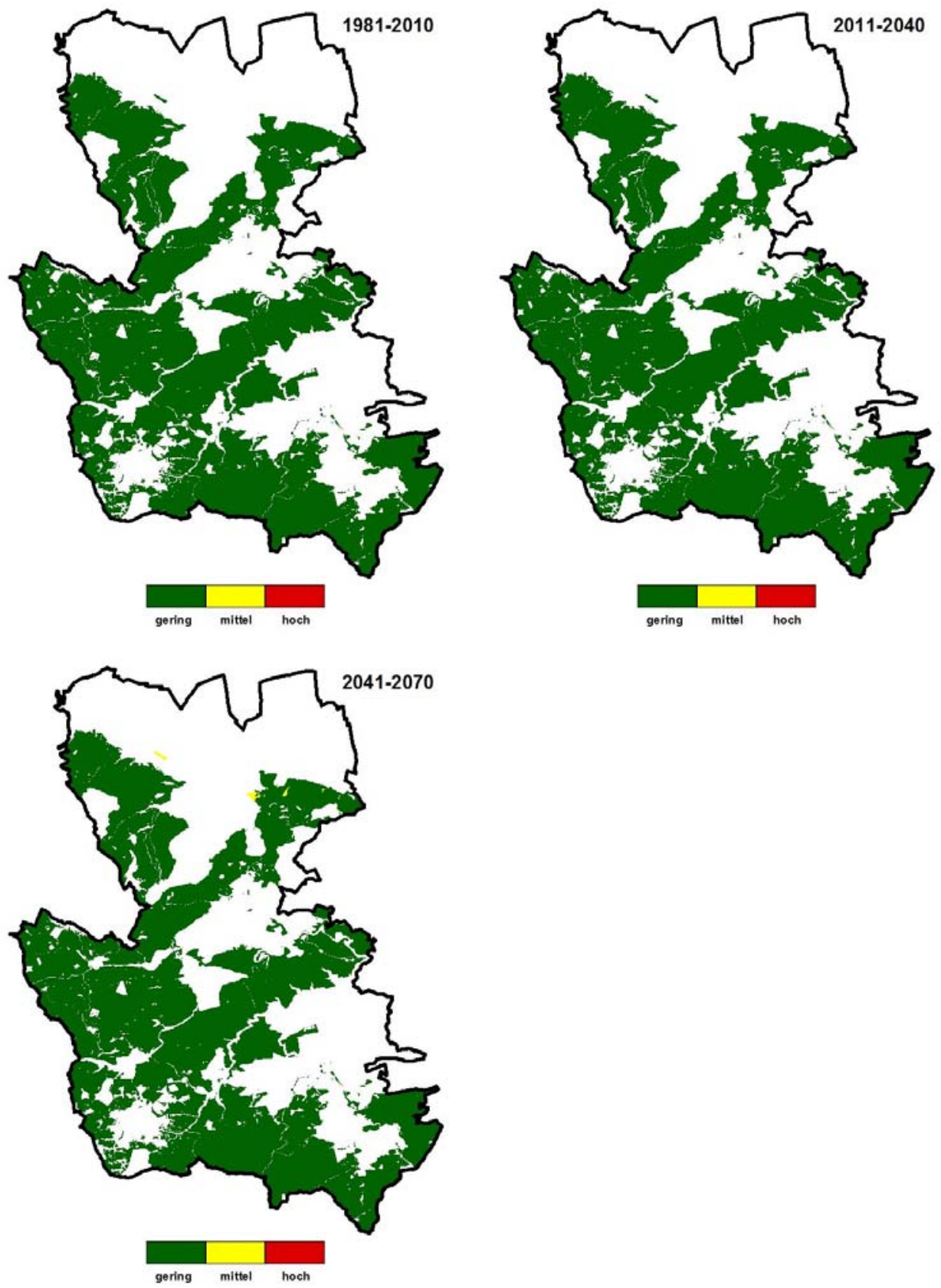

Anhang 1: $\quad$ Trockenstressrisiko für die Kiefer in der Region Oberharz. 
Fichte
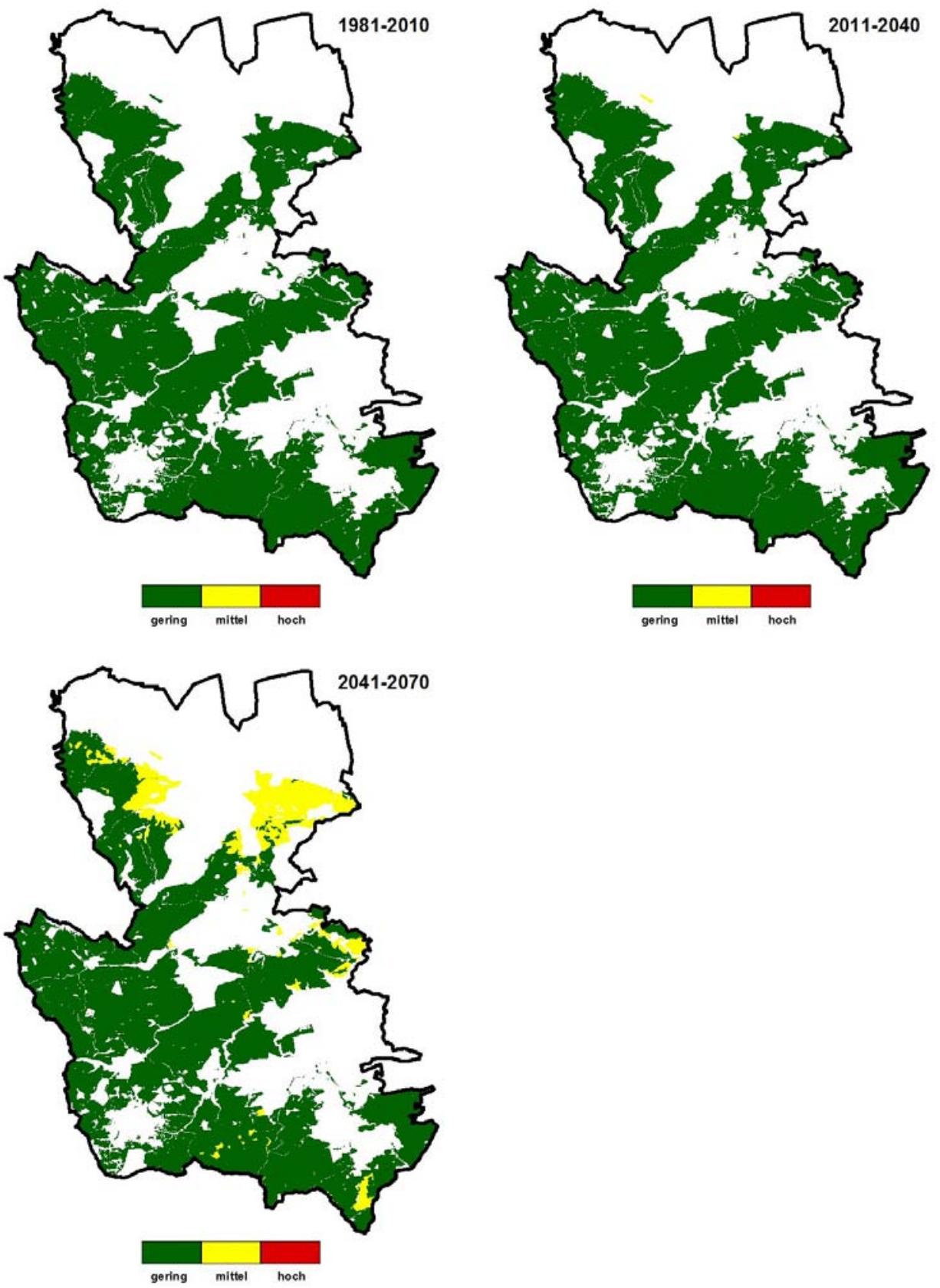

Anhang 2: $\quad$ Trockenstressrisiko für die Fichte in der Region Oberharz. 
Buche
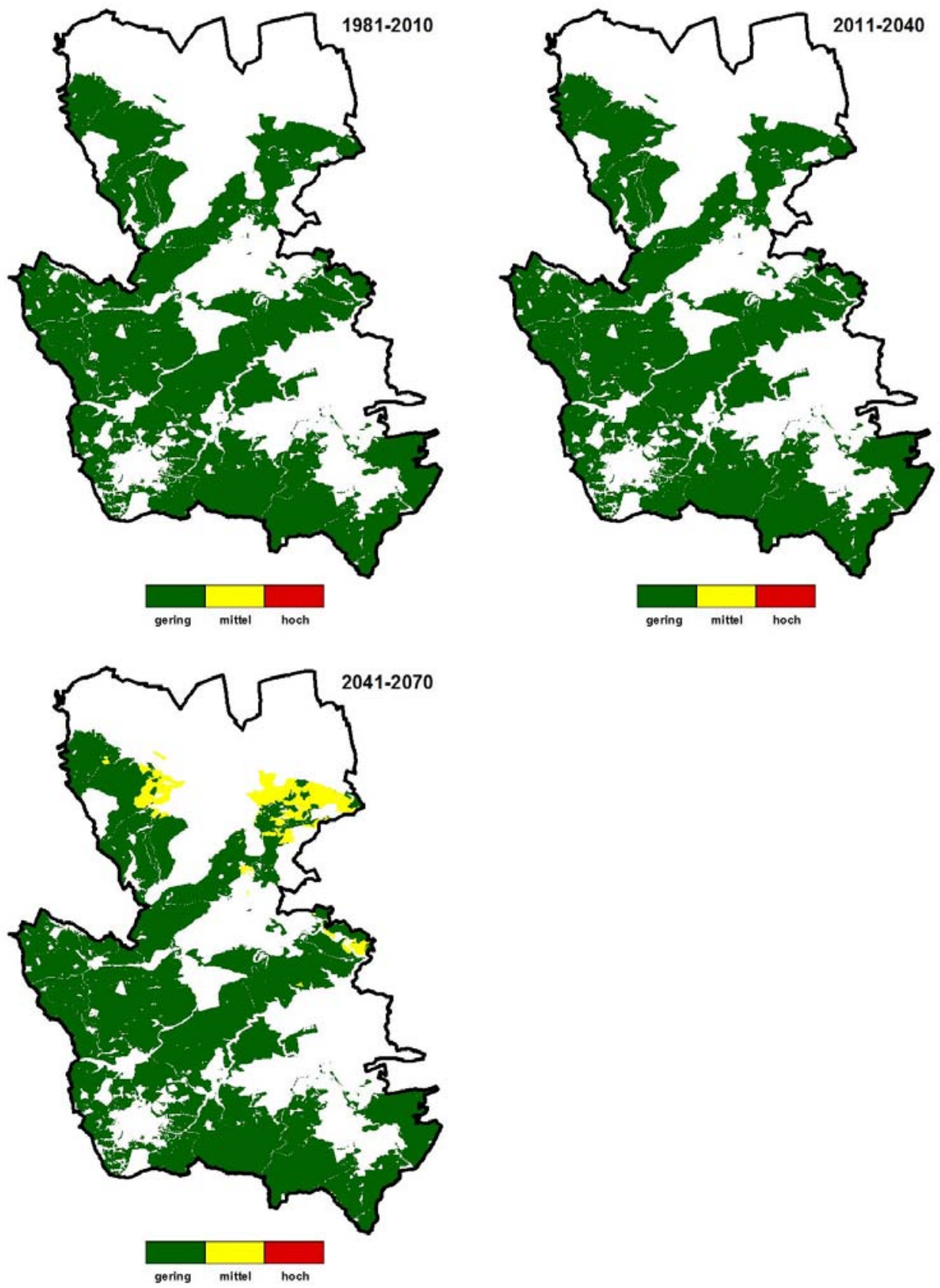

Anhang 3: $\quad$ Trockenstressrisiko für die Buche in der Region Oberharz. 
Eiche und Douglasie
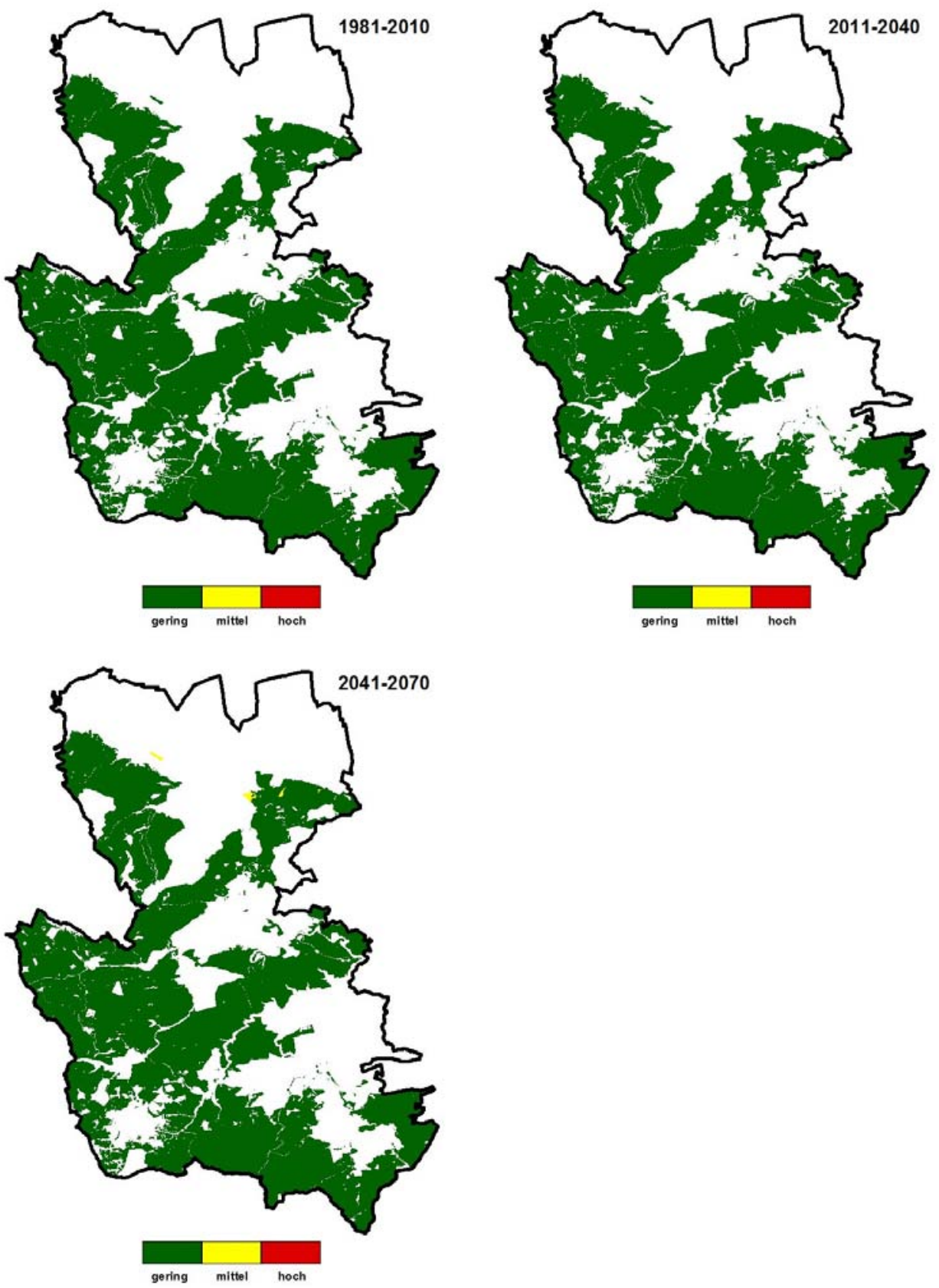

Anhang 4: $\quad$ Trockenstressrisiko für die Eiche und Douglasie in der Region Oberharz. 


\section{Kiefer}
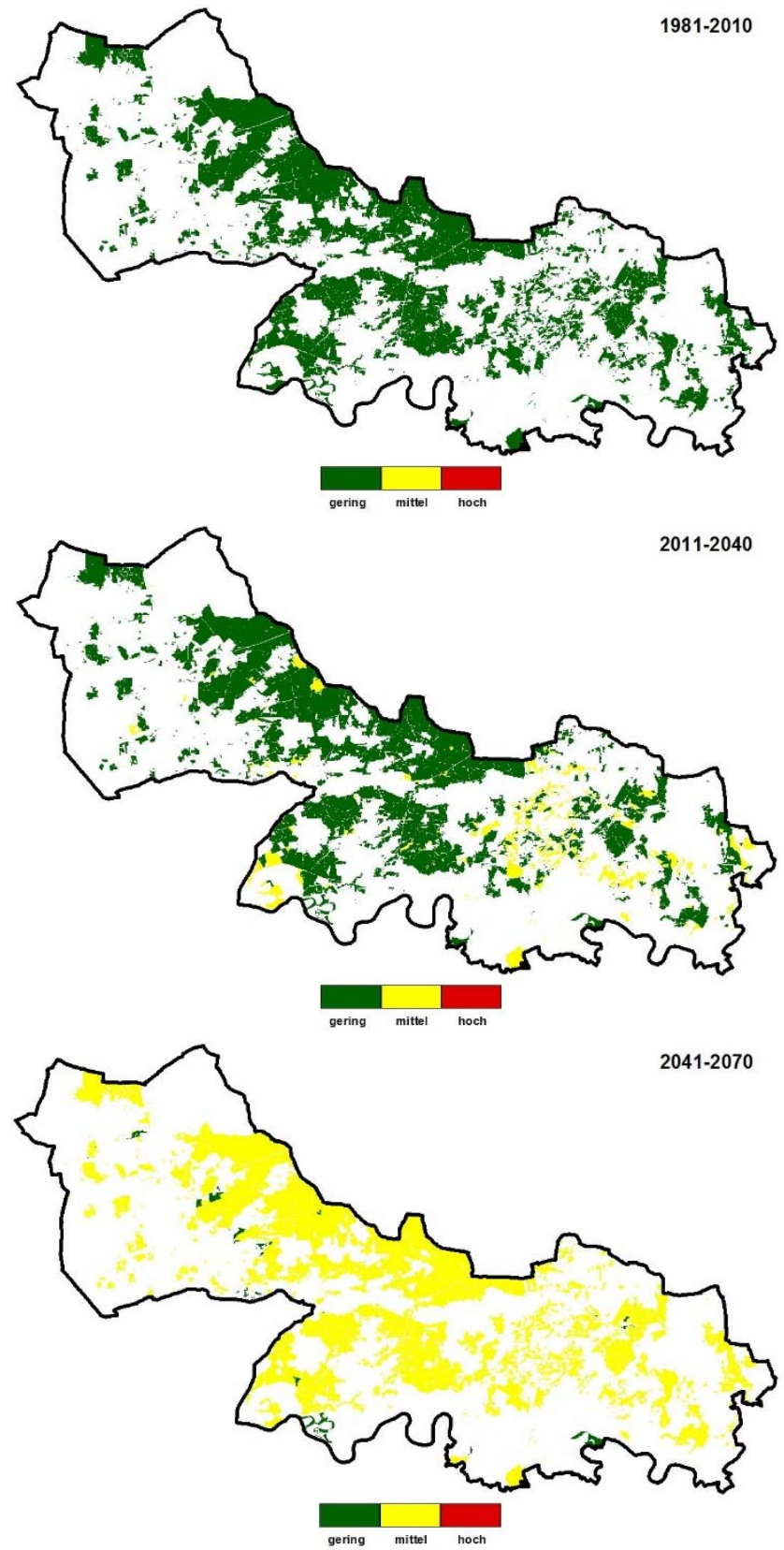

Anhang 5: $\quad$ Trockenstressrisiko für die Kiefer in der Region Fläming 
Fichte
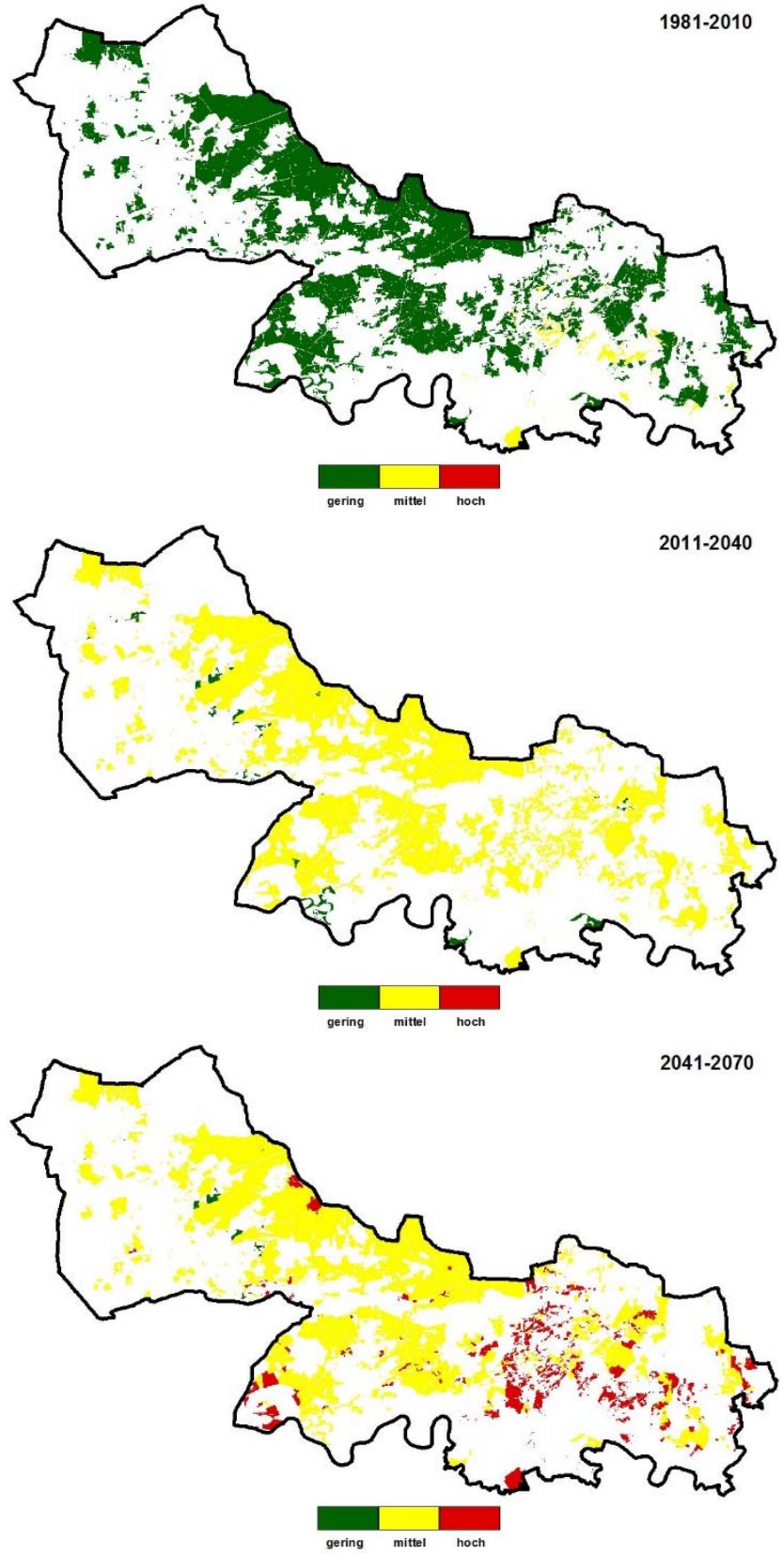

Anhang 6: $\quad$ Trockenstressrisiko für die Fichte in der Region Fläming 


\section{Buche}
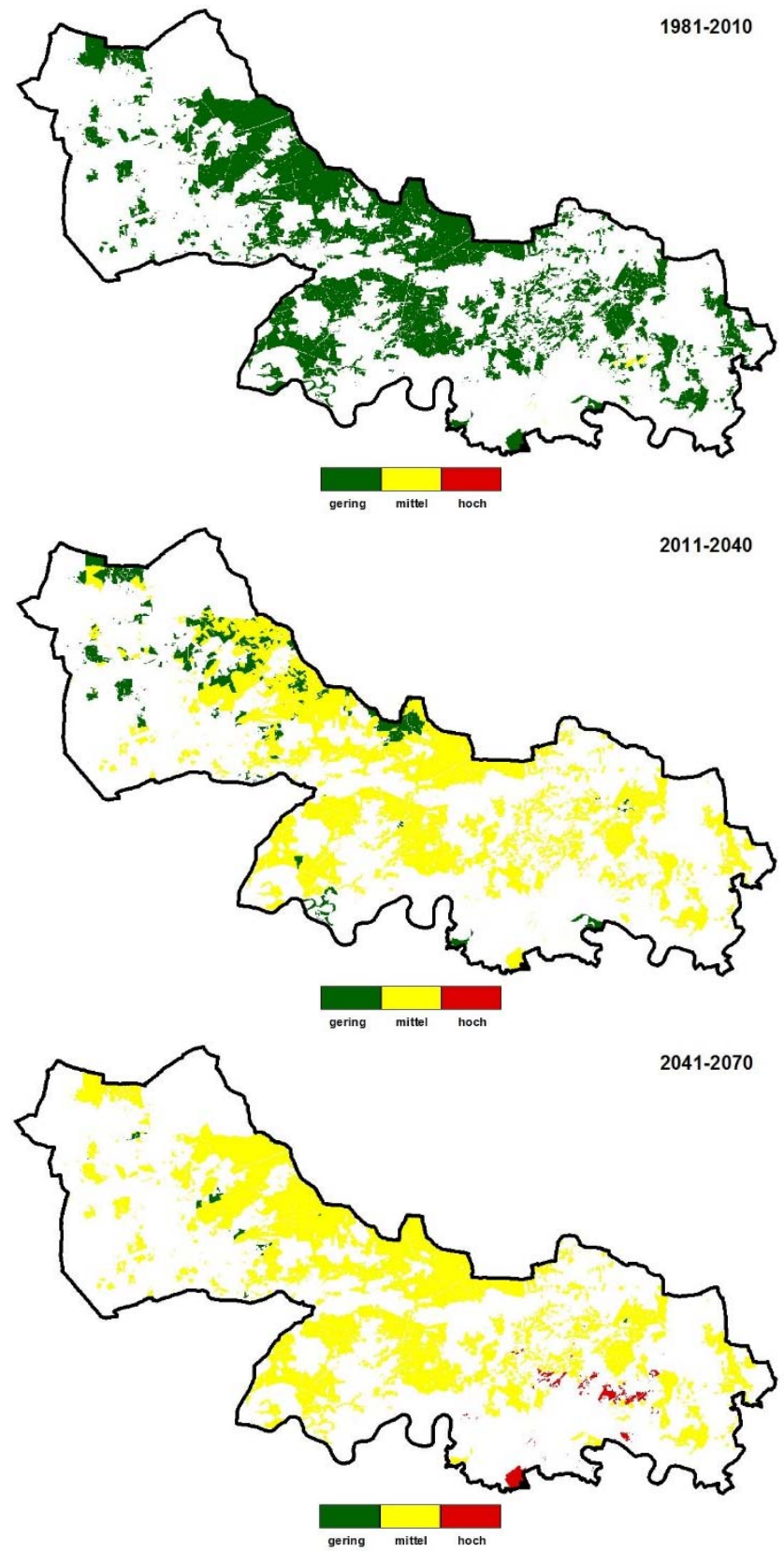

Anhang 7: Trockenstressrisiko für die Buche in der Region Fläming 
Eiche und Douglasie
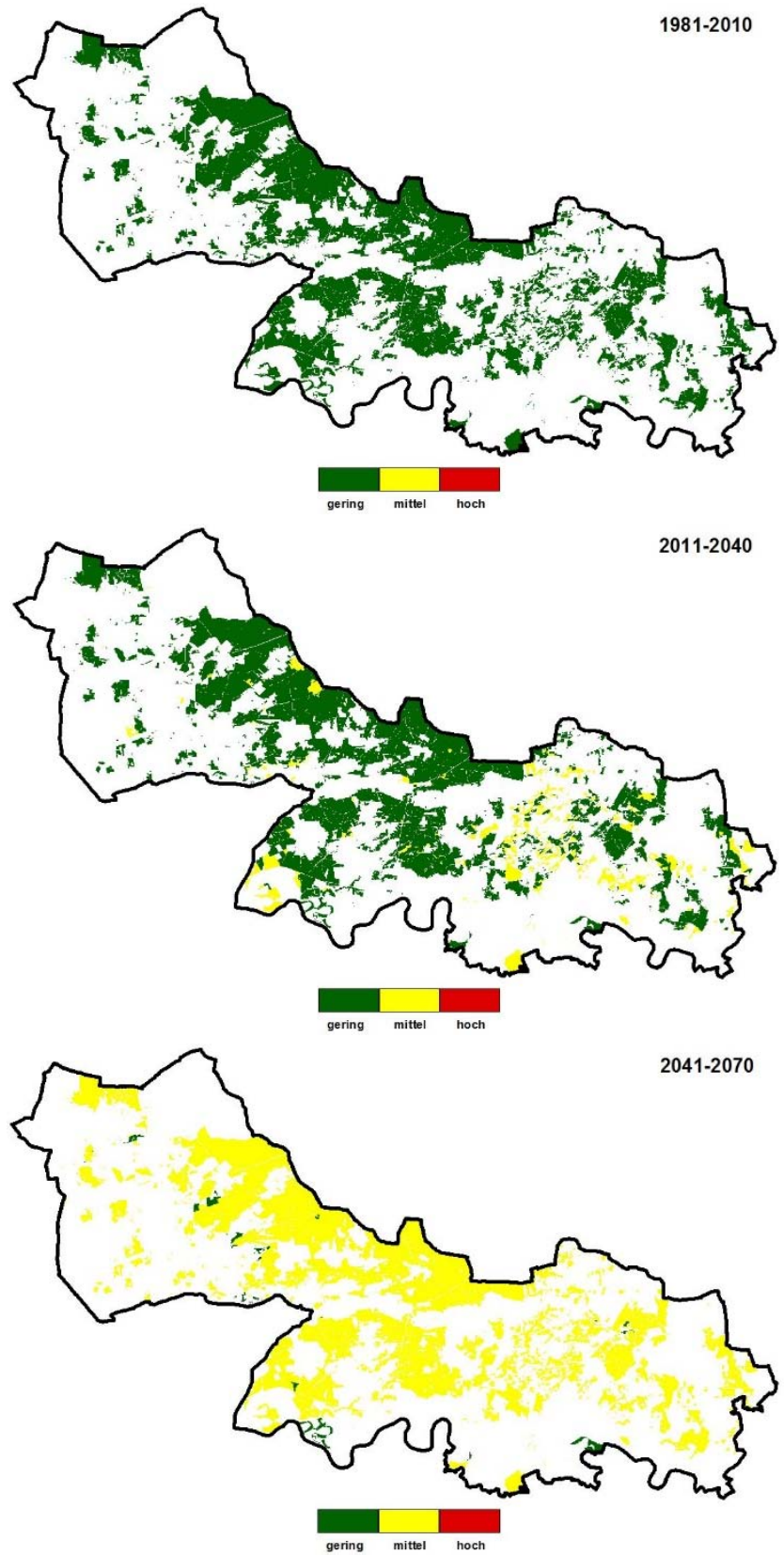

Anhang 8: Trockenstressrisiko für die Eiche und Douglasie in der Region Fläming 


\section{Kiefer}
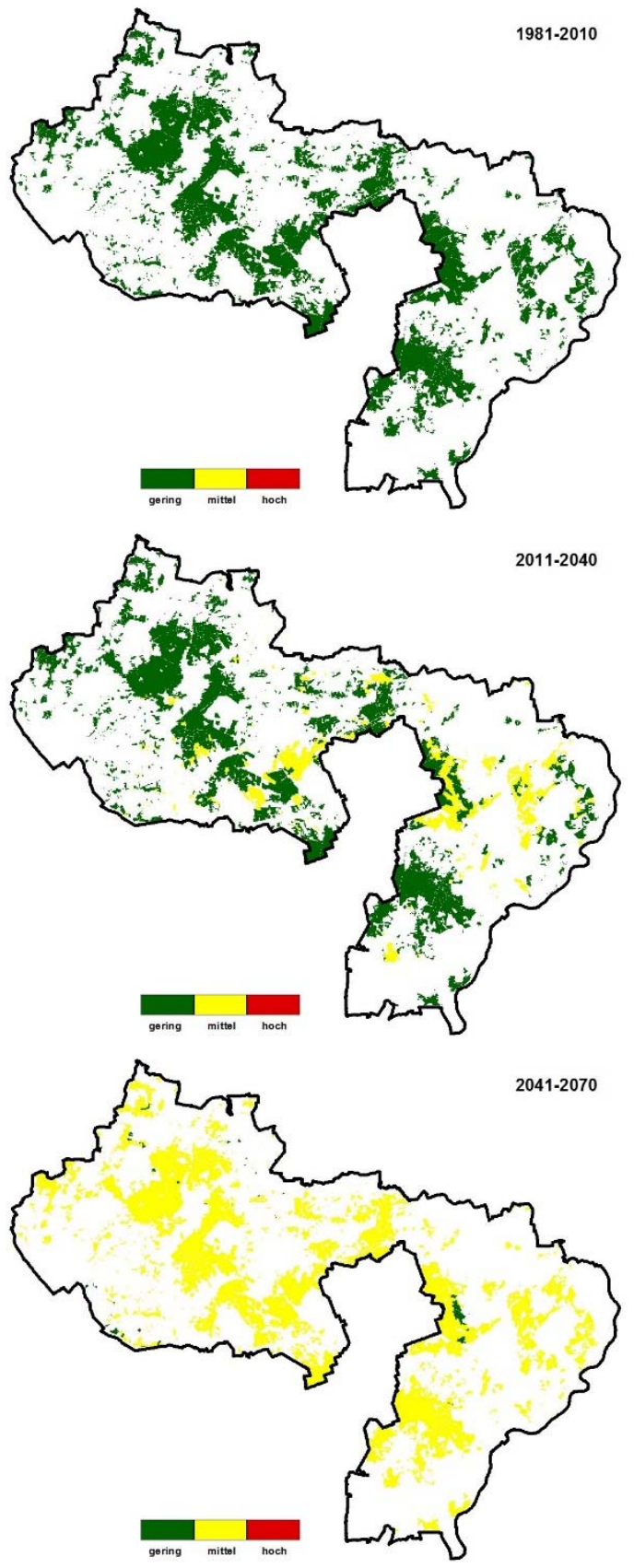

Anhang 9: $\quad$ Trockenstressrisiko für die Kiefer in der Region Colbitz-Letzlinger Heide 
Fichte
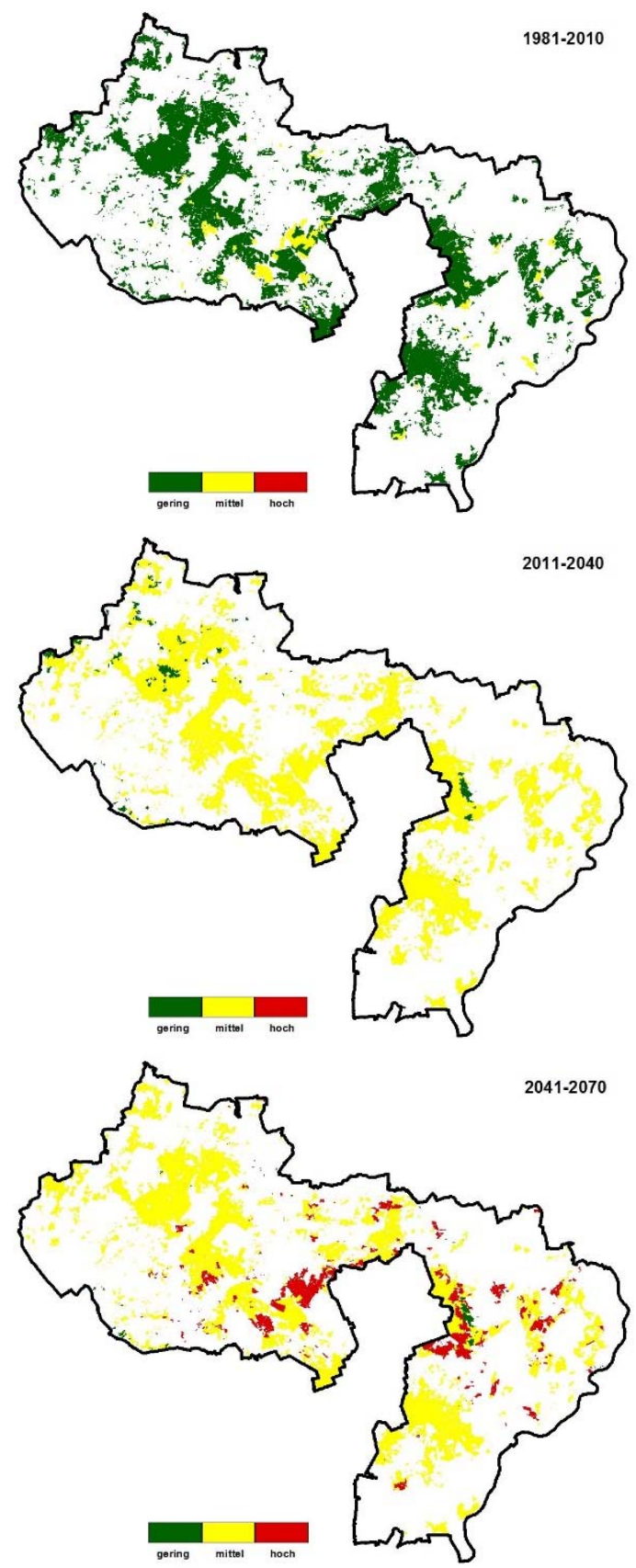

Anhang 10: Trockenstressrisiko für die Fichte in der Region Colbitz-Letzlinger Heide 


\section{Buche}
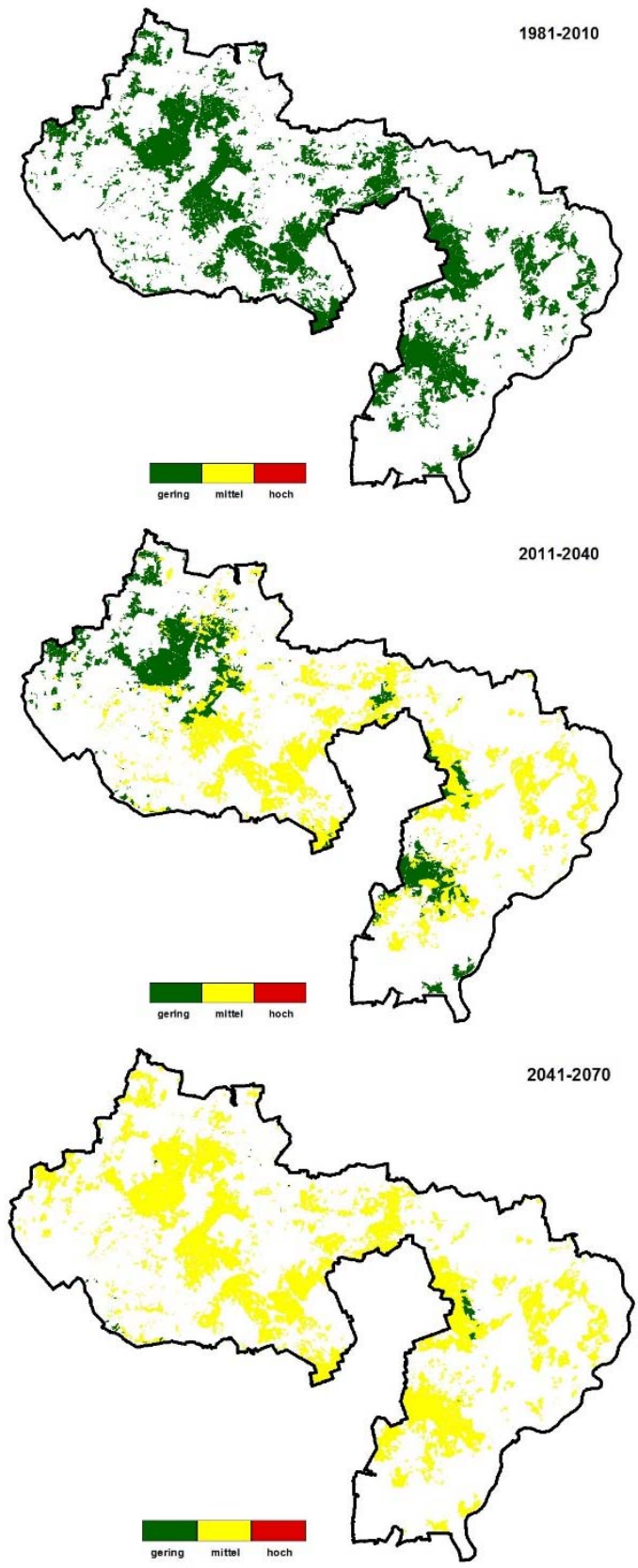

Anhang 11: Trockenstressrisiko für die Buche in der Region Colbitz-Letzlinger Heide 
Eiche und Douglasie
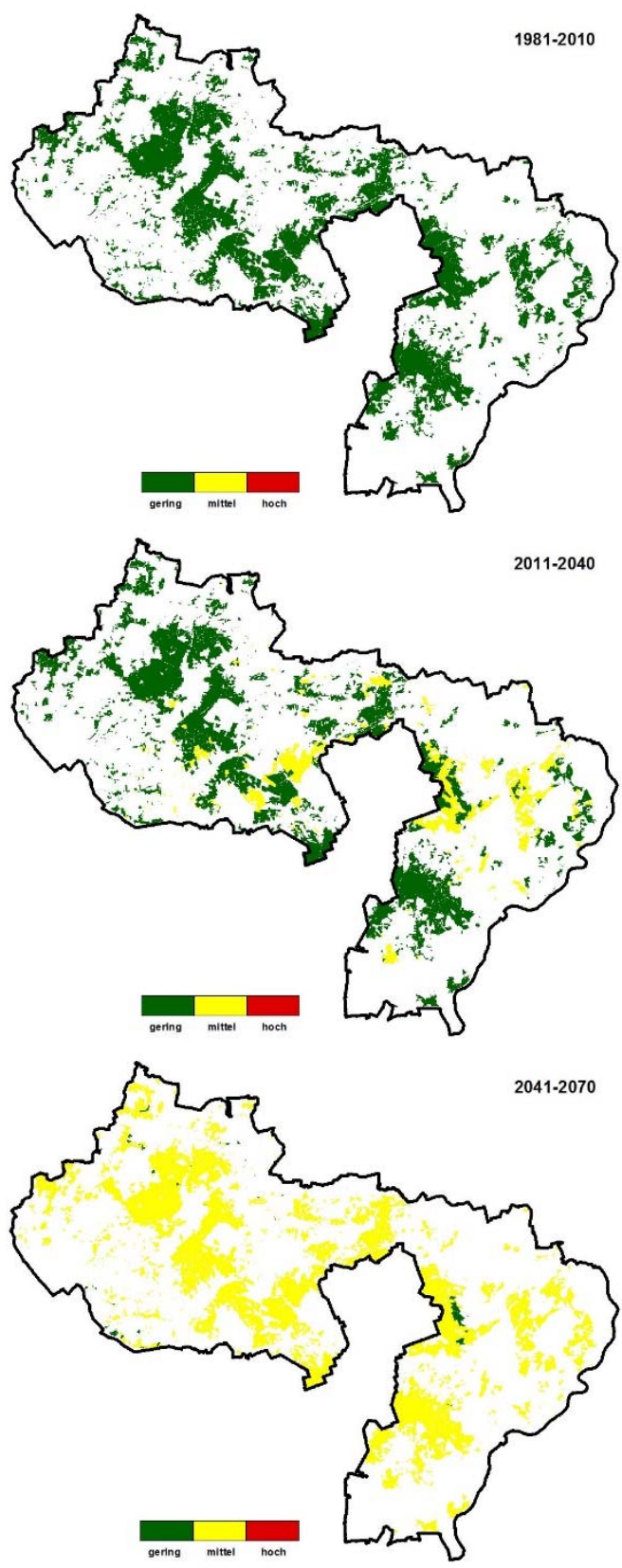

Anhang 12: Trockenstressrisiko für die Eiche und Douglasie in der Region Colbitz-Letzlinger Heide 



\section{Beiträge aus der Nordwestdeutschen Forstlichen Versuchsanstalt}

Band

1 (2007) Clusterstudie Forst und Holz Niedersachsen. Burkhard Rüther, Jan Hansen, Agatha Ludwig, Hermann Spellmann, Jürgen Nagel, Bernhard Möhring, Matthias Dieter. 92 S.

2 (2008) Die Waldkiefer - Fachtagung zum Baum des Jahres 2007. Nordwestdeutsche Forstliche Versuchsanstalt (Hrsg.). 98 S.

3 (2008) Ergebnisse angewandter Forschung zur Buche. Nordwestdeutsche Forstliche Versuchsanstalt (Hrsg.). 343 S.

4 (2008) Ergebnisse des westdeutschen IUFRO-Küstentannen-Provenienzversuches im Alter 27. Hans-Martin Rau, Armin König, Wolfhard Ruetz, Hendrik Rumpf, Egbert Schönfelder. $62 \mathrm{~S}$.

5 (2010) Fichtenherkunftsversuch von 1962 und IUFRO-Fichtenherkunftsversuch von 1972. Ergebnisse von mehr als 30jähriger Beobachtung in Deutschland. Mirko Liesebach, Hans-Martin Rau, Armin O. König. 467 S.

(2011) Kohlenstoffstudie Forst und Holz Niedersachsen. René Wördehoff, Hermann Spellmann, Jan Evers, Jürgen Nagel. 92 S.

7 (2012) Das digitale Luftbild. Ein Praxisleitfaden für Anwender im Forst- und Umweltbereich. Arbeitsgruppe Forstlicher Luftbildinterpreten (Hrsg.). $84 \mathrm{~S}$.

8 (2012) Züchtung und Ertragsleistung schnellwachsender Baumarten im Kurzumtrieb. Erkenntnisse aus drei Jahren FastWood, ProLoc und Weidenzüchtung. Fachtagung vom 21. bis 22.09.2011 in Hann. Münden. Nordwestdeutsche Forstliche Versuchsanstalt (Hrsg.). $430 \mathrm{~S}$.

9 (2012) SILVAQUA - Auswirkungen forstlicher Bewirtschaftsungsmaßnahmen auf den Zustand von Gewässern in bewaldeten Einzugsgebieten am Beispiel der Oker im Nordharz. Nordwestdeutsche Forstliche Versuchsanstalt (Hrsg.). 226 S.

10 (2013) Waldentwicklungsszenarien für das Hessische Ried. Entscheidungsunterstützung vor dem Hintergrund sich beschleunigt ändernder Wasserhaushalts- und Klimabedingungen und den Anforderungen aus dem europäischen Schutzgebietssystem Natura 2000. Nordwestdeutsche Forstliche Versuchsanstalt (Hrsg.). 397 S. 
Band

11 (2014) Waldwachstumskundliche Softwaresysteme auf Basis von TreeGrOSS - Anwendung und theoretische Grundlagen. Jan Hansen, Jürgen Nagel. 224 S.

12 (2015) Synopse der Hauptmerkmale der forstlichen Standortskartierungsverfahren der Nordwestdeutschen Bundesländer. Wolfgang Schmidt, Volker Stüber, Thomas Ullrich, Uwe Paar, Jan Evers, Klaus Dammann, Thomas Hövelmann, Marcus Schmidt. 136 S.

13 (2015) Pilotstudie zu den lokalen Auswirkungen des Klimawandels auf die Forstwirtschaft in ausgewählten Regionen SachsenAnhalts. Stefan Fleck, Matthias Albert, Pavel Plašil, Ralf Nagel, Johannes Sutmöller, Bernd Ahrends, Matthias Schmidt, Jan Evers, Jan Hansen, Marc Overbeck, Wolfgang Schmidt, Hermann Spellmann, Henning Meesenburg. $221 \mathrm{~S}$.

Alle Bände der „Beiträge der NW-FVA“ sind auch als freie Onlineversion über die Homepage der NW-FVA (www.nw-fva.de), des Verlags sowie über den Göttinger Universitätskatalog (GUK) bei der Niedersächsischen Staats- und Universitätsbibliothek Göttingen (http://www.sub.uni-goettingen.de) erreichbar.

Es gelten die Lizenzbestimmungen der Onlineversion. 
Sämtliche Klimaprojektionen lassen für Sachsen-Anhalt einen deutlichen Temperaturanstieg bei gleichzeitig veränderter saisonaler Niederschlagsverteilung erwarten. Von den Folgen des Klimawandels wird insbesondere die langfristig handelnde Forstwirtschaft betroffen sein, da sich die Produktionsgrundlagen, die Risiken und die Ertragsaussichten wesentlich verändern. In der vorliegenden Pilotstudie sind in ausgewählten Regionen Sachsen-Anhalts auf der Basis räumlich hoch aufgelöster Klima-, Standort-, Bestandes- und Risikoinformationen Entscheidungshilfen für das operationale Handeln von Forstbetrieben entwickelt worden. Als Modellregionen dienen der Oberharz, der Fläming und die Colbitz-Letzlinger Heide.

ISBN: 978-3-86395-239-6

ISSN: 1865-6994 